\title{
Nutrition and Celiac Disease
}

Edited by
Carlo Catassi and Alessio Fasano

Printed Edition of the Special Issue Published in Nutrients 
Carlo Catassi and Alessio Fasano (Eds.)

Nutrition and Celiac Disease 
This book is a reprint of the special issue that appeared in the online open access journal Nutrients (ISSN 2072-6643) in 2013 (http://www.mdpi.com/journal/nutrients/special_issues/celiac_disease).

\section{Guest Editors}

Professor Carlo Catassi

Department of Pediatrics, Università Politecnica delle Marche

Ancona, Italy

Professor Alessio Fasano

Mucosal Immunology and Biology Research Center, Massachusetts General Hospital East

Charlestown, MA, USA

Editorial Office

MDPI AG

Klybeckstrasse 64

Basel, Switzerland

Publisher

Shu-Kun Lin

Production Editor

Martyn Rittman

\section{Edition 2014}

MDPI • Basel, Switzerland

ISBN 978-3-906980-64-5

(C) 2014 by the authors; licensee MDPI, Basel, Switzerland. All articles in this volume are Open Access distributed under the Creative Commons Attribution 3.0 license (http://creativecommons.org/licenses/by/3.0/), which allows users to download, copy and build upon published articles even for commercial purposes, as long as the author and publisher are properly credited, which ensures maximum dissemination and a wider impact of our publications. However, the dissemination and distribution of copies of this book as a whole is restricted to MDPI, Basel, Switzerland. 


\section{Table of Contents}

Preface VII

\section{General Review}

Carlo Catassi, Julio C. Bai, Bruno Bonaz, Gerd Bouma, Antonio Calabrò, Antonio Carroccio, Gemma Castillejo, Carolina Ciacci, Fernanda Cristofori, Jernej Dolinsek, Ruggiero Francavilla, Luca Elli, Peter Green, Wolfgang Holtmeier, Peter Koehler, Sibylle Koletzko, Christof Meinhold, David Sanders, Michael Schumann, Detlef Schuppan, Reiner Ullrich, Andreas Vécsei, Umberto Volta, Victor Zevallos, Anna Sapone and Alessio Fasano Non-Celiac Gluten Sensitivity: The New Frontier of Gluten Related Disorders Reprinted from Nutrients 2013, 5, 3839-3853 http://www.mdpi.com/2072-6643/5/10/3839

\section{Maaike J. Bruins}

The Clinical Response to Gluten Challenge: A Review of the Literature

Reprinted from Nutrients 2013, 5, 4614-4641 16

http://www.mdpi.com/2072-6643/5/11/4614

\section{Pathophysiology}

Henrik Toft-Hansen, Christian Nielsen, Matteo Biagini, Steffen Husby and Søren T. Lillevang Lectin Staining Shows no Evidence of Involvement of Glycocalyx/Mucous Layer Carbohydrate Structures in Development of Celiac Disease

Reprinted from Nutrients 2013, 5(11), 4540-4552 
Juan P. Ortiz-Sánchez, Francisco Cabrera-Chávez and Ana M. Calderón de la Barca

Maize Prolamins Could Induce a Gluten-Like Cellular Immune Response in Some Celiac Disease Patients

Reprinted from Nutrients 2013, 5(10), 4174-4183

http://www.mdpi.com/2072-6643/5/10/4174

\section{Clinical issues}

Michele Di Stefano, Caterina Mengoli, Manuela Bergonzi and Gino Roberto Corazza

Bone Mass and Mineral Metabolism Alterations in Adult Celiac Disease: Pathophysiology and

Clinical Approach

Reprinted from Nutrients 2013, 5(11), 4786-4799

http://www.mdpi.com/2072-6643/5/11/4786

Rinaldo Pellicano, Claudio De Angelis, Davide Giuseppe Ribaldone, Sharmila Fagoonee and Marco Astegiano

2013 Update on Celiac Disease and Eosinophilic Esophagitis

Reprinted from Nutrients 2013, 5(9), 3329-3336

http://www.mdpi.com/2072-6643/5/9/3329

Antonella Diamanti, Teresa Capriati, Maria Sole Basso, Fabio Panetta, Vincenzo Maria Di Ciommo Laurora, Francesca Bellucci, Fernanda Cristofori and Ruggiero Francavilla

Celiac Disease and Overweight in Children: An Update

Reprinted from Nutrients 2014, 6(1), 207-220

http://www.mdpi.com/2072-6643/6/1/207

Nicolette J. Wierdsma, Marian A. E. van Bokhorst-de van der Schueren, Marijke Berkenpas, Chris J. J. Mulder and Ad A. van Bodegraven

Vitamin and Mineral Deficiencies Are Highly Prevalent in Newly Diagnosed Celiac Disease Patients

Reprinted from Nutrients 2013, 5(10), 3975-3992 103

http://www.mdpi.com/2072-6643/5/10/3975 


\section{Psycho-Social Aspects}

Carolina Ciacci, Monica Siniscalchi, Cristina Bucci, Fabiana Zingone, Ivonne Morra and Paola Iovino

Life Events and the Onset of Celiac Disease from a Patient's Perspective

Reprinted from Nutrients 2013, 5(9), 3388-3398 120

http://www.mdpi.com/2072-6643/5/9/3388

\section{Chiara Biagetti, Giulia Naspi and Carlo Catassi}

Health-Related Quality of Life in Children with Celiac Disease: A Study Based on the Critical Incident Technique

Reprinted from Nutrients 2013, 5(11), 4476-4485

http://www.mdpi.com/2072-6643/5/11/4476

\section{Preeti Rajpoot and Govind K. Makharia}

Problems and Challenges to Adaptation of Gluten Free Diet by Indian Patients with Celiac Disease

Reprinted from Nutrients 2013, 5(12), 4869-4879

http://www.mdpi.com/2072-6643/5/12/4869

Alexander Ross, Helen Shelley, Kim Novell, Elizabeth Ingham, Julia Callan, Robert Heuschkel, Mary-Anne Morris and Matthias Zilbauer

Assessing Quality Outcome Measures in Children with Coeliac Disease-Experience from Two UK Centres

Reprinted from Nutrients 2013, 5(11), 4605-4613

http://www.mdpi.com/2072-6643/5/11/4605

\section{Gluten-Free Diet and Human health}

SriHari Mahadev, Suzanne Simpson, Benjamin Lebwohl, Suzanne K. Lewis, Christina A. Tennyson and Peter H. R. Green

Is Dietitian Use Associated with Celiac Disease Outcomes?

Reprinted from Nutrients 2013, 5(5), 1585-1594 161

http://www.mdpi.com/2072-6643/5/5/1585 
Isabel Comino, María de Lourdes Moreno, Ana Real, Alfonso Rodríguez-Herrera, Francisco Barro and Carolina Sousa

The Gluten-Free Diet: Testing Alternative Cereals Tolerated by Celiac Patients 14

Reprinted from Nutrients 2013, 5(10), 4250-4268

http://www.mdpi.com/2072-6643/5/10/4250

Urszula Krupa-Kozak, Natalia Bączek and Cristina M. Rosell

Application of Dairy Proteins as Technological and Nutritional Improvers of Calcium-

Supplemented Gluten-Free Bread

Reprinted from Nutrients 2013, 5(11), 4503-4520

http://www.mdpi.com/2072-6643/5/11/4503

Francesca Penagini, Dario Dilillo, Fabio Meneghin, Chiara Mameli, Valentina Fabiano and

Gian Vincenzo Zuccotti

Gluten-Free Diet in Children: An Approach to a Nutritionally Adequate and

Balanced Diet

Reprinted from Nutrients 2013, 5(11), 4553-4565 208

http://www.mdpi.com/2072-6643/5/11/4553

Katri Kaukinen, Pekka Collin, Heini Huhtala and Markku Mäki

Long-Term Consumption of Oats in Adult Celiac Disease Patients 17

Reprinted from Nutrients 2013, 5(11), 4380-4389

http://www.mdpi.com/2072-6643/5/11/4380

Simona Gatti, Nicole Caporelli, Tiziana Galeazzi, Ruggiero Francavilla, Maria Barbato, Paola Roggero, Basilio Malamisura, Giuseppe Iacono, Andrea Budelli, Rosaria Gesuita, Carlo Catassi and Elena Lionetti

Oats in the Diet of Children with Celiac Disease: Preliminary Results of a Double-Blind, Randomized, Placebo-Controlled Multicenter Italian Study

Reprinted from Nutrients 2013, 5(11), 4653-4664 231

http://www.mdpi.com/2072-6643/5/11/4653 


\section{Preface}

During its 2.5 million years of evolution, the human species has evolved through major dramatic changes, mainly dictated by natural elements and, most importantly, by food availability. The diet of hunters and gatherers, hominids, was mainly based on fruit, vegetables, tubers, and occasionally meat and fish. Then, approximately 10,000 years ago, a drastic change in life style occurred, shifting from nomadic to settlers with domestication of animals and crops. A consequence of this change was the advent of wheat and other grains containing gluten-related proteins in human diet. This revolutionary transformation occurred at the Fertile Crescent, the modern-day Iraq, and spread from South to North and East to West at a speed of approximately 1 $\mathrm{km} /$ year. Ever since, the distribution of food goods became more and more uneven with wealthy countries getting more than necessary, while poor countries struggle with malnutrition and consequently this increased mortality. Unfortunately, the industrial revolution, rather than closing the gap, created even more inequalities that still exist today, leading to very different but equally worrisome pathologies, namely obesity in industrialized countries and famine in developing countries.

Beside nutritional problems secondary to quantitative imbalance, there are also nutritional pathologies triggered by specific dietary elements. Celiac disease (CD) is an autoimmune disorder occurring in genetically predisposed individuals, and triggered by the ingestion of dietary gluten, the major protein component in wheat, barley and rye. In many areas of the world, $\mathrm{CD}$ is one of the most common lifelong disorders affecting approximately $1 \%$ of the general population. Gluten is the typical example of a "friend" (food) becoming a foe (because it can cause an array of signs and symptoms in predisposed individuals). Gluten is a complex and ancient protein that represents the main component of wheat with a unique primary structure rich in proline and glutamine amino acid residues. For this reason, gluten is extremely difficult to digest. With more than 150,000 genes, wheat represents an extremely complicated component that evolved over the millennia to reach its present genetic and structural characteristics. The history of humans and the evolution of gluten related disorders are intertwined with the evolution of wheat and gluten: how they developed, how they continue to evolve, and how they affect humankind today around the world in a variety of gluten-related symptoms and disorders.

Almost 2,000 years ago, Aretaeus from Cappadocia reported what is believed to be the first description of a case of CD. It would take approximately another 1,800 years before CD resurfaced in medical literature. This time it was a British doctor, Samuel Gee, who officially placed celiac disease on the medical map in 1861 when he gave a famous lecture in London that provided the first modern description of $\mathrm{CD}$ :

"There is a kind of chronic indigestion which is met with in persons of all ages, yet is especially apt to affect children between one and five years old. Signs of the disease are yielded by the faces [sic]; being loose, not formed, but not watery; more bulky than the 
food taken would seem to account for; pale in colour [sic], as if devoid of bile; yeasty, frothy, an appearance probably due to fermentation; stinking, stench often very great, the food having undergone putrefaction rather than concoction."

Following his medical intuition, Gee described celiac disease as a malabsorption syndrome triggered by some unidentified foodstuff. He was right on target with his description, but not on target with the culprit of the disease. Gee's recommendation was to feed patients bread that was "cut thin and well toasted on both sides."

The real breakthrough that eventually led to the gluten-free diet as treatment came almost a century later thanks to the acumen of a Dutch physician, Willem-Karel Dicke. He noted that the mortality rate of children suffering from celiac disease before World War II decreased dramatically during the war from 30-35 percent to almost zero. Given the scarcity of wheat during the war, Dicke suspected that wheat flour was indeed responsible for the symptoms endured by the celiac children. He followed his intuition by performing a trial on a very limited number of children that proved him right. Since these pioneering studies and with the advent of specific and sensitive screening tools, we have appreciated that $\mathrm{CD}$ and other gluten related disorders are present worldwide and, like many other immune-mediated diseases, are increasing over time.

Gluten sensitivity (GS) was originally described in the 1980s and a recently "re-discovered" syndrome entity, characterized by intestinal and extra-intestinal symptoms related to the ingestion of gluten-containing food, in subjects that are not affected by either CD or a wheat allergy. Following the landmark work by Sapone and coworkers, describing the clinical and diagnostic features of GS in 2010, a rapidly increasing number of papers have been published by many independent groups, confirming that GS should definitely be included in the spectrum of glutenrelated disorders. However, many aspects of GS epidemiology, pathophysiology, clinical spectrum, and treatment are still unclear.

Treatment of $\mathrm{CD}$ and other gluten-relate disorders is based on the lifelong exclusion of glutencontaining cereals from the diet. The changes needed to begin and maintain a gluten-free diet (GFD) are substantial and have a major impact on daily life. Over the past 30 years, developments in the nutritional aspects of $\mathrm{CD}$ have been huge. Nutrient deficiencies have been described in celiac patients both before and after diagnosis, due to intestinal malabsorption and specific limitations of the GFD, respectively. The relationship between the level of gluten intake and intestinal damage has been analyzed leading to new Codex Alimentarius recommendations on the gluten threshold in gluten-free food. Contamination with gluten of the GFD is an important issue in $\mathrm{CD}$ management. The spectrum of cereal toxicity for $\mathrm{CD}$ patients has been investigated with practical implications on the GFD. The nutritional quality of gluten-free food is constantly improving thanks to the new scientific approach in the technology of gluten-free wheat substitutes. 
In this Nutrients Special Issue, a summary of contemporary issues on the management of CD and other gluten-related disorders is provided. This book capitalizes on the contribution of opinion leaders concerning the multidisciplinary ramifications of these disorders. We want to take this opportunity to thank all contributors to this book. This project would not have been possible without the expertise and invaluable contribution and technical support of the Nutrients editorial team.

Carlo Catassi, Alessio Fasano Guest Editors 
Reprinted from Nutrients. Cite as: Catassi, C.; Bai, J.C.; Bonaz, B.; Bouma, G.; Calabrò, A.; Carroccio, A.; Castillejo, G.; Ciacci, C.; Cristofori, F.; Dolinsek, J. Non-Celiac Gluten Sensitivity: The New Frontier of Gluten Related Disorders. Nutrients 2013, 5, 3839-3853.

Review

\section{Non-Celiac Gluten Sensitivity: The New Frontier of Gluten Related Disorders}

Carlo Catassi ${ }^{1}$, Julio C. Bai ${ }^{2}$, Bruno Bonaz ${ }^{3}$, Gerd Bouma $^{4}$, Antonio Calabrò ${ }^{5}$, Antonio Carroccio ${ }^{6}$, Gemma Castillejo ${ }^{7}$, Carolina Ciacci ${ }^{8}$, Fernanda Cristofori ${ }^{9}$, Jernej Dolinsek ${ }^{10}$, Ruggiero Francavilla ${ }^{9}$, Luca Elli ${ }^{11}$, Peter Green ${ }^{12}$, Wolfgang Holtmeier ${ }^{13}$, Peter Koehler ${ }^{14}$, Sibylle Koletzko ${ }^{15}$, Christof Meinhold ${ }^{16}$, David Sanders ${ }^{17}$, Michael Schumann ${ }^{18}$, Detlef Schuppan ${ }^{19,20}$, Reiner Ullrich ${ }^{18}$, Andreas Vécsei ${ }^{21}$, Umberto Volta $^{22}$, Victor Zevallos ${ }^{19}$, Anna Sapone ${ }^{23}$ and Alessio Fasano ${ }^{24, *}$

1 Department of Pediatrics, Università Politecnica delle Marche, Ancona 60121, Italy;

E-Mail: catassi@tin.it

2 Departamento de Medicina, Hospital de Gastroenterología "Dr. Carlos Bonorino Udaondo", Buenos Aires 1264, Argentina; E-Mail: jbai@intramed.net

3 Department of Gastroenterology and Liver Diseases, CHU Grenoble 38043, France;

E-Mail: bbonaz@chu-grenoble.fr

4 Department of Gastroenterology and Hepatology, Vrije Universiteit Medical Center, Amsterdam 1081 HV, The Netherlands; E-Mail: g.bouma@vumc.nl

5 Gastroenterology Unit, Department of Experimental and Clinical Biomedical Sciences, University of Florence, Florence 50134, Italy; E-Mail: a.calabro@dfc.unifi.it

6 Department of Internal Medicine, "Giovanni Paolo II" Hospital, Sciacca (AG) and University of Palermo, Sciacca 92019, Italy; E-Mail: acarroccio@hotmail.com

7 Pediatric Gastroenterology Unit, Hospital Universitari de Sant Joan de Reus, Universitat Rovira i Virgili, Tarragona 43204, Spain; E-Mail: gcv@tinet.cat

8 Department of Medicine and Surgery, University of Salerno, Baronissi Campus, Salerno 84081, Italy; E-Mail: cciacci@unisa.it

9 Interdisciplinary Department of Medicine, University of Bari, Bari 70124, Italy; E-Mails: fernandacristofori@gmail.com (F.C.); rfrancavilla@gmail.com (R.F.)

${ }^{10}$ Gastroenterology Unit, Department of Pediatrics, University Medical Centre Maribor, Maribor 2000, Slovenia; E-Mail: jernej.dolinsek@ukc-mb.si

11 Centro Prevenzione e Diagnosi Malattia Celiaca Fondazione IRCCS Ca Granda, Milan 20122, Italy; E-Mail: lucelli@yahoo.com

12 Department of Medicine, Celiac Disease Center, Columbia University Medical Center, New York, NY 10032, USA; E-Mail: pg11@columbia.edu

13 Division of Gastroenterology and Internal Medicine, Hospital Porz am Rhein, Köln 51149, Germany; E-Mail: w.holtmeier@khporz.de 
14 German Research Center for Food Chemistry, Leibniz Institute, Freising 85354, Germany; E-Mail: peter.koehler@tum.de

15 Division of Pediatric Gastroenterology and Hepatology, Dr. von Hauner Children's Hospital, University of Munich Medical Center, Munich 80337, Germany;

E-Mail: sybille.koletzko@med.uni-muenchen.de

16 Practice of Nutrition Therapy Meinhold \& Team, Köln 50674, Germany;

E-Mail:praxis@christof-meinhold.de

17 Department of Gastroenterology and Hepatology, Royal Hallamshire Hospital and University of Sheffield Medical School, Sheffield S10 2JF, UK; E-Mail: david.sanders@sth.nhs.uk

18 Department of Gastroenterology, Rheumatology and Infectiology, Charité University Medicine, Berlin 10203, Germany; E-Mails: michael.schumann@charite.de (M.S.); reiner.ullrich@charite.de (R.U.)

19 Department of Medicine I, University Medical Center, Johannes Gutenberg University Mainz, Mainz 55131, Germany; E-Mails: dschuppa@bidmc.harvard.edu (D.S.); zevallos@uni-mainz.de (V.Z.)

${ }^{20}$ Division of Gastroenterology and Celiac Center, Beth Israel Deaconess Medical Center and Harvard Medical School, Boston, MA 02215, USA

21 St. Anna Children's Hospital, Vienna 1090, Austria; E-Mail: andreas.vecsei@stanna.at

22 Department of Medical and Surgical Sciences, University of Bologna, Bologna 40138, Italy; E-Mail: uvolt@yahoo.com

23 Department of Gastroenterology, Second University of Naples, Naples 80136, Italy; E-Mail: annasapone@yahoo.it

24 Pediatric Gastroenterology and Nutrition, MassGeneral Hospital for Children, Boston, MA 02129, USA

* Author to whom correspondence should be addressed; E-Mail: afasano@partners.org; Tel.: +1-617-726-1450.

Received: 20 August 2013; in revised form: 17 September 2013 / Accepted: 18 September 2013 / Published: 26 September 2013

\begin{abstract}
Non Celiac Gluten sensitivity (NCGS) was originally described in the 1980s and recently a "re-discovered" disorder characterized by intestinal and extra-intestinal symptoms related to the ingestion of gluten-containing food, in subjects that are not affected with either celiac disease (CD) or wheat allergy (WA). Although NCGS frequency is still unclear, epidemiological data have been generated that can help establishing the magnitude of the problem. Clinical studies further defined the identity of NCGS and its implications in human disease. An overlap between the irritable bowel syndrome (IBS) and NCGS has been detected, requiring even more stringent diagnostic criteria. Several studies suggested a relationship between NCGS and neuropsychiatric disorders, particularly autism and schizophrenia. The first case reports of NCGS in children have been described. Lack of biomarkers is still a major limitation of clinical
\end{abstract}


studies, making it difficult to differentiate NCGS from other gluten related disorders. Recent studies raised the possibility that, beside gluten, wheat amylase-trypsin inhibitors and low-fermentable, poorly-absorbed, short-chain carbohydrates can contribute to symptoms (at least those related to IBS) experienced by NCGS patients. In this paper we report the major advances and current trends on NCGS.

Keywords: gluten sensitivity; celiac disease; wheat allergy; gluten-related disorders; gluten-free diet

\section{Introduction}

Gluten sensitivity (GS) was originally described in the 1980s [1] and a recently "re-discovered" syndrome entity, characterized by intestinal and extra-intestinal symptoms related to the ingestion of gluten-containing food, in subjects that are not affected with either celiac disease (CD) or wheat allergy (WA). Following the landmark work by Sapone and coworkers, describing the clinical and diagnostic features of GS in the year 2010 [2], a rapidly increasing number of papers have been published by many independent groups, confirming that GS should definitely be included in the spectrum of gluten-related disorders. However, many aspects of GS epidemiology, pathophysiology, clinical spectrum, and treatment are still unclear. Given the recent increase of the gluten-free market worldwide, partially sustained by individuals who claim a medical necessity to undertake a gluten-free diet (GFD), there is a need of "separating the wheat from the chaff" [3]. This goal will be achieved by (a) proper scientific information, (b) shared definitions, and (c) prospective, multi-center studies addressing the many unsolved issues on GS. In order to develop a consensus on new nomenclature and classification of gluten-related disorders, a panel of experts first met in London, in February 2011. The panel proposed a series of definitions and developed a diagnostic algorithm that has been recently published [4].

After the 2011 London Meeting, many new papers have been published on GS. Although its frequency in the general population is still unclear, epidemiological data have been generated that can help establish the magnitude of the problem. Clinical studies further defined the identity of GS and its possible implications in human disease. An overlap between the irritable bowel syndrome (IBS) and GS has been suspected, requiring even more stringent diagnostic criteria. The first case reports of GS in children have been described. Lack of biomarkers is still a major limitation of clinical studies, making the differential diagnosis with other gluten related disorders, as well conditions independent to gluten exposure, difficult.

Evaluation and discussion of this new information was the aim of a Second Expert Meeting on GS that was held in Munich, November 30-December 2, 2012. In this paper we report the major advances and current trends on GS, as presented and debated at the Munich meeting. 


\section{Nomenclature}

At least three papers have recently addressed the issue of defining gluten-related disorders [4-6]. Interestingly, one of these [4] ranks among the most frequently downloaded paper of the publishing journal (BMC Medicine), particularly by physicians, internists or general pediatricians, and directors of diagnostic labs. There is a general agreement that the term "gluten-related disorders" is the umbrella-term to be used for describing all conditions related to ingestion of gluten-containing food. $\mathrm{CD}$ is a chronic small intestinal, immune-mediated, enteropathy precipitated by exposure to dietary gluten and related prolamines in genetically predisposed individuals, characterized by specific autoantibodies against tissue transglutaminase 2 (anti-TG2) and endomysium (EMA). WA is an adverse immunologic reaction to wheat proteins. In the pathogenesis of WA, wheat specific $\operatorname{IgE}$ antibodies play a central role, however non-IgE-mediated WA does exist [7], and this form may be difficult to distinguish from GS.

GS, which this review will focus on primarily, is a condition in which symptoms are triggered by gluten ingestion, in the absence of celiac-specific antibodies and of classical celiac villous atrophy, with variable Human Leukocyte Antigen (HLA) status and variable presence of first generation anti-gliadin antibodies (AGA). The "labeling" of this disorder was a matter of debate among the panel experts. In order to avoid confusion with $\mathrm{CD}$, sometimes defined as gluten-sensitive enteropathy, "non celiac gluten sensitivity" (NCGS) appeared as an improved definition. Doubtless this is still too vague a terminology, simply reflecting the poor knowledge of the pathophysiology of this condition. As triggering cereal proteins could include fractions other than gluten (see Section 10 below) some panelists were in favor of "non-celiac wheat (protein) sensitivity", a terminology that would however conflict with the possibility that other gluten-containing cereals (rye, barley) may be offensive for the "gluten sensitive" patient. Bearing these limitations in mind, the experts' panel agreed that this entity can provisionally be defined as NCGS, a definition requiring refinement in the future.

\section{Epidemiology}

The overall prevalence of NCGS in the general population is still unknown, mainly because many patients are currently self-diagnosed and start a GFD without medical advice or consultation. However, new data confirm that this is not an uncommon disorder at all. In a region of New Zealand, $5 \%$ of children reported non-CD-related avoidance of gluten-containing food [8]. Gluten avoidance was associated with improvement of nonspecific behavioral and gastrointestinal complaints [9]. It remains to be elucidated how many children reporting gluten avoidance were indeed affected by NCGS, as the vast majority of the children involved in this study were not tested for CD nor underwent to an intestinal biopsy. In a US study performed on 7762 unselected persons aged six years or older who participated in the National Health and Nutrition Examination Survey (NHANES) 2009-2010, Digiacomo et al. found a 0.55\% prevalence of persons on a self-reported GFD. The prevalence was higher in females and older participants [10]. Many of the NHANES subjects on a GFD could indeed be affected by NCGS, however this is likely to be an underestimate as (a) the possible relationship between gastro-intestinal symptoms and gluten intake was not 
systematically explored in this population sample, and (b) the NHANES survey was conducted before NCGS was described in the medical literature.

The analysis of the epidemiology of IBS provides an indirect estimate of intestinal NCGS frequency. According to recent population-based surveys performed in Northern Europe, the prevalence of IBS in the general adult population is $16 \%-25 \%[11,12]$. In a selected (and, therefore, probably biased) series of adults with IBS, the frequency of NCGS, documented by a double-blind, placebo-controlled challenge, was $28 \%$ [13]. In the large study performed by Carroccio et al., 276 out of 920 (30\%) subjects with IBS-like symptoms, according to the Rome II criteria, suffered from wheat sensitivity or multiple food hypersensitivity, including wheat sensitivity [14]. Should a consistent proportion of IBS patients be affected with NCGS, the prevalence of NCGS in the general population could well be higher than CD (1\%).

Although risk factors for NCGS have not yet been identified, the disorder seems to be more common in females and in young/middle age adults. The prevalence of NCGS in children is still unknown.

\section{Clinical Picture and Natural History}

NCGS is characterized by symptoms that usually occur soon after gluten ingestion, disappear with gluten withdrawal and relapse following gluten challenge, within hours or few days. The "classical" presentation of NCGS is a combination of IBS-like symptoms, including abdominal pain, bloating, bowel habit abnormalities (either diarrhea or constipation), and systemic manifestations such as "foggy mind", headache, fatigue, joint and muscle pain, leg or arm numbness, dermatitis (eczema or skin rash), depression, and anemia [2,15]. When seen at the specialty clinic, many NCGS patients already report the causal relationship between the ingestion of gluten-containing food and worsening of symptoms. In children, NCGS manifests with typical gastrointestinal symptoms, such as abdominal pain and chronic diarrhea, while the extra-intestinal manifestations seem to be less frequent, the most common extra-intestinal symptom being tiredness [16].

During the last decade, several studies suggested a relationship between NCGS and neuropsychiatric disorders (see following paragraphs).

While it is undisputable that in some cases the positive effect of gluten withdrawal can be explained by a placebo effect, this is not the case in true NCGS. In a double-blind randomized placebo-controlled study design, Biesiekierski et al. found that IBS-like symptoms of NCGS were more frequent in the gluten-treated group (68\%) than in subjects on placebo (40\%) [13]. Furthermore a recent study found no significant differences between CD and NCGS patients regarding personality traits, level of somatization, quality of life, anxiety, and depressive symptoms. The somatization level was low in both diseases. Additionally, symptom increase after a gluten challenge was not related to personality in NCGS patients [17].

No major complication of untreated NCGS has so far been described; especially autoimmune comorbidity, as observed in $\mathrm{CD}$, has not been reported so far. However, natural history data on NCGS are still lacking. Therefore it is difficult to draw firm conclusions on the outcome of this condition. 


\section{NCGS and IBS: A Complex Relationship}

The complex relationship between IBS and dietary proteins has been recently reviewed [18]. Patients with CD often report symptoms compatible with IBS persisting after treatment with the GFD. In a recent meta-analysis the pooled prevalence of IBS-type symptoms in patients with treated CD was $38.0 \%$ (95\% CI, 27.0\%-50.0\%). The pooled odds ratio (OR) for IBS-type symptoms was higher in patients with $\mathrm{CD}$ than in controls $(5.60 ; 95 \% \mathrm{CI}, 3.23-9.70)$. In patients who were non-adherent with a GFD, the pooled OR for IBS-like symptoms, compared with those who were strictly adherent, was 2.69 (95\% CI, 0.75-9.56) [19].

That gluten ingestion may elicit gastrointestinal symptoms in non-CD patients has recently been shown in subjects affected with the D variant (diarrhea-predominant) of IBS, by Vazquez-Roque and coworkers. Subjects on a gluten containing diet (GCD) had more bowel movements per day, particularly those with HLA-DQ2 and/or DQ8 genotypes. The GCD was associated with higher small bowel permeability. Patients on the GCD had a small decrease in expression of zonula occludens 1 in small bowel mucosa, and significant decreases in expression of zonula occludens 1 , claudin-1, and occludin in rectosigmoid mucosa; again the effects of the GCD on expression were significantly greater in HLA-DQ2/8-positive patients. On the other hand, the GCD vs. the GFD had no significant effects on gastrointestinal transit or histology. It was concluded that gluten alters bowel barrier functions in patients with IBS-D, particularly in HLA-DQ2/8-positive patients. These data provided mechanistic explanations for the observation that gluten withdrawal may improve patient symptoms in IBS [20].

How specific the effect of gluten withdrawal from the diet of patients with IBS is, still remains to be elucidated. Besides gluten, wheat, and wheat derivatives contain other constituents that could play a role in triggering symptoms in IBS patients, e.g., amylase-trypsin inhibitors (ATIs, see below) and fructans. In a second study, Biesiekirski et al. reported on 37 patients with IBS/self-reported NCGS investigated by a double-blind crossover trial. Patients were randomly assigned to a period of reduced low-fermentable, poorly-absorbed, short-chain carbohydrates (fermentable oligo-, di-, and mono-saccharides and polyols $=$ FODMAPs) diet and then placed on either a gluten or whey proteins challenge. In all participants, gastrointestinal complaints consistently improved during reduced FODMAP intake, but significantly worsened to a similar degree when their diets included gluten or whey proteins [21]. FODMAPS list includes fructans, galactans, fructose, and polyols that are contained in several foodstuffs, including wheat, vegetables, and milk derivatives. These results raise the possibility that the positive effect of the GFD in patients with IBS is an unspecific consequence of reducing FODMAPs intake, given that wheat is one of the possible sources of FODMAPs. However, it should be stressed that FODMAPs cannot be entirely and exclusively responsible for the symptoms experienced by NCGS subjects, since these patients experience a resolution of symptoms while on a GFD despite continuing to ingest FODMAPs from other sources, like legumes (a much richer source of FODMPs than wheat). Nevertheless, based on the results reported by Biesiekirski et al. is also possible that there are IBS cases entirely due to FODMAPs that, therefore, cannot be classified as affected by NCGS [21]. 


\section{Is Autism Part of the NCGS Spectrum?}

Autism Spectrum Disorders (ASD) are chronic behavioral conditions, with onset before three years of age. ASD are one of the fastest growing developmental disabilities in the United States. They present with a wide range of stereotyped, repetitive behaviors, social and language impairment. Function and outcome is affected not only by core deficits but also by associated behaviors such as hyperactivity, aggression, anxiety, and depression. Many studies have indicated that behavioral therapy and medication may be at least partially helpful in the management of children with ASD. Research on the effect of diet and nutrition on autism has been increasing in the past two decades, particularly on the symptoms of hyperactivity and attention. One of the most popular interventions for ASD is the gluten free casein free (GFCF) diet.

The possible effect of the GFCF in children with autism is not due to underlying CD, since an association between these two conditions has never been clearly confirmed by serological screening studies [22]. It has been hypothesized that some symptoms may be caused by opioid peptides formed from the incomplete breakdown of foods containing gluten and casein. Increased intestinal permeability, also referred to as the "leaky gut syndrome," has been suspected in ASD to be part of the chain of events that allows these peptides to cross the intestinal membrane, enter the bloodstream, and cross the blood-brain barrier, affecting the endogenous opiate system and neurotransmission within the nervous system. The resulting excess of opioids is thought to lead to behaviors noted in ASD, and the removal of these substances from the diet could determine a change in autistic behaviors [23]. The leaky gut/autism connection has fuelled a strong debate within the scientific community, far from being settled. A recent study has reported a high percentage of abnormal intestinal permeability test (as established by the lactulose/mannitol ratio) among patients with autism $(36.7 \%)$ and their relatives $(21.2 \%)$ compared with normal subjects (4.8\%). Patients with autism on a reported GFCF diet had significantly lower intestinal permeability test values compared with those who were on an unrestricted diet and controls [24]. However, the degree of correlation between abnormal intestinal permeability to sugars (lactulose and mannitol) and proteins/peptides remains to be established. It should also be pointed out that, in a pilot study, Robertson et al. did not detect any changes in intestinal permeability in a small cohort of ASD children [25]. The finding of IgG class antibodies directed against food antigens is considered indirect evidence of increased intestinal permeability. Children with autism have significantly higher levels of $\operatorname{IgG}$ antibody (but not IgA) to gliadin compared with healthy controls, particularly in those with gastrointestinal symptoms [26]. Recent studies confirmed these findings and also reported an increase in antibodies directed to several other food allergens, including casein and whole milk [27].

Despite its popularity, the efficacy of the GFCF diet in improving autistic behavior remains not conclusively proven. A 2008 Cochrane review reported that only two small RCTs investigated the effect of GFCF diet in children with ASD $(n=35)$. There were only three significant treatment effects in favor of the diet intervention: overall autistic traits, mean difference $(M D)=-5.60$; social isolation, $\mathrm{MD}=-3.20$ and overall ability to communicate and interact, $\mathrm{MD}=1.70$. In addition three outcomes were not different between the treatment and control group while differences for ten outcomes could not be analyzed because data were skewed. The review concluded that the evidence 
for efficacy of these diets is poor, and large scale, good quality randomized controlled trials are needed [28].

By using a two-stage, randomized, controlled study of GFCF diet of children with ASD, Whiteley and coworkers recently reported significant group improvements in core autistic and related behaviors after eight and 12 months on diet. The results showed a less dramatic change between children having been on diet for eight and children in diet for 24 months, possibly reflective of a plateau effect [29].

The above data suggest that removing gluten from the diet may positively affect the clinical outcome in some children diagnosed with ASD, indicating that autism may be part of the spectrum of NCGS, at least in some cases. However, a word of caution is necessary to stress the fact that only a small, selected sub-group of children affected by ASD may benefit from an elimination diet. Additional investigations are required in order to identify phenotypes based on best- and non-response to dietary modifications and assess any biological correlates including anthropometry before considering a dietary intervention.

\section{Gluten-Related Disorders and Schizophrenia}

An association between schizophrenia and CD was noted in reports spanning back to the 1960s [30]. In 1986 a double-blind gluten-free/gluten-load controlled trial of 24 patients conducted by Vlissides et al. showed changes in symptom profile of schizophrenics in response to exclusion of gluten from the diet [31]. On the other hand, a small blind study conducted by Potkin et al. showed no differences in the clinical status of eight schizophrenic patients on a 5-week gluten challenge in an in-patient setting, as measured by the Brief Psychiatric Rating Scale [32]. A subsequent study by Storms et al. tested 26 schizophrenic patients on a locked ward assigned to either a gluten-free or high gluten diet. No differences were found between the groups on their performance in a battery of psychological tests [33]. A recent study using blood samples from the Clinical Antipsychotic Trials of Intervention Effectiveness (CATIE) found that 5.5\% of the subjects with schizophrenia had a high level of anti-tTG antibodies (compared to $1.1 \%$ in the healthy control sample) and $23.1 \%$ had AGA IgG positivity compared with $3.1 \%$ in controls. Interestingly enough, a large proportion of tTG positive subjects resulted EMA negative, questioning the possibility that their tTG positivity was related to $\mathrm{CD}$. Indeed, only $2 \%$ of schizophrenic patients fulfilled the $\mathrm{CD}$ diagnostic criteria (both anti-tTG and EMA positive), questioning the role of CD in schizophrenia [34]. Additional studies revealed that most of the $\mathrm{tTG}$ positive subjects were tTG- 6 positive, suggesting that these antibodies are more a biomarker of neuro-inflammation than CD [35]. This study indicated the existence of a specific immune response to gluten in some of these patients, probably related to NCGS. Other studies confirmed the high prevalence of antibodies to AGA among people with schizophrenia [36], however the exact mechanism underlying the observed improvement of symptoms in some patients with the GFD has remained elusive. Immunological mechanisms have been proposed, including the assertion that a subgroup of schizophrenics suffer from food intolerances that benefit from the adoption of a GFD. The beneficial effect of a GFD may also be achieved via circulating food-derived peptides (exorphins) exerting an influence on physiological processes in the brain (same mechanism as described in the autism paragraph). If it were true that a subset of schizophrenic patients did exhibit symptoms due to sensitivity to gluten, then not only would treatment for these individuals be easier and more efficient than neuroleptics but also their quality of life would improve. 
In summary, the role of NCGS in conditions affecting the nervous system remains a highly debated and controversial topic that requires additional, well-designed studies to establish the real role of gluten as a triggering factor in these diseases.

\section{Laboratory Evaluation}

So far no specific biomarker of NCGS has been identified. Recently, Volta and colleagues reported on the pattern of CD serology found in 78 untreated patients affected with NCGS. Many patients displayed an elevated prevalence of high titer, "first-generation" IgG AGA directed against native gliadin (56.4\%). The prevalence of IgG AGA detected in NCGS, although lower than that found in $\mathrm{CD}(81.2 \%)$, was much higher than other pathologic conditions such as connective tissue disorders $(9 \%)$ and autoimmune liver diseases $(21.5 \%)$ as well as in the general population and healthy blood donors $(2 \%-8 \%)$. On the other hand, the prevalence of IgA AGA in NCGS patients was very low (7.7\%). Noteworthy, the "best" CD markers, namely IgG deamidated gliadin peptide (DGP) antibodies, IgA tTGA, and IgA EMA, were always negative in NCGS patients, except for an isolated positivity at a very low titer for IgG DGP. The consistent negativity for IgG DGP, whose synthesis "in vivo" is an expression of the interaction between tissue transglutaminase and gliadin peptides, seems to exclude the involvement of adaptive immunity in NCGS pathogenesis. Interestingly enough, ELISA activities of IgA tTGA in NCGS patients were very low with $30 \%$ of them displaying values $<1 \mathrm{AU}$ (none of them had IgA deficiency) [15].

The CD-predisposing HLA-DQ2 and DQ8 genotypes are found in 50\% of NCGS patients, a prevalence that is lower than $\mathrm{CD}(95 \%)$ and only slightly higher than the general population $(30 \%)[4]$.

In the work of Sapone and coworkers all subjects (11 patients with NCGS, 13 with CD, and seven controls), underwent upper duodenal endoscopy for small intestinal biopsy. Those with NCGS revealed normal to mildly inflamed mucosa (Marsh 0 to 1), while all CD patients showed partial or subtotal villous atrophy with crypt hyperplasia. As expected, CD patients had increased numbers of CD3+ IELs (>50/100 enterocytes) compared to controls, while NCGS patients had a number of CD3+ IELs intermediate between CD patients and controls in the context of relatively conserved villus architecture. The numbers of TCR $-\gamma \delta$ IELs were only elevated in CD subjects $(>3.4 / 100$ enterocytes), while in NCGS patients the numbers of $\gamma \delta$ IELs were similar to those in controls [2]. Recently, activation of circulating basophils [14] and increased infiltration of duodenal lamina propria with eosinophils [37] have been described.

\section{Diagnosis}

NCGS diagnosis is sometimes suspected by the patients themselves based on food withdrawal and introduction. Physicians may then concur if there has been the exclusion of other forms of gluten-induced disease (CD and WA) by appropriate serological and/or biopsy tests. Specific IgE might normalize if the patients are already on GFD and this might be a potential pitfall in diagnosis of WA The finding that symptoms disappear after gluten elimination adds weight to the diagnosis of NCGS, which is definitely proven by a double-blind (or open) oral gluten challenge performed after at least three weeks of GFD. 
Based on a combination of clinical, biological, genetic and histological data, it is possible to differentiate the three gluten-related conditions (WA, CD, and NCGS), using recently published algorithms [4]. Since there is some degree of overlap between NCGS and other forms of wheat-exclusion responsive conditions (e.g., IBS responsive to low FODMAPs diet, non-IgE mediated WA), periodical patient reassessment (e.g., every 6-12 months), including an accurate dietary interview, is strongly recommended.

\section{Pathogenesis}

The pathophysiology of NCGS is under scrutiny. In the study conducted by Sapone et al. [2], NCGS subjects showed a normal intestinal permeability and claudin-1 and ZO-1 expression compared with celiac patients, and a significantly higher expression of claudin- 4 . In the same NCGS patients, the up-regulation of claudin- 4 was associated with an increased expression of toll-like receptor-2 and a significant reduction of T-regulatory cell marker FoxP3 relative to controls and CD patients. Additionally, an increase in IELs of the classes $\alpha$ and $\beta$, but no increase in adaptive immunity-related gut mucosal gene expression, including interleukin (IL)-6, IL-21, and interferon- $\gamma$ (IFN- $\gamma$ ), was detected in NCGS. These changes suggested an important role of the intestinal innate immune system in NCGS, without any involvement of the adaptive immune response. In a study aimed at exploring and comparing the early mucosal immunological events in CD and NCGS, Brottveit et al. confirmed that CD patients mounted a concomitant innate and adaptive immune response to gluten challenge. NCGS patients only showed increased IFN- $\gamma$ levels after gluten challenge and increased density of intraepithelial CD3(+) $\mathrm{T}$ cells at baseline [38]. These findings open the possibility of an adaptive component as well in the pathogenesis of NCGS.

The trigger/s of mucosal events leading to NCGS is not necessarily represented by the same array of gluten peptides responsible for CD development. Unlike the duodenal mucosa from patients with CD, upon incubation with gliadin, mucosa from patients with NCGS does not express markers of inflammation, and their basophils are not activated by gliadin [39]. In vitro studies suggest that wheat ATIs could play a major role as triggers of the innate immune response in intestinal monocytes, macrophages and dendritic cells eventually leading to NCGS. Wheat ATIs are a family of five or more homologous low-molecular-weight proteins highly resistant to intestinal proteolysis. They are known to be the major allergen responsible for baker's asthma. ATIs engage the TLR4-MD2-CD14 complex and lead to up-regulation of maturation markers and elicit release of pro-inflammatory cytokines in cells from celiac and non-celiac patients and in celiac patients' biopsies [40].

\section{Current and Future Trends}

The vast majority of celiac experts initially reacted with a great deal of skepticism to the concept of NCGS existence and the fact that it was a separate entity from CD. For those that witnessed the initial struggle of convincing health care professionals that $\mathrm{CD}$ was not confined within European boundaries this was a déjà $v u$. Indeed, we are now with NCGS where we probably were with CD forty years ago. In the 1980s we knew that CD existed, but we had little information on the mechanisms leading to the enteropathy, the genetic component of the disease, what kind of immune response was involved in the pathogenesis of the disease, its multifaceted clinical presentation, and 
its complication. We lacked robust screening tools to conduct well-design epidemiological studies and had little understanding on the most appropriate management of the disease and its complications. The confusion about NCGS stems from the few facts, and the many fantasies, currently available on this topic. The best testimonial of this concept is the comparison of the literature published on both conditions during the past 63 years. The publications on CD doubled every 20 years from approximately 2500 in the period of 1950-70 to 9500 in the period 1991-2010, with already more than 2000 papers published between 2011 and 2013. Conversely, there were almost no scientific reports on NCGS before 1970 and only a handful number of papers have been published ever since, most of them after 2005. The increase interest in NCGS is testified by the decreased NCGS/CD publication ratio that dropped from 1:438 in the period 1950-70 to 1:10 in the period 2010-13 (Table 1).

Table 1. Trends in publication on celiac disease (CD) and non-celiac gluten sensitivity (NCGS) during the last decades.

\begin{tabular}{cccc}
\hline Timeline & CD & NCGS & NCGS/CD ratio \\
\hline $1950-1970$ & 2632 & 6 & $1: 438$ \\
$1971-1990$ & 4915 & 118 & $1: 43$ \\
$1991-2010$ & 9498 & 733 & $1: 13$ \\
$2011-2013$ & 2014 & 188 & $1: 10$ \\
\hline
\end{tabular}

Given the limited literature on the topic, it should not come as a surprise that there are still numerous questions about NCGS that should be addressed. Is NCGS permanent or transitory? Is the threshold of sensitivity the same for everybody, or change from subject to subject and in the same subject over time? How frequent is NCGS? The range reported in the literature is between $0.5 \%$ and $6 \%$, based on poorly conducted studies and on definitions of the disease that varies widely from one report to another. Only recently, well-conducted studies based on double blind, placebo control design are providing evidence-based data on the prevalence of NCGS in specific clinical conditions, particularly IBS [13]. There is the strong need for more coordinated efforts to perform large multicenter studies for those conditions, including autism and schizophrenia, in which NCGS has been indicated as a possible cause in a subgroup of these patients. The lack of validated biomarkers for a diagnosis not based on exclusion criteria is judged to be of paramount importance by many experts in the field. Currently a large multicenter placebo-controlled study is underway to achieve this goal and, hopefully, will provide tools for a more correct diagnosis and for more rigorous studies to establish the prevalence of NCGS in specific conditions and in the general population. Recent studies raised the possibility that, beside gluten [13] and wheat ATIs [40], low-fermentable, poorly-absorbed, short-chain carbohydrates [21] can contribute to symptoms (at least those related to IBS) experienced by NCGS patients. These new findings need corroboration through additional studies involving larger numbers of subjects. If these studies will confirm these new findings, they will probably prompt a change in nomenclature from NCGS to wheat sensitivity to reflect the fact that, beside gluten, other components of wheat may be responsible for the symptoms reported by NCGS patients. 


\section{Acknowledgments}

We wish to express our gratitude to Jacqueline Pante (Schär, Merano, Italy) and Andrea Comaschi (Weber Shandwick, Milan, Italy) for logistic support in the organization of the Munich Meeting.

\section{Conflicts of interest}

Carlo Catassi received consulting fees from Schär and Menarini Diagnostics. Luca Elli and Anna Sapone received consulting fees from Schär. Peter Green is a member of the scientific advisory board of Alvine Pharmaceuticals and Alba Therapeutics. Alessio Fasano owns stock in Alba Therapeutics. The other authors declared no conflict of interest.

\section{References}

1. Cooper, B.T.; Holmes, G.K.; Ferguson, R.; Thompson, R.A.; Allan, R.N.; Cooke, W.T. Gluten-sensitive diarrhea without evidence of celiac disease. Gastroenterology 1981, 81, 192-194.

2. Sapone, A.; Lammers, K.M.; Mazzarella, G.; Mikhailenko, I.; Cartenì, M.; Casolaro, V.; Fasano, A. Differential mucosal IL-17 expression in two gliadin-induced disorders: Gluten sensitivity and the autoimmune enteropathy celiac disease. Int. Arch. Allergy Immunol. 2010, $152,75-80$.

3. Sanders, D.S.; Aziz, I. Non celiac wheat sensitivity: Separating the wheat from the chaff. Am. J. Gastroenterol. 2012, 107, 1908-1912.

4. Sapone, A.; Bai, J.C.; Ciacci, C.; Dolinsek, J.; Green, P.H.; Hadjivassiliou, M.; Kaukinen, K.; Rostami, K.; Sanders, D.S.; Schumann, M.; et al. Spectrum of gluten-related disorders: Consensus on new nomenclature and classification. BMC Med. 2012, 10, 13.

5. Ludvigsson, J.F.; Leffler, D.A.; Bai, J.C.; Biagi, F.; Fasano, A.; Green, P.H.; Hadjivassiliou, M.; Kaukinen, K.; Kelly, C.P.; Leonard, J.N.; et al. The Oslo definitions for coeliac disease and related terms. Gut 2013, 62, 43-52.

6. Mäki, M. Lack of consensus regarding definitions of coeliac disease. Nat. Rev. Gastroenterol. Hepatol. 2012, 9, 305-306.

7. Koletzko, S.; Niggemann, B.; Arato, A.; Dias, J.A.; Heuschkel, R.; Husby, S.; Mearin, M.L.; Papadopoulou, A.; Ruemmele, F.M.; Staiano, A.; et al. European Society of Pediatric Gastroenterology, Hepatology, and Nutrition. Diagnostic approach and management of cow's-milk protein allergy in infants and children: ESPGHAN GI Committee practical guidelines. J. Pediatr. Gastroenterol. Nutr. 2012, 55, 221-229.

8. Tanpowpong, P.; Ingham, T.R.; Lampshire, P.K.; Kirchberg, F.F.; Epton, M.J.; Crane, J.; Camargo, C.A., Jr.; New Zealand Asthma and Allergy Cohort Study Group. Coeliac disease and gluten avoidance in New Zealand children. Arch. Dis. Child. 2012, 97, 12-16.

9. Tanpowpong, P.; Broder-Fingert, S.; Katz, A.J.; Camargo, C.A., Jr. Predictors of gluten avoidance and implementation of a gluten-free diet in children and adolescents without confirmed celiac disease. J. Pediatr. 2012, 161, 471-475. 
10. Digiacomo, D.V.; Tennyson, C.A.; Green, P.H.; Demmer, R.T. Prevalence of gluten-free diet adherence among individuals without celiac disease in the USA: Results from the Continuous National Health and Nutrition Examination Survey 2009-2010. Scand. J. Gastroenterol. 2013, 48, 921-925.

11. Krosgaard, L.R.; Engsbro, A.L.; Bytzer, P. The epidemiology of irritable bowel syndrome in Denmark. A population-based survey in adults $<50$ years of age. Scand. J. Gastroenterol. 2013, 48, 523-299.

12. Breckan, R.K.; Asfeldt, A.M.; Straume, B.; Florholmen, J.; Paulssen, E.J. Prevalence, comorbidity, and risk factors for functional bowel symptoms: A population-based survey in Northern Norway. Scand. J. Gastroenterol. 2012, 47, 1274-1282.

13. Biesiekierski, J.R.; Newnham, E.D.; Irving, P.M.; Barrett, J.S.; Haines, M.; Doecke, J.D.; Shepherd, S.J.; Muir, J.G.; Gibson, P.R. Gluten causes gastrointestinal symptoms in subjects without celiac disease: A double-blind randomized placebo-controlled trial. Am. J. Gastroenterol. 2011, 106, 508-514.

14. Carroccio, A.; Mansueto, P.; Iacono, G.; Soresi, M.; D’Alcamo, A.; Cavataio, F.; Brusca, I.; Florena, A.M.; Ambrosiano, G.; Seidita, A.; et al. Non-celiac wheat sensitivity diagnosed by double-blind placebo-controlled challenge: Exploring a new clinical entity. Am. J. Gastroenterol. 2012, 107, 1898-1906.

15. Volta, U.; Tovoli, F.; Cicola, R.; Parisi, C.; Fabbri, A.; Piscaglia, M.; Fiorini, E.; Caio, G. Serological tests in gluten sensitivity (non celiac gluten intolerance). J. Clin. Gastroenterol. 2012, 46, 680-685.

16. Mastrototaro, L.; Castellaneta, S.; Gentile, A.; Fontana, C.; Tandoi, E.; Dellatte, S.; Romagnoli, V.; Catassi, C.; Francavilla R. Gluten sensitivity in children: Clinical, serological, genetic and histological description of the first paediatric series. Dig. Liver Dis. 2012, 44, S254-S255.

17. Brottveit, M.; Vandvik, P.O.; Wojniusz, S.; Løvik, A.; Lundin, K.E.; Boye, B. Absence of somatization in non-coeliac gluten sensitivity. Scand. J. Gastroenterol. 2012, 47, 770-777.

18. Boettcher, E.; Crowe, S.E. Dietary proteins and functional gastrointestinal disorders. Am. J. Gastroenterol. 2013, 108, 728-736.

19. Sainsbury, A.; Sanders, D.S.; Ford, A.C. Prevalence of irritable bowel syndrome-type symptoms in patients with celiac disease: A meta-analysis. Clin. Gastroenterol. Hepatol. 2013, 11, 359-365.

20. Vazquez-Roque, M.I.; Camilleri, M.; Smirk, T.; Murray, J.A.; Marietta, E.; O’Neill, J.; Carlson, P.; Lamsam, J. A controlled trial of gluten-free diet in patients with irritable bowel syndrome-diarrhea: Effects on bowel frequency and intestinal function. Gastroenterology 2013, 144, 903-911.

21. Biesiekirski, J.R.; Peters, S.L.; Newnham, E.D.; Rosella, O.; Muir, J.G.; Gibson, P.R. No effects of gluten in patients with self-reported non-celiac gluten sensitivity following dietary reduction of low-fermentable, poorly absorbed, short-chain carbohydrates. Gastroenterology 2013, 145, 320-328.

22. Batista, I.C.; Gandolfi, L.; Nobrega, Y.K.; Almeida, R.C.; Almeida, L.M.; Campos Junior, D.; Pratesi, R. Autism spectrum disorder and celiac disease: No evidence for a link. Arq. Neuropsiquiatr. 2012, 70, 28-33. 
23. Marcason, W. What is the current status of research concerning use of a gluten-free, casein-free diet for children diagnosed with autism? J. Am. Diet. Assoc. 2009, 109, 572.

24. De Magistris, L.; Familiari, V.; Pascotto, A.; Sapone, A.; Frolli, A.; Iardino, P.; Carteni, M.; de Rosa, M.; Francavilla, R.; Riegler, G.; et al. Alterations of the intestinal barrier in patients with autism spectrum disorders and in their first-degree relatives. J. Pediatr. Gastroenterol. Nutr. 2010, 51, 418-424.

25. Robertson, M.A.; Sigalet, D.L.; Holst, J.J.; Meddings, J.B.; Wood, J.; Sharkey, K.A. Intestinal permeability and glucagon-like peptide-2 in children with autism: A controlled pilot study. J. Autism Dev. Disord. 2008, 38, 1066-1071.

26. Lau, N.M.; Green, P.H.; Taylor, A.K.; Hellberg, D.; Ajamian, M.; Tan, C.Z.; Kosofsky, B.E.; Higgins, J.J.; Rajadhyaksha, A.M.; Alaedini, A. Markers of celiac disease and gluten sensitivity in children with autism. PLoS One 2013, 8, e66155.

27. De Magistris, L.; Picardi, A.; Siniscalco, D.; Riccio, M.P.; Sapone, A.; Cariello, R. Antibodies against food antigens in patients with autistic spectrum disorders. BioMed. Res. Int. 2013, 2013, 729349.

28. Millward, C.; Ferriter, M.; Calver, S.; Connell-Jones, G. Gluten- and casein-free diets for autistic spectrum disorder. Cochrane Database Syst. Rev. 2008, 2, CD003498.

29. Whiteley, P.; Haracopos, D.; Knivsberg, A.M.; Reichelt, K.L.; Parlar, S.; Jacobsen, J.; Seim, A.; Pedersen, L.; Schondel, M.; Shattock, P. The ScanBrit randomised, controlled, single-blind study of a gluten- and casein-free dietary intervention for children with autism spectrum disorders. Nutr. Neurosci. 2010, 13, 87-100.

30. Dohan, F.C. Cereals and schizophrenia data and hypothesis. Acta Psychiatr. Scand. 1966, 42, $125-152$.

31. Vlissides, D.M.; Venulet, A.; Jenner, F.A. A double-blind glutenfree/gluten-load controlled trial in a secure ward population. Br. J. Psychiatry 1986, 148, 447-452.

32. Potkin, S.G.; Weinberger, D.; Kleinman, J.; Potkin, S.G.; Weinberger, D.; Kleinman, J.; Nasrallah, H.; Luchins, D.; Bigelow, L.; Linnoila, M.; et al. Wheat gluten challenge in schizophrenic patients. Am. J. Psychiatry 1981, 138, 1208-1211.

33. Storms, L.H.; Clopton, J.M.; Wright, C. Effects of gluten in schizophrenics. Arch. Gen. Psychiatry 1982, 39, 323-327.

34. Cascella, N.G.; Kryszak, D.; Bhatti, B.; Gregory, P.; Kelly, D.L.; Mc Evoy, J.P.; Fasano, A.; Eaton, W.W. Prevalence of celiac disease and gluten sensitivity in the United States clinical antipsychotic trials of intervention effectiveness study population. Schizophr. Bull. 2011, 37, 94-100.

35. Cascella, N.G.; Santora, D.; Gregory, P.; Kelly, D.L.; Fasano, A.; Eaton, W.W. Increased prevalence of transglutaminase 6 antibodies in sera from schizophrenia patients. Schizophr. Bull. 2013, 39, 867-871.

36. Dickerson, F.; Stallings, C.; Origoni, A.; Vaughan, C.; Khushalani, S.; Leister, F.; Yang, S.; Krivogorsky, B.; Alaedini, A.; Yolken, R. Markers of gluten sensitivity and celiac disease in recent-onset psychosis and multi-episode schizophrenia. Biol. Psychiatry 2010, 68, 100-104.

37. Holmes, G. Non coeliac gluten sensitivity. Gastroenterol. Hepatol. Bed Bench 2013, 6, $115-119$. 
38. Brottveit, M.; Beitnes, A.C.; Tollefsen, S.; Bratlie, J.E.; Jahnsen, F.L.; Johansen, F.E.; Sollid, L.M.; Lundin, K.E. Mucosal cytokine response after short-term gluten challenge in celiac disease and non-celiac gluten sensitivity. Am. J. Gastroenterol. 2013, 108, 842-850.

39. Bucci, C.; Zingone, F.; Russo, I.; Morra, I.; Tortora, R.; Pogna, N.; Scalia, G.; Iovino, P.; Ciacci, C. Gliadin does not induce mucosal inflammation or basophil activation in patients with non-celiac gluten sensitivity. Clin. Gastroenterol. Hepatol. 2013, 11, 1294-1299.

40. Junker, Y.; Zeissig, S.; Kim, S.J.; Barisani, D.; Wieser, H.; Leffler, D.A.; Zevallos, V.; Libermann, T.A.; Dillon, S.; Freitag, T.L.; et al. Wheat amylase trypsin inhibitors drive intestinal inflammation via activation of toll-like receptor 4. J. Exp. Med. 2012, 209, 2395-2408. 
Reprinted from Nutrients. Cite as: Bruins, M.J. The Clinical Response to Gluten Challenge: A Review of the Literature. Nutrients 2013, 5, 4614-4641.

Review

\title{
The Clinical Response to Gluten Challenge: A Review of the Literature
}

\section{Maaike J. Bruins}

DSM Biotechnology Center, Alexander Fleminglaan 1, Delft 2613AX, The Netherlands;

E-Mail: maaike.bruins@dsm.com; Tel.: +31-613-207-601; Fax: +31-152-794-110

Received: 4 September 2013; in revised form: 1 November 2013 / Accepted: 6 November 2013 /

Published: 19 November 2013

\begin{abstract}
The aim of this review was to identify, evaluate and summarize all relevant studies reporting on the clinical response to gluten challenge by adult or pediatric patients with suspected or diagnosed coeliac disease (CD) on a gluten-free diet. We evaluated the effect of gluten challenge on changes in symptoms, intestinal mucosa histology, and serum antibodies. A systematic electronic search was performed for studies published as of 1966 using PubMed and Scopus databases. In the reviewed studies, doses ranged from 0.2 to $30 \mathrm{~g}$ /day of wheat gluten or comprised a gluten-containing diet. The onset of symptoms upon gluten intake varied largely from days to months and did not parallel serum antibody or histological changes. Within 3 months of gluten challenge, $70 \%-100 \%$ of pediatric CD patients became positive for AGA-IgA and EMA-IgA antibodies and 50\%-70\% for AGA-IgG. A limited number of trials suggest that no more than half of adult patients developed positive AGA-IgA, EMA-IgA, tTG-IgA or DGP-IgA/IgG titers. Approximately $50 \%-100 \%$ of pediatric and adult patients experienced mucosal relapse of gluten provocation within 3 months, which was preceded by increased mucosal intra-epithelial lymphocytes within several days of challenge. A 3-month high-dose gluten challenge should be suitable to diagnose the majority of CD patients. In some cases prolonged challenge may be needed to verify diagnosis. Combination testing for antibodies and mucosal histology may fasten the diagnosis.
\end{abstract}

Keywords: gluten challenge; coeliac disease; diagnosis

\section{Introduction}

Significant health complications may occur when coeliac patients remain on a normal gluten-containing diet. Diagnosis of coeliac disease (CD) should accurately be established before 
starting a person on a livelong gluten-free diet. In children and adults, diagnostic testing includes blood HLA-DQ2 and HLA-DQ8 testing, histological examination of small-intestinal biopsies and serum CD-specific antibodies [1-7]. The diagnosis is confirmed by decline in antibody levels after the exclusion of gluten from the diet. Clinical improvement and histological remission are also supportive clinical endpoint to confirm the disease. Gluten challenge is not necessary, except under unusual circumstances [4] where doubt exists about the initial diagnosis; for example, when the patient is on a gluten-free diet or presents with antibodies or complaints but, nonetheless, normal histology. Moreover, failure to respond to a gluten-free diet may raise doubt regarding the initial diagnosis. Examination of mucosal biopsy, however, involves a potential risk of misdiagnosis since it is subject to large method variability [8,9] and moderate-to-poor inter- and intra-observer reproducibility has been shown [10-12]. Unfortunately, histological findings in CD are characteristic but not specific as several disorders can produce comparable histopathological changes [13]. Over recent years, more reliable, specific and sensitive serological diagnostic tests and markers have become available. Small bowel histology remains the gold standard for diagnosis. Symptomatic relapse is not sufficient for a diagnosis of coeliac disease in isolation. Particularly in children in whom the initial biopsy was performed before two years of age, a gluten challenge may be necessary because of the risk of misdiagnosis due to confusion with other causes of enteropathy at this age [3]. In patients suspected of $\mathrm{CD}$ and following a gluten-free diet, diagnosis may be confirmed by reintroduction of gluten into the diet or by an oral gluten challenge followed by clinical relapse $[3,4,7,14]$.

Currently, the monitoring of parameters during a gluten challenge is largely empirical, particularly in those patients who remain asymptomatic, and the optimum duration and dose of a gluten challenge has not been established yet. Some guidelines propose a gluten diet/challenge until relapse, even for up to 2 years or longer if patients remain symptom free. The ESPGHAN guidelines recommend that daily gluten intake during gluten challenge should contain at least the normal amount of gluten intake for children (approximately $15 \mathrm{~g}$ /day) [4,15]. There is considerable inter-individual variability of clinical presentation among patients with CD [16,17] but also in clinical response time to gluten intake [17]. The large variability and lack of predictability in the response time and severity to gluten complicate defining recommendations regarding the duration and dose of necessary gluten challenge in the diagnostic setting as well as the clinical trial setting.

A standardized approach regarding the amount and duration of dietary gluten necessary to provoke a clinical response in children and adults could provide guidance to physicians and investigators. Therefore, the aim of this article was to perform a review of the literature reporting on the course of the clinical symptoms, serum CD autoantibodies, and intestinal histological changes in response to a gluten challenge in children and adults with diagnosed or suspected CD.

\section{Method}

The data sources used for this systematic review of references published between 1966 and July 2013 included PubMed and Scopus. Only publications in English were included. We included studies that evaluated the effect of oral gluten challenge in individuals with $\mathrm{CD}$ on clinical parameters, i.e., CD-specific antibodies, histology of small bowel mucosa biopsies, symptoms, and urinary sugar absorption test. We reviewed the studies that described the effect of a gluten challenge 
on clinical relapse in order to confirm diagnosis of $\mathrm{CD}$ in children or adults with diagnosed and/or suspected $\mathrm{CD}$. We also extracted valuable information of patients receiving a gluten challenge in the placebo arm of clinical trials testing CD-related therapies. Gluten challenge studies with the aim to determine the safe threshold of prolonged exposure to trace amounts of gluten were beyond the scope of this paper. Studies reporting on positive anti-gliadin antibodies (AGA), anti-endomysial antibodies (EMA), anti-tissue transglutaminase antibodies (tTG), and anti-deaminated gliadin peptide antibodies (DGP) were included. Anti-reticulin antibodies were not reviewed as this test has nowadays been replaced by the more reliable AGA test. While AGA antibodies have been in use for several decades. However, there is a wide variability in their diagnostic accuracy and both AGA-IgA and AGA-IgG have sensitivities and specificities inferior to tTG-IgA and DGP-IgA and are no longer included in the routine testing strategy for CD [5]. Positivity for CD-specific antibodies was defined as concentrations above the assay cutoff value, which varied among assays used in the different studies. The definition of abnormal mucosa histology of small bowel biopsies also varied depending on the biopsy rating scores used (e.g., villous height to crypt depth ratio, Marsh scores). Clinical symptoms in most studies comprised CD-specific symptoms including vomiting, abdominal pain or distension, obstipation, diarrhea, fatty or loose stool, anorexia, weight loss, and growth failure. Only in the summary table we specified whether symptoms constituted mild, moderate or severe symptoms. Studies that were excluded were studies investigating a single-dose gluten challenge, studies investigating oat challenge, rectal gluten challenge, transamidated, hydrolyzed or digested gluten, gluten-specific peptides, and ex-vivo studies. Since gluten intake at baseline is likely to influence the response to a gluten challenge, we excluded studies in which patients were on a normal gluten-containing diet or had positive baseline autoantibodies at start. Trials were categorized into trials enrolling pediatric or adult patients with mean age below 18 years or 18 years and older, respectively. Moreover, less patients suspected of having CD can be expected to respond to gluten than patients with a confirmed diagnosis of CD. Therefore, we classified trials according to "confirmed diagnosis based on a biopsy in the past", or "diagnosed based on inadequate grounds" referring to as "suspected CD". If possibly, results were reported separately for subgroups of patients with diagnosed and suspected CD in one study. When the amount of dietary gluten in bread was not reported [18-20], we estimated the gluten content, assuming that a slice of bread weighs $25-30 \mathrm{~g}$ and contains $8-11 \mathrm{~g} / 100 \mathrm{~g}$ of protein [15,21], which corresponds to approximately $2-3 \mathrm{~g}$ of gluten $[7,22]$. In some studies the gluten dose was expressed per $\mathrm{kg}$ of body weight. If body weight was not given, estimates were based on WHO child growth charts [23].

\section{Results}

Table 1 gives an overview of the included studies. In total, the following studies were identified that investigated the clinical effect of dietary gluten challenge; 16 trials with pediatric patients with biopsy-diagnosed $C D, 13$ trials with pediatric patients with suspected CD, 11 trials with biopsy-diagnosed adult CD patients and 3 with adolescent or adul Maaike J.t patients suspected of having $\mathrm{CD}$. Of the eleven trials with diagnosed adult patients, five reported on the clinical response to a placebo as part of a clinical intervention study [24-29]. In the studies included, a gluten challenge consisted of a gluten-containing diet, wheat-derived food products, wheat flour, or wheat gluten powder. The gluten doses ranged from 0.2 to $30 \mathrm{~g}$ /day and duration from 1 day to 8 years. 


\subsection{CD-Specific Symptoms in Pediatric Patients with Diagnosed or Suspected CD}

The response rates and onset of symptoms throughout the course of gluten challenge in the different studies was highly variable (Table 1); when a gluten-containing diet was given to children with diagnosed CD, $4 \%$ of them developed symptoms within 1-2 of weeks [30]. At least $10 \mathrm{~g} /$ day of

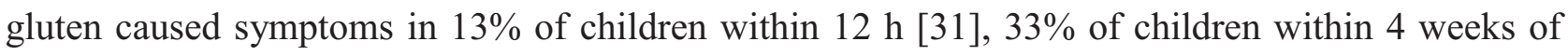
gluten challenge [32], and $60 \%$ of children within 3-months of challenge [33]. When given a gluten-containing diet or 3-15 g/day of gluten, $32 \%$ of children experienced symptoms within 4-5 months [34]. Smaller amounts of about $2 \mathrm{~g}$ /day of gluten caused symptoms in $4 \%$ children on the fourth day and in $25 \%$ of children after 6 months [18].

In children with suspected CD, a gluten-containing diet induced symptoms in about $26 \%-33 \%$ of children within between a few days to 13 months of gluten challenge [35,36], a gluten challenge of at least $10 \mathrm{~g} /$ day caused symptoms in approximately $24 \%-42 \%$ of children from 4 weeks to several [32,37-39] months of challenge, only few patients reported severe symptoms during gluten challenge [38]. A gluten-containing diet providing 5 to $15 \mathrm{~g}$ /day of gliadin caused symptoms in 59\% of children within 45 days of challenge [40]. About $32 \%$ of adolescents with diagnosed or suspected $\mathrm{CD}$ who received at least $10 \mathrm{~g} /$ day of gluten for 2.4 months to 2 years experienced abdominal symptoms at the time of appearance of antireticulin-IgA [41]. In 70\% of the cases, the mucosa relapsed before any symptoms had occurred [41]. Lower doses of 0.2-4.3 g/day of gluten, surprisingly triggered symptoms in $79 \%$ and $96 \%$ of children within 4 and 15 weeks, respectively [42]. No correlation was observed between time of appearance of symptoms and positive antibodies.

Summarizing, in most studies only few children with diagnosed or suspected CD respond by symptoms to a low or high gluten dose during the first 2 weeks. During prolonged low or high dose gluten challenge $24 \%-42 \%$ of children may experience symptoms, although in three studies higher response rates were reported of 60\% [33,40], and even 96\% [42]. Large variability exists in time of onset of symptoms during gluten challenge: symptoms appear almost immediately in some children while some do not develop symptoms until several months of challenge or develop no symptoms at all. Symptoms are generally mild to moderate. Some studies indicated that clinical symptoms are a very unreliable indicator of antibody response and mucosal relapse [34,41,42]. 


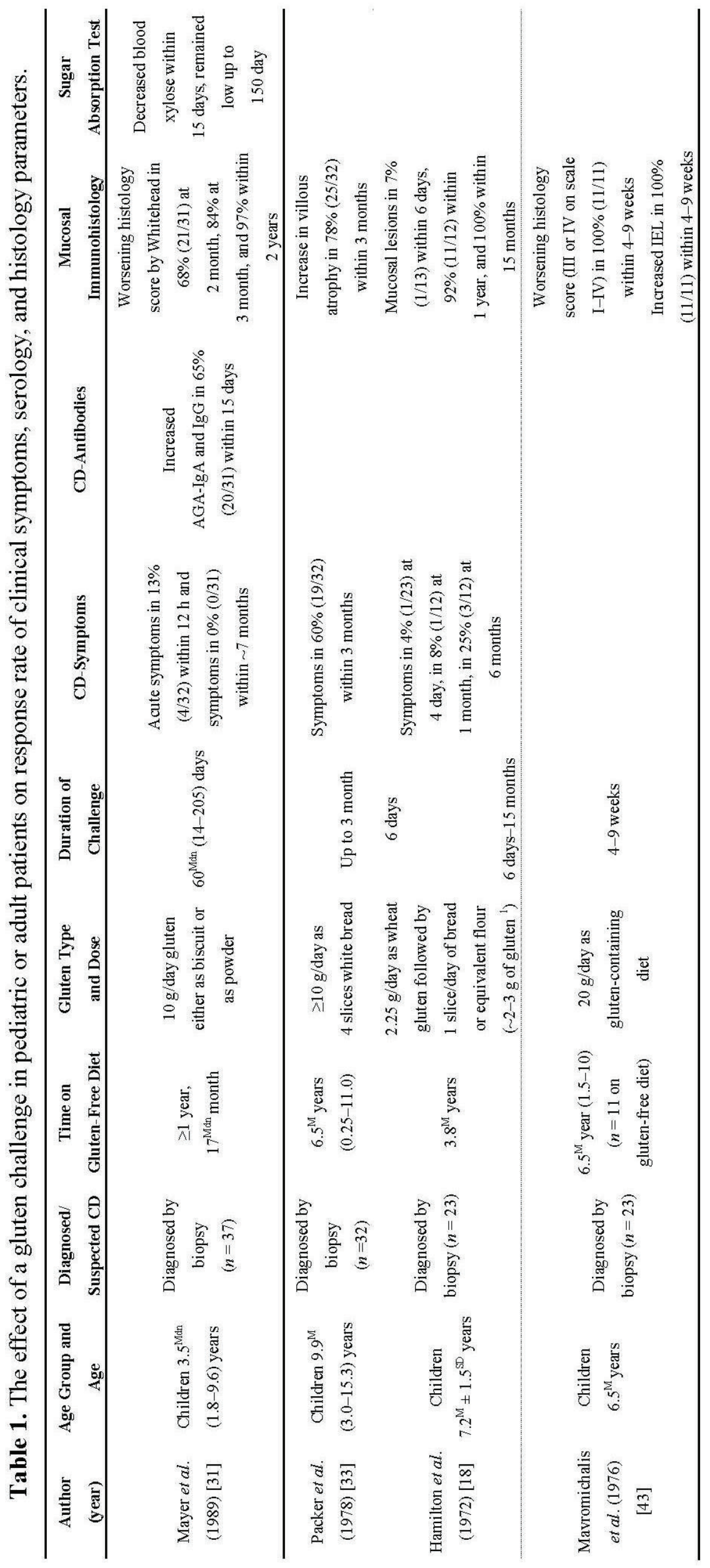




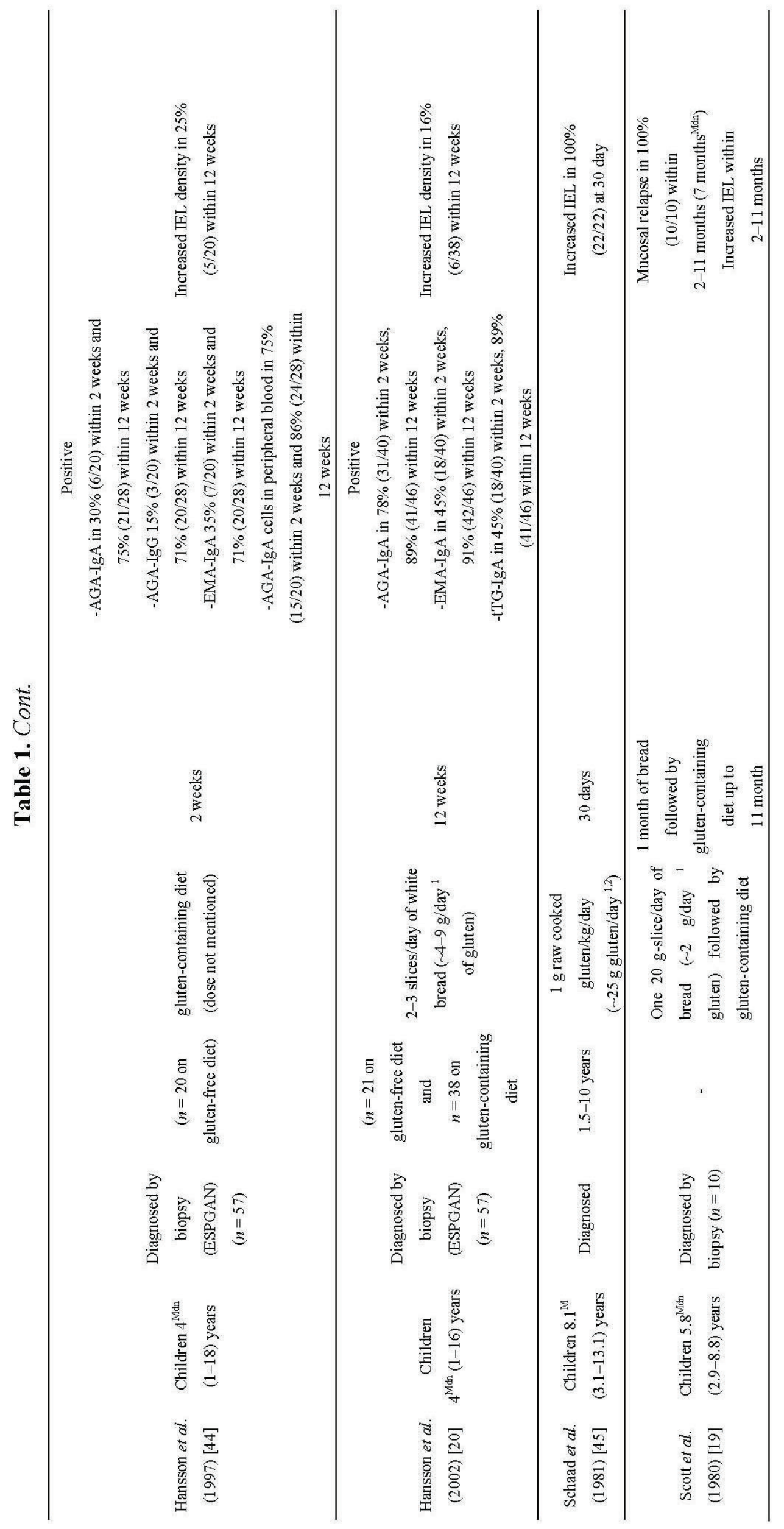




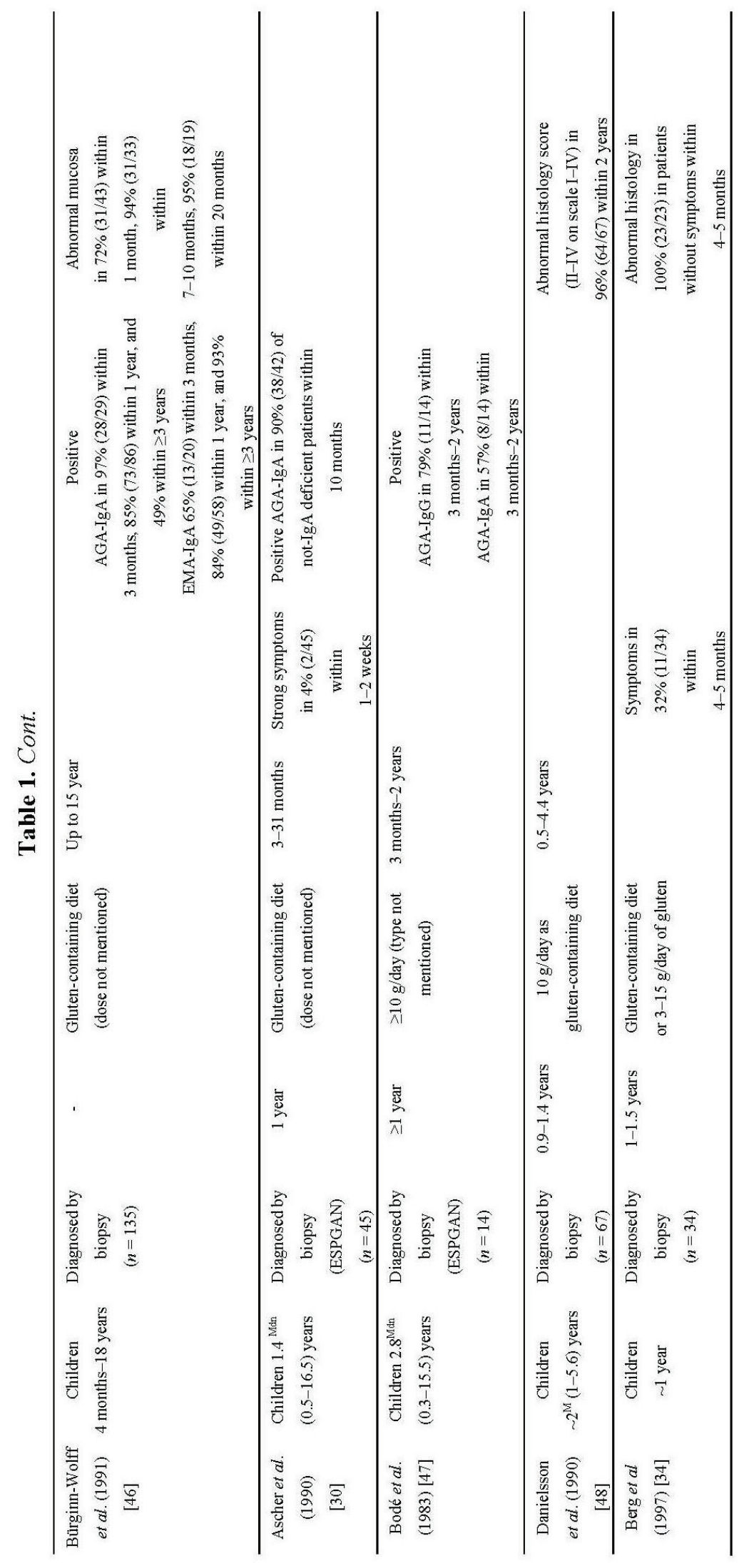




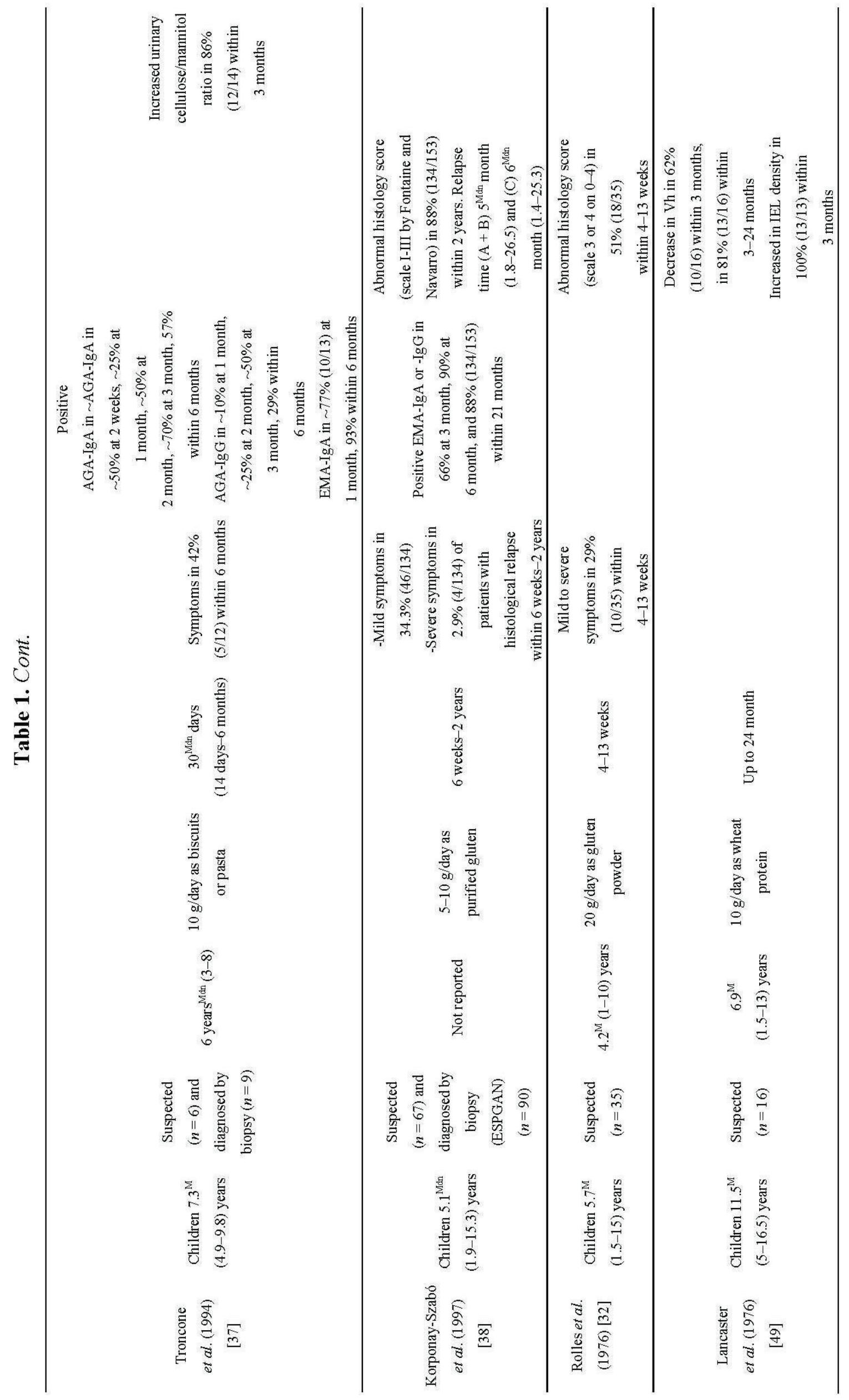




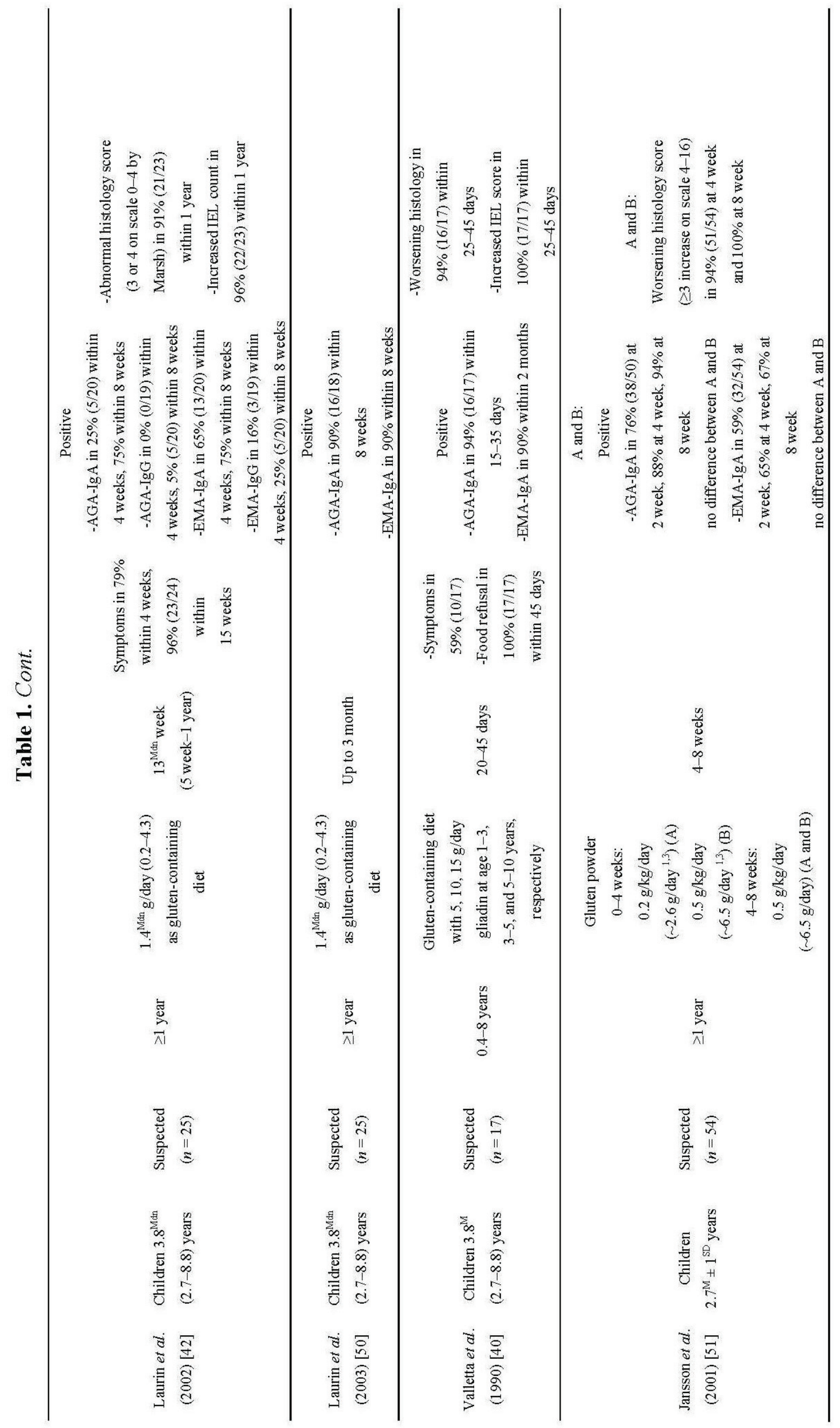




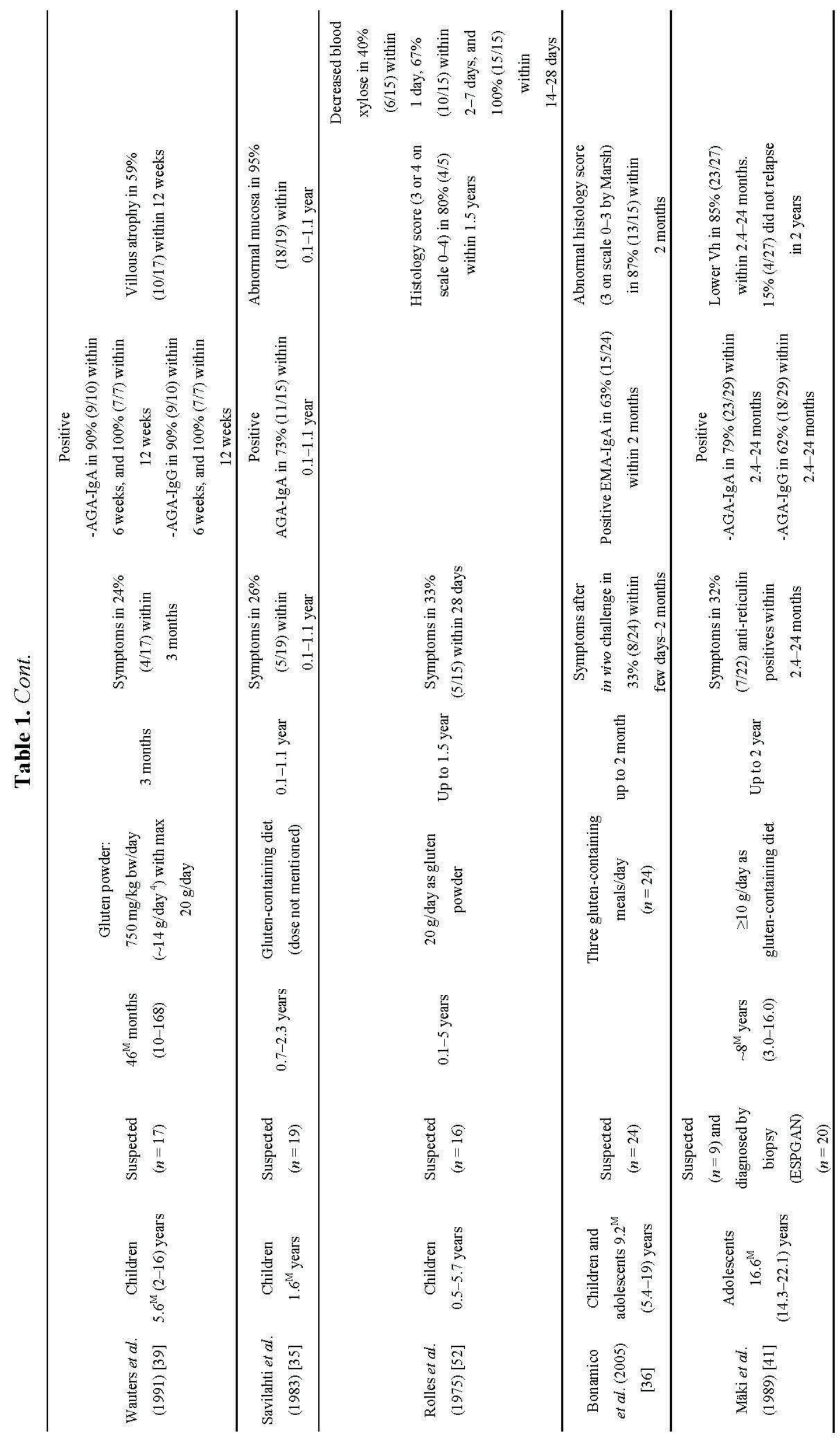




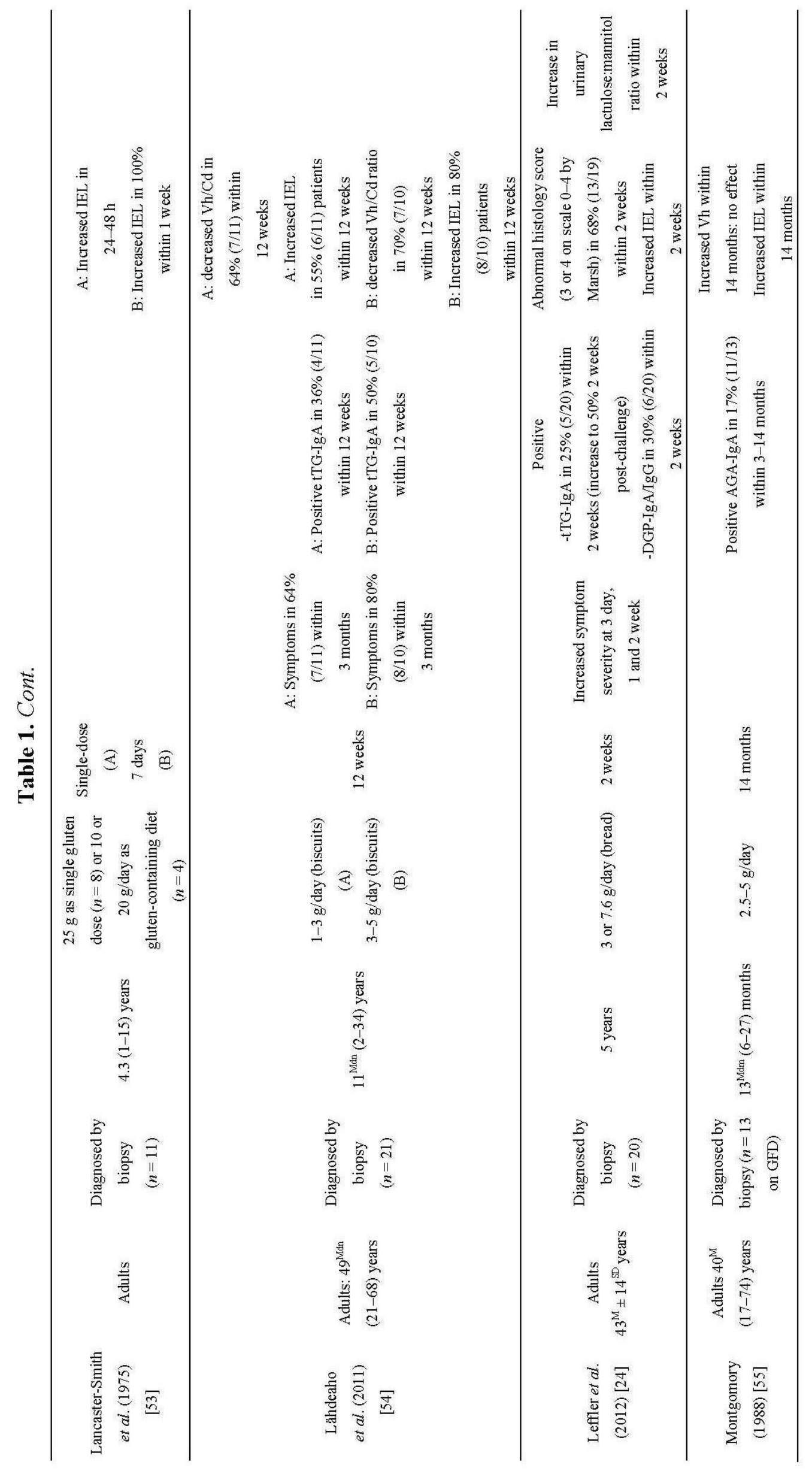




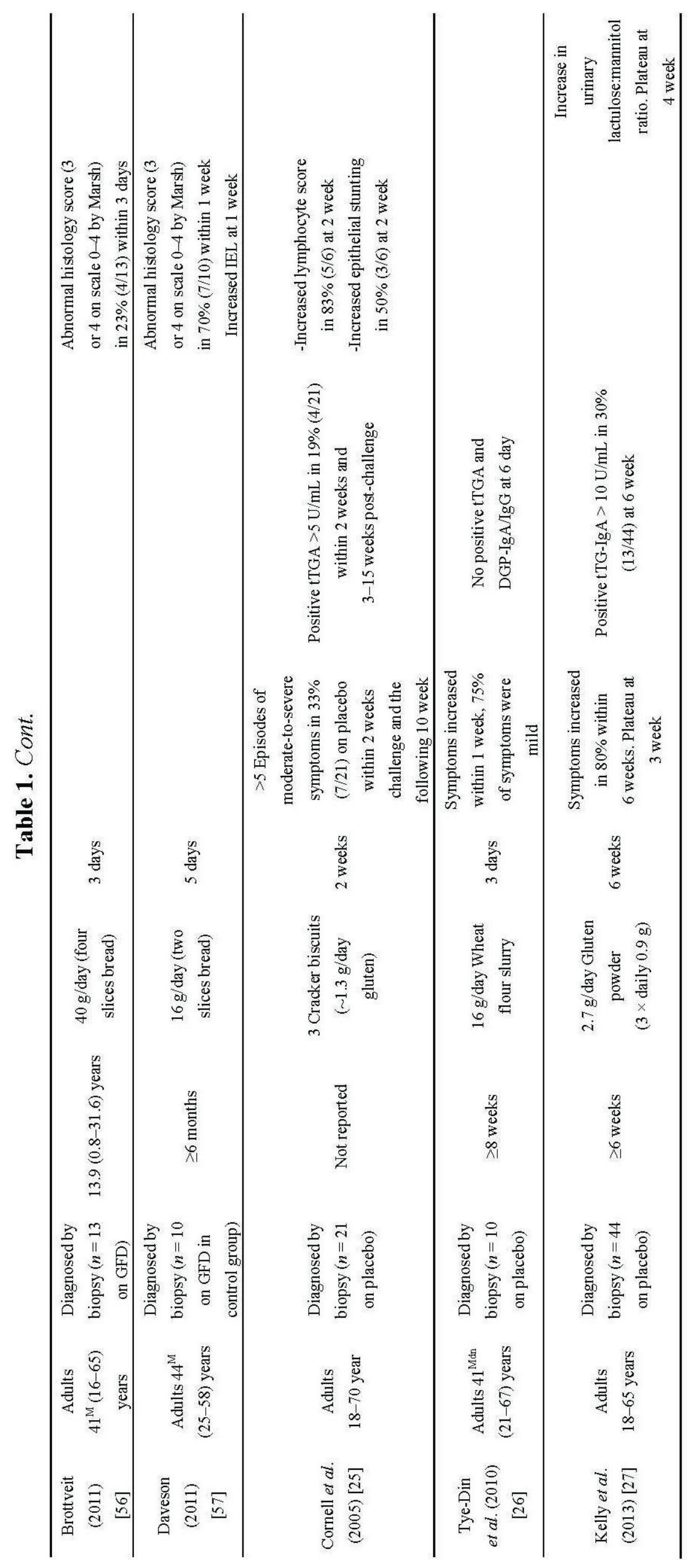




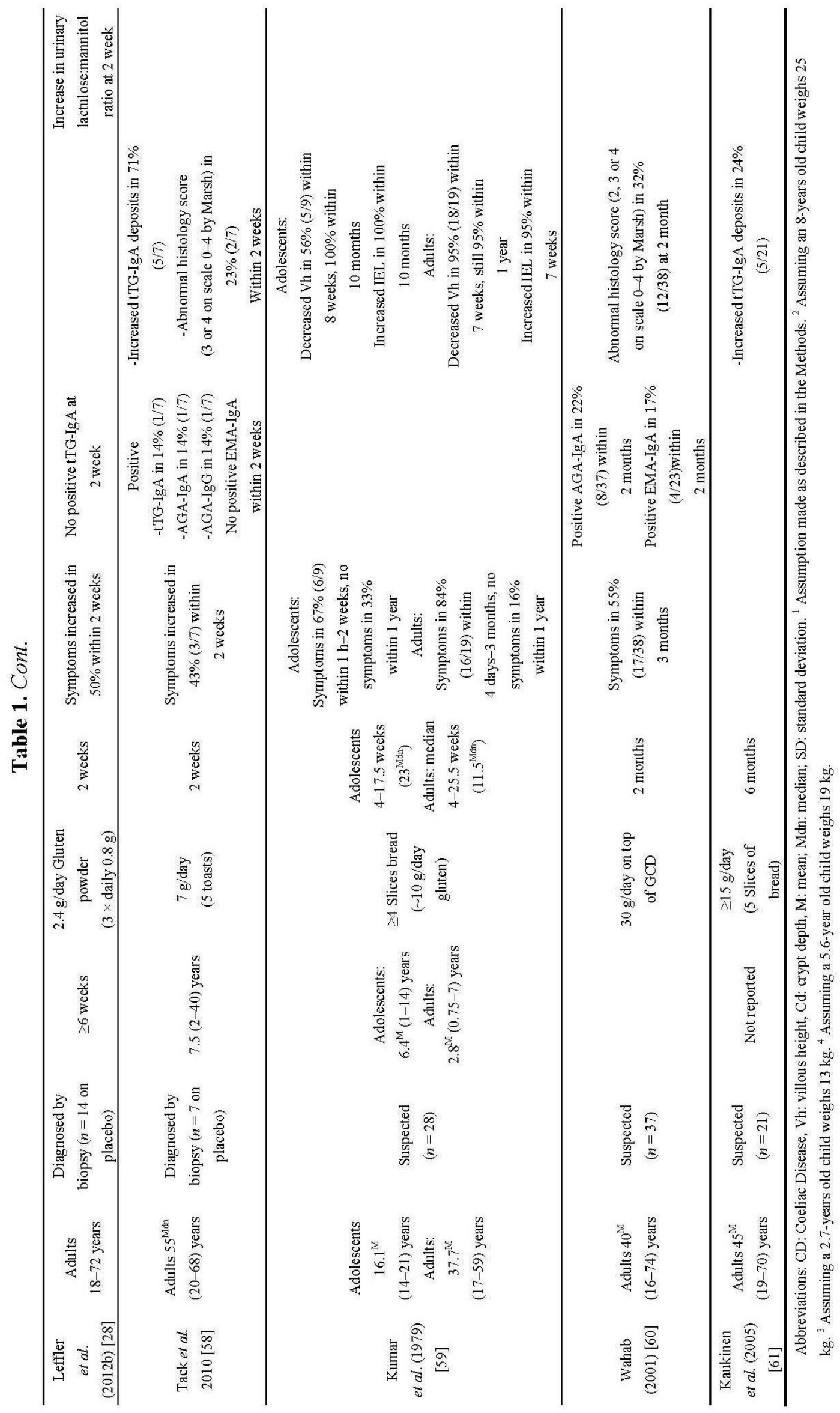




\subsection{CD-Specific Symptoms in Adults with Diagnosed or Suspected CD}

In three studies, effects were reported of gluten challenge on symptoms in diagnosed or suspected adult or adolescent CD patients [54,59,62]. In five clinical trials [25-28,58], the effects of gluten challenge given to diagnosed adult patients in the placebo arm were reported. Reintroduction of a gluten-containing diet induced gastrointestinal symptoms in $77 \%$ of patients suspected of CD between 1 and 8 months of challenge and $\mathrm{CD}$ was confirmed in $40 \%$ of these patients [62]. The diagnosis CD was nevertheless confirmed in $65 \%$ of the $33 \%$ patients who did not develop symptoms. Symptoms occurred in $67 \%$ of patients with confirmed diagnosis of CD. When diagnosed or suspected CD patients received 7 to $10 \mathrm{~g}$ /day of gluten, 43\% [58] and 67\% [59] reported symptoms within two weeks of challenge. Within 3 months $84 \%$ had experienced symptoms [59]. Most symptoms occurred after one week of challenge [59]. After a 2-week and 6-week challenge period with about $2.5 \mathrm{~g}$ /day of gluten three times daily, about $50 \%$ of patients [28] and $80 \%$ of patients [27] reported complaints, respectively. The severity of symptoms increased after 2 weeks [27,28] reaching a plateau at 3 weeks [27]. When diagnosed CD patients received a low (1-3 g/day) or a high (3-5 g/day) dose of gluten, $64 \%$ and $80 \%$ of them, respectively, reported symptoms within 3 months [54]. A 2 -week challenge of $\sim 1.3 \mathrm{~g}$ /day of gluten triggered symptoms in $66 \%$ of patients the following 12 weeks; about 33\% had more than five episodes of moderate to severe symptoms [25].

In summary, the number of adult patients reporting symptoms as well as the severity of symptoms may increase throughout gluten challenge. Within 3 months of gluten challenge, about $64 \%-80 \%$ of adult patients can be expected to experience symptoms. A proportion of patients with CD may never develop symptoms during gluten challenge. The onset of symptoms is rather unpredictable. The appearance of symptoms during gluten challenge is no indicator of CD.

\subsection{Antibodies in Pediatric Patients with Diagnosed or Suspected CD}

\subsubsection{AGA-IgA and AGA-IgG Antibodies}

Figure 1 illustrates the time course of children with diagnosed or suspected $\mathrm{CD}$ responding to a gluten challenge by positive AGA-IgA antibodies.

The proportion of children with diagnosed or suspected $\mathrm{CD}$ responding to gluten challenge by AGA-IgA antibodies varied widely. After 2 weeks, about $30 \%$ to $78 \%$ of children had responded to a challenge providing 3 to $15 \mathrm{~g}$ /day of gluten [20,31,44,63,64]. After 2 to 3 months of challenge with 4 to $14 \mathrm{~g}$ /day of gluten, about $70 \%-100 \%$ of children showed positive AGA-IgA antibodies in their serum $[20,44,46,63,64]$. No clear dose-response effect was observed between the different studies. In two studies with a low dose of gluten $(0.2-4.3 \mathrm{~g} /$ day), the percentage of children responding by AGA-IgA was $90 \%$ [50] or $75 \%$ after 2 months [42]. Within 10 months to 1 year, $73 \%-90 \%$ of children had developed AGA-IgA antibodies [30,35,46]. Interestingly, the percentage of CD children with AGA-IgA was highest (97\%) after a gluten consumption period of about 1 to 3 months and decreases thereafter to $85 \%$ at 1 year, and $49 \%$ after 3 years or more of gluten intake [46]. 
Figure 1. Percentage of pediatric patients with diagnosed or suspected coeliac disease (CD) showing an anti-gliadin antibodies (AGA)-IgA response to gluten over time.

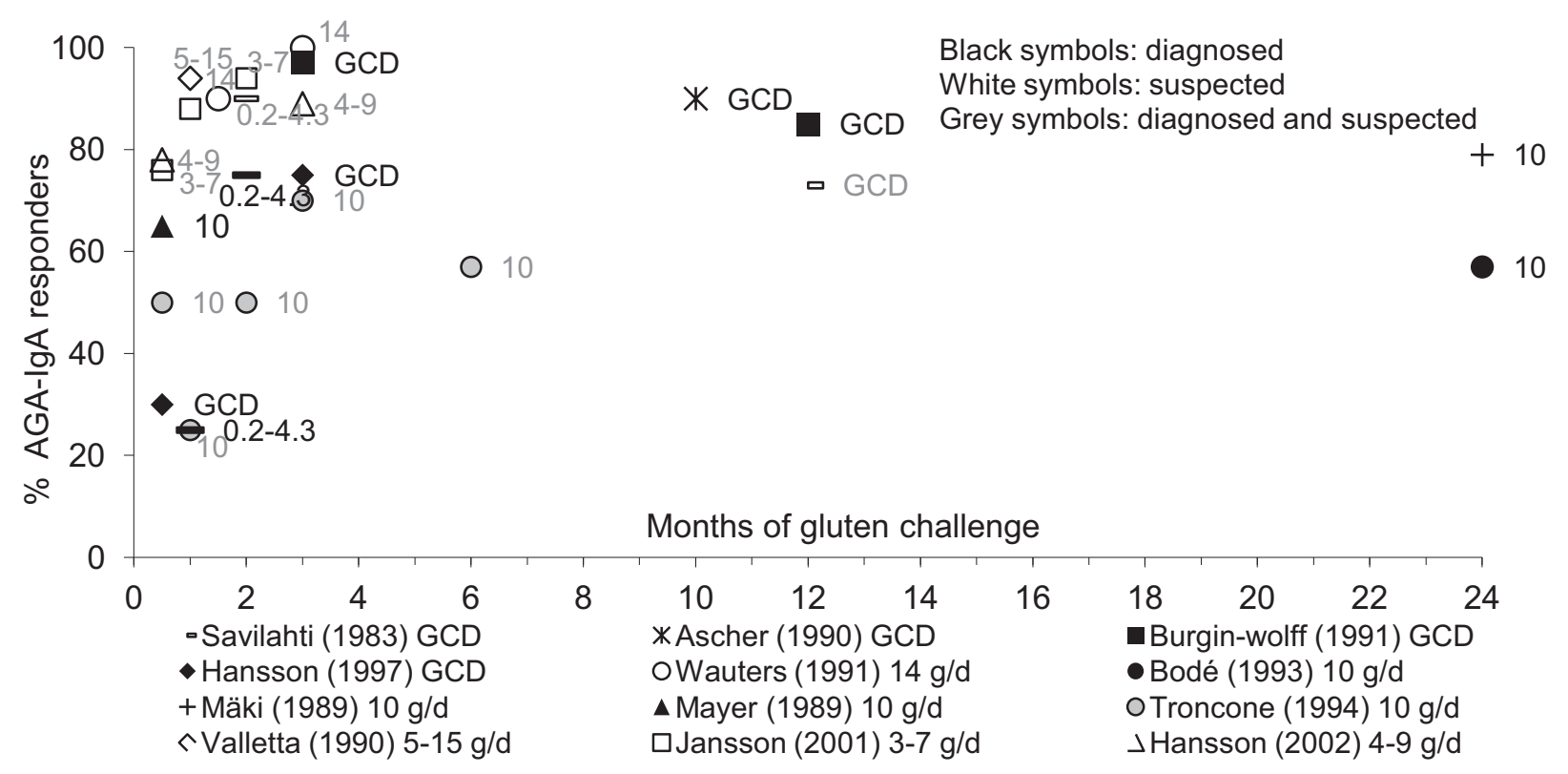

Figure 2 shows the proportion of diagnosed or suspected CD children responding with positive AGA-IgG antibodies to a gluten challenge.

Figure 2. Percentage of pediatric patients with diagnosed or suspected CD showing an AGA-IgG response to gluten over time.

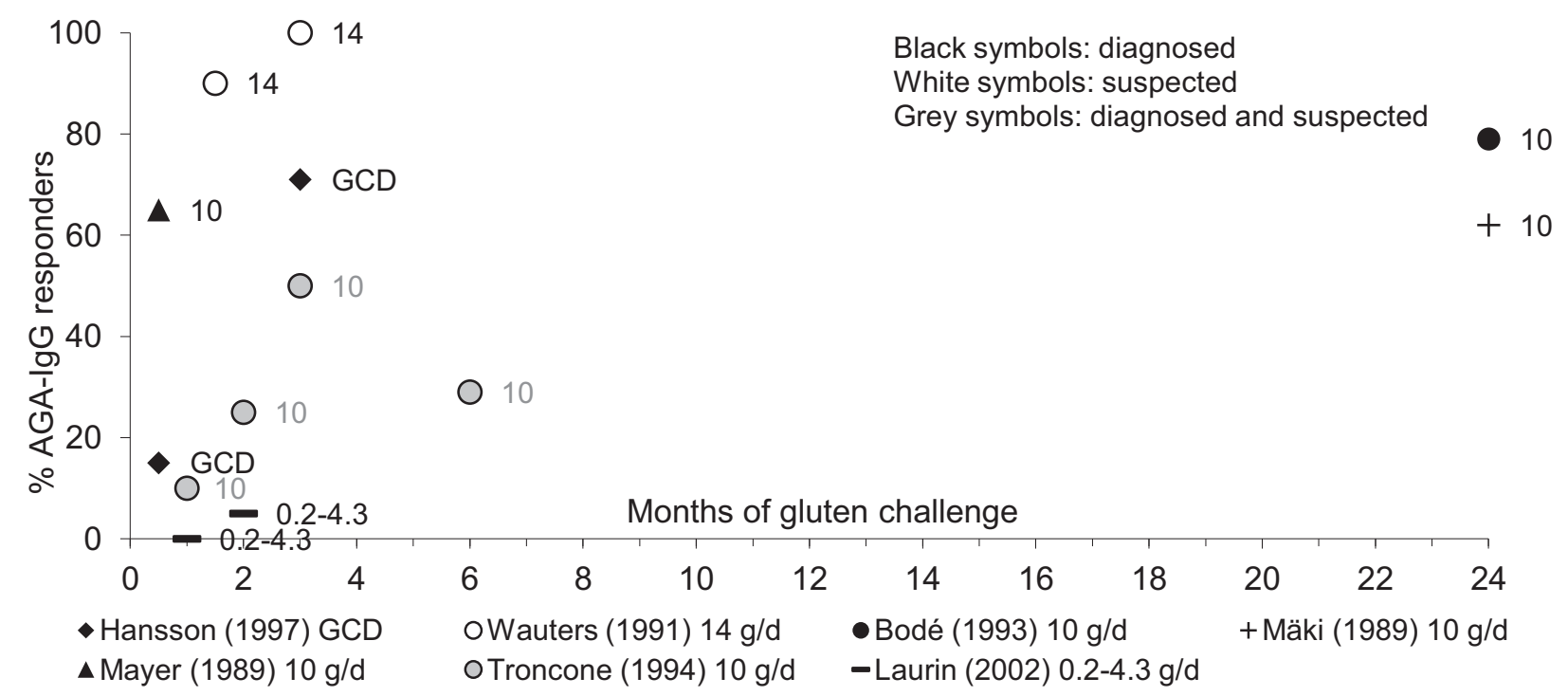

Whereas most studies showed less children responding by AGA-IgG than by AGA-IgA throughout the course of gluten challenge $[41,42,44,63]$, two studies showed similar response rates by AGA-IgA and AGA-IgG [31,39]. When children with CD were given a gluten-containing diet or 10 to $14 \mathrm{~g}$ /day of gluten, AGA-IgG rose significantly in $15 \%$ [44] or $65 \%$ [31] of children within 2 weeks and in $71 \%-100 \%$ of children within 3 months of challenge $[39,44]$. In two studies in which 
children received $10 \mathrm{~g} /$ day [63] or $0.2-4.3 \mathrm{~g} /$ day [42] of gluten, only $25 \%$ and $5 \%$ of children had responded by AGA-IgA after 2 months of gluten challenge, respectively.

\subsubsection{EMA-IgA Antibodies}

Figure 3 summarizes the proportion of children with diagnosed or suspected CD developing positive EMA-IgA during gluten challenge.

Figure 3. Percentage of pediatric patients with diagnosed or suspected CD showing an anti-endomysial antibodies (EMA)-IgA response to gluten over time.

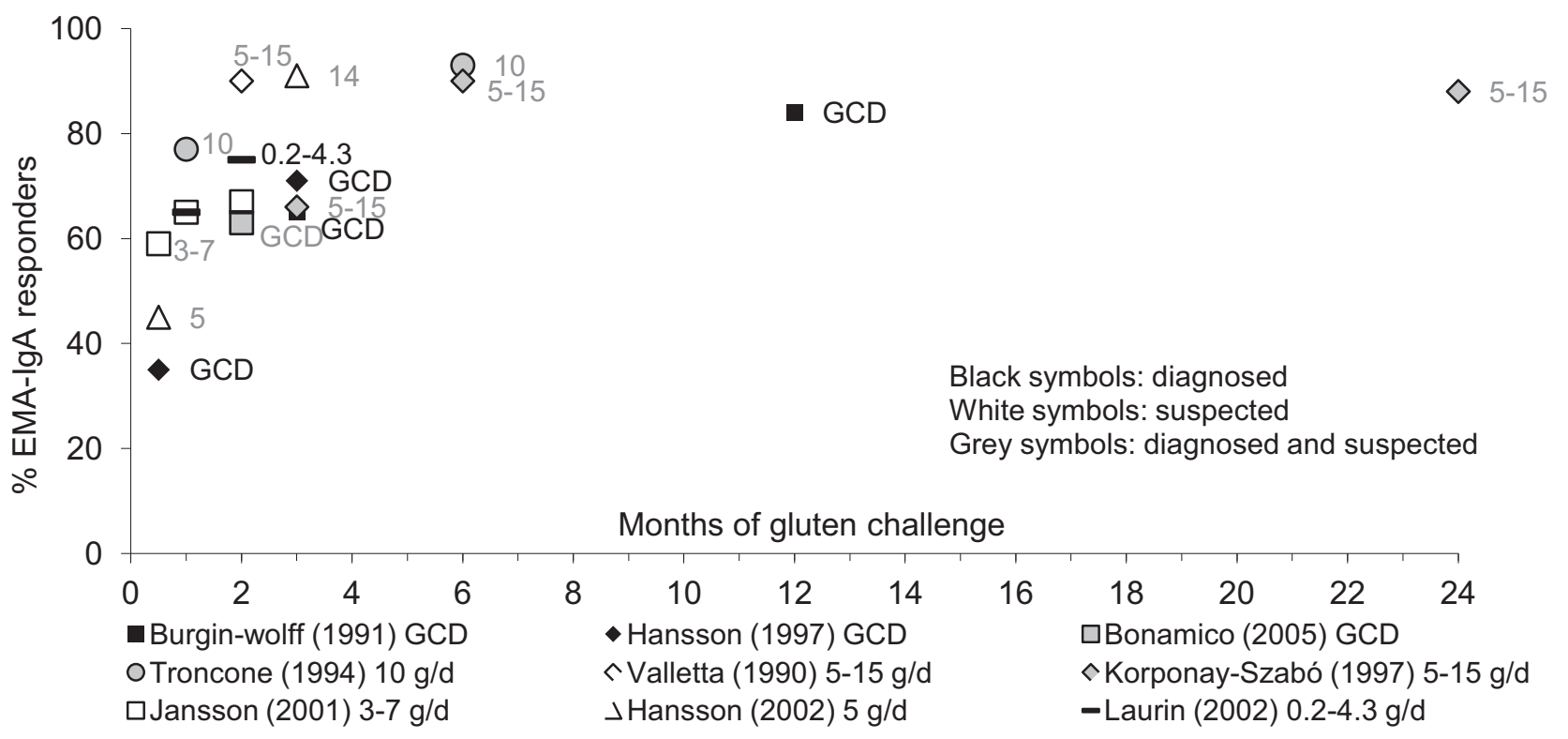

In a number of trials with children with diagnosed or suspected $C D$, results on serum EMA-IgA levels were reported during gluten challenge doses from 0.2 to $15 \mathrm{~g}$ /day of gluten or a gluten-containing diet. After 2 weeks of challenge, $35 \%$ to $59 \%$ of children showed positive EMA-IgA antibodies [20,36,40,42,44,46,63-65], $65 \%$ to $77 \%$ of children after 1 month $[42,51,63]$, between $63 \%$ and $100 \%$ of children became EMA-IgA positive between 2 and 3 months [20,36,38,40,42,44,46,51], while $84 \%$ to $93 \%$ of children had become positive from 6 months to 3 years of challenge [38,46,63]. Even small gluten amounts caused relapse by EMA-IgA [42]. There was no clear difference in time to EMA-IgA positivity between the different gluten doses.

\subsection{3. tTG-IgA Antibodies}

In one study, diagnosed CD children received 4-9 g/day of gluten [20]; positive tTG-IgA levels were detected in $45 \%$ and $89 \%$ of children within 2 and 12 weeks, respectively.

\subsubsection{Antibodies in Pediatric Patients: Summary}

In summary, the time it takes for children to relapse by antibodies with a gluten challenge is variable. Moderate-to-high gluten challenge doses given to children with diagnosed or suspected CD increased 
AGA-IgA, AGA-IgG and EMA-IgA to positive levels within the first few weeks. Within 3 months of challenge, the majority of children had developed AGA-IgA, AGA-IgG, EMA-IgA or tTG-IgA antibodies. Only few children relapsed by AGA-IgA, AGA-IgG and EMA-IgA after 1 year. No clear difference in relapse rate to gluten was observed between children with diagnosed and suspected CD. Conversion of AGA-IgA positive to negative tests has been reported to occur in some patients.

\subsection{Antibodies in Adult Patients with Diagnosed or Suspected CD}

\subsubsection{AGA-IgA and EMA-IgA Antibodies}

In four trials, AGA-IgA antibody titers in gluten-challenged adult patients with suspected or diagnosed CD were reported [26,29,55,60]. The AGA-IgA titers increased in 14\% of diagnosed patients in the placebo arm after a 2-week $7 \mathrm{~g}$ /day gluten challenge [58] and in $85 \%$ of diagnosed patients receiving 2.5-5 g/day of gluten for up to 14 months [55]. Increased AGA-IgA was observed in $22 \%$ of borderline patients receiving $30 \mathrm{~g}$ /day on top of a normal diet for up to 2 months [60], None of the diagnosed CD patients receiving 16 or $7 \mathrm{~g}$ /day of gluten developed positive EMA-IgA antibodies within 2 weeks [29,58]. In borderline patients, 17\% became EMA-IgA positive after a 2-month very high-dose gluten challenge [60].

\subsection{2. tTG-IgA and DGP-IgA/IgG Antibodies}

Figure 4 shows diagnosed adult CD patients responding by tTG-IgA throughout gluten challenge.

Figure 4. Percentage of adult patients with diagnosed CD showing a tTG-IgA response to gluten over time.

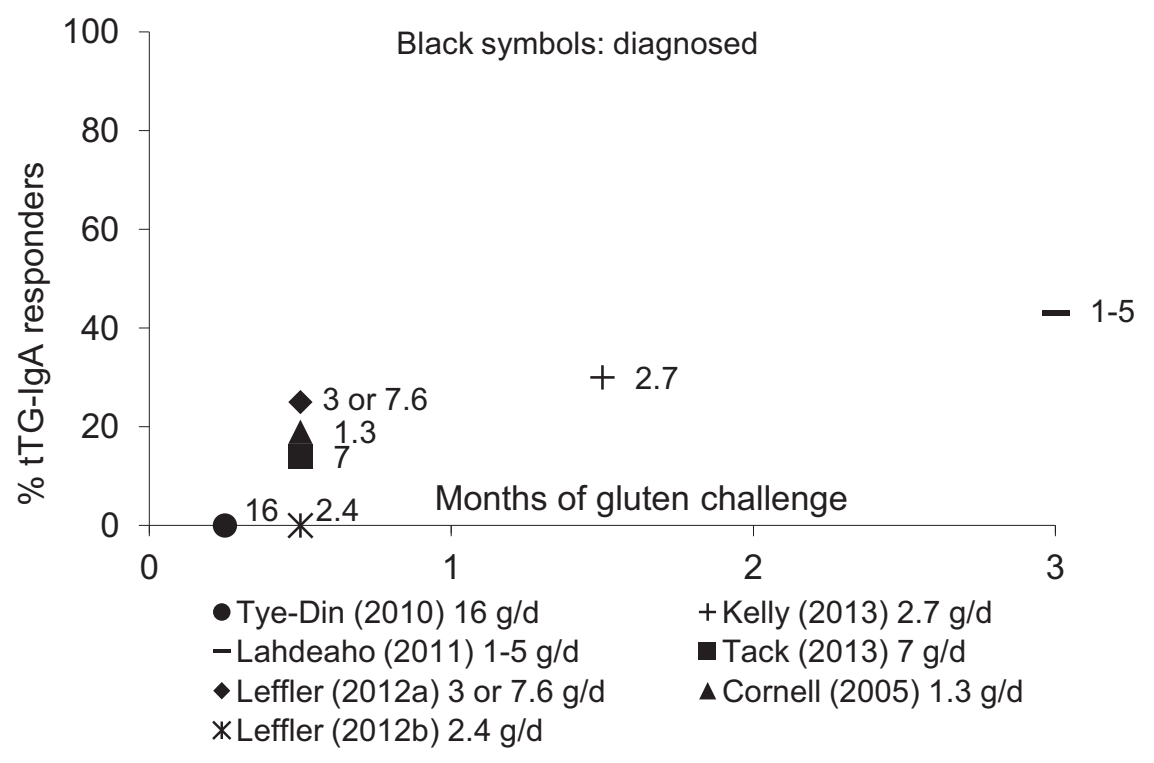

The effects of gluten challenge on tTG-IgA titers in diagnosed adult CD patients were reported either [24-29] or not [54] as part of a clinical trial. No positive tTG-IgA antibodies were observed at day 6 post-challenge in any of the diagnosed CD patients receiving $16 \mathrm{~g}$ of gluten for 3 days [26]. After a 2-week challenge with a dose from 1.3 to $7.6 \mathrm{~g} /$ day of gluten, tTG-IgA increased in $0 \%$ to $25 \%$ of 
adult diagnosed CD patients [24,25,28,29]. Longer gluten challenge form 6 weeks to 3 months increased the proportion of tTG-IgA-positive patients to 30\%-43\% [27,54]. A 3-day gluten challenge of $16 \mathrm{~g}$ /day did not increase DGP-IgA/IgG titers at day 6 [26], but 3 or $7.5 \mathrm{~g} /$ day induced positive DGP-IgA/IgG titers in $30 \%$ of diagnosed patients within two weeks and $45 \%$ the following two weeks [24].

\subsubsection{Antibodies in Adult Patients: Summary}

In summary, few diagnosed $\mathrm{CD}$ patients responded by AGA-IgA, EMA-IgA, tTG-IgA, or DGP-IgA/IgG antibodies after 2 weeks of gluten challenge. Within 6 weeks to 3 months of gluten challenge, still no more than $50 \%$ of patients became positive for these antibodies.

\subsection{Mucosal Immunohistology in Pediatric Patients with Diagnosed or Suspected CD}

\subsubsection{Mucosal IEL}

When children with diagnosed or suspected CD received 5 to $25 \mathrm{~g}$ /day of gluten, $91 \%$ to $100 \%$ of them developed increased mucosal IEL within 1 to 2 months [40,42,43,45,50]. Within 3 months of gluten challenge with $10 \mathrm{~g} /$ day, all children with suspected CD showed increased mucosal IEL counts [49]. In one study less children, $16 \%$ to $25 \%$, responded with increased IEL within 3 months of about $6 \mathrm{~g} /$ day of gluten challenge $[20,44]$. The authors of one study found that the gluten intake dose strongly correlated with the degree of inflammation in the biopsy, as expressed by IEL [42]. A gluten challenge increased IEL in mucosal biopsies before histological changes occurred.

\subsubsection{Mucosal Histology}

Figure 5 gives an overview of children with diagnosed or suspected CD developing changes in mucosal morphology throughout gluten challenge.

The proportion of children with diagnosed or suspected CD having abnormal mucosal histology gradually increased during the course of gluten challenge. Only $7 \%$ of children with diagnosed CD developed mucosal lesions after 1 week when a gluten challenge of 2-3 g/day of gluten was given [18]. However, when doses of 3 to $20 \mathrm{~g} /$ day of gluten were given, the proportion of children developing an abnormal small bowel mucosal histology scores within 1 month ranged from $72 \%$ to $100 \%[43,46,64]$. Mucosal relapse rates after 2 to 3 months of challenge ranged between $51 \%$ and $100 \%$ [31-33,36,39,49,64]. After 5 months to 2 years of gluten challenge, the majority of children have relapsed by mucosal abnormalities, with relapse rates of $79 \%$ to $100 \%$ reported in the different studies $[18,19,31,33,34,41,42,46,48,49,52]$. After 1 year low-dose gluten challenge $(0.2$ to $4.3 \mathrm{~g}$ /day gluten), the proportion of children showing abnormal small bowel mucosal histology scores did not differ from those receiving a higher dose challenge (5 g/day or higher). For some children it took 2 or even 8 years to relapse on gluten [48]. As expected, the intestinal mucosa relapse rate was higher in diagnosed than in suspected patients: within 3 months of gluten intake, respectively, about $60 \%$ and $80 \%$ had relapsed. 
Figure 5. Percentage of pediatric patients with diagnosed or suspected CD showing histological response to gluten over time.

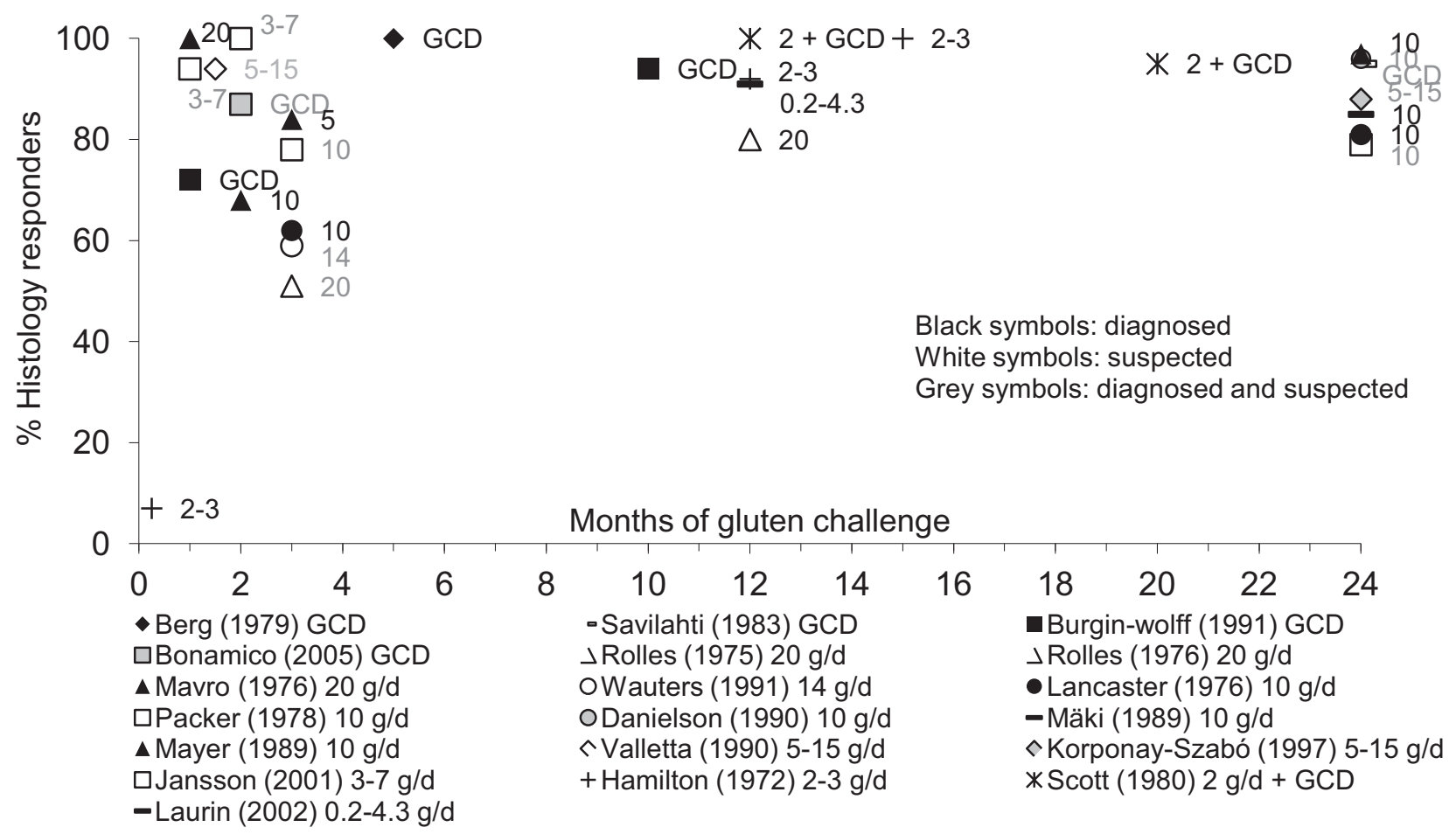

\subsubsection{Mucosal Immunohistology: Summary}

In summary, within 1 month of gluten exposure, mucosal IEL counts were increased in almost all children with diagnosed or suspected CD. The percentage of children developing moderate to severe mucosal histological abnormalities within 2 to 3 months of gluten challenge ranged between $51 \%-100 \%$. When child patients are biopsied after one week of challenge, only a minority show morphological relapse. The majority of children will have relapsed after 2 to 3 months of challenge, and only few children relapse thereafter.

\subsection{Mucosal Immunohistology in Adult Patients with Diagnosed or Suspected CD}

\subsubsection{Mucosal IEL}

A single 25 -g gluten challenge given to adult patients with proven CD increased IEL in the mucosal biopsy as soon as 24-48 $\mathrm{h}$ following challenge [49]. A one-week 10 to $20 \mathrm{~g}$ gluten-containing diet increased IEL density in the mucosal biopsy of all patients [49,57]. Lower gluten doses (3-7.6 g/day) also increased mucosal IEL of patients within 2 weeks [24]. Gluten challenges of 10-25 g/day increased mucosal IEL counts in $95 \%$ to $100 \%$ of adult or adolescent patients with diagnosed or suspected CD within 1 to 2 months [59], and 3 to 14-months [55,59]. Increased IEL were found in $55 \%$ and $80 \%$ of diagnosed CD patients receiving, respectively, $1-3$ $\mathrm{g} /$ day and 3-5 g/day of gluten for 3 months [54]. 


\subsubsection{Mucosal Histology}

Figure 6 illustrates the proportion of adult diagnosed or suspected CD patients responding by abnormal small bowel mucosal histology throughout a gluten challenge.

Figure 6. Percentage of adult patients with diagnosed or suspected CD showing histological response to gluten over time.

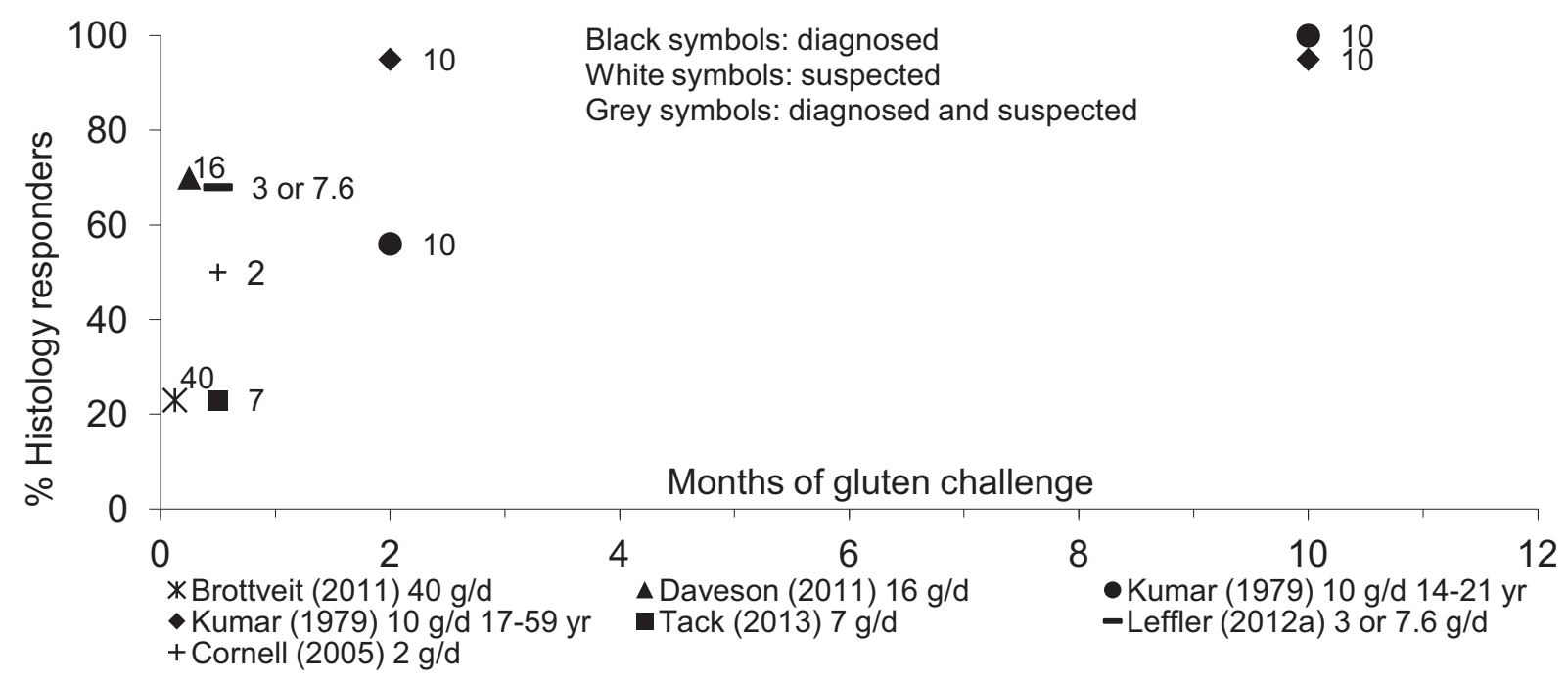

Increased biopsy Marsh scores were observed in 23\% of diagnosed CD patients receiving 40 $\mathrm{g} /$ day of gluten for 3 days [56], and in 70\% of diagnosed CD patients receiving $16 \mathrm{~g}$ /day of gluten for 5 days [57]. Gluten doses between 1 and $7 \mathrm{~g}$ /day induced abnormal histology scores in 23\% to $68 \%$ of adult diagnosed CD patients within 2 weeks [24,25,29], and $67 \%$ of patients within 3 months [54]. In adolescents and adult patients with suspected CD, $10 \mathrm{~g} /$ day of gluten triggered mucosal relapse in $56 \%$ and $95 \%$ of them within 2 months, and $95 \%$ and $100 \%$ of them within 1 year, respectively [59]. Less patients with borderline CD may respond to gluten; 32\% showed abnormal histology scores within 2 months of high-dose gluten challenge [60].

\subsubsection{Mucosal tTGA-IgA Deposits}

A gluten challenge of at least $15 \mathrm{~g} /$ day for 6 months induced positive tTGA-specific mucosal $\operatorname{IgA}$ deposits in $24 \%$ of suspected CD patients [61].

\subsubsection{Mucosal Immunohistology: Summary}

In summary, the results of gluten challenge on mucosal histology in adult patients are variable. More than two weeks of high-dose gluten challenge may be required to induce small intestinal mucosal morphology changes in the majority of patients. However, IEL can appear as early as 1 to 2 days after gluten challenge with increased counts in all patients after 4 weeks. Mucosal tTGA-IgA deposits is another marker appearing in the majority of patients within 2 weeks of challenge. 


\section{Discussion}

\subsection{Strength and Weaknesses}

To our knowledge, this is the first review giving an overview of gluten challenge studies in patients suffering from $\mathrm{CD}$ or suspected of having $\mathrm{CD}$ and the consequences on symptoms, mucosal damage and CD-specific antibodies. In this review, we excluded studies with patients who were on a regular gluten diet at the time of challenge, as their response to gluten may be lower and not representative for patients on a gluten-free diet. This review has, however, several limitations. The gluten challenges used in all studies were wheat-derived and hence, findings relate to wheat gluten. There is very limited data looking at the effect of barley hordein or rye secalin on CD outcomes in the published literature (e.g., [66,67]), but evidence exists that these prolamins induce effects different to wheat gluten, at least at an immunologic level. Moreover, the quoted gluten amounts in the publications were mostly estimates and probably not accurate. In a few studies, gluten amounts were analyzed by R5 ELISA probably providing better estimates. Although several studies looked at the effect of gluten challenge in pediatric patients, the number of studies with adult patients is limited. The clinical response to gluten is most likely larger in diagnosed CD patients than in patients suspected of having $\mathrm{CD}$ in whom part may not have $\mathrm{CD}$. Nevertheless, the results for both groups were combined in the figures. Furthermore, the participants in the different studies convey a heterogeneous group with respect to age, gluten dose, and time on a gluten-free diet, and criteria for diagnosis and are therefore difficult to compare. Also methodologies for measurement of antibodies, biopsies, and histology were different including the cutoff levels used to define antibody or histological positivity. Another limitation is that in most studies in the seventies to nineties, AGA-IgA and AGA-IgG antibodies were most commonly measured. However, particularly AGA-IgA has a poor sensitivity compared to newer antibodies such as EMA-IgA, tTG-IgA, and DGP-IgA/IgG which may have resulted in an underestimation of the patients responding to gluten by positive AGA-IgA titers.

\subsection{Occurrence of Symptoms in Response to Gluten}

Until recently, no proper guidelines for categorizing symptoms were available, making it difficult to compare the symptoms reported in the different studies. Moreover, symptoms in response to gluten are not $\mathrm{CD}$-specific as approximately half of non-coeliac patients also show exacerbation of symptoms during gluten challenge [62]. Gastrointestinal symptoms are not specific for CD. The predictive value of symptoms after gluten re-introduction or gluten challenge is very low $[34,38,39,41,62]$. In one study the positive predictive value of symptoms for having CD was $52 \%$ [62]. In diagnosed adult patients, the symptom response rate seems to range somewhere between $65 \%$ and $85 \%$ [26,59] and most symptoms seem to occur within 1 to 2 weeks [26,59]. Less children $(24 \%-42 \%)$ than adults $(64 \%-80 \%)$ reported symptoms throughout prolonged gluten challenge but this may strongly depend on the methodology used in the different studies.

In summary, symptoms upon gluten challenge are hard to predict and have low positive predictive value. Recently, a validated disease-specific symptom index for coeliac disease was developed, but it 
remains to be established whether this can be used as an independent outcome measure for the monitoring of coeliac disease [68].

\subsection{Occurrence of Antibodies in Response to Gluten}

The CD-specific antibody and mucosal response is more predictable than the appearance of symptoms. Nevertheless, considerable variation between patients exists in the time to serological relapse on gluten [17]. On average, about $70 \%-100 \%$ of diagnosed pediatric CD patients given a moderate to high-dose gluten challenge will have responded by AGA-IgA, EMA-IgA, and tTG-IgA antibodies within 3 months of moderate-to-high gluten intake. Less children responded to gluten by AGA-IgG than by AGA-IgA. Compared to diagnosed patients, slightly less patients suspected of CD developed positive antibodies, but the majority had responded by 3 months. Also low dose prolonged gluten challenge caused serological or histological relapse in children with (suspected) CD [25,69]. In these studies, mucosal changes to gluten correlated with the gluten dose given, suggesting a dose-dependent response to gluten. Histological relapse occurred faster in children receiving a larger gluten dose in children with diagnosed CD [51,70], also suggesting a dose-response effect. Therefore, when testing serological antibodies during gluten challenge of approximately $15 \mathrm{~g} /$ day on a 3 to 6 monthly basis as recommended by the current ESPGHAN recommendations [4], most cases of $\mathrm{CD}$ should be detected. While the majority relapses in three months, for a few patients it may take longer to relapse, and in rare cases it may take years to relapse. Conversion to antibody negativity during prolonged gluten intake has been reported, suggesting that in rare cases gluten tolerance may develop [46,71].

In adult patients, the few available studies suggest that no more than half of the patients develop positive serum antibodies (AGA-IgA, EMA-IgA, tTG-IgA, and DGP-IgA/IgG) in response to a 6-week to 3-month gluten challenge. The few available studies suggest that the AGA-IgA and EMA-IgA response rates of adult CD patients to high-dose gluten challenge was very low. This suggests a lower response in diagnosed adult than pediatric patients. Whether this lower antibody responsiveness to gluten in adults is due to a longer period of gluten withdrawal remains to be established.

\subsection{Occurrence of Histological Changes in Response to Gluten}

About $50 \%$ to $100 \%$ of children with diagnosed or suspected CD developed moderate to severe mucosal histological abnormalities within 2 to 3 months of gluten challenge. Comparable response rates were reported for adult patients. As can be expected, the average 3-month relapse rate in patients with diagnosed CD was generally higher than those with suspected CD. Some patients may still show histological relapse on gluten challenge continuing up to 1 or 2 years.

The earliest stages of gluten challenge include increased density of IEL in the mucosa, crypt hyperplasia, and finally, the development of villous atrophy [72], which was confirmed by the reviewed data. The gluten challenge studies showed that mucosal IEL infiltrates respond fast to gluten (days to weeks) whereas the CD-associated antibodies and mucosal morphological deterioration appeared later within weeks to years. In some studies, relapse by abnormal histology of the small bowel biopsy paralleled positive antibodies [38,40,41,51]. In other studies, antibodies and 
primarily AGA-IgA preceded the worsening of mucosal histology $[30,31,39,46]$ whereas in one study the mucosal changes preceded serum EMA-IgA positivity during gluten challenge [36]. The AGA-IgA antibodies may appear earlier than EMA-IgA during gluten challenge [37,46]. Both in adults and children, symptoms were unpredictable and did not coincide with histological or serological relapse. Within one month of gluten challenge, serological and histological relapse does not occur in all cases during challenge [31,32]. In contrast, increased mucosal IEL were reported in almost all diagnosed pediatric and adult patients within 1 month of gluten challenge $[43,45,49,53]$. High IEL counts in the mucosa are therefore a fast and sensitive marker of responsiveness to gluten although not specific for CD [42,73-75]. In addition, mucosal tTG-IgA deposits are considered to appear rapidly in response to gluten and are both a sensitive and specific marker of early stage CD present in biopsy samples with normal mucosal architecture $[61,76]$. Although not reviewed in this paper, tetramer staining of gluten-specific T-cells may be supportive in the diagnosis of CD due to the fast appearance after start of a gluten challenge [77].

\section{Conclusions}

To diagnose pediatric patients with suspected CD on a gluten-free diet, a moderate-to-high dose gluten challenge for up to 3 months should be sufficient to induce changes in mucosal histology and antibodies in the majority of patients. In adults on a gluten-free diet, histological and serological relapse rates to gluten may be slower and prolonged challenge may be considered if no relapse is observed. Moreover, testing for combinations of conventional and new early markers with high sensitivity and specificity will significantly shorten the time of gluten challenge to diagnose CD.

\section{Acknowledgments}

The author is an employee of DSM Biotechnology Center (Delft, The Netherlands). The author would like to acknowledge Sheryl Roumen (DSM Biotechnology Center) who substantially contributed to searching the literature and compiling the data.

\section{Conflicts of Interest}

The authors declare no conflict of interest.

\section{References}

1. Coeliac Disease: Recognition and Assessment of Coeliac Disease, 2009. Available online: http://www.nice.org.uk/nicemedia/pdf/cg86fullguideline.pdf (accessed on 7 August 2013).

2. Richey, R.; Howdle, P.; Shaw, E.; Stokes, T.; Guideline Development Group. Recognition and assessment of coeliac disease in children and adults: Summary of NICE guidance. BMJ 2009, $338, \mathrm{~b} 1684$.

3. Walker-Smith, J.A.; Guandalini, S.; Schmitz, J.; Shmerling, D.H.; Visakopi, J.L. Revised criteria for diagnosis of coeliac disease. Report of Working Group of European Society of Paediatric Gastroenterology and Nutrition. Arch. Dis. Child 1990, 65, 909-911. 
4. Husby, S.; Koletzko, S.; Korponay-Szabo, I.R.; Mearin, M.L.; Phillips, A.; Shamir, R.; Troncone, R.; Giersiepen, K.; Branski, D.; Catassi, C.; et al. European society for pediatric gastroenterology, hepatology, and nutrition guidelines for the diagnosis of coeliac disease. J. Pediatr. Gastroenterol. Nutr. 2012, 54, 136-160.

5. Rubio-Tapia, A.; Hill, I.D.; Kelly, C.P.; Calderwood, A.H.; Murray, J.A.; American College of Gastroenterology. ACG clinical guidelines: Diagnosis and management of celiac disease. Am.J. Gastroenterol. 2013, 108, 656-676.

6. Hill, I.D.; Dirks, M.H.; Liptak, G.S.; Colletti, R.B.; Fasano, A.; Guandalini, S.; Hoffenberg, E.J.; Horvath, K.; Murray, J.A.; Pivor, M.; et al. Guideline for the diagnosis and treatment of celiac disease in children: Recommendations of the North American society for pediatric gastroenterology, hepatology and nutrition. J. Pediatr. Gastroenterol. Nutr. 2005, 40, 1-19.

7. Murch, S.; Jenkins, H.; Auth, M.; Bremner, R.; Butt, A.; France, S.; Furman, M.; Gillett, P.; Kiparissi, F.; Lawson, M.; et al. Joint BSPGHAN and Coeliac UK guidelines for the diagnosis and management of coeliac disease in children. Arch. Dis. Child 2013, 98, 806-811.

8. Catassi, C.; Fasano, A. Is this really celiac disease? Pitfalls in diagnosis. Curr. Gastroenterol. Rep. 2008, 10, 466-472.

9. Ravelli, A.; Bolognini, S.; Gambarotti, M.; Villanacci, V. Variability of histologic lesions in relation to biopsy site in gluten-sensitive enteropathy. Am. J. Gastroenterol. 2005, 100, $177-185$.

10. Corazza, G.R.; Villanacci, V.; Zambelli, C.; Milione, M.; Luinetti, O.; Vindigni, C.; Chioda, C.; Albarello, L.; Bartolini, D.; Donato, F. Comparison of the interobserver reproducibility with different histologic criteria used in celiac disease. Clin. Gastroenterol. Hepatol. 2007, 5, 838-843.

11. Corazza, G.R.; Villanacci, V. Coeliac disease. J. Clin. Pathol. 2005, 58, 573-574.

12. Weile, B.; Hansen, B.F.; Hagerstrand, I.; Hansen, J.P.; Krasilnikoff, P.A. Interobserver variation in diagnosing coeliac disease. A joint study by Danish and Swedish pathologists. APMIS 2000, 108, 380-384.

13. Freeman, H.J. Refractory celiac disease and sprue-like intestinal disease. World J. Gastroenterol. 2008, 14, 828-830.

14. Guidelines for the Management of Patients with Coeliac Disease. British Society of Gastroenterology. Available online: www.bsg.org.uk (accessed on 8 April 2013).

15. United States Department of Agriculture, Agricultural Research Service. USDA National Nutrient Database for Standard Reference, Release 26. Available online: http://ndb.nal.usda.gov/ (accessed on 24 October 2013).

16. Setty, M.; Hormaza, L.; Guandalini, S. Celiac disease: Risk assessment, diagnosis, and monitoring. Mol. Diagn. Ther. 2008, 12, 289-298.

17. Akobeng, A.K.; Thomas, A.G. Systematic review: Tolerable amount of gluten for people with coeliac disease. Aliment. Pharmacol. Ther. 2008, 27, 1044-1052.

18. Hamilton, J.R.; McNeill, L.K. Childhood celiac disease: Response of treated patients to a small uniform daily dose of wheat gluten. J. Pediatr. 1972, 81, 885-893. 
19. Scott, H.; Ek, J.; Baklien, K.; Brandtzaeg, P. Immunoglobulin-producing cells in jejunal mucosa of children with coeliac disease on a gluten-free diet and after gluten challenge. Scand. J. Gastroenterol. 1980, 15, 81-88.

20. Hansson, T.; Dahlbom, I.; Rogberg, S.; Dannaeus, A.; Hopfl, P.; Gut, H.; Kraaz, W.; Klareskog, L. Recombinant human tissue transglutaminase for diagnosis and follow-up of childhood coeliac disease. Pediatr. Res. 2002, 51, 700-705.

21. Dutch Food Composition Database Online Version 2013/4.0. Available online: http://www.rivm.nl/en/Topics/Topics/D/Dutch_Food_Composition_Database (accessed on 24 October 2013).

22. Van Overbeek, F.M.; Uil-Dieterman, I.G.; Mol, I.W.; Köhler-Brands, L.; Heymans, H.S.; Mulder, C.J. The daily gluten intake in relatives of patients with coeliac disease compared with that of the general Dutch population. Eur. J. Gastroenterol. Hepatol. 1997, 9, 1097-1099.

23. World Health Organisation. The WHO Child Growth Standards. Available online: http://www.who.int/childgrowth/en/ (accessed on 7 August 2013).

24. Leffler, D.; Schuppan, D.; Pallav, K.; Najarian, R.; Goldsmith, J.D.; Hansen, J.; Kabbani, T.; Dennis, M.; Kelly, C.P. Kinetics of the histological, serological and symptomatic responses to gluten challenge in adults with coeliac disease. Gut 2012, 62, 996-1004.

25. Cornell, H.J.; Macrae, F.A.; Melny, J.; Pizzey, C.J.; Cook, F.; Mason, S.; Bhathal, P.S.; Stelmasiak, T. Enzyme therapy for management of coeliac disease. Anon. Scand. J. Gastroenterol. 2005, 40, 1304-1312.

26. Tye-Din, J.A.; Anderson, R.P.; Ffrench, R.A.; Brown, G.J.; Hodsman, P.; Siegel, M.; Botwick, W.; Shreeniwas, R. The effects of ALV003 pre-digestion of gluten on immune response and symptoms in celiac disease in vivo. Clin. Immunol. 2010, 134, 289-295.

27. Kelly, C.P.; Green, P.H.; Murray, J.A.; Dimarino, A.; Colatrella, A.; Leffler, D.A.; Alexander, T.; Arsenescu, R.; Leon, F.; Jiang, J.G.; et al. Larazotide acetate in patients with coeliac disease undergoing a gluten challenge: A randomised placebo-controlled study. Aliment. Pharmacol. Ther. 2013, 37, 252-262.

28. Leffler, D.A.; Kelly, C.P.; Abdallah, H.Z.; Colatrella, A.M.; Harris, L.A.; Leon, F.; Arterburn, L.A.; Paterson, B.M.; Lan, Z.H.; Murray, J.A. A randomized, double-blind study of larazotide acetate to prevent the activation of celiac disease during gluten challenge. Am. J. Gastroenterol. 2012, 107, 1554-1562.

29. Tack, G.J.; van de Water, J.M.; Bruins, M.J.; Kooy-Winkelaar, E.M.; van Bergen, J.; Bonnet, P.; Vreugdenhil, A.C.; Korponay-Szabo, I.; Edens, L.; von Blomberg, B.M.; et al. Consumption of gluten with gluten-degrading enzyme by celiac patients: A pilot-study. World $J$. Gastroenterol. 2013, 19, 5837-5847.

30. Ascher, H.; Lanner, A.; Kristiansson, B. A new laboratory kit for anti-gliadin IgA at diagnosis and follow-up of childhood celiac disease. J. Pediatr. Gastroenterol. Nutr. 1990, 10, 443-450.

31. Mayer, M.; Greco, L.; Troncone, R.; Grimaldi, M.; Pansa, G. Early prediction of relapse during gluten challenge in childhood celiac disease. J. Pediatr. Gastroenterol. Nutr. 1989, 8, 474-479.

32. Rolles, C.J.; McNeish, A.S. Standardised approach to gluten challenge in diagnosing childhood coeliac disease. Br. Med. J. 1976, 1, 1309-1311. 
33. Packer, S.M.; Charlton, V.; Keeling, J.W. Gluten challenge in treated coeliac disease. Arch. Dis. Child 1978, 53, 449-455.

34. Berg, N.O.; Lindberg, T. Incidence of coeliac disease and transient gluten intolerance in children in a Swedish urban community. Acta Paediatr. Scand. 1979, 68, 397-400.

35. Savilahti, E.; Viander, M.; Perkkio, M.; Vainio, E.; Kalimo, K.; Reunala, T. IgA antigliadin antibodies: A marker of mucosal damage in childhood coeliac disease. Lancet 1983, 1, 320-322.

36. Bonamico, M.; Sabbatella, L.; di Tola, M.; Vetrano, S.; Ferri, M.; Nenna, R.; Mariani, P.; Picarelli, A. Antiendomysial antibody detection in biopsy culture allows avoidance of gluten challenge in celiac children. J. Pediatr. Gastroenterol. Nutr. 2005, 40, 165-169.

37. Troncone, R.; Auricchio, R.; Granata, V. Issues related to gluten-free diet in coeliac disease. Curr. Opin. Clin. Nutr. Metab. Care 2008, 11, 329-333.

38. Korponay-Szabo, I.R.; Kovacs, J.B.; Lorincz, M.; Gorácz, G.; Szabados, K.; Balogh, M. Prospective significance of antiendomysium antibody positivity in subsequently verified celiac disease. J. Pediatr. Gastroenterol. Nutr. 1997, 25, 56-63.

39. Wauters, E.A.K.; Jansen, J.; Houwen, R.H.; Veenstra, J.; Ockhuizen, T. Serum IgG and IgA anti-gliadin antibodies as markers of mucosal damage in children with suspected celiac disease upon gluten challenge. J. Pediatr. Gastroenterol. Nutr. 1991, 13, 192-196.

40. Valletta, E.A.; Trevisiol, D.; Mastella, G. IgA anti-gliadin antibodies in the monitoring of gluten challenge in celiac disease. J. Pediatr. Gastroenterol. Nutr. 1990, 10, 169-173.

41. Maki, M.; Lahdeaho, M.L.; Hallstrom, O.; Viander, M.; Visakorpi, J.K. Postpubertal gluten challenge in coeliac disease. Arch. Dis. Child 1989, 64, 1604-1607.

42. Laurin, P.; Wolving, M.; Fälth-Magnusson, K. Even small amounts of gluten cause relapse in children with celiac disease. J. Pediatr. Gastroenterol. Nutr. 2002, 34, 26-30.

43. Mavromichalis, J.; Brueton, M.J.; McNeish, A.S.; Anderson, C.M. Evaluation of the intraepithelial lymphocyte count in the jejunum in childhood enteropathies. Gut 1976, 17, 600-603.

44. Hansson, T.; Dannaeus, A.; Kraaz, W.; Sjöberg, O.; Klareskog, L. Production of antibodies to gliadin by peripheral blood lymphocytes in children with celiac disease: The use of an enzyme-linked immunospot technique for screening and follow-up. Pediatr. Res. 1997, 41, 554-559.

45. Schaad, U.B.; Gaze, H.; Hadorn, B. Intraepithelial lymphocytes before and after gluten challenge in children with celiac disease. Am. J. Dis. Child 1981, 135, 272-273.

46. Burgin-Wolff, A.; Gaze, H.; Hadziselimovic, F.; Huber, H.; Lentze, M.J.; Nusslé, D.; Reymond-Berthet, C. Antigliadin and antiendomysium antibody determination for coeliac disease. Arch. Dis. Child 1991, 66, 941-947.

47. Bode, S.; Weile, B.; Krasilnikoff, P.A.; Gudmand-Høyer, E. The diagnostic value of the gliadin antibody test in celiac disease in children: A prospective study. J. Pediatr. Gastroenterol. Nutr. 1993, 17, 260-264.

48. Danielsson, L.; Stenhammar, L.; Astrom, E. Is gluten challenge necessary for the diagnosis of coeliac disease in young children? Scand. J. Gastroenterol. 1990, 25, 957-960. 
49. Lancaster Smith, M.; Packer, S.; Kumar, P.J.; Harries, J.T. Cellular infiltrate of the jejunum after re introduction of dietary gluten in children with treated coeliac disease. J. Clin. Pathol. 1976, 29, 587-591.

50. Laurin, P.; Fälth-Magnusson, K.; Sundqvist, T. Increase in nitric oxide urinary products during gluten challenge in children with coeliac disease. Scand. J. Gastroenterol. 2003, 38, 55-60.

51. Jansson, U.H.; Gudjonsdottir, A.H.; Ryd, W.; Kristiansson, B. Two different doses of gluten show a dose-dependent response of enteropathy but not of serological markers during gluten challenge in children with coeliac disease. Acta Paediatr. 2001, 90, 255-259.

52. Rolles, C.J.; Anderson, M.; McNeish, A.S. Confirming persistence of gluten intolerance in children diagnosed as having coeliac disease in infancy. Arch. Dis. Child 1975, 50, 259-263.

53. Lancaster-Smith, M.; Kumar, P.J.; Dawson, A.M. The cellular infiltrate of the jejunum in adult coeliac disease and dermatitis herpetiformis following the reintroduction of dietary gluten. Gut 1975, 16, 683-688.

54. Lahdeaho, M.L.; Maki, M.; Laurila, K.; Huhtala, H.; Kaukinen, K. Small-bowel mucosal changes and antibody responses after low- and moderate-dose gluten challenge in celiac disease. BMC Gastroenterol. 2011, 11, 129.

55. Montgomery, A.M.; Goka, A.K.; Kumar, P.J.; Farthing, M.J.; Clark, M.L. Low gluten diet in the treatment of adult coeliac disease: Effect on jejunal morphology and serum anti-gluten antibodies. Gut 1988, 29, 1564-1568.

56. Brottveit, M.; Raki, M.; Bergseng, E.; Fallang, L.E.; Simonsen, B.; Løvik, A.; Larsen, S.; Løberg, E.M.; Jahnsen, F.L.; Sollid, L.M.; et al. Assessing possible celiac disease by an HLA-DQ2-gliadin Tetramer Test. Am. J. Gastroenterol. 2011, 106, 1318-1324.

57. Daveson, A.J.; Jones, D.M.; Gaze, S.; McSorley, H.; Clouston, A.; Pascoe, A.; Cooke, S.; Speare, R.; Macdonald, G.A.; Anderson, R.; et al. Effect of hookworm infection on wheat challenge in celiac disease-A randomised double-blinded placebo controlled trial. PLoS One 2011, 6, e17366.

58. Tack, G.J.; van de Water, J.M.; Kooy-Winkelaar, E.M.; van Bergen, J.; Meijer, G.A.; von Blomberg, B.M.; Schreurs, M.W.; Bruins, M.J.; Edens, L.; Mulder, C.J.; et al. 379 Can prolyl endoprotease enzyme treatment mitigate the toxic effect of gluten in coeliac patients? Gastroenterology 2010, 138, S54.

59. Kumar, P.J.; O’Donoghue, D.P.; Stenson, K.; Dawson, A.M. Reintroduction of gluten in adults and children with treated coeliac disease. Gut 1979, 20, 743-749.

60. Wahab, P.J.; Crusius, J.B.A.; Meijer, J.W.; Mulder, C.J. Gluten challenge in borderline gluten-sensitive enteropathy. Am. J. Gastroenterol. 2001, 96, 1464-1469.

61. Kaukinen, K.; Peraaho, M.; Collin, P.; Partanen, J.; Woolley, N.; Kaartinen, T.; Nuutinen, T.; Halttunen, T.; Mäki, M.; Korponay-Szabo, I. Small-bowel mucosal transglutaminase 2-specific IgA deposits in coeliac disease without villous atrophy: A prospective and randomized clinical study. Scand. J. Gastroenterol. 2005, 40, 564-572.

62. Campanella, J.; Biagi, F.; Bianchi, P.I.; Zanellati, G.; Marchese, A.; Corazza, G.R. Clinical response to gluten withdrawal is not an indicator of coeliac disease. Scand. J. Gastroenterol. 2008, 43, 1311-1314. 
63. Troncone, R.; Caputo, N.; Micillo, M.; Maiuri, L.; Poggi, V. Immunologic and intestinal permeability tests as predictors of relapse during gluten challenge in childhood coeliac disease. Scand. J. Gastroenterol. 1994, 29, 144-147.

64. Jansson, U.H.; Kristiansson, B.; Magnusson, P.; Larsson, L.; Albertsson-Wikland, K.; Bjarnason, $\mathrm{R}$. The decrease of IGF-I, IGF-binding protein-3 and bone alkaline phosphatase isoforms during gluten challenge correlates with small intestinal inflammation in children with coeliac disease. Eur. J. Endocrinol. 2001, 144, 417-423.

65. Korponay-Szabo, I.; Kovacs, J.; Lorincz, M.; Körmendy, M.; Sashegyi, J. New cases of celiac disease detected by anti-endomysial antibody test in families of gluten-sensitive patients and among children examined for non-specific gastrointestinal complaints. Orv. Hetil. 1993, 134, 15-20.

66. Baker, P.G.; Read, A.E. Oats and barley toxicity in coeliac patients. Postgrad. Med. J. 1976, 52, 264-268.

67. Anand, B.S.; Piris, J.; Truelove, S.C. The role of various cereals in coeliac disease. Q. J. Med. 1978, 47, 101-110.

68. Leffler, D.A.; Dennis, M.; Edwards George, J.; Jamma, S.; Cook, E.F.; Schuppan, D.; Kelly, C.P. A validated disease-specific symptom index for adults with celiac disease. Clin. Gastroenterol. Hepatol. 2009, 7, 1328-1334.e3.

69. Anderson, R.P.; van Heel, D.A.; Tye-Din, J.A.; Barnardo, M.; Salio, M.; Jewell, D.P.; Hill, A.V.S. T cells in peripheral blood after gluten challenge in coeliac disease. Gut 2005, 54, $1217-1223$.

70. Catassi, C.; Rossini, M.; Ratsch, I.M.; Bearzi, I.; Santinelli, A.; Castagnani, R.; Pisani, E.; Coppa, G.V.; Giorgi, P.L. Dose dependent effects of protracted ingestion of small amounts of gliadin in coeliac disease children: A clinical and jejunal morphometric study. Gut 1993, 34, 1515-1519.

71. Hadziselimovic, F.; Bürgin-Wolff, A. P0429: Indication of gluten tolerance development in patients with coeliac disease. J. Pediatr. Gastroenterol. Nutr. 2004, 39, S218-S219.

72. Murray, J.A. The widening spectrum of celiac disease. Am. J. Clin. Nutr. 1999, 69, 354-365.

73. Chang, F.; Mahadeva, U.; Deere, H. Pathological and clinical significance of increased intraepithelial lymphocytes (IELs) in small bowel mucosa. APMIS 2005, 113, 385-399.

74. Collin, P.; Wahab, P.J.; Murray, J.A. Intraepithelial lymphocytes and coeliac disease. Best Pract. Res. Clin. Gastroenterol. 2005, 19, 341-350.

75. Ferguson, A.; Arranz, E.; O'Mahony, S. Clinical and pathological spectrum of coeliac disease-Active, silent, latent, potential. Gut 1993, 34, 150-151.

76. Koskinen, O.; Collin, P.; Korponay-Szabo, I.; Salmi, T.; Iltanen, S.; Haimila, K.; Partanen, J.; Mäki, M.; Kaukinen, K. Gluten-dependent small bowel mucosal transglutaminase 2-specific IgA deposits in overt and mild enteropathy coeliac disease. J. Pediatr. Gastroenterol. Nutr. 2008. $47,436-442$.

77. Raki, M.; Fallang, L.E.; Brottveit, M.; Bergseng, E.; Quarsten, H.; Lundin, K.E.; Sollid, L.M. Tetramer visualization of gut-homing gluten-specific T cells in the peripheral blood of celiac disease patients. Proc. Natl. Acad. Sci. USA 2007, 104, 2831-2836. 
Reprinted from Nutrients. Cite as: Toft-Hansen, H.; Nielsen, C.; Biagini, M.; Husby, S.; Lillevang, S.T. Lectin Staining Shows no Evidence of Involvement of Glycocalyx/Mucous Layer Carbohydrate Structures in Development of Celiac Disease. Nutrients 2013, 5, 4540-4552.

Article

\title{
Lectin Staining Shows no Evidence of Involvement of Glycocalyx/Mucous Layer Carbohydrate Structures in Development of Celiac Disease
}

\author{
Henrik Toft-Hansen ${ }^{1,2, *}$, Christian Nielsen ${ }^{2}$, Matteo Biagini ${ }^{3}$, Steffen Husby ${ }^{1}$ \\ and Søren T. Lillevang ${ }^{2}$
}

1 Hans Christian Andersen Children's Hospital, Odense University Hospital, Sdr. Boulevard 29, Odense 5000, Denmark; E-Mail: steffen.husby@rsyd.dk

2 Department of Clinical Immunology, Odense University Hospital, Sdr. Boulevard 29, Odense 5000, Denmark; E-Mails: christian.nielsen@rsyd.dk (C.N.); soren.lillevang@rsyd.dk (S.T.L.)

3 Department of Pathology, Odense University Hospital, J.B. Winsløws Vej 15, 2., Odense 5000, Denmark; E-Mail: matteo.biagini@rsyd.dk

* Author to whom correspondence should be addressed; E-Mail: htoft-hansen@health.sdu.dk; Tel.: +45-6541-1269; Fax: +45-6591-1862.

Received: 23 September 2013; in revised form: 29 October 2013 / Accepted: 6 November 2013 / Published: 18 November 2013

\begin{abstract}
The presence of unique carbohydrate structures in the glycocalyx/mucous layer of the intestine may be involved in a susceptibility to celiac disease (CD) by serving as attachment sites for bacteria. This host-microbiota interaction may influence the development of $\mathrm{CD}$ and possibly other diseases with autoimmune components. We examined duodenal biopsies from a total of 30 children, of which 10 had both celiac disease (CD) and type 1 diabetes (T1D); 10 had CD alone; and 10 were suspected of having gastrointestinal disease, but had normal duodenal histology (non-CD controls). Patients with both CD and T1D were examined before and after remission following a gluten-free diet. We performed lectin histochemistry using peanut agglutinin (PNA) and Ulex europaeus agglutinin (UEA) staining for Gal- $\beta(1,3)$-GalNAc and Fuc $\alpha 1-2$ Gal-R, respectively, of the glycocalyx/mucous layer. The staining was scored based on dissemination of stained structures on a scale from 0 to 3. Evaluation of the scores revealed no difference between biopsies obtained before and after remission in the group of children with both $\mathrm{CD}$ and T1D. A comparison of this pre-remission group with the children who had $\mathrm{CD}$ alone or the non-CD controls also showed no significant differences. Based on our material, we found no indication that the presence of
\end{abstract}


Gal- $\beta(1,3)-$ GalNAc or Fuc $\alpha 1-2$ Gal-R is involved in the susceptibility to CD, or that the disease process affects the expression of these carbohydrates.

Keywords: pediatric celiac disease; type 1 diabetes; duodenal biopsies; glycosylation; lectin staining

\section{Introduction}

The role of the adaptive immune system in celiac disease (CD) has been studied in detail over the past few decades, and its association with HLA-DQ2 and DQ8 is well established [1]. Association with other genomic regions has been demonstrated, but the individual impact of each region is small, and, in combination, these regions can only explain about $5 \%$ of the total genetic disease susceptibility [2]. Recently, there has been a surge of interest in the possible role of the microbiota in the etiology of $\mathrm{CD}$ and a wide range of other diseases, particularly with autoimmune components $[3,4]$.

The glycocalyx/mucous layer covers the gastrointestinal tract, and acts as a semi-permeable barrier between the lumen and the epithelium. Bacteria in the host microbiota may use carbohydrate structures in the glycocalyx/mucous layer as attachment sites [5]. Inherited differences in expression of certain carbohydrates could lead to a predisposition to colonization with particular strains of bacteria, which may influence the susceptibility to CD. Alternatively, the presence of certain bacteria independent of inherited factors could cause an environmental predisposition to CD by altering the expression of carbohydrates. Also, the disease process involving inflammation of the gut mucosa could affect the expression of carbohydrates [6].

Bacteria that are decreased or enriched in the gut of CD patients compared to healthy controls could be important in maintaining gut homeostasis in the healthy individuals or in compromising the epithelial function in $\mathrm{CD}$ [7]. It has been shown that rod-shaped bacteria were frequently found in the mucosa of pediatric CD patients with active disease, as well as in another group of pediatric CD patients in remission on a gluten-free diet (GFD), though not in controls with no known food intolerances [8]. Since the rod-shaped bacteria were also present in the children in remission, this indicates that $\mathrm{CD}$ patients could be genetically predisposed to colonization with these particular bacteria, perhaps due to the expression of particular carbohydrates.

The prevalence of CD in patients with Type 1 Diabetes (T1D) is estimated to be around $8 \%$ compared to around $1 \%$ in the general population [9]. CD and T1D share genetic risk factors with a strong association to HLA-DQ2 or DQ8 for both diseases [10]. According to Danish guidelines, all children diagnosed with T1D are screened by serology for the presence of CD-associated antibodies. In contrast to children with $\mathrm{CD}$ alone, biopsies are also taken after the implementation of a gluten-free diet in children with both diseases. By studying biopsies obtained from the same children with $\mathrm{CD}$ and T1D before and after remission, as well as children with $\mathrm{CD}$ alone or no $\mathrm{CD}$ at all, we aimed to investigate whether or not the expression of unique carbohydrate structures appeared to be genetically determined or affected by the inflammatory status of the tissue. 


\section{Materials and Methods}

\subsection{Study Design}

We obtained duodenal biopsies from a total of 30 children. Among this group, 10 had both celiac disease (CD) and type 1 diabetes (T1D) (aged 5-11 years, mean =8.6); 10 had CD but not T1D (aged $1-14$ years, mean $=5.9$ ); and 10 were non-CD controls, verified to have normal duodenal histology (aged 2-17, mean = 7.9), but who could have other gastrointestinal diseases, chiefly gastroesophageal reflux disease. The group with both CD and T1D were examined both before and after remission (2-4 years after first biopsy) following introduction of gluten-free diet (GFD). Biopsies were scored according to the Modified Marsh classification [11] (Table 1).

Table 1. Biopsy Marsh scores for individual patients. Group A consists of patients with both celiac disease (CD) and type 1 diabetes, so each patient has two scores: untreated and after remission (GFD). Group B patients have CD only, and Group C patients do not have $\mathrm{CD}$ (normal biopsies), but may have other diseases.

\begin{tabular}{ccccccc}
\hline $\begin{array}{c}\text { Group A } \\
\text { Patient ID }\end{array}$ & $\begin{array}{c}\text { CD + T1D } \\
\text { Untreated }\end{array}$ & $\begin{array}{c}\text { CD + T1D } \\
\text { GFD }\end{array}$ & $\begin{array}{c}\text { Group B } \\
\text { Patient ID }\end{array}$ & CD & $\begin{array}{c}\text { Group C } \\
\text { Patient ID }\end{array}$ & Non-CD \\
\hline A1 & 3A & 0 & B1 & 2 & C1 & 0 \\
A2 & 3C & 0 & B2 & 3B & C2 & 0 \\
A3 & 2 & 0 & B3 & 3 C & C3 & 0 \\
A4 & 2 & 0 & B4 & $3 B$ & C4 & 0 \\
A5 & 3B & 0 & B5 & $3 B$ & C5 & 0 \\
A6 & 3C & 0 & B6 & 3C & C6 & 0 \\
A7 & 3C & 0 & B7 & 3C & C7 & 0 \\
A8 & 3A & 0 & B8 & 3C & C8 & 0 \\
A9 & 3B & 0 & B9 & 3C & C9 & 0 \\
A10 & 3C & 0 & B10 & 3C & C10 & 0 \\
\hline
\end{tabular}

\subsection{Lectin Histochemistry}

We performed lectin histochemistry using peanut agglutinin (PNA) and Ulex europaeus agglutinin (UEA) staining carbohydrates in glycolipids or glycoproteins of the glycocalyx/mucous layer. PNA specifically binds the galactose sequence Gal- $\beta(1,3)$-GalNAc, and UEA specifically binds the fucose sequence Fucal-2Gal-R [12]. Briefly, duodenal biopsies were embedded and snap-frozen in liquid nitrogen immediately after gastroscopy. Cryosections were fixed, followed by blocking of endogen biotin with an Avidin Biotin kit (DAKO, Glostrup, Denmark) and then incubated with biotinylated lectin, rinsed, and followed by incubation with horse radish peroxidase (HRP)-conjugated streptavidin (DAKO). Bound lectins were visualized using the HRP substrate carbazol.

The staining was scored based on dissemination of stained structures in the epithelial cell layer on a scale from 0 to 3, where 0 represents no staining of the epithelial cell layer, 1 represents staining in the cytoplasm of epithelial cells, 2 represents staining of cytoplasm and glycocalyx, and 3 represents staining of cytoplasm and glycocalyx, in addition to villus goblet cells. The observed staining pattern 
was hierarchical as described. Staining of crypt goblet cells was not considered in the analysis. Intensity of staining was not included in the score.

\subsection{Ethics}

The study was approved by the Regional Committee for Biomedial Research (VF-20050134) and by the Danish Data Protection Agency (DOK 2709660991).

\subsection{Statistical Methods}

Statistical analysis was performed with non-parametrical tests using the GraphPad Prism software [13]. A statistical significance limit of $p<0.05$ was chosen.

\section{Results}

PNA staining patterns are illustrated in Figure 1 with examples of staining in samples from a CD patient (Figure 1a), a CD + T1D patient untreated and treated with a GFD (Figure 1b,c, respectively), and finally a non-CD patient (Figure 1d). Some degree of PNA staining was evident in all samples in all groups (Table 2) with a score of 1 as the lowest (Figure 1a), where only cytoplasmic was observed. Figure 1b,c show PNA staining of biopsies obtained from the same patient (A7) with both $\mathrm{CD}$ and T1D before and after treatment with GFD. In the case of patient A7, the score went up from 2 in the untreated state to 3 after treatment, but this was not a consistent trend for this group of patients as a whole. A score of 2 (Figure 1b) includes staining of the brush border as well as cytoplasm, and a score of 3 (Figure 1c,d) indicates additional staining in some, but not necessarily all villus goblet cells. The PNA staining in goblet cells appeared localized to spots inside the cells.

Figure 1. Peanut agglutinin (PNA) staining. (a) Patient B3 (CD, untreated), score 1; (b) Patient A7 (CD+T1D, untreated), score 2; (c) Patient A7 (CD + T1D, on GFD), score 3; (d) Patient C2 (non-CD), score 3. Thin arrows mark staining of cytoplasm of epithelial cells. Thick arrows mark staining of glycocalyx. Arrowheads mark staining in goblet cells.

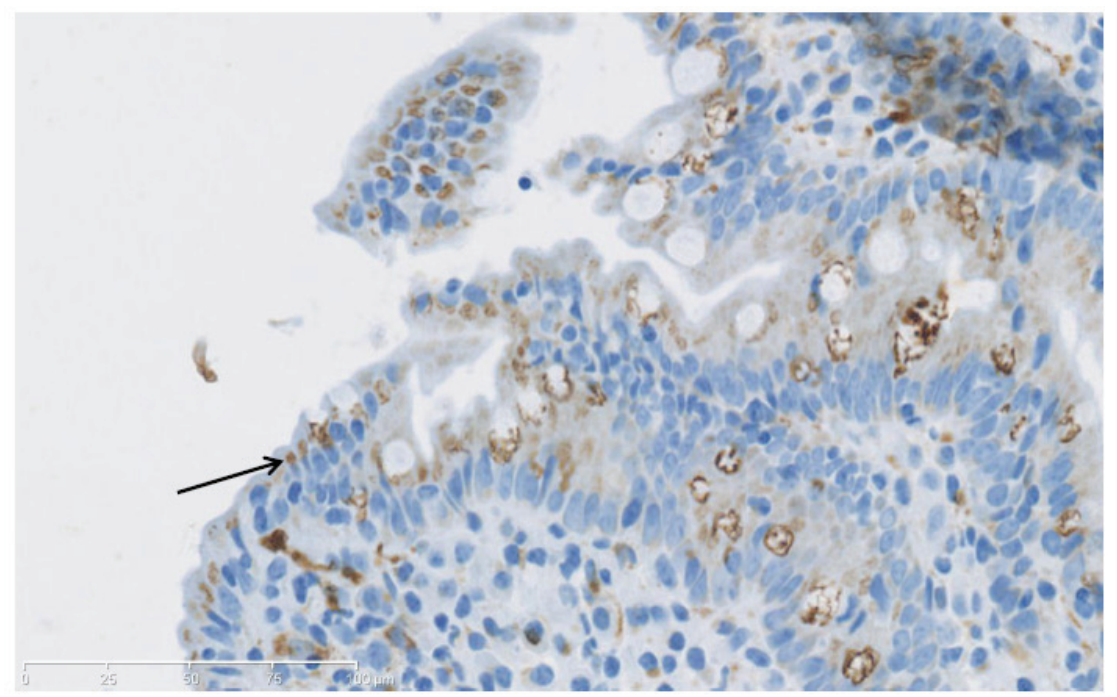

(a) 
Figure 1. Cont.

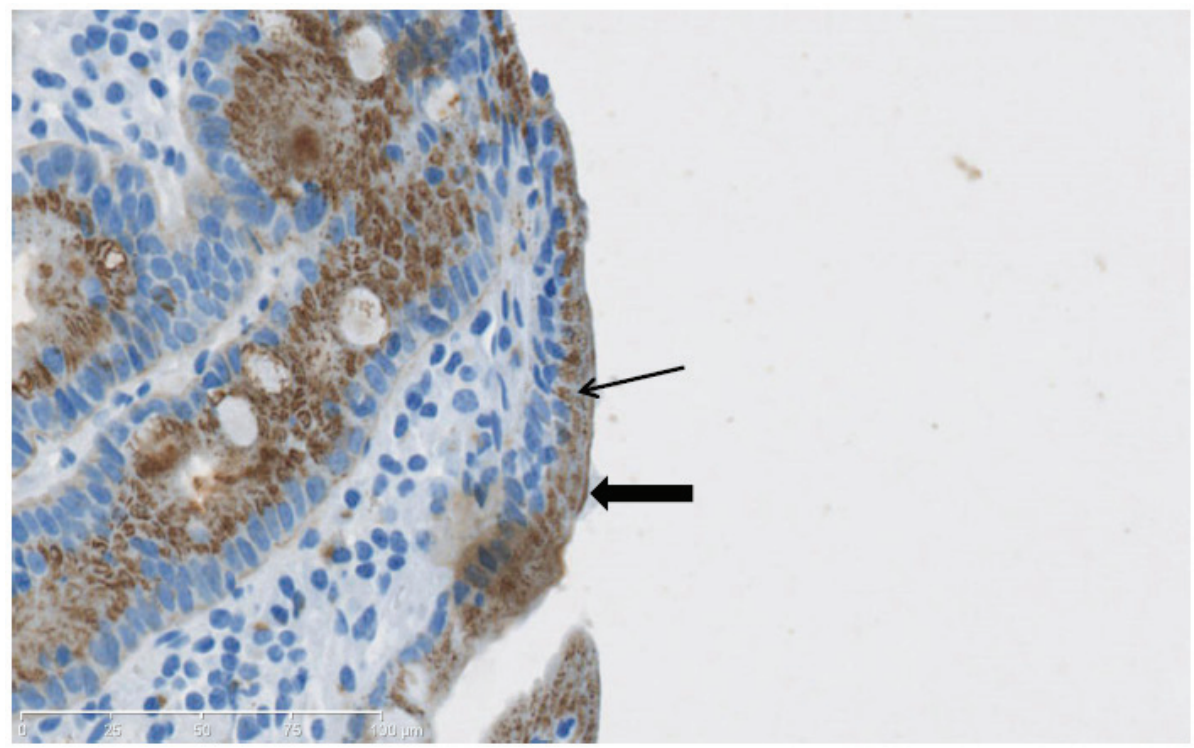

(b)

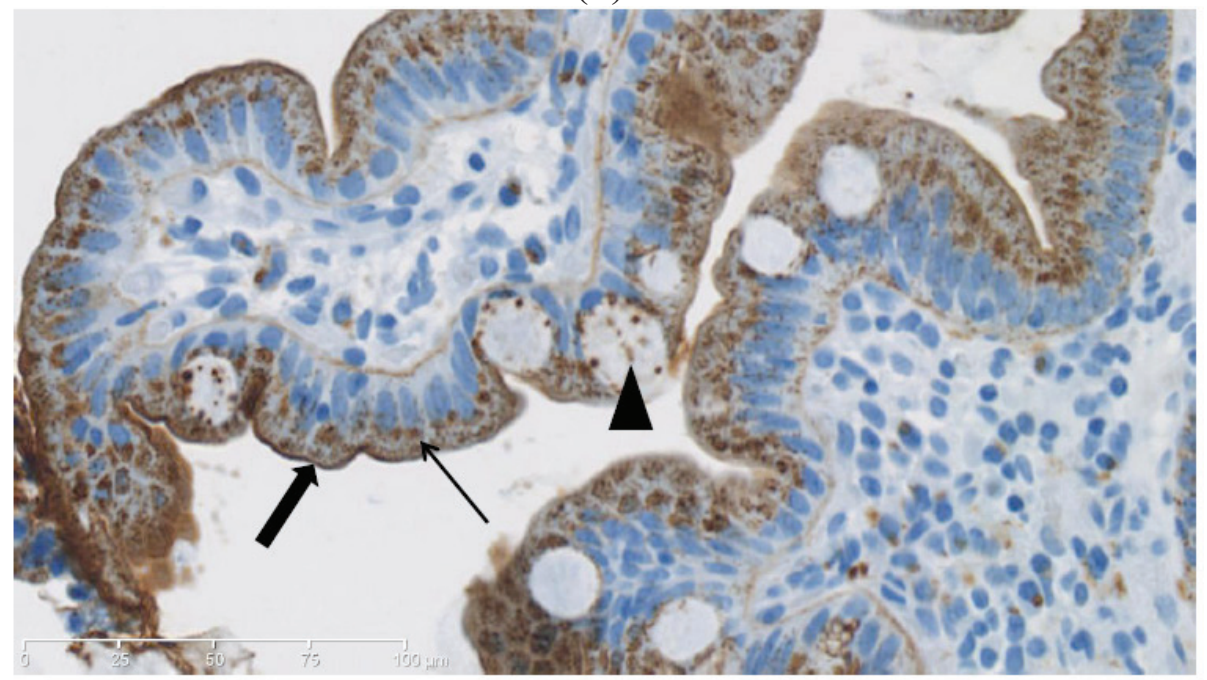

(c)

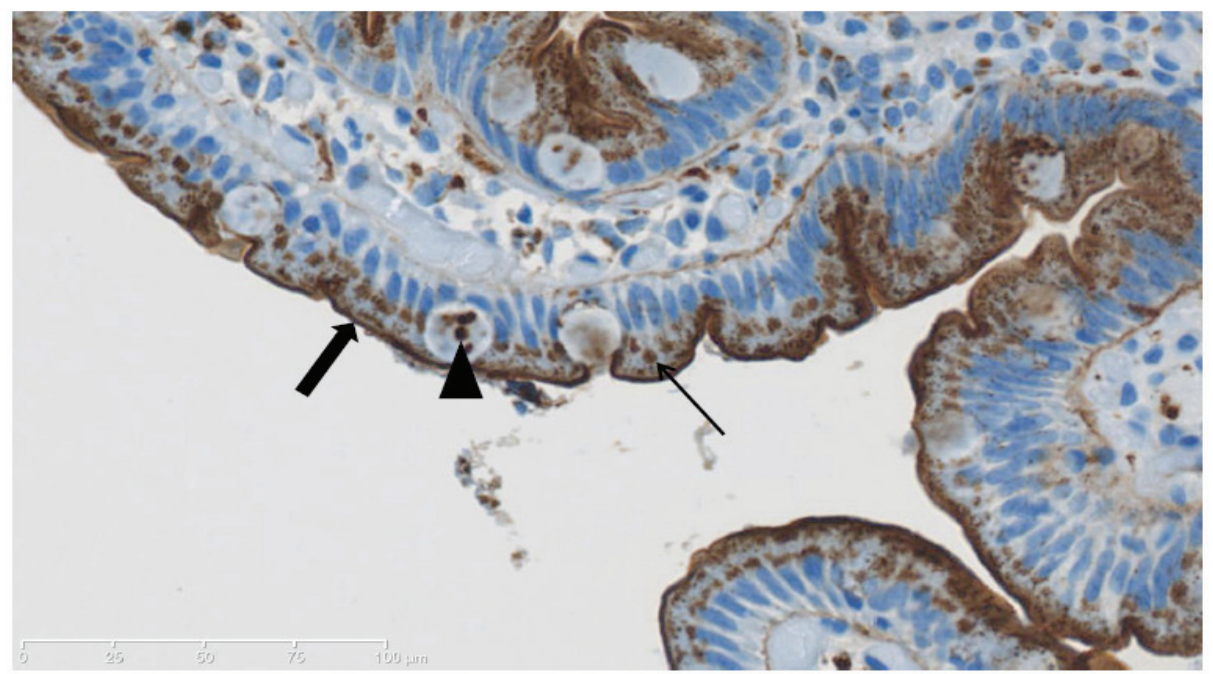

(d) 
Table 2. Peanut agglutinin staining scores. Stained sections of biopsies from patients in Groups A (before and after remission), B, and C were scored as follows: 0 represents no staining of the epithelial cell layer, 1 represents staining in the cytoplasm of epithelial cells, 2 represents staining of cytoplasm and glycocalyx, and 3 represents staining of cytoplasm and glycocalyx, as well as goblet cells.

\begin{tabular}{ccccc}
\hline Group & $\begin{array}{c}\mathbf{A} \\
\text { CD + T1D } \\
\text { Untreated }\end{array}$ & $\begin{array}{c}\text { A } \\
\text { CD + T1D GFD }\end{array}$ & B CD & $\begin{array}{c}\text { C } \\
\text { Non-CD }\end{array}$ \\
\hline 3 & 0 & 2 & 0 & 2 \\
2 & 5 & 4 & 1 & 0 \\
1 & 5 & 4 & 9 & 8 \\
0 & 0 & 0 & 0 & 0 \\
Median score & 1.5 & 2 & 1 & 1 \\
\hline
\end{tabular}

UEA staining patterns are illustrated in Figure 2 with samples from a non-CD patient, a CD + T1D patient before and after treatment with GFD, and a CD patient. UEA staining was observed in all patients except one: C4 (Figure 2a). A score of 1 (cytoplasmic staining alone) was not given to any samples (Table 3). In general, the UEA staining appeared more intense in comparison to PNA staining. This was especially evident in goblet cells (Figure 2b,d).

Figure 2. Ulex europaeus agglutinin (UEA) staining. (a) Patient C4 (non-CD), score 0; (b) Patient A1 (CD + T1D, untreated), score 3; (c) Patient A1 (CD + T1D, on GFD), score 2; (d) Patient B5 (CD), score 3. Thin arrows mark staining of cytoplasm of epithelial cells. Thick arrows mark staining of glycocalyx. Arrowheads mark staining in goblet cells.

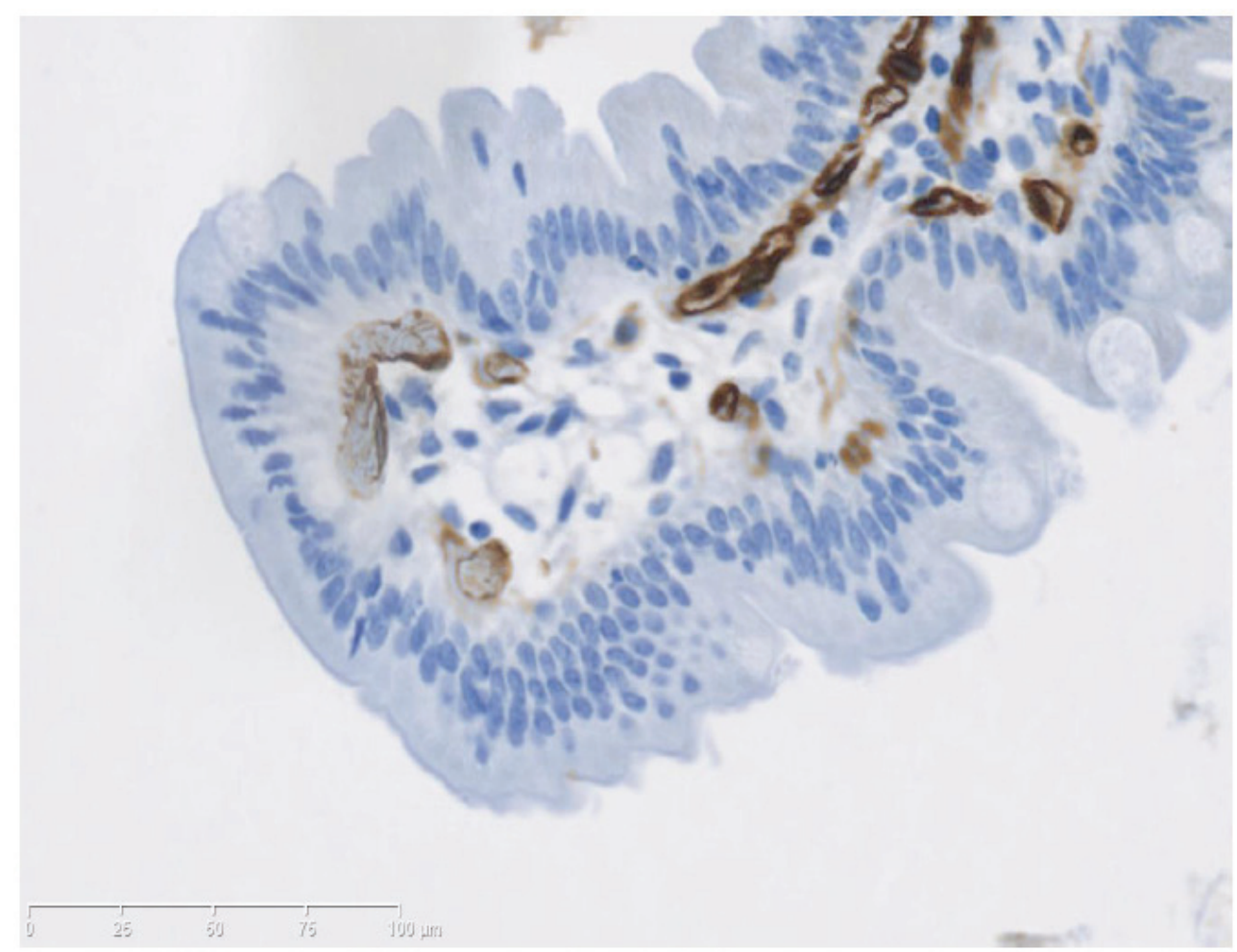

(a) 
Figure 2. Cont.

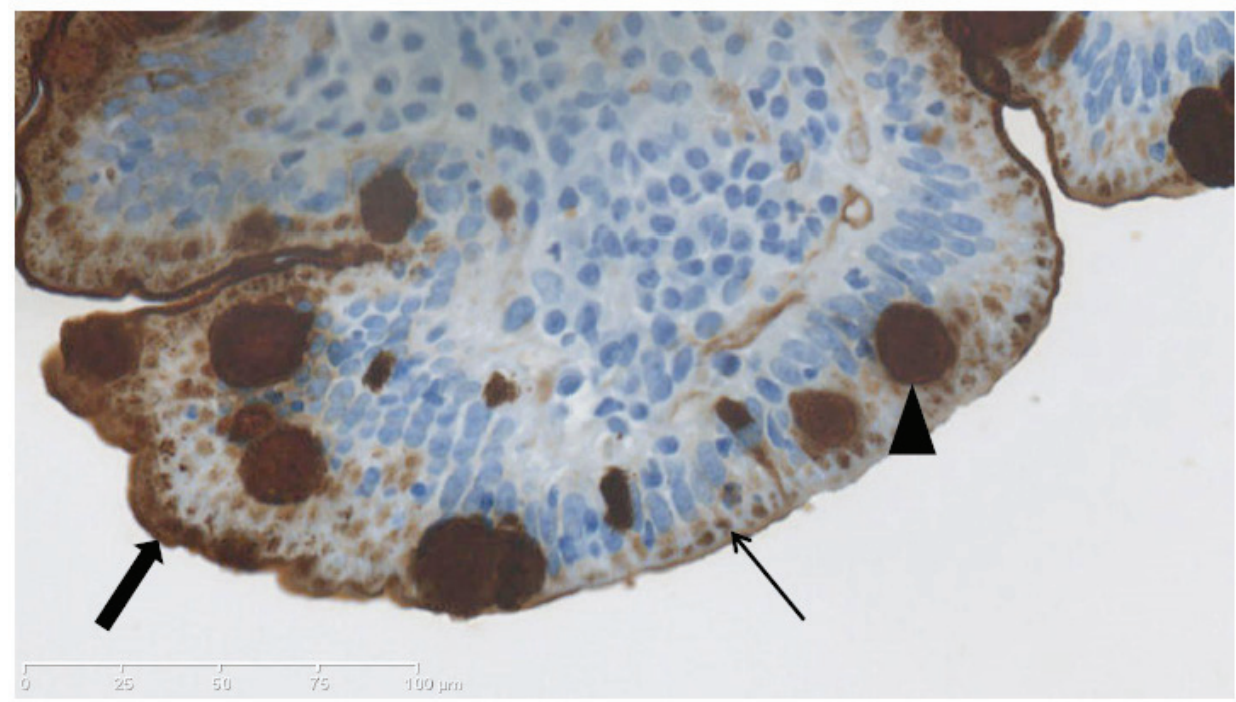

(b)

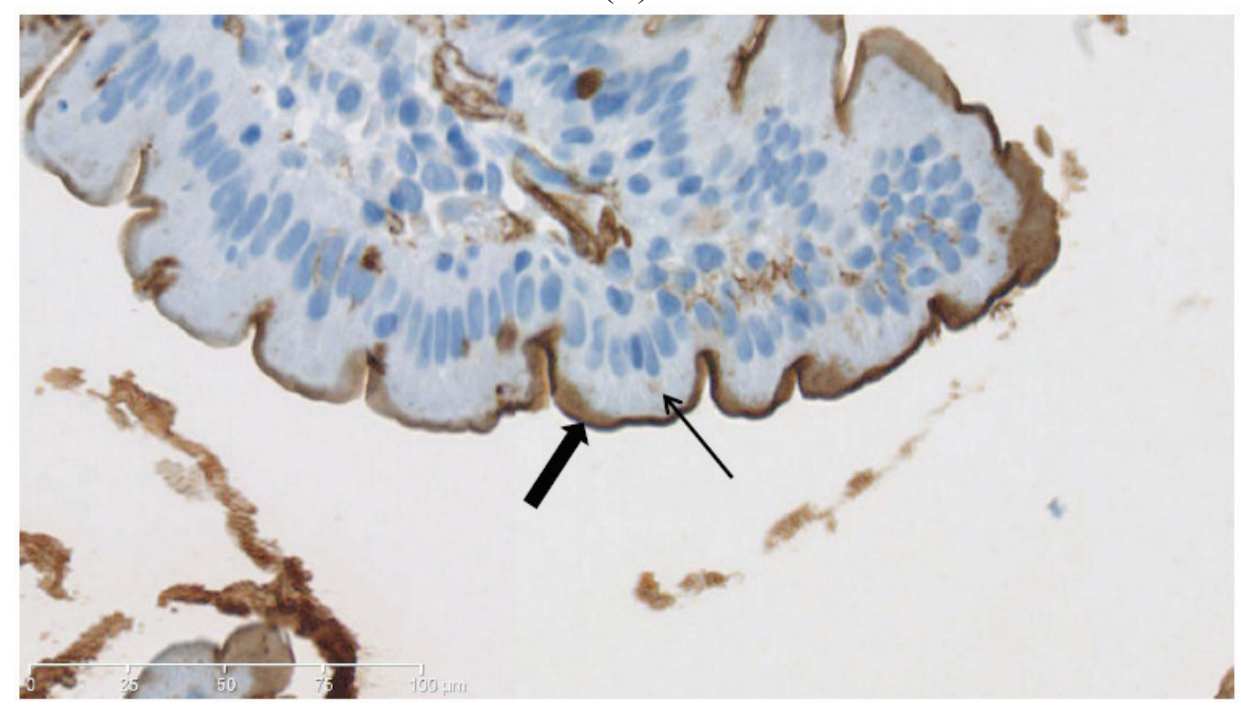

(c)

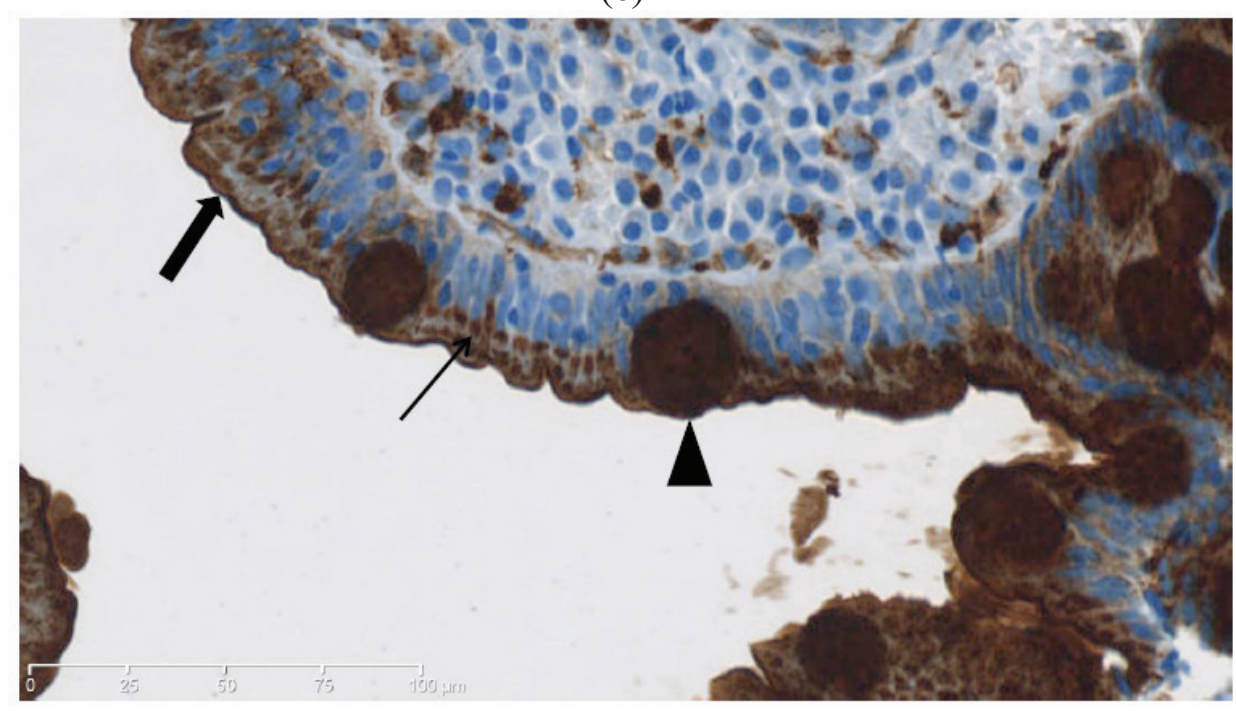

(d) 
Table 3. Ulex europaeus agglutinin lectin staining scores. Stained sections of biopsies from patients in Groups A (before and after remission), B, and C were scored as follows: 0 represents no staining of the epithelial cell layer, 1 represents staining in the cytoplasm of epithelial cells; 2 represents staining of cytoplasm and glycocalyx; and 3 represents staining of cytoplasm and glycocalyx, as well as goblet cells.

\begin{tabular}{|c|c|c|c|c|}
\hline Group & $\stackrel{\text { A }}{\text { CD + T1D Untreated }}$ & $\begin{array}{c}\text { A } \\
\text { CD }+ \text { T1D GFD }\end{array}$ & B CD & $\begin{array}{c}\text { C } \\
\text { Non-CD }\end{array}$ \\
\hline 3 & 5 & 4 & 10 & 5 \\
\hline 2 & 5 & 6 & 0 & 4 \\
\hline 1 & 0 & 0 & 0 & 0 \\
\hline 0 & 0 & 0 & 0 & 1 \\
\hline Median score & 2.5 & 2 & 3 & 2.5 \\
\hline
\end{tabular}

Scores from biopsy sections stained with PNA lectin are presented in Table 2. We performed three sets of statistical analysis to test if the scores reflected differences between the groups.

First, we compared the patients in Group A with both CD and T1D before (untreated) and after treatment with a GFD using the Wilcoxon signed-rank test for matched pairs. The median scores were 1.5 and 2 for the untreated and GFD-treated groups, respectively, with no statistically significant difference $(p=0.25)$.

Secondly, we compared the groups A untreated, B and C using the Kruskal-Wallis one-way ANOVA with Dunn's post test and found no statistically significant differences between any of the groups.

Finally, we combined the results from the two groups with inflamed tissue (A untreated and B, median $=1$ ) and compared with the combined results from the two groups without inflammation (A GFD and C, median =1) in a Mann-Whitney test and found no statistically significant difference $(p=0.38)$. Likewise, there was no difference between the combined groups with CD (A untreated and $\mathrm{B}$, median $=1)$ when compared with the non-CD group $\mathrm{C}($ median $=1)(p=0.88)$.

Scores from biopsy sections stained with UEA lectin are presented in Table 3. We performed statistical analyses analogous to the statistical tests described above for PNA lectin, and found no difference in the comparison between Group A untreated and GFD-treated ( $p=1.00$ ), no difference between any of the groups A untreated, B and C, and also no difference when the combined group with inflammation (median $=3$ ) was compared with the combined group without inflammation $($ median $=2)(p=0.09)$, or when the combined groups of CD patients (median $=3$ ) were compared with the non-CD group $\mathrm{C}($ median $=2.5)(p=0.23)$.

Combining the results in Tables 2 and 3 did not indicate any difference between the four groups in combined PNA/UEA staining with medians of 2, 2, 2.5 and 2, respectively.

\section{Discussion}

In our study, we found no difference in UEA and PNA lectin staining in biopsies from the same pediatric CD patients before and after remission. It should be noted that these children also had T1D, another autoimmune disease whereby the composition of commensals in the gastrointestinal 
tract could be of pathogenic importance [14]. Likewise, we found no difference when biopsies showing CD-associated inflammation were compared to biopsies with normal histology.

Earlier work has shown somewhat conflicting results regarding the reactivity of PNA in CD biopsies. PNA has been reported to not show any reactivity at all in patients or controls [15], to be upregulated in goblet cells of CD patients compared to controls [16], or downregulated in the glycocalyx of CD patients in a study where no reactivity in goblet cells was noted [8]. There is more agreement with regards to UEA reactivity, which was shown to be upregulated in goblet cells of CD patients in two of these studies $[8,15]$. Some methodological differences between these studies and our own could in part explain the discrepancies: whether it is children or adults being studied, the origin of the biopsy (jejunum as opposed to duodenum), and the specific structures included in the analysis (e.g., crypt goblet cells versus villus goblet cells).

It has been well described that the bacterial microbiota is important for the function of the mucosal immune system, and proper development of gut-associated lymphoid tissue is dependent on bacterial presence and influenced by the composition of the microbiota [17-19]. Colonization of germ-free mice with Bacteroides modulated the expression of numerousl genes involved in diverse intestinal functions including maintenance of intestinal permeability [20]. In rats, it was shown that the composition of the commensal microbiota can modulate intestinal permeability with certain bacterial strains increasing permeability, and other (probiotic) strains reducing permeability [21]. Colonization with such probiotic strains of bacteria could potentially reduce intestinal inflammation [22], and be beneficial in the context of celiac disease, whereas colonization with other strains could be detrimental.

Characterization of the bacterial population in the human gastrointestinal tract is currently intensely investigated [23], and it remains an important issue to define how the composition of the commensal microbiota is determined. It is clear that the exposure to diverse bacterial strains in different environments plays an important role, but a genetic predisposition to colonization with particular bacterial strains is also likely to influence the composition of the microbiota [24,25]. Such a genetic component could be the result of inherited differences in the expression pattern of unique carbohydrate structures in the glycocalyx/mucous layer. This could influence bacterial composition if bacteria can utilize carbohydrates for colonization, as was proposed for Bacteroides using glycans [26]. Recently, it was found that the genetically determined AB0 blood group system can modulate the composition of the human intestinal microbiota [27]. Furthermore, the secretor status encoded by the FUT2 gene, which defines the expression of the AB0 blood group antigens in the mucus, is associated with the composition of intestinal bifidobacteria [28]. If the AB0 antigens are present in the mucosal layer, they could facilitate bacterial colonization by acting as attachment sites or carbon sources. In a Finnish study, it was found that $14.7 \%$ of a healthy control population was homozygous for a nonsense mutation in the FUT2 gene leading to non-secretor status [29]. The non-secretor status was positively associated with CD susceptibility, and the frequency of non-secretors was increased to $18 \%$ in the CD population.

It appears likely that the composition of the intestinal microbiota is associated with the development of $\mathrm{CD}$ [30], either through a genetic predisposition, environmental influence, or infection with pathogens [31]. Several studies point to differences in the microbiota between CD patients with active disease, CD patients in remission, and normal controls [32-34]. Moreover, animal 
models have demonstrated how gluten-induced enteropathy can be modulated by the bacterial microbiota $[35,36]$. The presence of rod-shaped bacteria in intestinal mucosa was shown to be frequently associated with pediatric CD patients regardless of disease status (patients with active disease, compared to another group of patients in remission), but not with controls [8]. This was linked with differences in lectin staining of biopsies from the three groups in the study with UEA staining being more intense and widespread in CD patients (active CD or treated) compared to controls, and PNA staining being less widespread and intense in both groups of CD patients compared to controls. These findings could be explained by either a genetic predisposition to express certain carbohydrates in people who develop $\mathrm{CD}$, or by the effect that the components of the microbiota, such as the rod-shaped bacteria associated with CD patients, could have on glycosylation.

Although not evident in our study, there is indication that inflammatory processes can alter the expression of carbohydrates in the gut. A characterization of the human MUC2 mucin in colon revealed a complex glycosylation pattern in healthy individuals [37]. This pattern was altered with a shift towards smaller and less complex glycans in patients with active ulcerative colitis [38]. Interestingly, this effect was reversed after remission of disease, arguing against a genetic reason for the shift.

With respect to $\mathrm{CD}$ in future studies, it would be relevant to investigate other differences in mucus glycosylation that are not detected by UEA or PNA staining. Also, potential differences in other modifications, such as sulfation, could be relevant in CD [39]. Our limited study supports the notion that glycosylation is independent of disease activity as previously shown by Forsberg and colleagues [8]. In contrast to that study, however, we could not demonstrate a difference in lectin staining between CD patients, either with or without T1D or combined, when compared to non-CD controls.

\section{Conclusions}

In our study, we did not identify any differences in the staining pattern of the lectins PNA or UEA in duodenal biopsies between samples obtained before and after remission in the same children with both CD and T1D. Also, we did not observe any differences in lectin staining between children with both $\mathrm{CD}$ and $\mathrm{T} 1 \mathrm{D}$, children with $\mathrm{CD}$ alone, or non-CD controls. In conclusion, we found no indication that the presence of unique carbohydrate structures bound by PNA or UEA is involved in the susceptibility to $\mathrm{CD}$, or that the inflammation caused by the disease affects the glycosylation process.

\section{Acknowledgments}

The authors would like to thank laboratory technician Mette Hviid and the staff of the Department of Pathology, Odense University Hospital for their valuable assistance. Financial support for this study was provided by The Danish Council for Strategic Research, Programme Commission on Health, Food and Welfare, and The Novo Nordisk Research Foundation.

\section{Conflicts of Interest}

The authors declare no conflict of interest. 


\section{References}

1. Abadie, V.; Sollid, L.M.; Barreiro, L.B.; Jabri, B. Integration of genetic and immunological insights into a model of celiac disease pathogenesis. Annu. Rev. Immunol. 2011, 29, 493-525.

2. Dubois, P.C.; Trynka, G.; Franke, L.; Hunt, K.A.; Romanos, J.; Curtotti, A.; Zhernakova, A.; Heap, G.A.; Adany, R.; Aromaa, A.; et al. Multiple common variants for celiac disease influencing immune gene expression. Nat. Genet. 2010, 42, 295-302.

3. Kverka, M.; Tlaskalova-Hogenova, H. Two faces of microbiota in inflammatory and autoimmune diseases: Triggers and drugs. Acta Pathol. Microbiol. Immunol. Scand. 2013, 121, 403-421.

4. Le Chatelier, E.; Nielsen, T.; Qin, J.; Prifti, E.; Hildebrand, F.; Falony, G.; Almeida, M.; Arumugam, M.; Batto, J.M.; Kennedy, S.; et al. Richness of human gut microbiome correlates with metabolic markers. Nature 2013, 500, 541-546.

5. Robbe, C.; Capon, C.; Coddeville, B.; Michalski, J.C. Structural diversity and specific distribution of o-glycans in normal human mucins along the intestinal tract. Biochem. J. 2004, 384, 307-316.

6. Brinck, U.; Korabiowska, M.; Bosbach, R.; Gabius, H.J. Detection of inflammation- and neoplasia-associated alterations in human large intestine using plant/invertebrate lectins, galectin-1 and neoglycoproteins. Acta Anat. 1998, 161, 219-233.

7. Cheng, J.; Kalliomaki, M.; Heilig, H.G.; Palva, A.; Lahteenoja, H.; de Vos, W.M.; Salojarvi, J.; Satokari, R. Duodenal microbiota composition and mucosal homeostasis in pediatric celiac disease. BMC Gastroenterol. 2013, 13, 113.

8. Forsberg, G.; Fahlgren, A.; Horstedt, P.; Hammarstrom, S.; Hernell, O.; Hammarstrom, M.L. Presence of bacteria and innate immunity of intestinal epithelium in childhood celiac disease. Am. J. Gastroenterol. 2004, 99, 894-904.

9. Volta, U.; Tovoli, F.; Caio, G. Clinical and immunological features of celiac disease in patients with type 1 diabetes mellitus. Expert Rev. Gastroenterol. Hepatol. 2011, 5, 479-487.

10. Camarca, M.E.; Mozzillo, E.; Nugnes, R.; Zito, E.; Falco, M.; Fattorusso, V.; Mobilia, S.; Buono, P.; Valerio, G.; Troncone, R.; et al. Celiac disease in type 1 diabetes mellitus. Ital. J. Pediatr. 2012, 38, 10.

11. Antonioli, D.A. Celiac disease: A progress report. Mod. Pathol. 2003, 16, 342-346.

12. Roth, Z.; Yehezkel, G.; Khalaila, I. Identification and quantification of protein glycosylation. Int. J. Carbohydr. Chem. 2012, 2012, doi:10.1155/2012/640923.

13. GraphPad Prism, version 4.03 for Windows; GraphPad Software: San Diego, CA, USA, 2005.

14. Herold, K.C.; Vignali, D.A.; Cooke, A.; Bluestone, J.A. Type 1 diabetes: Translating mechanistic observations into effective clinical outcomes. Nat. Rev. Immunol. 2013, 13, 243-256.

15. Vecchi, M.; Torgano, G.; de Franchis, R.; Tronconi, S.; Agape, D.; Ronchi, G. Evidence of altered structural and secretory glycoconjugates in the jejunal mucosa of patients with gluten sensitive enteropathy and subtotal villous atrophy. Gut 1989, 30, 804-810.

16. Barresi, G.; Tuccari, G.; Tedeschi, A.; Magazzu, G. Lectin binding sites in duodeno-jejunal mucosae from coeliac children. Histochemistry 1988, 88, 105-112. 
17. Gronlund, M.M.; Arvilommi, H.; Kero, P.; Lehtonen, O.P.; Isolauri, E. Importance of intestinal colonisation in the maturation of humoral immunity in early infancy: A prospective follow up study of healthy infants aged 0-6 months. Arch. Dis. Childh. Fetal Neonatal Ed. 2000, 83, F186-F192.

18. Lanning, D.; Sethupathi, P.; Rhee, K.J.; Zhai, S.K.; Knight, K.L. Intestinal microflora and diversification of the rabbit antibody repertoire. J. Immunol. 2000, 165, 2012-2019.

19. Rhee, K.J.; Sethupathi, P.; Driks, A.; Lanning, D.K.; Knight, K.L. Role of commensal bacteria in development of gut-associated lymphoid tissues and preimmune antibody repertoire. $J$. Immunol. 2004, 172, 1118-1124.

20. Hooper, L.V.; Wong, M.H.; Thelin, A.; Hansson, L.; Falk, P.G.; Gordon, J.I. Molecular analysis of commensal host-microbial relationships in the intestine. Science 2001, 291, 881-884.

21. Garcia-Lafuente, A.; Antolin, M.; Guarner, F.; Crespo, E.; Malagelada, J.R. Modulation of colonic barrier function by the composition of the commensal flora in the rat. Gut 2001, 48, 503-507.

22. Bai, A.P.; Ouyang, Q.; Zhang, W.; Wang, C.H.; Li, S.F. Probiotics inhibit TNF- $\alpha$-induced interleukin-8 secretion of HT29 cells. World J. Gastroenterol. 2004, 10, 455-457.

23. Cox, M.J.; Cookson, W.O.; Moffatt, M.F. Sequencing the human microbiome in health and disease. Hum. Mol. Genet. 2013, doi: 10.1093/hmg/ddt398.

24. Benson, A.K.; Kelly, S.A.; Legge, R.; Ma, F.; Low, S.J.; Kim, J.; Zhang, M.; Oh, P.L.; Nehrenberg, D.; Hua, K.; et al. Individuality in gut microbiota composition is a complex polygenic trait shaped by multiple environmental and host genetic factors. Proc. Natl. Acad. Sci. USA 2010, 107, 18933-18938.

25. McKnite, A.M.; Perez-Munoz, M.E.; Lu, L.; Williams, E.G.; Brewer, S.; Andreux, P.A.; Bastiaansen, J.W.; Wang, X.; Kachman, S.D.; Auwerx, J.; et al. Murine gut microbiota is defined by host genetics and modulates variation of metabolic traits. PLoS One 2012, 7, e39191.

26. Lee, S.M.; Donaldson, G.P.; Mikulski, Z.; Boyajian, S.; Ley, K.; Mazmanian, S.K. Bacterial colonization factors control specificity and stability of the gut microbiota. Nature 2013, 501, 426-429.

27. Makivuokko, H.; Lahtinen, S.J.; Wacklin, P.; Tuovinen, E.; Tenkanen, H.; Nikkila, J.; Bjorklund, M.; Aranko, K.; Ouwehand, A.C.; Matto, J. Association between the abo blood group and the human intestinal microbiota composition. BMC Microbiol. 2012, 12, 94.

28. Wacklin, P.; Makivuokko, H.; Alakulppi, N.; Nikkila, J.; Tenkanen, H.; Rabina, J.; Partanen, J.; Aranko, K.; Matto, J. Secretor genotype (FUT2 gene) is strongly associated with the composition of bifidobacteria in the human intestine. PLoS One 2011, 6, e20113.

29. Parmar, A.S.; Alakulppi, N.; Paavola-Sakki, P.; Kurppa, K.; Halme, L.; Farkkila, M.; Turunen, U.; Lappalainen, M.; Kontula, K.; Kaukinen, K.; et al. Association study of FUT2 (rs601338) with celiac disease and inflammatory bowel disease in the finnish population. Tissue Antigens 2012, 80, 488-493.

30. Sanz, Y.; de Pama, G.; Laparra, M. Unraveling the ties between celiac disease and intestinal microbiota. Int. Rev. Immunol. 2011, 30, 207-218.

31. Plot, L.; Amital, H. Infectious associations of celiac disease. Autoimmun. Rev. 2009, 8, $316-319$. 
32. Collado, M.C.; Donat, E.; Ribes-Koninckx, C.; Calabuig, M.; Sanz, Y. Specific duodenal and faecal bacterial groups associated with paediatric coeliac disease. J. Clin. Pathol. 2009, 62, 264-269.

33. Di Cagno, R.; de Angelis, M.; de Pasquale, I.; Ndagijimana, M.; Vernocchi, P.; Ricciuti, P.; Gagliardi, F.; Laghi, L.; Crecchio, C.; Guerzoni, M.E.; et al. Duodenal and faecal microbiota of celiac children: Molecular, phenotype and metabolome characterization. BMC Microbiol. 2011, 11, 219.

34. Nistal, E.; Caminero, A.; Herran, A.R.; Arias, L.; Vivas, S.; de Morales, J.M.; Calleja, S.; de Miera, L.E.; Arroyo, P.; Casqueiro, J. Differences of small intestinal bacteria populations in adults and children with/without celiac disease: Effect of age, gluten diet, and disease. Inflamm. Bowel Dis. 2012, 18, 649-656.

35. Cinova, J.; de Palma, G.; Stepankova, R.; Kofronova, O.; Kverka, M.; Sanz, Y.; Tuckova, L. Role of intestinal bacteria in gliadin-induced changes in intestinal mucosa: Study in germ-free rats. PLoS One 2011, 6, e16169.

36. D’Arienzo, R.; Stefanile, R.; Maurano, F.; Mazzarella, G.; Ricca, E.; Troncone, R.; Auricchio, S.; Rossi, M. Immunomodulatory effects of Lactobacillus casei administration in a mouse model of gliadin-sensitive enteropathy. Scand. J. Immunol. 2011, 74, 335-341.

37. Larsson, J.M.; Karlsson, H.; Sjovall, H.; Hansson, G.C. A complex, but uniform o-glycosylation of the human MUC2 mucin from colonic biopsies analyzed by nanoLC/MSn. Glycobiology 2009, 19, 756-766.

38. Larsson, J.M.; Karlsson, H.; Crespo, J.G.; Johansson, M.E.; Eklund, L.; Sjovall, H.; Hansson, G.C. Altered o-glycosylation profile of MUC2 mucin occurs in active ulcerative colitis and is associated with increased inflammation. Inflamm. Bowel Dis. 2011, 17, 2299-2307.

39. Brockhausen, I. Sulphotransferases acting on mucin-type oligosaccharides. Biochem. Soc. Transact. 2003, 31, 318-325. 
Reprinted from Nutrients. Cite as: Ortiz-Sánchez, J.P.; Francisco, C.-C.; de la Barca, A.M.C. Maize Prolamins Could Induce a Gluten-Like Cellular Immune Response in Some Celiac Disease Patients. Nutrients 2013, 5, 4174-4183.

Review

\title{
Maize Prolamins Could Induce a Gluten-Like Cellular Immune Response in Some Celiac Disease Patients
}

\author{
Juan P. Ortiz-Sánchez ${ }^{1}$, Francisco Cabrera-Chávez ${ }^{2}$ and Ana M. Calderón de la Barca ${ }^{1, *}$
}

1 Department of Nutrition, Research Center for Food and Development (CIAD, A.C.), Carr. La Victoria, Km. 0.6, Hermosillo, Sonora 83304, Mexico; E-Mail: juanpedroo@gmail.com

2 Nutrition Sciences and Gastronomy Unit, University of Sinaloa, Culiacan, Sinaloa 80019, Mexico; E-Mail: fcabrera@uas.edu.mx

* Author to whom correspondence should be addressed; E-Mail: amc@ciad.mx;

Tel.: +52-662-289-2400 (ext. 288); Fax: +52-662-280-0094.

Received: 1 August 2013; in revised form: 2 October 2013 / Accepted: 10 October 2013 /

Published: 21 October 2013

\begin{abstract}
Celiac disease (CD) is an autoimmune-mediated enteropathy triggered by dietary gluten in genetically prone individuals. The current treatment for CD is a strict lifelong gluten-free diet. However, in some CD patients following a strict gluten-free diet, the symptoms do not remit. These cases may be refractory CD or due to gluten contamination; however, the lack of response could be related to other dietary ingredients, such as maize, which is one of the most common alternatives to wheat used in the gluten-free diet. In some CD patients, as a rare event, peptides from maize prolamins could induce a celiac-like immune response by similar or alternative pathogenic mechanisms to those used by wheat gluten peptides. This is supported by several shared features between wheat and maize prolamins and by some experimental results. Given that gluten peptides induce an immune response of the intestinal mucosa both in vivo and in vitro, peptides from maize prolamins could also be tested to determine whether they also induce a cellular immune response. Hypothetically, maize prolamins could be harmful for a very limited subgroup of CD patients, especially those that are non-responsive, and if it is confirmed, they should follow, in addition to a gluten-free, a maize-free diet.
\end{abstract}

Keywords: celiac disease; cellular immune response; maize prolamins; zeins

\section{Introduction}


Celiac disease (CD) is an immune-mediated enteropathy triggered by dietary wheat, rye and barley gluten (water-insoluble proteins) in genetically predisposed individuals [1]. Characteristic features of CD involve crypt hyperplasia, intra-epithelial lymphocytosis and villus atrophy of the intestinal mucosa. These injuries affect intestinal function and nutrient absorption, which can cause a variety of gastrointestinal and extra-intestinal symptoms [2].

Intestinal mucosa damage in $\mathrm{CD}$ patients begins with an innate response that leads to a cellular immune response [3]. First, prolamin peptides from gluten, which are resistant to human digestion, interact with a chemokine receptor, inducing zonulin release and a subsequent tight junction disassembly [4]. Then, the damaged barrier allows the arrival of gliadin peptides to the lamina propria, where tissue transglutaminase (tTG) deamidates specific glutamine residues to confer an overall negative charge. These peptides are bound to the human leucocyte antigen (HLA) DQ2 or DQ8 molecules, in antigen presenting cells, which present them to T-cells to develop the full immune response required for CD [5]. In addition to gluten peptides, self tTG is presented to T-cells, which triggers an auto-immune response. Therefore, $\mathrm{CD}$ is considered an autoimmune disease.

CD symptoms disappear in the majority of patients after dietary gluten withdrawal; however, in some patients, the symptoms are still present even after they adopt a strict gluten-free diet [6]. This is due to either refractory $\mathrm{CD}$ or to the presence of gluten as a contaminant or as a non-declared additive in foods [7]. Additionally, the lack of response to dietary gluten withdrawal in a very limited subgroup of patients, could be due to other dietary proteins present in the gluten-free diet, such as those from maize, which is a common alternative ingredient used in gluten-free diets.

It has been demonstrated that zeins, the maize prolamins, are able to induce an inflammatory response through contact with the mucosa in some CD patients [8]. Furthermore, IgA antibodies from some CD patients can recognize zeins [9], even after lime and/or enzymatic treatments [10]. Perhaps, in active $\mathrm{CD}$, peptides derived from zeins could exacerbate the immune response in the intestinal mucosa, because they have sequence characteristics and/or electronegative residues that resemble gluten peptides.

\section{Supporting Experimental Results}

Table 1 summarizes the similarities between maize prolamin peptides and wheat celiac-toxic gluten peptides that are involved in the pathogenesis of celiac disease. These results support the hypothesis that peptides from zeins that are resistant to human digestion are able to induce a celiac-like immune response in some $\mathrm{CD}$ patients by a similar mechanism to that triggered by wheat gluten peptides.

\subsection{Incomplete Protein Digestion}

Pepsin and trypsin, the main peptidases of the intestinal tract, cannot completely digest wheat gluten, because they are unable to cut its $15 \%$ proline-containing polypeptides $[11,12]$. The result is the release of peptides larger than nine amino acids, which are capable of eliciting innate and adaptive immune responses [13]. The proline content of zeins is also high (9\%) and, although zeins contain bonds that pepsin can cut, they also contain cysteine residues with disulfide bonds that 
obstruct digestion by pepsin [14]. All together, the ability of trypsin to digest zeins is low due to their low number of cleavage sites, low solubility [15] and secondary conformation [16].

Table 1. Similarities between maize prolamin peptides and wheat celiac-toxic gluten peptides that are involved in the pathogenesis of celiac disease (CD). NO: nitric oxide; NOS: nitric oxide synthase; HLA-DQ2 or DQ8: human leucocyte antigen molecules; IFN- $\gamma$ : interferon gamma.

\begin{tabular}{|c|c|c|}
\hline $\begin{array}{l}\text { Step in CD } \\
\text { Pathogenesis }\end{array}$ & $\begin{array}{l}\text { Characteristics of Celiac-Toxic } \\
\text { Peptides from Wheat Gluten }\end{array}$ & $\begin{array}{l}\text { Characteristics of Maize Prolamins } \\
\text { That Could be Inducers for CD }\end{array}$ \\
\hline $\begin{array}{l}\text { Incomplete } \\
\text { protein digestion }\end{array}$ & $\begin{array}{l}\text { Gastrointestinal peptidases do not digest the } \\
\text { proline-rich wheat gluten polypeptides completely, } \\
\text { which releases peptides larger than nine amino } \\
\text { acids }[11,12] \text {. }\end{array}$ & $\begin{array}{l}\text { Digestion of zeins is poor due to relatively } \\
\text { high concentrations of glutamine, proline } \\
\text { and cysteine residues [14-16]. }\end{array}$ \\
\hline $\begin{array}{l}\text { Innate imn } \\
\text { response }\end{array}$ & $\begin{array}{l}\text { Increased levels of NO were produced by } \\
\text { challenged granulocytes and NOS expression was } \\
\text { increased in enterocytes from CD patients' small } \\
\text { intestine biopsies }[17,18] \text {. }\end{array}$ & $\begin{array}{l}\text { Proteins from maize caused granulocyte } \\
\text { activation in a rectal challenge in six out of } \\
13 \mathrm{CD} \text { patients tested [8]. }\end{array}$ \\
\hline $\begin{array}{l}\text { Adaptive immune } \\
\text { response: } \\
\text { deamidation of } \\
\text { peptides by tTG }\end{array}$ & $\begin{array}{l}\text { Gluten peptides deamidated by tTG in the lamina } \\
\text { propria contain negative charges [19-21]. }\end{array}$ & $\begin{array}{l}\text { Maize prolamins deamidated by } \mathrm{TG} \\
\text { in vitro were better recognized than native } \\
\text { ones by IgA from some } \mathrm{CD} \\
\text { patients' sera [22]. }\end{array}$ \\
\hline $\begin{array}{l}\text { Adaptive response: } \\
\text { increased affinity } \\
\text { of HLA-DQ2/DQ8 } \\
\text { on antigen } \\
\text { presenting cells to } \\
\text { bind peptides }\end{array}$ & $\begin{array}{l}\text { HLA-DQ2 prefers negatively charged amino } \\
\text { acids from gluten peptides at the } \mathrm{p} 4, \mathrm{p} 6 \text { or } \mathrm{p} 7 \\
\text { positions in the peptide, while HLA-DQ8 prefers } \\
\text { them at positions } \mathrm{p} 1 \text { or } \mathrm{p} 9[20] .\end{array}$ & $\begin{array}{l}\text { Peptides from digested maize prolamins } \\
\text { have glutamine at positions } \mathrm{p} 1 \text { and } \mathrm{p} 9 \text { that } \\
\text { can be deamidated by tTG and bind to } \\
\text { HLA-DQ8 }[23,24] \text {. Other peptides can be } \\
\text { bound by HLA-DQ2 [10]. }\end{array}$ \\
\hline $\begin{array}{l}\text { Adaptive response: } \\
\text { processing and } \\
\text { presentation of } \\
\text { peptides }\end{array}$ & $\begin{array}{l}\text { After processing, the deamidated gluten peptides } \\
\text { are presented to T-cells. Then, B-cells are induced } \\
\text { to proliferate and produce antibodies [25]. }\end{array}$ & $\begin{array}{l}\text { T-cells from the intestine of one out of } \\
\text { seven CD patients stimulated by maize } \\
\text { prolamins and teff produced low IFN- } \gamma \text { as } \\
\text { compared to wheat, but higher than control } \\
\text { and other non-wheat grains [26]. } \\
\text { Additionally, IgA antibodies against maize } \\
\text { prolamins were detected in several CD } \\
\text { patients }[10,27] \text {. }\end{array}$ \\
\hline $\begin{array}{l}\text { Adaptive response: } \\
\text { role of antibodies } \\
\text { against dietary } \\
\text { prolamins }\end{array}$ & $\begin{array}{l}\text { Roles of tTG-specific antibodies induced by gluten } \\
\text { in CD patients could be: inhibiting epithelial cell } \\
\text { differentiation and inducing } \\
\text { their proliferation, increasing epithelial and } \\
\text { blood vessel permeability and affecting } \\
\text { angiogenesis [28]. }\end{array}$ & $\begin{array}{l}\text { Although the levels of antibodies against } \\
\text { gluten decrease in some CD patients } \\
\text { following a gluten-free diet, antibodies } \\
\text { against maize prolamins remained high } \\
\text { until both gluten and maize were } \\
\text { avoided }[29,30] \text {. }\end{array}$ \\
\hline $\begin{array}{l}\text { Adaptive response: } \\
\text { activation of } \\
\text { T-cells }\end{array}$ & $\begin{array}{l}\text { Activated T-cells drive the inflammatory } \\
\text { response that leads to the development of the } \\
\text { characteristic celiac lesions and the symptoms } \\
{[31] \text {. T-cells induce damage mostly by IFN- } \gamma} \\
\text { production [32]. }\end{array}$ & $\begin{array}{l}\text { Neither the intestinal lesions nor the CD } \\
\text { symptoms were alleviated with a } \\
\text { gluten-free diet when maize was } \\
\text { still eaten [29]. }\end{array}$ \\
\hline
\end{tabular}




\subsection{The Inflammatory Process}

Nitric oxide (NO) production is involved in the innate inflammatory response mediated by macrophages in $\mathrm{CD}$, and it has been detected in cultured gluten-challenged small intestine biopsies [17]. Additionally, there is an elevated expression of mRNA encoding the major inducible isoform of NO synthase II (iNOS) in untreated CD patients [18]. After rectal wheat gluten challenge in $\mathrm{CD}$ patients, granulocyte activation precedes NO production. Furthermore, some patients have been found to display signs of a similar inflammatory reaction after challenge with maize prolamins [8].

\subsection{Deamidation of the Peptides}

Gluten peptides are transported across the epithelial barrier to the lamina propria, where tTG changes the glutamine residues to glutamic acid. Antigen-presenting cells then process these negatively charged peptides and increase their affinity for the major histocompatibility complex (MHC) class II molecules, HLA-DQ2 and HLA-DQ8. These immunogenic peptide fragments can stimulate HLA-DQ2- and HLA-DQ8-restricted T-cells and trigger an adaptive response in the lamina propria [19-21]. Maize prolamins likely are also deamidated by tTG, because IgA from CD patients was more immunoreactive against maize prolamins extracted from maize bread, treated with microbial transglutaminase, than against maize prolamins from untreated bread [22].

\subsection{Affinity of HLA/DQ8 Molecules to Bind Peptides}

Adaptive responses to gluten initiate when dendritic cells phagocytose gliadin peptides and present them to undifferentiated $\mathrm{T}$ helper cells, whose activation is crucial for the development of CD. Peptide deamidation by tTG increases the affinity of HLA-DQ2/DQ8 for these peptides. HLA-DQ2 has an affinity for negatively charged amino acids at the p4, p6 or p7 positions in the peptide, while HLA-DQ8 has an affinity for those residues at positions $\mathrm{p} 1$ and $\mathrm{p} 9$ [23]. The primary amino acid sequences of maize zeins can fit into these HLA binding sites once they are deamidated. Through in silico analysis, Darewicz et al. [24] identified a high degree of homology between two zein peptides and the celiac-toxic peptides from prolamins found in wheat, barley and rye (gliadins, hordeins and secalins, respectively). Moreover, we have identified a peptide sequence ( $\alpha$-zein 58-91) that is resistant to complete digestion and which has characteristics that would allow it to bind to HLA-DQ8 [10]. In addition to this peptide, Table 2 provides the sequence of a 33-mer ( $\alpha 2$-gliadin 56-88) peptide that is a potent T-cell stimulator [19].

Table 2. Theoretical peptide sequences that bind to HLA-DQ2/DQ8 molecules. After deamidation by tTG [33], glutamine residues (underlined) became glutamic acid, which is an electronegative residue that binds to p4 and 6 in HLA-DQ2 and p1 and 9 in HLA-DQ8.

\begin{tabular}{ccccc}
\hline Food & Peptide & Sequence & Affinity & Reference \\
\hline Wheat & $\alpha$-Gliadin & LQLQPFPQPQLPYPQPQLPYPQPQLPYPQPQPF & HLA-DQ2 & {$[19]$} \\
Wheat & $\alpha$ Gliadin & LQLQPFPQPQLPYPQPQLPYPQPQLPYPQPQPF & HLA-DQ8 & {$[19]$} \\
Maize & $\alpha$-Zein & LQQAIAASNIPLSPLLFQQSPALSLVQSLVQTIR & HLA-DQ8 & {$[10]$} \\
\hline
\end{tabular}




\subsection{Processing and Presentation of Peptides}

Peptides of gliadin are deamidated by tTG, phagocytosed, processed and transported to the cell surface in dendritic cells via MHC class II molecules. Subsequently, the peptides are presented to infiltrated $\mathrm{T}$ helper cells that recognize deamidated peptides and trigger the proliferation of specific B-cells and the production of IgA anti-gliadin and anti-transglutaminase antibodies [25]. Some celiac patients contain B-cells that produce anti-maize prolamin IgA antibodies that do not cross-react with anti-wheat prolamins [10,27].

\subsection{Role of Antibodies}

After the DQ2-/DQ8-dependent activation of CD4+ T-cells, B-cells are stimulated and produce auto-antibodies. These auto-antibodies in the intestinal lumen could be involved in disease pathogenesis in various ways. For instance, they could be involved in inhibiting epithelial cell differentiation, augmenting epithelial cell proliferation, increasing epithelial and blood vessel permeability and affecting angiogenesis [28]. In some CD patients on a gluten-free diet, including maize-based foods, the anti-gliadin and anti-tTG antibody titers diminished, but the symptoms persisted [29,30]. Total symptom remission in these cases was achieved only with a gluten- and maize-free diet [30]. It is possible that partial production of anti-tTG antibodies, in addition to anti-zein antibodies, continued to affect the intestinal mucosa when dietary maize was present.

\subsection{Activation of T-Cells}

The activation of gliadin-reactive CD4+ T-cells results in the production of cytokines that drive an inflammatory response, which leads to the development of the characteristic CD lesions and symptoms [31]. Gluten-specific T-cells induce tissue damage mostly by the production of interferon (IFN) $\gamma$ [32]. There is some evidence of T-cells being simulated by maize prolamins: intestinal $\mathrm{T}$-cells cultured from $\mathrm{CD}$ patients were challenged with maize prolamins in vitro, and T-cells from one out of seven samples produced IFN- $\gamma$ as a result of T-cell stimulation [26]. Although this patient response was not specific, maize and teff peptides produced higher levels of IFN- $\gamma$ (145.6 and $154.4 \mathrm{pg} / \mathrm{mL}$, respectively) than the negative control $(10.9 \mathrm{pg} / \mathrm{mL})$ and others "non-toxic" grains $(\approx 110 \mathrm{pg} / \mathrm{mL})$.

Dietary gluten withdrawal has been demonstrated to induce mucosal recovery and the disappearance of CD symptoms. Nevertheless, some patients on gluten-free diet have forms of CD that do not respond to this diet. This could be due to a higher sensibility of these patients to "gluten-free" foods that still contain some traces of gluten [34] or to the presence of other cereal prolamins, such as those in maize in a very limited subgroup of CD patients.

\section{Potential Links between Zeins and CD}

Based on the similarities between wheat and maize prolamins discussed above, we can infer that the innate and adaptive responses to zeins would be similar to the response against gliadins in CD patients. Nevertheless, it is necessary to identify whether zeins contain immunodominant and minor epitopes similar to those found in gliadins after proteolysis. Some authors have found that there is no 
effect on T-cell activation or pro-inflammatory cytokine secretion when CD patient biopsies were treated with whole pepsin-trypsin digested prolamins from maize [26,35]. Therefore, there is a need to evaluate the effect of isolated immunogenic peptides from maize prolamins, which can be obtained by in silico analysis [10].

The evaluation of the response of immune cells to gliadins includes the increased expression of surface receptors and the production of different cytokines for both tissue and immune cells. Some of these receptors include HLA-DR (human leucocyte antigen), CD54 or ICAM-1 (intercellular adhesion molecule), CD3 (in mature T-cells), CD25 (interleukin-2 receptor) and CD69 (in activated T-cells and natural killer cells) [36,37]. Cytokines that would be produced include interferon gamma, interleukins (IL) 2 and 15 and zonulin [13,38-40]. To evaluate the immune response, an analysis of the protein expression of these markers can be performed after CD patient biopsies are challenged with zein peptides. These ex vivo digested-peptide challenge analyses are considered useful tools to evaluate the safety of non-gluten prolamins in a gluten-free diet $[26,40]$.

There is evidence that after a short gluten challenge in treated CD patients, gluten-specific T-cells are present in peripheral blood [41-44]. After this in vivo challenge, peripheral blood mononuclear cells can be isolated and activated with gluten peptides for quantitative detection of pro-inflammatory cytokines and direct detection of HLA-DQ2 tetramer specific for gliadins. For both cytokine measurements and the detection of an immune response, these techniques would be very useful in the evaluation of the effect of maize prolamins on the immune response in CD patients.

\section{Conclusions}

Although reaction to maize prolamins in CD patients appears to be a rare event, the confirmation that they play a role in the pathogenesis of $\mathrm{CD}$ will be useful information for the follow-up of some non-responsive celiac patients. It is estimated that approximately $10 \%$ to $18 \%$ of these cases are refractory $\mathrm{CD}$, which represents a more severe $\mathrm{CD}$, with a clear malignity and a less favorable prognosis [7]. Therefore, it is important to assess these clinical cases, because uncontrolled CD can lead to several malabsorption problems, osteoporosis and other autoimmune diseases [45].

Maize is one of the most commonly consumed grains in the gluten-free diet. Despite the low content of zeins in maize-containing foods compared with that of gliadins in wheat-containing foods, maize could be responsible for persistent mucosal damage in a very limited subgroup of CD patients. If our hypothesis is proven, zeins could be classified as harmful for some CD patients, especially those showing a poor response to a gluten-free diet.

\section{Acknowledgments}

Financial support was from the Mexican Conacyt (grant CB-2008-01-106227) and Ph.D. fellowship for J.P. Ortiz-Sanchez (101386). Authors are grateful to V. Mata-Haro, R. Sotelo-Mundo and M.I. Ortega for comments on the manuscript and to M.Sci. A. Bolaños for editing assistance.

\section{Conflicts of Interest}

The authors declare no conflict of interest. 


\section{References}

1. Ludvigsson, J.F.; Leffler, D.A.; Bai, J.C.; Biagi, F.; Fasano, A.; Green, P.H.R.; Hadjivassiliou, M.; Kaukinen, K.; Kelly, C.P.; Leonard, J.N.; et al. The Oslo definitions for celiac disease and related terms. Gut 2012, 62, 43-52.

2. Husby, S.; Koletzko, S.; Korponay-Szabó, I.R.; Miarin, M.L.; Phillips, A.; Shamir, R.; Troncone, R.; Giersiepen, K.; Branski, D.; Catassi, C.; et al. European Society for Pediatric Gastroenterology, Hepatology, and Nutrition guidelines for the diagnosis of coeliac disease. J. Pediatr. Gastroenterol. Nutr. 2012, 54, 136-160.

3. Abadie, V.; Sollid, L.M.; Barreiro, L.B.; Jabri, B. Integration of genetic and immunological insights into a model of celiac disease pathogenesis. Annu. Rev. Immunol. 2011, 29, 493-525.

4. Lammers, K.M.; Lu, R.; Brownley, J.; Lu, B.; Gerard, C.; Thomas, K.; Rallabhandi, P.; Shea-Donohue, T.; Tamiz, A.; Alkan, S.; et al. Gliadin induces an increase in intestinal permeability and zonulin release by binding to the chemokine receptor CXCR3. Gastroenterology 2008, 135, 194-204.

5. Sollid, L.M.; Qiao, S.W.; Anderson, R.P.; Gianfrani, C.; Koning, F. Nomenclature and listing of celiac disease relevant gluten T-cell epitopes restricted by HLA-DQ molecules. Immunogenetics 2012, 64, 455-460.

6. Lanzini, A.; Lanzarotto, F.; Villanacci, V.; Mora, A.; Bertolazzi, S.; Turini, D.; Carella, G.; Malagoli, A.; Ferrante, G.; Cesana, B.M.; et al. Complete recovery of intstinal mucosa occurs very rarely in adult celiac patients despite adherence to gluten-free diet. Aliment. Pharmacol. Ther. 2009, 29, 1299-1308.

7. Rubio-Tapia, A.; Murray, J.A. Classification and management of refractory celiac disease. Gut 2010, 59, 547-557.

8. Kristjansson, G.; Högman, M.; Venge, P.; Hällgren, R. Gut mucosal granulocyte activation precedes nitric oxide production: Studies in celiac patients challenged with gluten and corn. Gut 2005, 54, 769-774.

9. Cabrera-Chávez, F.; Rouzaud-Sánchez, O.; Sotelo-Cruz, N.; Calderón de la Barca, A.M. Bovine milk caseins and transglutaminase-treated cereal prolamins are differentially recognized by IgA of celiac disease patients according to their age. J. Agric. Food Chem. 2009, 57, 3754-3759.

10. Cabrera-Chávez, F.; Iameti, S.; Miriani, M.; Calderón de la Barca, A.M.; Mamone, G.; Bonomi, F. Maize prolamins resistant to peptic-tryptic digestion maintain immune-recognition by IgA from some celiac disease patients. Plant Food Hum. Nutr. 2012, 67, 24-30.

11. Shan, L.; Molberg, O.; Parrot, I.; Hausch, F.; Filiz, F.; Gray, G.; Sollid, L.; Khosla, C. Structural basis for gluten intolerance in celiac sprue. Science 2002, 297, 2275-2279.

12. Hausch, F.; Shan, L.; Santiago, N.; Gray, G.; Khosla, C. Intestinal digestive resistance of immunodominant gliadin peptides. Am. J. Physiol. Gastrointest. Liver Physiol. 2002, 283, G996-G1003.

13. Bernardo, D.; Garrote, J.A.; Fernández-Salazar, L.; Riestra, S. Is gliadin really safe for non-coeliac individuals? Production of interleukin 15 in biopsy culture from non-coeliac individuals challenged with gliadin peptides. Gut 2007, 56, 889-890. 
14. Shukla, R.; Cheryan, M. Zein: The industrial protein from corn. Ind. Crop. Prod. 2001, 13, 171-192.

15. Tschiersch, C.; Nikfardjam, M.P.; Schmidt, O.; Schwack, W. Degree of hydrolysis of some vegetable proteins used as fining agents and its influence on polyphenol removal from red wine. Eur. Food Res. Technol. 2010, 231, 65-74.

16. Zhang, B.; Luo, Y.; Wang, Q. Effect of acid and base treatments on structural, rheological, and antioxidant properties of $\alpha$-zein. Food Chem. 2011, 124, 210-220.

17. Beckett, C.G.; Dell'Olio, D.; Shidrawi, R.G.; Rosen-Bronson, S.; Ciclitira, P.J. Gluten-induced nitric oxide and pro-inflamatory citokine release by cultured coeliac small intestinal biopsies. Eur. J. Gastroenterol. Hepatol. 1999, 11, 529-536.

18. Daniels, I.; Cavill, D.; Murray, I.A.; Iargo, R.G. Elevated expression of iNOS mRNA and protein in celiac disease. Clin. Chim. Acta 2005, 356, 134-142.

19. Qiao, S.W.; Bergseng, E.; Molberg, O.; Xia, J.; Fleckenstein, B.; Khosla, C.; Sollid, L.M. Antigen presentation to celiac lesion-derivated $\mathrm{T}$ cells of a 33-mer gliadin peptide naturally formed by gastrointestinal digestion. J. Immunol. 2004, 173, 1757-1762.

20. Koning, F.; Gilissen, L.; Wijmenga, C. Gluten: A two-edged sword. Immunopathogenesis of celiac disease. Springer Semin. Immunopathol. 2005, 27, 217-232.

21. Ciccocioppo, R.; di Sabatino, A.; Corazza, G.R. The immune recognition of gluten in coeliac disease. Clin. Exp. Immunol. 2005, 140, 408-416.

22. Cabrera-Chávez, F.; Rouzaud-Sánchez, O.; Sotelo-Cruz, N.; Calderón de la Barca, A.M. Transglutaminase treatment of wheat and maize prolamins of bread increases the serum IgA reactivity of celiac disease patients. J. Agric. Food Chem. 2008, 56, 1387-1391.

23. Stepniak, D.; Wiesner, M.; de Ru, A.H.; Moustakas, A.K.; Drijfhout, J.W.; Papadopoulos, G.K.; van Veelen, P.A.; Koning, F. Large-scale characterization of natural ligands explains the unique gluten-binding properties of HLA-DQ2. J. Immunol. 2008, 180, 3268-3278.

24. Darewicz, M.; Dziuba, J.; Minkiewicz, P. Computational characterization and identification of peptides for in silico detection of potentially celiac-toxic proteins. Food Sci. Technol. Int. 2007, 13, 125-133.

25. Briani, C.; Samaroo, D.; Alaedini, A. Celiac disease: From gluten to autoimmunity. Autoimmun. Rev. 2008, 7, 644-650.

26. Bergamo, P.; Maurano, F.; Mazzarella, G.I.; Iaquinto, G.; Vocca, I.; Rivelli, A.R.; de Falco, E.; Gianfrani, C.; Rossi, M. Immunological evaluation of the alcohol-soluble protein fraction from gluten-free grains in relation to celiac disease. Mol. Nutr. Food Res. 2011, 55, 1266-1270.

27. Skerritt, J.H.; Devery, J.M.; Penttila, I.A.; LaBrooy, J.T. Cellular and humoral responses in coeliac disease. Protein extracts from different cereals. Clin. Chim. Acta 1991, 204, 109-122.

28. Caja, S.; Mäki, M.; Kaukinen, K.; Lindfors, K. Antibodies in celiac disease: Implications beyond diagnostics. Cell. Mol. Immunol. 2011, 8, 103-109.

29. Accomando, S.; Albino, C.; Montaperto, D.; Amato, G.M.; Corsello, G. Multiple food intolerance or refractory celiac sprue? Dig. Liver Dis. 2006, 38, 784-785. 
30. Calderón de la Barca, A.M.; Cabrera-Chávez, F. No Solo el Gluten Sino Otras Proteínas de la Avena, Maíz y Leche de Vaca Podrían Afectar También a Los Pacientes Celíacos. In Enfermedad Celíaca y Sensibilidad al Gluten no Celiaca; Rodrigo, L., Peña, A.S., Eds.; OmniaScience: Barcelona, Spain, 2013; pp. 89-101.

31. Green, P.H.R.; Cellier, C. Celiac disease. N. Engl. J. Med. 2007, 357, 1731-1743.

32. Nilsen, E.M.; Jahnsen, F.L.; Lundin, K.E.; Johansen, E. Gluten induces an intestinal cytokine response strongly dominated by interferon gamma in patients with celiac disease. Gastroenterology 1998, 115, 551-563.

33. Vader, L.W.; de Ru, A.; van der Wal, Y.; Kooy, Y.M.C.; Benckhuijsen, W.; Mearin, M.L.; Drijfhout, J.W.; van Veelen, P.; Koning, F. Specificity of tissue transglutaminase explains cereal toxicity in celiac disease. J. Exp. Med. 2002, 195, 643-649.

34. Dewar, D.H.; Donelly, S.C.; McLaughlin, S.D.; Johnson, M.W.; Ellis, H.J.; Ciclitira, P.J. Celiac disease: Manegement of persistent symptoms in patients on a gluten-free diet. World J. Gastroenterol. 2012, 18, 1348-1356.

35. Junker, Y.; Zeissig, S.; Seong-Jun, K.; Barisani, D.; Wieser, H.; Leffler, D.A.; Zevallos, V.; Libermann, T.A.; Dillon, S.; Freitag, T.L.; et al. Wheat amylase trypsin inhibitors drive intestinal inflammation via activation of toll-like receptor 4. J. Exp. Med. 2012, 209, 2395-2408.

36. Maiuri, L.; Ciacci, C.; Ricciardelli, I.; Vacca, L.; Raia, V.; Auricchio, S.; Picard, J.; Osman, M.; Quarantino, S.; Londei, M. Association between innate response to gliadin and activation of pathogenic T cells in coeliac disease. Lancet 2003, 362, 30-37.

37. Tortora, R.; Russo, I.; de Palma, G.D.; Luciani, A.; Rispo, A.; Zingone, F.; Iovino, P.; Capone, P.; Ciacci, C. In vitro gliadin challenge: Diagnostic accuracy and utility for the difficult diagnosis of celiac disease. Am. J. Gastroenterol. 2012, 107, 111-117.

38. Drago, S.; El Asmar, R.; di Pierro, M.; Clemente, M.G.; Tripathi, A.; Sapone, A.; Thakar, M.; Iacono, G.; Carroccio, A.; D’Agate, C.; et al. Gliadin, zonulin and gut permeability: Effects on celiac and non-celiac intestinal mucosa and intestinal cell lines. Scand. J. Gastroenterol. 2006, $41,408-419$.

39. Elli, L.; Roncoroni, L.; Hils, M.; Pasternack, R.; Barisani, D.; Terrani, C.; Vaira, V.; Ferrero, S.; Bardella, M.T. Immunological effects of transglutaminase-treated gluten in celiac disease. Hum. Immunol. 2012, 73, 992-997.

40. Zevallos, V.; Ellis, H.J.; Suligoj, T.; Herencia, L.I.; Ciclitira, P.J. Variable activation of immune response by quinoa (Chenopodium quinoa Willd.) prolamins in celiac disease. Am. J. Clin. Nutr. 2012, 96, 337-344.

41. Anderson, R.P.; van Heel, D.A.; Tye-Din, J.A.; Barnardo, M.; Salio, M.; Jewell, D.P.; Hill, A.V.S. T cells in peripheral blood after gluten challenge in coeliac disease. Gut 2005, 54, 1217-1223.

42. Raki, M.; Fallang, L.E.; Brottveit, M.; Bergseng, E.; Quarsten, H.; Lundin, K.E.A.; Sollid, L.M. Tetramer visualization of gut-homing gluten-specific T cells in the peripheral blood of celiac disease patients. Proc. Natl. Acad. Sci. USA 2007, 104, 2831-2836. 
43. Brottveit, M.; Raki, M.; Bergseng, E.; Fallang, L.E.; Simonsen, B.L.S.; Lovik, A.; Larsen, S.; Loberg, E.M.; Jahnsen, F.L.; Sollid, L.M.; et al. Assessing possible celiac disease by an HLA-DQ2-gliadin tetramer test. Am. J. Gastroenterol. 2011, 106, 1318-1324.

44. Camarca, A.; Radano, G.; di Mase, R.; Terrone, G.; Maurano, F.; Auricchio, S.; Troncone, R.; Greco, L.; Gianfrani, C. Short wheat challenge is a reproducible in-vivo assay to detect immune response to gluten. Clin. Exp. Immunol. 2012, 169, 129-136.

45. Rubio-Tapia, A.; Kyle, R.A.; Kaplan, E.L.; Johnson, D.R.; Page, W.; Erdtmann F.; Brantner, T.L.; Kim, W.R.; Phelps, T.K.; Lahr, B.D.; et al. Increased prevalence and mortality in undiagnosed celiac disease. Gastroenterology 2009, 137, 88-93. 
Reprinted from Nutrients. Cite as: di Stefano, M.; Mengoli, C.; Bergonzi, M.; Corazza, G.R. Bone Mass and Mineral Metabolism Alterations in Adult Celiac Disease: Pathophysiology and Clinical Approach. Nutrients 2013, 5, 4786-4799.

Review

\title{
Bone Mass and Mineral Metabolism Alterations in Adult Celiac Disease: Pathophysiology and Clinical Approach
}

\section{Michele Di Stefano, Caterina Mengoli, Manuela Bergonzi and Gino Roberto Corazza *}

1st Department of Internal Medicine, University of Pavia, IRCCS “S.Matteo" Hospital Foundation, Pavia 27100, Italy; E-Mails: m.distefano@unipv.it (M.S.); caterina.mengoli@libero.it (C.M.); manuela.bergonzi@libero.it (M.B.)

* Author to whom correspondence should be addressed; E-Mail: gr.corazza@smatteo.pv.it; Tel.: +39-0382-502973; Fax: +39-0382-502618.

Received: 9 September 2013; in revised form: 13 November 2013 / Accepted: 14 November 2013 / Published: 22 November 2013

\begin{abstract}
Osteoporosis affects many patients with celiac disease (CD), representing the consequence of calcium malabsorption and persistent activation of mucosal inflammation. A slight increase of fracture risk is evident in this condition, particularly in those with overt malabsorption and in postmenopausal state. The adoption of a correct gluten-free diet (GFD) improves bone derangement, but is not able to normalize bone mass in all the patients. Biomarkers effective in the prediction of bone response to gluten-free diet are not yet available and the indications of guidelines are still imperfect and debated. In this review, the pathophysiology of bone loss is correlated to clinical aspects, defining an alternative proposal of management for this condition.
\end{abstract}

Keywords: celiac disease; osteoporosis; gluten-free diet; bone densitometry

\section{Introduction}

Osteoporosis is a condition characterized by low bone mass and micro-architectural deterioration of bone tissue resulting in enhanced bone fragility and an increase in fracture risk [1]. It affects more than 75 million people in developed countries, causing 8.9 million fractures annually worldwide. Osteoporotic fractures account for 2.8 million disability-adjusted life years annually: to make this even clearer, this index is higher than other conditions, such as breast cancer, gastric cancer and hypertension [2]. There is a general agreement in the literature that more than $75 \%$ of untreated adult 
celiac disease $(C D)$ patients with an overt malabsorption syndrome at diagnosis suffer from a loss of bone mass [3-9], and this complication also affects about half the patients with subclinical CD, presenting with minimal, transient and apparently unrelated symptoms [3,9], or asymptomatic patients diagnosed because of their first-degree kinship [4].

Moreover, an increased prevalence of celiac disease in osteoporotic patients was reported [10-12] and, even if this result was not confirmed by others [13-15], all previous results show the importance of the problem from a clinical point of view. On the contrary, the value of active screening for CD in patients with otherwise unexplained bone loss is still under debate.

Accordingly, there is no doubt that $\mathrm{CD}$ is a condition at high risk for secondary osteoporosis, and the evaluation of bone mass and mineral metabolism is thus very important in the clinical management of these patients. In the last few years, while the mechanisms of bone derangement in CD have been extensively studied, less attention has been paid to the clinical management of this complication: there is in fact very little information available on the timing of the first bone mineral density (BMD) measurement, on follow-up frequency, even on the best treatment options.

\section{Bone Damage and Mineral Metabolism Derangement in Celiac Disease}

Intestinal malabsorption and inflammation contribute to the pathophysiology of bone damage in CD. Villous atrophy is responsible for alterations of intestinal absorption, and a negative calcium balance was shown in CD patients due to several mechanisms: malabsorption of calcium in untreated patients [16], partially reversible after gluten-free diet (GFD) [17]; the reduction of calcium intake [18] also due to a secondary lactose intolerance [19]; and the reduction of intestinal calcium absorption due to its binding to intraluminal unabsorbed fatty acids [16]. Hypocalcemia can induce a compensatory increase of serum levels of parathyroid hormone (PTH), in turn responsible for an increase of bone turnover [7,20]: in untreated CD, serum PTH correlates with markers of both bone synthesis, such as osteocalcin, as well as resorption, like telopeptide of type I collagen (ICTP) [5]. Bone resorption is faster than bone neoformation, resulting in net bone loss and a high turnover osteoporosis [21]. The increase of serum PTH enhances the activity of the renal enzyme 1- $\alpha$-hydroxylase, which converts 25 -vitamin D into 1,25 vitamin $\mathrm{D}$, in order to improve calcium absorption at intestinal level. However, this effort is ineffective, mainly due to the lack in immature enterocytes of celiac mucosa of calbindin [22], a vitamin D-dependent calcium-binding protein, minimizing the role of vitamin D malabsorption [23]. Finally, high levels of 1,25 vitamin D might have the paradoxical effect of increasing bone resorption, as shown in patients with chronic renal failure [4]. Accordingly, vitamin D functions are rarely impaired in untreated $C D$, as hyperconversion of metabolite 25 -vitamin $\mathrm{D}$ guarantees adequate levels of the active form 1,25 vitamin D.

Intestinal malabsorption could also lead to some deficits of other minerals, fat and water soluble vitamins that could affect normal bone metabolism. In particular, low levels of zinc were described in non-treated celiac patients [24], and related to low levels of insulin-like growth factor, that are subsequently responsible for derangement in bone metabolism, growth and immune function [25].

This complex network of events is present in both overt symptomatic and subclinical CD to be a disease that is below the threshold of clinical detection without signs or symptoms sufficient to trigger CD testing in routine practice - or it is silent, equivalent to asymptomatic CD patients, even 
if the extent of bone loss and the alterations of serum levels of indices of bone and mineral metabolism may be less severe than in CD patients with overt malabsorption [3].

More recently, much evidence has also suggested the role of both local and systemic inflammation in the pathophysiology of bone loss in $\mathrm{CD}$, characterized by a chronic increase of both mucosal and serum pro-inflammatory cytokines, in particular TNF $\alpha$, IL-1 and IL-6 [26,27]. IL-1 and TNF $\alpha$ stimulate osteoclastogenesis and bone resorption [28]; IL-6 has a pivotal role in bone resorption by recruiting osteoclast precursors and stimulating their differentiation [29]. In untreated CD patients, serum IL-6 levels inversely correlate with BMD [27] and directly with PTH and ICTP levels, a marker of bone resorption [30]. Recently, the existence of a complex cytokine imbalance in CD patients, affecting both osteoclast and osteoblast activity was shown: cultures of peripheral blood mononuclear cells of healthy donors with sera of untreated CD patients result in an increase in osteoclast number and IL-6 levels, together with an inhibition of IL-12 and IL-18 [31], two cytokines showing an in vitro inhibitory effect on osteoclastogenesis and osteoclast activity [32,33].

In the last 15 years, great attention has been given to the RANKL/RANK/osteoprotegerin pathway, that is today considered the main signaling system in bone metabolism. The receptor activator of nuclear factor $\kappa \mathrm{B}$ ligand (RANKL) is expressed and secreted by osteoblasts; it binds RANK, located on the surface of osteoclast precursors, to induce the differentiation of these cells into mature osteoclasts, promoting bone resorption. Osteoprotegerin (OPG) is also secreted by osteoblasts; it acts as a decoy receptor for RANK and blocks RANK-RANKL interaction [34]. In CD patients, an increased level of OPG and RANKL was described, with an OPG/RANKL ratio significantly lower than controls. Moreover, the OPG/RANKL ratio was correlated with spine BMD [35] and with IL-6 levels [31].

The pathophysiological role of autoantibodies against OPG is also debated, as in a recent paper the presence of these antibodies was detected in a man with $\mathrm{CD}$, high bone turnover and severe osteoporosis not responsive to GFD and to calcium and vitamin D supplementation [36]. This observation was not confirmed by a subsequent study on a large cohort of CD patients on GFD [37].

Further factors are linked to endocrine and reproductive disorders, commonly part of CD clinical presentation. In particular, early menopause and periods of amenorrhea could occur in women, due partly to malnutrition and partly to hormonal imbalance, and could worsen the severity of osteoporosis [38]. In men, hypogonadism was described, due to a reversible androgen resistance [39] and to hyperprolactinemia [40], and considered a possible adjunctive factor risk for osteoporosis [41]. Finally, CD is frequently associated with autoimmune thyroiditis and type I diabetes mellitus [42]: both these disorders are at high risk for osteoporosis $[43,44]$.

\subsection{Effect of GFD}

Strict adherence to GFD allows BMD improvement but it is not able to normalize it in all cases. Mucosal recovery does not appear to be the only determining variable: in fact, with the same histological response, bone mass normalization is present in celiacs on GFD since early infancy [45] but not always in patients on GFD for the same length of time but diagnosed at a later age. In particular, normalization of BMD levels in childhood CD may be complete as early as after two years of GFD [46]. On the contrary, in adults, many cross-sectional studies demonstrated higher BMD levels in treated $v s$. untreated CD patients but still lower than in healthy volunteers [3-9,47-50]; also 
the prevalence of alterations of indices of bone and mineral metabolism is lower in treated $v s$. untreated patients $[47,51,52]$. These results were shown in patients on GFD for a median duration of 28.5 months [5], in patients on GFD from a mean of 3.6 years [47] and in a group of patients treated for a mean of 16 years, a very long period of GFD [52]. It is therefore evident that the early onset of bone damage, probably before achieving bone mass peak, is an important time point determining GFD-induced bone mass gain. Even longitudinal studies are of little help here, as the longest period of GFD evaluated was 5 years [53]. On the other hand, these studies have provided important information on the kinetic of bone mass recovery with the start of GFD and correlations with the modifications of bone-mineral metabolism parameters. Following a GFD with optimal compliance for a period of one year allows a significant improvement of BMD values, ranging from 5\% [6] to $8 \%$ [3] according to different studies. These results were confirmed in a larger cohort of patients enrolled at diagnosis and restudied after one year of GFD [8]. In a two-year study, GFD improved not only bone mass but also serum levels of indices of bone and mineral metabolism. BMD improvement was more evident after two years than after one year of GFD, suggesting that a period longer than one year was necessary to point out intrinsic capacities of an individual patient to recover bone mass. Serum levels of propeptide of type I procollagen (PICP) at diagnosis proved to be a strong predictor of bone mass gain after two years, suggesting the possibility of selecting the group of patients with high levels of bone matrix formation activity that is more likely to readily respond to GFD [54]. In a three-year study, BMD increased in $92 \%$ of CD patients in GFD with a mean bone mass gain around $3 \%-4 \%$ per year. However, only $12 \%$ of patients showed a normalization of BMD. In particular, in a small group of patients, it was evident that relatively good bone mass gain was present during the first year, but was negligible in the subsequent study period [55]. These observations agree with a five-year study showing femoral and lumbar BMD values at five years similar to BMD values at one-year follow-up both in men and women, with the exception of trochanter values, which proved to be higher at five-year measurement than one-year values [53].

In summary, BMD values normalize only in children, when diagnosed early in infancy and if they follow a long-term GFD with optimal adherence. On the contrary, BMD values in adults show a good improvement in the first period, generally around two years, after the institution of a GFD; the improvement is then generally unsatisfactory and treatment with a mineral-active drug should probably be considered. Nevertheless, CD patients show a wide range of response to GFD and risk factors for osteoporosis include old age at diagnosis and the degree of osteopenia in late diagnosis, compliance to GFD, menstrual status, i.e., late age at menarche, early menopause, periods of amenorrhea, low body mass index (BMI), low dietary calcium intake, inadequate physical activity and use of glucocorticoids $[4,52,56,57]$. What appears to emerge is that as age progresses and, in women as menopause approaches, the ability to recover bone mass seems to diminish, being greatest in childhood and lowest in peri- and postmenopausal women. In this latter subgroup of patients, waiting two or three years to determine the extent of GFD-induced bone mass gain could thus be incorrect and the start of treatment with a mineral-active drug should be earlier, probably at diagnosis.

The availability of predictive markers of GFD-induced bone mass gain could be a solution for this problem, but the mechanism responsible for the unsatisfactory improvement is not completely clear. A persistent reduction of fractional calcium absorption was shown in patients on GFD, besides the improvement of intestinal mucosa architecture [17], and in a subgroup of patients the persistence of a 
secondary hyperparathyroidism and a significant correlation between serum PTH levels and femoral BMD were shown [17]. The possible role of secondary hyperparathyroidism was suggested by other papers [20,58] but also disproved [59], and the proposed pathophysiological mechanisms for the persistent raise of serum PTH were residual villous atrophy leading to calcium malabsorption [20], a reduction of calcium intake [18], but also a slow reversal of parathyroid hyperplasia [60]. Partial adherence to GFD [55] and incomplete mucosal recovery [57] could also have a role in subgroups of treated patients.

Circulating factors secondary to persistent activation of the mucosal immune system could directly interfere with osteoclastogenesis and osteoblast activity. It was shown that in patients following GFD for a mean period of 40 months [31] the prevalence of bone damage is around $40 \%$, and circulating levels of cytokines (IL-6, IL-1beta, TNF-alfa, TNF-beta, IL-12, IL-18, RANK-L, OPG) are significantly lower than in untreated patients, but significantly higher than in healthy volunteers. In particular, the osteoclastogenic activity of sera from patients on long-term GFD proved to be still significantly higher than sera of healthy volunteers and serum cytokine levels were not correlated to PTH levels [31]. An altered ratio between RANKL and OPG in untreated patients normalizes to healthy volunteer levels in patients on GFD [31,35]. Accordingly, the dietary treatment with GFD alone is not able to completely control the increased osteoclast differentiation and activity present in CD, as confirmed by a strong correlation between OPG/RANKL ratio and BMD [35], and the mechanism responsible for bone damage does not involve PTH. Finally, while a three-year period of GFD determines a significant decrease of IL 6, which is significantly inversely correlated at diagnosis with lumbar BMD, it cannot normalize IL-1 $\beta$ and IL-1 receptor antagonist serum levels [27].

Therefore, persistent inflammation in treated CD patients could have a role in the persistence of bone mass derangement. In particular, the predominant mechanism responsible for bone derangement seems different between short-term and long-term treated CD patients: in the period immediately after diagnosis, the malabsorption of calcium and the consequent hormonal and vitamin $\mathrm{D}$ alterations appear to be the prevalent pathophysiological mechanism, their correction allowing a satisfactory bone mass gain, comparable to the effect of administering mineral-active drugs in postmenopausal osteoporosis [61]. Unfortunately, the extent of the loss of bone mass in untreated CD is very often higher than the extent of the recovery induced just by GFD in the early stages of treatment and, once the GFD-induced metabolic surge that occurs in this phase is over, persistent bone loss seems due to the persistent activation of a local mechanism, related to chronic inflammation. Preliminary data from our group confirm this hypothesis, as an in-depth evaluation of hormonal and local factors suggests that high levels of OPG and low levels of PICP select the subgroup of CD patients with a persistent reduction of bone mass, despite strict adherence to GFD and architectural villi reconstitution [62]. If confirmed, these markers might be used to identify those patients who need mineral-active treatment associated with gluten-free diet.

\section{Fracture Risk}

$\mathrm{BMD}$ is only one of the factors that contribute to establishing the extent of fracture risk in osteoporotic patients. Other factors are related to bone mechanical characteristics, such as stiffness of 
cortical bone, but also to inadequacy of protectors from trauma (body mass, fat and muscle compartments) and to neuromuscular dysfunction [63].

Several studies pointed out the prevalence of fracture in celiac population, but with a very important heterogeneity in methods (study design, sample selection, fracture data collection) and cohorts studied (treated/untreated CD), making available data often inconsistent and difficult to interpret [64]. Most authors agree on the increased prevalence of fracture in CD patients [63,65-71] and a recent meta-analysis evaluating a total of 20,955 CD patients and 96,777 controls described a risk of fracture $43 \%$ greater in CD [72]. Data on fractures were collected by mailed questionnaires, by personal interviews or by medical records; consequently, results on peripheral fractures might be more easily estimated and axial fractures underestimated. Only a cross-sectional study explored the existence of asymptomatic vertebral fractures by spinal X-ray and did not find an increase of vertebral fractures in $\mathrm{CD}$ patients [68]. However, if $\mathrm{CD}$ patients are subdivided according to the clinical presentation, peripheral fracture risk proves to be higher than controls in patients with overt malabsorption symptom, while it is similar to the general population in subclinical and silent presentation [71]. These data were confirmed in a more recent case-control study, pointing out a higher peripheral fracture risk also in men and underlining again the importance of adherence to GFD [63]. However, to confirm what was said above with respect to pathophysiology, in a population-based study in Olmsted County, CD patients showed a fracture risk twice that of controls, and this figure persisted unchanged during GFD [66].

In general, however, large population-based studies should be interpreted with care, since, for example, in one study on the fracture risk in CD patients on a cohort of 1021 celiac patients, a possible misclassification of patients could have accounted for the negativity of results, as data were extracted from the National Patient Discharge Register, known for a low estimated validity of diagnosis of CD (78\%) [73].

It is, finally, likely that, in addition to just BMD measurement, assessment of the physical characteristics of bone, such as its elasticity, can add something to our understanding of the mechanisms that favor fractures [74,75]. Unfortunately, no studies are available that correlate bone ultrasound densitometry parameters with fracture risk in $\mathrm{CD}$ patients.

\section{Clinical Management}

Only a limited number of international recommendations are available on the clinical management of osteoporosis in $\mathrm{CD}$, probably due to the lack of sufficient data on patient follow-up and the role of the menopause. As already stated, there is no clinical or biochemical marker to select the subgroup of patients not responding to GFD alone with an improvement of bone mass and which will be characterized by a high risk of fractures; accordingly, we are not able, as yet, to optimize both treatment and timing of BMD follow-up measurement.

In 2000, the British Society of Gastroenterology published the guidelines for osteoporosis in CD [76]. General advice aimed at modifying lifestyle factor risk was provided, such as enhancing physical activity, stopping smoking, avoiding alcohol excess. Moreover, a daily calcium intake of 1500 $\mathrm{mg}$, even by pharmacological supplementation, and vitamin D supplementation, if inadequate serum levels were evident, was suggested. Bone densitometry was recommended at diagnosis for all patients, to detect osteoporosis early and to obtain the greatest possible benefit from treatment, or at 
least at menopausal age for women and at the age of 55 years for men. Postmenopausal women with normal BMD should repeat densitometry after two years. Osteoporotic postmenopausal women and men over 55 years should be offered treatment and bone densitometry yearly to monitor treatment efficacy.

The subsequent awareness of an ultimately low absolute risk of fracture, even if higher than in the general population [77], determined strong criticism of the extensive use of bone densitometry at diagnosis, suggesting that BMD measurement should be restricted to patients with high short-term fracture risk, such as patients non-compliant with GFD or who failed to respond to dietary treatment, on glucocorticoid therapy, with untreated hypogonadism, older age, low BMI, and previous fragility fracture [78]. This led to reconsideration of the guidelines and the proposal of BMD measurement only in clinically non-responder patients, especially those with low BMI, in menopausal women and after 55 years for men [79]. In 2003, the American Gastroenterological Association guidelines on osteoporosis in gastrointestinal disease suggested that bone densitometry should be performed in adults with newly diagnosed celiac disease after one year of GFD, to allow for stabilization of bone density, implementation of GFD, calcium and vitamin D supplementation as needed, and, if necessary, bisphosphonates and hormonal therapy were strongly encouraged in osteoporotic patients [80].

A Canadian Position Statement on evaluation and management of skeletal health in CD was recently published [81]. BMD measurement was suggested at diagnosis only in adults with classic $\mathrm{CD}$, and after one year of GFD in adults with asymptomatic or silent CD. The latter group of patients should be considered for earlier BMD evaluation in the presence of risk factors such as menopause, older age, history of fragility fracture, unexplained iron deficiency anemia, vitamin D deficiency/insufficiency, and high titers for $\mathrm{CD}$ serological markers. Indications for follow-up were also given: BMD should be re-evaluated after one year of GFD in the presence of osteopenia/osteoporosis at diagnosis, and after two years in cases of documentation of normal bone mass. The assessment of bone and mineral metabolism by dosing serum calcium, albumin, 1,25 dihydoxicolecalciferol and PTH levels should be repeated every six months until normalization.

Clinical application of the Canadian guidelines does not, however, seem to allow substantial resource savings, and is thus very similar to the earlier British proposal. In patients with asymptomatic or silent $\mathrm{CD}$ with the aforementioned risk factors, early prescription of bone densitometry is indicated, since anemia and vitamin D alterations show a very high prevalence also in this subgroup. Considering the prevalence of bone loss in patients with and without overt malabsorption symptoms, BMD measurement could provide more important information in asymptomatic than in clinically overt malabsorber patients: bone loss is highly prevalent in overt malabsorption, and these patients could undergo mineral-active therapy as of diagnosis; conversely, asymptomatic/silent patients should be screened for bone loss presence. Moreover, the most important risk factor at diagnosis seems to be the age of the patient, and patients well over the age of peak bone mass could be treated without measuring BMD, while those patients below or shortly after the age of the peak should undergo BMD measurement. This approach seems the most correct one in optimizing the use of bone densitometry, but the problem remains for the free dispensing of the mineral-active drug, which in Italy depends on the presence of a pathological fracture. Optimization of this phase could be achieved by performing a radiological study of the lumbar spine in patients most at risk, such as 
symptomatic subjects, peri- and postmenopausal women and men over the age of 55, in order to detect vertebral fractures, together with a complete case history for previous fractures. In Italy (Lombardia Region), the cost of lumbar and femoral densitometry is €88.66 (€44.33 for each segment), while the cost of a lumbar spine $\mathrm{X}$-ray is only $€ 34.80$.

In conclusion, there is no general agreement on the correct timing of bone densitometry in celiac patients; screening at diagnosis seems to be not justified in all patients and the proposed alternative approach is explained in Figure 1.

Figure 1. Diagnostic and therapeutic approach to $C D$ patients without a previous fracture.

$\begin{array}{ccc}\begin{array}{c}\text { CD with } \\ \text { overt malabsorption }\end{array} & \begin{array}{c}\text { Asymptomatic/silent } \\ \text { CD patients } \\ \text { Before }\end{array} & \begin{array}{c}\text { After } \\ \text { peak bone mass }\end{array} \\ & \text { peak bone mass } \\ \text { Densitometry } & \begin{array}{c}\text { Absence of additional } \\ \text { risk factors }\end{array}\end{array}$

Abnormal Normal

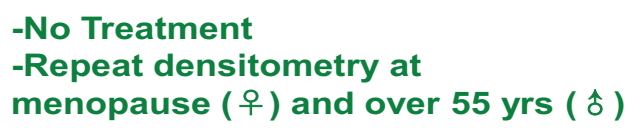

Dorsolumbar spine X-ray

Fracture +ve $\quad$ Fracture -ve

Treatment offered Treatment

By Health System charged to pts

Repeat Densitometry every two years

Treatment with a mineral-active drug in association to GFD, even without BMD measurement, should be prescribed to patients at high short-term risk of fracture, including symptomatic patients, asymptomatic patients in peri and postmenopausal period, men older than 55 years, low calcium intake, low BMI, poor compliance to GFD, or unresponsiveness to GFD following steroid therapy; in these cases, measure BMD after two years of treatment. To allow the free dispensing of the drug, 
patients should be screened for the presence of fracture and X-ray of the spine could be adequate to this aim. Young asymptomatic patients with a normal BMD should be re-evaluated at peri-menopausal period (female patients) or over 55 years (male patients).

As regards the choice of the drug for the treatment of osteoporosis in $\mathrm{CD}$, there are no longitudinal studies dealing with this topic and we have no information on which to base our choice in the individual subgroups of patients. The current approach is clearly based on post-menopausal osteoporosis treatment with a choice between a weekly administration of alendronate and a monthly administration of ibandronate. The use of denosumab, a decoy receptor for RANKL able to reduce the activation of the osteoclast system, seems very interesting, also on the basis of the data obtained on the RANK/RANKL/OPG system. The results of the first studies are awaited in order to define the best strategy for the different types of CD patients.

\section{Conclusions}

In conclusion, in the pathophysiology of bone derangement in CD patients, both malabsorption and the persistent activation of inflammation at intestinal level are important, in a two-step model. Biomarkers with a predictive role of the normalization of BMD levels are needed and the evaluation of the RANK/RANKL/OPG system could offer some inputs on this topic.

\section{Conflicts of Interest}

The authors declare no conflict of interest.

\section{References}

1. Consensus development conference: Prophylaxis and treatment of osteoporosis. Osteoporos. Int. 1991, 1, 114-117.

2. WHO Scientific Group on the Assessment of Osteoporosis at Primary Health Care Levels. Summary Meeting Report 2004; World Health Organization: Brussels, Belgium, 2007; pp. $1-17$.

3. Corazza, G.R.; Di Sario, A.; Cecchetti, A.; Jorizzo, R.A.; Di Stefano, M.; Minguzzi, L.; Brusco, G.; Bernardi, M.; Gasbarrini, G. Influence of pattern of clinical presentation and gluten-free diet on bone mass and metabolism in adult coeliac disease. Bone 1996, 18, 525-530.

4. Mazure, R.; Vazquez, H.; Gonzalez, D.; Mautalen, C.; Pedreira, S.; Boerr, L.; Bai, J.C. Bone mineral affectation in asymptomatic adult patients with celiac disease. Am. J. Gastroenterol. 1994, 89, 2130-2134.

5. Corazza, G.R.; di Sario, A.; Cecchetti, A.; Tarozzi, C.; Corrao, G.; Bernardi, M.; Gasbarrini, G. Bone mass and metabolism in patients with celiac disease. Gastroenterology 1995, 109, $122-128$.

6. McFarlane, X.A.; Bhalla, A.K.; Robertson, D.A.F. Effect of gluten free diet on osteopenia in adults with newly diagnosed celiac disease. Gut 1996, 39, 180-184. 
7. Keaveny, A.P.; Freaney, R.; McKenna, M.J.; Masterson, J.; O’Donoghue, D.P. Bone remodeling indices and secondary hyperparathyroidism in celiac disease. Am. J. Gastroenterol. 1996, 91, 1226-1231.

8. Valdimarsson, T.; Löfman, O.; Toss, G.; Ström, M. Reversal of osteopenia with diet in adult coeliac disease. Gut 1996, 38, 322-327.

9. Mustalahti, K.; Collin, P.; Sievänen, H.; Salmi, J.; Mäki, M. Osteopenia in patients with clinically silent coeliac disease warrants screening. Lancet 1999, 354, 744-745.

10. Duerksen, D.R.; Leslie, W.D. Positive celiac disease serology and reduced bone mineral density in adult women. Can. J. Gastroenterol. 2010, 24, 103-107.

11. Stenson, W.F.; Newberry, R.; Lorenz, R.; Baldus, C.; Civitelli, R. Increased prevalence of celiac disease and need for routine screening among patients with osteoporosis. Arch. Intern. Med. 2005, 165, 393-399.

12. Armagan, O.; Uz, T.; Tascioglu, F.; Colak, O.; Oner, C.; Akgun, Y. Serological screening for celiac disease in premenopausal women with idiopathic osteoporosis. Clin. Rheumatol. 2005, 24, 239-243.

13. Drummond, F.J.; Annis, P.; O’Sullivan, K.; Wynne, F.; Daly, M.; Shanahan, F.; Quane, K.A.; Molloy, M.G. Screening for asymptomatic celiac disease among patients referred for bone densitometry measurement. Bone 2003, 33, 970-974.

14. Mather, K.J.; Meddings, J.B.; Beck, P.L.; Scott, R.B.; Hanley, D.A. Prevalence of IgA-antiendomysial antibody in asymptomatic low bone mineral density. Am. J. Gastroenterol. 2001, 96, 120-125.

15. Karakan, T.; Ozyemisci-Taskiran, O.; Gunendi, Z.; Atalay, F.; Tuncer, C. Prevalence of IgA-antiendomysial antibody in a patient cohort with idiopathic low bone mineral density. World J. Gastroenterol. 2007, 21, 2978-2982.

16. Molteni, N.; Bardella, M.T.; Vezzoli, G.; Pozzoli, E.; Bianchi, P. Intestinal calcium absorption as shown by stable strontium test in celiac disease before and after gluten-free diet. Am. J. Gastroenterol. 1995, 90, 2025-2028.

17. Pazianas, M.; Butcher, G.P.; Subhani, J.M.; Finch, P.J.; Ang, L.; Collins, C.; Heaney, R.P.; Zaidi, M.; Maxwell, J.D. Calcium absorption and bone mineral density in celiacs after long term treatment with gluten-free diet and adequate calcium intake. Osteoporos. Int. 2005, 16, $56-63$.

18. Kinsey, L.; Burden, S.T.; Bannermann, E. A dietary survey to determine if patients with celiac disease are meeting current healthy eating guidelines and how their diet compares to that of the british general population. Eur. J. Clin. Nutr. 2008, 62, 1333-1342.

19. Plotkin, G.R.; Isselbacher, K.J. Secondary disaccharidase deficiency in adult celiac disease (Nontropical Sprue) and other malabsorption states. N. Engl. J. Med. 1964, 271, 1033-1037.

20. Valdimarsson, T.; Toss, G.; Löfman, O.; Ström, M. Three years' follow-up of bone density in adult coeliac disease: Significance of secondary hyperparathyroidism. Scand. J. Gastroenterol. 2000, 35, 274-280.

21. Corazza, G.R.; di Stefano, M.; Maurino, E.; Bai, J.C. Bones in celiac disease: Diagnosis and treatment. Best Pract. Res. Clin. Gastroenterol. 2005, 19, 453-465. 
22. Staun, M.; Jarnum, S. Measurement of the 10,000-molecular weight calcium-binding protein in small-intestinal biopsy specimens from patients with malabsorption syndromes. Scand. J. Gastroenterol. 1988, 23, 827-832.

23. Colston, K.W.; Mackay, A.G.; Finlayson, C.; Wu, J.C.; Maxwell, J.D. Localisation of Vitamin $\mathrm{D}$ receptor in normal human duodenum and in patients with coeliac disease. Gut 1994, 35, $1219-1225$.

24. Valdimarsson, T.; Arnqvist, H.J.; Toss, G.; Järnerot, G.; Nyström, F.; Ström, M. Low circulating insulin-like growth factor $\mathrm{i}$ in coeliac disease and its relation to bone mineral density. Scand. J. Gastroenterol. 1999, 34, 904-908.

25. Jameson, S. Coeliac disease, insulin-like growth factor, bone mineral density, and zinc. Scand. J. Gastroenterol. 2000, 35, 894-896.

26. Kontakou, M.; Przemioslo, R.T.; Sturgess, R.P.; Limb, A.G.; Ciclitira, P.J. Expression of tumour necrosis factor-alpha, interleukin-6, and interleukin-2 mRNA in the jejunum of patients with coeliac disease. Scand. J. Gastroenterol. 1995, 30, 456-463.

27. Fornari, M.C.; Pedreira, S.; Niveloni, S.; González, D.; Diez, R.A.; Vázquez, H.; Mazure, R.; Sugai, E.; Smecuol, E.; Boerr, L.; et al. Pre- and post-treatment serum levels of cytokines IL-1beta, IL-6, and IL-1 receptor antagonist in celiac disease. Are they related to the associated osteopenia? Am. J. Gastroenterol. 1998, 93, 413-418.

28. Wei, S.; Kitaura, H.; Zhou, P.; Ross, F.P.; Teitelbaum, S.L. IL-1 mediates TNF-induced osteoclastogenesis. J. Clin. Investig. 2005, 115, 282-290.

29. Raisz, L.G. Local and systemic factors in the pathogenesis of osteoporosis. N. Engl. J. Med. 1988, 318, 818-828.

30. Di Stefano, M.; Sciarra, G.; Jorizzo, R.A.; Grillo, R.L.; Cecchetti, L.; Speziale, D.; Pichini, L.; Gasbarrini, G.; Corazza, G.R. Local and gonadal factors in the pathogenesis of coeliac bone loss. Ital. J. Gastroenterol. 1997, 29, 31.

31. Taranta, A.; Fortunati, D.; Longo, M.; Rucci, N.; Iacomino, E.; Aliberti, F.; Facciuto, E.; Migliaccio, S.; Bardella, M.T.; Dubini, A. Imbalance of Osteoclastogenesis-regulating Factors in Patients with Celiac Disease. J. Bone Miner. Res. 2004, 19, 1112-1121.

32. Horwood, N.J.; Elliott, J.; Martin, T.J.; Gillespie, M.T. IL-12 alone and in synergy with IL-18 inhibits osteoclast formation in vitro. J. Immunol. 2001, 166, 4915-4921.

33. Yamada, N.; Niwa, S.; Tsujimura, T.; Iwasaki, T.; Sugihara, A.; Futani, H.; Hayashi, S.; Okamura H.; Akedo, H.; Terada, N. Interleukin-18 and interleukin-12 synergistically inhibit osteoclastic bone-resorbing activity. Bone 2002, 6, 901-908.

34. Khosla, S. Minireview: The OPG/RANKL/RANK system. Endocrinology 2001, 142, 5050-5055.

35. Fiore, C.E.; Pennisi, P.; Ferro, G.; Ximenes, B.; Privitelli, L.; Mangiafico, R.A.; Santoro, F.; Parisi, N.; Lombardo, T. Altered osteoprotegerin/RANKL ratio and low bone mineral density in celiac patients on long-term treatment with gluten-free diet. Horm. Metab. Res. 2006, 38, 417-422.

36. Riches, P.L.; McRorie, E.; Fraser, W.D.; Determann, C.; van't Hof, R.; Ralston, S.H. Osteoporosis associated with neutralizing autoantibodies against osteoprotegerin. N. Engl. J. Med. 2009, 361, 1459-1465. 
37. Larussa, T.; Suraci, E.; Nazionale, I.; Leone, I.; Montalcini, T.; Abenavoli, L.; Imeneo, M.; Pujia, A.; Luzza, F. No evidence of circulating autoantibodies against osteoprotegerin in patients with celiac disease. World J. Gastroenterol. 2012, 18, 1622-1627.

38. Ozgör, B.; Selimoğlu, M.A. Coeliac disease and reproductive disorders. Scand. J. Gastroenterol. 2010, 45, 395-402.

39. Green, J.R.; Goble, H.L.; Edwards, C.R.; Dawson, A.M. Reversible insensitivity to androgens in men with untreated gluten enteropathy. Lancet 1977, 1, 280-282.

40. Farthing, M.J.; Rees, L.H.; Dawson, A.M. Male gonadal function in coeliac disease: III. Pituitary regulation. Clin. Endocrinol. 1983, 19, 661-671.

41. Stazi, A.V.; Trecca, A.; Trinti, B. Osteoporosis in celiac disease and in endocrine and reproductive disorders. World J. Gastroenterol. 2008, 14, 498-505.

42. Di Sabatino, A.; Corazza, G.R. Coeliac disease. Lancet 2009, 373, 1480-1493.

43. Mosekilde, L.; Eriksen, E.F.; Charles, P. Effects of thyroid hormones on bone and mineral metabolism. Endocrinol. Metab. Clin. 1990, 19, 35-63.

44. Hofbauer, L.C.; Brueck, C.C.; Singh, S.K.; Dobnig, H. Osteoporosis in patients with diabetes mellitus. J. Bone Miner. Res. 2007, 22, 1317-1328.

45. Mora, S.; Barera, G.; Beccio, S.; Menni, L.; Proverbio, M.C.; Bianchi, C.; Chiumello, G. A prospective, longitudinal study of the long-term effect of treatment on bone density in children with celiac disease. J. Pediatr. 2001, 139, 516-521.

46. Mora, S.; Barera, G.; Ricotti, A.; Weber, G.; Bianchi, C.; Chiumello, G. Reversal of low bone density with a gluten-free diet in children and adolescents with celiac disease. Am. J. Clin. Nutr. 1998, 67, 477-481.

47. Bodé, S.; Hassager, C.; Gudmand-Høyer, E.; Christiansen, C. Body composition and calcium metabolism in adult treated coeliac disease. Gut 1991, 32, 1342-1345.

48. Valdimarsson, T.; Toss, G.; Ross, I.; Löfman, O.; Ström, M. Bone mineral density in coeliac disease. Scand. J. Gastroenterol. 1994, 29, 457-461.

49. Walters, J.R.; Banks, L.M.; Butcher, G.P.; Fowler, C.R. Detection of low bone mineral density by dual energy x-ray absorptiometry in unsuspected suboptimally treated coeliac disease. Gut 1995, 37, 220-224.

50. McFarlane, X.A.; Bhalla, A.K.; Reeves, D.E.; Morgan, L.M.; Robertson, D.A. Osteoporosis in treated adult coeliac disease. Gut 1995, 36, 710-714.

51. Ferretti, J.; Mazure, R.; Tanoue, P.; Marino, A.; Cointry, G.; Vazquez, H.; Niveloni, S.; Pedreira, S.; Mauriño, E.; Zanchetta, J.; et al. Analysis of the structure and strength of bones in celiac disease patients. Am. J. Gastroenterol. 2003, 98, 382-390.

52. González, D.; Mazure, R.; Mautalen, C.; Vazquez, H.; Bai, J. Body composition and bone mineral density in untreated and treated patients with celiac disease. Bone 1995, 16, 231-234.

53. Kemppainen, T.; Kröger, H.; Janatuinen, E.; Arnala, I.; Lamberg-Allardt, C.; Kärkkäinen, M.; Kosma, V.M.; Julkunen, R.; Jurvelin, J.; Alhava, E.; et al. Bone recovery after a gluten-free diet: A 5-year follow-up study. Bone 1999, 25, 355-360.

54. Corazza, G.R.; di Stefano, M.; Jorizzo, R.A.; Cecchetti, L.; Minguzzi, L.; Gasbarrini, G. Propeptide of Type I procollagen is predictive of posttreatment bone mass gain in adult celiac disease. Gastroenterology 1997, 113, 67-71. 
55. Bai, J.C.; Gonzalez, D.; Mautalen, C.; Mazure, R.; Pedreira, S.; Vazquez, H.; Smecuol, E.; Siccardi, A.; Cataldi, M.; Niveloni, S.; et al. Long-term effect of gluten restriction on bone mineral density of patients with coeliac disease. Aliment. Pharm. Ther. 1997, 11, 157-164.

56. Pistorius, L.R.; Sweidan, W.H.; Purdie, D.W.; Steel, S.A.; Howey, S.; Bennett, J.R.; Sutton, D.R. Coeliac disease and bone mineral density in adult female patients. Gut 1995, 37, 639-642.

57. Kemppainen, T.; Kröger, H.; Janatuinen, E.; Arnala, I.; Kosma, V.M.; Pikkarainen, P.; Julkunen, R.; Jurvelin, J.; Alhava, E.; Uusitupa, M. Osteoporosis in adult patients with celiac disease. Bone 1999, 24, 249-255.

58. Selby, P.L.; Davies, M.; Adams, J.E.; Mawer, E.B. Bone loss in celiac disease is related to secondary hyperparathyroidism. J. Bone Miner. Res. 1999, 14, 652-657.

59. Lemieux, B.; Boivin, M.; Brossard, J.H.; Lepage, R.; Picard, D.; Rousseau, L.; D’amour, P. Normal parathyroid function with decreased bone mineral density in treated celiac disease. Can. J. Gastroenterol. 2001, 15, 302-307.

60. Christensen, M.S.; Nielsen, H.E.; Torring, S. Hypercalcemia and parathyroid function after renal transplantation. Acta Med. Scand. 1977, 201, 35-39.

61. Liberman, U.A.; Weiss, S.R.; Bröll, J.; Minne, H.W.; Quan, H.; Bell, N.H.; Rodriguez-Portales, J.; Downs, R.W., Jr.; Dequeker, J.; Favus, M. Effect of oral alendronate on bone mineral density and the incidence of fractures in postmenopausal osteoporosis. N. Engl. J. Med. 1995, 333, 1437-1443.

62. Di Stefano, M.; Mengoli, C.; Tomarchio, O.; Bergonzi, M.; Pagani, E.; de Amici, M.; Ilardo, D.; Vattiato, C.; Biagi, F.; Zanaboni, A.; et al. Increased Osteoprotegerin and Decreased COOH-Terminal Propeptide of Type I Collagen Select Celiac Disease Patients on Long Term Gluten Free Diet with Persisting Low Bone Mass. In Proceedings of UEGW (21st United European Gastroenterology Week), Belin, Germany, 12-16 October 2013.

63. Sánchez, M.I.; Mohaidle, A.; Baistrocchi, A.; Matoso, D.; Vázquez, H.; González, A.; Mazure, R.; Maffei, E.; Ferrari, G.; Smecuol, E.; et al. Risk of fracture in celiac disease: Gender, dietary compliance, or both? World J. Gastroenterol. 2011, 17, 3035-3042.

64. Bianchi, M.L.; Bardella, M.T. Bone in celiac disease. Osteoporos. Int. 2008, 19, 1705-1716.

65. West, J.; Logan, R.F.; Card, T.R.; Smith, C.; Hubbard, R. Fracture risk in people with celiac disease: A population-based cohort study. Gastroenterology 2003, 125, 429-436.

66. Jafri, M.R.; Nordstrom, C.W.; Murray, J.A.; van Dyke, C.T.; Dierkhising, R.A.; Zinsmeister, A.R.; Melton, L.J., III. Long-term fracture risk in patients with celiac disease: A population-based study in Olmsted County, Minnesota. Dig. Dis. Sci. 2008, 53, 964-971.

67. Ludvigsson, J.F.; Michaelsson, K.; Ekbom, A.; Montgomery, S.M. Coeliac disease and the risk of fractures-A general population-based cohort study. Aliment. Pharm. Ther. 2007, 25, 273-285.

68. Vasquez, H.; Mazure, R.; Gonzalez, D.; Flores, D.; Pedreira, S.; Niveloni, S.; Smecuol, E.; Mauriño, E.; Bai, J.C. Risk of fractures in celiac disease patients: A cross-sectional, case-control study. Am. J. Gastroenterol. 2000, 95, 183-189.

69. Fickling, W.E.; McFarlane, X.A.; Bhalla, A.K.; Robertson, D.A. The clinical impact of metabolic bone disease in coeliac disease. Postgrad. Med. J. 2001, 77, 33-36. 
70. Davie M.W.; Gaywood, I.; George, E.; Jones, P.W.; Masud, T.; Price, T.; Summers, G.D. Excess non-spine fractures in women over 50 years with celiac disease: A cross-sectional, questionnaire-based study. Osteoporos. Int. 2005, 16, 1150-1155.

71. Moreno, M.L.; Vazquez, H.; Mazure, R.; Smecuol, E.; Niveloni, S.; Pedreira, S.; Sugai, E.; Mauriño, E.; Gomez, J.C.; Bai, J.C. Stratification of bone fracture risk in patients with celiac disease. Clin. Gastroenterol. Hepatol. 2004, 2, 127-134.

72. Olmos, M.; Antelo, M.; Vazquez, H.; Smecuol, E.; Mauriño, E.; Bai, J.C. Systematic review and meta-analysis of observational studies on the prevalence of fractures in coeliac disease. Dig. Liver Dis. 2008, 40, 46-53.

73. Vestergaard, P.; Mosekilde, L. Fracture risk in patients with celiac disease, crohn's disease, and ulcerative colitis: A nationwide follow-up study of 16,416 patients in denmark. Am. J. Epidemiol. 2002, 156, 1-10.

74. Glüer, C.C. Quantitative ultrasound techniques for the assessment of osteoporosis: Expert agreement on current status. The international quantitative ultrasound consensus group. $J$. Bone Miner. Res. 1997, 12, 1280-1288.

75. Njeh, C.F.; Boivin, C.M.; Langton, C.M. The role of ultrasound in the assessment of oeteoporosis: A review. Osteoporos. Int. 1997, 7, 7-22.

76. Scott, E.M.; Gaywood, I.; Scott, B.B. Guidelines for osteoporosis in coeliac disease and inflammatory bowel disease. Gut 2000, 46, I1-I8.

77. Thomason, K.; West, J.; Logan, R.F.; Coupland, C.; Holmes, G.K. Fracture experience of patients with coeliac disease: A population based survey. Gut 2003, 52, 518-522.

78. Compston, J. Is fracture risk increased in patients with coeliac disease? Gut 2003, 52, 459-460.

79. Lewis, N.R.; Scott, B.B. Should patients with coeliac disease have their bone mineral density measured? Eur. J. Gastroenterol. Hepatol. 2005, 17, 1065-1070.

80. American Gastroenterological Association. American gastroenterological association medical position statement: Guidelines on osteoporosis in gastrointestinal diseases. Gastroenterology 2003, 124, 791-794.

81. Fouda, M.A.; Khan, A.A.; Sultan, M.S.; Rios, L.P.; McAssey, K.; Armstrong, D. Evaluation and management of skeletal health in celiac disease: Position statement. Can. J. Gastroenterol. 2012, 26, 819-829. 
Reprinted from Nutrients. Cite as: Pellicano, R.; de Angelis, C.; Ribaldone, D.G.; Fagoonee, S.; Astegiano, M. 2013 Update on Celiac Disease and Eosinophilic Esophagitis. Nutrients 2013, 5, 3329-3336.

Review

\title{
2013 Update on Celiac Disease and Eosinophilic Esophagitis
}

\author{
Rinaldo Pellicano ${ }^{1, *}$, Claudio De Angelis ${ }^{1}$, Davide Giuseppe Ribaldone ${ }^{1}$, Sharmila Fagoonee ${ }^{2}$ \\ and Marco Astegiano ${ }^{1}$
}

1 Department of Gastro-Hepatology, Molinette Hospital, C.so Bramante 88, 10126 Turin, Italy;

E-Mails: eusdeang@hotmail.com (C.D.); davrib_1998@yahoo.com (D.G.R.);

mastegiano@cittadellasalute.to.it (M.A)

2 Molecular Biotechnology Center, University of Turin, 10126 Turin, Italy;

E-Mail: sharmila.fagoonee@unito.it

* Author to whom correspondence should be addressed; E-Mail: rinaldo_pellican@hotmail.com;

Tel.: +0039-011-633-3565; Fax: +0039-011-633-3623.

Received: 15 July 2013; in revised form: 31 July 2013 / Accepted: 1 August 2013 /

Published: 22 August 2013

\begin{abstract}
Celiac disease is a chronic, immune-mediated disorder, characterized by small intestinal inflammation and villous atrophy after the ingestion of gluten by genetically susceptible individuals. Several extraintestinal manifestations have been associated to celiac disease. Eosinophilic esophagitis is a primary disorder of the esophagus characterized by upper gastrointestinal symptoms, absence of gastroesophageal reflux disease and more than 15 eosinophils per high-power field in biopsy specimens. Both celiac disease and eosinophilic esophagitis are caused by aberrant, but distinct, immune responses to ingested antigens and can be responsive to restricted food intake. The aim of this review is to assess whether there is an association between these two pathologies. In the majority of the studies examined, including the studies in pediatric population, the prevalence of eosinophilic esophagitis in subjects with celiac disease was about 10-times that of the general population. We suggest searching for eosinophilic esophagitis in all children undergoing endoscopy for suspicious celiac disease.
\end{abstract}

Keywords: celiac disease; eosinophilic esophagitis; food allergy; autoimmune disorders 


\section{Introduction}

Celiac disease is a chronic, immune-mediated disorder, characterized by malabsorption of nutrients after the ingestion of wheat gluten or related proteins from rye and barley by genetically susceptible individuals expressing the human leukocyte antigen (HLA) class II molecules DQ2 or DQ8 [1] resulting in villus atrophy of the small intestinal mucosa. Prompt clinical and histologic improvement is observed following strict adherence to a gluten-free diet, and clinical and histologic relapse occurs when gluten is reintroduced [2]. Several extraintestinal manifestations, including anemia, osteopenia, neurologic symptoms, menstrual abnormalities, infertility, recurrent spontaneous abortions, growth retardation, dermatitis herpetiformis, aphthous stomatitis, dental defects, have been associated with celiac disease [3].

Eosinophilic esophagitis was first described in 1978 [4]; however, it became recognized as a distinct clinical entity in 1995 [5]. It is a chronic inflammatory primary disorder of the esophagus, presenting with dysphagia and symptoms mimicking those of gastroesophageal reflux disease, including vomiting, regurgitation, nausea and epigastric pain. This disorder is characterized by esophageal mucosal biopsy containing more than 15 eosinophils per high-power field (Figure 1) and absence of gastroesophageal reflux disease, as shown by normal $\mathrm{pH}$ monitoring or lack of response to high-dose proton pump inhibitory therapy [6].

Figure 1. Classic histological findings of eosinophilic esophagitis: hypereosinophilia, usually with $>15-20$ eosinophils per high-powered field. Eosinophils in the squamous mucosa are visible (arrows). (Courtesy of Dr. Ezio David, MD, Molinette Hospital, Turin, Italy.)

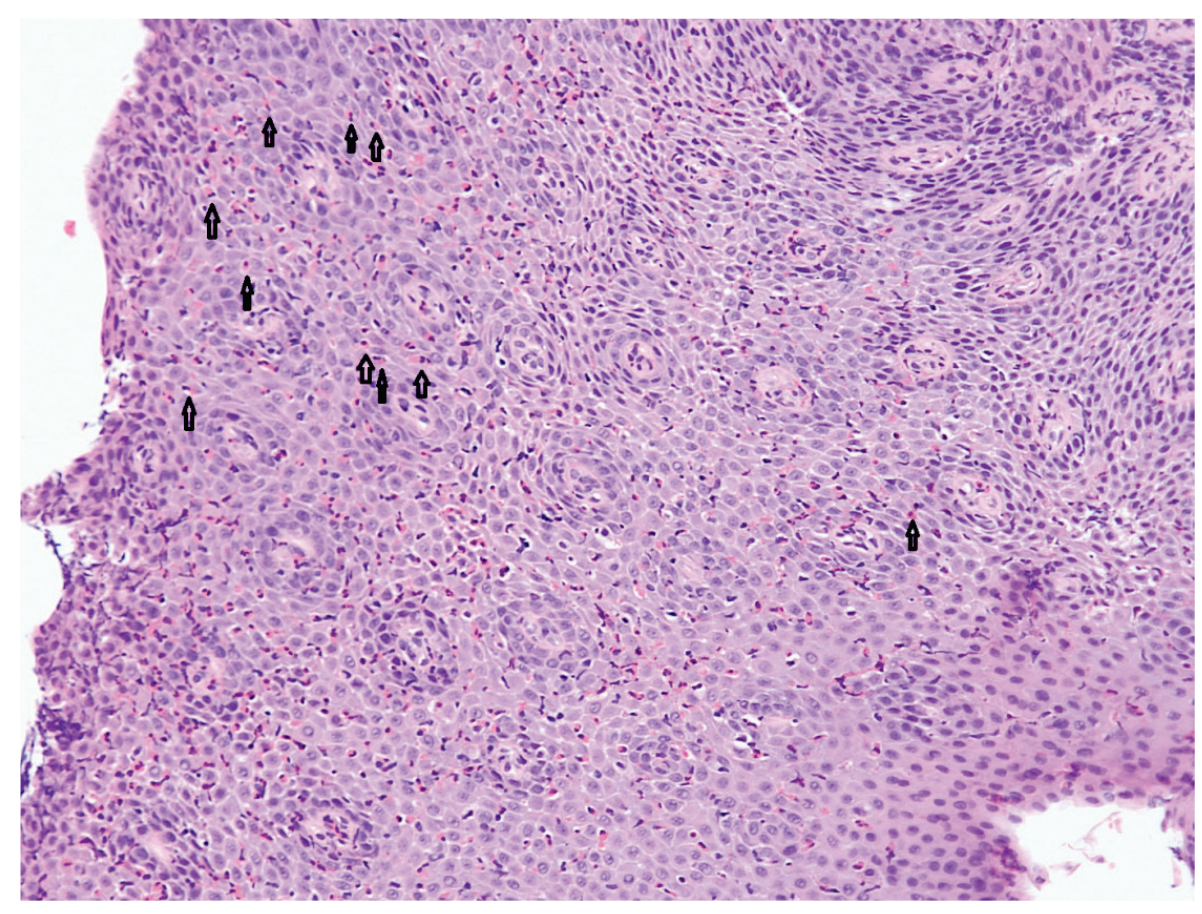

There are some classic endoscopic features including adherent whitish plaques, esophageal concentric rings, linear furrowing, but the esophagus can appear only slightly altered in some patients [7]. The squamous epithelium of the esophagus is normally devoid of eosinophils, but 
various disorders cause eosinophils to infiltrate the esophageal epithelium: parasitic infections, autoimmune disease, vasculitis, medications, gastroesophageal reflux disease [8]. When first described, eosinophilic esophagitis was believed to be a predominantly pediatric condition; however, it is now commonly diagnosed in adults as well as in children [9]. The clinical presentation of eosinophilic esophagitis may vary depending on age: younger children, generally present with non-specific symptoms of gastroesophageal reflux disease, abdominal pain or failure to thrive [10]; older children often present with dysphagia and esophageal food impaction [11]; however, asymptomatic low-grade counts of epithelial eosinophils (less than 15 eosinophils per high-power field) may be more common than has been estimated, but are of uncertain clinical significance [12]. A subset of patients with eosinophilic esophagitis responds to acid suppressive therapy, indicating some overlap between eosinophilic esophagitis and gastroesophageal reflux disease [6]; this is now considered as a separate entity labelled "proton pump inhibitors (PPI) responsive oesophageal eosinophilia" [13] In children, eosinophilic esophagitis has been shown to be associated with IgEand non-IgE-mediated food allergy, and the majority of cases respond to elemental diets or specific food protein elimination [6]. By contrast, in adults the response to dietary interventions is less predictable, and the treatment more commonly relies on swallowed corticosteroid aerosols [6]. The poor response rate to dietary interventions in adults may be due to a lower prevalence of food allergy, and sensitization to inhalant allergens may play a more significant etiological role [6]. The data about the incidence of eosinophilic esophagitis range from 0.5 cases per 10,000 [14] to 1 in 10,000 in Ohio, USA [15] and the prevalence range from 0.89/10,000 in Western Australia [16] to four cases per 10,000 in Ohio, USA [15] and 5.5 cases per 10,000 in Olmsted County, Minnesota, USA [17]. The incidence seems to be increasing in both adults and children, though it is as yet unclear whether this is solely attributable to increasing awareness and detection of the disease or whether it represents a genuine phenomenon [18]. There is a male predominance, with $76 \%$ of adult and $66 \%$ of pediatric cases being diagnosed in males [6]. Since both celiac disease and eosinophilic esophagitis are caused by aberrant, but distinct, immune responses to ingested antigens and can be responsive to food elimination diets, the objective of our study was to verify if there is an association between these two conditions.

\section{Experimental Section}

Articles regarding the association of these two diseases were identified through MEDLINE search using the terms "celiac disease or celiac sprue or gluten AND eosinophilic esophagitis". The search was also performed using reference lists from published articles. The titles of these publications and their abstracts were scanned in order to eliminate duplicates and irrelevant articles. The final date of the MEDLINE search was June 19, 2013.

\section{Results}

The search identified 30 publications (from November 2001 to May 2013) on this subject. We read the abstracts of all articles and selected the 13 original articles in which associations between the two diseases were addressed; three were excluded because there were no data about the prevalence of the diseases. Celiac disease and eosinophilic esophagitis have been described in the same patient for 
the first time in a case report of 2007 [19] in a 7-year old black male with reactive airway disease, eczema and type 1 diabetes mellitus referred to the gastroenterology clinic for positive celiac serologic findings in recurrent abdominal pain. The eosinophilic esophagitis responded to an elimination diet with normalization of esophageal histology and subsequently recurred with reintroduction of cow's milk protein (while celiac disease's histology remained in remission with gluten-free diet). A second report of three associated cases was described in the same year [20] with a reported prevalence of eosinophilic esophagitis in patients with celiac disease of $9 \%$, nine times higher than that expected in the general population (1:100) [21]. In one patient eosinophilic esophagitis disappeared after gluten-free diet; in the other two cases, the gluten-free diet did not have any effect on the eosinophilic infiltrate, but both subjects were not compliant to the gluten-free diet. Another study [22] reported a 35\% of prevalence of celiac disease in 17 patients affected by eosinophilic esophagitis investigated for upper gastrointestinal symptoms, with a significant clinical and histological remission on gluten-free diet compared to the group of patients with eosinophilic esophagitis without celiac disease. In an Australian study regarding seven children with eosinophilic esophagitis and celiac disease [23], no patients with food allergy were reported; the prevalence of eosinophilic esophagitis in the cohort of patients with celiac disease was 3.1\%; two of seven patients who underwent repeated endoscopic examinations showed improved duodenal histology but persistent eosinophilic esophagitis on gluten-free diet. In another Australian study published in 2010 [24], the prevalence of esophageal eosinophilia in children with celiac disease who had concurrent esophageal biopsies was 8.2\% (10 of 121), 60\% males, 30\% had normal-appearing esophageal mucosa at endoscopy; children who had undergone repeated endoscopic examinations showed recovery of duodenal mucosa but no resolution of esophageal eosinophilia on a gluten-free diet alone. The association of celiac and eosinophilic esophagitis may not be a true association but a matter of biased enrollment in the above studies: regarding HLA DQ2 and/or DQ8, a study [25] showed that these alleles were not present in eosinophilic esophagitis at a greater rate than in healthy controls. But a recent study [26] demonstrated, a clear association between celiac disease and eosinophilic esophagitis in both pediatric and adult populations: the standardized incidence ratio of eosinophilic esophagitis in patients with celiac disease was 16.0 (95\% CI, 8.7-25.5). A general population-based study on adults [27] did not find any association between eosinophilic esophagitis and celiac disease, whereas the latest one [28] showed a prevalence of $1.2 \%$ of eosinophilic esophagitis in children with celiac disease (Table 1).

Table 1. Published study about prevalence of eosinophilic esophagitis (EoE) in patients affected by celiac disease (CD).

\begin{tabular}{cccccc}
\hline Study & Country & $\begin{array}{c}\text { Prevalence of EoE } \\
\text { in CD population }\end{array}$ & $\begin{array}{c}\text { \% of Pediatric } \\
\text { patients }\end{array}$ & $\begin{array}{c}\text { \% of Male in } \\
\text { EoE patients }\end{array}$ & Population \\
\hline A [22] & Australia & $3.1 \%(7$ of 221$)$ & 100 & 43 & Tertiary center \\
B [23] & Australia & $8.2 \%(10$ of 121$)$ & 100 & 60 & Tertiary center \\
C [25] & USA & $0.97 \%(14$ of 1439$)$ & 20.6 & 57 & Tertiary center \\
D [27] & Canada & $1.2 \%(3$ of 245$)$ & 100 & 100 & General population \\
\hline
\end{tabular}




\section{Discussion}

Both celiac disease and eosinophilic esophagitis are distinct clinical entities except for a few minor similarities. Celiac disease is a Th1 mediated disorder which aligns with autoimmunity, triggered by the ingestion of food containing gluten and affects females:males at a ratio of 2:1 [29]. By contrast, eosinophilic esophagitis has been shown to be a Th2-mediated disorder, which is triggered by exposure to dietary allergens causing infiltration of the esophageal mucosa by $\mathrm{T}$ lymphocytes, mast cells and eosinophils and predominates in males, with a 3:1 ratio to females. There is also an overexpression of eotaxin-3 and interleukin-5 [6] in the latter. Affliction of $8 \%$ of first-degree relatives of patients with celiac disease is similar to that reported in $10 \%$ of first-degree relatives of patients with eosinophilic esophagitis [30]. The genetic basis for celiac disease (i.e., HLA DQ2) is well established and differs from that of eosinophilic esophagitis [25] whose etiology is far from clear, but in nearly $50 \%$ of the cases, it is associated with an allergy to food or to aeroallergens [15]. In children, IgE-dependent mechanism for eosinophilic esophagitis is supported; for instance, it was shown that affected patients have IgE sensitization to a wide variety of foods, although not all patients had evidence of food-specific IgE [31]. In one study, a higher level of IgE sensitization to food allergens was observed in patients with eosinophilic esophagitis alone compared to patients with both pathologies, and this led the authors to hypothesize that patients with both pathologies have elevated esophageal eosinophils for reasons different from allergy, with a significant clinical and histological remission on gluten-free diet [22]. Increased intestinal mucosal permeability secondary to celiac disease has been suggested as a contributing factor in the development of atopy $[32,33]$. Damaged intestinal barrier may expose the local intestinal immune system to macromolecules and lead to transport of these undigested proteins to other body sites, hence facilitating development of hypersensitivity reactions in a predisposed individual in and away from the gastrointestinal tract [23]. By contrast, in adults, the response to dietary interventions is less predictable, and treatment more commonly relies on swallowed corticosteroid aerosols [6]. The clinical significance of eosinophilic esophagitis as an incidental finding is uncertain; if the main treatment goal is suppression of clinical symptoms, asymptomatic eosinophilic esophagitis may not require any therapy, but as the natural history of eosinophilic esophagitis in largely unknown, it is unclear what proportion is at risk of developing esophageal strictures and dysphagia in the long term [34].

\section{Conclusions}

In summary, although there are fundamental differences in the pathophysiological mechanisms involved in eosinophilic esophagitis and celiac disease, these conditions may coexist and the prevalence is higher than anticipated. Our review highlights the importance of obtaining routine esophageal biopsies in children undergoing endoscopy for diagnosis of celiac disease irrespective of whether the esophagus appears normal or abnormal at endoscopy; however, asymptomatic low-grade counts of epithelial eosinophils are of uncertain clinical significance.

\section{Conflicts of Interest}

The authors declare no conflict of interest. 


\section{References}

1. Fasano, A. Clinical presentation of celiac disease in the pediatric population. Gastroenterology 2005, 128, 68-73.

2. Farrell, R.J.; Kelly, C.P. Celiac sprue. N. Engl. J. Med. 2002, 346, 180-188.

3. Ribaldone, D.G.; Astegiano, M.; Fagoonee, S.; Rizzetto, M.; Pellicano, R. Epilepsy and celiac disease. Panminerva Med. 2011, 53, 213-216.

4. Landres, R.T.; Kuster, G.G.; Strum, W.B. Eosinophilic esophagitis in a patient with vigorous achalasia. Gastroenterology 1978, 74, 1298-1301.

5. Kelly, K.J.; Lazenby, A.J.; Rowe, P.C.; Yardley, J.H.; Perman, J.A.; Sampson, H.A. Eosinophilic esophagitis attributed to gastroesophageal reflux: Improvement with amino acid-based formula. Gastroenterology 1995, 109, 1503-1512.

6. Furuta, G.T.; Liacouras, C.A.; Collins, M.H.; Gupta, S.K.; Justinich, C.; Putnam, P.E.; Bonis, P.; Hassall, E.; Straumann, A.; Rothenberg, M.E.; et al. Eosinophilic esophagitis in children and adults: A systematic review and consensus recommendations for diagnosis and treatment. Gastroenterology 2007, 133, 1342-1363.

7. Nurko, S.; Teitelbaum, J.E.; Husain, K.; Buonomo, C.; Fox, V.L.; Antonioli, D.; Fortunato, C.; Badizadegan, K.; Furuta, G.T. Association of Schatzki ring with eosinophilic esophagitis in children. J. Pediatr. Gastroenterol. Nutr. 2004, 38, 436-441.

8. Feldman, M.; Friedman, L.S.; Brandt, L.J. Gastrointestinal and Liver Disease, 9th ed.; Saunders Elsevier: Philadelphia, PA, USA, 2010; p. 427.

9. Potter, J.W.; Saeian, K.; Staff, D.; Massey, B.T.; Komorowski, R.A.; Shaker, R.; Hogan, W.J. Eosinophilic esophagitis in adults: An emerging problem with unique esophageal features. Gastrointest. Endosc. 2004, 59, 355-361.

10. Orenstein, S.R.; Shalaby, T.M.; di Lorenzo, C.; Putnam, P.E.; Sigurdsson, L.; Mousa, H.; Kocoshis, S.A. The spectrum of pediatric eosinophilic esophagitis beyond infancy: A clinical series of 30 children. Am. J. Gastroenterol. 2000, 95, 1422-1430.

11. Desai, T.K.; Stecevic, V.; Chang, C.H.; Goldstein, N.S.; Badizadegan, K.; Furuta, G.T. Association of eosinophilic inflammation with esophageal food impaction in adults. Gastrointest. Endosc. 2005, 61, 795-801.

12. Ronkainen, J.; Talley, N.J.; Aro, P.; Storskrubb, T.; Johansson, S.E.; Lind, T.; Bolling-Sternevald, E.; Vieth, M.; Stolte, M.; Walker, M.M.; et al. Prevalence of oesophageal eosinophils and eosinophilic oesophagitis in adults: The population-based Kalixanda study. Gut 2007, 56, 615-620.

13. Liacouras, C.A.; Furuta, G.T.; Hirano, I.; Atkins, D.; Attwood, S.E.; Bonis, P.A.; Burks, A.W.; Chehade, M.; Collins, M.H.; Dellon, E.S.; et al. Eosinophilic esophagitis: Updated consensus recommendations for children and adults. J. Allergy Clin. Immunol. 2011, 128, 3-20.

14. Hruz, P.; Straumann, A.; Bussmann, C.; Heer, P.; Simon, H.U.; Zwahlen, M.; Beglinger, C.; Schoepfer, A.M. Escalating incidence of eosinophilic esophagitis: A 20-year prospective, population-based study in Olten County, Switzerland. J. Allergy Clin. Immunol. 2011, 128, $1349-1350$. 
15. Noel, R.J.; Putnam, P.E.; Rothenberg, M.E. Eosinophilic esophagitis. N. Engl. J. Med. 2004, 351, 940-941.

16. Cherian, S.; Smith, N.M.; Forbes, D.A. Rapidly increasing prevalence of eosinophilic oesophagitis in Western Australia. Arch. Dis. Child. 2006, 91, 1000-1004.

17. Prasad, G.A.; Alexander, J.A.; Schleck, C.D.; Zinsmeister, A.R.; Smyrk, T.C.; Elias, R.M.; Locke, G.R.; Talley, N.J. Epidemiology of eosinophilic esophagitis over three decades in Olmsted County, Minnesota. Clin. Gastroenterol. Hepatol. 2009, 7, 1055-1061.

18. Straumann, A.; Simon, H.U. Eosinophilic esophagitis: Escalating epidemiology? J. Allergy Clin. Immunol. 2005, 115, 418-419.

19. Kagalwalla, A.F.; Shah, A.; Ritz, S.; Melin-Aldana, H.; Li, B.U. Cow's milk protein-induced eosinophilic esophagitis in a child with gluten-sensitive enteropathy. J. Pediatr. Gastroenterol. Nutr. 2007, 44, 386-388.

20. De Angelis, P.; Dall'Oglio, L.; di Leo, G.; Ventura, A. Eosinophilic oesophagitis and coeliac disease: Is it just a casual association? Gut 2007, 56, 1029-1030.

21. Verzegnassi, F.; Bua, J.; Tommasini, A.; Not, T.; Kiren, V.; Baldas, V.; Santon, D.; Trevisiol, C.; Berti, I; Neri, E.; et al. Mass screening for coeliac disease using antihuman transglutaminase antibody assay. Arch. Dis. Child. 2004, 89, 512-515.

22. Quaglietta, L.; Coccorullo, P.; Miele, E.; Pascarella, F.; Troncone, R.; Staiano, A. Eosinophilic oesophagitis and coeliac disease: Is there an association? Aliment. Pharmacol. Ther. 2007, 26, 487-493.

23. Ooi, C.Y.; Day, A.S.; Jackson, R.; Bohane, T.D.; Tobias, V.; Lemberg, D.A. Eosinophilic esophagitis in children with celiac disease. J. Gastroenterol. Hepatol. 2008, 23, 1144-1148.

24. Leslie, C.; Mews, C.; Charles, A.; Ravikumara, M. Celiac disease and eosinophilic esophagitis: A true association. J. Pediatr. Gastroenterol. Nutr. 2010, 50, 397-399.

25. Lucendo, A.J.; Arias, Á.; Pérez-Martínez, I.; López-Vázquez, A.; Ontañón-Rodríguez, J.; González-Castillo, S.; De Rezende, L.C.; Rodrigo, L. Adult patients with eosinophilic esophagitis do not show an increased frequency of the HLA-DQ2/DQ8 genotypes predisposing to celiac disease. Dig. Dis. Sci. 2011, 56, 1107-1111.

26. Thompson, J.S.; Lebwohl, B.; Reilly, N.R.; Talley, N.J.; Bhagat, G.; Green, P.H. Increased incidence of eosinophilic esophagitis in children and adults with celiac disease. J. Clin. Gastroenterol. 2012, 46, 6-11.

27. Ludvigsson, J.F.; Aro, P.; Walker, M.M.; Vieth, M.; Agréus, L.; Talley, N.J.; Murray, J.A.; Ronkainen, J. Celiac disease, eosinophilic esophagitis and gastroesophageal reflux disease, an adult population-based study. Scand. J. Gastroenterol. 2013, 48, 804-814.

28. Stewart, M.J.; Shaffer, E.; Urbanski, S.J.; Beck, P.L.; Storr, M.A. The association between celiac disease and eosinophilic esophagitis in children and adults. BMC Gastroenterol. 2013, $13,96$.

29. Green, P.H.; Cellier, C. Celiac disease. N. Engl. J. Med. 2007, 357, 1731-1743.

30. Rothenberg, M.E. Eosinophilic gastrointestinal disorders (EGID). J. Allergy Clin. Immunol. 2004, 113, 11-28. 
31. Spergel, J.M.; Beausoleil, J.L.; Mascarenhas, M.; Liacouras, C.A. The use of skin prick tests and patch tests to identify the causative foods in eosinophilic esophagitis. J. Allergy Clin. Immunol. 2002, 109, 363-368.

32. Van Elburg, R.M.; Uil, J.J.; Mulder, C.J.; Heymans, H.S. Intestinal permeability in patients with coeliac disease and relatives of patients with coeliac disease. Gut 1993, 34, 354-357.

33. Bischoff, S.C.; Mayer, J.; Nguyen, Q.T.; Stolte, M.; Manns, M.P. Immunohistological assessment of intestinal eosinophil activation in patients with eosinophilic gastroenteritis and inflammatory bowel disease. Am. J. Gastroenterol. 1999, 94, 3521-3529.

34. Heine, R.G. Eosinophilic esophagitis in children with celiac disease: New diagnostic and therapeutic dilemmas. J. Gastroenterol. Hepatol. 2008, 23, 993-994. 
Reprinted from Nutrients. Cite as: Diamanti, A.; Capriati, T.; Basso, M.S.; Panetta, F.; Di Ciommo Laurora, V.M., Bellucci, F.; Cristofori, F.; Francavilla, R. Celiac Disease and Overweight in Children: An Update. Nutrients 2014, 6, 207-220.

Review

\title{
Celiac Disease and Overweight in Children: An Update
}

\author{
Antonella Diamanti ${ }^{1, *}$, Teresa Capriati ${ }^{1,2}$, Maria Sole Basso ${ }^{1}$, Fabio Panetta ${ }^{1}$, \\ Vincenzo Maria Di Ciommo Laurora ${ }^{3}$, Francesca Bellucci ${ }^{1}$, Fernanda Cristofori ${ }^{2}$ and \\ Ruggiero Francavilla ${ }^{2}$
}

1 Gastroenterology-Hepatology and Nutrition Unit, "Bambino Gesù” Children's Hospital, Piazza Sant'Onofrio 4, Rome 00165, Italy; E-Mails: teresa.capriati@gmail.com (T.C.); msole.basso@opbg.net (M.S.B.); fabio.panetta@opbg.net (F.P.); francesca.bellucci@opbg.net (F.B.)

2 Gastroenterology Unit, Pediatric Clinic of University, Piazza Giulio Cesare 11, Bari 70124, Italy; E-Mails: fernandacristofori@gmail.com (F.C.); rfrancavilla@gmail.com (R.F.)

3 Epidemiology and Biostatistics Unit, "Bambino Gesù” Children's Hospital, Piazza Sant'Onofrio 4, Rome 00165, Italy; E-Mail: vmaria.diciommo@opbg.net

* Author to whom correspondence should be addressed; E-Mail: antonella.diamanti@opbg.net; Tel.: +39-06-6859-2329; Fax: +39-06-6859-3889.

Received: 11 October 2013; in revised form: 19 December 2013 / Accepted: 20 December 2013 / Published: 2 January 2014

\begin{abstract}
The clinical presentation of celiac disease in children is very variable and differs with age. The prevalence of atypical presentations of celiac disease has increased over the past 2 decades. Several studies in adults and children with celiac disease indicate that obesity/overweight at disease onset is not unusual. In addition, there is a trend towards the development of overweight/obesity in celiac patients who strictly comply with a gluten-free diet. However, the pathogenesis and clinical implications of the coexistence of classic malabsorption (e.g., celiac disease) and overweight/obesity remain unclear. This review investigated the causes and main clinical factors associated with overweight/obesity at the diagnosis of celiac disease and clarified whether gluten withdrawal affects the current trends of the nutritional status of celiac disease patients.
\end{abstract}

Keywords: celiac disease; overweight; obesity; gluten free diet 


\section{Introduction}

Celiac disease (CD) is a life-long condition that affects the small intestine in genetically susceptible individuals [1]. The global prevalence ranges from $1 \%$ to $2 \%[2,3]$. In children, the symptoms upon CD presentation are highly variable and are influenced by age. Very young children often present with "classic" symptoms including diarrhea, abdominal distension, and growth retardation [4-6]. Diarrhea and malabsorption represent the typical presentation of CD in young children [7], while abdominal pain, vomiting, and constipation are atypical gastrointestinal symptoms more common in older children and teenagers. Furthermore, in children, CD can be diagnosed on the basis of the occurrence of extra-intestinal conditions such as arthritis, neurological diseases, and anemia [8,9] or on the basis of screening procedures in the absence of gastrointestinal symptoms (typical or atypical) and in child or adolescent with CD- associated conditions [10].

The presentation of $\mathrm{CD}$ has changed over time. In the last 2 decades, diarrhea and malabsorption have progressively decreased as the mode of CD onset among both adults and children, whereas atypical manifestations have increased. Interestingly, many reports indicate that $\mathrm{CD}$ can be associated with overweight or normal weight; hence, malnutrition is not always present at CD presentation $[4,6,11,12]$. Therefore, $\mathrm{CD}$ and obesity can coexist during both childhood and adolescence. After the first 2 cases reported by Semeraro [13] and Conti-Nibali [14] in 1986 and 1987, respectively, there have been several reports of the coexistence of CD and obesity/overweight in children and adolescents in the last 2 decades [15-19].

At present, in pediatric [20-27] and adult [28-33] case series of CD, the body mass index (BMI) at diagnosis is within the normal range in many patients. Nevertheless, the pathogenesis and clinical implications of the coexistence of $\mathrm{CD}$ and overweight/obesity remain unclear. The clinical relevance of this association is highlighted by the observation that $\mathrm{CD}$ patients with normal weight or overweight at diagnosis have a higher risk of developing obesity after starting a gluten-free diet (GFD), which definitely improves intestinal absorption in these patients. Moreover, the GFD regimen appears to be associated with high lipid and protein intake, particularly in adolescents [20,29].

The key studies concerning the pathogenesis and clinical evidence of the association between CD and overweight/obesity in subjects aged $<18$ years are discussed below. This review investigated the causes and main clinical factors associated with overweight/obesity at CD diagnosis. In addition, this review aims to clarify if gluten withdrawal affects the trend of the nutritional status of CD patients.

\section{Clinical Evidence of $C D$ and Overweight/Obesity}

\subsection{Summary of the Main Case Reports}

The first pediatric case report [13] by Semeraro et al., in 1986 describes an obese 14-year-old girl who had been diagnosed with $\mathrm{CD}$ at the age of 1 year on the basis of a clinical condition characterized by malabsorption, diarrhea, and stunted growth (i.e., weight in the 7th percentile). The girl was started on a GFD, and had a normal weight at 2 years of age; however, she was overweight at 5 years of age and obese at 10 . She had a negative family history for endocrine diseases and CD but a positive family history for obesity. 
There are other reports of the development of obesity in children on a GFD who initially had malabsorption. For example Czaja-Bulsa et al. [15] describe the case of an 18-year-old boy with growth failure (i.e., <3rd percentile) and chronic diarrhea following gluten introduction and before $\mathrm{CD}$ diagnosis. However, after gluten withdrawal, his weight increased to the 97 th percentile at 5 years of age despite persistent mucosal atrophy. More recently, Balamtekin et al. [19] reported a similar case of a 21-month-old child with the classic condition of malabsorption (i.e., chronic diarrhea, failure to thrive, and abdominal distension) at CD onset. After 11 years on a GFD, the child became obese (weight, $>97$ th percentile).

Meanwhile, there are other reports of children with overweight/obesity at the time of CD diagnosis. The first published report describes a 5-year-old girl with obesity, short stature, and recurrent abdominal pain. The diagnosis of $\mathrm{CD}$ was suspected on the basis of family history, i.e., a sister with CD. A GFD attenuated the symptoms and improved height and weight growth [14]. Furthermore, in 2001, Franzese et al. [16] reported the case of a patient with steatohepatitis associated with obesity resistant to a low-calorie diet, in which CD was diagnosed on the basis of moderate persistent hypertransaminasemia. In 2006, Oso and Fraser [17] diagnosed CD in an obese teenager who had recurrent episodes of diarrhea, especially after eating spaghetti. At diagnosis, blood tests revealed low iron, GFD feeding normalized iron level, and the symptoms disappeared. However, the patient continued to gain weight (10 kg over 6 months) during follow-up. In 2009, Arslan et al. [18] reported the case of a 7-year-old obese patient with CD (weight, $>95$ th percentile; weight/height ratio, 167\%) suspected of having Hashimoto's thyroiditis and affected by hypochromic anemia unresponsive to iron therapy. Moreover, Balamtekin et al. [19] describe the case of a 17-year-old obese girl with weight $>97$ th percentile and a BMI of 32.9 with epigastric pain and vomiting. $\mathrm{CD}$ was diagnosed on the basis of the gastrointestinal symptoms, and the symptoms disappeared after a GFD was started. Nevertheless, her weight continued to increase.

\subsection{Summary of Case Series}

At present, few case series have been published on this topic. Valletta et al. [24] report the prevalence of overweight (BMI $z$-score $>+1$ ) and obesity (BMI $z$-score $>+2$ ) to be $11 \%$ and 3\%, respectively, in 149 children newly diagnosed with CD between 1991 and 2007. The authors found that after initiating a GFD, the BMI $z$-score increased significantly and the percentage of overweight subjects almost doubled. In a retrospective study, Venkatasubramani et al. [22] report 5\% of patients had a BMI > 95th percentile among 143 patients with CD diagnosed between 1986 and 2003. Among the obese patients, the most common symptoms at onset were abdominal pain, diabetes, and diarrhea.

Brambilla et al. [25] compared 150 children with CD on a GFD with 288 healthy sex- and age-matched children. They also retrospectively evaluated changes in BMI from CD diagnosis to the last clinical evaluation. The median BMI of CD patients was significantly lower than that of the healthy controls. In particular, children with CD were less frequently overweight or obese $(12 \% v s$. $23.3 \%)$ and more frequently underweight (16\% vs. $4.5 \%)$ than the controls. However, after GFD feeding, the number of underweight subjects decreased significantly, while the number of overweight subjects increased slightly.

Reilly et al. [26] studied 142 children with newly diagnosed CD from 2000 to 2008. Nearly $19 \%$ of patients had a high BMI at diagnosis (12.6\% overweight and $6 \%$ obese), while $74.5 \%$ had a 
normal BMI. Meanwhile, the BMI of $75 \%$ of the patients with high BMI at diagnosis decreased on a GFD. Among patients with a normal BMI at diagnosis, weight $z$-scores increased significantly after diet treatment and 13\% became overweight. Interestingly, in that survey, the initial symptom in $28 \%$ of overweight $\mathrm{CD}$ patients was abdominal pain and the diagnosis was made on the basis of the screening test in a asymptomatic portion of the population by $28 \%$. Venkatasubramani et al. [22] also found abdominal pain is one of the most common features of CD presentation in overweight patients. Another important aspect of their survey results is that the $\mathrm{CD}$ diagnosis was made on the basis of the screening test in at least $25 \%$ of overweight patients. Brambilla et al. [25] suggest that identifying $\mathrm{CD}$ patients on the basis of screening tests, and not symptoms, may increase the probability of finding overweight or obese subjects at $\mathrm{CD}$ diagnosis.

In a cross-sectional multicenter study, Norsa et al. [27] enrolled 114 children with CD in serologic remission, who were on a GFD for at least 1 year. The anthropometric measurements at diagnosis revealed that $9.6 \%, 76.3 \%, 8.8 \%$, and $5.3 \%$ were underweight (BMI $<5$ th percentile), had normal weight $(\mathrm{BMI}=5-85$ th percentile), were overweight $(\mathrm{BMI}=85-95$ th percentile), and were obese $($ BMI $>95$ th percentile), respectively. After gluten withdrawal, the prevalence of overweight and obesity increased to $11.4 \%$ and $8 \%$, respectively.

In a prospective case-control study, Barera et al. [34] found reduced fat mass, decreased bone mineral content, and lower lean body mass in the limbs of 29 children newly diagnosed with CD compared to healthy controls; all patients were normalized (i.e., approaching corresponding parameters in the control population) on a GFD. Table 1 summarizes the main results of the abovementioned reports.

Table 1. Prevalence of overweight/obesity in CD.

\begin{tabular}{|c|c|c|c|c|}
\hline $\begin{array}{l}\text { Author } \\
\text { (Year) }\end{array}$ & $\begin{array}{c}\text { Country } \\
\text { (Sample Size) }\end{array}$ & $\begin{array}{l}\text { Overweight/Obesity } \\
\text { at Presentation (\%) }\end{array}$ & $\begin{array}{c}\text { Overweight/Obesity after } \\
\text { Initiating a GFD (\%) }\end{array}$ & Reference \\
\hline Aurangzeb (2010) & $\begin{array}{l}\text { Australia \& New } \\
\text { Zealand }(n=25)\end{array}$ & $20.8 / 0$ & $\mathrm{ND} / \mathrm{ND}$ & {$[21]$} \\
\hline $\begin{array}{l}\text { Venkatasubramani } \\
\qquad(2010)\end{array}$ & $\begin{array}{l}\text { Milwaukee, WI, } \\
\text { USA }(n=143)\end{array}$ & $\mathrm{ND} / 5$ & $\mathrm{ND} / 3$ & [22] \\
\hline Balamtekin (2010) & $\begin{array}{l}\text { Ankara, Turkey } \\
\quad(n=220)\end{array}$ & $\mathrm{ND} / 0.5$ & $\mathrm{ND} / \mathrm{ND}$ & [23] \\
\hline $\begin{array}{l}\text { Valletta et al. } \\
\qquad(2010)\end{array}$ & Italy $(n=149)$ & $11 / 3$ & $21 / 4$ & {$[24]$} \\
\hline Reilly et al. (2010) & $\begin{array}{l}\text { NY, USA } \\
(n=142)\end{array}$ & $12.6 / 6$ & $20 / 4$ & {$[26]$} \\
\hline Norsa et al. (2011) & $\begin{array}{l}\text { Italy } \& \text { Israel } \\
\quad(n=114)\end{array}$ & $8.8 / 5.3$ & $11.5 / 8.8$ & [27] \\
\hline $\begin{array}{l}\text { Brambilla et al. } \\
\text { (2011) }\end{array}$ & Italy $(n=150)$ & $11.3 / 0.7$ & $9.4 / 0$ & {$[25]$} \\
\hline
\end{tabular}

GFD: gluten-free diet; ND: not done.

A clarification regarding the methodology of these studies should be made: in adults and children, the main criterion for defining overweight/obesity is BMI (or Quetelet index), which is calculated by dividing weight (in $\mathrm{kg}$ ) by height (in $\mathrm{m}$ ) squared. BMI is an expression of the weight "adjusted" to 
stature and is an index of adiposity; it is most strongly correlated with body fat and less correlated with stature. Despite its limitations, BMI is easy to calculate and widely used, especially in large-scale studies, to assess the risks of diseases. The internationally accepted age- and sex-standardized threshold values of BMI for nutritional status in adults are those proposed by the World Health Organization [35]. However, the curves of children's weight and height vary with growth, following development during puberty (with its consequences on body composition), and sex. Therefore, references for different age groups (i.e., the distribution of percentiles with cut-off points) are necessary. There are many different percentile tables based on data from reference populations that also have very different anthropometric characteristics. Ideally, the study population should be compared with tables based on national curves. Alternatively, the International Obesity Task Force (IOTF), which is the main organization of childhood obesity scholars, have validated tables with mean percentiles derived from cross-sectional studies of different populations (e.g., the USA, Brazil, Hong Kong, Singapore, Holland, and Great Britain) to enable international comparisons [36].

In this regard, the abovementioned studies have a discrete methodological heterogeneity. Although all are based on the calculation of BMI, they used different categorizations in various case series, such as the BMI percentile, BMI $z$-score, and IOTF cut-off point. In addition, different studies were conducted on geographically diverse populations, and only a few studies compared the case population with a control population $[21,25]$.

\section{Pathogenetic Link between CD and Overweight/Obesity}

\subsection{Overweight and Obesity in Newly Diagnosed CD Patients: The "Compensatory” Hypothesis}

Semeraro first hypothesized that the atrophy of the duodenum-jejunum in CD patients could be compensated by enhanced absorption in the distal intestinal segments [13]. The fat absorption coefficient could in fact be preserved in a patient with a partially atrophic bowel [13]. This process could be similar to that occurring in the residual bowel after surgical resection, which involves structural changes that lead to an increased absorptive attitude of the intestine. The intestinal adaptation consists of morphological changes of the mucosa, including increased villus height, crypt depth, and epithelial cell number. In CD patients, atrophy determines the loss of normal intestinal function. This can hypothetically induce increased absorption of the functionally preserved intestinal tract. If this process overcompensates, it could lead to the extraction of energy exceeding the child's needs, thus increasing the risk of overweight/obesity [13].

This compensatory hypothesis appears to be supported by some of the first published cases of adolescents affected by CD who continued to present with overweight or obesity despite persistent villous atrophy on jejunal biopsies $[14,15]$. The compensatory surface area of the small intestine appears to increase with patient age. Therefore, the intestine may develop the ability to absorb an adequate amount of compensatory energy [13]. This notion is corroborated by the particular distribution of symptoms upon CD diagnosis, which appears to be related to age [5-7]. Children aged less than 2 years often exhibit the classic CD presentation, which includes malabsorption. In contrast, older children, adolescents, and adults often present with atypical symptoms. This appears to be concordant with the compensatory hypothesis. In fact, the classic symptoms may be due to a lack of 
intestinal adaptation, which is less developed in young children as mentioned above. The absence of intestinal adaptation induces the occurrence of severe and classic symptoms including malabsorption and celiac crisis, which can be found in very young children newly diagnosed with CD. As intestinal adaptation is a time-dependent phenomenon, the probability that an individual's mucosa is modified increases with age. Therefore, CD symptoms could be attenuated in older children and adolescents.

Concordant with this hypothesis, there is no correlation between the presentation of $\mathrm{CD}$ and the degree of villous atrophy [37] or the extent of the intestine involved as visualized through video-capsule endoscopic procedures [38]. The morphological appearance of the mucosa may be unrelated to its functional expression responsible for the severity of the presenting symptoms.

In addition, the nutritional status of the underlying population is clearly very important for the correct interpretation of BMI in children with $\mathrm{CD}$ at diagnosis. CD may indeed develop in patients with overweight/obesity, reflecting an individual's predisposition (i.e., genetic, nutritional, and environmental factors). The worldwide prevalence of overweight/obesity in children has increased over the last 2 decades; an estimated 60 million children will be overweight or obese by 2020 [39]. In this scenario, the symptoms of malabsorption that could manifest in overweight patients at CD onset may reduce the prevalence of overweight/obesity in $\mathrm{CD}$ patients compared to the reference population but increase it in comparison to what is usually expected in CD patients.

\subsection{The Effect of Gluten Withdrawal on Overweight/Obese CD Patients}

Overweight or obesity may develop in CD patients after gluten withdrawal. The main surveys on children discussed above report the normalization of BMI in underweight and overweight patients on a GFD, although they also report the development of overweight and obesity independent of baseline nutritional status [21-27,34]. In consideration of the abovementioned "compensatory" hypothesis, it can be supposed the mucosal healing following gluten withdrawal is responsible for the normalization of BMI in both underweight and overweight patients as a result of the recovery of energy balance. Therefore, the restoration of the absorptive functions of the whole bowel could constitute a physiological redistribution of the absorptive attitude in whole bowel mucosa. This could result in an increased energetic yield in patients with symptoms of malabsorption. However, in patients with a mucosa adapted to supply a higher energetic yield, the improved absorptive function of the whole bowel could induce the normalization of caloric balance. Nevertheless, it remains to be determined if a GFD itself is a cause of the development of overweight/obesity in CD patients.

The unpalatability of some gluten-free foods may induce a preference toward hyperproteic and hyperlipidemic foods $[20,29,40]$. This may consequently lead to increased energy intake followed by excessive weight gain [41]. Mariani et al. [20] examined the eating habits and diet composition of 47 adolescents with CD and compared them to those of 47 healthy age-matched control subjects. They divided the CD patients into 2 subgroups according to compliance with a GFD: group 1A patients rigorously adhered to a GFD, while group 1B patients did not comply with a GFD. Compared to Recommended Dietary Allowances, total energy, lipid, and protein intake were higher and carbohydrate intake was lower in CD patients and controls. Total caloric intake and lipid and protein consumption were higher in group 1A than in group 1B. As a consequence, overweight/obesity was more frequent in group $1 \mathrm{~A}(72 \%)$ than in group $1 \mathrm{~B}(51 \%)$ and the controls $(47 \%)$. 
Several studies confirm long-term GFDs may not be nutritionally balanced. Indeed, there is clinical evidence indicating high simple sugar, protein, and saturated fat intake as well as low complex carbohydrate and fiber intake in such diets $[20,42,43]$. Concordant with this pediatric evidence, higher total caloric [44], carbohydrate, and fat [45] intake is reported among adults with CD than among healthy control subjects. In contrast, a few studies in adults [46,47] and children [48] report reduced caloric intake in CD patients on a GFD.

Besides increased total caloric intake, the macronutrient composition of the diet may be involved in the pathogenesis of overweight and obesity in patients with CD. Carbohydrates are the major energy source in the diet of children in developed countries and are the dietary components that most strongly affect blood glycemia. Both the quantity and type of carbohydrates are the determinants of postprandial glycemia [49]. The glycemic index (GI) is a parameter used to classify foods according to their postprandial glycemic response [50].

Many gluten-free foods are characterized by a GI higher than that of equivalent gluten-containing foods [50,51], although this is refuted by some authors [52]. Gluten-free foods have a higher GI, because gluten protein does not allow the easy access of amylase to hydrolyze starch granules in the lumen of the small intestine [50]. However, many foods with a high GI have been shown to only slightly increase blood glucose and vice versa $[51,53]$. Thus, the GI provides a measurement of the quality but not the quantity of the carbohydrates consumed. Meanwhile, blood glycemia is influenced by the synergistic interaction between the quantity and quality of carbohydrates. Therefore, epidemiological studies are utilizing a new concept to assess outcomes as a result of glucose metabolism: the glycemic load (GL). The GL may be calculated with the product of GI (as a percentage) of available carbohydrates, representing both the quality and quantity of carbohydrates consumed. The GL may be interpreted as a measure of insulin requested in free-living conditions, because the amount of carbohydrates consumed at each meal usually varies in such conditions $[51,54,55]$. Nevertheless, if the blood glucose response to food is a determinant of body weight remains controversial [56].

Several studies conducted in overweight or obese children show discordant results regarding the associations of GI and GL with obesity. One cross-sectional study reports no association of body fat with GI or GL [57]. Others studies show positive associations of GI and GL with waist circumference, BMI, and the sum of 4 skinfolds [58,59]. However, other cohort studies report inconclusive results [60-62]. A meta-analysis [63] that identified six eligible randomized clinic trials including a total of 202 participants concludes that low-GI or low-GL diets confer marked benefits on weight, BMI, total fat mass, and lipid profile. Regardless, further research on long-term improvements is required. A more recent systematic review provides evidence that long-term interventions with a low-GI/GL diet confer beneficial effects on fasting insulin and pro-inflammatory markers such as C-reactive protein; such interventions might prove to be helpful in the primary prevention of obesity-associated diseases [64]. These aspects could help explain the occurrence of overweight/obesity in celiac patients on a GFD.

On the other hand, several studies evaluating the effects of a GFD on metabolic control, growth, and nutritional status in celiac patients with type I diabetes provide a natural model of the interactions between diet, glycemic response, and nutritional status, demonstrating how this interrelationship can be much more complex. However, these studies have completely discordant results. Some studies 
[65] indicate improvements in BMI and glycosylated hemoglobin (HbA1c) levels in patients with $\mathrm{CD}$ and type I diabetes on a GFD. Meanwhile, Novòa Medina et al. [66] report no effect on the metabolic control, height, or weight of such patients. Other studies evaluated the influence of GFDs on metabolic parameters including insulin dose, HbA1c, glucose excretion, and hypoglycemic episodes. Saadah et al. [67] report that a GFD resulted in a significant improvement of growth and influenced diabetic control, particularly higher insulin levels in patients with $\mathrm{CD}$ than the levels at baseline. Other authors $[68,69]$ found no significant difference in the insulin dose, HbA1c, 24-h urinary glucose excretion, or the number of hypoglycemic episodes. Abid et al. [70] found that a GFD reduced gastrointestinal symptoms in the short term and particularly episodes of severe hypoglycemia in children with type I diabetes with $\mathrm{CD}$; however, there were no changes in the standard deviation scores for height, weight, BMI, or the average HbAlc before and after GFD consumption. Furthermore, epidemiological studies show energy intake is predictor of weight gain [71]. Thus, the GI and GL of the previous meal can theoretically influence energy intake in the next meal. A recent meta-analysis on this topic suggests that the GI, but not the GL might influence the energy intake of the next meal [72]. This may be because low-GI foods result in sustained blood glucose levels and hunger is delayed as compared with that after a high-GI meal [73]. Furthermore, recent evidence suggests energy intake is associated with changes in the resting metabolic rate [74]. The mechanism involved in this phenomenon may be the specific effect of blood glucose level on satiety (i.e., the glucostatic theory) or of other stimuli (e.g., peptides) involved in the control of appetite. Insulin and glucose stimulate the release of the leptin hormone that produces satiety and suppress the release of the ghrelin hormone that stimulates the appetite. Regardless, this does not precisely characterize relationships among GI, satiogenic leptin, and appetitic ghrelin. Furthermore, several gastrointestinal hormones called incretins are involved in the physiological control of hunger and satiety; they are involved in glucose metabolism and can act on pancreatic beta cells to stimulate insulin secretion. Among these hormones there is glucagon-like peptide-1 (GLP-1), which acts directly on the central nervous system and indirectly by slowing gastric emptying, inhibiting appetite and food intake, and inducing body weight reduction. The stimulation of insulin secretion by incretins is typically glucose dependent and manifests when glycemic levels are high but not when normal or low. Therefore, incretins have the potential to reduce hyperglycemia without causing hypoglycemia. A recent study revealed children with $\mathrm{CD}$ have a secretion pattern of gut-brain axis hormones that differs from that of controls. Alterations in this axis were more pronounced in children with both CD and type I diabetes mellitus; nevertheless, the roles of these gut-brain axis hormones in food intake and glycemic control in patients with $\mathrm{CD}$ and type I diabetes mellitus must be clarified [75].

Overall existing clinical evidence explains the variability of the anthropometric trends in $C D$ patients after gluten withdrawal. However, it does not clarify why some CD patients develop overweight/obesity after beginning a GFD. Furthermore, as is the case in newly diagnosed CD patients, the global trend toward increased overweight/obesity could explain why CD patients on a GFD may become overweight. The changes in nutritional habits that induce the development of obesity are probably shared by CD patients and the general population. Table 2 summarizes the main pathogenetic links between CD and overweight/obesity. 
Table 2. Suggested pathogenetic links between CD and overweight/obesity.

\begin{tabular}{|c|c|c|}
\hline $\begin{array}{l}\text { Time of } \\
\text { Overweight/Obesity } \\
\text { Diagnosis in } \\
\text { Celiac Patients } \\
\end{array}$ & Pathogenetic Link & Reference \\
\hline $\begin{array}{l}\text { Overweight/Obesity } \\
\text { at CD Presentation }\end{array}$ & $\begin{array}{l}\text { "Compensatory hypothesis": high energetic yield due } \\
\text { to the slow adaptation of the atrophic mucosa } \\
\text { Global trend toward overweight/obesity in children } \\
\text { Diagnosis not based on clinical symptoms but on } \\
\text { screening test }\end{array}$ & $\begin{array}{c}{[13]} \\
{[39]} \\
{[25,26]}\end{array}$ \\
\hline $\begin{array}{l}\text { Overweight/Obesity } \\
\text { on a GFD }\end{array}$ & $\begin{array}{l}\text { "Compensatory hypothesis": normalization of caloric } \\
\text { balance due to the restoration of mucosal functions } \\
\text { Worldwide trend toward overweight/obesity in children } \\
\text { Unpalatability of gluten-free foods, prompting the } \\
\text { consumption of foods with high caloric content (i.e., fat } \\
\text { and protein). } \\
\text { High GI/GL of GFD? } \\
\text { Altered secretion of gut-brain axis hormones? }\end{array}$ & $\begin{array}{c}{[13]} \\
{[39]} \\
{[20,29,40]} \\
{[49-74]} \\
{[75]}\end{array}$ \\
\hline
\end{tabular}

CD: celiac disease; GFD: gluten-free diet; GI: glycemic index; GL: glycemic load.

\section{Conclusions}

Overweight/obesity is more common in children with $\mathrm{CD}$ than previously recognized. The prevalence of overweight in $\mathrm{CD}$ patients at diagnosis ranges from $8.8 \%$ to $20.8 \%$ [21,24-27], whereas that in CD patients on a GFD ranges from $9.4 \%$ to $21 \%$ [23-26]. Meanwhile, the prevalence of obesity in $\mathrm{CD}$ patients at diagnosis ranges from $0 \%$ to $6 \%$ [21-27], whereas that in CD patients on a GFD ranges from $0 \%$ to $8.8 \%$ [22,24-27]. Overweight/obesity is more frequent in newly diagnosed CD patients diagnosed on the basis of abdominal pain $[22,26]$ and on the basis of screening procedures $[25,26]$. During follow-up, it is possible the unpalatability of gluten-free foods leads a preference for foods with high caloric fat and protein contents. However, the occurrence of overweight may be explained by the global trend toward overweight/obesity in children [39] including CD patients. An unconfirmed but nonetheless interesting hypothesis is that the development of overnutrition status is due to the compensatory high energetic yield secondary to the slow functional adaptation of the atrophic mucosa [13]. Therefore, mounting evidence suggests CD should be considered even in overweight/obese children in appropriate clinical settings.

\section{Conflicts of Interest}

The authors declare no conflict of interest.

\section{References}

1. Green, P.H.; Cellier, C. Celiac disease. N. Engl. J. Med. 2007, 357, 1731-1743. 
2. Hill, I.D.; Dirks, M.H.; Liptak, G.S.; Colletti, R.B.; Fasano, A.; Guandalini, S.; Hoffenberg, E.J.; Horvath, K.; Murray, J.A.; Pivor, M.; et al. Guideline for the diagnosis and treatment of celiac disease in children: Recommendations of the North American Society for Pediatric Gastroenterology, Hepatology and Nutrition. J. Pediatr. Gastroenterol. Nutr. 2005, 40, 1-19.

3. Mearin, M.L.; Ivarsson, A.; Dickey, W. Coeliac disease: Is it time for mass screening? Best Pract. Res. Clin. Gastroenterol. 2005, 19, 441-452.

4. Rampertab, S.D.; Pooran, N.; Brar, P.; Singh, P.; Green, P.H. Trends in the presentation of celiac disease. Am. J. Med. 2006, 119, e9-e14.

5. Vivas, S.; Ruiz de Morales, J.M.; Fernandez, M.; Hernando, M.; Herrero, B.; Casqueiro, J.; Gutierrez, S. Age-related clinical, serological, and histopathological features of celiac disease. Am. J. Gastroenterol. 2008, 103, 2360-2365.

6. Telega, G.; Bennet, T.R.; Werlin, S. Emerging new clinical patterns in the presentation of celiac disease. Arch. Pediatr. Adolesc. Med. 2008, 162, 164-168.

7. Llorente-Alonso, M.; Fernandez-Acenero, M.J.; Sebastian, M. Gluten intolerance: Gender and age-related features. Can. J. Gastroenterol. 2006, 20, 719-722.

8. Branski, D.; Troncone, R. Celiac disease: A reappraisal. J. Pediatr. 1998, 133, 181-187.

9. Ludvigsson, J.F.; Ansved, P.; Falth-Magnusson, K.; Hammersjö, J.A.; Johansson, C.; Edvardsson, S.; Ljungkrantz, M.; Stenhammar, L.; Ludvigsson, J. Symptoms and signs haven changed in Swedish children with coeliac disease. J. Pediatr. Gastroenterol. Nutr. 2004, 38, 181-186.

10. Husby, S.; Koletzko, S.; Korponay-Szabó, I.R.; Mearin, M.L.; Phillips, A.; Shamir, R.; Troncone, R.; Giersiepen, K.; Branski, D.; Catassi, C.; et al. European Society for Pediatric Gastroenterology, Hepatology, and Nutrition Guidelines for the diagnosis of coeliac disease. $J$. Pediatr. Gastroenterol. Nutr. 2012, 54, 136-160.

11. McGowan, K.E.; Castiglione, D.A.; Butzner, J.D. The changing face of childhood celiac disease in North America: Impact of serological testing. Pediatrics 2009, 124, 1572-1578.

12. Lo, W.; Sano, K.; Lebwohl, B.; Diamond, B.; Green, P.H. Changing presentation of adult celiac disease. Dig. Dis. Sci. 2003, 48, 395-398.

13. Semeraro, L.A.; Barwick, K.W.; Griboski, J.D. Obesity in celiac disease. J. Clin. Gastroenterol. 1986, 8, 177-180.

14. Conti Nibali, S.; Magazzù, G.; De Luca, F. Obesity in a child with untreated coeliac disease. Helv. Paediatr. Acta 1987, 42, 45-48.

15. Czaja-Bulsa, G.; Garanty-Bogacka, B.; Syrenicz, M.; Gebala, A. Obesity in an 18-year-old boy with untreated celiac disease. J. Pediatr. Gastroenterol. Nutr. 2001, 32, 226.

16. Franzese, A.; Iannucci, M.P.; Valerio, G.; Ciccimarra, E.; Spaziano, M.; Mandato, C.; Vajro, P. Atypical celiac disease presenting as obesity-related liver dysfunction. J. Pediatr. Gastroenterol. Nutr. 2001, 33, 329-332.

17. Oso, O.; Fraser, N.C. A boy with coeliac disease and obesity. Acta Paediatr. 2006, 95, 618-619.

18. Arslan, N.; Esen, I.; Demircioglu, F.; Yilmaz, S.; Unuvar, T.; Bober, E. The changing face of celiac disease: A girl with obesity and celiac disease. J. Paediatr. Child Health 2009, 45, 317-318.

19. Balamtekin, N.; Demir, H.; Baysoy, G.; Uslu, N.; Yuce, A. Obesity in adolescents with celiac disease: Two adolescents and two different presentations. Turk. J. Pediatr. 2011, 53, 314-316. 
20. Mariani, P.; Viti, M.G.; Montouri, M.; La Vecchia, A.; Cipolletta, E.; Calvani, L.; Bonamico, M. The gluten free diet: A nutritional risk factor for adolescents with celiac disease? J. Pediatr. Gastroenterol. Nutr. 1998, 27, 519-523.

21. Aurangzeb, B.; Leach, S.T.; Lemberg, D.A.; Day, A.S. Nutritional status of children with coeliac disease. Acta Padiatr. 2010, 99, 1020-1025.

22. Venkatasubramani, N.; Telega, G.; Werlin, S.L. Obesity in pediatric celiac disease. J. Pediatr. Gastroenterol. Nutr. 2010, 51, 295-297.

23. Balamtekin, N.; Uslu, N.; Baysoy, G.; Usta, Y.; Demir, H.; Saltik-Temizel, I.N.; Ozen, H.; Gürakan, F.; Yüce, A. The presentation of celiac disease in 220 Turkish children. Turk. J. Pediatr. 2010, 52, 239-244.

24. Valletta, E.; Fornaro, M.; Cipolli, M.; Conte, S.; Bissolo, F.; Danchielli, C. Celiac disease and obesity: Need for nutritional follow-up after diagnosis. Eur. J. Clin. Nutr. 2010, 64, 1371-1372.

25. Brambilla, P.; Picca, M.; Dilillo, D.; Meneghin, F.; Cravidi, C.; Tischer, M.C.; Vivaldo, T.; Bedogni, G.; Zuccotti, G.V. Changes of body mass index in celiac children on a gluten-free diet. Nutr. Metab. Cardiovasc. Dis. 2013, 23, 177-182.

26. Reilly, N.R.; Aguilar, K.; Hassid, B.G.; Cheng, J.; Defelice, A.R.; Kazlow, P.; Bhagat, G.; Green, P.H. Celiac disease in normal-weight and overweight children: Clinical features and growth outcomes following a gluten-free diet. J. Pediatr. Gastroenterol. Nutr. 2011, 53, $528-531$.

27. Norsa, L.; Shamir, R.; Zevit, N.; Verduci, E.; Hartman, C.; Ghisleni, D.; Riva, E.; Giovannini, M. Cardiovascular disease risk factor profiles in children with celiac disease on gluten-free diets. World J. Gastroenterol. 2013, 19, 5658-5664.

28. West, J.; Logan R.F.; Card, T.R.; Smith, C.; Hubbard, R. Risk of vascular disease in adults with diagnosed coeliac disease: A population-based study. Aliment. Pharmacol. Ther. 2004, 20, $73-79$.

29. Dickey, W.; Kearney, N. Overweight in celiac disease: Prevalence, clinical characteristics, and effect of a gluten-free diet. Am. J. Gastroenterol. 2006, 101, 2356-2359.

30. Olén, O.; Montgomery, S.M.; Marcus, C.; Ekbom, A.; Ludvigsson, J.F. Coeliac disease and body mass index: A study of two Swedish general population-based registers. Scand. J. Gastroenterol. 2009, 44, 1198-1206.

31. Cheng, J.; Brar, P.S.; Lee, A.R.; Green, P.H. Body mass index in celiac disease: Beneficial effect of a gluten-free diet. J. Clin. Gastroenterol. 2010, 44, 267-271.

32. Kabbani, T.A.; Goldberg, A.; Kelly, C.P.; Pallav, K.; Tariq, S.; Peer, A.; Hansen, J.; Dennis, M.; Leffler, D.A. Body mass index and the risk of obesity in coeliac disease treated with the gluten-free diet. Aliment. Pharmacol. Ther. 2012, 35, 723-729.

33. Tucker, E.; Rostami, K.; Prabhakaran, S.; Al Dulaimi, D. Patients with celiac disease are increasingly overweight or obese on presentation. J. Gastrointest. Liver Dis. 2012, 21, 11-15.

34. Barera, G.; Mora, S.; Brambilla, P.; Ricotti, A.; Menni, L.; Beccio, S.; Bianchi, C. Body composition in children with celiac disease and the effects of a gluten-free diet: A prospective case-control study. Am. J. Clin. Nutr. 2000, 72, 71-75.

35. Bailey, K.V.; Ferro-Luzzi, A. Use of body mass index of adults in assessing individual and community nutritional status. Bull. World Health Org. 1995, 73, 673-680. 
36. Cole, T.J.; Bellizzi, M.C.; Flegal, K.M.; Dietz, W.H. Establishing a standard definition for child overweight and obesity worldwide: International survey. BMJ 2000, 6, 1240-1243.

37. Brar, P.; Kwon, G.Y.; Egbuna, I.I.; Holleran, S.; Ramakrishnan, R.; Bhagat, G.; Green, P.H. Lack of correlation of degree of villous atrophy with severity of clinical presentation of coeliac disease. Dig. Liver Dis. 2007, 39, 26-29.

38. Murray, J.A.; Rubio-Tapia, A.; van Dyke, C.T.; Brogan, D.L.; Knipschield, M.A.; Lahr, B.; Rumalla, A.; Zinsmeister, A.R.; Gostout, C.J. Mucosal atrophy in celiac disease: Extent of involvement, correlation with clinical presentation, and response to treatment. Clin. Gastroenterol. Hepatol. 2008, 6, 186-193.

39. De Onis, M.; Blössner, M.; Borghi, E. Global prevalence and trends of overweight and obesity among preschool children. Am. J. Clin. Nutr. 2010, 92, 1257-1264.

40. Ferrara, P.; Cicala, M.; Tiberi, E.; Spadaccio, C.; Marcella, L.; Gatto, A.; Calzolari, P.; Castellucci, G. High fat consumption in children with celiac disease. Acta Gastroenterol. Belg. 2009, 72, 296-300.

41. Kupper, C. Dietary guidelines and implementation for celiac disease. Gastroenterology 2005, $128, \mathrm{~S} 121-\mathrm{S} 127$.

42. Ohlund, K.; Olsson, C.; Hernell, O.; Ohlund, I. Dietary shortcomings in children on a gluten-free diet. J. Hum. Nutr. Diet. 2010, 23, 294-300.

43. Hopman, E.G.; Le Cessie, S.; von Blomberg, B.M.; Mearin, M.L. Nutritional management of the gluten-free diet in young people with celiac disease in The Netherlands. J. Pediatr. Gastroenterol. Nutr. 2006, 43, 102-108.

44. Kemppainen, T.; Uusitupa, M.; Janatuinen, E.; Järvinen, R.; Julkunen, R.; Pikkarainen, P. Intakes of nutrients and nutritional status in coeliac patients. Scand. J. Gastroenterol. 1995, 30, $575-579$.

45. Capristo, E.; Addolorato, G.; Mingrone, G.; de Gaetano, A.; Greco, A.V.; Tataranni, P.A.; Gasbarrini, G. Changes in body composition, substrate oxidation, and resting metabolic rate in adult celiac disease patients after a 1-year gluten-free diet treatment. Am. J. Clin. Nutr. 2000, 72, $76-81$.

46. Björkman, A.C.; Mobacken, H.; Kastrup, W.; Andersson, H. Changes in food consumption and its nutritional quality when on a gluten-free diet for dermatitis herpetiformis. Hum. Nutr. Appl. Nutr. 1985, 39, 124-129.

47. Bardella, M.T.; Fredella, C.; Prampolini, L.; Molteni, N.; Giunta, A.M.; Bianchi, P.A. Body composition and dietary intakes in adult celiac disease patients consuming a strict gluten-free diet. Am. J. Clin. Nutr. 2000, 72, 937-939.

48. Dell'Olio, D.; Palma, L.; Malorgio, E.; Ansaldi Balocco, N. What do celiac children eat? Dietary analysis of a group of children with celiac disease on a diet. Minerva Gastroenterol. Dietol. 1995, 41, 269-273.

49. American Diabetes Association. Nutrition recommendations and interventions for diabetes. A position statement of the American Diabetes Association. Diabetes Care 2008, 31, S61-S74.

50. Jenkins, D.J.A.; Thorne, M.J.; Wolever, T.M.S.; Jenkins, A.L.; Venketschwer, R.; Thompson, L.U. The effect of starch-protein interaction in wheat on the glycemic response and rate of in vitro digestion. Am. J. Clin. Nutr. 1987, 45, 946-951. 
51. Foster-Powell, K.; Holt, S.H.A.; Brand-Miller, J.C. International table of glycemic index and glycemic load values. Am. J. Clin. Nutr. 2002, 76, 5-56.

52. Packer, S.C.; Dornhorst, A.; Frost, G.S. The glycaemic index of a range of gluten-free foods. Diabetes 2000, 17, 657-660.

53. Monro, J.A. Glycaemic glucose equivalent: Combining carbohydrate content, quantity and glycaemic index of foods for precision in glycaemia management. Asia Pac. J. Clin. Nutr. 2002, 11, 217-225.

54. Salmerón, J.; Ascherio, A.; Rimm, E.B.; Colditz, G.A.; Spiegelman, D.; Jenkins, D.J.; Stampfer, M.J.; Wing, A.L.; Willett, W.C. Dietary fiber, glycemic load, and risk of NIDDM in men. Diabetes Care 1997, 20, 545-550.

55. Salmerón, J.; Manson, J.E.; Stampfer, M.J.; Colditz, G.A.; Wing, A.L.; Willett, W.C. Dietary fiber, glycemic load, and risk of non-insulin-dependent diabetes mellitus in women. JAMA 1997, 277, 472-477.

56. Livesey, G. Low-glycaemic diets and health: implications for obesity Satellite Symposium on "The role of low-glycaemic diets in obesity and health". Proc. Nutr. Soc. 2005, 64, 105-113.

57. Das, S.K.; Gilhooly, C.H.; Golden, J.K.; Pittas, A.G.; Fuss, P.J.; Dallal, G.E.; McCrory, M.A.; Saltzman, E.; Roberts, S.B. Long term effects of energy-restricted diets differing in glycemic load on metabolic adaptation and body composition. Open Nutr. J. 2007, 85, 1023-1030.

58. Barba, G.; Sieri, S.; Russo, M.D.; Donatiello, E.; Formisano, A.; Lauria, F.; Sparano, S.; Nappo, A.; Russo, P.; Brighenti, F.; et al. Glycaemic index and body fat distribution in children: The results of the ARCA project. Nutr. Metab. Cardiovasc. Dis. 2012, 22, 28-34.

59. Nielsen, B.M.; Bjørnsbo, K.S.; Tetens, I.; Heitmann, B.L. Dietary glycaemic index and glycaemic load in Danish children in relation to body fatness. Br. J. Nutr. 2005, 94, 992-997.

60. Buyken, A.E.; Trauner, K.; Günther, A.L.; Kroke, A.; Remer, T. Breakfast glycemic index affects subsequent daily energy intake in free-living healthy children. Am. J. Clin. Nutr. 2007, $86,980-987$.

61. Buyken, A.E.; Cheng, G.; Günther, A.L.; Liese, A.D.; Remer, T.; Karaolis-Danckert, N. Relation of dietary glycemic index, glycemic load, added sugar intake, or fiber intake to the development of body composition between ages 2 and 7 year. Am. J. Clin. Nutr. 2008, 88, 755-762.

62. Boye, K.R.; Dimitriou, T.; Manz, F.; Schoenau, E.; Neu, C.; Wudy, S.; Remer, T. Anthropometric assessment of muscularity during growth: Estimating fat-free mass with 2 skinfold-thickness measurements is superior to measuring mid-upper arm muscle area in healthy prepubertal children. Am. J. Clin. Nutr. 2002, 76, 628-632.

63. Thomas, D.E.; Elliott, E.J.; Baur, L. Low glycaemic index or low glycaemic load diets for overweight and obesity. Cochrane Database Syst. Rev. 2007, 18, CD005105.

64. Schwingshackl, L.; Hoffmann, G. Long-term effects of low glycemic index/load vs. high glycemic index/load diets on parameters of obesity and obesity-associated risks: A systematic review and meta-analysis. Nutr. Metab. Cardiovasc. Dis. 2013, 23, 699-706.

65. Acerini, C.L.; Ahmed, M.L.; Ross, K.M.; Sullivan, P.B.; Bird, G.; Dunger, D.B. Coeliac disease in children and adolescents with IDDM: Clinical characteristics and response to gluten-free diet. Diabet. Med. 1998, 15, 38-44. 
66. Nóvoa Medina, Y.; López-Capapé, M.; Lara Orejas, E.; Alonso Blanco, M.; Camarero Salces, C.; Barrio Castellanos, R. Impact of diagnosis of celiac disease on metabolic control of type 1 diabetes. Ann. Pediatr. 2008, 68, 13-17.

67. Saadah, O.I.; Zacharin, M.; O’Callaghan, A.; Oliver, M.R.; Catto-Smith, A.G. Effect of gluten-free diet and adherence on growth and diabetic control in diabetics with coeliac disease. Arch. Dis. Child 2004, 89, 871-876.

68. Marchese, A.; Lovati, E.; Biagi, F.; Corazza, G.R. Coeliac disease and type 1 diabetes mellitus: Epidemiology, clinical implications and effects of gluten-free diet. Endocrine 2013, 43, 1-2.

69. Savilahti, E.; Simell, O.; Koskimies, S.; Rilva, A.; Akerblom, H.K. Celiac disease in insulin-dependent diabetes mellitus. J. Pediatr. 1986, 108, 690-693.

70. Abid, N.; McGlone, O.; Cardwell, C.; McCallion, W.; Carson, D. Clinical and metabolic effects of gluten free diet in children with type 1 diabetes and coeliac disease. Pediatr. Diabetes 2011, $12,322-325$.

71. Stubbs, C.O.; Lee, A.J. The obesity epidemic: both energy intake and physical activity contribute. Med. J. Aust. 2004, 181, 489-491.

72. Rouhani, M.H.; Salehi-Abargouei, A.; Azadbakht, L. Effect of glycemic index and glycemic load on energy intake in children. Nutrition 2013, 29, 1100-1105.

73. Anderson, G.H.; Woodend, D. Effect of glycemic carbohydrates on short-term satiety and food intake. Nutr. Rev. 2003, 61, S17-S26.

74. Caudwell, P.; Finlayson, G.; Gibbons, C.; Hopkins, M.; King, N.; Näslund, E.; Blundell, J.E. Resting metabolic rate is associated with hunger, self-determined meal size, and daily energy intake and may represent a marker for appetite. Am. J. Clin. Nutr. 2013, 97, 7-14.

75. Papastamataki, M.; Papassotiriou, I.; Bartzeliotou, A.; Vazeou, A.; Roma, E.; Chrousos, G.P.; Kanaka-Gantenbein, C. Incretins, amylin and other gut-brain axis hormones in children with coeliac disease. Eur. J. Clin. Investig. 2013, 44, 74-82. 
Reprinted from Nutrients. Cite as: Wierdsma, N.J.; van Bokhorst-de van der Schueren, M.A.E.; Berkenpas, M.; Mulder, C.J.J.; van Bodegraven, A.A. Vitamin and Mineral Deficiencies Are Highly Prevalent in Newly Diagnosed Celiac Disease Patients. Nutrients 2013, 5, 3975-3992.

Article

\title{
Vitamin and Mineral Deficiencies Are Highly Prevalent in Newly Diagnosed Celiac Disease Patients
}

\author{
Nicolette J. Wierdsma ${ }^{1, *}$, Marian A. E. van Bokhorst-de van der Schueren ${ }^{1}$, \\ Marijke Berkenpas ${ }^{1}$, Chris J. J. Mulder ${ }^{2}$ and Ad A. van Bodegraven ${ }^{2}$
}

1 Department of Nutrition and Dietetics, VU University Medical Centre, P.O. Box 7057, Amsterdam 1007 MB, The Netherlands; E-Mails: m.vanbokhorst@vumc.nl (M.A.E.B.S.); m.berkenpas@vumc.nl (M.B.)

2 Department of Gastroenterology, Celiac Centre Amsterdam, VU University Medical Centre, Amsterdam 1007 MB, The Netherlands; E-Mails: cjmulder@vumc.nl (C.J.J.M.); v.bodegraven@vumc.nl (A.A.B.)

* Author to whom correspondence should be addressed; E-Mail: N.Wierdsma@vumc.nl; Tel.: +31-20-444-3410; Fax: +31-20-444-4143.

Received: 19 July 2013; in revised form: 13 September 2013 / Accepted: 13 September 2013 / Published: 30 September 2013

\begin{abstract}
Malabsorption, weight loss and vitamin/mineral-deficiencies characterize classical celiac disease (CD). This study aimed to assess the nutritional and vitamin/mineral status of current "early diagnosed" untreated adult CD-patients in the Netherlands. Newly diagnosed adult CD-patients were included $(n=80,42.8 \pm 15.1$ years) and a comparable sample of 24 healthy Dutch subjects was added to compare vitamin concentrations. Nutritional status and serum concentrations of folic acid, vitamin $\mathrm{A}, \mathrm{B}_{6}, \mathrm{~B}_{12}$, and (25-hydroxy) $\mathrm{D}$, zinc, haemoglobin $(\mathrm{Hb})$ and ferritin were determined (before prescribing gluten free diet). Almost all CD-patients (87\%) had at least one value below the lower limit of reference. Specifically, for vitamin A, 7.5\% of patients showed deficient levels, for vitamin $\mathrm{B}_{6} 14.5 \%$, folic acid 20\%, and vitamin $\mathrm{B}_{12} 19 \%$. Likewise, zinc deficiency was observed in $67 \%$ of the CD-patients, $46 \%$ had decreased iron storage, and 32\% had anaemia. Overall, 17\% were malnourished $(>10 \%$ undesired weight loss), $22 \%$ of the women were underweight (Body Mass Index (BMI) < 18.5), and $29 \%$ of the patients were overweight (BMI $>25)$. Vitamin deficiencies were barely seen in healthy controls, with the exception of vitamin $\mathrm{B}_{12}$. Vitamin/mineral deficiencies were counter-intuitively not associated with a (higher) grade of histological intestinal damage or (impaired) nutritional status. In conclusion, vitamin/mineral deficiencies are
\end{abstract}


still common in newly "early diagnosed" CD-patients, even though the prevalence of obesity at initial diagnosis is rising. Extensive nutritional assessments seem warranted to guide nutritional advices and follow-up in CD treatment.

Keywords: vitamins; minerals; celiac disease; deficiency; adult; Body Mass Index

\section{Introduction}

Celiac disease (CD) is the most common food intolerance in the Western population, and currently represents a major health care issue. The prevalence of $\mathrm{CD}$ has been estimate to be $0.5 \%-1 \%$ in different parts of the world [1]. CD is an inflammatory, immune-mediated chronic disease of the mucosa of the proximal small intestine due to irreversible gluten intolerance in genetically susceptible individuals. Gluten refers to a set of amino acid sequences found in the prolamine fraction of wheat, barley and rye. The characteristic histopathological finding is a varying degree of villous atrophy and crypt hyperplasia, primarily in the duodenum and jejunum, with inflammatory changes leading to malabsorption. The first-line, and up-till-now only, treatment is a lifelong strict adherence to a gluten free diet (GFD). All other treatment modalities suppress the intestinal inflammatory response and do not treat the intolerance $[2,3]$.

$\mathrm{CD}$ is a multi-system disorder which leads to striking differences in its clinical presentation. When present, gastrointestinal symptoms, including clinically evident malabsorption, may facilitate the diagnosis of CD. Over the last few decades, there appeared to be a changing clinical presentation of CD from the classical malabsorptive picture (diarrhoea, malabsorption and weight loss) towards one of a non-classical presentation with milder, non-specific symptoms such as tiredness, hematologic abnormalities, constipation and/or abdominal distension [4-6]. Nowadays, many patients present with no or only minor extra-intestinal symptoms. Indeed, microcytic or macrocytic anaemia, or folate deficiency may occasionally be the only clinical symptom to suggest $\mathrm{CD}$. This leads to a great extent of underdiagnoses in several countries [7]. Currently, 20\%-40\% of newly diagnosed CD-patients are even classified as overweight (Body Mass Index (BMI) $>25 \mathrm{~kg} / \mathrm{m}^{2}$ ) instead of the anticipated underweight [8-12], although the prevalence of obesity seems lower than in the general population [13]. This makes the diagnosis of CD challenging. Greater clinical awareness-especially improved serological testing since the late 1990s, including that for anti-tissue transglutaminase (tTG) antibodies $[14,15]$ - and the appearance of specialized centres has led to earlier recognition of CD-patients.

In the classically presenting CD-patients, malabsorption is frequently encountered [16], and micronutrient deficiencies may arise. Indeed, several studies demonstrate these deficiencies with varying results [17-21].

Our group recently demonstrated the specific functional insufficiency of the proximal small bowel in CD-patients by means of the citrulline generation test, in which the citrulline peak after glutamine administration and its conversion into citrulline in the enterocyte was delayed due to a decreased functional intestinal mass as a consequence of inflammatory changes [22]. 
Deficiencies of water-soluble vitamins, like B-vitamins, would be expected since they are absorbed in the proximal small bowel, which is the most prominent site affected in CD-patients. However, available data, in particular regarding vitamin $\mathrm{B}_{2}$ and $\mathrm{B}_{6}$ deficiencies, do not support this in untreated CD-patients [17,23]. Table 1 shows an overview of older and more recent literature on vitamin and mineral deficiencies in adult CD-patients.

Table 1. Literature overview on vitamin and mineral status in newly diagnosed adult celiac disease (CD)-patients.

\begin{tabular}{|c|c|c|}
\hline Author, year & Patient Group & Outcome \\
\hline \multicolumn{3}{|c|}{ Till 2005} \\
\hline Hallert,1981 [24] & CD (Folate) & $\begin{array}{l}\text { Decreased serum folate concentration abnormality in } \\
\text { adult CD. Predictive value of low folate for advice } \\
\text { jejunal biopsy. }\end{array}$ \\
\hline Stene-Larsen, 1988 [25] & $\mathrm{CD}(n=3)$ & $\begin{array}{l}\text { Vitamin } \mathrm{B}_{12} \text { malabsorption by } \mathrm{CD} \text { is emphasized as a } \\
\text { pathogenic mechanism of megaloblastic anaemia. }\end{array}$ \\
\hline Crofton, 1990 [20] & $\begin{array}{c}\text { Untreated } \mathrm{CD}(n=8) \text { and } \\
\text { healthy controls }(n=5)(\mathrm{Zinc})\end{array}$ & $\begin{array}{l}\text { Impaired turnover and loss of endogenous zinc in mild } \\
\text { untreated CD. Zinc levels normal. }\end{array}$ \\
\hline & Untreated CD $(n=40)$ & Nutritional status quite good in both groups. $15 \%-38 \%$ \\
\hline Kemppainen, 1995 [26] & $\begin{array}{l}\text { CD in remission }(n=52) \\
\text { (Nutritional status) }\end{array}$ & $\begin{array}{l}\text { deficiencies }\left(\mathrm{HB} \text {, ferritin, iron or } \mathrm{B}_{12}\right) \text { in untreated } \mathrm{CD} \\
\text { compared to } 0 \%-20 \% \text { in remission } \mathrm{CD} \text {. }\end{array}$ \\
\hline Kemppainen, 1998 [27] & $\begin{array}{l}\text { Untreated CD }(n=40) \\
\text { (Nutritional status) }\end{array}$ & $\begin{array}{l}\text { Anthropometric and biochemical nutritional status } \\
\text { acceptable. Low ferritin and folate (enterocyte) levels, } \\
\text { but normalised after } 1 \text { year GFD. }\end{array}$ \\
\hline Alwitry, 2000 [23] & Celiac disease (Vitamin A) & Case report in vitamin A deficiency and eye deviation \\
\hline Dohlo 2001 [187 & Untreated CD $(n=39)$ & $41 \%$ B12 deficient $(<220 \mathrm{ng} / \mathrm{L}), 41 \%$ anaemic, and \\
\hline Danere, 2001 [10] & (Vitamin B12) & $31 \%$ folate deficient $\mathrm{f}$ the $\mathrm{B}_{12}$ deficient $\mathrm{CD}$-patients. \\
\hline Dickey, 2002 [28] & $\mathrm{CD}(n=159)($ Vitamin B12) & $\begin{array}{l}\text { Low serum } \mathrm{B}_{12} \text { is common in } \mathrm{CD}(12 \%) \text { and is not due } \\
\text { to autoimmune gastritis. } 10 \% \text { of } \mathrm{B}_{12} \text { deficient group } \\
\text { had atrophic gastritis. Advice to know } \mathrm{B}_{12} \text { level before } \\
\text { folate supplementation. }\end{array}$ \\
\hline Hozyasz, 2003 [29] & $\begin{array}{l}\text { Untreated CD Polish }(n=18) \\
\text { remission on GFD } \\
(n=12)(\text { Vitamin E) }\end{array}$ & $\begin{array}{l}\text { All untreated CD-patients had reduced vitamin E } \\
\text { levels. Vitamin A comparable to treated CD. }\end{array}$ \\
\hline \multicolumn{3}{|c|}{$2005-2013$} \\
\hline Harper, 2007 [30] & $\begin{array}{l}\text { Untreated ( } 3 \text { mo after diagnosis) } \\
\qquad \mathrm{CD}(n=405) \text { (Anaemia) }\end{array}$ & $\begin{array}{l}\text { Iron deficiency in } 31 \% \text { of male and } 19 \% \text { of females, } \\
\text { folate in } 12 \%, \mathrm{~B}_{12} 5 \% \text { and anaemia in } 20 \% \text { of } \\
\text { CD-patients. Anaemia can not only be explained by } \\
\text { nutritional deficiencies. }\end{array}$ \\
\hline Dickey, 2008 [17] & $\begin{array}{l}\text { Untreated } \mathrm{CD}(n=35) \text {, } \\
\text { persistent villous atrophy } \\
\quad(n=34, \text { recovered } \\
(n=41) \text { (B-vitamins })\end{array}$ & $\begin{array}{l}\text { No compromised } \mathrm{B}_{2} \text { and } \mathrm{B}_{6} \text { in } 3 \text { groups. } \\
\text { Homocysteine concentrations are inversely associated } \\
\text { with serum and red cell folate and with } \mathrm{B}_{12} \text {. }\end{array}$ \\
\hline
\end{tabular}


Table 1. Cont.

\begin{tabular}{|c|c|c|}
\hline Henri-Bhargava, 2008 [19] & CD (Vitamin E, copper) & $\begin{array}{l}\text { Neurological impairment due to vitamin } \mathrm{E} \text { and } \\
\text { copper deficiencies in } \mathrm{CD} \text {. }\end{array}$ \\
\hline Bergamaschi, 2008 [21] & $\begin{array}{l}\text { Untreated } \mathrm{CD}(n=150) \\
\quad \text { after } 1 \text { year GFD } \\
(n=53) \text { (Anaemia) }\end{array}$ & $\begin{array}{l}34 \% \text { anaemia at diagnosis. Iron, vitamin deficiencies } \\
\text { and anaemia of chronic disease are common in CD. } \\
\text { GFD treatment improves anaemia. }\end{array}$ \\
\hline Lerner, 2012 [31] & $\begin{array}{l}\mathrm{CD}(\text { Spanish })(n=22) \text { and CD } \\
\text { children }(n=120)(\text { Spanish, } \\
\text { Israeli), (Vitamin D) }\end{array}$ & $\begin{array}{l}\text { Vitamin D levels correlate negatively with age. } 55 \% \\
\text { of Adult CD-patients had vitamin D deficiency } \\
(25 \text {-hydroxy }<20 \mathrm{ng} / \mathrm{mL}) \text { and } \\
\text { should be supplemented. }\end{array}$ \\
\hline
\end{tabular}

It has been suggested that the current "early diagnosis" of CD might be associated with less vitamin and mineral deficiencies at the moment of diagnosis than the classical CD. Mineral and trace-element status of untreated CD-patients has not been widely studied. There is even a lack of recent reports in the literature (see Table 1) indicating which deficiencies should be checked in newly diagnosed celiacs in Western Europe. Therefore, we aimed to measure essential serum nutritional variables in order to assess the prevalence of vitamin and mineral deficiencies in untreated adult CD-patients from a tertiary referral Celiac Disease Centre, consuming a (gluten containing) standard Dutch (Western) diet before diagnosis. Secondly, we studied the nutritional status and differences in prevalence of vitamin and mineral deficiencies between patients with different grades of intestinal histological damage, nutritional status, and sex.

\section{Materials and Methods}

\subsection{Patients}

Eighty consecutively diagnosed adult patients (aged 18-75 years) with newly diagnosed CD were recruited from the Outpatient Clinic of the VU University Medical Centre, Amsterdam, the Netherlands, during the period 2005-2012. All patients consumed a normal (gluten-containing) Dutch-Western diet until inclusion. The mean daily gluten intake in the Netherlands is around $13 \mathrm{~g}$ [32]. Duodenal biopsy specimens were harvested to determine the grade of histological damage due to gluten sensitive enteropathy as classified by Marsh [33] and modified by Rostami [34,35]. Gastric (corpus) biopsies were routinely harvested to determine whether atrophic gastritis was present. CD associated antibodies, i.e., anti-endomysial antibodies (EMA) and anti-tTG antibodies, were determined [36,37]. In addition, HLA-genotyping was performed, to analyse the presence of $\mathrm{DQ}_{2}$ and $\mathrm{DQ}_{8}$ (heterozygote or homozygote) as a prerequisite for a definitive diagnosis [38]. The diagnosis of $\mathrm{CD}$ was based on these histopathological, serological and genetic criteria.

Histopathological classification according to Marsh was used: intraepithelial lymphocytosis, crypt hyperplasia and villous atrophy Marsh IIIA, B and C (respectively, partial, subtotal and total villous atrophy) with or without elevated antibodies. Also, a group was added with low grade histopathological abnormalities Marsh I or Marsh II (lymphocytic enteritis with crypt hyperplasia) with gluten-dependent disorders, in case of elevated antibodies and the HLA-DQ 2 or HLA-DQ 8 genotype.

Blood samples were collected as part of routine clinical care. Patients were excluded if they had an established or suspected gastrointestinal abnormality other than CD, such as inflammatory bowel 
disease (IBD). A representative sample of 24 healthy Dutch subjects (comparable for sex, age and $\mathrm{BMI}$ ) was added to compare concentrations of vitamin $\mathrm{A}, \mathrm{B}_{6}$, folic acid and $\mathrm{B}_{12}$ (see control subjects characteristics [39]). Both groups were studied in the same period, were living in a similar environment, and measurements were performed at the same clinical chemistry laboratory.

The study protocol was approved by the Medical Ethics Committee (2005, project code 05.153) of the VU University Medical Centre Amsterdam, The Netherlands.

\subsection{Nutritional Status}

Patient characteristics and demographic data (including age (year), body height $(\mathrm{m})$, body weight $(\mathrm{kg})$ and self-reported involuntary weight loss in the past 1 and 6 months) were collected, BMI was calculated and biochemical analyses were performed following diagnosis and before any dietary advice to initiate a GFD. Patients were subsequently classified as "malnourished" when they unintentionally lost more than $10 \%$ of their bodyweight in the past 6 months or more than $5 \%$ in the past month prior to diagnosis or as having "risk of malnutrition" when $5 \%-10 \%$ of the bodyweight was unintentionally lost in the 6 months before diagnosis. Moreover, patients were classified into 3 groups on the basis of baseline BMI; less than $18.5 \mathrm{~kg} / \mathrm{m}^{2}$ (underweight), $18.5-25.0 \mathrm{~kg} / \mathrm{m}^{2}$ (normal weight) and more than $25 \mathrm{~kg} / \mathrm{m}^{2}$ (overweight, or even "obese" in case of BMI above $30 \mathrm{~kg} / \mathrm{m}^{2}$ ) (according to the definition of the World Health Organisation (2000)).

\subsection{Biochemical Analysis}

Fasting venous blood samples were drawn and subsequently analysed at the endocrine and clinical chemistry laboratories of the VU University Medical Centre, Amsterdam. Samples for serum folic acid and serum vitamin $B_{12}$ were analysed by competitive immunoassay (Luminescence, Abbott, IL, USA). Serum vitamin $\mathrm{B}_{6}$ and vitamin A status were determined by high-performance liquid chromatography (HPLC). Vitamin (25-hydroxy) D was assessed with a competitive binding protein assay (Diasorin, Stillwater, MN, USA). Zinc status was assessed using Flame Atomic Absorption Spectroscopy (FAAS), serum haemoglobin by colorimetric methods (Cell Dyn Sapphire, Abbott, IL, USA) and ferritin values by electro-chemiluminescence immunoassay "ECLIA" (Roche, Mannheim, Germany) or "ACS CENTAUR” (Bayer, Mijdrecht, The Netherlands).

Vitamin $\mathrm{B}_{6}$ and folic acid (both proximally absorbed) and vitamin $\mathrm{B}_{12}$ (distally absorbed) were considered to represent the water-soluble vitamin status. Vitamin A and vitamin (25-hydroxy) D were considered to represent the fat-soluble vitamin status, although sun exposure, even in The Netherlands, may have a strong influence on serum levels of vitamin D. Haemoglobin and ferritin levels below the reference ranges listed were used to establish a respective diagnosis of anaemia, iron-deficiency or iron-deficiency anaemia when both haemoglobin and ferritin were below reference ranges. Patients with a serum value below the lower limit of the reference value were considered "deficient". Reference values for the different parameters are displayed in Table 3. 


\subsection{Statistical Analysis}

Data were tested for normal distribution and presented as means \pm SD. The percentage of patients with values below the reference value and absolute number of deficient patients were additionally calculated for all assessed serum vitamin and mineral concentrations.

Data were analysed for the total group, and in stratified subgroups by sex, histological damage (Marsh classification) and BMI. To determine differences with regard to gender, a Student's $t$-test was applied in case of continuous data and Pearson's Chi-Square tests $\left(\chi^{2}\right)$ in the case of comparing proportions ( $\%$ of deficient patients). Analysis of variance (ANOVA), with a Bonferroni correction when a statistical significant difference was achieved, was used to compare more than two groups. A Mann-Whitney U test (Wilcoxon) or in case of more than 2 variables, a Kruskal-Wallis test was applied for variables not found in a normal distribution. The level of statistical significance was determined a priori at $p<0.05$. Statistical analyses were performed using SPSS (Statistical Package for Social Sciences Inc., Chicago, IL, USA-Windows version 20.0).

\section{Results}

\subsection{Patient Characteristics and Nutritional Status}

Patient characteristics are shown in Table 2; two-thirds of the population was female. Male patients were significantly older than female patients $(p=0.006)$. Approximately $46 \%(37 / 80)$ of the patients had partial villous atrophy (Marsh IIIA) and 42.5\% showed subtotal (20/80) or total villous atrophy (14/80) (Marsh IIIB or IIIC, respectively). Histologically and serologically atrophic (corpus) gastritis was ruled out by a pathologist experienced in intestinal histology. Some of the CD patients reported to have used vitamin and mineral supplements before diagnosis of CD was made: 18 (22.5\%) a multivitamin, folic acid or vitamin B-complex, 7 (8.8\%) iron supplements and 14 (17.5\%) a calcium supplement. The anthropometric data of 24 healthy controls were comparable to those of the patients included.

Patients had, on average, lost $2.4 \%( \pm 6.3 \%)$ of their bodyweight during the 6 months prior to diagnosis. Approximately $17 \%$ was classified as malnourished ( $>10 \%$ weight loss) and $5 \%$ as being at risk of malnutrition (5\%-10\% previous weight loss). Six out of 80 patients $(7.5 \%)$, and only females, were classified as underweight, whereas $29 \%(23 / 80)$ of patients were classified as overweight (female:male ratio $=1: 1)$. Of the overweight patients, $26 \%$ were even obese $(6 / 80$ of the total group, with female:male ratio $=1: 1$ ). 
Table 2. Patient characteristics of untreated adult CD-patients by gender and compared to healthy controls.

\begin{tabular}{|c|c|c|c|c|c|}
\hline \multicolumn{5}{|c|}{ CD Patients } & \multirow{2}{*}{$\frac{\text { Healthy Controls * }}{24}$} \\
\hline$N$ & & & 80 & & \\
\hline Sex & & All & Female (52) & Male (28) & F14/M11 \\
\hline Age (year) & mean $\pm \mathrm{SD}$ (range) & $42.8 \pm 15.1(18-75)$ & $39.5 \pm 14.3^{\wedge}$ & $49.1 \pm 14.9$ & $43.0 \pm 12.9$ \\
\hline Height (m) & mean $\pm \mathrm{SD}$ & $1.73 \pm 0.1$ & $1.68 \pm 0.09^{\wedge}$ & $1.80 \pm 0.08$ & $1.76 \pm 0.07 * \mathrm{a}$ \\
\hline Weight (kg) & mean $\pm \mathrm{SD}$ & $70.6 \pm 15.3$ & $66.0 \pm 14.3^{\wedge}$ & $79.1 \pm 13.8$ & $75.5 \pm 11.6$ \\
\hline BMI $\left(\mathrm{kg} / \mathrm{m}^{2}\right)$ & mean $\pm \mathrm{SD}$ & $23.6 \pm 4.0$ & $23.2 \pm 4.2$ & $24.3 \pm 3.6$ & $24.1 \pm 2.6$ \\
\hline \multirow{7}{*}{$\begin{array}{c}\text { Marsh } \\
\text { classification } \\
N(\%)\end{array}$} & $<18.5$ & $6(7.5 \%)$ & $6(22.5 \%)$ & 0 & 0 \\
\hline & $18.5-25$ & $51(63.8 \%)$ & $31(59.6 \%)$ & $20(71.4 \%)$ & $18(72 \%)$ \\
\hline & $>25$ & $23(28.8 \%)$ & $15(28.8 \%)$ & $8(28.6 \%)$ & $27(28 \%)$ \\
\hline & $\mathrm{I} / \mathrm{II} \#$ & $9(11.3 \%)$ & $6(11.5 \%)$ & $3(10.7 \%)$ & \\
\hline & IIIA & $37(46 \%)$ & $23(44.2 \%)$ & $14(50.0 \%)$ & \\
\hline & IIIB & $20(25 \%)$ & $10(19.2 \%)$ & $10(35.7 \%)$ & \\
\hline & IIIC & $14(17.5 \%)$ & $13(25 \%)$ & $1(3.6 \%)$ & \\
\hline \multirow[t]{3}{*}{ Antibodies $N(\%)$} & Negative & $17(21.3 \%)$ & $11(21.1 \%)$ & $6(22.0 \%)$ & \\
\hline & doubtful & $1(1.3 \%)$ & $1(1.9 \%)$ & 0 & \\
\hline & weak positive & $5(6.3 \%)$ & $5(9.6 \%)$ & 0 & \\
\hline \multirow[t]{3}{*}{ EMA } & positive & $14(17.5 \%)$ & $10(19.2 \%)$ & $4(14.3 \%)$ & \\
\hline & strong positive & $38(47.5 \%)$ & $23(44.2 \%)$ & $15(53.6 \%)$ & \\
\hline & n.d. & $5(6.3 \%)$ & $2(3.8 \%)$ & $1(3.6 \%)$ & \\
\hline \multirow{6}{*}{ tTG } & Negative & $16(20 \%)$ & $9(17.3 \%)$ & $7(25 \%)$ & \\
\hline & doubtful & $4(5 \%)$ & $3(5.8 \%)$ & $1(3.6 \%)$ & \\
\hline & weak positive & $10(12.5 \%)$ & $9(17.3 \%)$ & $1(3.6 \%)$ & \\
\hline & positive & $18(22.5 \%)$ & $12(23.1 \%)$ & $6(21.4 \%)$ & \\
\hline & strong positive & $31(38.8 \%)$ & $19(36.5 \%$ & $12(42.9 \%)$ & \\
\hline & n.d. & $1(1.3 \%)$ & 0 & $1(3.6 \%)$ & \\
\hline \multirow{2}{*}{$\mathrm{tTG}(\mathrm{U} / \mathrm{mL})$} & mean $\pm \mathrm{SD}$ & $171 \pm 402$ & $124 \pm 378$ & $274 \pm 445$ & \\
\hline & (range) & $(3.2-2500)$ & $(4-2500)$ & (3.2-1999) & \\
\hline \multirow{5}{*}{ CD genotypes } & $\mathrm{DQ}_{2 \text { (hetero-/homozygote) }}$ & $62(77.5 \%)_{(56 / 6)}$ & $42(80.8 \%)_{(37 / 5)}$ & $20(71.4 \%)_{(19 / 1)}$ & \\
\hline & $\mathrm{DQ}_{8 \text { (hetero-/homozygote) }}$ & $5(6.3 \%)_{(3 / 2)}$ & $3(5.8 \%)_{(2 / 1)}$ & $2(7.1 \%)_{(1 / 1)}$ & \\
\hline & $\mathrm{DQ}_{2}$ and $\mathrm{DQ}_{8}$ & $3(3.8 \%)$ & $2(3.8 \%)$ & $1(3.6 \%)$ & \\
\hline & $\mathrm{DQ}_{2}$ nor $\mathrm{DQ}_{8}$ & $3(3.8 \%)$ & $1(1.9 \%)$ & $2(7.1 \%)$ & \\
\hline & n.d. & $7(8.8 \%)$ & $4(7.7 \%)$ & $3(14.3 \%)$ & \\
\hline
\end{tabular}

BMI: Body Mass Index, EMA: anti-endomysial antibodies, tTG: anti-tissue transglutaminase, $\wedge$ Significantly different from men $(p<0.05)$ by Student's $t$-test, n.d. (not determined), \# low grade histopathological abnormalities with $\mathrm{HLA}-\mathrm{DQ}_{2}$ and/or $\mathrm{DQ}_{8}$ and elevated antibodies (EMA and/or tTG), * variables NS (not statistically significant from CD-patients $(p<0.05)$ by Mann-Whitney $\mathrm{U}$ test $),{ }^{* \mathrm{a}}$ statistical trend $p=0.05$. 


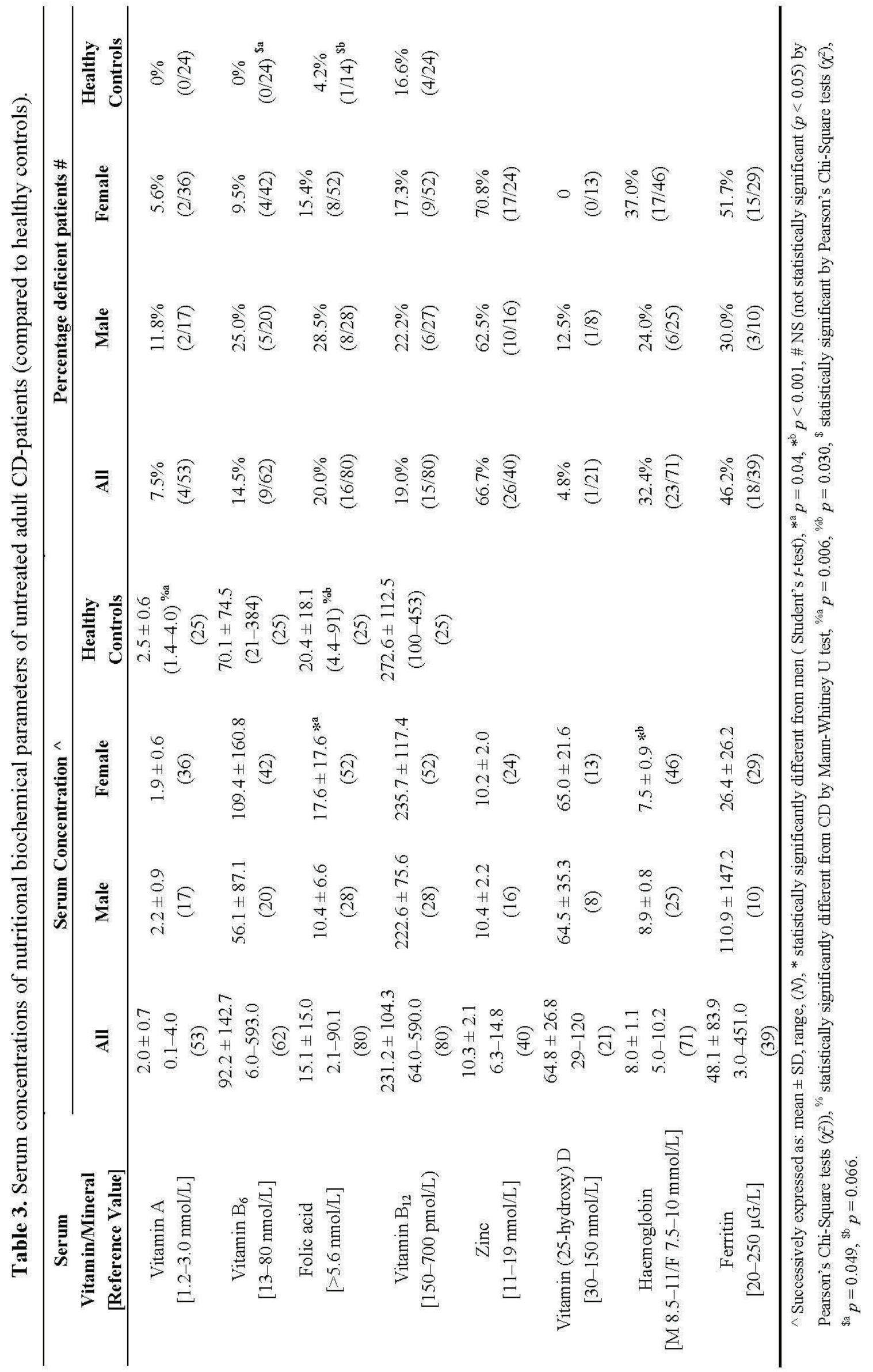




\subsection{Biochemical Analyses}

Serum concentrations of vitamins and minerals of the untreated CD-patients are shown in Table 3. CD-patients were most frequently deficient for folic acid $(20 \%, 16 / 80)$, followed by vitamin $\mathrm{B}_{12}$ $(19 \%, 15 / 79)$, vitamin $\mathrm{B}_{6}(14.5 \%, 9 / 62)$, vitamin A $(7.5 \%, 4 / 53)$ and vitamin (25-hydroxy) D (4.5\%, $1 / 21)$, respectively. Approximately 67\% (26/39) of the patients had zinc deficiency, $32.4 \%$ (23/71) had anaemia, $46.2 \%$ (18/39) had insufficient iron storage evidenced by low ferritin and 25\% (8/40) had iron-deficiency anaemia. CD-patients had lower values of vitamin A and folic acid than healthy controls. Overall, vitamin deficiencies were barely seen in healthy controls, with the exception of vitamin $\mathrm{B}_{12}$. None of the healthy controls showed deficient levels (below the reference values) for vitamin $\mathrm{A}$ and vitamin $\mathrm{B}_{6}$ and only one for folic acid.

Ten patients $(12.5 \%)$ were not deficient for any of the assessed vitamins and minerals. These patients had similar base-line characteristics to the rest of the $\mathrm{CD}$ group. The remaining 70 patients $(87.5 \%)$ were deficient in at least one of the nutritional parameters and $43(53.8 \%)$ for two or more parameters.

Table 3 depicts the proportion of deficient patients, stratified by sex. As anticipated, serum haemoglobin concentrations were lower in women than in men $(p<0.001)$. While, on the other hand, serum folic acid concentrations were lower $(p=0.040)$ in men when compared to women. No statistically significant difference was found for any of the other nutritional parameters when comparing males and females. Notwithstanding, multivitamin use (including folic acid or vitamin B supplement)

at their own volition or prescribed by the GP was more prevalent in women than men (30\% vs. $13 \%)$. A statistical trend was seen for vitamin $\mathrm{B}_{6}$, suggesting that men might be more often deficient than women $(p=0.097)$.

\subsection{Association between Vitamin and Mineral Concentrations and Histological Damage (Marsh-Classification)}

Table 4 depicts the mean serum vitamin and mineral values by Marsh strata. No statistically significant differences were found between the serum vitamin and mineral concentrations across the four Marsh strata, except for ferritin. Serum ferritin values decreased when the villous atrophy score increased $(p=0.041)$.

Table 4. Mean serum concentrations of vitamins and minerals $( \pm \mathrm{SD})$ in untreated

CD-patients by Marsh stratum.

\begin{tabular}{ccccc}
\hline \multirow{2}{*}{$\begin{array}{c}\text { Serum } \\
\text { Vitamin/Mineral }\end{array}$} & I/II \# & MIIA & IIIB & IIIC \\
\cline { 2 - 5 } & $(\boldsymbol{n}=\mathbf{9})$ & $(\boldsymbol{n}=\mathbf{3 7})$ & $(\boldsymbol{n}=\mathbf{2 0})$ & $(\boldsymbol{n}=\mathbf{1 4})$ \\
\hline \multirow{2}{*}{ Vitamin A (nmol/L) } & $2.3 \pm 0.4$ & $2.0 \pm 0.8$ & $1.9 \pm 0.44$ & $1.9 \pm 0.8$ \\
& $(4 / 9)$ & $(28 / 37)$ & $(11 / 20)$ & $(10 / 14)$ \\
Vitamin $\mathrm{B}_{6}(\mathrm{nmol} / \mathrm{L})$ & $129.8 \pm 115.0$ & $107.6 \pm 170.7$ & $55.7 \pm 43.5$ & $86.8 \pm 163.9$ \\
& $(7 / 9)$ & $(29 / 37)$ & $(15 / 20)$ & $(11 / 14)$ \\
Folic acid $(\mathrm{nmol} / \mathrm{L})$ & $14.1 \pm 8.5$ & $14.7 \pm 12.9$ & $13.9 \pm 15.5$ & $18.4 \pm 22.4$ \\
& $(9 / 9)$ & $(37 / 37)$ & $(20 / 20)$ & $(14 / 14)$ \\
\hline
\end{tabular}


Table 4. Cont.

\begin{tabular}{ccccc}
\hline & $282.4 \pm 151.7$ & $225.2 \pm 91.1$ & $216.5 \pm 80.2$ & $234.7 \pm 131.8$ \\
Vitamin $\mathrm{B}_{12}(\mathrm{pmol} / \mathrm{L})$ & $(9 / 9)$ & $(37 / 37)$ & $(20 / 20)$ & $(14 / 14)$ \\
& $89.3 \pm 27.0$ & $52.7 \pm 16.4$ & $63.0 \pm 31.4$ & $69.2 \pm 29.2$ \\
Vitamin (25-hydroxy) D (nmol/L) & $(3 / 9)$ & $(7 / 37)$ & $(6 / 20)$ & $(5 / 14)$ \\
& $11.0 \pm 1.7$ & $10.8 \pm 2.3$ & $9.8 \pm 1.7$ & $8.8 \pm 1.7$ \\
Zinc (nmol/L) & $(5 / 9)$ & $(19 / 37)$ & $(11 / 20)$ & $(5 / 14)$ \\
& $8.5 \pm 0.5$ & $8.0 \pm 1.2$ & $8.0 \pm 1.1$ & $7.9 \pm 1.0$ \\
Haemoglobin $(\mathrm{mmol} / \mathrm{L}) *$ & $(7 / 9)$ & $(33 / 37)$ & $(18 / 20)$ & $(13 / 14)$ \\
& $123.8 \pm 167.3$ & $47.6 \pm 66.3$ & $18.8 \pm 17.6$ & $17.2 \pm 22.0$ \\
Ferritin $(\mu \mathrm{G} / \mathrm{L})^{\wedge}$ & $(6 / 9)$ & $(18 / 37)$ & $(10 / 20)$ & $(5 / 14)$ \\
\hline
\end{tabular}

\# Low grade histopathological abnormalities with $\mathrm{HLA}-\mathrm{DQ}_{2}$ and/or $\mathrm{DQ}_{8}$ and elevated antibodies (EMA and/or tTG), * gender specific Females I/II $8.2 \pm 0.29$; IIIA $7.4 \pm 0.95$; IIIB $7.3 \pm 0.84$; IIIC $7.8 \pm 0.97$ and Male I/II $8.9 \pm 0.78$; IIIA $8.9 \pm 0.96$; IIIB $9.0 \pm 0.67$; IIIC $8.8 \pm 0.72, \wedge$ statistically significantly different $(p=0.041)$ by Kruskal-Wallis test.

\subsection{Association between Vitamin and Mineral Concentrations and Nutritional Status}

Table 5 depicts the mean serum concentrations of vitamins and minerals per BMI stratum. A trend was observed for underweight patients having a slightly higher serum folic acid concentration than patients with normal weight or overweight patients $(p=0.058)$. Besides, patients with $>10 \%$ unintentional weight loss in the past 6 months had higher vitamin A and (a trend for) higher vitamin $\mathrm{B}_{6}(p=0.09)$ levels than patients without weight loss. Otherwise, no differences were observed between the different classes of nutritional status.

Table 5. Mean serum concentrations of vitamins and minerals $( \pm \mathrm{SD})$ in untreated CD-patients by nutritional status (BMI stratum and unintentional weight loss).

\begin{tabular}{|c|c|c|c|c|c|}
\hline \multirow[b]{2}{*}{$\begin{array}{c}\text { Serum } \\
\text { Vitamin/Mineral }\end{array}$} & \multicolumn{3}{|c|}{ BMI Stratum $\left(\mathrm{kg} / \mathrm{m}^{2}\right)^{\wedge}$} & \multicolumn{2}{|c|}{ Weight Loss (\% in past 6 months) } \\
\hline & $\begin{array}{l}\text { Under-Weight } \\
\qquad \begin{array}{c}(<18.5) \\
(n=6)\end{array}\end{array}$ & $\begin{array}{c}\text { Normal Weight } \\
\begin{array}{c}(18.5-25.0) \\
(n=51)\end{array}\end{array}$ & $\begin{array}{c}\text { Over-Weight } \\
(>25) \\
(n=23)\end{array}$ & $\begin{array}{l}\text { Well-Nourished } \\
\begin{array}{c}(0 \%-10 \%) \\
(n=64)\end{array}\end{array}$ & $\begin{array}{l}\text { Mal-Nourished } \\
\qquad \begin{array}{l}(>10 \%) \\
(n=13)\end{array}\end{array}$ \\
\hline Vitamin A (nmol/L) & $1.7 \pm 0.7(5)$ & $2.0 \pm 0.7(35)$ & $2.0 \pm 0.6(13)$ & $1.8 \pm 0.6(40)$ & $2.6 \pm 0.7 \#(11)$ \\
\hline Vitamin $\mathrm{B}_{6}(\mathrm{nmol} / \mathrm{L})$ & $\begin{array}{c}128.5 \pm 207.8 \\
(6)\end{array}$ & $99.3 \pm 146.2(39)$ & $63.1 \pm 109.0$ & $68.1 \pm 102.0(48)$ & $206 \pm 240.8(11)$ \\
\hline Folic acid (nmol/L) & $33.0 \pm 29.9(6)$ & $14.5 \pm 13.4(51)$ & $11.8 \pm 10.0(23)$ & $13.4 \pm 11.2(64)$ & $23.5 \pm 27.1(13)$ \\
\hline Vitamin $\mathrm{B}_{12}(\mathrm{pmol} / \mathrm{L})$ & $216.3 \pm 57.3(6)$ & $\begin{array}{c}226.2 \pm 114.5 \\
(51)\end{array}$ & $246.0 \pm 90.4(23)$ & $\begin{array}{c}227.6 \pm 104.0 \\
(64)\end{array}$ & $250.8 \pm 117.7(13)$ \\
\hline $\begin{array}{c}\text { Vitamin (25-hydroxy) } \\
\text { D (nmol/L) }\end{array}$ & $52.0(1)$ & $63.4 \pm 28.0(11)$ & $68.0 \pm 27.9(9)$ & $67.1 \pm 27.1(17)$ & $55.0 \pm 26.5(4)$ \\
\hline Zinc (nmol/L) & $8.8 \pm 0.3(2)$ & $10.3 \pm 2.1(26)$ & $10.6 \pm 2.2(12)$ & $10.5 \pm 2.1(31)$ & $9.8 \pm 2.1(7)$ \\
\hline Haemoglobin $(\mathrm{mmol} / \mathrm{L})$ & $7.6 \pm 0.7(6)$ & $8.0 \pm 1.1(45)$ & $8.2 \pm 1.2(20)$ & $8.1 \pm 1.1(56)$ & $7.8 \pm 1.4(12)$ \\
\hline Ferritin $(\mu \mathrm{G} / \mathrm{L})$ & $25.0 \pm 2.1(3)$ & $34.9 \pm 38.4(26)$ & $89.0 \pm 151.9(10)$ & $48.5 \pm 90.4(33)$ & $46.2 \pm 38.3(5)$ \\
\hline
\end{tabular}

$\wedge$ NS (not statistically significant $(p<0.05)$ by Kruskal-Wallis test, \# statistically significantly different from well-nourished patients (Student $t$-test), $p=0.001$. 


\section{Discussion}

The present study showed that the majority of an "early diagnosis" adult untreated CD patient group (with so-called non-classical presentation) in the Netherlands, had at least one, and often several, serum vitamin or mineral deficiencies at diagnosis. Almost 90\% of CD-patients were found to be deficient in at least one or more of the assessed nutritional parameters, and half of patients were deficient for two or more nutritional serum variables. This was observed to be unrelated to severity of clinical presentation, nutritional status or (semi-quantified) histopathological damage (score).

In this study, no statistically significant difference in serum vitamin and mineral concentration was found between men and women, although (multi)vitamin use prior to diagnosis was more prevalent in women than men (30\% vs. 13\%). Information is lacking whether they took the vitamins at their own volition, or if they were prescribed by the general practitioner. In addition, the assessed serum deficiencies were independent of Marsh stratum (Table 4), nutritional status (Table 5) and age (data not shown). Folic acid deficiency was observed in $20 \%$ of the untreated CD-patients in this study. The prevalence of folate deficiency varies from $18 \%$ to $90 \%$ in varying older and newer reports of CD-patients $[17,18,24,26]$. In studies from Scotland and Finland, folate deficiency was reported in 42\% [18] and 37\% [27] of the untreated CD-patients, respectively. The difference in prevalence might at least partially be explained by technical aspects of measurement of "folate" (which is the natural form of folic acid) and "folic acid" since bioavailability of folic acid is twice that of folate [40]. Macrocytic anaemia in untreated CD-patients is usually caused by folate deficiency. In two large European studies, anaemia, mostly attributed to malabsorption, was reported to be present in $20 \%-34 \%$ of untreated CD-patients [20,30]. This corresponds with our results in which $25 \%$ of the CD-patients suffered from iron-deficiency anaemia.

The presence of vitamin $\mathrm{B}_{6}$ deficiency has been reported in two studies, albeit in children with "acute celiac disease". A decreased pyridoxal phosphate was reported in serum samples and in duodenal mucosa, suggestive of vitamin $\mathrm{B}_{6}$ deficiency $[41,42]$ and indicative for decreased levels of vitamin $\mathrm{B}_{6}$ in untreated $\mathrm{CD}$ children. We found water-soluble vitamin deficiencies $\left(\mathrm{B}_{6}\right.$, folic acid and $\mathrm{B}_{12}$ ) in approximately one in seven $\left(\mathrm{B}_{6}\right)$ to one in five (folic acid and $\mathrm{B}_{12}$ ) untreated CD-patients. This was despite the fact that more than $20 \%$ of the patients were using a prescribed or over-the-counter multivitamin/vitamin B-complex or a folic acid supplement before diagnosis.

Vitamin $B_{12}$ deficiency was frequently observed in our CD-patient group (19\%), in accordance with earlier studies, notably also in those without atrophic gastritis [17,18,26-28]. Intriguingly, this vitamin is typically absorbed in the terminal ileum. Apparently, the distal small bowel is functionally more affected than previously believed, based on patho-histological analysis of distal small bowel biopsy samples [43]. Vitamin $\mathrm{B}_{12}$ deficiency in untreated CD-patients has been confirmed in several previously conducted European studies [18,25-28], ranging from $12 \%$ up to $41 \%$. It may be hypothesized that vitamin $\mathrm{B}_{12}$ deficiency is a result of a dysfunctional intrinsic factor [44]. Dickey reported that low vitamin $B_{12}$ concentrations in $C D$ were not due to auto-immune gastritis [28]. In this study, none of the patients had histologically or serologically demonstrated atrophic gastritis and, therefore, this was unlikely to be responsible for vitamin $\mathrm{B}_{12}$ deficiencies in $\mathrm{CD}$.

In various small studies in Europeans, deficiencies of the fat soluble vitamins A [23,27], E [19,29] and D [45] in untreated CD-patients have been previously reported. The latter has been associated 


\section{4}

with osteomalacia. In clinical practice, many believe that the clinically relevant lower limit for vitamin $\mathrm{D}$ deficiency should be increased. As a consequence, the displayed (relatively low) deficiency percentages can therefore be an underestimation. The observed $7.5 \%$ of vitamin A deficient patients is less than the percentage reported in Finland (14\%) for this vitamin [27]. From healthy subject studies, it is known that the vitamin A body storage is usually stable and sufficient for approximately one to two years [46]. This is confirmed by our healthy sample, in which none of the subjects showed a deficiency.

A majority of our untreated CD-patients had a zinc deficiency, which can probably be explained by increased endogenous losses of zinc, rather than abnormal zinc absorption [20]. Clinical relevance of zinc deficiency remains inconclusive and additional research is warranted. However, it is known that $\mathrm{CD}$ is associated with a wide array of skin lesions and manifestations, which may be partly ascribed to zinc deficiency [47-50], and cell mediated immunity and antioxidant buffer capacity may be compromised due to it as well [51].

Possible explanations for the high prevalence of nutritional deficiencies in untreated CD-patients might be an insufficient nutritional intake. This is supported by the presence of malnutrition in this cohort: $17 \%$ was malnourished based on the usual definition of $>10 \%$ involuntary body weight loss prior to diagnosis and $7.5 \%$ had a BMI $<18.5 \mathrm{~kg} / \mathrm{m}^{2}$. However, this seemed unlikely since patients did not report any changes (intentional or involuntary) in their habitual diet before diagnosis. Moreover, the observed presence of high serum folate levels, particularly in those classified as underweight, were contradictory as well. On the other hand, increased faecal losses of nutrients as a result of malabsorption might (partially) explain the high prevalence of deficiencies. However, since most patients did not report any clinical sign of malabsorptive diarrhoea, losses via the stools were thought to be of limited importance as well. Nevertheless, the observed findings in this study, sharing deficiencies in water and fat soluble vitamins, zinc and iron, indicate that maldigestion, malabsorption or a structurally moderately inadequate intake might have been present long before the clinical diagnosis of CD was established. It is known that the delay in diagnosing CD can be more than a decade [52]. One may hypothesize that this might be due to functional changes of the intestinal tract or due to changes in the intestinal microbiome [53]. In this study, even patients with mild CD (low grade pathohistological abnormalities) showed nutritional deficiencies and weight loss. A finding that was recently corroborated in a large Italian cohort showed that mild (histopathological) enteropathy did not necessarily mean mild intestinal dysfunction since, also in this group, alterations in bone marrow density and laboratory parameters were reported [54]. Remarkably, only female patients presented as underweight (approximately one in four females), while the female-male ratio was similar in the overweight and obese patients. A statistical trend for higher serum vitamin A and B concentrations was observed in patients classified as malnourished (low BMI or $>10 \%$ unintentional weight loss prior to diagnosis). This may be explained by their more so-called classical CD presentation, which may trigger use of supplements due to a greater physicians' or patients' awareness when observing clinical signs of malnutrition.

Some considerations arise when interpreting the presented results: not all data were available in all subjects and some subgroups were relatively small, precluding detection of small effects or the drawing of firm conclusions in subgroups. Vitamin or mineral supplement use, whether at the patient's own initiative or prescribed by the GP, was based on self-reported information and can 
therefore be underestimated if patients forget to mention these "medicinal supplements". However, real numbers and frequencies of deficiencies or anaemia might be underestimated, since some patients were already taking these supplements before the initial diagnosis of CD, potentially leading to misclassification as "non-deficient" in this study. Besides, serum values of almost all vitamins or minerals do not fully represent total body stock or physiological function.

Based on our experience and supported by a recently published guideline on CD [3], we suggest monitoring body weight at diagnosis and nutritional serum parameters; at least vitamin $\mathrm{B}_{6}$, folic acid, $\mathrm{B}_{12}$ and zinc and in any case (25-hydroxy) D of the fat soluble vitamins (due to its connection with presence of osteomalacia). Moreover, we suggest follow-up until serum values are at satisfying levels or upon indication (for instance, if bone density deviations, chronic diarrhoea, or skin lesions are present). Practically, a standard complete multivitamin supplement (100\%-300\% of RDA) should be considered for every newly diagnosed CD-patient. Continuation time has yet to be determined, since patients are at risk for vitamin deficiencies even after 10 years of a GFD [55]. Evidently, hypervitaminosis should be avoided, in particular regarding pyridoxine and iron [56,57]. It is demonstrated that bone-mineral density and nutritional status can improve after a GFD treatment [58] as well as that of general well-being, which can improve after vitamin B supplementation in CD-patients on a GFD [59].

\section{Conclusions}

In conclusion, deficiencies of vitamins or minerals are frequently observed in untreated adult Dutch CD-patients using a Western diet, although they are currently diagnosed earlier than in the previous century. This was observed even in obese patients. Almost $90 \%$ of the newly diagnosed CD-patients had one or more nutritional deficiencies. Malnutrition, expressed as an involuntary weight loss or being underweight, was found in $16 \%$ and $7.5 \%$ of patients, respectively, while overweight status (BMI $>25 \mathrm{~kg} / \mathrm{m}^{2}$ ) was present in almost $30 \%$ of the patients. Therefore, these results indicate that extensive nutritional assessment of body weight and serum nutritional parameters should be an integral part of celiac disease treatment to guide nutritional advices and follow-up in CD-treatment by means of an adequately composed individual-based, gluten free diet.

\section{Acknowledgments}

The authors thank the Department of Clinical Chemistry of the VU University Medical Centre in Amsterdam for their help with the analyses. And student Priyantha Malhoe for her assistance at the beginning of this manuscript.

\section{Authorship Statement}

NJW, AAvB and CJJM designed the protocol, NJW and MB conducted the research, NJW analyzed the data, NJW and AAvB wrote the paper, and all authors critically reviewed and approved the final version of the paper. NJW is primarily responsible for the contents of the paper and is acting as the submission's guarantor. 


\section{Financially Support}

This study was funded in part by a grant received from the Dutch Society for Gastroenterology (Gastrostart). Gastrostart had no involvement in any of the study tasks.

\section{Conflicts of interest}

The authors declare no conflict of interest.

\section{References}

1. Gujral, N.; Freeman, H.J.; Thomson, A.B. Celiac disease: Prevalence, diagnosis, pathogenesis and treatment. World J. Gastroenterol. 2012, 18, 6036-6059.

2. Green, P.; Cellier, C. Celiac disease. N. Engl. J. Med. 2007, 357, 1731-1743.

3. Bai, J.; Fried, M.; Corazza, G.; Schuppan, D.; Farthing, M.; Catassi, C.; Greco, L.; Cohen, H.; Ciacci, C.; Eliakim, R.; et al. World Gastroenterology Organisation global guidelines on celiac disease. J. Clin. Gastroenterol. 2013, 47, 121-126.

4. Tack, G.; Verbeek, W.H.; Schreurs, M.; Mulder, C.J.J. The spectrum of celiac disease: Epidemiology, clinical aspects and treatment. Nat. Rev. Gastroenterol. Hepatol. 2010, 7, 204-213.

5. Pare, P.; Douville, P.; Caron, D.; Lagace, R. Adult celiac sprue: Changes in the pattern of clinical recognition. J. Clin Gastroenterol. 1988, 10, 395-400.

6. Corazza, G.; Frisoni, M.; Treggiari, E.; Valentini, R.; Filipponi, C.; Volta, U.; Gasbarrini, G. Subclinical celiac sprue. Increasing occurence and clues to its diagnosis. J. Clin. Gastroenterol. 1993, 16, 16-21.

7. Gupta, R.; Reddy, D.; Makhari, G.; Sood, A.; Ramakrischna, B.; Yaccha, S.; Thapa, B.; Banerjee, R.; Anuradha, S.; Dutta, U.; et al. Indian task force for celiac disease: Current status. World J. Gastroenterol. 2009, 15, 6028-6033.

8. Cheng, J.; Brar, P.S.; Lee, A.R.; Green, P.H. Body mass index in celiac disease: Beneficial effect of a gluten-free diet. J. Clin. Gastroenterol. 2010, 44, 267-271.

9. Ukkola, A.; Maki, M.; Kurppa, K.; Collin, P.; Huhtala, H.; Kekkonen, L.; Kaukinen, K. Changes in body mass index on a gluten free diet in coeliac disease: A nationwide study. Eur. J. Int. Med. 2012, 23, 384-388.

10. Sinniah, R.; Roche, H.M. Letter: Rising incidence of obesity in the coeliac population-A malady or maladaptation? Alliment. Pharmacol. Ther. 2012, 35, 1483-1484.

11. Tucker, E.; Rostami, K.; Prabhakaran, S.; al Dulaimi, D. Patients with coeliac disease are increasingly overweight or obese on presentation. J. Gastrointest. Liver Dis. 2012, 21, 11-15.

12. Tikkakoski, S.; Savilahti, E.; Kolho, K. Undiagnosed coeliac disease and nutritional deficiencies in adults screened in primary health care. Scand. J. Gastroenterol. 2007, 42, 60-65.

13. Kabbani, T.; Goldberg, A.; Kelly, C.; Pallav, K.; Tariq, S.; Peer, A.; Hansen, J.; Dennis, M.D.; Leffler, D. Body mass index and the risk of obesity in coeliac disease treated with gluten-free diet. Alliment. Pharmacol. Ther. 2012, 35, 723-729. 
14. Evans, K.E.; Leeds, J.S.; Sanders, D.S. Be vigilant for patients with coeliac disease. Practitioner 2009, 253, 19-22.

15. Rostami, N.M.; Rostami, K.; Pourhoseingholi, M.A.; Nazemalhosseini, M.E.; Habibi, M.; Dabiri, H.; Zali, M.R. Atypical presentation is dominant and typical for coeliac disease. J. Gastrointest. Liver Dis. 2009, 18, 285-291.

16. McGough, N.; Cummings, J.H. Coeliac disease: A diverse clinical syndrome caused by intolerance of wheat, barley and rye. Proc. Nutr. Soc. 2005, 64, 434-450.

17. Dickey, W.; Ward, M.; Whittle, C.R.; Kelly, M.T.; Pentieva, K.; Horigan, G.; Patton, S.; McNulty, H. Homocysteine and related B-vitamin status in coeliac disease: Effects of gluten exclusion and histological recovery. Scand. J. Gastroenterol. 2008, 43, 682-688.

18. Dahele, A.; Ghosh, S. Vitamin B12 deficiency in untreated celiac disease. Am. J. Gastroenterol. 2001, 96, 745-750.

19. Henri-Bhargava, A.; Melmed, C.; Glikstein, R.; Schipper, H.M. Neurologic impairment due to vitamin E and copper deficiencies in celiac disease. Neurology 2008, 71, 860-861.

20. Crofton, R.W.; Aggett, P.J.; Gvozdanovic, S.; Gvozdanovic, D.; Mowat, N.A.; Brunt, P.W. Zinc metabolism in celiac disease. Am. J. Clin. Nutr. 1990, 52, 379-382.

21. Bergamaschi, G.; Markopoulos, K.; Albertini, R.; di Sabatino, A.; Biagi, F.; Ciccocioppo, R.; Arbustini, E.; Corazza, G.R. Anemia of chronic disease and defective erythropoietin production in patients with celiac disease. Haematologica 2008, 93, 1785-1791.

22. Peters, J.H.; Wierdsma, N.J.; Teerlink, T.; van Leeuwen, P.A.; Mulder, C.J.; van Bodegraven, A.A. The citrulline generation test: Proposal for a new enterocyte function test. Aliment. Pharmacol. Ther. 2008, 27, 1300-1310.

23. Alwitry, A. Vitamin A deficiency in coeliac disease. Br. J. Ophthalmol. 2000, 84, 1079-1080.

24. Hallert, C.; Tobiasson, P.; Walan, A. Serum folate determinations in tracing adult coeliacs. Scand. J. Gastroenterol. 1981, 16, 263-267.

25. Stene-Larsen, G.; Mosvold, J.; Ly, B. Selective vitamin B12 malabsorption in adult coeliac disease. Report on three cases with associated autoimmune diseases. Scand. J. Gastroenterol. 1988, 23, 1105-1108.

26. Kemppainen, T.; Uusitupa, M.; Janatuinen, E.; Jarvinen, R.; Julkunen, R.; Pikkarainen, P. Intakes of nutrients and nutritional status in coeliac patients. Scand. J. Gastroenterol. 1995, 30, $575-579$.

27. Kemppainen, T.A.; Kosma, V.M.; Janatuinen, E.K.; Julkunen, R.J.; Pikkarainen, P.H.; Uusitupa, M.I. Nutritional status of newly diagnosed celiac disease patients before and after the institution of a celiac disease diet-Association with the grade of mucosal villous atrophy. Am. J. Clin. Nutr. 1998, 67, 482-487.

28. Dickey, W. Low serum vitamin B12 is common in coeliac disease and is not due to autoimmune gastritis. Eur. J. Gastroenterol. Hepatol. 2002, 14, 425-427.

29. Hozyasz, K.K.; Chelchowska, M.; Laskowska-Klita, T. Vitamin E levels in patients with celiac disease. Med. Wieku Rozwoj 2003, 7, 593-604.

30. Harper, J.W.; Holleran, S.F.; Ramakrishnan, R.; Bhagat, G.; Green, P.H. Anemia in celiac disease is multifactorial in etiology. Am. J. Hematol. 2007, 82, 996-1000. 
31. Lerner, A.; Shapira, Y.; Agmon-Levin, N.; Pacht, A.; Ben-Ami Shor, D.; Lopez, H.; Sanchez-Castanon, M.; Shoenfeld, Y. The clinical significance of $25 \mathrm{OH}$-vitamin D status in celiac disease. Clin. Rev. Allergy Immunol. 2013, 42, 322-330.

32. Van Overbeek, F.M.; Uil-Dieterman, I.G.A.; Mol, I.W.; Kohler-Brands, L.; Heymans, H.S.A.; Mulder, C.J.J. The daily gluten intake in relatives of patients with coeliac disease compared with that of the general Dutch population. Eur. J. Gastroenterol. Hepatol. 1997, 9, 1097-1099.

33. Marsh, M.N. Gluten, major histocompatibility complex and the small intestine. A molecular and immunobiologic approach to the spectrum of gluten sensitivity ("celiac sprue"). Gastroenterology 1992, 102, 330-354.

34. Rostami, K. From microenteropathy to villous atrophy: What is treatable? Dig. Liver Dis. 2003, $35,758-759$.

35. Rostami, K.; Kerckhaert, J.; Tiemessen, R.; von Blomberg, B.M.; Meijer, J.W.; Mulder, C.J. Sensitivity of antiendomysium and antigliadin antibodies in untreated celiac disease: Disappointing in clinical practice. Am. J. Gastroenterol. 1999, 94, 888-894.

36. Hopper, A.D.; Hadjivassiliou, M.; Hurlstone, D.P.; Lobo, A.J.; McAlindon, M.E.; Egner, W.; Wild, G.; Sanders, D.S. What is the role of serologic testing in celiac disease? A prospective, biopsy-confirmed study with economic analysis. Clin. Gastroenterol. Hepatol. 2008, 6, 314-320.

37. Wahab, P.J.; Meijer, J.W.; Mulder, C.J. Histologic follow-up of people with celiac disease on a gluten-free diet: Slow and incomplete recovery. Am. J. Clin. Pathol. 2002, 118, 459-463.

38. Al-Toma, A.; Goerres, M.S.; Meijer, J.W.; Pena, A.S.; Crusius, J.B.; Mulder, C.J. Human leukocyte antigen-DQ2 homozygosity and the development of refractory celiac disease and enteropathy-associated T-cell lymphoma. Clin. Gastroenterol. Hepatol. 2006, 4, 315-319.

39. Wierdsma, N.J.; Peters, J.H.C.; van Bokhorst-de van der Schueren, M.A.E.; Mulder, C.J.J.; Metgod, I.; van Bodegraven, A.A. Bomb calorimetry, the gold standard for assessment of intestinal absorption capacity: Normative values in healthy ambulant adults. J. Hum. Nutr. Diet. 2013, doi: 10.1111/jhn.12113.

40. Suitor, C.W.; Bailey, L.B. Food folate vs. synthetic folic acid: A comparison. J. Am. Diet. Assoc. 1999, 99, 285.

41. Reinken, L.; Zieglauer, H. Vitamin B-6 absorption in children with acute celiac disease and in control subjects. J. Nutr. 1978, 108, 1562-1565.

42. Reinken, L.; Zieglauer, H.; Berger, H. Vitamin B6 nutriture of children with acute celiac disease, celiac disease in remission, and of children with normal duodenal mucosa. Am. J. Clin. Nutr. 1976, 29, 750-753.

43. Hadithi, M.; Al-Toma, A.; Oudejans, J.J.; van Bodegraven, A.A.; Mulder, C.J.J.; Jacobs, M. The value of double-balloon enteroscopy in patients with refractory celiac disease. Am. J. Gastroenterol. 2007, 102, 987-996.

44. Fedosov, S.N.; Fedosova, N.U.; Krautler, B.; Nexo, E.; Petersen, T.E. Mechanisms of discrimination between cobalamins and their natural analogues during their binding to the specific B12-transporting proteins. Biochemistry 2007, 46, 6446-6458.

45. Deressa, E.; Wammer, A.C.; Falch, J.A.; Jahnsen, J. Bone metabolism in patients with newly diagnosed caeliac disease. Tidsskr. Laegeforen. 2006, 126, 1201-1204. 
46. Tanumihardjo, S.A. Assessing vitamin A status: Past, present and future. J. Nutr. 2004, 134, 290S-293S.

47. Loche, F.; Bazex, J. Celiac disease associated with cuteanous sarcodosic granuloma. Rev. Med. Interne 1997, 18, 975-978.

48. Tasanen, K.; Raudasoja, R.; Kallioinen, M.; Ranki, A. Erythema elevatum diutinum in association with coeliac disease. Br. J. Dermatol. 1997, 136, 624-627.

49. Collin, P.; Reunala, T. Recognition and management of the cutaneous manifestations of celiac disease: A guide for dematologists. Am. J. Clin. Dermatol. 2003, 4, 13-20.

50. Addolorato, G.; Parente, A.; de Lorenzi, G.; D’angelo di Paola, M.E.; Abenavoli, L.; Leggio, G.; Capristo, E.; de Simone, C.; Rotoli, M.; Rapaccini, G.; et al. Rapid regression of psoriasis in a coeliac patient after gluten-free diet. A case report and review of the literature. Digestion 2003, 68, 9-12.

51. Prasad, A. Discovery of human zinc deficiency: Its impact on human health and disease. Adv. Nutr. 2013, 4, 176-190.

52. Nordstrom, F.; Lindholm, L.; Sandström, O.; Nordyke, K.; Ivarsson, A. Delay to celiac disease diagnosis and its implications for health-related quality of life. BMC Gastroenterol. 2011, 11, 118.

53. Blaser, M.J.; Falkow, S. What are the consequences of the disappearing human microbiota? Nat. Rev. Microbiol. 2009, 7, 887-894.

54. Zanini, B.; Caselani, F.; Magni, A.; Turini, D.; Ferraresi, A.; Lanzarotto, F.; Villanacci, V.; Carabellese, N.; Ricci, C.; Lanzini, A. Celiac disease with mild enteropathy is not mild disease. Clin. Gastroenterol. Hepatol. 2013, 11, 253-258.

55. Hallert, C.; Grant, C.; Grehn, S.; Granno, C.; Hulten, S.; Midhagen, G.; Strom, M.; Svensson, H.; Valdimarsson, T. Evidence of poor vitamin status in coeliac patients on a gluten-free diet for 10 years. Aliment. Pharmacol. Ther. 2002, 16, 1333-1339.

56. Snodgrass, S. Vitamin neurotoxicity. Mol. Neurobiol. 1992, 6, 41-73.

57. Gdynia, H.; Müller, T.; Sperfeld, A.; Kühnlein, P.; Otto, M.; Kassubek, J.; Ludolph, A.C. Severe sensorimotor neuropathy after intake of highest dosages of vitamin B6. Neuromuscul. Disord. 2008, 18, 156-158.

58. Sategna-Guidetti, C.; Grosso, S.B.; Grosso, S.; Mengozzi, G.; Aimo, G.; Zaccaria, T.; Di Stefano, M.; Isaia, G.C. The effects of 1-year gluten withdrawal on bone mass, bone metabolism and nutritional status in newly-diagnosed adult coeliac disease patients. Aliment. Pharmacol. Ther. 2000, 14, 35-43.

59. Hallert, C.; Svensson, M.; Tholstrup, J.; Hultberg, B. Clinical trial: B vitamins improve helath in patients with coeliac disease living on a gluten-free diet. Alliment. Pharmacol. Ther. 2009, 29, 811-816. 
Reprinted from Nutrients. Cite as: Ciacci, C.; Siniscalchi, M.; Bucci, C.; Zingone, F.; Morra, I.; Iovino, P. Life Events and the Onset of Celiac Disease from a Patient's Perspective. Nutrients 2013, 5, 3388-3398.

Concept Paper

\title{
Life Events and the Onset of Celiac Disease from a Patient's Perspective
}

\section{Carolina Ciacci *, Monica Siniscalchi, Cristina Bucci, Fabiana Zingone, Ivonne Morra and Paola Iovino}

Department of Medicine and Surgery, University of Salerno, via S.Allende 84081, Salerno 84081, Italy; E-Mails: monicasiniscalchi@aliceposta.it (M.S.); cristinabucci@hotmail.it (C.B.); z.fabiana@hotmail.it (F.Z.); ivonne.morra@gmail.com (I.M.); piovino@unisa.it (P.I.)

* Author to whom correspondence should be addressed; E-Mail: cciacci@unisa.it; Tel.: +39-0-8996-5032; Fax: +39-0-8996-9642.

Received: 7 June 2013; in revised form: 9 August 2013 / Accepted: 12 August 2013 /

Published: 28 August 2013

\begin{abstract}
Stressful events have been investigated in various immune-mediated diseases but not in celiac disease. Our aim was to examine the relationship of stressful events assessed by the standardized interview of Paykel with the diagnosis of celiac disease in comparison to patients, with a diagnosis of gastroesophageal reflux disease used as the control group. Adults with celiac disease $(n=186)$ reported more frequent and more severe life events in the years prior to the diagnosis than control patients $(n=96)(67.2 \%$ vs. 37.5\%, $p<0.001$, mean Paykel score 11.5 vs. 13.4, $p=0.001$, respectively). Findings were not significantly different between celiac disease and control patients for the time lapse between the event and the diagnosis (mean 5.5 vs. 5.7 months). Pregnancy was defined as a negative event by $20.3 \%$ of celiac women, but never by control women. Findings were confirmed when analyses were repeated in the subgroup of patients of both groups with diagnosis made within one year of onset of symptoms. Data indicate that, before diagnosis, the number of stressful events in celiac disease was more frequent although less severe than in the control group suggesting that life events may favor the clinical appearance of celiac disease or accelerate its diagnosis.
\end{abstract}

Keywords: celiac disease; life events; symptoms; onset of disease; stress; pregnancy 


\section{Introduction}

There has recently been an increasing awareness of the importance of psychosocial factors on the course of the celiac diseases (CD) [1,2]. Several studies investigated the association between life events and the development of chronic diseases [3], in particular gastrointestinal diseases such as inflammatory bowel disease [4-7] or in general other chronic diseases such as multiple sclerosis [8], rheumatoid arthritis [9], diabetes [10], skin diseases [11], mania [12], bipolar [13] and affective disorder [14]. Overall, there is an increasing evidence of the role of stressful life events in the onset of immune-mediated diseases [6,15], although the reviews point out the weakness of the majority of the published studies because of methodological issues (study design, controls, scale and timing) and small-sample size. Studies on the relationship between CD and stressful life events are, to our best knowledge, not available. Theoretically, a life event could relate to the onset of CD through at least two mechanisms. Firstly, a stressful life event pushes the person to seek medical consultation, because pre-existing symptoms become more important in their perception. Secondly, affecting the primary mechanism of the disease itself, for instance in the case of $\mathrm{CD}$, through an event inducing psychological modulation of symptoms in a genetically-determined immunological response to gluten. Methodologically standardized questionnaires are considered an adequate tool for investigation about life events when administered by specialized personnel because it is difficult to collect reliable and measurable information about life events and to assess their impact on disease [11]. Conversely, the notable limitation of those studies that explored the role of stressful life events in the onset of immune-mediated diseases, is that the patient may contribute to the misinterpretation of events due to several factors such as the misperception of reality and the altered individual sensitivity that could be features of premorbid personality and/or psychic disturbances. Poor social network and unemployment are additional important factors of vulnerability. Moreover, the impact of life events may be retrospectively the object of interpretation as the patient tries to find an explanation for his/her illness. However, the use of a preformed list of events, of adequate control subjects and, more importantly, limiting the time lapse from the event and the onset of symptoms as much as possible to reduce the recall bias, should guarantee the reliability of the findings.

To explore our hypothesis that there is a possible relationship between life events and the onset of $\mathrm{CD}$, we examined the prevalence of life events prior to the diagnosis in adult $\mathrm{CD}$ patients compared to patients with another chronic gastroenterological disease.

\section{Experimental Section}

\subsection{Patients}

\subsubsection{Group}

Patients with newly diagnosed CD and adult age ( $>18$ years) were consecutively recruited in the study at the Gastroenterology Unit, University of Naples "Federico II", which is a tertiary center for food intolerance and celiac disease. CD was diagnosed based on anti-human tissue transglutaminase and antiendomysium positivity in presence of normal total IgA level and on a positive gluten-related damage at well-performed intestinal biopsies [16,17]. Exclusion criteria were as follows: 
asymptomatic $\mathrm{CD}$ diagnosed in first-degree relatives of a celiac patient; older age ( $>75$ years); signs/symptoms suggesting the onset of $\mathrm{CD}$ in pediatric age (history of recurrent gastrointestinal symptoms, reported failure to thrive and/or presence of dental enamel defects or dental hypoplasia); reported use of cannabis or other drugs; alcohol abuse; previous diagnoses of brain disorders (e.g., epilepsy, atassia, etc.); previous diagnoses of depression or the actual existence of a depressed mood or of depression as assessed by the modified Zung Depression Rating [18,19].

\subsubsection{Control Group}

Among gastrointestinal diseases, gastroesophageal reflux disease (GERD) was chosen as the control disease for its similarities to CD in at least two aspects: both of them are chronic diseases and symptoms are subtle in early stages and become progressively more evident over the time up to a climax when often upper endoscopy is performed to obtain a definitive diagnosis [20]. Conversely, GERD is independent from immunological disorders. In fact, the immune-mediated aspects in GERD are usually absent or of minor importance than in CD. Thus, patients with GERD symptoms undergoing upper endoscopy at the Federico II Gastrointestinal Unit that revealed non erosive esophageal disease (NERD) or esophagitis (Los Angeles grade A) were enrolled in this study as a control group. During upper endoscopy, GERD patients underwent jejunal biopsy in order to exclude $\mathrm{CD}$ or other causes of malabsorption. Inclusion criteria for GERD were age between 18 to 75 years and negative serum antitransglutaminase antibodies and no IgA deficiency. Exclusion criteria were similar to those for CD patients including signs/symptoms suggesting the onset of GERD in pediatric age; reported use of cannabis or other drugs; alcohol abuse; previous diagnoses of brain disorders (e.g., epilepsy, atassia, etc.); previous diagnoses of depression or the actual existence of a depressed mood or of depression as assessed by the modified Zung Depression Rating [18,19].

All patients gave their written informed consent and the study protocol was approved by the Ethic Committee (Diagnosis and Follow-up of Celiac Disease protocol).

\subsection{Assessment of Symptoms}

A previously published questionnaire was used to routinely assess the presence of gastrointestinal symptoms such as abdominal pain, diarrhea, constipation, dyspepsia-like symptoms, GERD symptoms and weight loss $[21,22]$.

\subsection{Assessment of Life Events}

Information about incident events in the last year prior to the diagnosis were collected with the use of "The Interview for Recent Life Events" of Paykel [23-26] administered by a specialized psychologist (MS) who was unaware of the final diagnosis. The interview categorizes life events of moderate to severe degree in 10 groups as follows: employment, education, financial status, somatic health, loss (death of close relatives), living place, relationship, criminality, family and social problems, marital problems. In the Paykel scale, there are several possibilities to point to a personal disease (admission to the hospital, severe disease, minor disease, pregnancy, abortion, childbirth) although the interview does not allow for specification of the diagnosis or pathological pregnancy. When an event was reported, information was collected also about the date of the event. In the case 
of multiple reported events, analyses on the date and the quality of the event were based on the event defined as "dominant" by the participant. All events, dependent or independent from illness were recorded. The interview requires approximately 25-70 min, depending on the number of events, the difficulties in obtaining information from subjects and the complexity of the reported event. The Paykel's interview includes the pregnancy as a possible stressful event. Thus, data were collected also on previous pregnancies. The Paykel's interview also allows an assessment of the level of independence of the event in a 5-point scale in relation to the illness under consideration and the negative objective impact, and a subjective judgement of the expected stressfulness of the event for an average person, also in a 5-point rating scale. Moreover, the Paykel's interview attributes an objective normative value for each event (the higher the score, the more severe is an event).

\subsection{Statistical Analysis}

ANOVA were used for analyses on continuous data and reported as means \pm standard deviation (SD). Differences in frequencies between groups were calculated with the $\chi^{2}$ square test. Odds ratios (OR) \pm $95 \%$ confidence interval (CI) were used to analyze the risk of having life events in CD compared to the control group. The SPSS software package for Windows [27] was used for statistical analysis.

\section{Results}

Two-hundred and twenty-nine patients newly diagnosed adult patients with celiac disease were eligible. A total of 186 (mean age \pm SD: $37.6 \pm 12.5$ years) fulfilling the inclusion criteria were enrolled in the study, and 43 patients were not included because of exclusion criteria. The majority of CD patients were feminine gender $(n=152,81.7 \%)$, with age $<40$ years $(n=117,63 \%)$. Ninety-six GERD patients were selected as the control group. They were similar for gender (women $n=70$, $72.9 \%, \chi^{2} 1.842, p=0.2$ ) and age (mean age \pm SD $36.2 \pm 11.8$ years, $p=0.4$ ) distribution to the $\mathrm{CD}$ group. As for the BMI, as expected, it was higher in GERD than in CD patients (mean BMI \pm SD: $24.7 \pm 3.1$ in GERD patients and $22.3 \pm 3.8$ in CD patients, $p=0.000$ ), but none of them showed a BMI greater than 30 . None of them refused to answer the questionnaires.

$\mathrm{CD}$ and control group did not differ in the length of the period with symptoms prior to diagnosis $(4.87 \pm 5.8 v s .5 .56 \pm 3.0$ years, $p=0.4)$. Gastrointestinal symptoms (diarrhea and/or abdominal pain) were present in $43 \%$ of $\mathrm{CD}$ and $22 \%$ of control group patients $\left(\chi^{2} 11.402, p<0.001\right)$. Dyspepsia was present in $41 \%$ of CD and $62 \%$ of control group patients $\left(\chi^{2} 9.956, p=0.002\right)$. Weight loss was present in $24 \%$ of $\mathrm{CD}$ and $12 \%$ of control group patients $\left(\chi^{2} 5.677, p=0.017\right)$. GERD symptoms were present in $46 \%$ of CD and $100 \%$ of Control group patients $\left(\chi^{2} 77.640, p<0.001\right)$.

CD patients were statistically more likely to have a life event prior to the diagnosis (OR $3.495 \%$ CI 1.766-6.606) compared to patients in the GERD group although the severity of the event assessed by the Paykel's interview was significantly higher in control group patients than CD (Table 1). In the comparison between the two diseases, differences were not significant in the time lapse between the date of the event and the date of the diagnosis. Due to the higher prevalence of women in both groups a gender-analysis was performed. A significantly higher prevalence of events was observed in women in the CD group than in the control group $\left(71.1 \%\right.$ vs. $\left.34.3 \%, \chi^{2} 16.727, p<0.001\right)$, while men did not differ in the frequency of events between groups $\left(50.0 \% v s .46 .2 \%, \chi^{2} 0.056, p=0.8\right)$. 
Pregnancies were reported in $79 \mathrm{CD}$ and in 22 control group women. Number of pregnancies/women was $1.03 \pm 1.13$ for celiac women and $1.00 \pm 0.9$ for disease control women $(p=0.9)$. Additional analyses were done to exclude that pregnancy could be a major determinant of the difference in the prevalence of events between $\mathrm{CD}$ and control disease. To address this possibility, the comparison between women with $\mathrm{CD}$ and women with control disease was repeated after exclusion of events related to pregnancy. However, a significant difference in the prevalence of events between the $\mathrm{CD}$ and the control group was confirmed in this additional analysis $(67.4 \% \mathrm{vs}$. $\left.34.3 \%, \chi^{2} 12.769, p<0.001\right)$. Furthermore, $20.3 \%$ of CD women who experienced a pregnancy indicated the pregnancy itself as a stressful event, while no women who experienced a pregnancy in the control disease group did $(p=0.02)$.

Table 1. Reported number of events according to the Paykel's interview, time lapse from the event to the diagnosis, and normative values (the standardized weight of each event in the scale) in coeliac disease (CD) and control gastroesophageal reflux disease (GERD) subjects.

\begin{tabular}{cccc}
\hline Variables & CD & GERD & $\boldsymbol{p}$ \\
\hline Number of patients & 186 & 96 & \\
\% with events & $67.2 \%$ & $37.5 \%$ & $<0.001 *$ \\
Normative value of event, Paykel score (means \pm SD) & $11.5 \pm 4.8$ & $13.4 \pm 4.3$ & $0.001 * *$ \\
Time lapse between event and diagnosis (months, means \pm SD) & $5.5 \pm 4.1$ & $5.7 \pm 6.2$ & $0.9 * *$ \\
\hline
\end{tabular}

$\mathrm{SD}=$ standard deviation; $*$ Chi square test, ** ANOVA.

Table 2 shows the distribution of life events by the 10 categories of the Paykel's interview. The two most common categories of events in CD patients were somatic health problems and loss (prevalence $>17 \%$ ). Distribution of events was similar in the Control group. CD patients reported most frequently gastrointestinal symptoms, and diagnosis of severe anemia, osteoporosis, and thyroiditis among somatic health problems.

Table 2. Distribution of life events among the 10 areas identified by the Paykel Scale in $\mathrm{CD}$ and control (GERD) subjects.

\begin{tabular}{|c|c|c|c|}
\hline Variables & CD $n(\%)$ & GERD $n(\%)$ & $p^{*}$ \\
\hline Number and percentage of patients reporting an event & $125(67.2)$ & $36(37.5)$ & $<0.001$ \\
\hline \multicolumn{4}{|c|}{ Type of Event } \\
\hline Employment & $12(9.6)$ & $2(5.6)$ & 0.6 \\
\hline Education & $14(11.2)$ & $0(0.0)$ & 0.08 \\
\hline Financial status & $7(5.6)$ & $6(16.7)$ & 0.07 \\
\hline Somatic health & $39(31.2)$ & $10(27.8)$ & 0.9 \\
\hline Loss & $22(17.6)$ & $6(16.7)$ & 0.9 \\
\hline Living place & $7(5.6)$ & $2(5.6)$ & 0.7 \\
\hline Sentimental life & $4(3.2)$ & $2(5.6)$ & 0.9 \\
\hline Criminality & $1(0.8)$ & $0(0.0)$ & 0.5 \\
\hline Family and society & $8(6.4)$ & $2(5.6)$ & 0.8 \\
\hline Matrimonial problems & $11(8.8)$ & $6(16.7)$ & 0.3 \\
\hline
\end{tabular}

$n=$ number; $*$ Chi square test. 
Since most of somatic health problems might be considered dependent from the illness under consideration, analyses were repeated with the exclusion of the patients reporting health-related events. CD patients reported more frequently events independent from the disease when compared to the control disease group (86/147 (58.5\%) vs. 26/86 (30.2\%), $\left.\chi^{2} 10.655, p=0.001\right)$.

Table 3 describes the patients' opinions about the possible relationship between the reported event and the disease development. Findings were not significantly different between the CD group and Control group $\left(\chi^{2} 1.132, p=0.9\right)$ and indicated that only a minority of the patients pointed out that the event could have a role in the development of the disease.

Table 3. Patients' opinion about the effect of the reported event on the development of the disease Scale in CD and control (GERD) subjects according to the Paykel scale.

\begin{tabular}{|c|c|c|}
\hline Variables & CD & GERD \\
\hline Number of patients reporting an event & 125 & 36 \\
\hline \multicolumn{3}{|c|}{ 5-Point Rate Scale } \\
\hline No effect & $52.8 \%$ & $55.6 \%$ \\
\hline Probably no effect & $20.8 \%$ & $11.1 \%$ \\
\hline Uncertain & $16.8 \%$ & $22.2 \%$ \\
\hline Probably some effect & $5.6 \%$ & $5.6 \%$ \\
\hline Sure effect & $4.0 \%$ & $5.6 \%$ \\
\hline
\end{tabular}

Celiac patients were interviewed extensively about the life events that occurred during the year prior to the onset of symptoms. Since symptoms typically precede diagnosis by months or even years in this condition, we aimed to explore specifically whether limiting the time lapse from the diagnosis and the onset of symptoms as much as possible changes the previous findings. Then, the analyses were repeated selecting subgroups of CD and GERD patients in whom symptoms begun not more than 12 months before diagnosis. In this subgroup, CD patients reported a higher prevalence of life events than GERD patients $\left(40 / 56,71.4 \%\right.$ vs. $\left.18 / 48,37.5 \%, \chi^{2} 12.062, p=0.01\right)$. This prevalence became even stronger with the exclusion of patients who experienced a pregnancy and consequently reported events $\left(\chi^{2} 8.181, p=0.006\right)$.

\section{Discussion}

The main finding of the present study is that $\mathrm{CD}$ patients reported more frequent stressful events in the years prior to diagnosis and this result is even more significant when the analysis is limited to CD patients with onset of symptoms in the year preceding the diagnosis. In fact, also in this subgroup during the 12 months prior to CD diagnosis there is a significant higher prevalence of life events in comparison to patients with GERD, even higher than that found in the whole CD group (71.4\% vs. 67.2\%).

Reflux disease had been chosen as a control group in this study for several symptomatological similarities with $\mathrm{CD}$, but for its known independence from immunological mechanisms. Moreover, it has already been demonstrated that in practice total stress scores are found to be highly correlated with number of events, since, as previously stated, most reported events are from a comparatively narrow midrange of scores [24].

Other indices related to the event - type and time before diagnosis - were similar between CD patients and GERD patients, although severity of the events, assessed by the normative values was 
higher in GERD patients than CD. Somatic health problems and death of a close relative were the most frequent events. CD patients complain of some other co-morbidities, such as thyroid diseases and diabetes, that could obscure the association between life events and the onset of disease. With the exclusion of patients reporting those health problems as "stressful events", the number of stressful events in celiac disease was still greater than that reported in the GERD patients. Among CD patients, the frequency of stressful events prior to diagnosis was significantly higher in women than men. Analyzing the type of events, we noticed that pregnancy was reported as a stressful event in $(20.5 \%)$ cases by celiac women but never by women in the control group. This could at least in part explain the higher percentage of stressful events reported by CD women. Pregnancy is a peculiar moment in the fertile life of an undiagnosed celiac woman [28] also because pregnancy may disclose the relative "subclinical" deficiency of iron or other nutrients by increasing the metabolic demand and also may favor the development of the so-called "celiac crisis" that is an acute phase of open malabsorption leading to gluten-related auto-antibodies search [29]. One cannot exclude, however, that during pregnancy a combination of somatic and psychological factors may equally contribute to the request for medical help by the celiac women who are not yet aware of their disease. In the present study, pregnancy was one the events which could be selected by the patient in the Paykel's interview. It is possible that celiac women could have perceived their pregnancy as a negative event more frequently than women with the control disease because of the metabolic imbalance associated with malabsorption. To address this possibility, the comparison between CD women and GERD group women was repeated after exclusion of events related to pregnancy and our results demonstrated that celiac women still remained more sensitive to psychosocial stressors.

As symptoms due to gluten intolerance may precede by years the diagnosis of celiac disease, a separate analysis of data from a subgroup of subjects in whom the diagnosis was made within one year from the onset of symptoms was planned and the results fully confirmed the above findings.

The interpretation of the results of this study is complex because the available evidence indicates that the relationship between life stress events and the course of a disease is likely dependent on various factors. Little is known about how these factors, individually or in combination, are related to any disease activity. The nature and strength of these inter-relationships have strong clinical implications not for a single disease but for understanding the pathogenesis and the course of several pathologies. Patients with a long-lasting, obscure, oscillating illness experience various stressful events, which affect life patterns and give rise to conflicts. So the patients might become entangled in a vicious circle where life events accelerate the appearance of a disease or its symptoms which, in turn, facilitates the occurrence of life events, as for instance the loss of work or the onset of economic problems. It is well known that CD patients often experience altered psychological behavior during their life [30-32]. Other, alternative possibilities are that an event could push a person to a medical consultation because pre-existing symptoms become more important in his/her perception or that the event affected the primary immunological mechanism of the disease. The first possibility however is not in accordance with our findings of this subgroup of celiac patients in whom diagnosis was made close to the onset of symptoms who reported a greater number of life events compared to controls.

Moreover, in the case of $C D$, we know that $C D$ may run undiagnosed for years and that subtle symptoms may be underestimated by patients until after treatment. In those cases the evaluation of 
the precise time of onset of symptoms and effect of stressful events on the diagnosis the might be hard to estimate.

Psychological stress has been repeatedly reported to increase disease activity in gastrointestinal diseases [33] and recent studies have confirmed that adverse life events, chronic stress, and depression increase the likelihood of relapse in patients with quiescent IBD seemingly through changes in hypothalamic-pituitary-adrenal axis function, alterations in bacterial-mucosal interactions, mucosal mast cells and mediators such as corticotrophin releasing factor [7,34]. These observations were not confirmed by a population-based studies focused on the role of life events in determining the onset of inflammatory bowel diseases [35,36].

There are several limitations in our study. First of all, the lack of sample size calculation that might lead to a misinterpretation of results. This factor could be less of a concern in the majority of results since significant differences were reported. Another point is that our sample group might not represent all CD patients since they were studied in a tertiary care center. Despite the possibility through the Paykel's scale to point out major and minor health problems, for the lack of information about the precise diagnosis we were unable to assess if those events were related to the pathogenesis of $\mathrm{CD}$, as suggested in previous studies [37,38]. Lastly, the recall bias as pointed out in the introduction, although we tried to reduce it limiting the time lapse from the onset of symptoms and the diagnosis to one year in all CD and GERD patients.

\section{Conclusions}

In conclusion, our study indicates that life-events are associated to some degree with recent diagnosis of celiac disease in adults. The number of the events and not their severity appear as the determinant factor. Our data indicate that stressful events preceding celiac disease diagnosis are particularly frequent among celiac women, including pregnancy, which is defined as a stressful event only by celiac women and not by control women with gastroesophageal reflux. Altogether, also the present data support the need for psychological support in celiac disease, particularly in women at the time of celiac disease diagnosis $[39,40]$.

\section{Acknowledgments}

This study was sponsored by Regione Campania educational grants. Monica Siniscalchi's work was supported by a grant from Regione Campania, Assessorato alla Sanità. The Authors are grateful to Steve Hart for revising the manuscript.

\section{Conflicts of Interest}

The authors declare no conflict of interest.

\section{References}

1. Addolorato, G.; Capristo, E.; Ghittoni, G.; Valeri, C.; Mascianà, R.; Ancona, C.; Gasbarrini, G. Anxiety but not depression decreases in coeliac patients after one-year gluten-free diet: A longitudinal study. Scand. J. Gastroenterol. 2001, 36, 502-506. 
2. Hallert, C.; Grännö, C.; Hultén, S.; Midhagen, G.; Ström, M.; Svensson, H.; Valdimarsson, T. Living with coeliac disease: Controlled study of the burden of illness. Scand. J. Gastroenterol. 2002, 37, 39-42.

3. Renzaho, A.M.; Houng, B.; Oldroyd, J.; Nicholson, J.M.; D’Esposito, F.; Oldenburg, B. Stressful life events and the onset of chronic diseases among australian adults: Findings from a longitudinal survey. Eur. J. Public Health 2013, in press.

4. Vidal, A.; Gomez-Gil, E.; Sans, M.; Portella, M.J.; Salamero, M.; Pique, J.M.; Panes, J. Life events and inflammatory bowel disease relapse: A prospective study of patients enrolled in remission. Am. J. Gastroenterol. 2006, 101, 775-781.

5. Wolters, F.L.; Russel, M.G.; Sijbrandij, J.; Schouten, L.J.; Odes, S.; Riis, L.; Munkholm, P.; Langholz, E.; Bodini, P.; O'Morain, C.; et al. Disease outcome of inflammatory bowel disease patients: General outline of a Europe-wide population-based 10-year clinical follow-up study. Scand. J. Gastroenterol. Suppl. 2006, 243, 46-54.

6. Maunder, R.G.; Levenstein, S. The role of stress in the development and clinical course of inflammatory bowel disease: Epidemiological evidence. Curr. Mol. Med. 2008, 8, 247-252.

7. Mawdsley, J.E.; Rampton, D.S. Psychological stress in ibd: New insights into pathogenic and therapeutic implications. Gut 2005, 54, 1481-1491.

8. Brown, R.F.; Tennant, C.C.; Sharrock, M.; Hodgkinson, S.; Dunn, S.M.; Pollard, J.D. Relationship between stress and relapse in multiple sclerosis: Part II. Direct and indirect relationships.

Mult. Scler. 2006, 12, 465-475.

9. Geenen, R.; van Middendorp, H.; Bijlsma, J.W. The impact of stressors on health status and hypothalamic-pituitary-adrenal axis and autonomic nervous system responsiveness in rheumatoid arthritis. Ann. NY. Acad. Sci. 2006, 1069, 77-97.

10. Beveridge, R.M.; Berg, C.A.; Wiebe, D.J.; Palmer, L.D. Mother and adolescent representations of illness ownership and stressful events surrounding diabetes. J. Pediatr. Psychol. 2006, 31, 818-827.

11. Picardi, A.; Abeni, D. Stressful life events and skin diseases: Disentangling evidence from myth. Psychother. Psychosom. 2001, 70, 118-136.

12. Kennedy, S.; Thompson, R.; Stancer, H.C.; Roy, A.; Persad, E. Life events precipitating mania. Br. J. Psychiatry 1983, 142, 398-403.

13. Hammen, C.; Gitlin, M. Stress reactivity in bipolar patients and its relation to prior history of disorder. Am. J. Psychiatry 1997, 154, 856-857.

14. Johnson, L.; Andersson-Lundman, G.; Aberg-Wistedt, A.; Mathe, A.A. Age of onset in affective disorder: Its correlation with hereditary and psychosocial factors. J. Affect. Disord. 2000, 59, 139-148.

15. Rampton, D.S. The influence of stress on the development and severity of immune-mediated diseases. J. Rheumatol. Suppl. 2011, 88, 43-47.

16. Iovino, P.; Pascariello, A.; Russo, I.; Galloro, G.; Pellegrini, L.; Ciacci, C. Difficult diagnosis of celiac disease: Diagnostic accuracy and utility of chromo-zoom endoscopy. Gastrointest. Endosc. 2013, 77, 233-240. 
17. Marsh, M.N. Gluten, major histocompatibility complex, and the small intestine. A molecular and immunobiologic approach to the spectrum of gluten sensitivity ("celiac sprue"). Gastroenterology 1992, 102, 330-354.

18. Ciacci, C.; Iavarone, A.; Mazzacca, G.; de Rosa, A. Depressive symptoms in adult coeliac disease. Scand. J. Gastroenterol. 1998, 33, 247-250.

19. Zung, W.W. From art to science. The diagnosis and treatment of depression. Arch. Gen. Psychiatry 1973, 29, 328-337.

20. Green, P.H. The many faces of celiac disease: Clinical presentation of celiac disease in the adult population. Gastroenterology 2005, 128, S74-S78.

21. Amato, G.; Limongelli, P.; Pascariello, A.; Rossetti, G.; del Genio, G.; del Genio, A.; Iovino, P. Association between persistent symptoms and long-term quality of life after laparoscopic total fundoplication. Am. J. Surg. 2008, 196, 582-586.

22. Santonicola, A.; Siniscalchi, M.; Capone, P.; Gallotta, S.; Ciacci, C.; Iovino, P. Prevalence of functional dyspepsia and its subgroups in patients with eating disorders. World J. Gastroenterol. 2012, 18, 4379-4385.

23. Paykel, E.S. Methodological aspects of life events research. J. Psychosom. Res. 1983, 27, 341-352.

24. Paykel, E.S. The interview for recent life events. Psychol. Med. 1997, 27, 301-310.

25. Paykel, E.S. The evolution of life events research in psychiatry. J. Affect. Disord. 2001, 62, 141-149.

26. Canestrari, R. Versione Italiana Della Scala di Paykel per Gli Eventi Stressanti. In Nuovi Metodi in Psicometria; Organizzazioni Speciali: Firenze, Italy, 1982.

27. SPSS 15.0 Command Syntax Reference 2006; SPSS Inc.: Chicago, IL, USA, 2006.

28. Santonicola, A.; Iovino, P.; Cappello, C.; Capone, P.; Andreozzi, P.; Ciacci, C. From menarche to menopause: The fertile life span of celiac women. Menopause 2011, 18, 1125-1130.

29. Smecuol, E.; Maurino, E.; Vazquez, H.; Pedreira, S.; Niveloni, S.; Mazure, R.; Boerr, L.; Bai, J.C. Gynaecological and obstetric disorders in coeliac disease: Frequent clinical onset during pregnancy or the puerperium. Eur. J. Gastroenterol. Hepatol. 1996, 8, 63-89.

30. Ciacci, C.; Iavarone, A.; Siniscalchi, M.; Romano, R.; de Rosa, A. Psychological dimensions of celiac disease: Toward an integrated approach. Dig. Dis. Sci. 2002, 47, 2082-2087.

31. De Rosa, A.; Troncone, A.; Vacca, M.; Ciacci, C. Characteristics and quality of illness behavior in celiac disease. Psychosomatics 2004, 45, 336-342.

32. Ciacci, C.; de Rosa, A.; de Michele, G.; Savino, G.; Squillante, A.; Iovino, P.; Sabbatini, F.; Mazzacca, G. Sexual behaviour in untreated and treated coeliac patients. Eur. J. Gastroenterol. Hepatol. 1998, 10, 649-651.

33. Stanghellini, V. Relationship between upper gastrointestinal symptoms and lifestyle, psychosocial factors and comorbidity in the general population: Results from the domestic/international gastroenterology surveillance study (digest). Scand. J. Gastroenterol. Suppl. 1999, 231, 29-37.

34. Mawdsley, J.E.; Rampton, D.S. The role of psychological stress in inflammatory bowel disease. Neuroimmunomodulation 2006, 13, 327-336. 
35. Fritze, J.; Schneider, B.; Maurer, K. Additive effects, but no synergistic interaction of stressful life-events and genetic loading in affective disorders. J. Neural Transm. 1996, 103, 1221-1229.

36. Lerebours, E.; Gower-Rousseau, C.; Merle, V.; Brazier, F.; Debeugny, S.; Marti, R.; Salomez, J.L.; Hellot, M.F.; Dupas, J.L.; Colombel, J.F.; et al. Stressful life events as a risk factor for inflammatory bowel disease onset: A population-based case-control study. Am. J. Gastroenterol. 2007, 102, 122-131.

37. Riddle, M.S.; Murray, J.A.; Porter, C.K. The incidence and risk of celiac disease in a healthy US adult population. Am. J. Gastroenterol. 2012, 107, 1248-1255.

38. Maple, J.T.; Pearson, R.K.; Murray, J.A.; Kelly, D.G.; Lara, L.F.; Fan, A.C. Silent celiac disease activated by pancreaticoduodenectomy. Dig. Dis. Sci. 2007, 52, 2140-2144.

39. Addolorato, G.; de Lorenzi, G.; Abenavoli, L.; Leggio, L.; Capristo, E.; Gasbarrini, G. Psychological support counselling improves gluten-free diet compliance in coeliac patients with affective disorders. Aliment. Pharmacol. Ther. 2004, 20, 777-782.

40. Siniscalchi, M.; Iovino, P.; Tortora, R.; Forestiero, S.; Somma, A.; Capuano, L.; Franzese, M.D.; Sabbatini, F.; Ciacci, C. Fatigue in adult coeliac disease. Aliment. Pharmacol. Ther. 2005, 22, 489-494. 
Reprinted from Nutrients. Cite as: Biagetti, C.; Naspi, G.; Catassi, C. Health-Related Quality of Life in Children with Celiac Disease: A Study Based on the Critical Incident Technique. Nutrients 2013, 5, 4476-4485.

Article

\title{
Health-Related Quality of Life in Children with Celiac Disease: A Study Based on the Critical Incident Technique
}

\section{Chiara Biagetti *, Giulia Naspi and Carlo Catassi}

Department of Pediatrics, Università Politecnica delle Marche, Via Corridoni 11, Ancona 60123, Italy; E-Mails: giulia.naspi@live.it (G.N.); catassi@tin.it (C.C.)

* Author to whom correspondence should be addressed; E-Mail: chiara.biagetti@libero.it; Tel.: +39-071-5962-370; Fax: +39-071-36281.

Received: 9 September 2013; in revised form: 1 November 2013 / Accepted: 6 November 2013 / Published: 12 November 2013

\begin{abstract}
Celiac Disease (CD) is a chronic autoimmune disease triggered by dietary gluten. Gluten avoidance, which is the only available treatment for $\mathrm{CD}$, could impact on quality of life of children with $\mathrm{CD}$. We present the results of a qualitative study on the emotional impact of gluten free diet (GFD) on the everyday life of children affected with CD. We investigated 76 celiac patients aged 2-18 years (average age: 9.5 years). By using the Critical Incident Technique (CIT), we defined emotions related to difficulties and awkward situations experienced by the patients. Written answers to open-ended questions from either children (older than 8 years) and parents (children younger than 8 years) were analyzed qualitatively. We found 80 dilemmas experienced in three different arenas (food situations at school, meals at home, meals outside) and characterized lived experiences of children with $\mathrm{CD}$ in everyday life (specific emotions, difficulties in relationships and in management of daily life). Children with $\mathrm{CD}$ experience strong emotions related to the GFD, permeating several aspects of everyday life. These dilemmas may be missed by a conventional, questionnaire-based approach to the psycho-social consequences of $\mathrm{CD}$ treatment.
\end{abstract}

Keywords: quality of life; celiac disease; gluten free diet; children; lived experiences; psycho-social aspects 


\section{Introduction}

Celiac Disease (CD) is a permanent autoimmune disorder triggered by dietary gluten in genetically predisposed individuals. It is a frequent condition affecting about $1 \%$ of the general population. Gluten is a protein complex found in cereals that are diffusely consumed in most countries, i.e., wheat, rye and barley. The only treatment that is currently available for CD is the lifelong avoidance of gluten from the diet (so called gluten-free diet $=$ GFD). This treatment is effective but limits the patients' food choice and influences the patient's lifestyle and quality of life (QOL) [1].

During the last 10 years the interest on how patient perceive the impact of chronic diseases and how the health state is modified by the therapeutic intervention is getting increasing attention in medical and health care settings. Health-Related QOL (HRQOL) is a multidimensional concept including physical, emotional, social and cognitive domains. What matters in HRQOL is the way patients feel about their functioning, not their functioning itself [2].

Few data are available about QOL of children on GFD. Dietary restrictions can be difficult to accept and follow, especially for screening-detected patients and during adolescence, a life period characterized by lower adherence to the dietary treatment [3]. Nevertheless recent studies suggest that adolescents with screening-detected CD have similar HRQOL as their peers without CD, both at diagnosis and after one year of treatment [4]. A study on the long-term health and QOL after mass-screening for childhood disease reported a similar QOL after 10 years follow-up between children on GFD and controls [5]. Early CD diagnosis seems to be associated with better physical health, lower CD-associated burden and fewer social problems [6]. Other studies used disease-specific questionnaire in order to elicit specific problems related to the GFD. In 2001 Kolsteren et al. reported that the QOL of children with CD was similar to that of an healthy age-matched population using the TACQOL-COE-DIET (Technisch Natuurkundig Onderzoek-Academisch Ziekenhuis Leiden Children's Quality Of Life Celiac Disease Gluten Free Diet) questionnaire [7]. Using the CDDUX (Celiac Disease Dutch Children) quality of life questionnaire, CD children had a lower QOL than the healthy reference group. The CDDUX tool is a "bottom-up" questionnaire developed using a focus group-based approach that allow to perceive the situation from the child point of view; this kind of questionnaire could have elicited more specific information about those aspects of life that are typically influenced by the disease $[8,9]$.

The variability of results yielded by different questionnaires and the insufficient data about QOL in children with CD need further investigations.

An alternative method of investigating the QOL is based on the Critical Incident Technique (CIT), that is a qualitative research tool (open-ended questions). Studies in adults with CD showed that negative lived experiences and feelings of adults with celiac disease occur frequently and have an impact on daily life. Patients" "dilemmas" are related to awkward situations or the need to cope difficulties in social relationships and management of daily life in order to adhere to GFD, as reported by Sverker and colleagues [10]. Given the interesting results reported in adults with CD by using the CIT approach, in the present study we aimed to investigate the impact of CD and the GFD on the HRQOL and the social and emotional world of children with $\mathrm{CD}$, using this qualitative method of research. 


\section{Experimental Section}

\subsection{Patients}

Eighty children (aged 2-18) with biopsy-proven CD on the GFD by at least one year were invited to participate in the study when seen at the Gastroenterology Outpatient Clinic (Department of Pediatrics, Università Politecnica delle Marche, Ancona, Italy) for follow up, between 2006 and 2009. To assess the compliance to the GFD, determination of CD serological markers was performed in the three months preceding the enrolment in this study. Children under 2 years and/or affected by type 1 diabetes (T1D) were excluded. Children with CD showing elevated levels of serological markers were included in the study. Parents were informed about the study's risks and benefits and agreed to participate through a written informed consent. The study protocol was approved by the Ethical Committee of the Università Politecnica delle Marche, Ancona, Italy.

\subsection{Instrument}

We used the Critical Incident Technique (CIT), a qualitative research instrument representing a practical and structured way of collecting and analyzing information about human activities and their significance to the involved individuals. This tool is useful to yield rich, contextualized data that reflect real-life experiences in a flexible way, to meet the specific situation at hand [11]. It requires collection of brief, written, factual reports of "critical" actions or experiences in response to explicit situations or problems in defined fields. In this study we defined a "critical incident" as a "perplexing or awkward situation perceived by a patient to cause disturbances in his/her everyday life" [10]. With this phenomenological approach, the goal of the study is to define the individuals' own world. The method is sensitive to minor problems that are however important for the individual ("dilemmas"). We collected dilemmas until redundancy appeared, in order to carry out a meaningful qualitative analysis as suggested by Sverker and co-workers [10].

We chose to collect data through written answer to the following open-ended question:

- Could you describe the last occasion in which you thought: "If I were not affected by CD" (children older than 8 years)

- In your opinion what are the major difficulties your child had to cope while following the GFD? (parents of children under 8 years)

A sheet reporting the questions was delivered to patients (children older than 8 years) or parents (children younger than 8 years). They were invited to write their answers. The interviews were administered at the end of the follow-up visit, in a quiet and empathetic environment. Participants were leaved alone during the test and the interviewer came back at the end of the test; at this time answers were read aloud and sometimes the interviewer asked follow-up questions, to help the youngest patients if their answers were not precise, not tying themselves to a specific incident, or to better understand the single situation or experience, or to know other experiences or dilemmas perceived by the patients, all related to $\mathrm{CD}$ and the GFD. All the answers to the follow-up questions were then written by the participants. 


\subsection{CIT Data Analysis}

To analyze the data obtained by the CIT we used the method previously described by Sverker et al. [10]. First the examiner read several times each written answer, in order to become familiar with the data. From each interview the examiner abstracted one or more dilemmas and listed them in different categories. Some informants described more than one critical incident and identified dilemmas were listed in three main categories. The next step was to find an appropriate label for each dilemma and category; then the examiner identified three arenas in which dilemmas were experienced. An initial classification was presented by the first author (C.B.) and then discussed and revised within the research group.

\section{Results}

\subsection{CIT Interviews}

Eighty children were initially screened. Three patients were excluded because of lack of parental consent and one patient was excluded because of co-morbidity (T1D), so seventy-six children agreed to participate and were included in the study. Only two patients had abnormal values of the serological CD markers. The demographic characteristics of the study group are described in Table 1. Twenty-one patients did not report difficulties related to the GFD, 11 out of 33 symptomatic patients (33\%), and 10 out of 43 asymptomatic patients $(23 \%)(p=0.3$, chi square test).

Table 1. Main characteristics of study group.

\begin{tabular}{|c|c|}
\hline & Patients with CD $(n=76)$ \\
\hline \multicolumn{2}{|l|}{ Age (years) } \\
\hline median $(95 \% \mathrm{CI})$ & $8.7(7.4-9.9)$ \\
\hline 25th-75th percentile & $6.1-12.8$ \\
\hline \multicolumn{2}{|l|}{ Age below 8 years } \\
\hline$[n(\%)]$ & $33(44 \%)$ \\
\hline \multicolumn{2}{|l|}{ Gender } \\
\hline Male $[n(\%)]$ & $18(23.7 \%)$ \\
\hline \multicolumn{2}{|l|}{ Associated disorders ${ }^{\text {a }}$} \\
\hline$[n(\%)]$ & $16(21.1 \%)$ \\
\hline \multicolumn{2}{|l|}{ Age at CD diagnosis (years) } \\
\hline median $(95 \% \mathrm{CI})$ & $3.5(2.6-4.4)$ \\
\hline 25th-75th percentile & $1.9-7.0$ \\
\hline \multicolumn{2}{|l|}{ Duration of GFD (years) } \\
\hline median $(95 \% \mathrm{CI})$ & $3.9(3.2-4.5)$ \\
\hline 25 th -75 th percentile & $1.7-5.4$ \\
\hline Typical CD at diagnosis ${ }^{\mathrm{b}}[n(\%)]$ & $33(43.4 \%)$ \\
\hline No reported dilemmas related to GFD $[n(\%)]$ & $21(27.6 \%)$ \\
\hline
\end{tabular}


We collected a total of 80 dilemmas experienced by 55 patients, some patients reporting more than one dilemma. We identified three main categories of dilemmas (emotions, relationships and management of daily life) that patients experienced in three different arenas: (1) meals at school; (2) meals at home; (3) food situations and meals outside home (Figure 1).

We didn't find any significant difference on dilemmas distribution between symptomatic and asymptomatic patients. There were no noticeable differences in reported dilemmas between parents and children.

Figure 1. Lived experiences of dilemmas $(n)$ in everyday life among children with celiac disease.

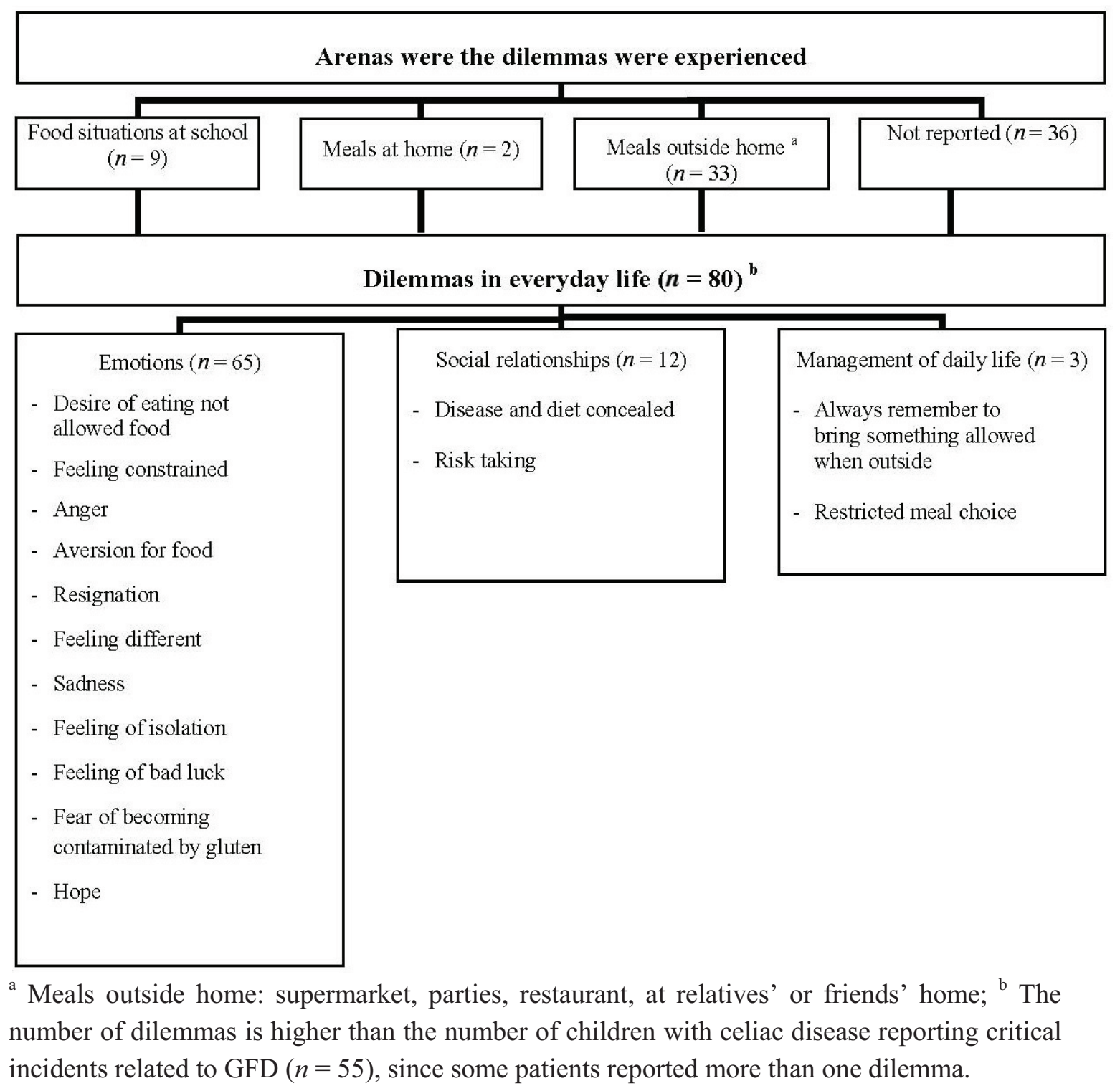

\subsection{Emotions}

The lived experiences of children with $\mathrm{CD}$ were characterized by specific emotions, as summarized in Figure 1. The most frequently reported emotion was the desire of eating gluten containing foods that were not allowed; sometimes it was just to try, but often the desire raised just because it was forbidden to share or taste the same food that the parents or friends were eating. 
Sometimes this emotion was caused by avoidance of food that children liked very much before starting the GFD.

"The most important difficulty my son meets is being not allowed to share the same "gluttonies" as their cousins do when we are outside home all together." (5 years)

What is interesting in children's reports is the kind of adjectives used to describe food: the gluten containing items are "great", "good", "tasty", while GFD is reported as "different", "often crumbled", "smelly", "cold".

Gluten avoidance was associated with specific moods, e.g., sense of restriction and constraint, sadness, anger, sacrifice and resignation:

"My son doesn't accept the imposition of "his food." (7 years)

"When I can't eat what my brother or my friends are eating at school parties, I get angry because what I can do is just see them eating their snacks or meals, but I can't share. I don't show my anger to my friends, I don't want others know what I feel." (8 years)

"Every time I see other children eating I get angry and I eat the same foods they are eating, or I go to the supermarket and I buy all the things I'd like to eat, without thinking about gluten content." (13 years)

"Last Monday I went to the supermarket with my father and I had to give up a candy I liked very much." (8 years)

This diet restriction could lead to food refusal in youngers or conscious transgressions in adolescents. In particular adolescents sometimes chose to eat gluten containing food just to conceal their intolerance to their mates (see social relationship).

Children and adolescents reported to feel different when they could not do the same things in a peer group, since they had to eat something they brought or they had to "say no". Sometimes isolation was a disappointing adults' fault, because someone didn't know appropriate strategies to cope with a child on the GFD.

"I wished I wasn't celiac when I went for a walk with my friends last Saturday. They bought hot pizza, while I had to eat my cold and 'different' pizza, that I brought with me from home. If I were not a celiac I could eat the same pizza that my friends eat." (10 years)

"When we eat at school, I have to sit alone in a different table; sometimes I think I have a bad luck because I can't eat what my friends eat, while they can eat their food and also my food." (9 years)

Older children and adolescents were more frequently conscious of their intolerance and of risks related to gluten intake. They could experience fear of becoming contaminated by gluten:

"It happened twice that my friends at school brought a slice of pizza near to my mouth for joke, and I had a great fear of getting ill for this." (13 years)

An interesting aspect of children's world of lived experiences is a great hope for the future:

"My daughter is hopeful and she's waiting for new therapies." (7 years)

"My son doesn't accept his new diet and he hopes that when he will be older he will eat all kind of food." (7 years) 


\subsection{Relationships}

GFD strongly influences social relationships. Children, particularly adolescents, did not want to reveal their food intolerance to their mates. They did not want their diet be the subject of conversations, because they did not want to feel different when they were in a peer group. For this reason they sometimes decided to eat gluten containing food consciously taking risks, while others chose not to go out with friends for lunch or dinner, to avoid the problem:

"At school I don't eat 'my snack' that my mother puts in my backpack because I don't want my mates know about my gluten intolerance." (10 years)

"Two weeks ago on Saturday I was at dinner with my mates: everyone had a pizza and I also had a pizza, not to be forced to answer questions about my diet and not to feel different." (16 years)

\subsection{Management of Daily Life}

The GFD and the related need to avoid some food from the diet necessarily impact on the management of daily life. In particular adolescents, more than children, reported practical difficulties related to the adherence to the GFD. Some adolescent described restricted meal choice when dining at restaurant or at the supermarket. Schools were often organized to offer something to eat, much less frequently offered gluten-free snacks. This situation was awkward for some and sometimes led to giving up going out for dinner or find alternative strategies, like always remembering to bring some gluten free food with them.

"Last summer I was in a great bakery in Sicily; there were a lot of cakes, but I could only eat a little chocolate." (12 years)

"Last week at school I forgot my snack; all my mates bought snack at automatic machines, but I couldn't. I was really hungry!'” (12 years)

"It was difficult to learn what kind of food I can buy at the supermarket and it is also really difficult to find new food at the supermarket, just to have different choices." (12 years)

\section{Discussion}

Most studies focusing on the QOL of children with CD or adolescents on treatment with the GFD reported no differences between patients and healthy reference groups. This "optimistic" view is in contrast with follow-up studies suggesting that the adherence to the dietary treatment is often poor, particularly in adolescents, as a consequence of the heavy psycho-social burden imposed by the GFD on daily life. Poor adherence to the treatment is likely to be the cause of the incomplete recovery of the small intestinal mucosa that is frequently found at follow-on intestinal biopsies [12]. In turn, persistent intestinal damage may cause long-term CD complications, such as osteoporosis and intestinal lymphoma [1]. In this study only two patients had abnormal levels of serological CD markers. We think that our sample of children with CD was somewhat selected because these patients regularly attended our Gastroenterology Outpatient Clinic. Furthermore it should be highlighted that minimal transgression to the gluten-free diet do not necessarily lead to abnormal antibody levels. 
In our opinion questionnaires used to measure the QOL are not always the appropriate instrument to catch and characterize the psycho-social discomfort associated with the long-term dietary treatment of CD. By using a qualitative method of investigation (the CIT) we found that most children and adolescents (72\%) reported and clearly described their difficulties in following the GFD. This method highlighted the specific problems related to the GFD and their emotional and social impact on everyday life of children and adolescents with CD. Since the CIT focused on specific problems related to the GFD, a comparison with a control group was not possible in this study.

Interestingly many of our findings in $\mathrm{CD}$ children/adolescents are similar to those found in $\mathrm{CD}$ adults by Sverker et al. using the same method of investigation [10]. Our study could present a report bias, given that many questionnaires were answered by the parents and not directly by the children (when younger than 8). However we could not find any noticeable differences in reported dilemmas between parents and children. Children with CD reported to experience dilemmas in three different "arenas" (contexts): meals at school, meals at home, food situations and meals outside home. Unlike adults, travel and purchase were not mentioned by anyone of the participants. This is not an unexpected finding, given that these arenas are usually managed by the patients' parents.

In $C D$ children we identified the same three main categories of dilemmas as experienced by adults: emotions, relationships and management of daily life. Children with $\mathrm{CD}$ described more emotions than adults, with strong feelings like anger, sadness or sense of diversity that could negatively impact their quality of life. Also social relationships were deeply influenced by CD and the GFD, but interestingly children with CD did not feel forgotten or neglected, probably because parents provided all their food-consumption needs. Management of daily life can be difficult for children and even more difficult for adolescents affected with $\mathrm{CD}$, however we found only few dilemmas in this category. The influence of parents and the family environment played a primary role in children's lives, and probably reduced dilemmas and difficult situations that could become more important later in life.

\section{Conclusions}

Our data clearly show that a part of the difficulties that children experience in coping with the GFD are not related to the quality of gluten-free food itself, that has improved over the last years, but could be influenced by other environmental factors, such as poor awareness of CD treatment in the general population and catering staff, and low availability of gluten-free food in most restaurants and cafeterias. These problems could be easily overcome by targeted information campaigns and staff training.

Our study shows that having a chronic illness like CD and following a GFD can negatively impact the QOL of affected children, a dimension that is not easily measured by standardized psycho-social questionnaires. A qualitative analysis of the psycho-social dilemmas, as performed by the CIT, provides information on many aspects of the difficulties that children with CD have to cope with, because of a limited food choice. These psychological and social aspects should be taken into account in the management of children and adolescents affected with CD. 


\section{Acknowledgments}

Authors are grateful to the children and parents who participated in the study.

\section{Conflicts of Interest}

Chiara Biagetti and Giulia Naspi declare no conflict of interest. Carlo Catassi has served as consultant for Menarini Diagnostics and Schar.

\section{References}

1. Fasano, A.; Catassi, C. Clinical practice. Celiac disease. N. Engl. J. Med. 2012, 367, 2419-2426.

2. Gill, T.M.; Feinstein, A.R. A critical appraisal of the quality of quality-of-life measurements. J. Am. Med. Assoc. 1994, 272, 619-626.

3. Fabiani, E.; Taccari, L.M.; Ratsch, I.M.; di Giuseppe, S.; Coppa, G.V.; Catassi, C. Compliance with gluten-free diet in adolescents with screening-detected celiac disease: A 5-year follow-up study. J. Pediatr. 2000, 136, 841-843.

4. Nordyke, K.; Norstrom, F.; Lindholm, L.; Stenlund, H.; Rosen, A.; Ivarsson, A. Health-related quality of life in adolescents with screening-detected celiac disease, before and one year after diagnosis and initiation of gluten-free diet, a prospective nested case-referent study. BMC Public Health 2013, 13, doi:10.1186/1471-2458-13-142.

5. Van Koppen, E.J.; Schweizer, J.J.; Csizmadia, C.G.; Krom, Y.; Hylkema, H.B.; van Geel, A.M.; Koopman, H.M.; Verloove-Vanhorick, S.P.; Mearin, M.L. Long-term health and quality-of-life consequences of mass screening for childhood celiac disease: A 10-year follow-up study. Pediatrics 2009, 123, e582-e588.

6. Wagner, G.; Berger, G.; Sinnreich, U.; Grylli, V.; Schober, E.; Huber, W.D.; Karwautz, A. Quality of life in adolescents with treated coeliac disease: Influence of compliance and age at diagnosis. J. Pediatr. Gastroenterol. Nutr. 2008, 47, 555-561.

7. Kolsteren, M.M.; Koopman, H.M.; Schalekamp, G.; Mearin, M.L. Health-related quality of life in children with celiac disease. J. Pediatr. 2001, 138, 593-595.

8. Van Doorn, R.K.; Winkler, L.M.; Zwinderman, K.H.; Mearin, M.L.; Koopman, H.M. CDDUX: A disease-specific health-related quality-of-life questionnaire for children with celiac disease. J. Pediatr. Gastroenterol. Nutr. 2008, 47, 147-152.

9. Vrijmoet-Wiersma, C.M.; Kooloos, V.M.; Koopman, H.M.; Kolk, A.M.; van der Laan, I.; Grootenhuis, M.A.; Egeler, R.M. Health-related quality of life, cognitive functioning and behaviour problems in children with Langerhans cell histiocytosis. Pediatr. Blood Cancer 2009, $52,116-122$.

10. Sverker, A.; Hensing, G.; Hallert, C. "Controlled by food"-Lived experiences of coeliac disease. J. Hum. Nutr. Diet. 2005, 18, 171-180.

11. Flanagan, J.C. The critical incident technique. Psychol. Bull. 1954, 51, 327-358. 
12. Lanzini, A.; Lanzarotto, F.; Villanacci, V.; Mora, A.; Bertolazzi, S.; Turini, D.; Carella, G.; Malagoli, A.; Ferrante, G.; Cesana, B.M.; et al. Complete recovery of intestinal mucosa occurs very rarely in adult coeliac patients despite adherence to gluten-free diet. Aliment. Pharmacol. Ther. 2009, 29, 1299-1308. 
Reprinted from Nutrients. Cite as: Rajpoot, P.; Govind K. Makharia, G.K. Problems and Challenges to Adaptation of Gluten Free Diet by Indian Patients with Celiac Disease. Nutrients 2013, 5, 4869-4879.

Review

\title{
Problems and Challenges to Adaptation of Gluten Free Diet by Indian Patients with Celiac Disease
}

\section{Preeti Rajpoot and Govind K. Makharia *}

Departments of Gastroenterology and Human Nutrition, All India Institute of Medical Sciences, New Delhi 110029, India; E-Mail: prajpoot15@gmail.com

* Author to whom correspondence should be addressed;

E-Mail: govindmakharia@gmail.com or govindmakharia@aiims.ac.in;

Tel.: +91-11-2658-8091 or +91-11-2659-6546; Fax: +91-11-2658-8641 or +91-11-2658-8663.

Received: 11 October 2013; in revised form: 5 November 2013 / Accepted: 20 November 2013 / Published: 27 November 2013

\begin{abstract}
Celiac disease is emerging in India and has become a public health problem. Almost 6-8 million Indians are estimated to have celiac disease. While there is a large pool of patients with celiac disease in India, until now, only a fraction of them have been diagnosed. With increasing awareness about celiac disease amongst health care providers and the general population, a massive increase in the number of patients with celiac disease is expected now and in the subsequent decade in India. While the number of patients with celiac disease is increasing, the country's preparedness towards the emerging epidemic of this disease is minimal. There are a number of issues, which requires urgent attention. Some of the key issues include increased awareness amongst health care professionals and the general public about the disease and its management, team-based management of patients with celiac disease, proper counseling and supervision of patients, training of dietitians in the management of patients with celiac disease, industrial production of reliable and affordable gluten-free food, and food labeling for gluten contents.
\end{abstract}

Keywords: nutrition; India; wheat; barriers; compliance, counseling 


\section{Introduction}

Celiac disease $(\mathrm{CeD})$ is an immune-mediated enteropathy caused by exposure to gluten in genetically susceptible individuals $[1,2]$. Once thought to be a rare disease and believed to occur only in Western Europe, $\mathrm{CeD}$ is now a global disease and affects almost $0.6 \%-1 \%$ of the world's population [3,4]. After Europe, America (both North and South), and the Middle East, it is now emerging in the East, including many Asian countries [5-13]. Also, once thought to be a disease of children and therefore to be managed mainly by pediatricians; CeD is now known to affect all the age groups including the elderly [14]. While CeD is emerging, the level of the awareness it too low amongst health care professionals, even amongst those most closely involved such as general physicians, family physicians, internists, gastroenterologist and pathologists [7,9].

\section{Emergence of CeD in India}

An increase in number of patients with $\mathrm{CeD}$ has been observed from many centers in India including ours $[15,16]$. Furthermore, two community based prevalence studies have been reported, both from the Northern part of India. In the first report from Ludhiana (Punjab), a questionnaire based survey of 4347 school children (3-17 years), Sood et al. [17] reported prevalence of CeD to be 1 in 310. In another community-based study including 10,488 subjects, both children and adults, we reported the prevalence of CeD in the Northern part of India to be 1.04\% ( 1 in 96) and the prevalence of seropositivity (anti-tTG ab) to be $1.44 \%$ (1 in 69) [18]. Based on these two general population based studies, 5-8 millions of Indians are expected to have $\mathrm{CeD}$. Of such a large pool of patients, only a fraction has been diagnosed to have CeD. The results of these studies suggest that $\mathrm{CeD}$ is a much greater problem in India than has been previously thought [18].

At present, only those with the most typical manifestations of CeD come to clinical attention and are ultimately diagnosed to have CeD. Now screening programs within populations indicate that celiac disease is under-diagnosed and what we detect clinically represents only the tip of the iceberg. With an appreciation of the existence of an iceberg of CeD in any society, and with increased awareness of $\mathrm{CeD}$, patients with even milder symptoms are likely to be diagnosed in the coming years $[19,20]$. Currently, most celiac specific serology ELISA kits in India are imported from Europe. Their diagnostic cut-off values of antibody concentrations are based on Caucasian population data. With the difference and diversity in gluten ingestion, the cut-off values for a positive test in India may not be similar to those reported in the Caucasians.

\section{Evolution of Dietary Management of CeD}

Dietary management was a mainstay of treatment of $\mathrm{CeD}$ even in the early part of the 20th century [21]. During 1930s, clinical improvement was observed with several differing diets including an oyster diet suggested by Gee and the banana diet popularized by Haas [22]. Stools of such patients were quite greasy and worsening of their diarrhea after a carbohydrate diet led to another dietary approaches such as reduction or almost complete elimination of dietary fat or carbohydrates. A remarkable observation by a Dutch pediatrician, Willem Dicke [23], gave the birth to an idea from listening to one of his child patients' mothers. The mother of the patient told Willem 
Dicke that her child used to become better if he did not eat porridge. From a clinical observation of one child and through years of clinical questioning and dietary therapy, he concluded that wheat was the toxic agent leading to CeD [23,24]. Toward the end of World War II, the so-called "winter of starvation" when even bread was not available in Holland; children with CeD paradoxically improved even though they were consuming a starvation diet (almost devoid of wheat products). When bread was airdropped in Holland, deterioration was noticed in these children [23]. Such an observation further strengthened the idea that some of the ingredients of wheat were the toxic agents for CeD.

\section{What Is Gluten?}

The protein content of wheat varies between $8 \%$ and $17 \%$, depending on the genetic make-up and external factors associated with the crop. When wheat flour is washed with water, the insoluble protein fraction forms a viscoelastic protein mass, called gluten. Gluten, which comprises roughly $78 \%$ to $85 \%$ of the total wheat protein, is a very large complex mainly composed of polymeric (multiple polypeptide chains linked by disulphide bonds and monomeric (single-chain polypeptides) proteins. Gluten plays a key role in determining the unique-baking quality of wheat by conferring water absorption capacity, cohesively, viscosity and elasticity on dough [25-27].

Gluten is classified into two main fractions according to their solubility in aqueous alcohols: the fraction which is soluble in aqueous alcohol is gliadin and those insoluble are called glutenins. Both the fractions consist of numerous, closely related polypeptides that are rich in glutamine and proline amino acids. Gliadins are mainly monomeric proteins with molecular weights around 28,000-55,000 and they are further subclassified as $\alpha / \beta$-, $\gamma$ - and $\omega$-type according to differences in their primary structures. Glutenins consist of glutenin subunits of high $\left(M_{\mathrm{W}} 67,000-88,000\right)$ or low molecular weight $\left(M_{\mathrm{W}} 32,000-35,000\right)$ that are connected by intermolecular disulphide bonds. Non-covalent bonds such as hydrogen bonds, ionic bonds and hydrophobic bonds bind gliadins and glutenins, which provide structure and physical properties of the gluten. Glutenins confer elasticity, while gliadins mainly confer viscosity and extensibility to the gluten complex [25-27].

Gliadins and glutenins contain domains with numerous repetitive sequences rich in these amino acids [28]. The gliadins have high proline and glutamine content and humans inherently lack endopeptidases to cleave bonds between proline and glutamines. The incomplete digestion of gliadin by digestive tract enzymes leads to the generation of many polypeptides, which are immunogenic to patients genetically susceptible to $\mathrm{CeD}$.

\section{What Is a Gluten Free Diet (GFD)?}

The absence of gluten in natural and processed foods represents a key aspect of the GFD (gluten free diet). The current Codex Standard for gluten free foods was adopted by the Codex Alimentarius Commission of the World Health Organization (Geneva, Switzerland) and by the Food and Agricultural Organization (Rome, Italy) in 1976 and amended in 1983. In 2000, the Codex Alimentarius Commission of the World Health Organization and the Food and Agriculture Organization described gluten free foods with a gluten level not exceeding 20 ppm and consisting of, 
or made only from ingredients which do not contain any prolamines from wheat or any Triticum species, such as spelt, kamut or durum wheat, rye, barley, oats, or their crossbred varieties $[29,30]$.

Gluten intake varies from population to population and depends upon dietary practices. In a double blind, placebo controlled prospective study; Catassi et al. [31] demonstrated that an intake of as little as $50 \mathrm{mg}$ of gluten per day for 3 months was sufficient to cause a significant decrease in the intestinal mucosal mucosal villous height/crypt depth ratio. Furthermore, a daily intake of gluten of lower than $10 \mathrm{mg}$ is unlikely to produce significant histological abnormalities [32]. The GFD with the threshold at less than $20 \mathrm{ppm}$ of gluten ensures an intake of less than $50 \mathrm{mg} /$ day and provides a sufficient safety margin [33].

\section{Indian Dietary Habits and Gluten in the Indian Diet}

India is a country of diversity in terms of culture, language, living standards and dietary practices. Until a few decades ago, Indians used to prefer natural foods to refined foods. In general, Indian families cook most of their meals at home on a daily basis and eat freshly cooked warm food. Most Indians do not prefer frozen or packaged food and tend to prepare their food from fresh ingredients. Indians are also less likely to visit restaurants. The family and social bond is very strong and they like to have food together with family members. Most of the meals contain a combination of cereals, pulses, spices and vegetables. Those who can afford, also uses dairy products, fruits and non-vegetarian food.

The "Green Revolution" in 1970s and onwards, promoted record grain promoted and ensured self-sufficiency in cereal grains [34,35]. During the past two decades, a shift from traditional to modern technologies, globalization, industrialization, constant travels across the world, and a fast growing economy led to the use of processed and fast foods at least in the urbanized part of India. While on one hand, there is poverty and hunger causing under-nutrition and its related disorders; a substantial increase in the intake of fast food is on the other hand leading to over-nutrition related disorders such as obesity and diabetes [36-38]. Therefore, the effects of malabsorption secondary to $\mathrm{CeD}$ could be severe in those who already have poor nutritional status due tounderlying poverty and ignorance.

Gluten intake varies from population to population and depends upon dietary practices. Wheat is the staple cereal in the northern part of India and flat bread made from wheat flour is the one of the most important constituents of almost every meal. In the southern and northeastern part of India, rice is a staple cereal. A typical North Indian diet, where flat bread is the usual meal, contains about 25-30 $\mathrm{g}$ of gluten per day; whereas average gluten intake in the West varies from 10 to $20 \mathrm{~g} /$ day [39].

\section{Barriers in Maintaining a Strict GFD in India}

There are many barriers to maintenance of a GFD; some of them are universal and some of them are unique to Indian patients with $\mathrm{CeD}$. Patients with $\mathrm{CeD}$ are challenged with barriers in maintenance of a strict GFD because of factors such as inadequate information and education about the disease, food contamination, and inadequate/no food labeling on the packaged food items $[40,41]$. 
Because of viscoelastic properties, as discussed earlier, gluten is used extensively in the food industry. In fact, efforts have been made to increase the gluten content of the wheat and globally the gluten industry is very big. It may be surprising to know that gluten is present in daily use items such as lipsticks, postage stamps, beer, ice-creams, sweets, confectionary foods spreads and seasonings, soups and sauces, malted beverages and many more [42]. Due to the lack of gluten labeling on food items in India, it is difficult for anyone to know if a particular food product is gluten free or not $[43,44]$.

Contamination of food with gluten is another concern. The contamination of food with gluten can occur: during milling if the same mill is used without proper cleaning for grinding gluten-containing and gluten-free grains; at the grocery store, if the same spatula is used to pick gluten containing and gluten free grains/flours; at factories producing commercial food products if same production line and equipment are used for both gluten-containing and GF (gluten free) food products; during preparation of commercial food products where gluten is added as fillers, stabilizing agents or processing aids such as thickener in soups, canned vegetables and other processed foods; at home, if the same utensils are used for storing, cooking and handling (rolling pin, surface griddle, dusters and oil for frying) gluten-containing and GF cereal flours and products; and while eating out, if addition of thickeners fillers/binders had been used which may unintentionally contain gluten.

Successful management of CeD requires a team approach, including patient, family, physicians, and dietitian. After a diagnosis is made, all the patients should be referred to a dietician for nutritional assessment, diet education, meal planning, and assistance with the social and emotional adaptation to the GF lifestyle. A delay in referral, or no referral at all, increases the likelihood of the patient obtaining inaccurate information from the Internet, health food stores, alternative health practitioners, family, friends, and other sources, which may be outdated, inaccurate, and/or conflicting. This results in confusion, frustration, and insufficient knowledge regarding $\mathrm{CeD}$ and the GFD. The poorly informed patients might unnecessarily restrict certain foods, thus limiting the variety and nutritional quality of their diet [45].

Adherence to GFD is the most critical factor for remission of CeD. Adherence to GFD is complex and is influenced by knowledge, country or region of residence, availability of GF food, determination, and social support [46-49]. Furthermore, attitude and behaviors of significant others such as family, teachers and friends also affect the decision to comply with GFD. Compliance to GFD is best maintained when residing at home; and eating out with friends or at school are the most difficult places to comply with GFD [40]. Lack of background awareness about the CeD and its strict dietary restriction in the community creates a problem for the patient and the family of patients with $\mathrm{CeD}[1,40]$. Furthermore, it has been observed that girls face compliance issues after marriage. The compliance to GFD is better if CeD is diagnosed in their early part of life compared to those in whom the diagnosis is made later in life. Patients having minimal symptoms or those diagnosed on screening comply less well than those who have overt symptoms [50,51]. As the patient grows from childhood to adulthood and become asymptomatic, the compliance to GFD gets worse. Non-appearance of acute symptoms after inadvertent or deliberate ingestion of gluten might make the patient more confident and induce them to try gluten at other occasions also. Many patients in remission might try gluten to see what happens to them with gluten intake [46-50]. 
Because of the perception that $\mathrm{CeD}$ is uncommon in India and a low absolute number of patients with $\mathrm{CeD}$ in India, the need for making a GFD available has not been perceived. The diagnosis of CeD presently is limited to only at the secondary and tertiary care centers and often more so in the northern part of India. There is some small-scale production of GFD (mainly flour and biscuits) food in certain sectors in the northern part of India, which relies upon mixing of non-wheat and non-barley cereals. There is a possibility of contamination at various levels such as during harvesting, storage and packaging of grain bags by the farmers. In fact all of these should be taken into consideration before the grains are used for making gluten free flour. Whichever gluten free flour/products are available do not undergo rigorous quality check for their gluten contents. The production of GFD in India till now has been small-scale industry based only, and the varieties and choices of GFD are also extremely limited.

Availability of GF foods is a factor, which determines compliance to GFD. Furthermore, difficulty in obtaining GF food also interrupts compliance to GFD. Even in countries where CeD is common, 10\%-15\% patients report difficulty in getting a continuous supply of GF food [52]. Non-availability of GFD outside their home environment restricts their travel, occupation and profession.

GF food items are considerably more expensive than regular gluten-containing food [53]. A nutritionally-balanced GF market basket, based on foods typically consumed by the Scottish population, costs more than a standard market basket [54]. Therefore, patients with $\mathrm{CeD}$ have additional financial requirement for purchasing of GFD for their living.

\section{Measures to Break the Barriers to GFD}

\subsection{Patient Education and Awareness}

The management of $\mathrm{CeD}$ is unique and different from the treatment of other medical or surgical diseases. At present, life-long and complete avoidance of gluten from the diet is the most effective treatment of $\mathrm{CeD}$. While prescribing GFD is easy; the key to the success is the dietary counseling by a dietician and maintenance of compliance by the patient [44,46-48]. Like any other chronic disease management, education of the patients and their families about the disease and dietary restrictions is of immense importance. It is generally not possible to explain everything about the dietary restrictions in one visit from the nutrition specialist. The understanding and maintenance of GFD requires consistent supervision and guidance, which is best provided to the patients and families on multiple visits. At every visit to the hospital/clinic, the level of compliance to gluten avoidance should be checked and appropriate guidance should be provided.

\subsection{Training of Nutritionist in CeD Management and Counseling of Patients}

The dietary councilor should have sufficient knowledge about the GF food and food products. It is not only about prescribing GFD but it is essential to provide for the patient a specific well-balanced diet. The dietitian is the most qualified health care professional to provide nutrition therapy. Dietitians have extensive academic and practical experience including in-depth knowledge of 
nutrition, nutritional needs, nutrition composition and food preparation information and educational factors that affect food and nutrition behavior of people. They are also skilled to translate scientific information into laymen's terms and assist individuals in gaining knowledge, self-understanding, improved decision making, and behavioral changes. Although other health care professionals can disseminate nutrition advice, they do not have the training in nutrition sciences and food composition to be able to translate complex medical nutrition concepts and issues into attainable dietary changes $[42,44,46-48]$.

\subsection{Celiac Disease Support Groups}

Celiac disease support groups provides a platform for patients to discuss their problems amongst themselves and learn from each other, provides information about GF products and their availability, but also can act as an advocacy for gluten labeling and other issues to the Government and regulatory bodies [55-57]. Furthermore, it has been observed that the adherence to a GFD increases when individuals are members of a patient support group [55-59].

\subsection{Development of Reliable GFD at a Large Scale}

While the number of patients with $\mathrm{CeD}$ is small at present in India, the absolute number of patients is rising day after day. As awareness about the disease increase, there is a likelihood of an exponential increase in the number of patients with $\mathrm{CeD}$ and hence the demand of GFD is likely to increase in the near future. Therefore, there is need for large-scale industrial level production of reliable and affordable GF food, including choices of food products ranging from snacks, flour, sweets, ice-creams and ready-to-eat packets.

All GF food products should be tested for their quality before releasing for patient's use. In fact, there should be certified gluten check laboratories where food items may be checked for their gluten contents. This is an important step to ensure quality of food for its gluten content until the gluten labeling legislation is enforced.

These foods, which are available at present in India, are not labeled for their gluten content. As mentioned above, gluten is used extensively in the food industry. Furthermore, even a small amount of gluten can maintain the disease activity [31-33]. It is therefore essential that food products, which are available, should be labeled for gluten content.

\section{Combining Diabetes and Gluten Free Dietary Management Guidelines}

Eight to fifteen percent of patients with $\mathrm{CeD}$ have type I diabetes [58,59]. Planning of a dietary management for such patients is a challenge for endocrinologists and dietitians. Disclosure of dietary restrictions with a diagnosis of $\mathrm{CeD}$ in a patient with type I diabetes may be received as a shock to the patient and the family. The objectives of diet planning for such patients are to provide a balanced nutritive diet with restrictions posed by diabetes and CeD. A healthy eating plan for diabetes should always be individualized based on the patient's need and metabolic outcome goals (HbA1C, weight/height, lipid profile, blood pressure, etc.) [60]. It is therefore essential that rather than making two dietary plans for control of $\mathrm{CeD}$ and diabetes, the dietitian should make one unified dietary plan for both the co-existing conditions. 


\section{Conclusions}

$\mathrm{CeD}$ is a public health problem and 6-8 million Indians are estimated to have $\mathrm{CeD}$. While there is a large pool of patients with $\mathrm{CeD}$ in India, only a fraction of them are currently diagnosed. With increasing awareness about $\mathrm{CeD}$ amongst health care providers and the general population, a massive increase in the number of patients with $\mathrm{CeD}$ is expected in the present and subsequent decade in India. While the number of patients with $\mathrm{CeD}$ is increasing, the country's preparedness towards this disease management is extremely minimal. There are a number of issues, which require urgent attention. Some of the key issues include team-based management of patients with $\mathrm{CeD}$, proper counseling and supervision of patients, training of dietitians in the management of patients with $\mathrm{CeD}$, industrial production of reliable and affordable GF food, food labeling for gluten contents and increase in awareness amongst health care professionals and the general public about the disease and its management.

\section{Conflicts of Interest}

The authors declare no conflict of interest.

\section{References}

1. Fasano, A.; Catassi, C. Clinical practice. Celiac disease. N. Engl. J. Med. 2012, 367, 2419-2426.

2. Schuppan, D.; Junker, Y.; Barisani, D. Celiac disease: From pathogenesis to novel therapies. Gastroenterology 2009, 137, 1912-1933.

3. Rewers, M. Epidemiology of celiac disease: What are the prevalence, incidence, and progression of celiac disease? Gastroenterology 2005, 128, S47-S51.

4. Fasano, A.; Berti, I.; Gerarduzzi, T.; Not, T.; Colletti, R.B.; Drago, S.; Elitsur, Y.; Green, P.H.; Guandalini, S.; Hill, I.D.; et al. Prevalence of celiac disease in at-risk and not-at-risk groups in the United States: A large multicenter study. Arch. Intern. Med. 2003, 163, 286-292.

5. Cataldo, F.; Montalto, G. Celiac disease in the developing countries: A new and challenging public health problem. World J. Gastroenterol. 2007, 13, 2153-2159.

6. Khoshoo, V.; Bhan, M.K.; Jain, R.; Phillips, A.D.; Walker-Smith, J.A.; Unsworth, D.J.; Stintzing, G. Coeliac disease as cause of protracted diarrhoea in Indian children. Lancet 1988, 1, 126-127.

7. Sood, A.; Midha, V.; Sood, N.; Malhotra, V. Adult celiac disease in northern India. Indian J. Gastroenterol. 2003, 22, 124-126.

8. Makharia, G.K.; Baba, C.S.; Khadgawat, R.; Lal, S.; Tevatia, M.S.; Madan, K.; Dattagupta, S. Celiac disease: Variations of presentations in adults. Indian J. Gastroenterol. 2007, 26, 162-166.

9. Bhattacharya, M.; Kapoor, S.; Dubey, A.P. Celiac disease presentation in a tertiary referral centre in India: Current scenario. Indian J. Gastroenterol. 2013, 32, 98-102.

10. Wu, J.; Xia, B.; von Blomberg, B.M.; Zhao, C.; Yang, X.W.; Crusius, J.B.; Peña, A.S. Coeliac disease: Emerging in China? Gut 2010, 59, 418-419. 
11. Wang, X.Q.; Liu, W.; Xu, C.D.; Mei, H.; Gao, Y.; Peng, H.M.; Yuan, L.; Xu, J.J. Celiac disease in children with diarrhea in 4 cities in China. J. Pediatr. Gastroenterol. Nutr. 2011, 53, 368-370.

12. Watanabe, C.; Komoto, S.; Hokari, R.; Kurihara, C.; Okada, Y.; Hozumi, H.; Higashiyama, M.; Sakuraba, A.; Tomita, K.; Tsuzuki, Y.; et al. Prevalence of serum celiac antibody in patients with IBD in Japan. J. Gastroenterol. 2013, doi:10.1007/s00535-013-0838-6.

13. Arshad, H.; Ahmad, Z. Histologic findings in biopsies/resection specimens from the small intestine with special emphasis on celiac disease: Experience from a developing country in South

Asia. Ann. Diagn. Pathol. 2012, 16, 436-440.

14. Singh, P.; Shergill, S.; Makharia, G.K. Celiac disease in older adults. J. Gastrointest. Liver Dis. 2013, 22, 359-360.

15. Sood, A.; Midha, V.; Sood, N.; Kaushal, V.; Puri, H. Increasing incidence of celiac disease in India. Am. J. Gastroenterol. 2001, 96, 2804-2805.

16. Sharma, M.; Singh, P.; Agnihotri, A.; Das, P.; Mishra, A.; Verma, A.K.; Ahuja, A.; Sreenivas, V.; Khadgawat, R.; Gupta, S.D.; et al. Celiac disease: A disease with varied manifestations in adults and adolescents. J. Dig. Dis. 2013, 14, 518-525.

17. Sood, A.; Midha, V.; Sood, N.; Avasthi, G.; Sehgal, A. Prevalence of celiac disease among school children in Punjab, North India. J. Gastroenterol. Hepatol. 2006, 21, 1622-1625.

18. Makharia, G.K.; Verma, A.K.; Amarchand, R.; Bhatnagar, S.; Das, P.; Goswami, A.; Bhatia, V.; Ahuja, V.; Datta Gupta, S.; Anand, K. Prevalence of celiac disease in the northern part of India: A community based study. J. Gastroenterol. Hepatol. 2011, 26, 894-900.

19. Leffler, D.; Vanga, R.; Mukherjee, R. Mild enteropathy celiac disease: A wolf in sheep's clothing? Clin. Gastroenterol. Hepatol. 2013, 11, 259-261.

20. Zanini, B.; Caselani, F.; Magni, A.; Turini, D.; Ferraresi, A.; Lanzarotto, F.; Villanacci, V.; Carabellese, N.; Ricci, C.; Lanzini, A. Celiac disease with mild enteropathy is not mild disease. Clin. Gastroenterol. Hepatol. 2013, 11, 253-258.

21. Gee, S.J. On the celiac affection. St. Bartholomew's Hosp. Rep. 1888, 24, 17-20.

22. Haas, S.V. The value of the banana in the treatment of coeliac disease. Am. J. Dis. Child. 1924, 24, 421-423.

23. Yan, D.; Holt, P.R. Willem Dicke. Brilliant clinical observer and translational investigator. Discoverer of the toxic cause of celiac disease. Clin. Transl. Sci. 2009, 2, 446-448.

24. Dicke, W.K.; Weijers, H.A.; van de Kamer, J.H. Coeliac disease. II. The presence in wheat of a factor having a deleterious effect in cases of coeliac disease. Acta Paediatr. 1953, 42, 34-42.

25. Anjum, F.M.; Khan, M.R.; Din, A.; Saeed, M.; Pasha, I.; Arshad, M.U. Wheat gluten: High molecular weight glutenin subunits - Structure, genetics, and relation to dough elasticity. J. Food Sci. 2007, 72, R56-R63.

26. Wieser, H. Chemistry of gluten proteins. Food Microbiol. 2007, 24, 115-119.

27. Shewry, P.R.; Halford, N.G.; Belton, P.S.; Tatham, A.S. The structure and properties of gluten: An elastic protein from wheat grain. Philos. Trans. R. Soc. Lond. B 2002, 357, 133-142.

28. Kagnoff, M.F. Celiac disease: Pathogenesis of a model immunogenetic disease. J. Clin. Investig. 2007, 11, 41-49. 
29. Hischenhuber, C.; Crevel, R.; Jarry, B.; Mäki, M.; Moneret-Vautrin, D.A.; Romano, A.; Troncone, R.; Ward, R. Review article: Safe amounts of gluten for patients with wheat allergy or coeliac disease. Aliment. Pharmacol. Ther. 2006, 23, 559-575.

30. Kupper, C. Dietary guidelines and implementation for celiac disease. Gastroenterology 2005, 128, S121-S127.

31. Catassi, C.; Fabiani, E.; Iacono, G.; D’Agate, C.; Francavilla, R.; Biagi, F.; Volta, U.; Accomando, S.; Picarelli, A.; de Vitis, I.; et al. A prospective, double-blind, placebo-controlled trial to establish a safe gluten threshold for patients with celiac disease. Am. J. Clin. Nutr. 2007, $85,160-166$.

32. Lanzini, A.; Lanzarotto, F.; Villanacci, V.; Mora, A.; Bertolazzi, S.; Turini, D.; Carella, G.; Malagoli, A.; Ferrante, G.; Cesana, B.M.; et al. Complete recovery of intestinal mucosa occurs very rarely in adult coeliac patients despite adherence to gluten-free diet. Aliment. Pharmacol. Ther. 2009, 29, 1299-1308.

33. Akobeng, A.K.; Thomas, A.G. Systematic review: Tolerable amount of gluten for people with coeliac disease. Aliment. Pharmacol. Ther. 2008, 27, 1044-1052.

34. Singh, G.P. Dr B.C. Dasgupta Memorial Oration. Green revolution and after: The public health perspective. Indian J. Public Health 2006, 50, 138-146.

35. Science in India. Green revolution: Caring for a continent. Nature 1984, 308, 598-599.

36. Gera, T.; Khetarpaul, N. Food consumption pattern and nutrient intake of Indian obese males. Nutr. Health 2000, 14, 205-216.

37. Shobana, R.; Snehalatha, C.; Latha, E.; Vijay, V.; Ramachandran, A. Dietary profile of urban south Indians and its relations with glycaemic status. Diabetes Res. Clin. Pract. 1998, 42, 181-186.

38. Mohan, V. Why are Indians more prone to diabetes? J. Assoc. Physicians India 2004, 52, 468-474.

39. Price, S. Understanding the importance to health of a balanced diet. Nurs. Times 2005, 101, $30-31$.

40. Hall, N.J.; Rubin, G.; Charnock, A. Systematic review: Adherence to a gluten-free diet in adult patients with coeliac disease. Aliment. Pharmacol. Ther. 2009, 30, 315-330.

41. Haines, M.L.; Anderson, R.P.; Gibson, P.R. Systematic review: The evidence base for long-term management of coeliac disease. Aliment. Pharmacol. Ther. 2008, 28, 1042-1066.

42. García-Manzanares, A.; Lucendo, A.J. Nutritional and dietary aspects of celiac disease. Nutr. Clin. Pract. 2011, 26, 163-173.

43. Saturni, L.; Ferretti, G.; Bacchetti, T. The gluten-free diet: Safety and nutritional quality. Nutrients 2010, 2, 16-34.

44. See, J.; Murray, J.A. Gluten-free diet: The medical and nutrition management of celiac disease. Nutr. Clin. Pract. 2006, 21, 1-15.

45. Case, S. The gluten-free diet: How to provide effective education and resources. Gastroenterology 2005, 128, S128-S134.

46. Olsson, C.; Hörnell, A.; Ivarsson, A.; Sydner, Y.M. The everyday life of adolescent coeliacs: Issues of importance for compliance with the gluten-free diet. J. Hum. Nutr. Diet. 2008, 21, $359-367$. 
47. Nasr, I.; Leffler, D.A.; Ciclitira, P.J. Management of celiac disease. Gastrointest. Endosc. Clin. N. Am. 2012, 22, 695-704.

48. Niewinski, M.M. Advances in celiac disease and gluten-free diet. J. Am. Diet. Assoc. 2008, 108, 661-672.

49. Addolorato, G.; de Lorenzi, G.; Abenavoli, L.; Leggio, L.; Capristo, E.; Gasbarrini, G. Psychological support counselling improves gluten-free diet compliance in coeliac patients with affective disorders. Aliment. Pharmacol. Ther. 2004, 20, 777-782.

50. Fabiani, E.; Taccari, L.M.; Rätsch, I.M.; di Giuseppe, S.; Coppa, G.V.; Catassi, C. Compliance with gluten-free diet in adolescents with screening-detected celiac disease: A 5-year follow-up study. J. Pediatr. 2000, 136, 841-843.

51. Murray, J.; Watson, T.; Clearman, B.; Mitros, F. Effect of a gluten-free diet on gastrointestinal symptoms in celiac disease. Am. J. Clin. Nutr. 2004, 79, 669-673.

52. Zarkadas, M.; Cranney, A.; Case, S.; Molloy, M.; Switzer, C.; Graham, I.D.; Butzner, J.D.; Rashid, M.; Warren, R.E.; Burrows, V. The impact of a gluten-free diet on adults with coeliac disease: Results of a national survey. J. Hum. Nutr. Diet. 2006, 19, 41-49.

53. Lee, A.R.; Ng, D.L.; Zivin, J.; Green, P.H. Economic burden of a gluten-free diet. J. Hum. Nutr. Diet. 2007, 20, 423-430.

54. Stevens, L.; Rashid, M. Gluten-free and regular foods: A cost comparison. Can. J. Diet. Pract. Res. 2008, 69, 147-150.

55. Silvester, J.A.; Rashid, M. Long-term management of patients with celiac disease: Current practices of gastroenterologists in Canada. Can. J. Gastroenterol. 2010, 24, 499-509.

56. Leffler, D.A.; Edwards-George, J.; Dennis, M.; Schuppan, D.; Cook, F.; Franko, D.L.; Blom-Hoffman, J.; Kelly, C.P. Factors that influence adherence to a gluten-free diet in adults with celiac disease. Dig. Dis. Sci. 2008, 53, 1573-1581.

57. NIH Consensus Development Conference on Celiac Disease. NIH Consens State Sci Statements. Available online: http://consensus.nih.gov/2004/2004celiacdisease118html.htm (accessed on 21 November 2013).

58. Camarca, M.E.; Mozzillo, E.; Nugnes, R.; Zito, E.; Falco, M.; Fattorusso, V.; Mobilia, S.; Buono, P.; Valerio, G.; Troncone, R.; et al. Celiac disease in type 1 diabetes mellitus. Ital. J. Pediatr. 2012, 38, 10 .

59. Franzese, A.; Lombardi, F.; Valerio, G.; Spagnuolo, M.I. Update on coeliac disease and type 1 diabetes mellitus in childhood. J. Pediatr. Endocrinol. Metab. 2007, 20, 1257-1264.

60. Scaramuzza, A.E.; Mantegazza, C.; Bosetti, A.; Zuccotti, G.V. Type 1 diabetes and celiac disease: The effects of gluten free diet on metabolic control. World Diabetes 2013, 4, 130-134. 
Reprinted from Nutrients. Cite as: Ross, A.; Shelley, H.; Novell, K.; Ingham, E. Callan, J.; Heuschkel, R.; Morris, M.-A.; Zilbauer, M. Assessing Quality Outcome Measures in Children with Coeliac Disease-Experience from Two UK Centres. Nutrients 2013, 5, 4605-4613.

Article

\title{
Assessing Quality Outcome Measures in Children with Coeliac Disease-Experience from Two UK Centres
}

\author{
Alexander Ross ${ }^{1, \dagger}$, Helen Shelley ${ }^{1, \dagger}$, Kim Novell ${ }^{1}$, Elizabeth Ingham ${ }^{2}$, Julia Callan ${ }^{1}$,
} Robert Heuschkel ${ }^{1}$, Mary-Anne Morris ${ }^{2, \dagger}$ and Matthias Zilbauer ${ }^{1, *}$

1 Department of Paediatric Gastroenterology, Addenbrooke's Hospital, Cambridge University Hospitals, Cambridge, CB2 0QQ, UK; E-Mails: Alexander.Ross@addenbrookes.nhs.uk (A.R.); Helen.Shelley@addenbrookes.nhs.uk (H.S.); Kim.Novell@addenbrookes.nhs.uk (K.N.); Julia.Callan@addenbrookes.nhs.uk (J.C.); Robert.Heuschkel@addenbrookes.nhs.uk (R.H.)

2 Department of Paediatric Gastroenterology, Norfolk and Norwich University Hospital, Norwich, NR4 7UY, UK; E-Mails: Elizabeth.Ingham@nhs.net (E.I.);

Mary-Anne.Morris@nnuh.nhs.uk (M.-A.M.)

$\dagger$ These authors contributed equally to this work.

* Author to whom correspondence should be addressed; E-Mail: mz304@cam.ac.uk; Tel.: +44-(0)-1223-336889.

Received: 30 August 2013; in revised form: 2 November 2013 / Accepted: 8 November 2013 / Published: 19 November 2013

\begin{abstract}
Improved diagnosis of coeliac disease has increased incidence and therefore burden on the health care system. There are no quality outcome measures (QOM) in use nationally to assess hospital management of this condition. This study applied QOM devised by the East of England paediatric gastroenterology network to 99 patients reviewed at two tertiary hospitals in the Network, to assess the quality of care provided by nurse led and doctor led care models. The average performance across all QOM was 96.2\% at Addenbrooke's Hospital (AH), and 98.7\% at Norfolk and Norwich Hospital (NNUH), whilst 95\% $(n=18)$ of QOM were met. Patient satisfaction was high at both sites (uptake of questionnaire 53 of 99 patients in the study). The study showed a comparably high level of care delivered by both a nurse and doctor led service. Our quality assessment tools could be applied in the future by other centres to measure standards of care.
\end{abstract}


Keywords: quality outcome measures; coeliac disease; outpatient management; patient satisfaction

\section{Introduction}

Coeliac disease affects up to $0.5 \%-1 \%$ of all children in Europe and North America, with a large number of cases being undiagnosed [1-3]. With the implementation of simple widespread diagnostic screening tools, the incidence has increased rapidly in recent years, leading to an increasing burden on the healthcare system [3].

Overall, it is very well established that a lifelong gluten free diet results in symptom resolution and normalisation of tissue transglutaminase (tTG) in the majority of patients $[4,5]$, and remains the mainstay of treatment. Hence, patient care includes the involvement of primary, secondary and tertiary healthcare institutions as well as several health care professionals such as dieticians, nurses, and doctors. The ultimate aim is to optimise resources and expertise in order to provide the highest quality patient care.

In the East of England, patients are managed within the East of England Paediatric Gastroenterology Network (EEPGN), which operates two tertiary centres (Addenbrooke's hospital, Cambridge University Hospitals, and Norfolk and Norwich University Hospital) and fifteen secondary care sites. Approximately 100 patients with coeliac disease are reviewed annually at Addenbrooke's hospital (AH) and 100 at Norfolk and Norwich University Hospital (NNUH) in specialist "coeliac clinics" run on a monthly basis at each site. These clinics are set up to deliver a review of coeliac related symptoms and general health, assessment of growth and nutritional status, understanding, adherence and support to optimise compliance with the diet. Food diaries are completed prior to each clinic, with blood results taken prior to each clinic so that results are available during the consultation. Following diagnosis, patients are reviewed as outpatients at three, six, and twelve months, and annually thereafter. At NNUH annual review is conducted by Doctors and Dieticians, however at $\mathrm{AH}$ review is predominantly run by Dieticians and Specialist Nurses, with doctors available for consultation if required. Once patients reach the age of sixteen years, their care is transitioned on to the adult gastroenterology or primary care services.

Assessment of Quality Outcome Measures (QOM) is growingly important to assess efficiency and quality of service delivery, however there are currently no formally stated national QOM for paediatric coeliac disease. It has previously been shown that measures of care quality can be beneficial in both acute [6], and chronic [7] conditions in Paediatric care, and that quality of care correlates with patient satisfaction [8]. The main aim of our study was to use a set of QOM devised by the EEPGN, which were based upon national guidelines [5] to assess quality of care delivered by each hospital. The QOM were developed and approved by the EEPGN steering group comprising Paediatricians, Paediatric Gastroenterologists, Paediatric Specialist Nurses and Dietitians with representation from the two tertiary and five of the secondary care units. The QOM were designed to represent acceptable quality of care, be patient centered and be applicable to a range of service models across tertiary and secondary units. These QOM were then used to assess 
the quality of care and patient/parent satisfaction at the two main tertiary centres each offering a different service care model.

\section{Experimental Section}

All patients $(n=99)$ seen in the annual review clinic at both centres during a six month period $(01 / 11 / 10$ to $31 / 05 / 11)$ were included in the study. Three major areas of QOM were assessed; diagnosis and initial patient management, the annual clinical review process, and transition of care from paediatrics to adult medicine.

The "annual clinical review process" and "transition of care from paediatrics to adult medicine" data were collected using a proforma completed during the clinic session. The proforma was designed to reflect the 2009 NICE guidelines (CG86) for the management of coeliac disease [5]. The proforma consisted of thirteen questions regarding patient care, and was completed by the assessing clinicians during each appointment (Appendix 1). The Data relating to each patient's "diagnosis and initial management" was extracted retrospectively from patient documentation using an extraction tool based consisting of the six areas outlined for this subsection (Appendix 1).

"Patient satisfaction" was assessed using an anonymised questionnaire designed by paediatric gastroenterologists, dieticians, and nurse specialists within the East of England Paediatric Gastroenterology network, which was completed by parents of children attending an annual review clinic. Parents were allowed to complete each questionnaire in their own time after the clinic appointment had taken place, and handed in the data upon completion. There were eleven questions included in the questionnaire. Uptake of the questionnaire was 53 of the 99 (54\%) patients included in the study ( $\mathrm{AH} n=20$, NNUH $n=33$ ). Each assigned QOM had a predefined target of $90 \%$ concordance.

A table of each area assessed, and the outcome for each area can be found in Appendix 1.

The original data was collected as part of a clinical audit and therefore in accordance with Department of Health (UK), did not require formal ethical approval [9].

\section{Results}

\subsection{Quality Outcome Measures}

The initial focus of our investigation was to compare the quality outcome measures at each site to assess the quality of care delivered by each system, i.e., nurse and dietician (AH) versus doctor and dietician (NNUH) led care. The average performance across all QOMs was 96.2\% at AH, and $98.7 \%$ at NNUH.

For "diagnosis and initial management", six areas were assessed, with the average achievement in each standard as $96.3 \%$ at NNUH, and $92.3 \%$ at $\mathrm{AH}$. As demonstrated in Figure 1, AH met the $90 \%$ target set in each subsection, whilst NNUH achieved compliance in five of the six targets (Figure 1). The QOM target that was not achieved was "performance of biopsy within four weeks of positive serology result".

To assess the quality delivered at the "annual clinical review of patients" eleven areas were assessed. The average percentage of compliance across each standard was $98.3 \%$ at $\mathrm{AH}$, and $100 \%$ 
at NNUH. NNUH therefore met the 90\% target in each of the eleven QOMs, however AH did not achieve the target, "tTG less than twice upper limit of normal within two years of diagnosis" (Figure 1).

Efficient referral of children to adult services is essential as it preserves continuity of ongoing care and provides vital information to the adult team. To assess the quality of patient referral at AH and NNUH two QOMs were assessed, "discussion of referral", and "completed referral before sixteen years of age". There were two referral cases at AH, and five at NNUH. In 100\% of cases at $\mathrm{AH}$ and NNUH, referral was discussed during a clinic appointment. Of the five cases at NNUH, four were appropriately referred to adult services, however in one exceptional case at NNUH it was decided with the patient's carer that the child should remain under paediatric care for the next year due to multiple significant co-morbidities.

Figure 1. Comparison of the percentage of targets reached in each quality outcome measures (QOM) section between Addenbrooke's Hospital (AH) (solid bars), and Norfolk and Norwich Hospital (NNUH) (hashed bars), showing the majority to targets being reached in each section.

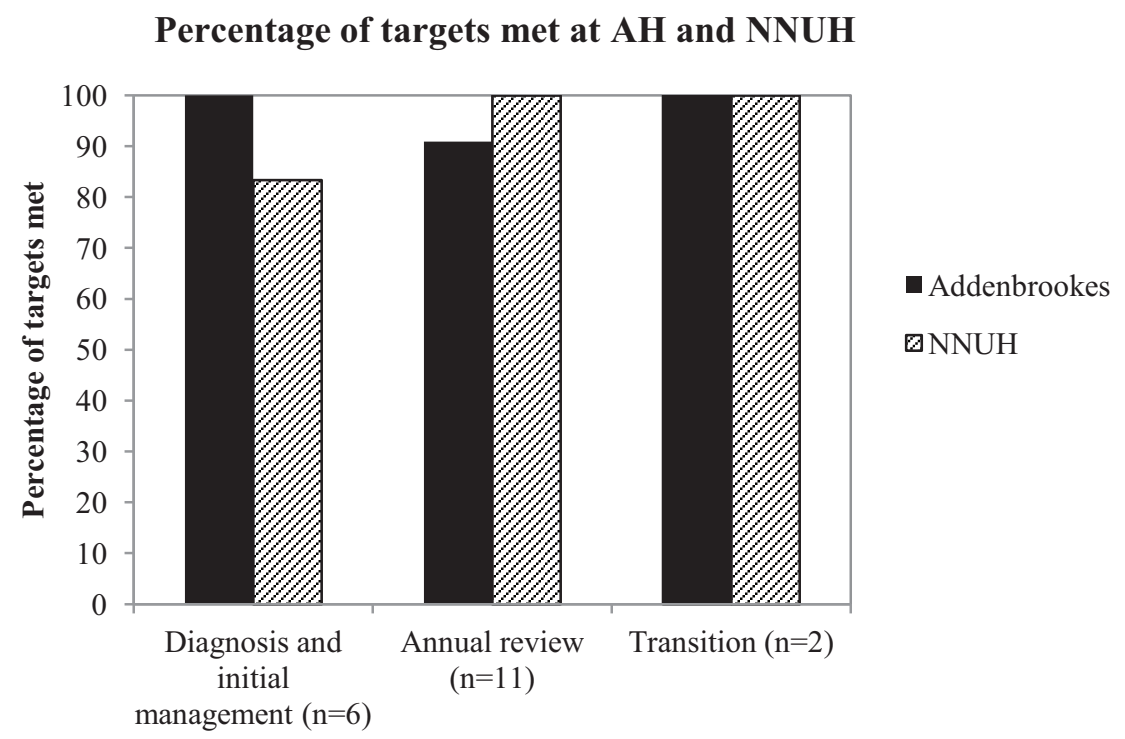

\subsection{Parent and Patient Satisfaction}

In addition to the EEPGN quality of care assessment, it is important that parents and patients perceive the healthcare service provided as being of a high standard. Therefore, a parent and patient satisfaction survey was used to assess the satisfaction at each site. The overall satisfaction ratings were comparably high at both sites, with $88 \%(n=29)$ of responses at NNUH and $75 \%(n=15)$ at AH rated as "very satisfied" (Figure 2). 3\% $(n=1)$ of responses at NNUH and $5 \%(n=1)$ at $\mathrm{AH}$ were rated as dissatisfied, and in all cases the cause listed was long waiting times when attending clinic.

The combined data for AH and NNUH showed that the best reviewed areas during consultation were patient diet $(98 \%, n=52)$, symptoms $(92 \%, n=49)$, and growth $(96 \%, n=51)$ (Figure 3$)$. The less well covered areas were updating patients about gluten free diet options, and availability 
of food samples. Interestingly, when questioned on which areas parents would like to be covered, the greatest response was for gluten free food samples to be provided during the appointment (61\%). The area that parents least wanted to discuss was review of diet $(23 \%)$.

Figure 2. Comparison of parent satisfaction levels at AH (solid bars) and NNUH (hashed bars). Satisfaction levels are mostly very high in both sites, with slightly higher levels noted in NNUH.

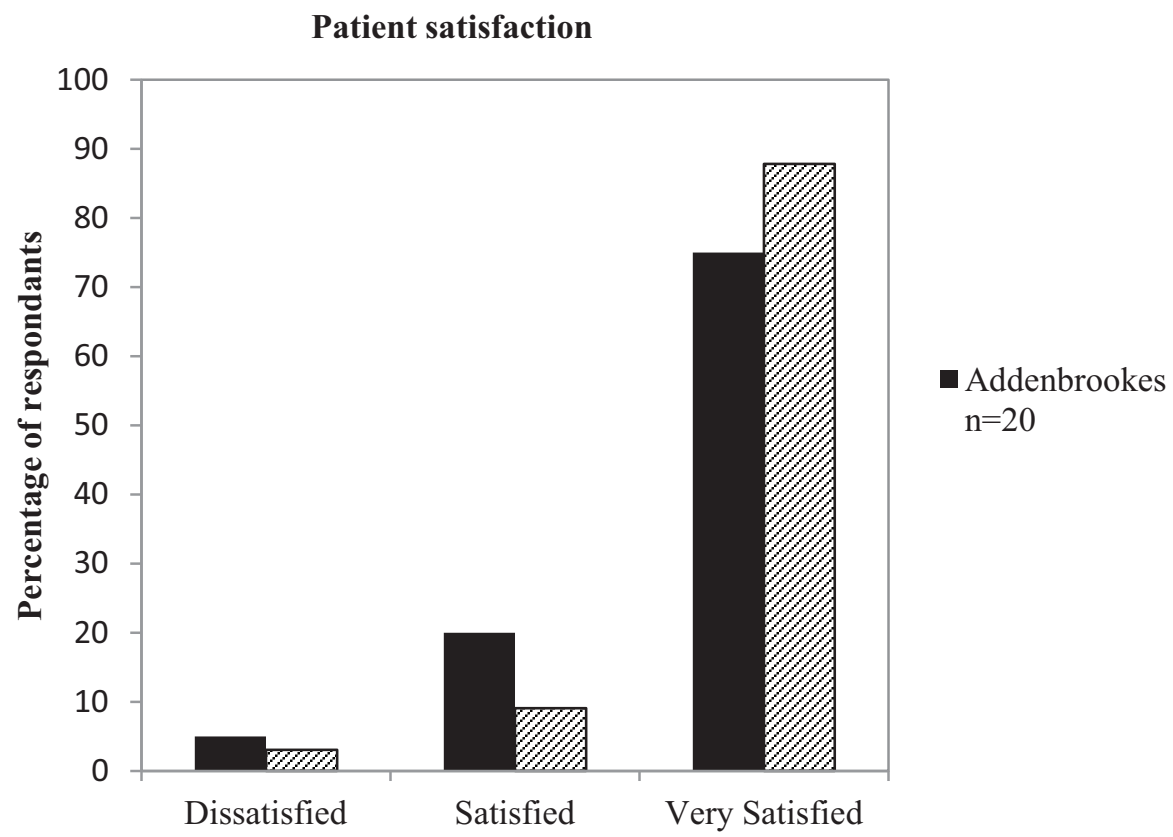

Figure 3. Bar graph displaying the percentage of topics patients would like to discuss (hashed bars) contrasted to the areas actually discussed during the appointment. Data contains answers from all patients both at $\mathrm{AH}$ and $\mathrm{NNUH}$.

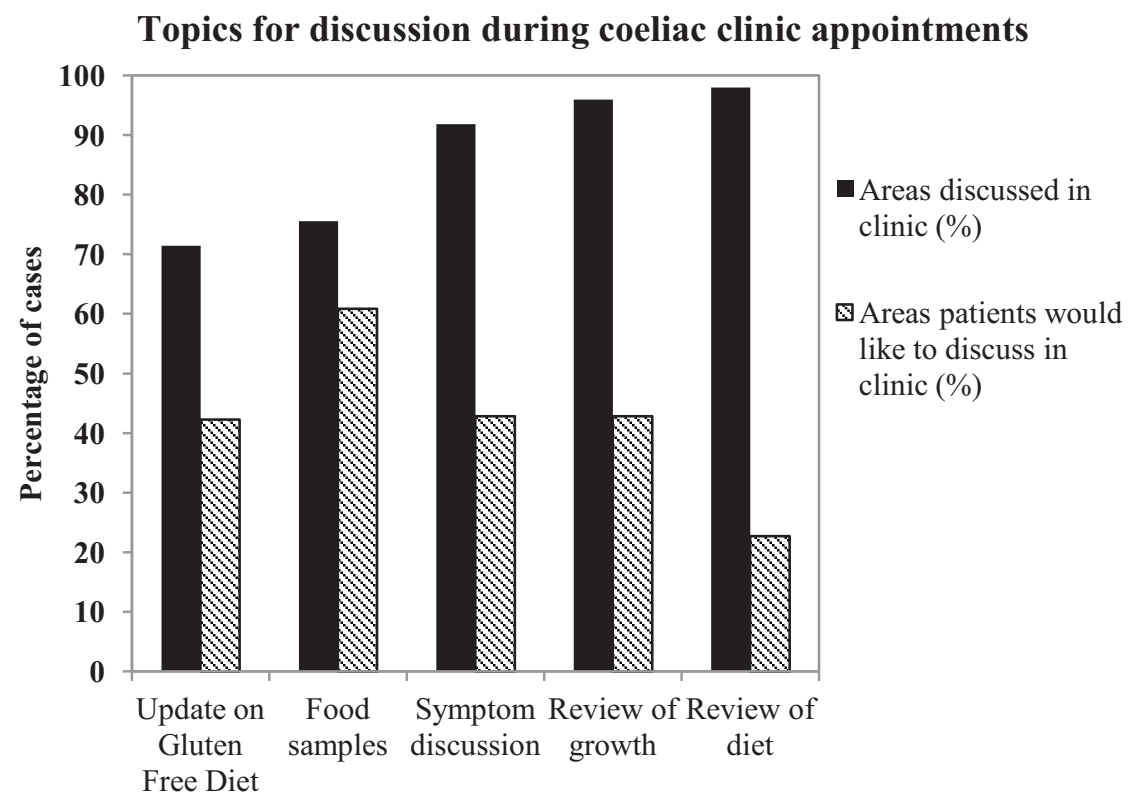




\section{Discussion}

Numbers of children and adolescents diagnosed with coeliac disease are rapidly increasing in most European countries including the UK [3]. Given substantial financial restrictions to most healthcare systems new cost-effective solutions to provide high quality patient care are urgently required. Addenbrooke's hospital adopted an alternative approach to the doctor led coeliac clinic by running a predominantly dietician and nurse led service with doctors offering advice in specific circumstances. In contrast, a second tertiary centre in the east of England ran the model of a primarily doctor led service. In this study we assessed and compared the quality of both models using QOM and parent satisfaction. Results of our study demonstrate that both approaches provide similarly high standards of care.

The missed target in the "diagnosis and initial management" subsection was "diagnostic biopsy within four weeks of positive serology at NNUH". This was due to waiting times for endoscopy, preventing more prompt scheduling of biopsy. Our data has prompted a review of patient pathway to meet this target. Subsequent to our study there have been changes to the diagnostic guidelines provided by the British Society of Paediatric Gastroenterology, Hepatology and Nutrition (BSPGHAN) allowing the diagnoses of coeliac disease to be made without the requirement of a small bowel biopsy. This has the potential to improve endoscopic capacity in both units as well as delivering cost savings to the healthcare economy [10].

Within the "annual clinical review of patients" subset of QOM, the only missed target at the two sites was to achieve a tTG value less than twice the upper limit of normal within two years. This was the case for five patients at $\mathrm{AH}$, despite NNUH achieving $100 \%$ in this target. The most likely cause of elevated tTG following diagnosis is poor adherence to a gluten free diet as refractory coeliac disease is rare [4]. As a learning point arising from our data, increasing focus has now been placed on the provision and support for parents and patients in adhering to a gluten free diet at $\mathrm{AH}$ further highlighting the vital role of nurse specialists and dieticians in the long term management of children with coeliac disease.

Uptake of the voluntary questionnaire was low across both centres, and was lower at $\mathrm{AH}(41 \%$, $n=20)$ than NNUH $(66 \%, n=33)$. It is possible that patients and their parents that had received a low quality of service might not have wanted to give feedback, and this must be considered when comparing the highly positive feedback at the two centres. Excellent overall satisfaction ratings were recorded at both sites, and this correlation with good QOM scores reflects previous research into patient satisfaction [8]. The cause stated for all dissatisfied parents was waiting times, which were up to two hours long. Waiting times can be very difficult to control in the clinical setting, however given that $50 \%$ of patients would be happy to be seen in a non-coeliac clinic, it may be possible to further outsource patient care according to specific requirements such as dietetic advice or practical guidance, which could be provided by trained general practitioners, healthcare visitor or nurse specialists. Care could then be reassessed using further application of QOM.

An additional area that was found to carry potential for future service improvement was the topic of clinic consultation. Specifically, parents most frequently voiced the desire for gluten free food samples to be provided during clinic. In contrast, the need to discuss and review current dietary issues was much less pertinent to parents in the study, whilst the dietician's impression of 
nutritional adequacy and gluten exclusion implied this was an important issue. Providing a large variety of gluten free food options is essential in aiding the ability of patients to adhere to a gluten free diet as well as improving overall quality of life. However the financial burden of Coeliac Disease to families and health care economy is considerable [11], and in our experience children's taste preferences for products are hard to predict. The provision of samples of new and different products for children to try before requesting on prescription could potentially improve compliance and reduce wastage. Hence, it may be beneficial for the future development of the service to explore the options of working together with industry in providing a greater exposure to new products on the market.

What defines excellence in care provision is still open to discussion, and the relative importance in each QOM in reflecting care quality remains subjective. The conclusions of this study are also limited by the study size; future investigation into care quality would benefit from studying patient care over a longer period of time, allowing for more detailed analysis of the cohort and estimation of cost saving. Additionally, guidelines are subject to change and therefore QOM would need to be regularly assessed and redesigned to reflect such changes.

\section{Conclusions}

In summary, using predefined QOM we were able to demonstrate that an equally high standard of care for children with coeliac disease can be delivered both via a nurse and dietician led, and a doctor and dietician led service. The QOM and parent satisfaction results also offer further insight into ways of further developing the service to meet the demands of an increasing patient population. We have now extended our study to several secondary care sites within the EEPGN, with preliminary data suggesting comparably high outcomes at these sites.

\section{Conflicts of Interest}

The authors declare no conflict of interest.

\section{References}

1. Evans, K.E.; Sanders, D.S. Celiac disease. Gastroenterol. Clin. N. Am. 2012, 41, 639-650.

2. Fasano, A.; Berti, I.; Gerarduzzi, T.; Not, T.; Colletti, R.B.; Drago, S.; Elitsur, Y.; Green, P.H.; Guandalini, S.; Hill, I.D.; et al. Prevalence of celiac disease in at-risk and not-at-risk groups in the United States: A large multicenter study. Arch. Intern. Med. 2003, 163, 286-292.

3. Kang, J.Y.; Kang, A.H.; Green, A.; Gwee, K.A.; Ho, K.Y. Systematic review: Worldwide variation in the frequency of coeliac disease and changes over time. Aliment. Pharmacol. Ther. 2013, 38, 226-245.

4. Roshan, B.; Leffler, D.A.; Jamma, S.; Dennis, M.; Sheth, S.; Falchuk, K.; Najarian, R.; Goldsmith, J.; Tariq, S.; Schuppan, D.; et al. The incidence and clinical spectrum of refractory celiac disease in a North American referral center. Am. J. Gastroenterol. 2011, 106, 923-928.

5. National Institute for Health and Clinical Excellence (2009). Coeliac Disease: Recognition and Assessment of Coeliac Disease. Available online: http://www.nice.org.uk/CG86 (accessed on 1 August 2013). 
6. Stang, A.S.; Straus, S.E.; Crotts, J.; Johnson, D.W.; Guttmann, A. Quality indicators for high acuity pediatric conditions. Pediatrics 2013, 132, 752-762.

7. Gurvitz, M.; Marelli, A.; Mangione-Smith, R.; Jenkins, K. Building quality indicators to improve care for adults with congenital heart disease. J. Am. Coll. Cardiol. 2013, doi:10.1016/j.jacc.2013.07.099.

8. Llanwarne, N.R.; Abel, G.A.; Elliott, M.N.; Paddison, C.A.; Lyratzopoulos, G.; Campbell, J.L.; Roland, M. Relationship between clinical quality and patient experience: Analysis of data from the english quality and outcomes framework and the National GP Patient Survey. Ann. Fam. Med. 2013, 11, 467-472.

9. Department of Health (UK). Governance Arrangements for Research Ethics Committees, 2011. Available online: https:/www.gov.uk/government/uploads/system/uploads/attachment_data/ file/213753/dh_133993.pdf (accessed on 7 November 2011).

10. Murch, S.; Jenkins, H.; Auth, M.; Bremner, R.; Butt, A.; France, S.; Furman, M.; Gillett, P.; Kiparissi, F.; Lawson, M.; et al. Joint BSPGHAN and Coeliac UK guidelines for the diagnosis and management of coeliac disease in children. Arch. Dis. Child. 2013, 98, 806-811.

11. Stevens, L.; Rashid, M. Gluten-free and regular foods: A cost comparison. Can. J. Diet. Pract. Res. 2008, 69, 147-150.

\section{Appendix}

Appendix 1. Summary table of each quality outcome measure and percentage of cases in which each measure was met at $\mathrm{AH}, \mathrm{NNUH}$, and the target set for both sites.

Percentage cases in which standards met

\begin{tabular}{llccc}
\hline & & AH & NNUH & Target \\
\hline $\mathbf{1}$ & Diagnosis and initial management & & & \\
\hline i & Biopsy within 4 weeks of referral & 90 & 78 & 90 \\
ii & Carer informed within 5 days & 100 & 100 & 90 \\
iii & Dietetic review within 2 weeks & 91 & 100 & 90 \\
iv & Information pack sent within 2 weeks & 91 & 100 & 90 \\
v & Coeliac UK & 91 & 100 & 90 \\
vi & Team details provided & 91 & 100 & 90 \\
\hline $\mathbf{2}$ & Annual review & & & \\
\hline i & Annual appointments offered & 100 & 100 & 90 \\
ii & Annual attendance & 94 & 100 & 90 \\
iii & Non-attenders seen within 6 months & 100 & 100 & 90 \\
iv & Proforma completed & 100 & 100 & 90 \\
v & Understanding of GFD assessed & 98 & 100 & 90 \\
vi & GF prescriptions reviewed & 100 & 100 & 90 \\
vii & Growth assessed & 100 & 100 & 90 \\
viii & Bloods taken annually & 100 & 100 & 90 \\
ix & tTG less than twice upper limit of normal within 2 years & 89 & 100 & 90 \\
x & Coeliac UK membership & 100 & 100 & 90 \\
\hline
\end{tabular}


Appendix 1. Cont.

Percentage cases in which standards met

\begin{tabular}{llccc}
\hline & & AH & NNUH & Target \\
\hline xi & Iron and calcium intake & 100 & 100 & 90 \\
\hline $\mathbf{3}$ & Transfer of care & & & \\
\hline i & Transition discussed & 100 & 100 & 90 \\
ii & Smooth transition process & 100 & 100 & 90 \\
\hline
\end{tabular}


Reprinted from Nutrients. Cite as: SriHari, M.; Simpson, S.; Lebwohl, B.; Lewis, S.K.; Tennyson, C.A.; Green, P.H.R. Is Dietitian Use Associated with Celiac Disease Outcomes? Nutrients 2013, 5, 1585-1594.

Article

\title{
Is Dietitian Use Associated with Celiac Disease Outcomes?
}

\author{
SriHari Mahadev, Suzanne Simpson, Benjamin Lebwohl, Suzanne K. Lewis, \\ Christina A. Tennyson and Peter H. R. Green *
}

Celiac Disease Center, Columbia University College of Physicians and Surgeons, 180 Fort Washington Avenue, Suite 934, New York, NY 10032, USA; E-Mails: sm3455@columbia.edu (S.M.); sms2246@columbia.edu (S.S.); b1114@columbia.edu (B.L.); sk13@columbia.edu (S.K.L.); ct2398@columbia.edu (C.A.T.)

* Author to whom correspondence should be addressed; E-Mail: pg11@columbia.edu; Tel.: +1-212-342-4529; Fax: +1-212-342-0447.

Received: 1 April 2013; in revised form: 6 May 2013 / Accepted: 6 May 2013 /

Published: 15 May 2013

\begin{abstract}
A gluten-free diet (GFD) is the treatment for celiac disease (CD), but due to its complexity, dietitian referral is uniformly recommended. We surveyed patients with CD to determine if dietitian use is associated with quality of life, symptom severity, or GFD adherence. The survey utilized three validated CD-specific instruments: the CD quality of life (CD-QOL), CD symptom index (CSI) and CD adherence test (CDAT). Four hundred and thirteen patients with biopsy-proven $\mathrm{CD}$ were eligible for inclusion. The majority $(77 \%)$ were female and mean BMI was 24.1. Over three-quarters of patients $(326,79 \%)$ had seen a dietitian, however, 161 (39\%) had seen a dietitian only once. Age, sex, and education level were not associated with dietitian use; nor was BMI (24.6 vs. $24.0, p=0.45)$. On multivariate analysis, adjusting for age gender, education, duration of disease, and body mass index, dietitian use was not associated with CD-QOL, CSI, or CDAT scores. Our survey did not show an association between dietitian use and symptom severity, adherence, or quality of life. Delay in diagnosis was associated with poorer outcomes. This is a preliminary study with several limitations, and further prospective analysis is needed to evaluate the benefits and cost-effectiveness of dietitian-referral in the care of celiac disease patients.
\end{abstract}

Keywords: celiac disease; dietary services; quality of life; quality improvement 


\section{Introduction}

Celiac disease (CD) is a common multi-system autoimmune disease, affecting approximately $1 \%$ of people worldwide [1]. Predisposed individuals develop an immune response to gluten, a protein found in the cereal grains: wheat, barley and rye. Autoimmune intestinal damage is the cardinal feature of CD, and typically involves villous atrophy, crypt hyperplasia, and increased intraepithelial lymphocytes [2]. Symptoms may be subclinical, varying from gastrointestinal upset to severe malabsorption $[3,4]$. Skin, nervous system, and multisystem involvement is also recognized. Strict avoidance of gluten-containing foods can reverse both enteric and extra-intestinal manifestations of the disease.

$\mathrm{CD}$ is unique in that its treatment consists of a dietary intervention: lifelong exclusion of gluten. A gluten-free diet (GFD) is highly effective at improving symptoms of $\mathrm{CD}$ in the majority of patients [5]. Nevertheless, a significant proportion remains symptomatic, and lack of strict adherence to GFD is the primary cause [6]. Those patients with celiac disease who follow a GFD frequently have persistent villous atrophy which may result from persistent gluten contamination and, in rare cases, can predispose patients to serious sequelae including T-cell lymphoma [7].

Although straightforward in principle, strict avoidance of gluten is challenging in practice. Gluten-containing products are ubiquitous and contamination may occur both consciously and unintentionally [8]. Poor labeling can make it difficult to determine which foods are gluten free, and options may be limited when eating out and traveling [9]. Moreover, a GFD is significantly more expensive, and may be deficient in certain nutrients, when compared to a regular diet [10,11]. Given the complexity of maintaining a strict GFD, multiple guidelines recommend dietetic referral for patients diagnosed with CD [12-15]. Dietitian involvement was recommended in the National

Institutes of Health (NIH) Consensus Development Conference on celiac disease (2004) [16]. There is evidence that when asked to choose among several referral options, patients themselves express a preference for dietetic follow-up [17]. Nevertheless, availability of expert dietetic counselors is limited and may impact upon patient outcomes [18]. Membership in celiac advocacy groups, and regular dietetic follow-up has previously been reported to be correlate with higher rates of GFD adherence $[19,20]$. However there have been no studies that have directly examined the impact of dietitian use on celiac disease outcomes in the United States. As such, we sought to determine if dietitian use was associated with quality of life, symptom severity, or GFD adherence in patients with celiac disease.

\section{Methods}

Approval was obtained from the Institutional Review Board of Columbia University Medical Center prior to initiation of the study.

Adults $(\geq 18$ years of age) with celiac disease were recruited to participate in a survey either via email or in-person. A link to an online questionnaire, hosted by SurveyMonkey, was distributed by the Celiac Disease Center of Columbia University to an email list of patients affiliated with the Center. In addition, the questionnaire was administered to attendees at celiac support group conferences in Iowa, California, and New York, and patients additionally completed the questionnaire on-paper during an office visit. Data was collected between November 2010 and July 
2011. Prior to distribution, the questionnaire was administered to a group of ten patients and subsequently modified for clarity.

The survey consisted of questions on demographics, celiac disease onset, symptoms, and dietitian use. It also included three validated celiac disease-specific instruments to assess quality of life, disease activity, and GFD adherence respectively [21-23]. Patients were asked how many times they had seen a dietitian: never, once, or more than once. Patients were excluded from the analysis if they did not have biopsy-proven celiac disease, or omitted the items on gender, age, or dietitian use.

The celiac disease-specific quality of life instrument (CD-QOL) was used to assess quality of life [21]. This validated instrument consists of 20 questions across four clinically relevant subscales (celiac disease-related limitations, dysphoria, health concerns, and inadequate treatment), and asks the respondent to indicate the frequency of celiac disease-related symptoms over the previous 30 days. The questions are graded on a 5-point Likert scale labeled 1 through 5, where $1=$ not at all, $2=$ slightly, $3=$ moderately, $4=$ quite a bit, and $5=$ a great deal. The responses were reverse-coded and summed, with a higher score (up to a maximum of 100) suggestive of higher quality of life. No clear cut-off point has been established to dichotomize CD-QOL scores; hence membership in the lowest quartile of CD-QOL was taken to indicate poorer quality of life.

The Celiac Symptom Index (CSI) was employed to assess celiac disease-specific symptom severity [23]. The CSI consists of 16 questions on a 5-point Likert scale; with scores $\leq 30$ and $\geq 45$ suggestive of clinical remission and ongoing active disease respectively. For the purpose of our analysis; a single cutoff of $\geq 35$ (suggestive of ongoing disease) was used to dichotomize patients.

The Celiac Disease Dietary Adherence Test (CDAT) was used to assess adherence to a GFD [22]. This validated 7-question instrument employs a 5-point Likert scale, with additive scores ranging from 7 to 35, where higher scores indicate worse adherence. For the purposes of dichotomization, scores $\geq 13$ were taken to be indicative of poor adherence.

Univariate analysis was used to identify associations between demographics, dietitian use, and CD-QOL, CSI, and CDAT. The Chi-square and Fisher exact tests were used to compare proportions of categorical variables. The Mann-Whitney $U$ test was used to compare continuous variables. Logistic regression was performed to develop a multivariate model identifying variables predictive of three outcomes as determined by these validated scores: poor quality of life, high symptom activity, and poor adherence. Two-sided $p$-values $<0.05$ were considered significant. All statistical calculations were performed with SAS 9.2 (Cary, NC, USA).

\section{Results}

\subsection{Patient Characteristics}

Of 600 respondents, 413 with biopsy-proven celiac disease were eligible for inclusion (Table 1). Roughly equal numbers of patients completed the survey online (47\%) versus on paper (49\%). The majority (77\%) of subjects were female, with almost one-quarter over the age of $60(24 \%)$. The cohort was highly educated, with $88 \%$ having attained a college degree, and $40 \%$ having a graduate or higher degree. 
Table 1. Patient characteristics.

\begin{tabular}{|c|c|}
\hline & $n(\%)$ \\
\hline Total number & 413 \\
\hline \multicolumn{2}{|l|}{ Age } \\
\hline $18-30$ & $80(19)$ \\
\hline $31-40$ & $67(16)$ \\
\hline $41-50$ & $75(18)$ \\
\hline $51-60$ & $93(23)$ \\
\hline $61-70$ & $67(16)$ \\
\hline$>70$ & $31(8)$ \\
\hline \multicolumn{2}{|l|}{ Gender } \\
\hline Male & $94(23)$ \\
\hline Female & $319(77)$ \\
\hline \multicolumn{2}{|l|}{ Educational level } \\
\hline High school or less & $43(10)$ \\
\hline College & $197(48)$ \\
\hline Graduate school & $167(40)$ \\
\hline \multicolumn{2}{|l|}{ Presentation } \\
\hline Classical & $166(40)$ \\
\hline Atypical & $204(49)$ \\
\hline None & $32(8)$ \\
\hline \multicolumn{2}{|l|}{ Years since diagnosis } \\
\hline$<1$ & $55(13)$ \\
\hline $1-4$ & $149(36)$ \\
\hline $5-10$ & $115(28)$ \\
\hline$>10$ & $93(23)$ \\
\hline \multicolumn{2}{|c|}{ Delay to diagnosis in years } \\
\hline$<1$ & $51(12)$ \\
\hline $1-4$ & $149(36)$ \\
\hline $5-10$ & $115(28)$ \\
\hline$>10$ & $93(23)$ \\
\hline Mean Body Mass Index & 24.1 \\
\hline \multicolumn{2}{|c|}{ Symptoms improved on GFD } \\
\hline Yes & $288(70)$ \\
\hline Somewhat & $73(18)$ \\
\hline No & $28(7)$ \\
\hline \multicolumn{2}{|l|}{ Filled in survey } \\
\hline Online & $195(47)$ \\
\hline Paper & $204(49)$ \\
\hline
\end{tabular}

\subsection{Disease Characteristics}

Regarding CD presentation, almost half of patients (49\%) reported atypical symptoms of fatigue, anemia or osteoporosis; $40 \%$ reported classical diarrhea-predominant symptoms, and $8 \%$ reported no symptoms. Most patients' symptoms were either improved (70\%) or somewhat improved (13\%) with GFD. Mean body mass index (BMI) was in the normal-weight range (24.1). The median time 
since diagnosis was 5-10 years, and the median delay from onset of symptoms to diagnosis was also $5-10$ years.

\subsection{Dietitian Use}

Of the 413 patients in the analysis, 326 (79\%) reported having seen a dietitian, but $161(39 \%)$ had only seen a dietitian once. One hundred and sixty-four patients (40\%) agreed with the statement "it is hard to find a dietitian knowledgeable about GFD". One hundred and ninety-one patients (46\%) reported gaining weight since starting a GFD. Demographic factors, including age, sex, and education level, were not associated with dietitian use (Table 2). There was no significant difference in BMI between patients who had and had not seen a dietitian (24.0 vs. 25.6, $p=0.45)$. Patients who had not seen a dietitian were more likely to agree with the statement "health insurance limits my ability to see a dietitian" (55\% vs. $43 \% ; p=0.04)$.

Table 2. Patient characteristics by dietitian use.

\begin{tabular}{lccc}
\hline & Not seen $\boldsymbol{n}(\boldsymbol{\%})$ & Seen $\boldsymbol{n}(\boldsymbol{\%})$ & $\boldsymbol{p}$ \\
\hline Total number & $87(100)$ & $326(100)$ & \\
Age $\geq 60$ & $20(23)$ & $78(24)$ & 0.96 \\
Female gender & $72(83)$ & $247(76)$ & 0.21 \\
College educated & $75(86)$ & $289(89)$ & 0.53 \\
Symptoms improved on GFD & $63(72)$ & $225(69)$ & 0.54 \\
Mean BMI & 25.6 & 24.0 & 0.45 \\
\hline
\end{tabular}

\subsection{Quality of Life, Disease Activity, and Dietary Adherence}

Dietitian use was not associated with CD-QOL, CDAT, or CSI on univariate analysis (Table 3). Multivariate analysis (Table 4) identified two covariates associated with low CD-QOL, indicative of poor quality of life: a long delay ( $>10$ years $v s .<1$ year) from symptom onset to CD diagnosis (OR 3.92, 95\% CI 1.45-0.63), and underweight vs. normal weight (OR 3.46, 1.12-10.68). Older age $(>60)$ and time since diagnosis $(>10$ years $v s .<1$ year) were protective for disease activity as measured by CSI (OR $0.35,95 \%$ CI $0.17-0.71$ and OR $0.34,95 \%$ CI $0.13-0.87$ respectively). Dietitian use was not associated with CD-QOL, CSI, or CDAT scores on multivariate analysis (see Table 4).

Table 3. Mean validated scores and use of a dietitian.

\begin{tabular}{lccc}
\hline & Not seen & Seen & $\boldsymbol{p}$ \\
\hline CD-QOL & 75.4 & 72.6 & 0.08 \\
CSI & 33.5 & 33.3 & 0.62 \\
CDAT & 12.9 & 12.1 & 0.11 \\
\hline
\end{tabular}


Table 4. Multivariate analysis of factors associated with low celiac disease quality of life (CD-QOL), high CD symptom index (CSI), and high CD adherence test (CDAT).

\begin{tabular}{lcccccc}
\hline \multirow{2}{*}{\multicolumn{1}{c}{ Covariate }} & \multicolumn{2}{c}{ Low CD-QOL } & \multicolumn{2}{c}{ High CSI } & \multicolumn{2}{c}{ High CDAT } \\
& OR & $\mathbf{9 5 \%}$ CI & OR & $\mathbf{9 5 \% ~ C I ~}$ & OR & 95\% CI \\
\hline Seen a dietitian & 1.49 & $0.68-3.24$ & 0.86 & $0.45-1.63$ & 0.85 & $0.47-1.55$ \\
Older age $(>60)$ & 0.60 & $0.26-1.37$ & 0.35 & $0.17-0.71$ & 0.53 & $0.28-1.01$ \\
Male gender & 1.29 & $0.61-2.72$ & 1.24 & $0.58-2.64$ & 0.92 & $0.48-1.77$ \\
College educated & 3.65 & $0.97-13.7$ & 1.33 & $0.53-3.38$ & 0.74 & $0.32-1.71$ \\
Time since diagnosis ${ }^{1}$ & 0.38 & $0.13-1.10$ & 0.34 & $0.13-0.87$ & 0.48 & $0.20-1.13$ \\
Delayed diagnosis ${ }^{1}$ & 3.92 & $1.45-10.63$ & 2.08 & $0.98-4.41$ & 1.05 & $0.53-2.07$ \\
Underweight $v s$. normal $^{3}$ & 3.46 & $1.12-10.68$ & 2.52 & $0.79-8.04$ & 1.90 & $0.66-5.51$ \\
Overweight $v s$. normal & 1.31 & $0.62-2.75$ & 0.60 & $0.31-1.16$ & 0.66 & $0.36-1.21$ \\
Obese $v s$. normal & 0.86 & $0.33-2.23$ & 0.75 & $0.32-1.76$ & 0.65 & $0.29-1.46$ \\
\hline
\end{tabular}

\section{Discussion}

To our knowledge, this is the first study to examine dietitian use and outcomes as measured by validated CD-specific instruments. We were surprised at the lack of association between dietitian use, dietary adherence and quality of life. The study has several limitations, including selection bias, self-reported outcomes, and poor generalizability, however prior literature addressing these issues is similarly limited. While our findings are preliminary, they should provide impetus to further study the role of dietary counseling in CD management.

Several studies have examined dietitian use in $\mathrm{CD}$, with frequently divergent results. Ukkola et al. [24] surveyed 698 newly diagnosed CD patients on their perceptions of living with CD, and found no correlation between dietitian follow-up and patients' knowledge of GFD or experience of their disease. A similar proportion of patients in this cohort $(76 \%)$ had received dietitian counseling as in our sample (79\%). The authors note that despite a lack of correlation, patients often requested more detailed dietary counseling when asked to indicate in their own words their wishes/needs, suggesting insufficient dietitian contact.

One outcome measure that has been demonstrated to be associated with dietitian use is improved GFD adherence, but this was not seen in our cohort. Wylie et al. [25] concluded in a prospective cohort of 99 patients that annual review within the context of a dietitian-led celiac clinic can significantly improve adherence as well as other nutritional markers. In a systemic review of 38 studies examining factors associated with adherence to GFD, Hall et al. [19] also concluded that regular dietetic follow-up and annual review in a dietitian-led clinic can improve adherence. Membership of a patient support group has been associated with adherence, and studies with cohorts recruited from patient support groups trend towards higher adherence rates $(66 \%-90 \%$ strict adherence) than clinical samples (42\%-91\%) [26]. There are several possible reasons why these results might diverge from that of our study. First, dietitian use was common in our study (approaching 80\%), leading to a small sample size for patients who had not seen a dietitian, and hence decreased ability to detect a difference. Our cohort was very highly educated, with almost $90 \%$ of patients having a college degree or higher qualification, as compared to a US-wide average of 
$28 \%$ [27]. It may be that university-educated patients may have less to gain from dietitian referral: they are more health-literate at baseline, and are better equipped to seek out and utilize information from other sources in addition to their health professional. As such, the high prevalence of highly educated patients in our cohort may have reduced the effect size of dietitian exposure. Hall et al. [19] included studies that assessed GFD adherence via a trained nutritionist assessment, which may be more sensitive for differences than use of the self-reported CDAT questionnaire. Last, cultural factors can influence response to dietary interventions. In a cross-sectional survey from 2004, Butterworth et al. [20] noted that South Asian patients with CD were much less likely than Caucasians to be members of a support group (53\% vs. $80 \%, p=0.02)$, were more frequently dissatisfied with dietetic advice $(30 \% v s .6 \%, p=0.01)$, and exhibited a trend towards poorer dietetic follow-up ( $31 \%$ vs. $60 \%, p=\mathrm{NS}$ ). Dietetic follow-up correlated with GFD compliance for Caucasians but not for South Asians in that study. It is unclear to what extent these cultural issues apply to our cohort.

Although dietitian use was common in this study, almost half of these patients had only seen a dietitian once, possibly at diagnosis, falling short of $\mathrm{CD}$ treatment guidelines. Guidelines published by several authorities generally recommend annual dietitian review. The American Gastroenterological Association position statement on diagnosis and management of CD advises consultation with an experienced dietitian at initiation of a treatment plan and ongoing evaluation at regular intervals by a health care team including a dietitian [12]. Several other organizations also publish guidelines recommending ongoing dietitian involvement, including the NIH, the United States Department of Health and Human Services Agency for Healthcare Research and Quality, the National Institute for Health and Clinical Excellence in the United Kingdom, and the World Gastroenterology Association [14,16,28,29]. Given a median time since diagnosis of 5-10 years in this cohort, dietitian involvement fell well short of recommendations in a significant proportion of patients and insufficient dietitian follow-up may have contributed to the lack of effect on outcomes.

A long delay of $>10$ years from symptom onset to diagnosis of CD was associated with poor quality of life in this cohort. Prior studies have addressed the influence of delayed diagnosis on CD outcomes. In a survey of over 1000 patients with CD from Sweden, the mean delay to diagnosis from first symptoms was 9.7 years, and 5.8 years from the first doctor visit [30]. A long delay was associated with lower quality-adjusted life year scores prior to treatment; however delay in diagnosis had no effect on scores following initiation of GFD. The study concluded that untreated CD resulted in poor quality of life, which returned to baseline with treatment. Our findings of persistent quality of life impairment even following initiation of GFD are novel and warrant further study.

The limitations of this study include recruitment from a tertiary referral center (the Celiac Disease Center of Columbia University) and support groups, leading to a cohort that is likely to have greater health literacy than average. The sample size for patients who had not seen a dietitian was low, limiting our power to detect a difference in $\mathrm{CD}$ outcomes. The actual response rate of the survey is unable to be determined as the survey link may have been electronically forwarded by subjects to other members of support groups or known contacts with celiac disease. In addition we did not have information on the quality, nor expertise and practice setting of the dietitians used by the respondents. 


\section{Conclusions}

In this survey of patients with celiac disease, more than $20 \%$ of respondents had never seen a dietitian, and 39\% only saw a dietitian once. Dietitian follow-up fell short of published guidelines, which may relate to insurance access issues. Dietitian exposure was not associated with symptom severity, adherence, or quality of life, while delay in diagnosis was associated with poorer quality of life. Further prospective analysis is needed to evaluate the benefits and cost-effectiveness of dietitian referral in the care of patients with celiac disease.

\section{Acknowledgments}

The Celiac Disease Center thanks its supporters for their ongoing contribution.

\section{Conflicts of Interest}

Competing interests: The authors have no competing interests to disclose.

Financial interests: The authors have none to declare.

Funding: No sources of funding were used

Ethical approval: This study was approved by the institution review board of Columbia University Medical Center, reference number AAAF2497.

Guarantor: PG.

Contribution: SM, SS, BL, SL, PG, CT designed research; SM, SS, CT conducted research; SM, BL, CT analyzed data; SM, BL, PG, CT wrote paper; SM, CT had primary responsibility for final content.

All authors read and approved the final manuscript.

\section{References}

1. Green, P.H.R.; Cellier, C. Celiac disease. N. Engl. J. Med. 2007, 357, 1731-1743.

2. Oberhuber, G.; Granditsch, G.; Vogelsang, H. The histopathology of coeliac disease: Time for a standardized report scheme for pathologists. Eur. J. Gastroenterol. Hepatol. 1999, 11, 1185-1194.

3. Corazza, G.R.; Frisoni, M.; Treggiari, E.A.; Valentini, R.A.; Filipponi, C.; Volta, U.; Gasbarrini, G. Subclinical celiac sprue. Increasing occurrence and clues to its diagnosis. J. Clin. Gastroenterol. 1993, 16, 16-21.

4. Ciacci, C.; Cirillo, M.; Sollazzo, R.; Savino, G.; Sabbatini, F.; Mazzacca, G. Gender and clinical presentation in adult celiac disease. Scand. J. Gastroenterol. 1995, 30, 1077-1081.

5. Murray, J.A.; Watson, T.; Clearman, B.; Mitros, F. Effect of a gluten-free diet on gastrointestinal symptoms in celiac disease. Am. J. Clin. Nutr. 2004, 79, 669-673.

6. Abdulkarim, A.S.; Burgart, L.J.; See, J.; Murray, J.A. Etiology of nonresponsive celiac disease: Results of a systematic approach. Am. J. Gastroenterol. 2002, 97, 2016-2021.

7. Cellier, C.; Delabesse, E.; Helmer, C.; Patey, N.; Matuchansky, C.; Jabri, B.; Macintyre, E.; Cerf-Bensussan, N.; Brousse, N. Refractory sprue, coeliac disease, and enteropathy-associated T-cell lymphoma. French Coeliac Disease Study Group. Lancet 2000, 356, 203-208. 
8. Ciacci, C.; Mazzacca, G. Unintentional gluten ingestion in celiac patients. Gastroenterology 1998, 115, 243.

9. Zarkadas, M.; Cranney, A.; Case, S.; Molloy, M.; Switzer, C.; Graham, I.D.; Butzner, J.D.; Rashid, M.; Warren, R.E.; Burrows, V. The impact of a gluten-free diet on adults with coeliac disease: Results of a national survey. J. Hum. Nutr. Diet. 2006, 19, 41-49.

10. Stevens, L.; Rashid, M. Gluten-free and regular foods: A cost comparison. Can. J. Diet. Pract. Res. 2008, 69, 147-150.

11. Thompson, T.; Dennis, M.; Higgins, L.A.; Lee, A.R.; Sharrett, M.K. Gluten-free diet survey: Are Americans with coeliac disease consuming recommended amounts of fibre, iron, calcium and grain foods? J. Hum. Nutr. Diet. 2005, 18, 163-169.

12. AGA Institute. AGA Institute Medical Position Statement on the Diagnosis and Management of Celiac Disease. Gastroenterology 2006, 131, 1977-1980.

13. Centre for Clinical Practice at NICE (UK). Coeliac Disease: Recognition and Assessment of Coeliac Disease; National Institute for Health and Clinical Excellence: London, UK, 2009.

14. Bai, J.; Zeballos, E.; Fried, M.; Corazza, G.R.; Schuppan, D.; Farthing, M.; Catassi, C.; Greco, L.; Cohen, H.; Krabshuis, J.H. WGO-OMGE practice guideline celiac disease. World Gastroenterol. News 2005, 10, 1-8.

15. Hill, I.D.; Dirks, M.H.; Liptak, G.S.; Colletti, R.B.; Fasano, A.; Guandalini, S.; Hoffenberg, E.J.; Horvath, K.; Murray, J.A.; Pivor, M.; Seidman, E.G. North American Society for Pediatric Gastroenterology, Hepatology and Nutrition Guideline for the diagnosis and treatment of celiac disease in children: Recommendations of the North American Society for Pediatric Gastroenterology, Hepatology and Nutrition. J. Pediatr. Gastroenterol. Nutr. 2005, 40, 1-19.

16. NIH Consensus Development Conference on Celiac Disease; U.S. Department of Health and Human Services: Bethesda, MD, USA, 2004.

17. Bebb, J.R.; Lawson, A.; Knight, T.; Long, R.G. Long-term follow-up of coeliac disease-What do coeliac patients want? Aliment. Pharmacol. Ther. 2006, 23, 827-831.

18. Nelson, M.; Mendoza, N.; McGough, N. A survey of provision of dietetic services for coeliac disease in the UK. J. Hum. Nutr. Diet. 2007, 20, 403-411.

19. Hall, N.J.; Rubin, G.; Charnock, A. Systematic review: Adherence to a gluten-free diet in adult patients with coeliac disease. Aliment. Pharmacol. Ther. 2009, 30, 315-330.

20. Butterworth, J.R.; Banfield, L.M.; Iqbal, T.H.; Cooper, B.T. Factors relating to compliance with a gluten-free diet in patients with coeliac disease: Comparison of white Caucasian and South Asian patients. Clin. Nutr. 2004, 23, 1127-1134.

21. Dorn, S.D.; Hernandez, L.; Minaya, M.T.; Morris, C.B.; Hu, Y.; Leserman, J.; Lewis, S.; Lee, A.; Bangdiwala, S.I.; Green, P.H.R.; Drossman, D.A. The development and validation of a new coeliac disease quality of life survey (CD-QOL). Aliment. Pharmacol. Ther. 2010, 31, 666-675.

22. Leffler, D.A.; Dennis, M.; Edwards George, J.B.; Jamma, S.; Magge, S.; Cook, E.F.; Schuppan, D.; Kelly, C.P. A simple validated gluten-free diet adherence survey for adults with celiac disease. Clin. Gastroenterol. Hepatol. 2009, 7, 530-536. 
23. Leffler, D.A.; Dennis, M.; Edwards George, J.; Jamma, S.; Cook, E.F.; Schuppan, D.; Kelly, C.P. A validated disease-specific symptom index for adults with celiac disease. Clin. Gastroenterol. Hepatol. 2009, 7, 1328-1334.

24. Ukkola, A.; Mäki, M.; Kurppa, K.; Collin, P.; Huhtala, H.; Kekkonen, L.; Kaukinen, K. Patients' experiences and perceptions of living with coeliac disease-Implications for optimizing care. J. Gastrointestin. Liver Dis. 2012, 21, 17-22.

25. Wylie, C.; Geldart, S.; Winwood, P. Dietitian-led coeliac clinic: A successful change in working practice in modern healthcare. Gastroenterol. Today 2005, 15, 11-12.

26. Leffler, D.A.; Edwards George, J.; Dennis, M.; Schuppan, D.; Cook, F.; Franko, D.L.; Blom-Hoffman, J.; Kelly, C.P. Factors that influence adherence to a gluten-free diet in adults with celiac disease. Dig. Dis. Sci. 2008, 53, 1573-1581.

27. Educational Attainment in the United States: 2009; U.S. Department of Commerce, Economics and Statistics Administration, U.S. Census Bureau: Washington, DC, USA, 2012; pp. 1-17.

28. Rostom, A.; Dube, C.; Cranney, A.; Saloojee, N.; Sy, R.; Garritty, C.; Sampson, M.; Zhang, L.; Yazdi, F.; Mamaladze, V.; et al. Celiac disease. Evid. Rep. Technol. Assess. (Summ.) 2004, $104,1-6$.

29. Richey, R.; Howdle, P.; Shaw, E.; Stokes, T.; Guideline Development Group. Recognition and assessment of coeliac disease in children and adults: Summary of NICE guidance. BMJ 2009, $338, \mathrm{~b} 1684$.

30. Norström, F.; Lindholm, L.; Sandström, O.; Nordyke, K.; Ivarsson, A. Delay to celiac disease diagnosis and its implications for health-related quality of life. BMC Gastroenterol. 2011, 11, 118 . 
Reprinted from Nutrients. Cite as: Dufrene, K.; DeBiasi, R.L.; Colberg-Poley, A.M. The Gluten-Free Diet: Testing Alternative Cereals Tolerated by Celiac Patients. Nutrients 2013, 5, 4250-4268.

Review

\title{
The Gluten-Free Diet: Testing Alternative Cereals Tolerated by Celiac Patients
}

\author{
Isabel Comino ${ }^{1}$, María de Lourdes Moreno ${ }^{1}$, Ana Real ${ }^{1}$, Alfonso Rodríguez-Herrera ${ }^{2}$, \\ Francisco Barro ${ }^{3}$ and Carolina Sousa ${ }^{1, *}$
}

1 Departamento de Microbiología y Parasitología, Facultad de Farmacia, Universidad de Sevilla, c/Profesor García González 2, 41012 Sevilla, Spain; E-Mails: icomino@us.es (I.C.); 1moreno@us.es (M.L.M.); arc@us.es (A.R.)

2 Instituto Hispalense de Pediatría, c/Guadalbullón 2, 41013 Sevilla, Spain;

E-Mail: alfonsorodriguez@ihppediatria.com

3 Instituto de Agricultura Sostenible (C.S.I.C.), Alameda del Obispo s/n, 14004 Córdoba, Spain; E-Mail: fbarro@ias.csic.es

* Author to whom correspondence should be addressed; E-Mail: csoumar@us.es; Tel.: +34-954-556-452; Fax: +34-954-628-162.

Received: 29 August 2013; in revised form: 7 October 2013 / Accepted: 15 October 2013 / Published: 23 October 2013

\begin{abstract}
A strict gluten-free diet (GFD) is the only currently available therapeutic treatment for patients with celiac disease, an autoimmune disorder of the small intestine associated with a permanent intolerance to gluten proteins. The complete elimination of gluten proteins contained in cereals from the diet is the key to celiac disease management. However, this generates numerous social and economic repercussions due to the ubiquity of gluten in foods. The research presented in this review focuses on the current status of alternative cereals and pseudocereals and their derivatives obtained by natural selection, breeding programs and transgenic or enzymatic technology, potential tolerated by celiac people. Finally, we describe several strategies for detoxification of dietary gluten. These included enzymatic cleavage of gliadin fragment by Prolyl endopeptidases (PEPs) from different organisms, degradation of toxic peptides by germinating cereal enzymes and transamidation of cereal flours. This information can be used to search for and develop cereals with the baking and nutritional qualities of toxic cereals, but which do not exacerbate this condition.
\end{abstract}

Keywords: celiac disease; gluten-free diet; cereals; pseudocereals; gluten detoxification 


\section{Introduction}

Celiac disease is a food intolerant related syndrome that, despite being under-diagnosed, is one of the most frequent chronic gastrointestinal disorders. It develops in genetically predisposed individuals in whom unidentified environmental factors (infections, changes in microbial flora, etc.) can trigger intolerance to gluten contained in wheat, barley, rye and oats $[1,2]$. Gluten is a complex mixture of proteins called prolamins. This protein fraction has specific name: wheat prolamins are termed gliadins and glutenins, barley prolamins are hordeins, rye prolamins are secalin and those from oats are avenins. A common characteristic of these proteins is the presence of multiple proline and glutamine residues, making them resistant to gastrointestinal digestion and more exposed to deamination by tissue transglutaminase.

Several epitopes responsible for the toxicity of gluten have been identified based on their ability to stimulate proliferation of gluten-responsive $\mathrm{T}$ cells in celiac patient-derived small intestine biopsies. Considering only wheat, in the Immune Epitope Database (IEDB) [3] can be found 190 T-cell stimulatory epitopes related to celiac disease. Of these, 94 epitopes are located in $\alpha$-gliadin genes, 74 in $\gamma$-gliadin genes, 12 in $\omega$-gliadin genes, 8 in low molecular weight (LMW) glutenin genes, and 2 in high molecular weight (HMW) genes.

The most accepted model for explaining the immunopathogenesis of celiac disease is the two-signal model, characterized by a first innate immune response and a subsequent secondary adaptive response, which will promote a histological lesion characterized by a massive intraepithelial infiltration of lymphocytes, crypt hyperplasia and villous atrophy [2]. The ingestion of these proteins leads to the inflammation, atrophy, and hyperplasia of the small-intestinal crypts of the celiac patient. However, this disease not only affects the gut, but it is a systemic disease that may cause injury to the skin, liver, joints, brain, heart, and other organs.

Celiac disease goes in remission when the patients are put on a gluten-exclusion diet, and patients relapse when gluten is reintroduced into the diet [1,2]. Complying with a GFD is difficult and affects the patients' quality of life, but a strict diet is critical to reduce morbidity and mortality [4].

Gluten has many special characteristics that favor its use in various food products. Because a large amount of gluten is generated during the manufacture of starch, it has a relatively low price. This may turn out to be problematic for people on a GFD, since gluten proteins may be found in unexpected sources such as meat, fish or milk products. This is the reason why alternative approaches to the GFD are actively sought [5], which include the search for and development of new cereals or gluten with no or low immunogenic content. In this article, we will review the current status of alternative cereals and their derivatives obtained by natural selection, breeding programs and transgenic or enzimatic technology, which may be potential tolerated by patients with celiac disease.

\section{Natural Varieties of Cereal and Pseudocereals Suitable for Patients with Celiac Disease}

\subsection{Wheat and Barley}

Cultivated wheat is genetically very complex due to its origin from ancestral diploid species through a process of natural hybridization and subsequent polyploidization. The two wheat species 
of agricultural importance, the pasta wheat, and the bread wheat, are tetraploid (two genomes, $\mathrm{AABB}$ ) and hexaploid (three genomes, AABBDD), respectively (Figure 1). The tetraploids originated in nature through spontaneous hybridization of two diploid species, each donor genomes $\mathrm{A}$ and $\mathrm{B}$, between 0.5 and 2 million years ago. Bread wheat (AABBDD) originated in the fields, about 8000 years ago, through spontaneous hybridization between durum wheat (AABB) and Aegilops tauschii, the diploid donor of the D genome (Figure 1).

One species of wheat, Triticum aestivum, is predominantly used in the modern industrialized world, due to its increased protein production as well as its hardiness in colder climates. It has also been determined that the proteins that are the most immunogenic for celiac disease reside in the gliadin fraction of T. aestivum. However, there are almost 20 other species of wheat that are either not being cultivated by modern societies or are cultivated in select regions of the world [6]. With such a large number of wheat species available, a significant amount of research has been focused on the exploration of different species and cultivars of wheat as an alternative to a strict GFD for celiac patients [7].

Figure 1. Diagram showing the evolutionary relationships among species of wheat and related species of Aegilops. The dotted line separates the wild and domesticated species of wheat. Ancestral or unknown species are surrounded by a double dashed rectangle. Ploidy level and the number of chromosomes are indicated. Nomenclature according to van Slageren [8].

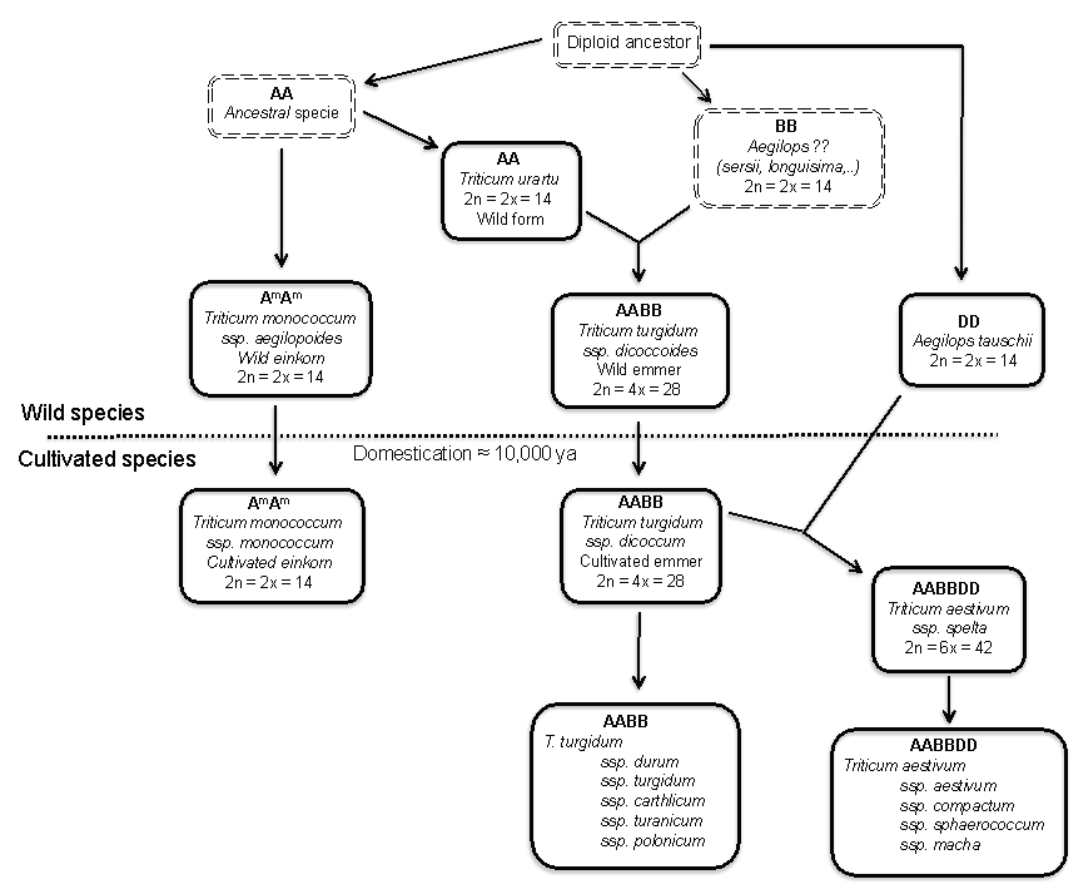

Attempts have been made to quantify the toxicity of a range of bread wheat and pasta wheat varieties and of species that contain only one of the three genomes of bread wheat $[9,10]$. Using specific T-cell clones and monoclonal antibodies, the results demonstrated that large quantitative differences exist in the presence of toxic gluten peptides, with some cultivars completely lacking particular harmful peptides [11]. Diploid wheat species are among the suitable candidates for their low capability to activate intestinal $\mathrm{T}$ cell responses in celiac patients [11,12]. Compared with 
tetraploid and hexaploid wheat, commonly used in the making of bread and pasta, the ancient diploid Triticum monococcum ssp. monococcum wheat showed a marked reduction, or even a lack, of toxicity in vitro cellular assays [13,14]. Gianfrani et al. [15] compared the immunological properties of 2 lines of diploid monococcum wheat, Monlis and ID331, with those of T. aestivum. They found that both lines activate celiac T cell response. However, ID331 was less effective to activate the innate immune pathways. The reduced ability of some diploid wheat lines to in vitro activate the innate immune response in celiac mucosa could render these cultivars less active in inducing celiac disease. However, more analyses are required to explore their potential use as new dietary opportunities for celiac patients.

Some approaches were performed to remove celiac toxic proteins in barley. Double-null hybrid plants, largely devoid of both B- and C-hordeins, were produced by conventional crossing [16]. Barley is a diploid and unlike the situation in bread wheat, the genetics of hordeins are relatively straightforward. There are four protein families of hordeins: B-, C-, D- and $\gamma$-hordeins, with the Band C-hordeins together accounting for over $90 \%$ of barley hordeins. Isolation of hordein double-null barley lines from hybrids of Risø 56 and Risø 1508 has produced a line which does not accumulate B- or C-hordein and only has $3 \%$ of wild type hordein along with a 20 -fold reduction in reactivity in T-cell assays [16,17]. In addition, studies carried out demonstrated that some malting lines (Hordeum vulgare) were less immunogenic compared with wild lines (Hordeum chilense) [18]. These findings could raise the prospect of breeding barley species with low levels of harmful gluten, and the attractive goal of developing non-toxic barley cultivars with a potential use in the manufacture of beverages as consumed worldwide as are the beers. However, nothing is known about the variability in celiac toxicity of other species or varieties of toxic cereals such as rye.

\subsection{Oats}

Cultivated oats are hexaploid cereals belonging to the genus Avena L., which is found worldwide in almost all agricultural environments [19]. Recently, oats have been receiving increasing interest as human food, mainly because the cereal could be suitable for consumptions by celiac patients. Several varieties of oats are available. It is a rich source of protein, contains a number of important minerals, lipids, $\beta$-glucan, a mixed-linkage polysaccharide, which forms an important part of oat dietary fiber, and also contains various other phytoconstituents like avenanthramides, an indole alkaloid-gramine, flavonoids, flavonolignans, triterpenoid saponins, sterols, and tocols. Traditionally oats have been in use since long and are considered as stimulant, antispasmodic, antitumor, diuretic, and neurotonic. Oat possesses different pharmacological activities like antioxidant, anti-inflammatory, antidiabetic, anticholesterolaemic, etc. [20].

The presence of oats in a GFD is still a subject of controversial. Oats differ from other cereals in their prolamin content. The percentage of proline and glutamine (amino acids abundant in toxic regions) in avenin is lower than in other toxic cereals. Some clinical researchers state that patients with celiac disease tolerate oats without signs of intestinal inflammation [21]. According to the Codex Alimentarius for food for special dietary use for persons intolerant to gluten, CODEX STAN118-1979 (revised 2008, [22]), oats can be tolerated by most but not all people who are intolerant to gluten. Moreover, according to the Commission Regulation (EC) No 41/2009 [23] concerning the composition and labeling of foodstuffs suitable for people intolerant to gluten, a 
major concern is the contamination of oats with wheat, rye or barley that can occur during grain harvesting, transport, storage and processing. Therefore, the risk of gluten contamination in products containing oats should be taken into consideration with regard to labeling of those products. In contrast, other studies confirmed the toxicity of oats in certain types of patients with celiac disease. Arentz-Hansen et al. [24] described the intestinal deterioration suffered by some patients with celiac disease following the consumption of oats while on a GFD. Avenin can trigger an immunological response in these patients similar to the response produced by the gluten of wheat, rye or barley. The monitoring of 19 adult patients with celiac disease who consumed 50 $\mathrm{g} /$ day of oats over 12 weeks showed that one of the subjects was sensitive to oats. Therefore, it is critical to clarify either qualitatively or quantitatively the potential immunotoxicity of oats to patients with celiac disease $[21,24]$.

Comparison of the different studies are complicated by the different study designs, the different conditions used in the testing, the number of subjects included in each study and the reporting of the purity control of the oat material used in the clinical trials. Another relevant factor in different designs is the absence of information on the oat variety used. Silano et al. [25] investigated the immunogenic effect of avenins from four oat cultivars using peripheral lymphocytes from patients with CD. All the varieties of oats tested (Lampton, Astra, Ava, and Nave) by these investigators were immunogenic with differences in their capacity to induce a response. However, other study confirmed that Avena genziana and Avena potenza do not display in vitro activities related to CD pathogenesis [26].

The utility of the G12 antibody to identify potentially toxic oat varieties for celiac patients has been reported [27]. This finding allowed classification of oat varieties into three groups based in their degree of affinity for the G12 antibody: a highly recognized group, one of moderate recognition, and one with no reactivity [27]. These results were confirmed by MALDI-TOF, SDS-PAGE and western blot by showing that the number, relative intensity of the peaks and protein profile obtained for the nine oat varieties differ from one another. The potentially immunotoxicity of the different types of oats was determined by $\mathrm{T}$ cell proliferation and interferon $\gamma$ release. The reactivity that $\mathrm{T}$-cells isolated from celiac patients exhibited with three oat varieties (one from each of the classified groups) correlated directly with the moAb G12 reactivity. The diversity observed in the reactivity to the different oat cultivars suggests variations in the avenin composition, and therefore in the amount of immunotoxic epitopes similar to the 33-mer present in these varieties. This gives a rational explanation for why only some oats trigger an immunological response.

In comparison with wheat gliadins, the avenins have been little studied, and the number of full avenin genes present at the moment in the databases is limited and from few genotypes, so that the variability of avenin genes in oats is not well represented. It has recent been known that, like wheat, oat grains have both monomeric and polymeric avenins [28]. A direct correlation between the immunogenicity of the different varieties of oats and the presence of the specific peptides with a higher/lower potential immunotoxicity has been found, that could explain why certain varieties of oats are toxic for celiac patients and other not [28]. The incorporation of some varieties of oats in food products not only may improve the nutritional quality but also may provide a treatment for various illnesses and would be welcomed by patients with celiac disease. 


\subsection{Other Cereals and Pseudocereals}

It is well-known that the high nutritional value of gluten containing cereals and the viscoelastic network generated by the gluten that enables an excellent aerated structure in food products. In contrast, cereal based gluten-free products can be rich in carbohydrates and fats, and they have deficiencies in macronutrients and micronutrients. In consequence, long time adherence to GFD could induce nutrients deficiencies. Different proteins have been proposed as alternative for both playing the polymer role and increasing the nutritional value of gluten-free products. The incorporation of other ingredients/nutrients like 3-omega lipids, specific proteins, etc. is an alternative to improve the nutritional composition of gluten-free products.

Figure 2. Taxonomic relation of known non-toxic cereals, minor cereals and pseudocereals in the context of celiac disease.

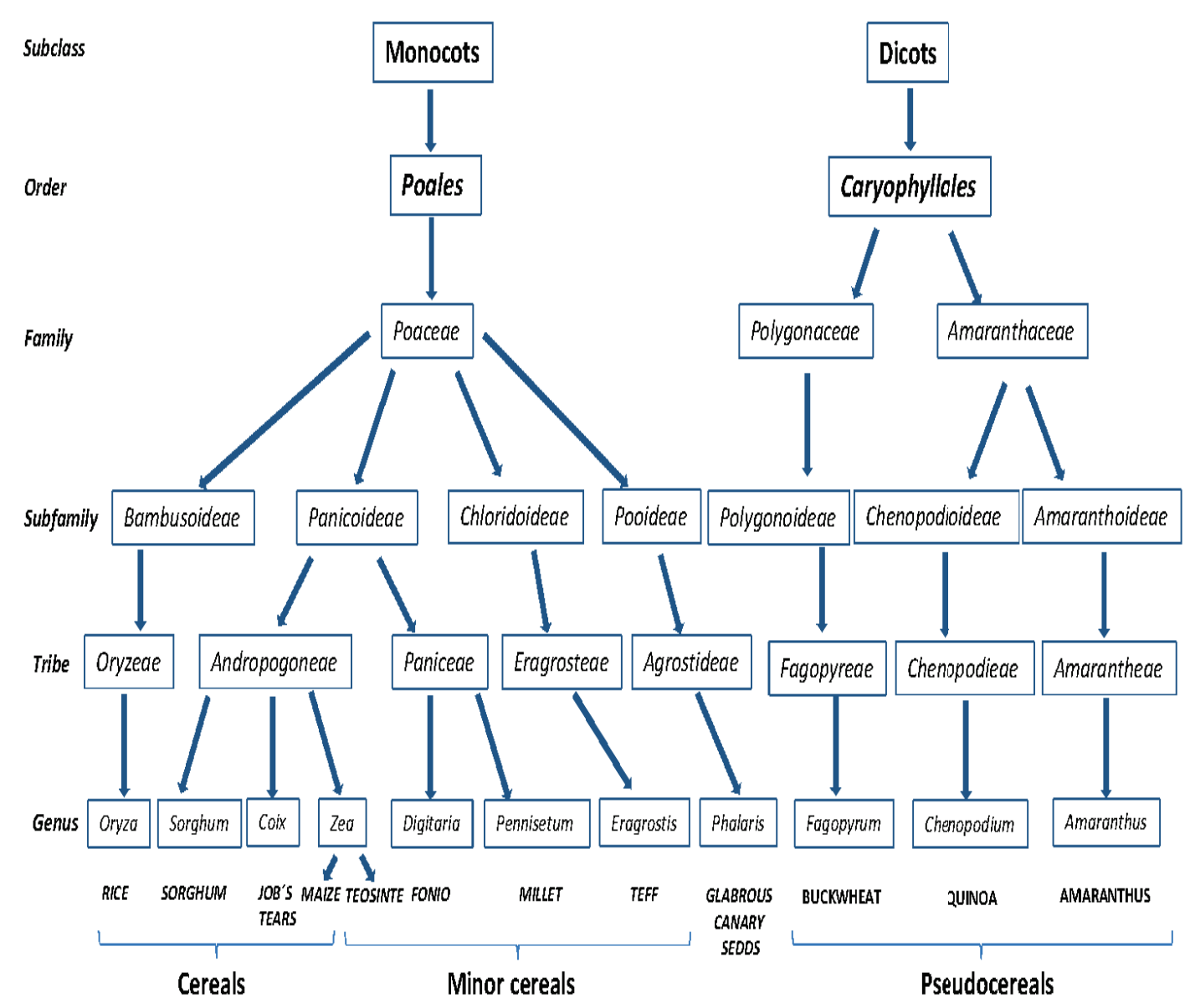

It is noteworthy that many grains (members of the grass family) that are closely related to wheat, rye and barley are considered toxic based on taxonomy. Furthermore, some studies focused on the protein homology in grains have supported molecular evidences [28,29]. However, member belonging to other tribes that appear to be related to corn, are considered safe (Figure 2) and can serve as substitutes and provide flours for cooking and baking for celiac and gluten-sensitive individuals. There are protein studies in support of this conclusion, although the studies are not sufficiently complete to provide more than guidance. 
Non-gluten-containing sources frequently used in product formulation include cereals (rice, corn and sorghum), minor cereals (fonio, teff, millet, and job's tears) and pseudocereals (buckwheat, quinoa and amaranth). As the environmental conditions for growing these grains are variable, availability of regular supplies is not always assured.

\subsubsection{Rice, Maize and Sorghum}

Rice is the seed of the monocot plant of the genus Oryza and of the grass family Poaceae (formally Graminae), which includes twenty wild species and two cultivated ones, Oryza sativa (Asian rice) and Oryza glaberrima (African rice). Rice is one of the most important foods in the human diet and extended cereal crop. Rice is mainly consumed as white grain, but in the last decade, dozens of products containing rice as an ingredient have appeared on the food market [30]. There has been a notable increase in the use of rice flour in the formulation of gluten-free products for their hypoallergenic qualities or hypoallergenicity in spite of it is necessary to use a hydrocolloid, emulsifier, enzyme or protein to confer viscoelastic properties [30,31].

Maize (Zea mays subsp. mays L), also known as corn, is considered as a safe cereal for celiac patients. It is used as alternative to elaborate gluten-free foodstuffs. Some celiac patients considered refractory to the treatment with a GFD improved when a corn-free diet was prescribed [32]. However, some studies have showed the certain maize prolamins (zeins) contain amino acid sequences that resemble the wheat gluten immunodominant peptides and their integrity after gastrointestinal proteolysis is unknown [33]. Darewickz et al. [34] detected amino acid sequences with a high degree of identity to the celiac-toxic peptides in maize prolamins (zeins). This could be because the zeins, like other storage proteins, have its origin in the alpha-amylase inhibitors [35].

Sorghum (genus of numerous species of grasses) is a drought-and heat-tolerant cereal grain that grows in semiarid conditions. Whereas sorghum traditionally has been used primarily as animal feed in western countries, nearly $40 \%$ of the world sorghum production is used for human food in Africa and India. Immunological studies and in vitro and in vivo challenges of sorghum food products have supported that sorghum might provide a good basis for gluten-free foods [36]. In a recent study, Pontieri et al. [37] by using in silico approaches and biochemical/immunochemical experiments have demonstrated that sorghum can be definitively considered safe for consumption by people with celiac disease for the absence of toxic gliadin-like peptides.

\subsubsection{Minor Cereals}

Minor cereals, so called because they are less common and are only grown in a few small regions of the world, included fonio, teff, millet, teosinte and Job's tears [38].

Fonio (Digitaria exilis) is a typically cereal in Sudan or Ethiopia where it is considered to be the tastiest of all cereals [38]. Fonio can survive in poor soil conditions such as sandy and acidic soils and its composition is similar to that of other millets: limited in lysine, but rich in methionine [39].

Teff (Eragrostis tef) is the smallest of all grains in the world and it is classified on the basis of seed color, ranging from milky white to almost black. Teff is a cereal traditionally grown in Ethiopia and used to make injera or flat bread. 
Millet refers to a number of different species of the Pennisetum genus, all of which are small-grained, annual cereal grasses. The most important type for food consumption is pearl millet that is similar in texture to rice flour [38].

Job's tears (Coix lacryma-jobi), also known as Chinese Pearl Barley, is a type of millet wild tropical Asian grass related to maize. Job's tears is naturally gluten-free, but similar to other grains it may be contaminated during processing by comingling with gluten grains such as wheat. It is used as a source of food and drinks.

\subsubsection{Pseudocereals}

Pseudocereals are non-grasses plants which grains are used in the same way that true cereals. Pseudocereals seeds can be ground into flour and then to produce derived products like bread and pasta. Recently, the use of pseudocereals producing small grain-like seeds like amaranthus and quinoa (Amaranthaceae family), belonging to dicotyledons (Magnoliopsida class), have been considered for the preparation of gluten-free food products because the lack toxic seed proteins and have high nutritional value [40]. However, the believed lack of toxicity for most of these pseudocereals was based on their taxonomical classification rather than a direct evaluation of their inmunostimulator activity.

Several studies affirmed that amaranth and quinoa have high quality protein in terms of digestibility, efficiency ratio and nutrition balance, almost equivalent to that of milk protein casein $[41,42]$. Additionally, these pseudocereals are also rich in polyunsaturated fatty acids (high linolenic:linoleic acid ratio) and bioactive compounds such as $\gamma$ - and $\beta$-tocopherol, polyphenols and flavonoids.

The genus Amaranthus L. contains more than 60 species; A. caudatus, A. cruentus and A. hypochondriacus are those most used for human nutrition. Amaranth proteins consist mainly of albumins and globulins, where prolamins, the toxic proteins for celiac patients, are very scarce. The essential amino acids content is high in amaranth seeds and the amino acid composition is better balanced than in most cereals. It is a good source of riboflavin, vitamin E, calcium, magnesium and irons, among minerals [40]. Studies focused on investigate from the molecular point of view the protein patterns from different amaranth cultivars to verify their suitability for the diet of subjects suffering from celiac disease, suggested that amaranth may be safely included in a GFD. However, controlled clinical studies are necessary to confirm the results and support the inclusion in the celiac's diet [40].

Quinoa (Chenopodium quinoa) is an Andean grain that has been consumed for thousands of years in South America and was a staple of the Incas. There are hundreds of varieties of quinoa, ranging in color from white to red and purple to black [38]. Quinoa has a high biological value (83\%) because of its high concentration of proteins $(<23 \%)$, providing all of the essential amino acids [43-45]. Quinoa has important applications in the food and pharmaceutical industries. Due to its excellent nutritional value and a potential for production in various climates, quinoa has been classified as one of the humanity's most promising crops [46]. Several studies to examine the suitability of quinoa for patients with celiac disease have been carried out in last years and concluded that quinoa could be a safe addition to a GFD. However, 2 cultivars had celiac-toxic epitopes that could activate the adaptive and innate immune responses in some patients with celiac disease [45]. A complete in vivo 
characterization of quinoa protein reactivity is needed to recommend their consumption by patients with celiac disease $[45,47,48]$.

Buckwheat (Fagopyrum spp.) is botanically classified as a fruit and it is thought to have originated in China. It can be consumed as grains or as flour. The toasted grains are known as Kasha. Buckwheat is a highly nutritious pseudocereal known as a dietary source of protein with favorable amino acid composition and vitamins [49], starch, and dietary fiber [50], essential minerals (Steadman and others 2001), and trace elements [51]. Two species of buckwheat are cultivated for food consumption, Fagopyrum esculentum or common buckwheat and Fagopyrum tartaricum or tartary buckwheat [52]. Common buckwheat is primarily consumed in Asian countries. However, consumption in western countries including the United States is increasing due to it is the substitute for wheat flour for gluten-sensitive patients and as a health food because of its nutrient content $[53,54]$. It has been reported cases of buckwheat allergy in Japan, Korea and Europe [55].

\subsubsection{Other Cereals}

An alternative grain that may potentially be considered for celiac patients is glabrous canary seed (Phalaris canarienses L.) that belongs to the Poaceae (Gramineae) family. In a recent study carried out by Boye et al. [30] confirmed that glabrous canary seeds were a good alternative gluten-free cereal and reported three techniques able to be used to support gluten-free labeling of products that contain it.

\section{Modified Harmless Cereal Varieties}

\subsection{Gluten Detoxification by Biotechnological Methods}

The use of genetic engineering to down-regulate gene expression by RNA interference (RNAi) technology [56] is now routine in many crops, including wheat, and is therefore an attractive opportunity for reducing the immunotoxic components of gluten and, hence the incidence of gluten-related allergies and intolerance in wheat. Several groups have taken advantage of the possibilities that the RNAi technology offers for the silencing of multigene families, and they have addressed the down-regulation of more than one group of gliadins and/or glutenins.

This technology was applied to down-regulated the expression of $\alpha$-gliadins [57], $\gamma$-gliadins [58,59], $\omega$-gliadins [60], all gliadins [61], and gliadins and LMW-GS [62] in bread wheat. These examples show the usefulness of RNAi to silence specific genes corresponding to gluten proteins, which are the known sources of immunogenic epitopes. However, only transgenic lines deficient in $\gamma$-gliadins [58] and in all three gliadin fractions [61] have been tested by monoclonal antibodies and $\mathrm{T}$-cell assays for these transgenic lines to be used in foodstuff tolerated by many patients with celiac disease or other gluten-related pathologies.

Results reported by Gil-Humanes et al. [58] and Piston et al. [59] used two hpRNA construct to silence the $\gamma$-gliadins in two genotypes of the bread wheat cv "Bobwhite". They reported 18 transgenic lines with reduction of the $\gamma$-gliadin fraction, from 65 to $97 \%$ depending of the transgenic line [59]. The reduction of $\gamma$-gliadins was also accompanied by an increase in other storage proteins, in particular, the $\omega$ - and $\alpha$-gliadins, and the HMW-GS and LMW-GS. However, the total gliadin 
content did not show significant differences in the transgenic lines relative to the wild types. Later, two hpRNA constructs designed using a chimeric sequence encompassing highly conserved genes among $\alpha-, \omega-$, and $\gamma$-gliadins were reported [61]. They showed that the chimeric fragment was able to effectively down-regulate the expression of genes from all three gliadin groups. The gliadin composition of the transgenic lines, determined by RP-HPLC, showed a significant reduction of the gliadin content in all of the transgenic lines, ranging from $70 \%$ to $88 \%$. Overall, the gluten proteins were decreased up to $56 \%$ while the non-gluten proteins albumins and globulins were increased in some transgenic lines [63], as consequence, the total nitrogen content of the grain was not significantly affected.

These lines hold good potential to be used in foodstuff tolerated by many patients with celiac disease or other gluten-related pathologies. The competitive ELISA system based on monoclonal antibody [64] is a good assay for quantifying the amount of gluten in foods [65]. For transgenic lines with $\gamma$-gliadins silenced $[58,61]$, gliadin content (ppm) did not decrease significantly but increased for some lines as consequence of the compensatory effects with other gliadins, specifically $\alpha$ - and $\omega$-gliadins. However, when gluten proteins from transgenic lines, deficient in all three groups of gliadins, were tested by the monoclonal antibody, there was a significant reduction of the gliadin content in all of the transgenic lines, with the average reduction of $92 \%$ and a range between $90 \%$ and 98\% [61]. Total gluten proteins were extracted for T-cell assays, treated with recombinant human TG2, and tested in serial dilution for stimulation of DQ2- and DQ8-restricted T-cell clones of celiac patients [61]. The transgenic lines deficient only in $\gamma$-gliadins retained high amounts of $\alpha$ - and $\omega$-gliadins, and the DQ2- $\gamma$-VII-specific T-cell clone gave strong response to the total gluten extract from those lines [61]. In contrast, a pronounced reduction in proliferative responses was seen in some transgenic lines deficient in all three gliadin fractions. There was about a 2-log reduction in the expression of the DQ2- $\alpha$-II epitope in these transgenic lines. The responses of the T-cell clones specific for the other epitopes (DQ2- $\gamma$-VII, DQ8- $\alpha$-I, and DQ8- $\gamma-\mathrm{I}$ ) were at or below detection level for the highest concentration of gluten protein tested. They concluded that transgenic lines containing around $30 \%$ of $\omega$-gliadin, $10 \%$ of $\alpha$-gliadin, and only $1 \%$ of $\gamma$-gliadin compared with their wild-type control, were particularly inefficient to stimulate the celiac disease lesion-derived $\mathrm{T}$ cells [61].

One important question is how quality is affected by the silencing of gliadins, or other gluten proteins. The rheological and gluten properties, and baking quality of wheat lines with the $\alpha$-gliadins down-regulated were reported [66]. They showed that the deficiency of $\alpha$-gliadins did not substantially affect the baking performance of wheat flour, although breads made using flour from lines with $\alpha$-gliadins silenced had lower volumes $(-11 \%)$ compared to that of wild type breads. The mixing properties of 18 transgenic lines with the $\gamma$-gliadins down-regulated were determined by using the mixograph and sodium dodecyl sulfate sedimentation (SDSS) test [59]. They concluded that the reduction of $\gamma$-gliadins seems not to have a direct effect on the mixing and bread-making properties of wheat dough, but the compensatory effect on the synthesis of the other prolamins can provide stronger doughs with improved overmixing resistance. Although gliadins are not the main component affecting the bread-making quality of wheat, it is unknown the effect that the silencing of all three groups of gliadins [61], or gliadins and LMW-GS [62] will have on wheat quality. Preliminary results reported [61] based on SDSS test, showed that most of transgenic lines with $\omega$-, 
$\gamma$ - and $\alpha$-gliadins down regulated had SDSS volumes comparable with those of wild types, and five lines had SDSS volume values significantly lower than wild types. However, SDSS volumes of these five lines were still comparable with those of the medium-quality bread wheat. The addition of non-toxic oat flour [67], or other flours from gluten-free cereals or pseudocereals, such as sorghum, buckwheat and quinoa [37,68], might compensate the lack of gliadins, enhancing the quality of the new wheat varieties.

\subsection{Gluten Detoxification by Enzymatic Methods}

Food proteins are usually degraded into small peptides and amino acids by gastric, pancreatic and brushborder enzymes. However, gluten proteins are highly resistant to complete proteolytic digestion due to their high proline and glutamine content. Since the pioneering experiments carried out by Frazer et al. [69], who determined that celiac-toxic proteins could be partially hydrolyzed by gastrointestinal enzymes without loss toxicity, several strategies have been considered for detoxification of dietary gluten. Some of these strategies have been based on treatment with special peptidases that hydrolyze toxic protein and peptides to nontoxic fragments.

The beginning for the enzymatic strategies was the findings that the toxicity was abolished by complete acidic hydrolysis [70]. However, researches about gluten detoxification were not developed until 21 st century. The approaches included enzymatic cleavage of gliadin fragment by PEP from different organisms, degradation of toxic peptides by germinating cereal enzymes and transamidation of cereal flours [5].

\subsubsection{Prolyl Endopeptidases}

Shan et al. [71] were the first in propose that PEPs could catalyze breakdown of gluten peptides and thereby diminish its toxic effects. This hypothesis was based on that the abundance and location of proline residues is a crucial factor for the gastrointestinal resistance, and the unique ability of these enzymes to hydrolyze the peptide bond on the carboxyl side of a proline residue. Since then, further studies have shown that the fermentation of wheat, rye and barley flours with selected peptidases cause a significant decrease of gluten toxicity. The PEPs are widely distributed in bacteria, fungi, animals and plants, but it is known that lactic acid bacteria (lactobacilli) have a very complex peptidase system [72]. Lactobacilli species isolated from sourdoughs have been screened with respect to gluten degradation, finding that a pool of peptidases is needed to degraded $\alpha$-gliadin fragments [73]. Studies based on use lactobacillus as microbial inoculum during fermentation of flour mixture has shown a potential ability to hydrolyze wheat prolamins by in vitro and in vivo assays [74]. Despite that, the gluten concentration remains high, therefore studies based on more complex formulas were developed. Recently, the combination of lactobacilli and fungal peptidases has been selected to eliminate the toxicity of wheat flour during long-time fermentation. Thus, food processing by selected proteases opens new perspectives toward an efficient approach to eliminate gluten toxicity. 


\subsubsection{Germinating Cereals}

The role of the proline- and glutamine-rich storage proteins of cereals is to supply the embryo with nitrogen and amino acids during the first period of seedling development. Therefore, it is likely that endogenous cereal proteases synthesized during germination would be capable of extensively hydrolyzing these proteins [75]. Given evidences about this capacity, the use of proteases from germinating wheat seeds was proposed to create safe cereal products for celiac patients [76,77]. The analysis of protein content by RP-HPLC during kernels germination of wheat, rye and barley demonstrated a remarkable degradation of prolamins. In further experiments, protease extracts from these germinated cereals cleave peptides rapidly into non-toxic fragments with less than nine amino acids [75]. Comparative studies of proteases efficacy from different cereals by in vitro models have revealed that barley enzymes were superior in diminish the toxicity of gliadin and secalin, but there were only minor differences between the three enzyme mixtures (oats, wheat and barley) [78]. On the other hand, it should be pointed out that germinating cereal proteases have distinct advantages in comparison to bacterial and fungal peptidases. These enzymes derive from a naturally safe food source being excellent alternatives to recombinant proteases, which might not be accepted by many celiac patients. Indeed, the production of germinated cereals, just like the extraction of highly active proteases, is simple and well-established technological process [75]. Altogether, these enzymes from germinating cereals might be utilized in food processing to develop high quality food safe for celiac patients.

\subsubsection{Transamidation}

The enzyme transglutaminase (TG) catalyzes two classes of reactions, transamidation and deamidation. The first one transforms the primary $\gamma$-carboxamide group to a secondary one, and in the second reaction, the glutaminyl residue is converted to a glutamyl residue [79]. Tissue transglutaminase 2 (tTG) has been describe as one of the key factor in the immunopathogenesis of celiac disease because of gluten peptides increase their immunogenicity due to the deamidation [80]. Considering that only the transamidation might be use for gluten detoxification, several studies have been focused to know the ratio between these two reactions [81,82]. It seems that the ratio can vary considerably depending on different factors like the presence of primary amines, the peptide sequence as well as enzyme concentration. In general terms, it has been found highest rate of deamidation in tTG, however, the few studies carry out with TGs from other origins have shown a considerably lower deamidation versus transamidation activity in microbial TG (mTG) $[83,84]$. Based on that, Gianfrani et al. [85] treated wheat flour with $\mathrm{mTG}$ and lysine methyl ester to abolish gluten activity, detecting the decrease of the activity mediated by T-cell. Thus, they suggested a food-grade enzyme and an appropriate amine donor to block the $\mathrm{T}$ cell-mediated gliadin activity [85]. Recently, Mazzarella et al. [86] has shown in a randomized single blinded trial that transamidated gluten reduced the number of clinical relapses in challenged patients with no changes of baseline values for serological/mucosal celiac markers and an unaltered kidney function. Other application has been use the mTG from $S$. mobaraensis to detoxify cereal-based beverages since the beer treatment with mTG will lead to crosslinking of residual gluten peptide. If that aggregate exceeds a certain MW, they lose their solubility and can be removed from the beer resulting in 
beverages with gluten content below $20 \mathrm{mg} / \mathrm{kg}$ [87]. Therefore, although $\mathrm{mTG}$ is not able to degrade gluten, it could be used to immunodetoxify gluten.

\section{Conclusions}

Currently the only treatment for celiac disease is a lifelong GFD. However, adherence to the GFD is not easy, due to the ubiquitous nature of gluten, cross-contamination of foods and social constraints. While many patients are content with their GFD, others would welcome alternative treatments and/or food products that would allow more flexibility.

Here, we review the status of potential alternative cereals and pseudocereals and their derivatives under consideration for celiac disease. Diploid wheat species appear to be among the suitable candidates for their low capability to activate intestinal $\mathrm{T}$ cell responses in celiac patients. Compared with tetraploid and hexaploid wheats commonly used in the making of bread and pasta, the ancient diploid Triticum monococcum ssp. monococcum wheat showed a marked reduction of toxicity in vitro assays. Moreover, the use of genetic engineering to down-regulate gene expression by RNAi technology represents an attractive opportunity for reducing the immunotoxic components of wheat. Simultaneous silencing of the full complement of gliadins results effective for the reduction of T-cell epitopes in celiac disease.

Several studies have been demonstrated that oat immunogenicity for patients with celiac disease varies according to the cultivars. The incorporation of some varieties of oats in food products not only may improve the nutritional quality, but may provide a treatment for various illnesses and would be welcomed by patients with celiac disease.

Non-gluten-containing sources frequently used in product formulation include cereals (rice, corn and sorghum), minor cereals (fonio, teff, millet, and job's tears) and pseudocereals (buckwheat, quinoa and amaranth). However, new studies seem to show that certain cereals and pseudocereals such as corn and quinoa, traditionally considered safe for celiac patients, could activate the immune response in some celiac patients.

More recently, studies have been showed several strategies for detoxification of dietary gluten based on treatment with special peptidases that hydrolyze toxic protein and peptides to nontoxic fragments. These included enzymatic cleavage of gliadin fragment by PEPs from different organisms, degradation of toxic peptides by germinating cereal enzymes and transamidation of cereal flours. Food processing by selected proteases opens new perspectives toward an efficient approach to eliminate gluten toxicity, which could allow the development of foods with reduced or absent levels of gluten.

\section{Acknowledgments}

This work was supported by the Spanish Ministry of Economy and Competitiveness (AGL2010-19643-C02-02 and TRA2009_0047), the European Regional Development Fund (FEDER) and Junta de Andalucía (Project P09AGR-4783).

\section{Conflicts of Interest}

The authors declare no conflict of interest. 


\section{References}

1. Sollid, L.M. Coeliac disease: Dissecting a complex inflammatory disorder. Nat. Rev. Immunol. 2002, 2, 647-655.

2. Bernardo, D.; Peña, A.S. Developing strategies to improve the quality of life of patients with gluten intolerance in patients with and without coeliac disease. Eur. J. Intern. Med. 2012, 23, $6-8$.

3. Immune Epitope Database and Analysis Resourse. Available online: http://www.iedb.org/ (accessed on 14 October 2013).

4. Corrao, G.; Corazza, G.R.; Bagnardi, V.; Brusco, G.; Ciacci, C.; Cottone, M.; Sategna Guidetti, C.; Usai, P.; Cesari, P.; Pelli, M.A.; et al. Mortality in patients with coeliac disease and their relatives: A cohort study. Lancet 2001, 358, 356-361.

5. Rashtak, S.; Murray, J.A. Review article: Coeliac disease, new approaches to therapy. Aliment. Pharmacol. Ther. 2012, 35, 768-781.

6. Feldman, M.; Levy, A.A. Genome evolution in allopolyploid wheat-A revolutionary reprogramming followed by gradual changes. J. Genet. Genomics 2009, 36, 511-518.

7. Marietta, E.V.; Murray, J.A. Testing the safety of alternative wheat species and cultivars for consumption by celiac patients. Am. J. Clin. Nutr. 2012, 96, 1247-1248.

8. Van Slageren, M.W. Wild wheats: A Monograph of Aegilops L. and Amblyopyrum (Jaub. \& Spach) Eig (Poaceae); Wageningen Agriculture University: Wageningen, The Netherlands, 1994.

9. Auricchio, S.; de Ritis, G.; de Vincenzi, M.; Occorsio, P.; Silano, V. Effects of gliadin-derived peptides from bread and durum wheats on small intestine cultures from rat fetus and coeliac children. Pediatr. Res. 1982, 16, 1004-1010.

10. Van Herpen, T.W.; Goryunova, S.V.; van der Schoot, J.; Mitreva, M.; Salentijn, E.; Vorst, O.; Schenk, M.F.; van Veelen, P.A.; Koning, F.; van Soest, L.J.; et al. Alpha-Gliadin genes from the $\mathrm{A}, \mathrm{B}$, and $\mathrm{D}$ genomes of wheat contain different sets of celiac disease epitopes. BMC Genomics 2006, 10, 7-11.

11. Spaenij-Dekking, L.; Kooy-Winkelaar, Y.; van Veelen, P.; Drijfhout, J.W.; Jonker, H.; van Soest, L.; Smulders, M.J.; Bosch, D.; Gilissen, L.J.; Koning, F. Natural variation in toxicity of wheat: Potential for selection of nontoxic varieties for celiac disease patients. Gastroenterology 2005, 129, 797-806.

12. Molberg, O.; Uhlen, A.K.; Jensen, T.; Flaete, N.S.; Fleckenstein, B.; Arentz-Hansen, H.; Raki, M.; Lundin, K.E.; Sollid, L.M. Mapping of gluten T-cell epitopes in the bread wheat ancestors: Implications for celiac disease. Gastroenterology 2005, 128, 393-401.

13. Pizzuti, D.; Buda, A.; D’Odorico, A.; D’Incà, R.; Chiarelli, S.; Curioni, A.; Martines, D. Lack of intestinal mucosal toxicity of Triticum monococcum in coeliac disease patients. Scand. J. Gastroenterol. 2006, 41, 1305-1311.

14. Vincentini, O.; Maialetti, F.; Gazza, L.; Silano, M.; Dessi, M.; de Vincenzi, M.; Pogna, N.E. Environmental factors of coeliac disease: Cytotoxicity of hulled wheat species Triticum monococcum, T. turgidum ssp. dicoccum and T. aestivum ssp. spelta. J. Gastroenterol. Hepatol. 2007, 22, 1816-1822. 
15. Gianfrani, C.; Maglio, M.; Rotondi Aufiero, V.; Camarca, A.; Vocca, I.; Iaquinto, G.; Giardullo, N.; Pogna, N.; Troncone, R.; Auricchio, S.; et al. Immunogenicity of monococcum wheat in celiac patients. Am. J. Clin. Nutr. 2012, 96, 1339-1345.

16. Tanner, G.J.; Howitt, C.A.; Forrester, R.I.; Campbell, P.M.; Tye-Din, J.A.; Anderson, R.P. Dissecting the T-cell response to hordeins in coeliac disease can develop barley with reduced immunotoxicity. Aliment. Pharmacol. Ther. 2010, 32, 1184-1191.

17. Tanner, G.J.; Blundell, M.J.; Colgrave, M.L.; Howitt, C.A. Quantification of hordeins by ELISA: The correct standard makes a magnitude of difference. PLoS One 2013, 8, e56456.

18. Comino, I.; Real, A.; Gil-Humanes, J.; Pistón, F.; de Lorenzo, L.; Moreno, M.L.; López-Casado, M.Á.; Lorite, P.; Cebolla, A.; Torres, M.I.; et al. Significant differences in coeliac immunotoxicity of barley varieties. Mol. Nutr. Food. Res. 2012, 56, 1697-1707.

19. Suttie, J.M.; Reynolds, S.G. Fodder oats: A world overview, 2004. Food and Agriculture Organization of the United Nations (FAO). Available online: http://www.fao.org/docrep/008/ y5765e/y57 65e00.htm (accessed on 16 October 2012).

20. Eppendorfer, W.H. Nutritive value of oat and rye grain protein as influenced by nitrogen and amino acid composition. J. Sci. Food Agric. 2006, 28, 152-156.

21. Pulido, O.; Gillespie, Z.; Zarkadas, M.; Dubois, S.; Vavasour, E.; Rashid, M.; Switzer, C.; Godefroy, S.B. Introduction of oats in the diet of individuals with coeliac disease: A systematic review. Adv. Food Nutr. Res. 2009, 57, 235-285.

22. Codex Alimentarius International Food Standars. Available online: http://www.codexalimentarius.net/web/more_info.jsp?id_sta=291 (accessed on 28 August 2013).

23. COMMISSION REGULATION (EC) No 41/2009 of 20 January 2009 Concerning the Composition and Labelling of Foodstuffs Suitable for People Intolerant to Gluten. Available online: http://eur-lex.europa.eu/LexUriServ/LexUriServ.do?uri=OJ:L:2009:016:0003:0005:EN:PDF (accessed on 21 January 2009).

24. Arentz-Hansen, H.; Fleckenstein, B.; Molberg, Ø.; Scott, H.; Koning, F.; Jung, G.; Roepstorff, P.; Lundin, K.E.; Sollid, L.M. The molecular basis for oat intolerance in patients with coeliac disease. PLoS Med. 2004, 1, 84-92.

25. Silano, M.; di Benedetto, R.; Maialetti, F.; de Vincenzi, A.; Calcaterra, R.; Cornell, H.J.; de Vincenzi, M. Avenins from different cultivars of oats elicit response by coeliac peripheral lymphocytes. Scand. J. Gastroenterol. 2007, 42, 1302-1305.

26. Maglio, M.; Mazzarella, G.; Barone, M.V.; Gianfrani, C.; Pogna, N.; Gazza, L.; Stefanile, R.; Camarca, A.; Colicchio, B.; Nanayakkara, M.; et al. Immunogenicity of two oat varieties, in relation to their safety for celiac patients. Scand. J. Gastroenterol. 2011, 46, 1194-1205.

27. Comino, I.; Real, A.; de Lorenzo, L.; Cornell, H.; López-Casado, M.Á.; Barro, F.; Lorite, P.; Torres, M.I.; Cebolla, A.; Sousa, C. Diversity in oat potential immunogenicity: Basis for the selection of oat varieties with no toxicity in celiac disease. Gut 2011, 60, 915-922.

28. Real, A.; Comino, I.; de Lorenzo, L.; Merchán, F.; Gil-Humanes, J.; Giménez, M.J.; López-Casado, M.Á.; Torres, M.I.; Cebolla, Á.; Sousa, C.; et al. Molecular and immunological characterization of gluten proteins isolated from oat cultivars that differ in toxicity for celiac disease. PLoS One 2012, 7, e48365. 
29. Tye-Din, J.A.; Stewart, J.A.; Dromey, J.A.; Beissbarth, T.; van Heel, D.A.; Tatham, A.; Henderson, K.; Mannering, S.I.; Gianfrani, C.; Jewell, D.P.; et al. Comprehensive, quantitative mapping of T cell epitopes in gluten in celiac disease. Sci. Transl. Med. 2010, 2, 41 ra51.

30. Rosell, C.M.; Marco, C. Rice. In Gluten Free Cereal Products and Beverages; Arendt, E.K., dal Bello, F., Eds.; Elsevier Science: London, UK, 2008; pp. 81-100.

31. Boye, J.I.; Achouri, A.; Raymond, N.; Cleroux, C.; Weber, D.; Koerner, T.B.; Hucl, P.; Patterson, C.A. Analysis of Glabrous canary seeds by ELISA, Mass spectrometry, and western blotting for the absence of cross-reactivity with major plant food allergens. J. Agric. Food Chem. 2013, 61, 6102-6112.

32. Accomando, S.; Albino, C.; Montaperto, D.; Amato, G.M.; Corsello, G. Multiple food intolerance or refractory celiac sprue? Dig. Liver Dis. 2006, 38, 784-785.

33. Cabrera-Chávez, F.; Iamett, S.; Miriani, M.; Calderón de la Barca, A.M.; Mamone, G.; Bonomi, F. Maize prolamins resistant to peptic-tryptic digestion maintain immune-recognition by IgA from some celiac disease patients. Plant Foods. Hum. Nutr. 2012, 67, 24-30.

34. Darewicz, M.; Dziuba, J.; Minkiewicz, P. Computational characterization and identification of peptides for in silico detection of potentially celiac-toxic proteins. Food Sci. Technol. Int. 2007, 13, 125-133.

35. Shewry, P.R.; Napier, J.A.; Tatham, A.S. Seed storage proteins: Structures and biosynthesis. Plant Cell 1995, 7, 945.

36. Ciacci, C.; Maiuri, L.; Caporaso, N.; Bucci, C.; del Giudice, L.; Rita Massardo, D.; Pontieri, P.; di Fonzo, N.; Bean, S.R.; Ioerger, B.; et al. Celiac disease: In vitro and in vivo safety and palatability of wheat-free sorghum food products. Clin. Nutr. 2007, 26, 799-805.

37. Pontieri, P.; Mamone, G.; de Caro, S.; Tuinstra, M.R.; Roemer, E.; Okot, J.; de Vita, P.; Ficco, D.B.; Alifano, P.; Pignone, D.; et al. Sorghum, a healthy and gluten-free food for celiac patients as demonstrated by genome, biochemical, and immunochemical analyses. J. Agric. Food. Chem. 2013, 61, 2565-2571.

38. Saturni, L.; Ferretti, G.; Bacchetti, T. The gluten-free diet: Safety and nutritional quality. Nutrients 2010, 2, 16-34.

39. Arendt, E.K.; dal Bello, F. Gluten-Free Cereal, Products and Beverages (Food Science and Technology); Academic press: London, UK, 2011.

40. Ballabio, C.; Uberti, F.; di Lorenzo, C.; Brandolini, A.; Penas, E.; Restani, P. Biochemical and immunochemical characterization of different varieties of amaranth (Amaranthus L. ssp.) as a safe ingredient for gluten-free products. J. Agric. Food. Chem. 2011, 59, 12969-12974.

41. Ranhotra, G.S.; Gelroth, J.A.; Glaser, B.K.; Lorenz, K.J.; Johnson, D.L. Composition and protein nutritional quality of quinoa. Cereal. Chem. 1993, 70, 303-305.

42. Repo-Carrasco, R.; Esponiza, C.; Jacobsen, S.-E. Nutritional value and use of the Andean crops: Quinoa (Chenopodium quinoa) and kañiwa (Chenopodium pallidicaule). Food Rev. Int. 2003, 19, 179-189.

43. Abugoch, J.L.E. Quinoa (Chenopodium quinoa Wild.): Composition, Chemistry, Nutritional, and Functional Properties. In Advances in Food and Nutrition Research; Taylor, S.L., Ed.; Academic Press: Lincoln, NE, USA, 2009; Volume 58, pp. 1-31. 
44. Gonzalez, J.A.; Konishi, Y.; Bruno, M.; Valoy, M.; Prado, F.E. Interrelationships among seed yield, total protein and amino acid composition of ten quinoa (Chenopodium quinoa) cultivars from two different agroecological regions. J. Sci. Food Agric. 2012, 92, 1222-1229.

45. Zevallos, V.F.; Ellis, H.J.; Suligoj, T.; Herencia, L.I.; Ciclitira, P.J. Variable activation of immune response by quinoa (Chenopodium quinoa Wild.) prolamins in celiac disease. Am. J. Clin. Nutr. 2012, 96, 337-344.

46. Mäkinen, O.E.; Zannini, E.; Arendt, E.K. Germination of oat and quinoa and evaluation of the malts as gluten free baking ingredients. Plant Foods Hum. Nutr. 2013, 68, 90-95.

47. De Vincenzi, M.; Silano, M.; Luchetti, R.; Carratu, B.; Boniglia, C.; Pogna, N.E. Agglutinating activity of alcohol-soluble proteins from quinoa seed flour in celiac disease. Plant Foods Hum. Nutr. 1999, 54, 93-100.

48. Berti, C.; Ballabio, C.; Restani, P.; Porrini, M.; Bonomi, F.; Iametti, S. Immunochemical and molecular properties of proteins in Chenopodium quinoa. Cereal Chem. 2004, 81, 275-277.

49. Bonafaccia, G.; Marocchini, M.; Kreft, I. Composition and technological properties of the flour and bran from common and tartary buckwheat. Food Chem. 2003, 80, 9-15.

50. Skrabanja, V.; Kreft, I.; Golob, T.; Modic, M.; Ikeda, S.; Ikeda, K.; Kreft, S.; Bonafaccia, G.; Knapp, M.; Kosmelj, K. Nutrient content in buckwheat milling fractions. Cereal Chem. 2004, 81, 172-176.

51. Bonafaccia, G.; Gambelli, L.; Fabjan, N.; Kreft, I. Trace elements in flour and bran from common and Tartary buckwheat. Food Chem. 2003, 83, 1-5.

52. Ikeda, K. Buckwheat: Composition, chemistry and processing. Adv. Food Nutr. Res. 2002, 44, 395-434.

53. Stember, R.H. Buckwheat allergy. Allergy Asthma Proc. 2006, 27, 393-395.

54. Wieslander, G. Review on buckwheat allergy. Allergy 1996, 51, 661-665.

55. Panda, R.; Taylor, S.L.; Goodman, R.E. Development of a Sandwich Enzyme-Linked Immunosorbent Assay (ELISA) for detection of buckwheat residues in food. J. Food Sci. 2010, 75, 110-117.

56. Watanabe, Y. Overview of Plant RNAi. In Methods in Molecular Biology; Kodama, H., Komamine, A., Eds.; Humana Press: Totowa, NJ, USA, 2011; Volume 744, pp. 1-11.

57. Becker, D.; Wieser, H.; Koehler, P.; Folck, A.; Mühling, K.H.; Zörb, C. Protein composition and techno-functional properties of transgenic wheat with reduced $\alpha$-gliadin content obtained by RNA interference. J. Appl. Bot. Food Qual. 2012, 85, 23.

58. Gil-Humanes, J.; Pistón, F.; Hernando, A.; Álvarez, J.B.; Shewry, P.R.; Barro, F. Silencing of $\gamma$-gliadins by RNA interference (RNAi) in bread wheat. J. Cereal Sci. 2008, 48, 565-568.

59. Pistón, F.; Gil-Humanes, J.; Rodríguez-Quijano, M.; Barro, F. Down-Regulating $\gamma$-gliadins in bread wheat leads to non-specific increases in other gluten proteins and has no major effect on dough gluten strength. PLoS One 2011, 6, e24754.

60. Altenbach, S.B.; Allen, P.V. Transformation of the US bread wheat "Butte 86 " and silencing of omega-5 gliadin genes. GM Crops 2011, 2, 66-73.

61. Gil-Humanes, J.; Pistón, F.; Tollefsen, S.; Sollid, L.M.; Barro, F. Effective shutdown in the expression of celiac disease-related wheat gliadin T-cell epitopes by RNA interference. Proc. Natl. Acad. Sci. USA 2010, 107, 17023-17028. 
62. Wen, S.; Wen, N.; Pang, J.; Langen, G.; Brew-Appiah, R.A.T.; Mejias, J.H.; Osorio, C.; Yang, M.; Gemini, R.; Moehs, C.P.; et al. Structural genes of wheat and barley 5-methylcytosine DNA glycosylases and their potential applications for human health. Proc. Natl. Acad. Sci. USA 2012, 109, 20543-20548.

63. Gil-Humanes, J.; Pistón, F.; Shewry, P.R.; Tosi, P.; Barro, F. Suppression of gliadins results in altered protein body morphology in wheat. J. Exp. Bot. 2011, 62, 4203-4213.

64. Valdés, I.; García, E.; Llorente, M.; Méndez, E. Innovative approach to low-level gluten determination in foods using a novel sandwich enzyme-linked immunosorbent assay protocol. Eur. J. Gastroenterol. Hepatol. 2003, 15, 465-747.

65. Codex Alimentarius International Food Standars. Available online: http://www.codexalimentarius.org (accessed on 28 August 2013).

66. Wieser, H.; Koehler, P.; Folck, A.; Becker, D. Characterization of Wheat with Strongly Reduced $\alpha$-Gliadin Content. In Gluten Proteins; Lookhart, L.G., Ng, W.P.K., Eds.; AACC International: St Paul, MN, USA, 2006; pp. 13-16.

67. Van den Broeck, H.C.; Gilissen, L.J.W.J.; Smulders, M.J.M.; van der Meer, I.M.; Hamer, R.J. Dough quality of bread wheat lacking alpha-gliadins with celiac disease epitopes and addition of celiac-safe avenins to improve dough quality. J. Cereal Sci. 2011, 53, 206-216.

68. Alvarez-Jubete, L.; Auty, M.; Arendt, E.K.; Gallagher, E. Baking properties and microstructure of pseudocereal flours in gluten-free bread formulations. Eur. Food Res. Technol. 2010, 230, 437-445.

69. Frazer, A.C.; Fletcher, R.F.; Ross, C.A.C.; Shaw, B.; Sammons, H.G.; Schneider, R. Gluten-Induce enteropathy the effect of partially digested gluten. Lancet 1959, 2, 252-255.

70. Van de Kamer, J.H.; Weijers, H.A. Celiac disease. V. Some experiments on the cause of the harmful effect of wheat gliadin. Acta Pediatr. Scand. 1955, 44, 465-469.

71. Shan, L.; Molberg, Ø.; Parrot, I.; Hausch, F.; Gray, G.M.; Sollid, L.M.; Khosla, C. Structural basis for gluten intolerance in celiac sprue. Science 2002, 297, 2275-2279.

72. Di Cagno, R.; de Angelis, M.; Lavermicocca, P.; de Vincenzi, M.; Giovanini, C.; Faccia, M.; Gobbetti, M. Proteolysis by sourdough lactic acid bacteria: Effects on wheat flour protein fractions and gliadin peptides involved in human cereal intolerance. Appl. Environ. Microbiol. 2002, 68, 623-633.

73. Gerez, C.L.; Font de Valdez, G.; Rollan, G.C. Functionality of lactic acid bacteria peptidase activities in the hydrolysis of gliadin-like fragments. Appl. Microbiol. 2008, 47, 427-432.

74. Di Cagno, R.; de Angelis, M.; Auricchio, S.; Greco, L.; Clarke, C.; de Vincenzi, M.; Giovanini, C.; D’Archivio, M.; Landolfo, F.; Parrilli, G.; et al. Sourdough bread made from wheat and nontoxic flours and started with selected lactobacilli is tolerated in celiac sprue patients. Appl. Environ. Microbiol. 2004, 70, 1088-1096.

75. Hartmann, G.; Koehler, P.; Wieser, H. Rapid degradation of gliadin peptides toxic for celiac disease patients by proteases from germinating cereals. J. Cereal Sci. 2006, 44, 368-371.

76. Kiyosaki, T.; Matsumoto, I.; Asakura, T.; Funaki, J.; Kuroda, M.; Misaka, T.; Arai, S.; Abe, K. Gliadain, a gibberellin-inducible cysteine proteinase occurring in germinating seeds of wheat, Triticum aestivum L., specifically digests gliadin and is regulated by intrinsic cystatins. FEBS J. 2007, 274, 1908-1917. 
77. Michalcova, E.; Potoka, E.; Chmelova, D.; Ondrejovic, M. Study of wheat protein degradation during germination. J. Microbiol. Biotechnol. 2012, 1, 1439-1447.

78. Stenman, S.M.; Lindfors, K.; Venäläinen, J.I.; Hautala, A.; Mänistö, P.T.; Garcia-Horsman, J.A.; Kaukorvita-Norja, A.; Auriola, S.; Mauriala, T.; Mäki, M.; et al. Degradation of celiac disease-inducing rye secalin by germinating cereal enzymes: Diminishing toxic effects in intestinal epithelial cells. Clin. Exp. Immunol. 2010, 161, 242-249.

79. Wieser, H.; Koehler, P. Detoxification of gluten by means of enzymatic treatment. J. AOAC Int. 2012, 95, 356-363.

80. Van de Wal, Y.; Kooy, Y.; van Veelen, P.A.; Pena, S.; Mearin, L.; Papadopoulus, G.; Koning, F. Selective deamidation by tissue transglutaminase strongly enhances gliadin-specific $\mathrm{T}$ cell reactivity. J. Immunol. 1998, 15, 1585-1588.

81. Skovbjerg, H.; Koch, C.; Anthonsen, D.; Sjoestroem, H. Deamidation and cross-linking of gliadin peptides by transglutaminases and the relation to celiac disease. Biochim. Biophys. Acta 2004, 1690, 220-230.

82. Stamnaes, J.; Fleckenstein, B.; Sollid, L.M. The propensity for deamidation and transamidation of peptides by transglutaminase 2 is dependent on substrate affinity and reaction conditions. Biochim. Biophys. Acta 2008, 1784, 1804-1811.

83. Ohtsuka, T.; Umezawa, Y.; Nio, N.; Kubota, K. Comparison of deamidation activity of transglutaminases. J. Cereal Sci. 2001, 66, 25-29.

84. Nonaka, M.; Sawa, A.; Matsuura, Y.; Motoki, M.; Nio, N. Deamidation of several food proteins using free and immobilized $\mathrm{Ca}^{2+}$-independent microbial transglutaminase. Biosci. Biotechnol. Biochem. 1996, 60, 532-533.

85. Gianfrani, C.; Siciliano, R.A.; Facchiano, A.M.; Camarca, A.; Mazzeo, M.F.; Costantini, S.; Salvati, V.M.; Maurano, F.; Mazzarella, G.; Iaquinto, G.; et al. Transamidation of wheat flour inhibits the response to gliadin of intestinal T cells in celiac disease. Gastroenterology 2007, 133, 780-789.

86. Mazzarella, G.; Salvati, V.M.; Laquinto, G.; Stefanile, R.; Capobianco, F.; Luongo, D.; Bergamo, P.; Maurano, F.; Giardullo, N.; Malamisura, B.; et al. Reintroduction of gluten following flour transamidation in adult celiac patients: A randomized, controlled clinical study. Clin. Dev. Immunol. 2012, 2012, 329150.

87. Pasternack, R.; Marx, S.; Jordan, D. Prolamin-Reduced Beverages and Methods for the Preparation Thereof. Int. Pat. WO 2006051093 A1, 18 May 2006. 
Reprinted from Nutrients. Cite as: Krupa-Kozak, U.; Bączek, N.; Rosell, C.M. Application of Dairy Proteins as Technological and Nutritional Improvers of Calcium-Supplemented Gluten-Free Bread. Nutrients 2013, 5, 4503-4520.

Article

\title{
Application of Dairy Proteins as Technological and Nutritional Improvers of Calcium-Supplemented Gluten-Free Bread
}

\author{
Urszula Krupa-Kozak $^{1, *}$, Natalia Bączek ${ }^{1,2}$ and Cristina M. Rosell ${ }^{2}$
}

1 Department of Chemistry and Biodynamics of Food, Institute of Animal Reproduction and Food Research of the Polish Academy of Sciences, Tuwima St., 10, Olsztyn 10-748, Poland;

E-Mail: n.baczek@pan.olsztyn.pl

2 Cereal Group, Institute of Agrochemistry and Food Technology (IATA-CSIC), Av Agustin Escardino 7, Paterna, Valencia 46980, Spain; E-Mail: crosell@iata.csic.es

* Author to whom correspondence should be addressed; E-Mail: u.krupa-kozak@pan.olsztyn.pl; Tel.: +48-89-5234-618; Fax: +48-89-5240-124.

Received: 29 August 2013; in revised form: 29 October 2013 / Accepted: 4 November 2013 / Published: 14 November 2013

\begin{abstract}
Effect of dairy proteins on gluten-free dough behavior, and nutritional and technological properties of gluten-free bread was evaluated. Experimental doughs, containing dairy powders, showed low consistency. Obtained gluten-free breads were rich in proteins, and, regarding the energy value delivered by proteins, they could be considered as a source of proteins or high in proteins. Applied dairy proteins affected the technological properties of experimental breads causing a significant $(p<0.05)$ increase of the specific volume, crust darkening, and crumb lightness, depending on the dairy supplementation level, rather than the protein type. Dairy proteins incorporated at a $12 \%$ level, significantly $(p<0.05)$ decreased the hardness; nevertheless, the highest amount of proteins tested led to the opposite effect. These results indicate that milk proteins tested could be successfully added to gluten-free bread with beneficial effects on technological and nutritional properties.
\end{abstract}

Keywords: dairy proteins; gluten-free bread; dough consistency; technological properties; nutritional value; celiac disease 


\section{Introduction}

Celiac disease (CD) is a chronic immune-mediated intestinal disorder that develops in individuals having genetic predispositions with multiple contributing genes. The most important are HLA-DQ2 and HLA-DQ8, however, non-HLA genes also contribute to the development of CD. Approximately $1 \%$ of the worldwide population is suffering from $\mathrm{CD}$, and, thus, this disorder is classified as one of the most common food intolerances [1,2]. CD is related to permanent intolerance to gluten, a storage protein found in wheat (gliadin), rye (secalins), barley (hordeins), and, probably, in some oat (avenins) cultivars. A great deal is known on the sequential pathophysiological events driving the intestinal inflammatory cascade [3-5] The immune response in CD involves the adaptive, as well as the innate, and is characterized by the presence of anti-gluten and anti-transglutaminase 2 antibodies, lymphocytic infiltration in the epithelial membrane and the lamina propria, and expression of multiple cytokines and other signaling proteins. The disease leads to inflammation, villous atrophy, and crypt hyperplasia in the small intestine. Mentioned factors can contribute to malabsorption of several nutrients (iron, folic acid, calcium, and fat-soluble vitamins) [6], general malnutrition, and reduced body mass index (BMI) [7]. Currently, strict and life-long adherence to a gluten-free diet (GFD) remains the only effective treatment for CD.

Generally, gluten-free formulas and baked products are poor in proteins [8]. In traditional baking industry proteins derived from plants (proteins of soya) and animal origin (milk proteins and egg albumins) are frequently used [9,10]. Milk proteins are highly functional ingredients characterized by a significant nutritional value. They swell in a high level and are able to build up a network [11,12]. Next to the functional benefits, gluten-free products with milk proteins are affluent in calcium and proteins, and, thus, enriched in essential amino acids like lysine, methionine and tryptophan [13]. Milk proteins can be successfully added to gluten-free products with beneficial effects on the technological properties. Caseinates are good emulsifiers and stabilize the batter; isolated and concentrated whey proteins can form gels; high temperature skim milk powder exhibits high water-binding capacity [10]. Whey proteins increased the specific volume and decreased bread crumb hardness over time, while sodium caseinate demonstrated the opposite effect [14]. On the contrary, the addition of both - whey proteins concentrate and sodium caseinate to short biscuit formulation, raised hardness and intensified surface brownness [15]. To improve the nutritional value of gluten-free products by the addition of milk proteins particular attention should be paid to the lactose content [16]. Celiac patients are often susceptible to secondary lactose intolerance due to alterations of lactase secretion resulted from the villous atrophy [6]. The addition of high protein/low lactose dairy powders combined with optimal amount of water resulted in gluten-free breads rich in proteins, with dark crust and white crumb, good acceptability scores in sensory tests, an increase in loaf volume, and a decrease in crust and crumb hardness [17].

There is a justified need to improve the nutritional value of gluten-free products. The present study is a continuation of previous trials on the enhancement of the quality and nutritional value of gluten-free bread [18], this time focused on the fortification in proteins. The aim of the study was to enrich a gluten-free formulation, supplemented with calcium citrate in low-lactose dairy proteins, to evaluate its mixing and pasting behavior and to analyze the technological properties, overall quality, and sensory characteristics of obtained gluten-free bread. 


\section{Experimental Section}

\subsection{Materials}

Corn starch (Huici Leidan SA, Huarte, Spain), potato starch (EPSA, Valencia, Spain), pectin (E 440(i), ZPOW Pectowin, Jasło, Poland), calcium citrate (Sigma-Aldrich, St. Louis, MO, USA), commercial sunflower oil, dried yeast (Lessaffre, Valladolid, Spain), sugar, salt, and tap water were used as the basic ingredients. Calcium citrate was added in an amount to provide $0.6 \%$ of elementary calcium. The diary components used were: calcium caseinate (CAS; PZH SM Lacpol, Murowana Goślina, Poland), sodium caseinate (NAS; POCh, Gliwice, Poland), spray dried whey protein isolate (ISO; Carbery Ballineen, Ireland), and hydrolyzed whey proteins (OPT; Carbery Ballineen, Ireland). The amount of protein components was determined on the basis of nutrition and health claims made on foods in such a way that the final gluten-free product was either a source of protein or high in protein [19].

\subsection{Characteristic of Dairy Powders}

\subsubsection{Chemical Composition of Dairy Ingredients}

The moisture, crude proteins $(\mathrm{N} \times 6.25)$, and ash contents were evaluated using the standard methods [20-22]. The results presented are the mean values of at least two replicates.

\subsubsection{Physical and Functional Properties of Dairy Ingredients}

Particle size distribution was determined using a Mastersizer 2000 Particle Size Analyzer with a wet dispersion unit Hydro 2000 S (Malvern Instrument Ltd, Malvern, England). Samples (1-2 g) were suspended in isopropanol. In order to keep the sample suspended and homogenized, it was recirculated continuously through the measurement zone. Particle size distribution was assessed using the mean particle volume (D50) in six replicates for each sample.

The measurement of color was performed by using a Minolta colorimeter (Chroma Meter CR-400/410, Konica Minolta, Japan), equipped with a granular attachment after standardization with a white calibration plate. The color was expressed in accordance with CIE- $L * a * b *$ uniform color space (CIE-Lab). The parameters determined were lightness $L^{*}\left(L^{*}=0\right.$ [black] and $L^{*}=100$ [white]), $a^{*}\left(-a^{*}=\right.$ greenness and $+a^{*}=$ redness $)$, and $b^{*}\left(-b^{*}=\right.$ blueness and $+b^{*}=$ yellowness $)$. Values were the mean of nine replicates.

Water absorption index (WAI) and water solubility index (WSI) were determined according the method of Anderson et al. [23] at room temperature (RT) and after heating. Oil absorption capacity (OAC) was determined according to the method of Lin, Humbert, and Sosulski [24]. The values presented are the average of three measurements.

\subsection{Mixolab ${ }^{\circledR}$ Measurements}

Mixing and pasting behavior of the protein enriched gluten-free dough were evaluated using Mixolab $^{\circledR}$ (Chopin, Tripette et Renaud, Paris, France) [25]. The Mixolab ${ }^{\circledR}$ curves were recorded to evaluate the effect of different dairy powders and variable amount of them. Potato starch $(8.4 \mathrm{~g})$, 
pectin $(2.1 \mathrm{~g})$, calcium citrate $(3.0 \mathrm{~g})$, sugar (2.6 g), and salt (0.8 g) were added to corn starch (31.4 $\mathrm{g})$. The investigated protein powders $(6.3$ or $12.6 \mathrm{~g})$ were added substituting the appropriate amount of corn starch and $41.7 \mathrm{~mL}$ of water. For the assays, $90 \mathrm{~g}$ sample was placed into the Mixolab ${ }^{\circledR}$ bowl and mixed $\left(15 \mathrm{~min} / 30^{\circ} \mathrm{C}\right)$. Subsequently, the temperature was raised to $90{ }^{\circ} \mathrm{C}$ (increase of $4{ }^{\circ} \mathrm{C} / \mathrm{min}$ ) and at $90{ }^{\circ} \mathrm{C}$ was held for $8 \mathrm{~min}$. In the cooling phase the temperature was reduced until $50{ }^{\circ} \mathrm{C}$ (decrease of $4{ }^{\circ} \mathrm{C} / \mathrm{min}$ ), and $50^{\circ} \mathrm{C}$ was held for $2 \mathrm{~min}$. The mixing speed during the entire assay was $80 \mathrm{rpm}$. The values obtained are the mean of two replicates. Parameters recorded included: initial pasting temperature where initial increase of consistency was detected, maximum torque during heating for potato $(\mathrm{C} 3)$ and corn (Cß 3 starches, gelatinization rate for potato $(\beta 1)$ and corn $(\beta 2)$ starches, minimum dough torque during heating $(\mathrm{C} 4)$, cooking stability rate $(\gamma)$, final consistency after cooling until $50{ }^{\circ} \mathrm{C}(\mathrm{C} 5)$, and gelling rate $(\delta)$.

\subsection{Breadmaking Process}

Potato starch (16 g), pectin (4 g), and calcium citrate (5.8 g) were added to corn starch (60.3 g). The investigated protein powders (12 or $24 \mathrm{~g})$ were added substituting the appropriate amount of corn starch. Subsequently, sugar (5 g), dried yeast (5 g), and salt (1.5 g) were dissolved separately in tap water $(80 \mathrm{~mL})$ and added to the dry ingredients together with oil $(2.5 \mathrm{~g})$. The batter was mixed for 12 min in a Brabender farinograph SEW (Brabender OHG, Duisburg, Germany). The resulting batter was proofed for $20 \mathrm{~min}$ in a proofing cabinet $\left(35^{\circ} \mathrm{C} / 70 \%\right.$ relative humidity). Then, the batter was divided into $200 \mathrm{~g}$ samples, placed in baking tins and proofed for another $20 \mathrm{~min}$ under the same conditions. The baking was carried out in convection oven at $200{ }^{\circ} \mathrm{C}$ for 25 min (Eurofours type 10AB20W2, Gommergnies, France). The obtained loaves were divided into two groups: the first was analyzed directly after cooling to room temperature $(2 \mathrm{~h})$; and the second was packed in polyethylene bags and stored $24 \mathrm{~h}$ at ambient temperature $\left(22 \pm 2{ }^{\circ} \mathrm{C}\right)$ for crumb structure analysis and sensory evaluation.

\subsection{Characteristic of Gluten-Free Bread}

\subsubsection{Chemical Composition and Nutritional Value}

Bread moisture [20], proteins [21], ash [22], and fat [26] contents were determined following the standard methods. Total carbohydrates were determined by difference subtracting $100 \mathrm{~g}$ minus the sum of protein, ash, and fat expressed in $\mathrm{g} / 100 \mathrm{~g}$. The energy value was calculated by multiplying the amount of each macronutrient by the corresponding conversion factor $(4,9$, and 4 for protein, fat, and carbohydrates, respectively) [27]. The percentage of energy delivered by protein was calculated.

\subsubsection{Technological Parameters}

The bread loaf weight and volume (rapeseed displacement method) were determined. The specific volume and bake loss were calculated [18]. The height/width ratio of the central $10 \mathrm{~mm}$ slices was determined using Image J software (National Institutes of Health, Bethesda, MD, USA) [28]. The energy status of water in bread samples was measured as water activity $\left(a_{w}\right)$ using an Aqua Lab 
Series 3 (Decagon Devices Pullman, USA) at $22{ }^{\circ} \mathrm{C}$. The crust and crumb color was analyzed as previously described using a Minolta colorimeter. Values were the mean of nine replicates.

\subsubsection{Crumb Texture Properties}

Texture profile analysis (TPA test) of the 24-h stored bread crumbs was performed using Texture Analyzer TA-XT2i (Stable Micro Systems, Surrey, UK) equipped with a $30 \mathrm{~kg}$ load cell and $25 \mathrm{~mm}$ aluminum cylindrical probe. Three middle slices of each loaf were evaluated. A $10 \mathrm{~mm}$ thick bread slice underwent a double compression test up to $50 \%$ deformation of its original height at a crosshead speed of $1 \mathrm{~mm} / \mathrm{s}$ and a $30 \mathrm{~s}$ gap between compressions. From the two-bite texture profile curve the following texture parameters were obtained: hardness, adhesiveness, springiness, cohesiveness, chewiness, and resilience.

\subsection{Statistical Analysis}

One-way analysis of variance (ANOVA) and Fisher's least significant difference test (LSD) using Statistica 7.1 (StatSoft, Kraków, Poland) were applied to define significant differences $(p<0.05)$ between samples.

\section{Results and Discussion}

\subsection{Chemical, Physical and Functional Characteristic of Dairy Powders}

Detailed characteristics of selected dairy powders (NAS, CAS, ISO, OPT), including chemical composition, and physical and functional properties, are provided in order to evaluate the effect of each individual powder on the gluten-free batter and bread (Table 1). High concentration of proteins was confirmed for all dairy ingredients tested (Table 1). Total proteins content exceeded $85 \%$, with distinguishing concentration in sodium caseinate, containing over $94 \%$ of proteins. According to the suppliers, all dairy ingredients tested were poor in fat $(1.0 \%-1.5 \%)$ and lactose $(0.5 \%-2 \%)$. Caseinates (CAS and NAS) were richer in mineral compounds (ash; near $4 \%$ ) than whey proteins tested.

The measurement of particle size showed that the mean size of whey proteins particles was significantly higher in comparison with particles of caseinates tested, and ISO showed the highest particle size (Table 1). The color parameters of dairy powders indicated that they were very light, white, or creamy powders, characterized by high $L^{*}$ value, above 91, and in the case of NAS, near 94. The negative value of parameter $a^{*}$ (below-2) indicated greenish color and the positive $b^{*}$ values described the yellowish color, being significantly higher for the whey proteins.

The ability to bind and hold water without syneresis is critical in many foods, thus, hydration properties were determined in the dairy proteins. Results indicated that CAS followed by NAS showed the highest value of WAI at room temperature (Table 1), however, that trend was reversed when this parameter was determined after heating. Although caseins are relatively hydrophobic, they contain regions of high, medium, or low hydrophobicity [29] and they bind about $2 \mathrm{~g}$ water/g, which is typical of proteins [30]. Whey proteins in their native form exhibit little water-binding capacity [31]. Nevertheless, heat-denaturated whey proteins, although retaining most of their 
secondary structure, are linked together and can have a perceived hydration of over $10 \mathrm{~g}$ of water/g protein, compared with $0.2 \mathrm{~g}$ water/g protein for whey protein in their native globular state [29]. Processing has also a considerable impact on solubility of dairy proteins (Table 1). At room temperature, whey proteins tested were soluble in very high degree, however the heat treatment impaired the solubility of these proteins. WSI of ISO and OPT decreased by over $80 \%$ and $70 \%$, respectively. Whey proteins are susceptible to denaturation at temperature higher that $70{ }^{\circ} \mathrm{C}$. When heated, the tertiary structure of protein globules are destroyed, then unfolding of the protein molecules and new protein-protein interactions occur [32]. In case of both caseinates tested the opposite situation was observed. Here, after boiling the WSI values for CAS and NAS increased by more than $15 \%$. Analyzing the OAC, significant differences were found between the dairy proteins tested (Table 1). The highest OAC was observed for CAS, followed by ISO, and OPT, whereas NAS has the lowest oil absorption capacity, probably due to its highest protein concentration. According to Kinsella [33], the mechanism of fat absorption has been attributed mostly to the physical entrapment of oil, but as well may be influenced by lipophilicity of the protein concentrate. In protein powder foods fat binding can be influenced by the size of powder particles [34], however in the dairy proteins tested such correlation was not observed.

\subsection{Effect of Dairy Powders on the Gluten-Free Dough Characteristics}

The effect of the dairy powders at two different levels (12\% and $24 \%$ ) on the rheology of gluten-free dough was studied by using the Mixolab, where dough behavior subjected to shear and temperature constraints is recorded [25]. The Mixolab plots obtained in the presence of the dairy powders are shown in Figure 1.

Gluten-free doughs showed very low consistency during mixing, which only increased after heating when starches present in the recipe started to gelatinize. Consistency enhanced during heating till the rupture of the starch granules where the maximum consistency was detected. Further heating led to a decrease in the consistency derived from the starches stability during cooking and when temperature decreased the amylose retrogradation associated to cooling was observed as a consistency increase. This pattern agrees with previous description of the compounds changes reported by Rosell et al. [25]. The trends observed on the plots indicated that gluten-free dough rheology was clearly governed by starch changes during heating and cooling. The consistency of the gluten-free dough is greatly dependent on the amount of water or hydration, showing very low consistency during mixing when water adsorption is higher than $90 \%$ [35]. It must be remarked that during heating two consistency peaks were detected that were associated to the different gelatinization temperature of corn and potato starches. Matos and Rosell [36] also detected different peaks depending on the type of starch and their diverse pasting temperatures, being $65.4{ }^{\circ} \mathrm{C}$ for potato starch, $69.9{ }^{\circ} \mathrm{C}$ for corn starch and $70.2{ }^{\circ} \mathrm{C}$ for rice flour. In addition, Krupa-Kozak et al. [18] observed two different slopes during heating, the first one detected around 28-31 min corresponded to potato starch gelatinization, whereas the second one observed from 31 to 36 min was ascribed to corn starch gelatinization, which agrees with results of the present study. The patterns obtained during mixing, overmixing, pasting, and gelling greatly varied with the protein source and the level of proteins (Figure 1). It was not possible to record the consistency of the $24 \%$ NAS containing dough with the Mixolab due to its high consistency. The inclusion of the 
dairy proteins decreased the dough consistency during the heating-cooling stages, with the exception of NAS added at $12 \%$ that showed higher consistency after cooling. Bonet et al. [37] found that the addition of protein sources (gelatin, egg, and lupine) to wheat flour significantly changed the Mixolab plot and the effect was attributable to the nature of the proteins. The presence of different proteins and starches modifies protein-protein interactions and also the starch gelatinization and the gelling processes [25,35,38]. Regarding the level of the proteins added, dough consistencies decreased with increasing level of proteins.

Figure 1. Effect of dairy proteins on the gluten-free dough consistency determined by Mixolab ${ }^{\circledR}$ device. Control: unfortified gluten-free dough; CAS 12: gluten-free dough with $12 \%$ of calcium caseinate; CAS 24 : gluten-free dough with $24 \%$ of calcium caseinate; NAS 12: gluten-free dough with $12 \%$ of sodium caseinate; NAS 24: gluten-free dough with $24 \%$ of sodium caseinate: OPT 12: gluten-free dough with $12 \%$ of whey proteins hydrolysate; OPT 24: gluten-free dough with $24 \%$ of whey proteins hydrolysate; ISO 12: gluten-free dough with $12 \%$ of whey proteins isolate; ISO 24 : gluten-free dough with $24 \%$ of whey proteins isolate. C3: maximum torque during heating of potato starch; $\mathrm{C}: 3$ maximum torque during heating of corn starch; $\mathrm{C} 4$ : minimum dough torque during heating; $\mathrm{C}$ 5: final viscosity after cooling till $50{ }^{\circ} \mathrm{C} ; \beta 1$ : gelatinization rate for potato starch; $\beta 2$ : gelatinization rate for corn starch; $\gamma$ : cooking stability rate.

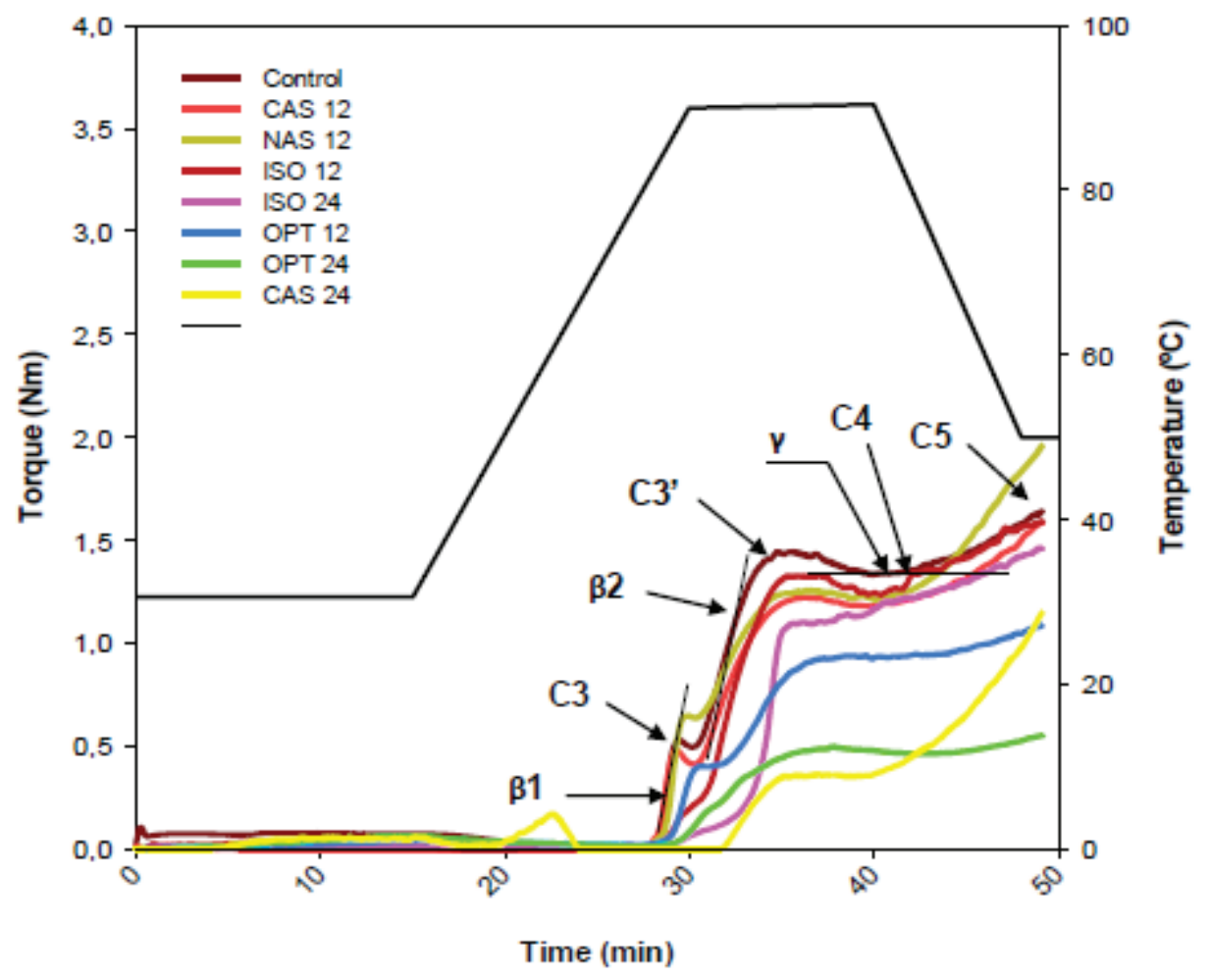

Primary and secondary parameters were extracted from the Mixolab curves to quantify the effect of the different dairy proteins on dough empirical rheology (Table 2). Proteins added to experimental doughs retarded the initial pasting temperature and the temperatures at which maximum dough consistency (C3 and C3) was obtained; likely due to proteins compete with the starch for the 
available water, limiting the starch granule swelling and, therefore, promoting a delay in the pasting process as has been observed with hydrocolloids [39]. Consistency associated to potato starch gelatinization (C3) decreased in the presence of proteins, with the exception of NAS 12, and was barely noticeable when increasing protein level up to $24 \%$. That effect was even more accentuated in the case of the consistency associated to corn starch gelatinization (C) B since water will be even more limited after proteins hydration and potato starch gelatinization. Nevertheless, no relationship could be established between the consistency parameters during heating and the hydration properties of the dairy powders, likely the interaction between starches and proteins affected the ability of the proteins and starches to bind water. The final dough consistency after cooling (C5) was also significantly affected with the addition of the different protein powders, therefore, dairy powders modified amylose chains crystallization and in consequence, starch gelling, and the effect was dependent on the nature of the protein. NAS added at $12 \%$ increased the final consistency, whereas ISO at $12 \%$ did not modify that parameter and the other proteins decreased it. When increasing levels of proteins were added, the effect was a reduction of the final consistency. Considering the particle size of the proteins and also the hydration properties of the dairy proteins, there was no relationship between those properties and the dough behavior during heating and cooling. Regarding the secondary parameters, all doughs showed very low cooking stability range (C4-C3), whereas the cooling setback or gelling (C4-C5) was increased with the inclusion of dairy proteins, with the exception of the OPT that decreased that value. Likely the hydrolyzed nature of this protein isolate hindered the amylose recrystallization, lowering the final dough consistency. The rate of the starches phenomena associated to heating $(\beta 1, \beta 2, \gamma)$ and cooling $(\delta)$ were significantly affected in the presence of proteins. In general, starch gelatinization was slowed down in the presence of proteins, with the exception of NAS-12 in the case of $\beta 1$ or ISO- 12 in the case of $\beta 2$. Again, no correlation could be established with the protein properties.

\subsection{Chemical Composition and Nutritional Value of the Gluten-Free Breads Containing Different} Dairy Powders

The addition of dairy proteins to gluten-free bread formulations is a common practice for increasing nutritional value, as in general, the commercial gluten-free formulas are poor in proteins $[35,40]$. Similarly to the results of our previous study [41], the control gluten-free bread, mainly composed of corn and potato starches, was poor in proteins (Table 3 ).

As was expected, the proteins content in all experimental gluten-free breads with dairy powders increased significantly $(p<0.05)$. Breads supplemented with $12 \mathrm{~g}$ of milk powders were more than five-times richer in protein than the control. The increase of the level of supplementation with dairy proteins (up to $24 \mathrm{~g}$ ) caused the further increase of protein content in the bread samples, especially in NAS 24 with sodium caseinate, which was ten-times richer in proteins than the control. Milk proteins have a high nutritional value $[10,13]$ and the addition of milk proteins and essential amino acids, such as lysine, methionine and tryptophan also increases the nutritional value of the bakery products $[14,15]$. Within bread samples with dairy proteins addition, a fat content was low ranging from 0.39 to 2.08 (Table 3 ) as all dairy powders added were relatively poor in fat (Table 1). However, comparing with the control, experimental breads were more affluent in minerals, especially when $24 \mathrm{~g}$ of calcium caseinate was included to formulation (CAS 24). There are inconsistencies about 
CD patients being overweight at diagnosis and gaining weight while on GFD $[42,43]$. Taking this into account, the calorie content of gluten-free products is of importance [44]. Energetic value (in kcal) of experimental gluten-free bread with dairy proteins ranged from 228 to 246, and was comparable to unfortified control $(239 \mathrm{kcal})$. Hager et al. [45] indicated that although the calorie content was higher in white and wholemeal wheat bread than in oat, buckwheat, maize, sorghum, teff, and rice, these breads were of inferior quality at the same time. In the present study the two levels of dairy proteins supplementation to experimental gluten-free bread were tested regarding the energy delivered by proteins (Table 3). In the case of experimental breads with lower amount (12 g) of caseinates and whey proteins powders, the proteins delivered around $15 \%$ or above $13 \%$ of energy, respectively. The higher threshold of proteins supplementation influenced further significant growth of energy delivered by proteins up to $26 \%$ in NAS 24 . According to the European Parliament regulation on nutrition and health claims made on foods, a claim that a food is a source of protein may only be applied to food product where at least $12 \%$ of the energy value of the food is provided by protein, whereas a claim that a food is high in protein may only be made where at least $20 \%$ of the energy value of the food is provided by protein [19]. Based on that regulation, all experimental gluten-free breads with $12 \mathrm{~g}$ dairy proteins addition can be recognized as a source of proteins, whereas all experimental gluten-free breads with $24 \mathrm{~g}$ dairy proteins addition are high in protein.

\subsection{Technological Parameters of Gluten-Free Breads with Dairy Powders}

In general, milk products have been described as volume-depressing contributory factors of wheat bread $[46,47]$. In this study, the addition of $12 \mathrm{~g}$ dairy proteins to the experimental gluten-free formulations increased significantly the specific volume of all breads, comparing with the control bread, with distinguishing results obtained in bread NAS 12 (Table 4). Similarly, Gallagher et al. [17] indicated that addition of high protein/low lactose dairy powders resulted in gluten-free breads of improved overall shape and volume. Additionally, in the case of increase of sodium caseinate concentration in gluten-free formulation, the further increase of specific volume of bread NAS 24 was observed. In all remaining samples, the used of increased amount of dairy proteins (up to $24 \mathrm{~g}$ ) affected specific volume in a different manner. Compared with the control, increased level of hydrolyzed whey proteins decreased significantly the volume of bread OPT 24 , whereas a higher concentration of whey proteins isolate increased the specific value of bread ISO 24. Specific volume of bread CAS 24 was similar to the control. The volume of bread with dairy powders depends on the powder type and level of addition. Breads with addition of dairy supplements showed higher height/width ratio of central slices in comparison with the unfortified control (Table 4). However, only in the case of breads with the $12 \mathrm{~g}$ of proteins in formulation, the increase in the height/width ratio of slice was significant. Analyzing relationship between specific volume and H/W ratio a linear positively correlation (Pearson's $r=0.49$ ) was found. 


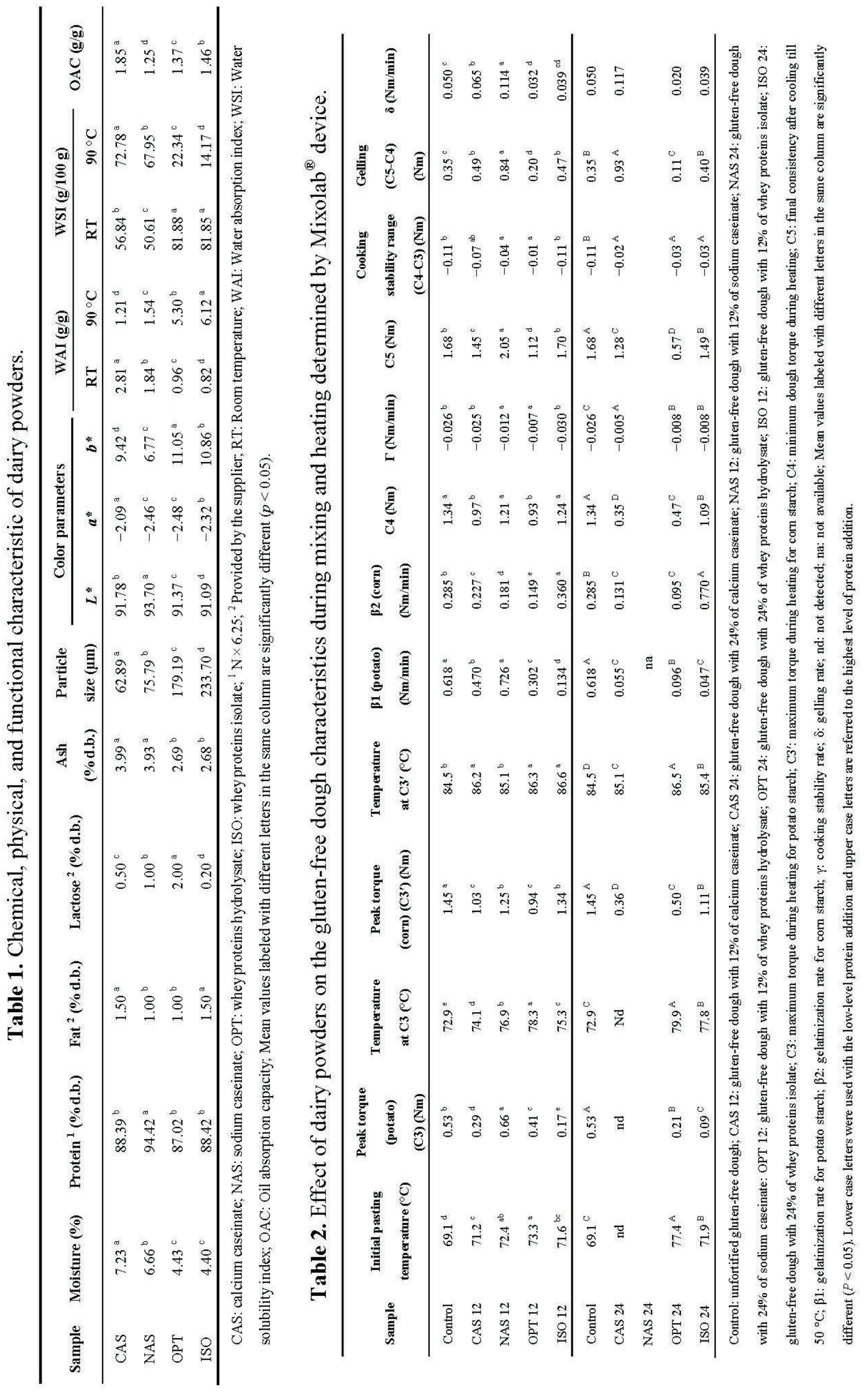


Table 3. Chemical composition and nutritional value of the gluten-free breads containing different dairy powders.

\begin{tabular}{ccccccc}
\hline Sample & $\begin{array}{c}\text { Moisture } \\
(\%)\end{array}$ & $\begin{array}{c}\text { Proteins } \\
(\% \text { as is) }\end{array}$ & $\begin{array}{c}\text { Fat } \\
(\% \text { as is })\end{array}$ & $\begin{array}{c}\text { Ash } \\
(\% \text { as is })\end{array}$ & $\begin{array}{c}\text { Energetic value } \\
(\text { kcal as is })\end{array}$ & $\begin{array}{c}\text { Energy delivered } \\
\text { by proteins (\%) }\end{array}$ \\
\hline Control & $39.97^{\mathrm{c}}$ & $1.51^{\mathrm{b}}$ & $1.14^{\mathrm{a}}$ & $1.82^{\mathrm{d}}$ & $239^{\mathrm{a}}$ & $2.53^{\mathrm{e}}$ \\
CAS 12 & $39.23^{\mathrm{e}}$ & $8.65^{\mathrm{a}}$ & $0.83^{\mathrm{c}}$ & $2.14^{\mathrm{a}}$ & $239^{\mathrm{a}}$ & $14.50^{\mathrm{b}}$ \\
NAS 12 & $41.64^{\mathrm{a}}$ & $8.57^{\mathrm{a}}$ & $0.39^{\mathrm{d}}$ & $1.82^{\mathrm{d}}$ & $228^{\mathrm{c}}$ & $15.03^{\mathrm{a}}$ \\
OPT 12 & $39.69^{\mathrm{d}}$ & $8.23^{\mathrm{a}}$ & $0.83^{\mathrm{c}}$ & $1.98^{\mathrm{b}}$ & $237^{\mathrm{b}}$ & $13.86^{\mathrm{c}}$ \\
ISO 12 & $40.09^{\mathrm{b}}$ & $8.07^{\mathrm{a}}$ & $1.04^{\mathrm{b}}$ & $1.92^{\mathrm{c}}$ & $237^{\mathrm{b}}$ & $13.61^{\mathrm{d}}$ \\
Control & $39.97^{\mathrm{C}}$ & $1.51^{\mathrm{C}}$ & $1.14^{\mathrm{D}}$ & $1.82^{\mathrm{D}}$ & $239^{\mathrm{C}}$ & $2.53^{\mathrm{D}}$ \\
CAS 24 & $40.59^{\mathrm{A}}$ & $14.49^{\mathrm{B}}$ & $1.29^{\mathrm{C}}$ & $2.20^{\mathrm{A}}$ & $235^{\mathrm{D}}$ & $24.63^{\mathrm{B}}$ \\
NAS 24 & $39.37^{\mathrm{D}}$ & $15.75^{\mathrm{A}}$ & $1.21^{\mathrm{CD}}$ & $2.15^{\mathrm{B}}$ & $240^{\mathrm{B}}$ & $26.25^{\mathrm{A}}$ \\
OPT 24 & $40.49^{\mathrm{B}}$ & $14.74^{\mathrm{B}}$ & $1.71^{\mathrm{B}}$ & $2.06^{\mathrm{C}}$ & $238^{\mathrm{C}}$ & $24.74^{\mathrm{B}}$ \\
ISO 24 & $39.06^{\mathrm{D}}$ & $14.39^{\mathrm{B}}$ & $2.08^{\mathrm{A}}$ & $2.12^{\mathrm{B}}$ & $246^{\mathrm{A}}$ & $23.43^{\mathrm{C}}$ \\
\hline
\end{tabular}

Control: unfortified gluten-free bread; CAS 12: gluten-free bread with $12 \%$ of calcium caseinate; CAS 24 : gluten-free bread with $24 \%$ of calcium caseinate; NAS 12: gluten-free bread with $12 \%$ of sodium caseinate; NAS 24: gluten-free bread with $24 \%$ of sodium caseinate: OPT 12: gluten-free bread with $12 \%$ of whey proteins hydrolysate; OPT 24: gluten-free bread with $24 \%$ of whey proteins hydrolysate; ISO 12 : gluten-free bread with $12 \%$ of whey proteins isolate; ISO 24 : gluten-free bread with $24 \%$ of whey proteins isolate; Mean values labeled with different letters in the same column are significantly different $(p<0.05)$. Lower case letters were used with the low-level protein addition and upper case letters are referred to the highest level of protein addition.

Applied dairy supplements, regardless of the amount, influenced the bake loss, defined as the amount of water and organic material lost during baking (Table 4). In comparison with the control, bake loss of all breads tested increased significantly. The opposite effect was observed only in bread OPT 24, where the value of bake loss was significantly reduced by higher concentration of hydrolyzed whey proteins. Crumb characteristic affects the rate of water transport [48]. Small size of crumb pore slowed down moisture migration [49], whereas a larger number of connections between gas cells would give a faster transport of water. Additionally, the number of connections of each gas cell increased with increased size of gas cell [50]. 


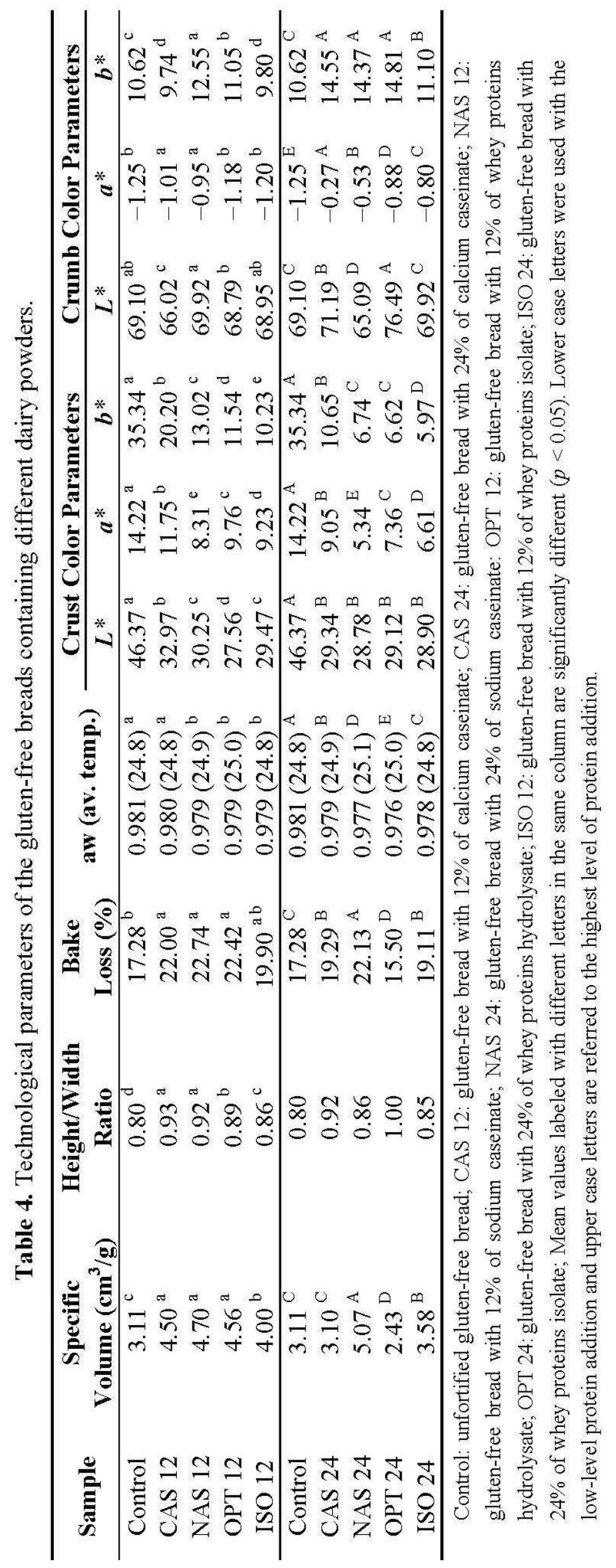


Incorporation of dairy powders affected the color of both, crust and crumb of experimental gluten-free breads (Table 4). Crust of the control bread was characterized by the highest lightness $\left(L^{*}=46.37\right)$, whereas the inclusion of dairy proteins resulted in crust darkening, influenced by the level of dairy proteins addition rather than the protein type. All gluten-free breads containing dairy supplements showed the significantly lower $L^{*}$ value. Additionally, in the case of experimental gluten-free breads containing $12 \mathrm{~g}$ of dairy proteins, the further crust color diversification was observed. Crust of bread CAS 12 and NAS 12, containing calcium or sodium caseinate, respectively, was significantly lighter in comparison with dark crust of breads containing whey proteins, with distinguishing OPT12 where $L^{*}$ value reached 27.56. The value of parameter $a^{*}$ (red hue) was positive for crust of all experimental breads (Table 4). Comparing with the control, the incorporation of dairy powders to gluten-free formulation affected the significant decrease in redness, especially in breads containing sodium caseinate at both levels tested (NAS 12 and NAS 24). Crust of the control gluten-free bread obtained the highest $b^{*}$ value (yellow hue). Whereas, the addition of dairy proteins to formulation produced a significant decrease of crust yellowness of breads obtained, however all the values were still positive. The crust yellowness was especially low in the case of breads with higher dairy proteins concentration. Observed darkening of crust color resulted probably from the Maillard browning, a chemical reaction between amino groups and reducing sugars. In the case of milk derivatives undergoing a high temperature treatment, lactose as a reducing sugar interacts mainly with lysine residues, resulting in the formation of brown melanoidins [51]. These non-enzymatic reactions are responsible for numerous changes on food properties. From the technological point of view, the brown crust formation on gluten-free bread is desirable and the resulting color, taste and flavor characteristics are generally experienced as pleasant. Crumb color was influenced by a level of dairy proteins addition. Lower concentration of proteins tested in the formulations resulted in bread of similar to control crumb lightness, with slightly distinguishing NAS 12 (Table 4). Only in CAS 12 the $L^{*}$ was significantly reduced. Whereas, the lightness of bread crumb supplemented with $24 \mathrm{~g}$ of dairy proteins was higher in comparison with crumb of breads with $12 \mathrm{~g}$ dairy proteins addition, except for crumb of bread NAS 24 . The $a^{*}$ values for the crumbs were all negative, with the lowest redness detected in the control crumb.

Comparing with the control, increasing concentration of dairy powders increased significantly the value of $a^{*}$ parameter of tested crumbs. This effect was especially visible in the case of breads containing calcium (CAS 24) and sodium (NAS 24) caseinate, where the $a^{*}$ value reached -0.27 and -0.53 , respectively. Yellowness $\left(b^{*}\right)$ of all crumb samples was positive. Similarly to redness, also the value of $b^{*}$ increased significantly with increased dairy proteins concentration. Gluten-free breads containing dairy powders had an appealing dark crust and white crumb appearance, and received good acceptability scores in sensory tests $[14,17]$. As a wide variety of dairy supplement are available, their application in baked product development need to be determined adequately. In addition to the type and the amount of dairy supplement, the choice must be based on their physicochemical and functional properties, which varies remarkably.

\subsection{Textural Parameters of Gluten-Free Breads with Dairy Powders}

The values obtained for the textural parameters of the bread crumbs are shown in Table 5. Wide variations in the crumb hardness (3.66 to $25.28 \mathrm{~N}$ ) were observed among the gluten-free bread 
samples. These results reflect large differences depending on type of proteins used. Dairy proteins incorporated at $12 \%$ level significantly $(p<0.05)$ decreased the hardness, with the exception of NAS 12. Nevertheless, the addition of increasing amounts of proteins led to the opposite effect and only ISO 24 remained softer than the control crumb. NAS at any level of addition led to harder crumbs and the same effect was observed in chewiness. Nunes et al. [14] analyzed the influence of low lactose dairy powders on gluten-free bread quality indicated that sodium caseinate had a negative impact on crumb hardness, whereas whey proteins demonstrated the ability to increase significantly the specific volume of the breads and decrease its the hardness.

Table 5. Texture profile analysis of the gluten-free bread crumbs containing different dairy powders.

\begin{tabular}{cccccc}
\hline Sample & Hardness (N) & Springiness & Cohesiveness & Chewiness (g) & Resilience \\
\hline Control & $9.44^{\mathrm{b}}$ & $1.002^{\mathrm{a}}$ & $0.454^{\mathrm{ab}}$ & $431.70^{\mathrm{b}}$ & $0.195^{\mathrm{a}}$ \\
CAS 12 & $3.66^{\mathrm{e}}$ & $1.007^{\mathrm{a}}$ & $0.460^{\mathrm{a}}$ & $169.80^{\mathrm{c}}$ & $0.186^{\mathrm{a}}$ \\
NAS 12 & $11.43^{\mathrm{a}}$ & $0.981^{\mathrm{ab}}$ & $0.427^{\mathrm{b}}$ & $475.84^{\mathrm{a}}$ & $0.188^{\mathrm{a}}$ \\
OPT 12 & $5.46^{\mathrm{c}}$ & $0.940^{\mathrm{c}}$ & $0.335^{\mathrm{c}}$ & $170.74^{\mathrm{c}}$ & $0.134^{\mathrm{b}}$ \\
ISO 12 & $4.20^{\mathrm{d}}$ & $0.972^{\mathrm{b}}$ & $0.366^{\mathrm{c}}$ & $152.99^{\mathrm{c}}$ & $0.141^{\mathrm{b}}$ \\
\hline Control & $9.44^{\mathrm{C}}$ & $1.002^{\mathrm{A}}$ & $0.454^{\mathrm{B}}$ & $431.70^{\mathrm{C}}$ & $0.195^{\mathrm{A}}$ \\
CAS 24 & $11.60^{\mathrm{B}}$ & $0.979^{\mathrm{AB}}$ & $0.486^{\mathrm{A}}$ & $568.38^{\mathrm{B}}$ & $0.196^{\mathrm{A}}$ \\
NAS 24 & $25.28^{\mathrm{A}}$ & $0.954^{\mathrm{B}}$ & $0.434^{\mathrm{B}}$ & $1071.26^{\mathrm{A}}$ & $0.184^{\mathrm{A}}$ \\
OPT 24 & $11.06^{\mathrm{B}}$ & $0.872^{\mathrm{C}}$ & $0.376^{\mathrm{C}}$ & $368.83^{\mathrm{D}}$ & $0.133^{\mathrm{B}}$ \\
ISO 24 & $6.35^{\mathrm{D}}$ & $0.959^{\mathrm{B}}$ & $0.446^{\mathrm{B}}$ & $282.34^{\mathrm{E}}$ & $0.181^{\mathrm{A}}$ \\
\hline
\end{tabular}

Control: unfortified gluten-free bread; CAS 12: gluten-free bread with $12 \%$ of calcium caseinate; CAS 24 : gluten-free bread with $24 \%$ of calcium caseinate; NAS 12: gluten-free bread with $12 \%$ of sodium caseinate; NAS 24: gluten-free bread with $24 \%$ of sodium caseinate: OPT 12: gluten-free bread with $12 \%$ of whey proteins hydrolysate; OPT 24 : gluten-free bread with $24 \%$ of whey proteins hydrolysate; ISO 12 : gluten-free bread with $12 \%$ of whey proteins isolate; ISO 24 : gluten-free bread with $24 \%$ of whey proteins isolate; Mean values labeled with different letters in the same column are significantly different $(p<0.05)$. Lower case letters were used with the low-level protein addition and upper case letters are referred to the highest level of protein addition.

Springiness is associated with a fresh, aerated and elastic product, thus high springiness is desirable in this type of products. Low springiness value is indicative of brittleness and this reflects the tendency of the bread to crumble when is sliced. Although the proteins addition decreased the springiness, the effect was only significant in the presence of the protein isolates (ISO and OPT) at both levels tested. Marco and Rosell [35] found springiness values that ranged from 0.77 to 0.94 when study the protein enrichment of rice based gluten-free breads, and later on Matos and Rosell [8] reported springiness values from 0.76 to 1.00 in commercial gluten free breads. Therefore, springiness values obtained in the present study agree with reported ones.

Cohesiveness characterizes the extent to which a material can be deformed before it ruptures, reflecting the internal cohesion of the material. Bread with high cohesiveness is desirable because it forms a bolus rather than disintegrates during mastication, whereas low cohesiveness indicates increased susceptibility of the bread to fracture or crumble [8]. In case of breads containing caseinates (CAS and NAS) values obtained for cohesiveness were similar to control, while whey 
proteins (OPT and ISO) decreased, significantly, the value of this parameter $(p<0.05)$. Very low resilience values were obtained for experimental gluten-free breads, especially for breads with whey proteins, indicating a low elasticity. Values obtained agreed with results reported for commercial gluten-free bread where resilience ranged from 0.09 to 0.84 [8].

\section{Conclusions}

The present study has shown that the application of low-lactose dairy proteins in a gluten-free formulation influenced considerably the characteristic of experimental doughs and breads. Gluten-free doughs containing dairy proteins tested showed very low consistency during mixing stage and decreased consistency during the heating-cooling stages. Experimental breads were significantly richer in proteins and more affluent in minerals than the control one. Energetic value of experimental gluten-free bread with dairy proteins was comparable to unfortified control, however regarding the energy delivered by proteins they can be recognized as a source of proteins or as high in protein. Addition of dairy proteins to the experimental gluten-free formulations increased significantly $(p<0.05)$ the specific volume of all breads, with distinguishing results obtained in bread NAS 24. Inclusion of dairy proteins resulted in crust darkening and crumb lightness, influenced by the level of dairy proteins addition rather than the protein type. Dairy proteins incorporated at a $12 \%$ level significantly $(p<0.05)$ decreased the hardness, with the exception of NAS 12. Nevertheless, the addition of increasing amounts of proteins led to the opposite effect. Obtained results suggest that dairy proteins tested in this study could be used successfully in gluten-free recipes in order to obtain gluten-free bread of a pleasant color, taste, and flavor characteristics, and improved technological and nutritional properties.

\section{Acknowledgments}

Authors acknowledge the financial support of the Spanish Ministry of Economy and Competitiveness (Project AGL2011-23802), the European Regional Development Fund (FEDER) and Generalitat Valenciana (Project Prometeo 2012/064) and Project REFRESH-Unlocking the potential of the Institute of Animal Reproduction and Food Research for strengthening integration with the European Research Area and regional development of the 7th Framework Programme EU-Research Potential-Capacities-FP7-REGPOT-2010-1-264103. The authors would like to thank to Józef Warechowski from the Faculty of Food Sciences of the University of Warmia and Mazury in Olsztyn for the particle size distribution analysis.

\section{Conflicts of Interest}

The authors declare no conflict of interest.

\section{References}

1. Fasano, A.; Berti, I.; Gerarduzzi, T.; Not, T.; Colletti, R.B.; Drago, S.; Elitsur, Y.; Green, P.H.; Guandalini, S.; Hill, I.D.; et al. Prevalence of celiac disease in at-risk and not-at-risk groups in the United States: A large multicenter study. Arch. Int. Med. 2003, 163, 286-292. 
2. Tommasini, A.; Not, T.; Kiren, V.; Baldas, V.; Santon, D.; Trevisiol, C.; Berti, I.; Neri, E.; Gerarduzzi, T.; Bruno, I.; et al. Mass screening for coeliac disease using anti-human transglutaminase antibody assay. Arch. Dis. Child. 2004, 89, 512-515.

3. Darewicz, M.; Dziuba, J.; Minkiewicz, P. Coeliac disease-Background, molecular, bioinformatics and analytical aspects. Food Rev. Int. 2008, 24, 311-329.

4. Lerner, A. New therapeutic strategies for celiac disease. Autoimmun. Rev. 2010, 9, 144-147.

5. Briani, C.; Samaroo, D.; Alaedini, A. Celiac disease: From gluten to autoimmunity. Autoimmun. Rev. 2008, 7, 644-650.

6. Murray, J.A. The widening spectrum of celiac disease. Am. J. Clin. Nutr. 1999, 69, 354-365.

7. Bianchi, M.L.; Bardella, M.T. Bone in celiac disease. Osteoporos. Int. 2008, 19, 1705-1716.

8. Matos, M.E.; Rosell, C.M. Relationship between instrumental parameters and sensory characteristics in gluten-free breads. Eur. Food Res. Technol. 2012, 235, 107-117.

9. Marco, C.; Rosell, C.M. Effect of different protein isolates and transglutaminase on rice flour properties. J. Food Eng. 2008, 84, 132-139.

10. Kenny, S.; Wehrle, K.; Stanton, C.; Arendt, E.K. Incorporation of dairy ingredients into wheat bread: Effects on dough rheology and bread quality. Eur. Food Res. Technol. 2000, 210, 391-396.

11. Stathopoulos, C.; O'Kennedy, B.T. A rheological evaluation of concentrated casein systems as replacement for gluten: Calcium effects. Int. J. Dairy Technol. 2008, 61, 397-402.

12. Stathopoulos, C.; O'Kennedy, B.T. The effect of salt on the rheology and texture of a casein based ingredient intended to replace gluten. Milchwissenschaft 2008, 63, 430-433.

13. Stathopoulos, C. Dairy-Based Ingredients. In Gluten-Free Cereal Products and Beverages; Arendt, E.K., Dal Bello, F., Eds.; Academic Press: San Diego, CA, USA, 2008; pp. 217-236.

14. Nunes, M.; Ryan, L.; Arendt, E.K. Effect of low lactose dairy powder addition on the properties of gluten-free batters and bread quality. Eur. Food Res. Technol. 2009, 229, 31-41.

15. Gallagher, E.; Kenny, S.; Arendt, E.K. Impact of dairy protein powders on biscuit quality. Eur. Food Res. Technol. 2005, 221, 237-243.

16. Ojetti, V.; Nucera, G.; Migneco, A.; Gabrielli, M.; Lauritano, C.; Danese, S.; Zocco, M.A.; Nista, E.C.; Cammarota, G.; de Lorenzo, A.; et al. High prevalence of celiac disease in patients with lactose intolerance. Digestion 2005, 71, 106-110.

17. Gallagher, E.; Kunkel, A.; Gormley, T.R.; Arendt, E.K. The effect of dairy and rice powder addition on loaf and crumb characteristics, and on shelf life (intermediate and long-term) of gluten-free breads stored in a modified atmosphere. Eur. Food Res. Technol. 2003, 218, 44-48.

18. Krupa-Kozak, U.; Altamirano-Fortoul, R.; Wronkowska, M.; Rosell, C.M. Breadmaking performance and technological characteristic of gluten-free bread with inulin supplemented with calcium salts. Eur. Food Res. Technol. 2012, 235, 545-554.

19. European Union. Regulation (EC) No 1924/2006 of the European Parliament and the Concil of 20 December 2006 on nutrition and health claims made on foods. Off. J. Eur. Union 2006, L404, 9-25.

20. ICC Standard Method 110/1, Determination of the Moisture Content of Cereals and Cereal Products (Practical method), 1976. Available online: http://www.icc.or.at/standard_methods (accessed on 12 November 2013). 
21. ICC Standard Method 105/2, Determination of Crude Protein in Cereals and Cereal Products for Food and for Feed, 1994. Available online: http://www.icc.or.at/standard_methods (accessed on 12 November 2013).

22. ICC Standard Method 104/1, Determination of Ash in Cereals and Cereal Products, 1990. Available online: http://www.icc.or.at/standard_methods (accessed on 12 November 2013).

23. Anderson, R.A.; Conway, H.F.; Pfeifer, V.F.; Griffin, E.L. Gelatinization of corn grits by roll and extrusion cooking. Cereal Sci. Today 1969, 14, 4-12.

24. Lin, M.J.Y.; Humbert, E.S.; Sosulski, F. Certain functional properties of sunflower meal products. J. Food Sci. 1974, 39, 368-370.

25. Rosell, M.C.; Collar, C.; Haros, M. Assessment of hydrocolloid effects on the thermo-mechanical properties of wheat using the Mixolab. Food Hydrocoll. 2007, 21, 452-462.

26. ICC Standard Methods, 136, Cereals and Cereal Products-Determination of Total Fat Content, 1984. Available online: http://www.icc.or.at/standard_methods (accessed on 12 November 2013).

27. Food and Agriculture Organization of the United Nations (FAO) recommendations. Food Energy-Methods of Analysis and Conversion Factors; Rome, Italy, 2003; pp. 57-60.

28. Gonzales-Barron, U.; Butler, F. A comparison of seven thresholding techniques with the k-means clustering algorithm for measurement of breadcrumb features by digital image analysis. J. Food Eng. 2006, 74, 268-278.

29. Carr, A.J.; Southward, C.R.; Creamer, L.K. Protein Hydratation and Viscosity of Dairy Proteins. In Advanced Dairy Chemistry. Proteins. Part B; Fox, P.F., McSweeney, P.L.H., Eds.; Kluver Academic/Plenum Publishers: New York, NY, USA, 2003; pp. 1289-1318.

30. Fox, P.F.; McSweeney, P.L.H. Dairy Chemistry and Biochemistry; Blackie Academic: London, UK, 1998.

31. Cayot, P.; Lorient, D. Food Proteins and their Applications; Damodaran, S., Paraf, A., Eds.; Marcel Decker: New York, NY, USA, 1997.

32. Mulvihill, D.M.; Ennis, M.P. Functional Milk Proteins: Production and Utilization. In Advanced Dairy Chemistry. Proteins. Part B; Fox, P.F., McSweeney, P.L.H., Eds.; Kluver Academic/Plenum Publishers: New York, NY, USA, 2003; pp. 1175-1219.

33. Kinsella, J.E. Functional properties of soybean proteins. J. Am. Oil Chem. Soc. 1979, 56, 242-258.

34. Zayas, J.F. Functionality of Proteins in Food; Zayas, J.F., Ed.; Springer: Berlin/Heidelberg, Germany, 1997.

35. Marco, C.; Rosell, C.M. Breadmaking performance of protein enriched gluten free breads. Eur. Food Res. Technol. 2008, 227, 1205-1213.

36. Matos, M.E.; Rosell, C.M. Quality indicators of rice based gluten-free bread-like products: Relationships between dough rheology and quality characteristics. Food Bioprocess Technol. 2013, 6, 2331-2341.

37. Bonet, A.; Blaszczak, W.; Rosell, C.M. Formation of homopolymers and heteropolymers between wheat flour and several protein sources by transglutaminase catalyzed crosslinking. Cereal Chem. 2006, 83, 655-662. 
38. Rosell, C.M.; Santos, E.; Collar, C. Physical characterization of fiber-enriched bread doughs by dual mixing and temperature constraint using the Mixolab ${ }^{\circledR}$. Eur. Food Res. Technol. 2010, 231, 535-544.

39. Rosell, C.M.; Yokoyama, W.; Shoemaker, C. Rheology of different hydrocolloids-rice starch blends. Effect of successive heating-cooling cycles. Carbohyd. Polym. 2011, 84, 373-382.

40. Wronkowska, M.; Soral-Śmietana, M. Buckwheat flour-a valuable component of gluten-free formulations. Pol. J. Food Nutr. Sci. 2008, 58, 59-63.

41. Krupa-Kozak, U.; Troszyńska, A.; Bączek, N.; Soral-Śmietana, M. Effect of organic calcium supplements on the technological characteristic and sensory properties of gluten-free bread. Eur. Food Res. Technol. 2011, 232, 497-508.

42. Valletta, E.; Fornaro, M.; Cipolli, M.; Conte, S.; Bissolo, F.; Danchielli, C. Celiac disease and obesity: Need for nutritional follow-up after diagnosis. Eur. J. Clin. Nutr. 2010, 64, 1371-1372.

43. Brambilla, P.; Picca, M.; Dilillo, D.; Meneghin, F.; Cravidi, C.; Tischer, M.C.; Vivaldo, T.; Bedoni, G.; Zuccotti, G.V. Changes of body mass index in celiac children on a gluten-free diet. Nutr. Metab. Cardiovasc. 2013, 23, 177-182.

44. Matos, M.E.; Rosell, C.M. Chemical composition and starch digestibility of different gluten-free breads. Plant Food Hum. Nutr. 2011, 66, 224-230.

45. Hager, A-S.; Wolter, A.; Czerny, M.; Bez, J.; Zannini, E.; Arendt, E.K.; Czerny, M. Investigation of product quality, sensory profile and ultrastructure of breads made from a range of commercial gluten-free flours compared to their wheat counterparts. Eur. J. Food Res. Technol. 2012, 235, 333-344.

46. Singh, N.; Kaur, B.I.; Singh, G.H. Effect of different additives on mixograph and bread making properties of Indian wheat flour. J. Food Eng. 2003, 56, 89-95.

47. Esteller, M.S.; Zancanaro, O.; Palmeira, C.N.S.; da Silva Lannes, S.C. The effect of kefir addition on microstructure parameters and physical properties of porous white bread. Eur. Food Res. Technol. 2006, 222, 26-31.

48. Roca, E.; Guillard, V.; Guilbert, S.; Gontard, N. Moisture migration in a cereal composite food at high water activity: Effects of initial porosity and fat content. J Cereal Sci. 2006, 43, 144-151.

49. Labbuza, T.P.; Hyman, C.R. Moisture migration and control in multi-domain foods Trends Food Sci. Technol. 1998, 9, 47-55.

50. Van Dalen, G.; Notenboom, P.; van Vliet, L.J.; Voortman, L.; Esveld, E. 3-D imaging analysis and modeling of porous cereal products using X-ray microtomography. Image Anal. Stereol. 2007, 26, 169-177.

51. Calligaris, S.; Manzocco, L.; Anese, M.; Nicoli, M.C. Effect of heat-treatment on the antioxidant and pro-oxidant activity of milk. Int. Dairy J. 2004, 14, 421-427. 
Reprinted from Nutrients. Cite as: Penagini, F.; Dilillo, D.; Meneghin, F.; Mameli, C.; Fabiano, V.; Zuccotti, G.V. Gluten-Free Diet in Children: An Approach to a Nutritionally Adequate and Balanced Diet. Nutrients 2013, 5, 4553-4565.

Review

\title{
Gluten-Free Diet in Children: An Approach to a Nutritionally Adequate and Balanced Diet
}

\section{Francesca Penagini *, Dario Dilillo, Fabio Meneghin, Chiara Mameli, Valentina Fabiano and Gian Vincenzo Zuccotti}

Department of Pediatrics, University of Milan, Luigi Sacco Hospital, Milan 20157, Italy;

E-Mails: dilillo.dario@hsacco.it (D.D.); meneghin.fabio@hsacco.it (F.M.); mameli.chiara@hsacco.it (C.M.); fabiano.valentina@hsacco.it (V.F.); gianvincenzo.zuccotti@unimi.it (G.V.Z.)

* Author to whom correspondence should be addressed; E-Mail: frapenagini@tiscali.it; Tel.: +0039-0239-042-268; Fax: +0039-0239-042-254.

Received: 25 October 2013; in revised form: 4 November 2013 / Accepted: 7 November 2013 / Published: 18 November 2013

\begin{abstract}
Gluten-free diet (GFD) is the cornerstone treatment for celiac disease (CD). GFD implies a strict and lifelong elimination from the diet of gluten, the storage protein found in wheat, barley, rye and hybrids of these grains, such as kamut and triticale. The absence of gluten in natural and processed foods, despite being the key aspect of GFD, may lead to nutritional consequences, such as deficits and imbalances. The nutritional adequacy of GFD is particularly important in children, this the age being of maximal energy and nutrient requirements for growth, development and activity. In recent years, attention has focused on the nutritional quality of gluten-free products (GFPs) available in the market. It is well recognized that GFPs are considered of lower quality and poorer nutritional value compared to the gluten-containing counterparts. The present review focuses on the nutritional adequacy of GFD at the pediatric age, with the aim being to increase awareness of the potential complications associated with this diet, to identify strategies in order to avoid them and to promote a healthier diet and lifestyle in children with CD.
\end{abstract}

Keywords: celiac disease; gluten-free diet; children; nutritional complications; balanced diet 


\section{Introduction}

Celiac disease (CD) is a chronic systemic autoimmune disorder caused by a permanent intolerance to gluten proteins in genetically susceptible individuals. Gluten is a general term used to describe a mixture of storage proteins, including prolamins, hordeins and secalins found in wheat, barley and rye, respectively. These proteins may exert a toxic effect on intestinal mucosa in genetically susceptible individuals by triggering an immune-mediated response, responsible for the typical villous atrophy and lymphocyte infiltrate in small intestine mucosa seen in CD. In fact, these proteins contain epitopes that undergo deamidation, an important process for the binding of the CD associated human leukocyte antigen (HLA) DQ2/DQ8 haplotypes with T-lymphocytes, activating an autoimmune response [1-3].

A lifelong strict gluten-free diet (GFD) is the only available treatment for CD. Adherence to GFD leads to regression of symptoms, normalization of histological and laboratory findings and reduces the risk of CD associated complications [4]. Within the range of gluten-free foods, a distinction must be made between those that are naturally gluten-free and those that are made gluten-free through a process of purification. There are several foods that are naturally gluten-free, such as rice, corn, potatoes and a number of different grains, seeds and legumes. Historically, rice, corn and potatoes have been the first natural substitutes for gluten-containing grains. Today, a number of different grains, including pseudo-cereals, offer increased variety, improved palatability to GFD and are a good source of carbohydrates, protein, dietary fiber, vitamins and polyunsaturated fatty acids $[5,6]$. The commercially available gluten-free (GF) products are processed foods purified of gluten. The elimination of this storage protein inevitably alters the macro- and micro-nutrient composition, thus the nutritional value. First, wheat is not only a major source of protein, but also of iron, folates and B vitamins (thiamin, riboflavin and niacin); in fact, GF products are often low in these nutrients, as opposed to their gluten containing equivalents [7-9]. The various gluten-free and gluten containing foods are listed in Table 1.

Table 1. Gluten-free and gluten containing cereals and other foods.

\begin{tabular}{cc}
\hline Gluten-Free Cereals & Gluten Containing Cereals \\
\hline Cereals and minor cereals & \\
Corn & \\
Rice & Wheat \\
Sorghum & Barley \\
Oats* & Rye \\
Teff & Kamut \\
Millet & Malt \\
Pseudo-cereals & Triticale \\
Amaranth & \\
Quinoa & \\
Buckwheat & \\
Vegetable foods & Bread \\
Vegetables & Pasta \\
Fruits & Snacks \\
Nuts & Biscuits \\
\hline
\end{tabular}


Table 1. Cont.

\begin{tabular}{cc}
\hline $\begin{array}{c}\text { Other plant foods } \\
\text { Potatoes } \\
\text { Tapioca }\end{array}$ & All foods produced with any of the \\
Soybean & gluten containing cereals \\
Vegetable oils & mentioned above. Numerous \\
Animal foods & foods available in supermarkets \\
Dairy products & and grocery stores, including meat \\
Egg & products, sweets and beer, \\
Meat & contain gluten. \\
Fish & \\
\hline$*$ Controversial; see paragraph on oats.
\end{tabular}

\subsection{Macronutrients}

Studies show that gluten-free products (GFPs) often have a greater carbohydrate and lipid content than their gluten containing equivalents [10-13]. Segura et al. analyzed the nutritional composition of a range of GF breads and found that these are starchy-based foods with a high glycemic index (estimated between 83.3 and 96.1 vs. 71 for white wheat flour bread), with low protein and high fat content [9]. With regards to lipid content and composition, Caponio et al. showed that commercially available GF biscuits are richer in saturated fatty acids compared to the gluten containing equivalents [13]. All these characteristics have a negative effect on health, and this should be seriously taken into account, because the limited choice of food products in the diet of children with CD induces a high consumption of packaged GFPs, such as snacks and biscuits.

\subsection{Micronutrients and Minerals}

It has been shown that some commercially available GFPs have a lower content of folates, iron and $\mathrm{B}$ vitamins or are not consistently enriched/fortified compared to their gluten containing counterparts [7-9]. Thompson [7,8] conducted two studies on US commercially available GFPs. The author analyzed the folate, iron and B vitamins (thiamin, riboflavin and niacin) composition of these products and compared them with the composition of their gluten containing counterparts, finding that GFPs were significantly lower in folates, iron and B vitamins.

\subsection{Dietary Fiber}

Some studies have reported that GFD is associated with a lower intake of dietary fiber than a standard gluten containing diet $[14,15]$. A study conducted in the USA on adults showed that the diet of CD patients on GFD was low in fiber intake [15]. This phenomenon is likely to be related to the composition of many GF foods made with starches and/or refined flours with low content in fiber. In fact, during the refining process, the outer layer of grain containing most of the fiber is removed, leaving only the starchy inner layer. 


\section{Nutritional Imbalances in Children with Celiac Disease Following a GFD}

Studies conducted in adults and children show that approximately $20 \%-38 \%$ of patients with CD have nutritional complications, such as calorie/protein imbalance, dietary fiber, mineral and vitamin deficiencies [16-19]. These complications may be encountered both at diagnosis and during follow-up, whilst on GFD [9,18]. At diagnosis, the deficiencies are often secondary to nutrient malabsorption due to mucosal damage. Studies show that the more pronounced the villous atrophy, the greater the nutritional deficiencies, with lower levels of iron, copper, folate, vitamin B-12 and zinc [19]. For CD patients on GFD, the nutritional complications are likely to be caused by the poor nutritional quality of the GFPs mentioned above and by the incorrect alimentary choices of CD patients. The most common nutritional deficiencies encountered in adults with $\mathrm{CD}$, at diagnosis and during GFD, are described in Table 2.

Table 2. Common nutrient deficiencies in adults with celiac disease (CD) at diagnosis and after Gluten-free diet (GFD). Modified from Cynthia Kupper [20].

\begin{tabular}{cccc}
\hline \multicolumn{4}{c}{ Common Nutrient Deficiencies in Subjects with Celiac Disease } \\
\hline At Diagnosis & GFD & GFD Products & Long-Term GFD \\
\hline Calorie/protein & Fiber & Fiber & Fiber \\
Fiber & Iron & Iron & \\
Iron & Calcium & & \\
Calcium & Vitamin D & & \\
Vitamin D & Magnesium & & \\
Magnesium & & & \\
Zinc & Folate, niacin, vitamin & Folate, niacin, vitamin & Folate, niacin, vitamin \\
Folate, niacin, & B12 & B12 & B12 \\
vitamin B12 & Riboflavin & Riboflavin & Riboflavin \\
Riboflavin & & &
\end{tabular}

Numerous studies focus their attention specifically on the nutrient intakes of CD children and adolescents on GFD. Mariani et al. [21] studied the nutritional habits of 47 adolescents (aged 10-20 years) affected by $\mathrm{CD}$, by means of a three-day alimentary diary. A comparison with the Italian and American recommended daily allowance (RDAs) was done for the intakes of macronutrients, fiber, iron and calcium. The results showed that CD subjects followed a high-protein and high-lipid diet with low intakes of carbohydrates, iron, calcium and fiber compared to the recommended daily intakes. Furthermore, Hopman et al. [22] studied the nutrient intake of 37 adolescents with CD (aged 13-16 years) following a strict GFD and compared the intakes with a control group in the same age category. In the CD group, the intake of saturated fat was significantly higher than recommended by both the American and Dutch RDAs; the intake of fiber and iron was significantly lower than recommended. Furthermore, the comparison between the two groups showed that the intake of fiber and iron was lower in the CD group compared to controls $(p<0.05)$. Öhlund et al. [23] conducted a study in 2010 on 30 children aged 4-17 years with CD and on GFD, using a five-day food record. High intakes of saturated fat and sucrose and low intakes of dietary fiber, vitamin D and magnesium compared to recommendations (New Nordic Nutrition Recommendations, 2004) was observed. 
An elegantly conducted study by Zuccotti et al. compared the dietary intake of CD children on GFD to a group of healthy children (healthy controls, HC) and evaluated the contribution of commercially available GFPs on the observed nutritional intake [24]. The study showed that the daily intake of vitamin D was significantly lower in the CD compared to the HC group (vitamin D median value $3.1 \mu \mathrm{g}+0.6$ vs. $3.1 \mu \mathrm{g}+2.8, p<0.01)$. With regards to macronutrients, the intake of simple sugars, fats and protein exceeded the national recommendations for health in both the CD and HC groups. The total daily energy intake was significantly higher in the CD group compared to HCs (8961.8 $\mathrm{KJ}_{\text {day }}{ }^{-1}$ vs. $\left.5761.0 \mathrm{KJ}_{\text {day }}{ }^{-1} ; p<0.001\right)$. In the CD group, the carbohydrate-derived energy was higher, while the lipid-derived energy was lower, compared to the HC group. Protein-derived energy did not differ between the two groups. In the CD group, the contribution of commercially available GFPs on daily energy intake was studied. The main finding was that these products provided $36.3 \%$ of the total daily energy intake $\left(3253.1 \mathrm{KJ}\right.$ day $^{-1}$ out of $\left.8961.8 \mathrm{KJ} \mathrm{day}^{-1}\right)$ in these patients. Analyzing the contribution of the macronutrients derived from GFPs on the total daily energy intake, it was observed that protein derived from GFPs accounted for $7.3 \%$ of the total energy derived from protein, representing $18 \%$ of total daily energy intake. With regards to lipids, GFPs contributed a median of $12.9 \mathrm{~g} \mathrm{day}^{-1}$ of the median total daily fat intake of $73.0 \mathrm{~g}$, which is equivalent to $17.7 \%$ of energy from fat. Furthermore, Mariani et al. [21] conducted a nutritional analysis of children with CD. The author found that children complying with a strict GFD had significantly greater nutritional imbalance in their diet than did children cheating on their GFD. More troubling, the incidence of children who were overweight or obese was more frequent (72\%) in the strict GFD group compared with the children not following a strict GFD (51\%) and healthy age-matched controls (47\%). A study conducted by Ferrara et al. [25] compared the caloric intake and fat consumption of 50 children with CD following a GFD with 50 healthy children. A significant increase in fat consumption was observed in children with $\mathrm{CD}$ compared to healthy children $(72.5+$ $37.2 \mathrm{~g} v s .52 .9+35.4 \mathrm{~g}$ per $100 \mathrm{~g}$ of food, $p<0.008)$. Furthermore, a significant difference in fat intake was observed between the two groups $(10.21+3.15 \mathrm{~g}$ per $100 \mathrm{~g}$ of food in CD group vs. 7.46 $+2.91 \mathrm{~g} / 100 \mathrm{~g}$ in control group, $p=0.004)$.

\section{Effects of Gluten-Free Diet on Anthropometric Parameters}

The scientific literature on anthropometric parameters in children with CD on GFD provide contrasting data. On the one hand, there is evidence that good compliance with GFD is associated with a positive effect on anthropometric parameters, including: the reduction of fat and the recovery of lean body mass [26], normalization of body mass index (BMI) in both previously underweight and overweight subjects [27] and acceleration of linear growth [28]. A study conducted in children with $\mathrm{CD}$ and obesity at diagnosis showed a significant reduction in BMI after 12 months of GFD [29]. Another study conducted by Brambilla et al. found a lower frequency of being overweight and of obesity in children with $\mathrm{CD}$, both at diagnosis and during GFD, compared to healthy controls [30]. Even though the frequency of being overweight and of obesity in the CD group increased on GFD, it remained lower than observed in the general population.

On the other hand, there are also studies that suggest that GFD may have a negative effect on body composition and anthropometric parameters in subjects with CD [31]. Mariani et al. [21] first reported the high prevalence of being overweight and of obesity in CD adolescents on GFD; the 
authors found that more than $50 \%$ of CD adolescents were overweight during GFD. However, in the latter paper, the authors used a relative body weight $>110 \%$, rather than BMI, to define being overweight, probably leading to an overestimation of being overweight. Furthermore, a study conducted by Valletta et al. showed that the frequency of being overweight in children with CD was nearly doubled after one year of GFD [32]. Potential explanations for the undesirable weight gain and obesity observed in these studies are possible overfeeding as the intestinal mucosa heals, consumption of less complex carbohydrates and fiber and more sugars, proteins and saturated fats in GFD. The conflicting data may in part be caused by differences in the timing of anthropometric assessment. Many children with CD, after introduction of GFD, may initially gain excessive weight and only thereafter start to show catch-up growth and normalization of weight. Even though the effect of GFD on body weight and BMI remains a controversial issue, it remains fundamental for pediatricians to be aware of the possible nutritional consequences of GFD for which early recognition can be crucial in the prevention of obesity-related complications.

\section{Dietary Advices for a Nutritionally Adequate and Balanced Diet in Children with Celiac Disease}

\subsection{Education and Compliance}

The first step towards a balanced diet starts from early education on CD and GFD, possibly provided by a skilled dietitian and/or by a physician with expert knowledge in CD. The diet is complicated and can be overwhelming if not presented using a thorough and proactive approach. Early education is fundamental to promote adherence to GFD. In fact, studies focusing on compliance to GFD indicate that adherence is compromised by a number of factors, including a lack of education and continued support by a physician and dietitian [33,34]. In a study conducted by Charalampopoulos et al. on determinants of the adherence of GFD in children with CD, baseline education was one of the main determinant factors influencing compliance, suggesting the importance for frequent reinforcement and an accurate explanation of dietary recommendations [34].

\subsection{Dietary Intake}

In $C D$ children on GFD, the recommended distribution of daily calorie intake for a healthy and balanced diet does not differ from that recommended to the general population. According to the dietary reference intake values (DRI), the total daily dietary calories should be ideally obtained as $55 \%$ from complex and simple carbohydrates, $15 \%$ from dietary protein and $25 \%-30 \%$ or less from lipids. The intake of unsaturated fat (monounsaturated and polyunsaturated) should be preferred. Monounsaturated and polyunsaturated fatty acids should provide more than $15 \%$ and $10 \%$ of total calories, respectively. They are found in foods, such as vegetable oils, nuts, seeds and fish high in lipid content, such as salmon, trout and herring. Thus, it is important to inform and recommend children with $\mathrm{CD}$ on the ideal distribution of the daily calorie intake. 


\subsection{Gluten-Free Foods}

It is advisable to prefer consumption of naturally GF foods, since it has been shown that they are more balanced and complete under both the macro- and micro-nutrient point of view. In fact, these foods are considered to have a higher nutritional value in terms of energy provision, lipid composition and vitamin content as opposed to the commercially purified GF products. Within the range of naturally GF foods, it is preferable to consume those rich in iron and folic acid, such as leafy vegetables, legumes, fish and meat. During explanation of naturally GF foods to patients, it is a good approach for healthcare professionals to bear in mind the local food habits and recipes of each country. This may provide tailored dietary advice, improving acceptance and compliance to GFD. Furthermore, increasing awareness on the availability of the local naturally GF foods may help promote their consumption, resulting in a more balanced and economically advantageous diet. Indeed, these aspects should always be addressed during dietary counseling. With regards to the commercially purified GFPs, it is recommended to pay special attention to the labeling and chemical composition. In European countries, the currently accepted definition of GF is the one designed by Codex Alimentarius (the gluten-free certification organization, 2007) [35]. The term "gluten-free" refers only to foods containing less than $20 \mathrm{ppm}$ of gluten. In addition, the claim "very low gluten" is used for foods, such as bread, produced using cereals that have been specially processed to remove most of the gluten and containing less than $30 \mathrm{mg}$ daily. Furthermore, some GFPs are enriched/fortified with vitamins and/or minerals, thus the choice of these products is preferred, to prevent the deficiencies associated with GFD, mentioned above. Clear labeling of GFPs and education of $\mathrm{CD}$ patients on how to interpret them is fundamental to helping CD subjects make safer and more informed food choices.

\subsection{Pseudo-Cereals and Minor-Cereals}

Pseudo-cereals, such as amaranth, quinoa and buckwheat, and other minor cereals represent a healthy alternative to frequently used ingredients in gluten-free products. They are a good source of carbohydrates, protein, dietary fiber, vitamins and polyunsaturated fatty acids [36]. In fact, the fiber content in these grains ranges from seven to $10 \mathrm{~g} / 100 \mathrm{~g}$ [37], which is higher compared to those of other plant foods and cereals and approximately the same as the content in wheat (fiber $9.5 \mathrm{~g} / 100 \mathrm{~g}$ ). Furthermore, they are a valid source of protein, as their content is superior to that of wheat in terms of the quantity and quality of proteins: in particular, lysine, arginine, histidine, methionine and cysteine can be found in high amounts [38,39]. Although the lipid content of pseudo-cereals is higher compared to other plant foods, they are characterized by a higher content of unsaturated fatty acids, in particular, $\alpha$-linolenic acid [40,41], beneficial for the prevention of cardiovascular diseases. In addition, higher concentrations of folic acid have been found in quinoa $(78.1 \mu \mathrm{g} / 100 \mathrm{~g})$ and amaranth $(102 \mu \mathrm{g} / 100 \mathrm{~g})$ with respect to wheat $(40 \mu \mathrm{g} / 100 \mathrm{~g})$. Furthermore, both amaranth and quinoa are also good sources of riboflavin, vitamin $\mathrm{C}$ and vitamin $\mathrm{E}$ [42]. In addition, pseudo-cereals permit a wider variety of foods, broadening the choice for $\mathrm{CD}$ children when selecting foods. Under the economic point of view, these grains offer a less expensive alternative with respect to standard gluten-free choices; also, this aspect could help increase dietary compliance by reducing the economic burden of the diet [43]. The nutritional advantages of pseudo-cereals are listed in Table 3. 
Table 3. Nutritional advantages of pseudo-cereals (amaranth, buckwheat and quinoa).

\begin{tabular}{c}
\hline Nutritional Characteristics of Amaranth, Buckwheat and Quinoa \\
\hline High fiber content, $7-10 \mathrm{~g} / 100 \mathrm{~g}$, approximately the same as wheat fiber $9.5 \mathrm{~g} / 100 \mathrm{~g}$. \\
High protein content, $10.9 \%-15.2 \%$ of dry mass $v s .11 .7 \%$ of dry mass in wheat. \\
High quality amino acids: lysine, arginine, histidine, methionine and cysteine. \\
Source of unsaturated fatty acids, in particular, $\alpha$-linolenic acid. \\
High content of folic acid: quinoa and amaranth, $78.1 \mu \mathrm{g} / 100 \mathrm{~g}$ and $102 \mu \mathrm{g} / 100 \mathrm{~g}$, respectively, $v s$. \\
$40 \mathrm{~g} / 100 \mathrm{~g}$ in wheat. \\
Source of vitamins: B2, B6, riboflavin, vitamin C and E. \\
Source of minerals: the content is twice as high as in other cereals.
\end{tabular}

\subsection{Oats}

The inclusion of oats in the GFD has been, for many years, and still is a matter of debate, because it was thought that avenin (the storage protein found in oats) was also toxic to CD patients. Moreover, attention has been focused on the issue of the frequent cross-contamination of oats with gluten-containing grains. Studies have demonstrated that when consumed in moderation, oats free from cross-contamination are well tolerated by most children $[44,45]$. Under the nutritional point of view, oats represent a good source of iron, dietary fiber, thiamin and zinc and, in addition, have a good palatability [46]. A study conducted by Størsund et al. in CD children suggests that oats may improve the nutritional value of GFD and, in view of the good palatability, may also help increase compliance [47]. Recently, Lee et al. demonstrated that adding three servings of gluten-free alternative grains, including oats, positively impacts the nutrient profile (fiber, thiamin, riboflavin, niacin, folate and iron) of the grain portion of the gluten-free diet [48].

\subsection{Vitamins and Minerals}

Meat, fish, fruit and vegetables are an important natural source of vitamins, minerals and trace elements. In view of the possible micronutrient deficiencies associated with GFPs, an appropriate consumption of these foods should be advised in children with CD. In particular, fruit and vegetables are low in energy and rich in vitamins and minerals; moreover, they contain phytochemicals and antioxidant compounds that exert a protective effect against diseases associated with oxidative damage [49]. The intake of at least five portions of fruit and vegetables a day should be recommended in children with CD. Minerals (calcium, phosphorus, sodium, potassium, chloride and magnesium) and trace elements (iron, zinc and selenium) are also contained in a significant amount in pseudo-cereals, in which the content can be twice as high as in other cereals. For example, in teff, iron and calcium contents (11-33 mg/100 g and 100-150 mg/100 g, respectively) are higher than those of wheat, barley, sorghum and rice.

\subsection{Nutritional Follow-Up}

Continuous long-term follow-up is crucial to promote adherence to GFD and for early identification of nutritional deficiencies and/or metabolic imbalances. Ideally, a skilled dietitian with knowledge in CD and GFD should be an integral part of the healthcare team. A child's nutritional 
status should be accurately assessed at diagnosis and at each follow-up, which ideally should be performed at six months post commencement of GFD and then annually, post-diagnosis. The evaluation of nutritional status should start from a thorough and accurate dietary history and include the assessment of anthropometric parameters (weight, height and body mass index). Adherence to GFD should be assessed, and information on how to safely broaden food choices and interpret food labeling should be given. Early identification and correction of nutritional deficiencies should be regularly addressed. Table 4 summarizes the key points of nutritional follow-up in children with CD.

Table 4. Recommended timing for nutritional follow-up. BMI: body mass index.

\begin{tabular}{|c|c|}
\hline \multicolumn{2}{|c|}{ Nutritional Follow-Up } \\
\hline When? & How? \\
\hline $\begin{array}{c}\text { Diagnosis } \\
6 \text { months post commencement of GFD } \\
\text { Annually post diagnosis }\end{array}$ & $\begin{array}{c}\text { Accurate dietary history } \\
\text { Evaluation of nutritional status } \\
\text { Anthropometric parameters, (weight, height, BMI) } \\
\text { Physical examination (attention to signs of malnutrition) }\end{array}$ \\
\hline
\end{tabular}

Figure 1. Schematic representation of proposed approach to a nutritionally adequate and balanced gluten-free diet.

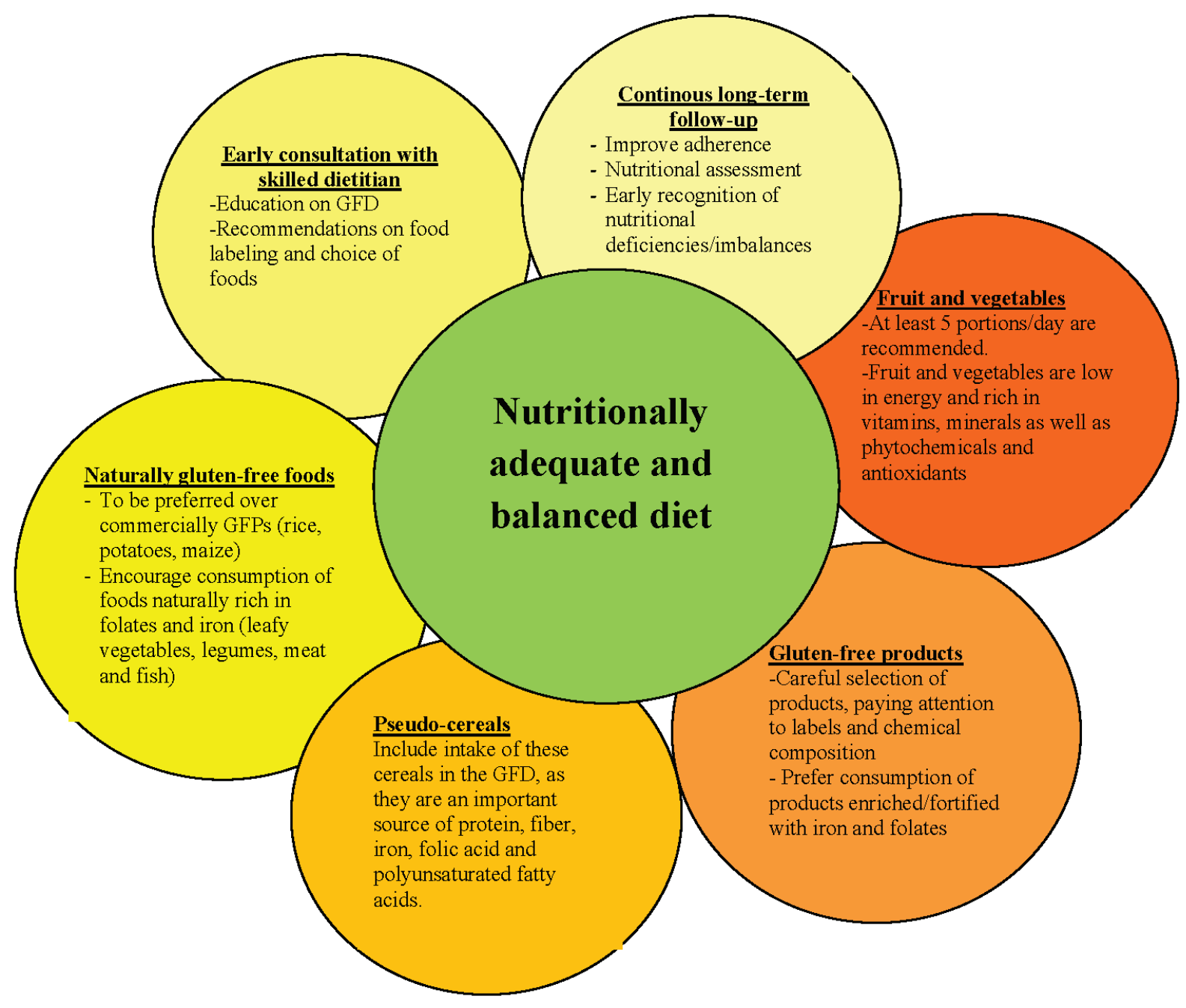




\section{Conclusions}

Gluten-free diet, the only available treatment for $\mathrm{CD}$, if not carried out with attention, may paradoxically lead to nutritional imbalances, which should be avoided, particularly at the pediatric age, the phase of maximal growth and development. Increasing awareness on the possible nutritional deficiencies associated with GFD may help healthcare professionals and families tackle the issue by starting from early education on GFD and clear dietary advice on how to choose the most appropriate gluten-free foods. Figure 1 summarizes, by means of a schematic representation, a proposed approach towards a nutritionally adequate and balanced gluten-free diet. Further studies on the technological and nutritional properties of the alternative cereals as wheat replacements are needed to confirm their role in improving the intake of protein, iron, calcium and fiber and reducing nutritional deficiencies in children with CD. Their role in the economic burden of the diet and their effect on compliance should also be further investigated. Furthermore, a promising field for gluten-free diet is food biotechnologies. By means of this science, it would be worth considering genetically modifying the amino acid sequence of gluten storage proteins, in order to make them free of those domains high in prolines and glutamines, which are responsible for the toxicity.

\section{Conflicts of Interest}

The authors declare no conflict of interest.

\section{References}

1. Sjöström, H.; Lundin, K.E.; Molberg, O.; Körner, R.; McAdam, S.N.; Anthonsen, D.; Quarsten, H.; Norén, O.; Roepstorff, P.; Thorsby, E.; et al. Identification of a gliadin T-cell epitope in celiac disease: General importance of gliadin deaminidation for intestinal $\mathrm{T}$ cell recognition. Scand. J. Immunol. 1998, 48, 111-115.

2. Maiuri, L.; Ciacci, C.; Ricciardelli, I.; Vacca, L.; Raia, V.; Auricchio, S.; Picard, J.; Osman, M.; Quarantino, S.; Londei, M. Association between innate response to gliadin and activation of pathogenic T cells in coeliac disease. Lancet 2003, 362, 30-37.

3. Mowat, A.M. Coeliac disease-A meeting point for genetics, immunology and protein chemistry. Lancet 2003, 361, 1290-1292.

4. Ciacci, C.; Cirillo, M.; Cavallaro, R.; Mazzacca, G. Long-term follow-up of celiac adults on gluten-free diet: Prevalence and correlates of intestinal damage. Digestion 2002, 66, 178-185.

5. Alvarez-Jubete, L.; Arendt, E.K.; Gallagher, E. Nutritive value and chemical composition of pseudocereals as gluten-free ingredients. Int. J. Food Sci. Nutr. 2009, 60, 240-257.

6. Vega-Gàlvez, A.; Miranda, M.; Vergara, J.; Uribe, E.; Puente, L.; Martìnez, E.A. Nutrition facts and functional potential of quinoa (Chenopodium quinoa willd.), an ancient Andean grain: A review. J. Sci. Food Agric. 2010, 90, 2541-2547.

7. Thompson, T. Thiamin, riboflavin, and niacin contents of the gluten-free diet: Is there cause for concern? J. Am. Diet. Assoc. 1999, 99, 858-862.

8. Thompson, T. Folate, iron, and dietary fiber contents of the gluten-free diet. J. Am. Diet. Assoc. 2000, 100, 1389-1396. 
9. Hallert, C.; Grant, C.; Grehn, S.; Grännö, C.; Hultén, S.; Midhagen, G.; Strön, M.; Svensson, H.; Valdimarsson, T. Evidence of poor vitamin status in celiac patients on a gluten-free diet for 10 years. Aliment. Pharmacol. Ther. 2002, 16, 1333-1339.

10. Segura, M.E.; Rosell, C.M. Chemical composition and starch digestibility of different gluten-free breads. Plant Foods Hum. Nutr. 2011, 66, 3, 224-230.

11. Jenkins, D.J.; Thorne, M.J.; Wolever, T.M.; Jenkins, A.L.; Rao, A.V.; Thompson, L.U. The effect of starch-protein interaction in wheat on the glycaemic response and rate of in vitro digestion.

Am. J. Clin. Nutr. 1987, 45, 946-951.

12. Berti, C.; Riso, P.; Monti, L.D.; Porrini, M. In vitro starch digestibility and in vivo glucose response of gluten-free foods and their gluten counterparts. Eur. J. Nutr. 2004, 43, 198-204.

13. Caponio, F.; Summo, C.; Clodoveo, M.L.; Pasqualone, A. Evaluation of the nutritional quality of the lipid fraction of gluten-free biscuits. Eur. Food Res. Technol. 2008, 223, 135-139.

14. Thompson, T.; Dennis, M.; Higgins, L.A.; Lee, A.R.; Sharrett, M.K. Gluten-free diet survey: Are Americans with celiac disease consuming recommended amounts of fibre, iron, calcium and grain food? J. Hum. Nutr. Diet. 2005, 18, 163-169.

15. Wild, D.; Robins, G.G.; Burley, V.J.; Howdle, P.D. Evidence of high sugar intake and low fiber and mineral intake in the gluten-free diet. Aliment. Pharmacol. Ther. 2010, 32, 573-581.

16. Kemppainen, T.; Uusitupa, M.; Janatuinen, E.; Järvinen, R.; Julkunen, R.; Pikkarainen, P. Intakes of nutrients and nutritional status in celiac patients. Scand. J. Gastroenterol. 1995, 30, 575-579.

17. Kinsey, L.; Burden, S.T.; Bannerman, E. A dietary survey to determine if patients with celiac disease are meeting current healthy eating guidelines and how their diet compares to that of British general population. Eur. J. Clin. Nutr. 2008, 62, 1333-1342.

18. Bardella, M.T.; Fredella, C.; Prampolini, L.; Molteni, N.; Giunta, A.M.; Bianchi, P.A. Body composition and dietary intakes in adult celiac disease patients consuming a strict gluten-free diet. Am. J. Clin. Nutr. 2000, 72, 937-939.

19. Jameson, S. Coeliac, insulin-like growth factor, bone mineral density, and zinc. Scand. J. Gastroenterol. 2000, 35, 894-896.

20. Kupper, C. Dietary guidelines and implementation for celiac disease. Gastroenterology 2005 , 128, S121-S127.

21. Mariani, P.; Viti, M.G.; Montuori, M.; La Vecchia, A.; Cipolletta, E.; Calvani, L.; Bonamico, M. The gluten-free diet: A nutritional risk factor for adolescents with celiac disease? J. Pediatr. Gastroenterol. Nutr. 1998, 27, 519-523.

22. Hopman, E.G.; le Cessie, S.; von Blomberg, B.M.; Mearin, M.L. Nutritional management of the gluten-free diet in young people with coeliac disease in The Netherlands. J. Paediatr. Gastroenterol. Nutr. 2006, 43, 102-108.

23. Öhlund, K.; Olsson, C.; Hernell, O.; Öhlund, L. Dietary shortcomings in children on a gluten-free diet. J. Hum. Nutr. Diet. 2010, 23, 294-300.

24. Zuccotti, G.; Fabiano, V.; Dilillo, D.; Picca, M.; Cravidi, C.; Brambilla, P. Intakes of nutrients in Italian children with celiac disease and the role of commercially available gluten-free products. J. Hum. Nutr. Diet. 2013, 26, 436-444. 
25. Ferrara, P.; Cicala, M.; Tiberi, E.; Spadaccio, C.; Marcella, L.; Gatto, A.; Calzolari, P.; Castelluccio, G. High fat consumption in children with celiac disease. Acta Gastroenterol. Belg. 2009, 72, 296-300.

26. Barera, G.; Mora, S.; Brambilla, P.; Ricotti, A.; Menni, L.; Beccio, S.; Bianchi, C. Body composition in children with celiac disease and the efffects of a gluten-free diet: A prospective case-control study. Am. J. Clin. Nutr. 2000, 72, 71-75.

27. Cheng, J.; Brar, P.S.; Lee, A.R.; Green, P.H.; Body mass index in celiac disease: Beneficial effect of a gluten-free diet. J. Clin. Gastroenterol. 2010, 44, 267-271.

28. Radlović, N.; Mladenović, M.; Leković, Z.; Zivanović, D.; Brdar, R.; Radlović, V.; Ristić, D.; Pavlović, M.; Stojsić, Z.; Vuletić, B.; et al. Effect of gluten-free diet on the growth and nutritional status of children with coeliac disease. Srp. Arh. Celok. Lek. 2009, 137, 632-637.

29. Venkatasubramani, N.; Telega, G.; Werlin, S.L. Obesity in pediatric celiac disease. J. Pediatr. Gastroenterol. Nutr. 2010, 51, 295-297.

30. Brambilla, P.; Picca, M.; Dilillo, D.; Meneghin, F.; Cravidi, C.; Tischer, M.C.; Vivaldo, T.; Bedogni, G.; Zuccotti, G.V. Changes of body mass index in celiac children on a gluten-free diet. Nutr. Metab. Cardiovasc. Dis. 2013, 23, 177-182.

31. Kabbani, T.A.; Goldberg, A.; Kelly, C.P.; Pallav, K.; Tariq, S.; Peer, A.; Hasen, J.; Dennis, M.; Leffler, D.A. Body mass index and the risk of obesity in coeliac disease treated with gluten-free diet. Aliment. Pharmacol. Ther. 2012, 35, 723-729.

32. Valletta, E.; Fornaro, M.; Cipolli, M.; Conte, S.; Bissolo, F.; Danchielli, C. Celiac disease and obesity: Need for nutritional follow-up after diagnosis. Eur. J. Clin. Nutr. 2010, 64, 1371-1372.

33. Roma, E.; Roubani, A.; Kolia, E.; Panayiotou, J.; Zellos, A.; Syriopoulou, V.P. Dietary compliance and life style of children with celiac disease. J. Hum. Nutr. Diet. 2010, 23, 176-182.

34. Charalampopoulos, D.; Panayiotou, J.; Chouliaras, G.; Zellos, A.; Kyritsi, E.; Roma, E. Determinants of adherence to gluten-free diet in Greek children with celiac disease: A cross-sectional study. Eur. J. Clin. Nutr. 2013, 67, 615-619.

35. Codex Alimentarius Commission. Draft Revised Codex Standard for Foods for Special Dietary Use for Persons Intolerant to Gluten Joint FAO/WHO Food Standards Programme; WHO: Geneva, Switzerland, 2007.

36. Dyner, L.; Drago, S.R.; PiÑeiro, A.; Sànchez, H.; Gonzàlez, R.; Villaamil, E.; Valencia, M.E. Composition and potential contribution of iron, calcium and zinc of bread and pasta made with wheat and amaranth flours. Arch. Latinoam. Nutr. 2007, 57, 69-77.

37. Ötles, S.; Cagindi, Ö. Cereal based functional foods and nutraceuticals. Acta Sci. Pol. Technol. Aliment. 2006, 5, 107-112.

38. Gorinstein, S.; Pawelzik, E.; Delgado-Licon, E.; Haruenkit, R.; Weisz, M.; Trakhtenberg, S. Characterization of pseudocereal and cereal proteins by protein and amino acid analyses. J. Sci. Food Agric. 2002, 82, 886-891.

39. Abdel-Aal, E.S.M.; Hucl, P. Amino acid composition and in vitro protein digestibility of selected ancient wheats and their products. J. Food Comp. Anal. 2002, 15, 737-747.

40. Yànez, E.; Zacarìas, I.; Granger, D.; Vàsquez, M.; Estèvez, A.M. Chemical and nutritional characterization of amaranthus (Amaranthus cruentus). Arch. Latinoam. Nutr. 1994, 44, 57-62. 
41. Adeyeye, A.; Ajewole, K. Chemical composition and fatty acid profiles of cereals in Nigeria. Food Chem. 1992, 44, 41-44.

42. Coulter, L.; Lorenz, K. Quinoa-composition, nutritional value, food applications. Lebensm. WissTechnol. 1990, 23, 203-207.

43. Stevens, L.; Rashid, M. Gluten-free and regular foods: A cost comparison. Can. J. Diet. Pract. Res. 2008, 69, 147-150.

44. Hoffenberg, E.J.; Haas, J.; Drescher, A.; Barnhurst, R.; Osberg, I.; Bao, F.; Eisenbarth, G. A trial of oats in children with newly diagnosed celiac disease. J. Pediatr. 2000, 137, 361-366.

45. Höberg, L.; Laurin, P.; Faith-Magnusson, K.; Grant, C.; Grodzinsky, E.; Jansson, G.; Ascher, H.; Browaldh, L.; Hammersjö, J.A.; Lindberg, E.; et al. Oats to children with newly diagnosed celiac disease: A randomized double-blind study. Gut 2004, 53, 649-654.

46. Sadiq Butt, M.; Tahir-Nadeem, M.; Khan, M.K.; Shabir, R.; Butt, M.S. Oat: Unique among the cereals. Eur. J. Nutr. 2008, 47, 68-79.

47. Størsund, S.; Hulthèn, L.R.; Lenner, R.A. Beneficial effects of oats in the gluten-free diet of adults with special reference to nutrient status, symptoms and subjective experiences. $B r . J$. Nutr. 2003, 90, 101-107.

48. Lee, A.R.; Ng, D.L.; Dave, E.; Ciaccio, E.J.; Green, P.H. The effect of substituting alternative grains in the diet on the nutritional profile of the gluten-free diet. J. Hum. Nutr. Diet. 2009, 22, 359-363.

49. Stevenson, D.E.; Hurst, R.D. Polyphenolic phytochemicals—Just antioxidants or much more? Cell Mol. Life Sci. 2007, 64, 2900-2916. 
Reprinted from Nutrients. Cite as: Kaukinen, K.; Collin, P.; Huhtala, H.; Mäki, M. Long-Term Consumption of Oats in Adult Celiac Disease Patients. Nutrients 2013, 5, 4380-4389.

Article

\title{
Long-Term Consumption of Oats in Adult Celiac Disease Patients
}

\section{Katri Kaukinen $^{1,2,3, *}$, Pekka Collin ${ }^{1,2}$, Heini Huhtala ${ }^{4}$ and Markku Mäki ${ }^{5}$}

1

Department of Gastroenterology and Alimentary Tract Surgery, Tampere University Hospital, Tampere FIN-33521, Finland; E-Mail: pekka.collin@uta.fi

2 School of Medicine, University of Tampere, Tampere FIN-33014, Finland

3 Department of Medicine, Seinäjoki Central Hospital, Seinäjoki FIN-60220, Finland

4 School of Health Sciences, University of Tampere, Tampere FIN-33014, Finland;

E-Mail: heini.huhtala@uta.fi

5 Center for Child Health Research, University of Tampere and Tampere University Hospital, Tampere FIN-33014, Finland; E-Mail: markku.maki@uta.fi

* Author to whom correspondence should be addressed; E-Mail: katri.kaukinen@uta.fi; Tel.: +358-50-318-6343; Fax: +358-3-364-1369.

Received: 29 August 2013; in revised form: 21 October 2013 / Accepted: 25 October 2013 / Published: 6 November 2013

\begin{abstract}
Many celiac disease patients tolerate oats, but limited data are available on its long-term consumption. This was evaluated in the present study, focusing on small-bowel mucosal histology and gastrointestinal symptoms in celiac adults maintaining a strict gluten-free diet with or without oats. Altogether 106 long-term treated celiac adults were enrolled for this cross-sectional follow-up study. Daily consumption of oats and fiber was assessed, and small-bowel mucosal morphology and densities of $\mathrm{CD} 3+, \alpha \beta+$ and $\gamma \sigma+$ intraepithelial lymphocytes determined. Gastrointestinal symptoms were assessed by a validated Gastrointestinal Symptom Rating Scale questionnaire. Seventy (66\%) out of the 106 treated celiac disease patients had consumed a median of $20 \mathrm{~g}$ of oats (range 1-100 g) per day for up to eight years; all consumed oat products bought from general stores. Daily intake and long-term consumption of oats did not result in small-bowel mucosal villous damage, inflammation, or gastrointestinal symptoms. Oat-consumers had a significantly higher daily intake of fiber than those who did not use oats. Two thirds of celiac disease patients preferred to use oats in their daily diet. Even long-term ingestion of oats had no harmful effects.
\end{abstract}


Keywords: celiac disease; gluten-free diet; morphology; oats; questionnaire; small-bowel

\section{Introduction}

Currently the only treatment for celiac disease is lifelong adherence to a strict gluten-free diet avoiding wheat-, rye-, and barley-derived prolamins. There is a large body of evidence to support the nutritional value [1-3] and safety of consumption of oats in the vast majority of both children and adults suffering from celiac disease and dermatitis herpetiformis [4-7]. There is, nevertheless, no consensus among scientists and nutrition experts as to whether oats can be unequivocally recommended for celiac patients [8-10]. One obvious concern is the safety of oats in long-term use. Even if products containing pure oats are nowadays on the market, many commercially available oat products are contaminated with wheat and barley during harvesting and milling processes [11,12]. Furthermore, some individuals may be intolerant even to pure oats, and case reports have shown that oat-intolerant celiac patients may have avenin-reactive T-cells in the small-bowel mucosa [13,14]. Oats has some wheat-like sequences in its protein structure, but as these are less frequent in oats than in other prolamins, it might take a considerably longer time to trigger a disease relapse [15]. Furthermore, limited data on the reasons for withdrawals among oat consumers in randomized trials implies some uncertainty $[5,16,17]$. Long-term follow-up studies on oats in celiac disease have been lacking, as the longest follow-up including small-bowel biopsies has been only five years [5].

Based on a statement from the scientific advisory board of the national Celiac Disease Society, consumption of oats has been allowed for celiac adults in Finland since 1997. The statement was extended in 1998 to apply to patients with dermatitis herpetiformis, and in 2000, also children. In consequence, about $70 \%$ of all celiac disease and dermatitis herpetifomis patients in Finland currently consume an oat-containing gluten-free diet [18]. In this cross-sectional study the aim was to assess the effects of long-term oat consumption on small-bowel mucosal villous morphology and inflammation and gastrointestinal symptoms in a series of celiac adults maintaining a strict gluten-free diet with or without oats.

\section{Methods}

\subsection{Subjects}

Altogether, 110 long-term treated celiac disease adults were invited to participate in a health survey comprising a follow-up small-bowel biopsy and clinical and dietary evaluation at the Department of Gastroenterology and Alimentary Tract Surgery, Tampere University Hospital, Finland. Patients found to be adhering to a strict gluten-free diet were eligible. Oat consumption was not an inclusion criterion. At diagnosis all patients had had biopsy-proven celiac disease, and after one year on a gluten-free diet clinical, serological or histological recovery was evident in all. The patients had been followed up in primary health care and no further routine small-bowel biopsies had been taken during the usual long-term surveillance. The study protocol was approved by the Ethical Committee of Tampere University Hospital. All subjects gave written informed consent. 


\subsection{Dietary Assessment}

A detailed dietary analysis and a history of occasional or regular consumption of gluten-containing products, oats, and fiber were assessed by means of an interview by a trained dietician and by a four-day record of food intake [4]. The duration of oat intake was also recorded.

\subsection{Small-Bowel Mucosal Morphology and Inflammation}

Altogether, six small-bowel biopsy specimens were taken from the distal part of the duodenum upon esophago-gastroduodenoscopy; the specimens were evaluated by the same investigator without prior knowledge of history or findings. Three biopsies were formalin-fixed and embedded in paraffin; 5- $\mu \mathrm{m}$-thick biopsy sections were stained with hematoxylin-eosin and studied under light microscopy. Morphometric analysis by measuring villous height and crypt depth ratio $(\mathrm{Vh} / \mathrm{CrD})$ was made in well-oriented biopsy samples as previously described [19], and $\mathrm{Vh} / \mathrm{CrD}>2$ was considered normal. For immunohistochemical stainings, three biopsies were freshly embedded in optimal temperature compound (OCT, Tissue-Tec, Miles Inc., Elkhart, IN, USA), snap-frozen in liquid nitrogen, and stored at $70{ }^{\circ} \mathrm{C}$. In 5 - $\mu$ m-thick frozen sections, $\mathrm{CD} 3+$ intraepithelial lymphocytes (IELs) were stained with monoclonal antibody Leu-4 (anti Leu-4 also known as anti CD3, Becton Dickinson, San Jose, CA, USA), $\alpha \beta+$ IELs with monoclonal $\beta F 1$ antibody (Endogen, Woburn, MA, USA) and $\gamma \delta+$ IELs with TCR $\gamma$ antibody (Endogen). IELs were counted with a $100 \times$ flat-field light microscope objective in randomly selected areas of surface epithelium and the density of IELs expressed as cells/millimeter of epithelium as previously described [19]. The reference values were set at 37 cells $/ \mathrm{mm}$ for $\mathrm{CD} 3+$, at 25 cells $/ \mathrm{mm}$ for $\alpha \beta+$, and at 4.3 for $\gamma \delta+$ IELs [19].

\subsection{Gastrointestinal Symptoms and Clinical Evaluation}

Gastrointestinal symptoms were evaluated by the Gastrointestinal Symptom Rating Scale (GSRS) questionnaire, which is also well-validated in celiac disease [17,20-22]. The questionnaire comprises, altogether, 15 items in five subdimensions describing: diarrhea (increased passage of stools, loose stools, urgent need for defecation), indigestion syndrome (borborygmus, abdominal distension, eructation, increased flatus), constipation (decreased passage of stools, hard stools, feeling of incomplete evacuation), abdominal pain (abdominal pain, nausea, and vomiting), and gastro-esophageal reflux (heart burn, acid regurgitation). Each item was graded from one to seven, a higher score indicating more gastrointestinal symptoms. In earlier studies, 95\% confidence intervals of GSRS total scores have been 1.8-2.2 in non-celiac controls [22]. The body mass index (BMI) was calculated as weight in kilograms divided by the square of height in meters (normal range: $\left.18.0-25.0 \mathrm{~kg} / \mathrm{m}^{2}\right)$.

\subsection{Serology and Chemical Analysis}

Serum IgA class endomysial antibodies (EmA) were determined using an indirect immunofluorescence method with human umbilical cord as substrate, and a dilution 1: $\geq 5$ was considered positive [4]. Serum IgA-class tissue transglutaminase antibodies (tTG-ab) were investigated by enzyme-linked immunosorbent assay (ELISA) (Celikey ${ }^{\circledR}$, Phadia, GmbH, Freiburg, 
Germany); the result was classified as positive when5.0 U/L. Blood hemoglobin level was measured using routine laboratory methods.

\subsection{Statistics}

Differences between patients consuming and avoiding oats were compared by Mann-Whitney U test or $t$-test, when appropriate. Data were given mainly as medians and range. Spearman's coefficient was used for correlation studies. A $p$-value of $<0.05$ was considered statistically significant. All statistical testing was performed using SPSS version 16.0 (SPSS Inc., Chicago, IL, USA).

\section{Results}

At the beginning of the study, the dietician observed that four celiac disease patients had committed minor dietary transgressions less than once a month, and they were excluded from further studies. The remaining 106 treated celiac disease patients, adhering to a strict gluten-free diet, were deemed eligible. As shown in Table 1, altogether $70(66 \%)$ out of the 106 treated celiac disease patients had preferred to consume oats in their otherwise strict gluten-free diet; 40 of them had taken oats for five years or more (up to eight years). All 70 patients used oat products purchased in local markets. The median daily intake of oats was $20 \mathrm{~g}$, but 10 patients consumed 50-100 g per day. The clinical picture of celiac disease at diagnosis, age at study onset and the duration of the gluten-free diet were no different in patients who decided not to take oats from those favoring an oat-containing gluten-free diet, but oat consumers were more likely to have a family history of celiac disease (Table 1).

Table 1. Demographic data and dietary history in 106 treated celiac disease (CD) patients.

\begin{tabular}{|c|c|c|}
\hline Characteristics & No Oats $n=36$ & Oats $n=70$ \\
\hline Female $(\%)$ & $25(69 \%)$ & $46(66 \%)$ \\
\hline Median age at time of study (range), years & $54(36-73)$ & $59(24-81)$ \\
\hline \multicolumn{3}{|l|}{ Symptoms and signs leading to the diagnosis of $\mathrm{CD}, n(\%)$} \\
\hline Abdominal symptoms & $29(81 \%)$ & $61(87 \%)$ \\
\hline Malabsorption, anemia, loss of weight & $17(47 \%)$ & $47(67 \%)$ \\
\hline Dermatitis herpetiformis & $4(11 \%)$ & $9(13 \%)$ \\
\hline Extraintestinal symptoms ${ }^{\text {a }}$ & $5(14 \%)$ & $11(16 \%)$ \\
\hline Screening of risk groups ${ }^{b}$ & $2(6 \%)$ & $4(6 \%)$ \\
\hline Family history of CD, $n(\%)$ & $10(28 \%)$ & $42(60 \%)^{c}$ \\
\hline Median duration of gluten-free diet (range), years & $10(1-28)$ & $8(1-41)$ \\
\hline Median duration of oat consumption after the diagnosis of $\mathrm{CD}$ (range), years & 0 & $5(0.5-8)$ \\
\hline Median (range) daily intake of oats, $g$ & 0 & $20(1-100)$ \\
\hline
\end{tabular}

a Osteoporosis, arthritis, polyneuropathy, ataxia, mild memory disturbances, depression, anxiety, fatigue, fibromyalgia, enamel defects in permanent teeth, elevated liver enzymes; ${ }^{\mathrm{b}}$ Family history of CD, population screening; ${ }^{c} p=0.002$ when compared to patients taking no oats; differences in sex, age, the difference between symptoms and duration of gluten-free diet was not statistically significant.

Altogether, 30 out of 36 subjects avoiding oats were diagnosed to have celiac disease before oat-containing gluten-free-products were permissible for adults with celiac disease; thus, after the 
diagnosis they all had been on a standard gluten-free diet without oats. Afterwards, one patients tried oats but he stopped using oats due to abdominal symptoms; the rest 29 did not want to start to consume oats again (partly do the fear of adverse effects). From the remaining six patients there were no data why they avoided oats.

Small-bowel mucosal villous morphology was normal in 103 (97\%) out of the 106 long-term treated celiac disease patients; two patients using, and one not using oats had abnormal villous structure. A high daily oat intake and a long duration of oat intake correlated with a better small-bowel mucosal $\mathrm{Vh} / \mathrm{CrD}$ ratio (Figure $1 \mathrm{~A}, \mathrm{~B}$ ). The densities of mucosal $\alpha \beta+$ IELs were not different between patients who did or did not consume oats (Figure 1C,D). Similarly, small-bowel mucosal CD3+ and $\gamma \sigma+$ IEL counts did not correlate with the daily oat intake $(r=0.134, p=0.170$ and $r=0.167, p=0.088$, respectively) or the duration of oat consumption $(r=0.029, p=0.773$ and $r=0.043, p=0.771$, respectively).

Figure 1. Small-bowel mucosal villous height crypt depth ratios in 106 treated celiac disease patients correlated with the daily oat intake (A) $r=0.251, p=0.009$ and the duration of oat consumption $(\mathbf{B}) r=0.252, p=0.012$. Correlations between densities of $\alpha \beta+$ intraepithelial lymphocytes and daily oat intake $(\mathbf{C})$, and between the cells and the duration of oat consumption (D) were not statistically significant $(r=0.152, p=0.119$ and $r=0.132, p=0.190$, respectively). GFD $=$ gluten-free diet. Black diamond $=$ no oats. Open diamond $=$ oat user.
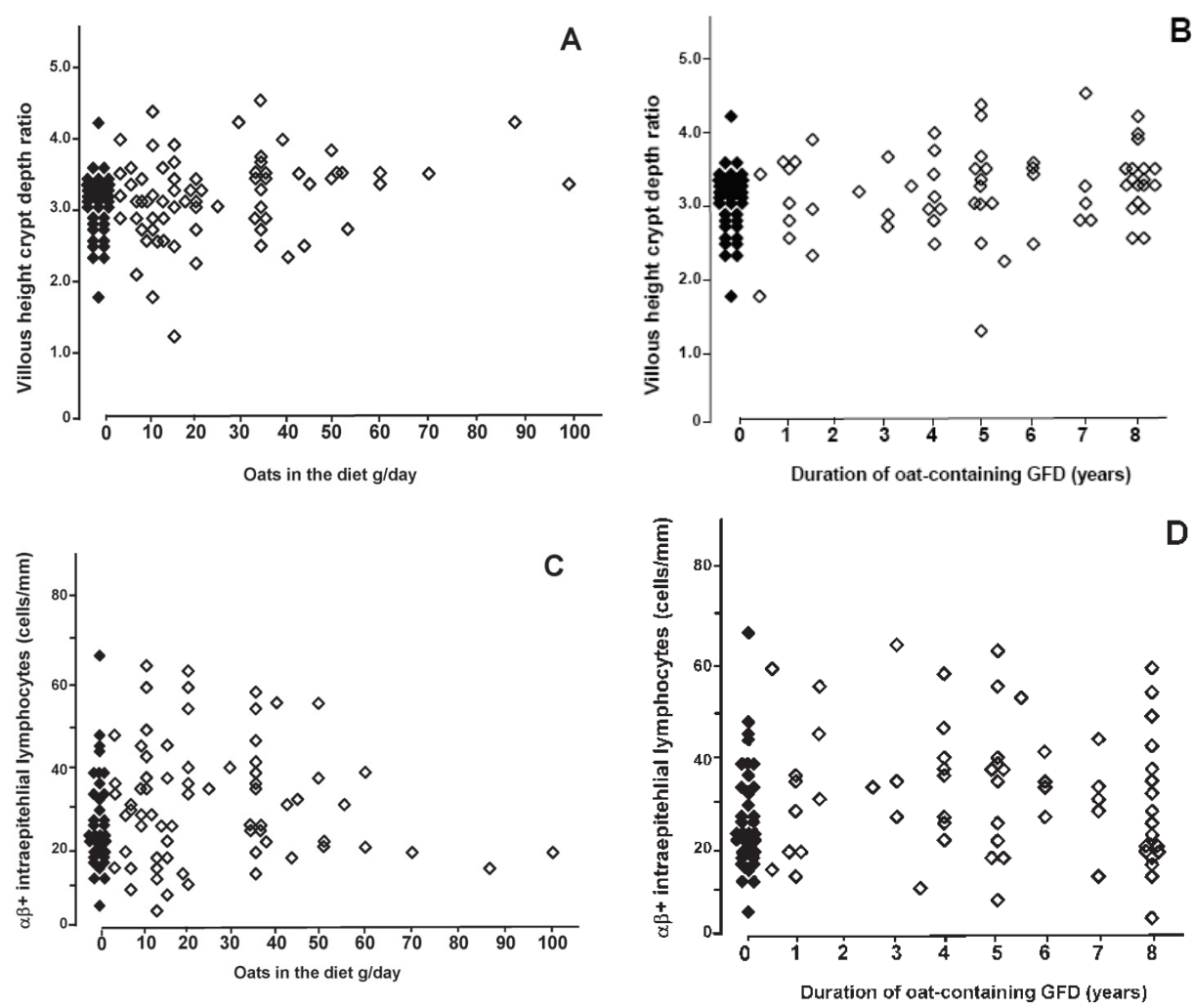

Based on GSRS total score, oat-consumers did not suffer more gastrointestinal symptoms than non-oat consumers (Figure 2A). In GSRS subdimensions, a higher oat intake and a long duration of oat intake correlated significantly with fewer complaints of indigestion (Figure 2B); there were no 
significant differences in other subdimensions (data not shown). Daily fiber intake was significantly higher in celiac disease patients who consumed oats than in those who did not $(p=0.013$; median $18.1 \mathrm{~g}$, range $7.6-50.9 \mathrm{~g} v \mathrm{~s}$. median $15.8 \mathrm{~g}$, range $6.0-23.3 \mathrm{~g}$ ). None of the 13 patients with dermatitis herpetiformis suffered from cutaneous rash involvement; nine of the 13 were taking oats. BMI was no different between those treated celiac disease patients who did or did not consume oats (median $25.5 \mathrm{~kg} / \mathrm{m}^{2}$, range $19.2-33.0 \mathrm{~kg} / \mathrm{m}^{2}$ vs. $26.6 \mathrm{~kg} / \mathrm{m}^{2}$, range $\left.19.0-34.6 \mathrm{~kg} / \mathrm{m}^{2} p=0.671\right)$. All treated celiac patients were negative for serum EmA- and tTG-abs (median 0.5 U/L, range 0-2.9 U/L). Blood hemoglobin levels did not correlate with the consumption of oats (data not shown).

Figure 2. Gastrointestinal symptom rating scale (GSRS) total score correlated negatively with daily oat intake in 106 treated celiac disease patients (A) $r=-0.220, p=0.025$, but not with the duration of oat consumption $(r=-0.166, p=0.101$ [data not shown]). A high daily oat intake and a long duration of oat intake correlated with less indigestion (B) $r=-0.313, p=0.003$ and $r=-0.232, p=0.037$ [data not shown], respectively. Black diamond $=$ no oats. Open diamond $=$ oat user.
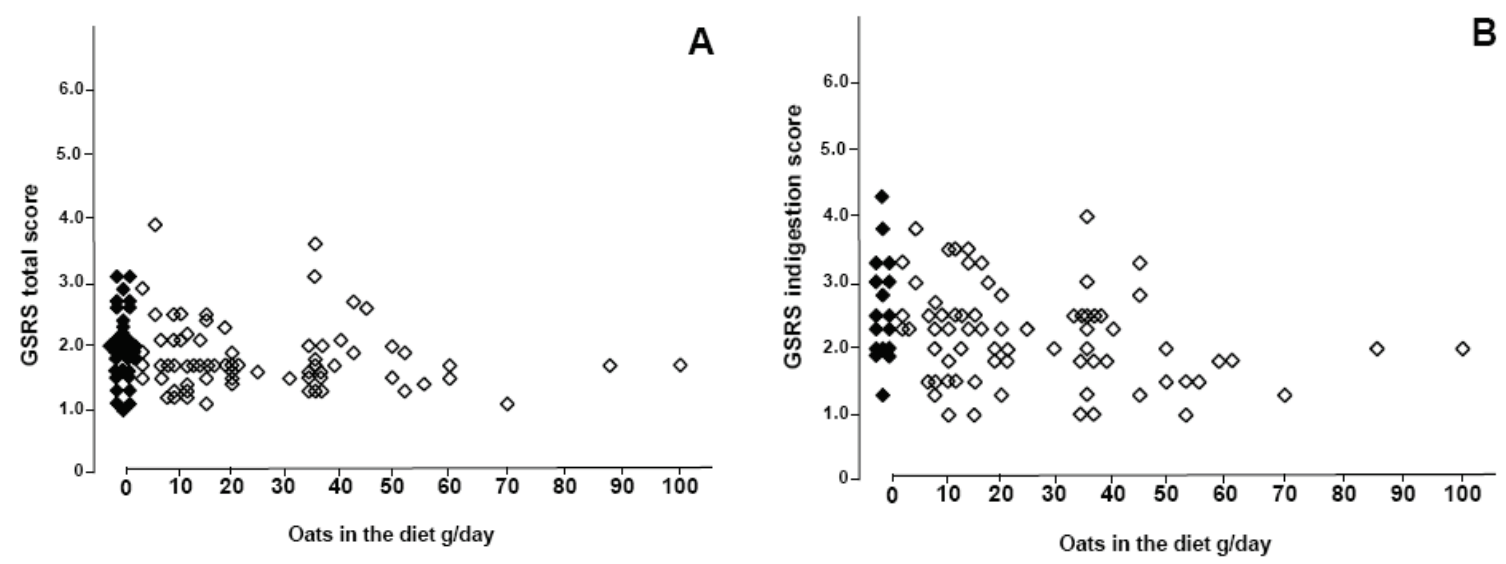

\section{Discussion}

In this large cross-sectional study we showed that in celiac disease long-term consumption of oats for up to eight years had no detrimental effect on symptoms, small-bowel mucosal villous morphology and inflammation, or on humoral response against tissue transgluatminase. In contrast, the mucosal morphology was even significantly better in subjects who had consumed oats in larger amounts or over a longer time-period than in those who did not take oats (Figure 1A,B). Our data confirm the results of an earlier five-year follow-up study in 23 celiac disease adults showing that the long-term ingestion of oats is safe [5].

Some studies have indicated that there is a subgroup of celiac patients who experience gastrointestinal symptoms more frequently on an oat-containing gluten-free diet than on a gluten-free diet without oats $[4,14,16,17]$. Flatulence and abdominal distension often occur soon after commencing an oat-containing diet, but in most cases the symptoms disappear gradually as consumption of oats continues [1]. This has been explained by an increased intake of fiber in oat products. Indeed, many non-celiac individuals develop similar symptoms when they suddenly start to consume oats [23]. In the current study celiac disease patients consuming oats, even up to $100 \mathrm{~g}$ 
per day, experienced no more gastrointestinal symptoms than those taking less or avoiding oats. Interestingly, patients who ingested high amounts of oats and for longer periods experienced less indigestion (Figure 2). In our series there were no oat-intolerant celiac disease patients who had previously consumed oats but discontinued due to symptoms. According to the literature, such patients may exist, but usually the occurrence of symptoms has not been associated with small-intestinal mucosal damage or inflammation [4,17]. Earlier, a Norwegian research group demonstrated that some oat-intolerant celiac disease patients have oat avenin-reactive T-cells in the small-intestinal mucosa $[13,14]$. Altogether, it would appear that even if most celiac patients tolerate oats, there might be some who have to avoid it in order to maintain remission. Interestingly, it has recently been shown that oat immunogenicity may vary between different oat cultivars [24]. In the current study it was impossible to trace which cultivars were used, as the patients were able to use a wide range of commercial oat products from the market. This notwithstanding, the patients remained in clinical and histological remission.

In the current study all celiac disease patients on an oat-containing gluten-free diet consumed ordinary oat products bought from general stores and meant for normal consumption. It has recently been shown that most commercially available oat products in both Europe and the United States are contaminated with wheat or barley, gluten levels ranging even from 200 to $8000 \mathrm{mg} / \mathrm{kg}$ (=ppm) when measured by the R5 antibody-based ELISA method [11,12]. The inevitable question is how to achieve a good clinical and histological response when consuming potentially gluten-contaminated oats. Firstly, in the long run, patients choose to consume relatively small doses of oats daily. If the product in question were contaminated even with $1000 \mathrm{ppm}$ gluten, a daily consumption of $20 \mathrm{~g}$ oats would have resulted in no more than $20 \mathrm{mg}$ gluten intake per day, an amount evidently tolerated by most celiac patients [25,26]. Secondly, according to earlier studies many oat products have been contaminated with barley [11]. There are only a few clinical studies on the toxicity of barley in celiac disease. Thirdly, the R5-ELISA method may overestimate barley contamination in oats, implying that in fact the oat products in question may have much lower contamination levels than reported [27]. It must also be considered that naturally gluten-free products, such as rice, corn, buckwheat, and soy may also be similarly contaminated with gluten [26,28]. Furthermore, there are studies showing that only $20 \%-73 \%$ of long-term treated patients adhering to a strict naturally gluten-free diet without oats evinced normal small-bowel mucosal villous architecture [29,30]. This implies that inadvertent gluten ingestion is not restricted to oats, but is also possible when consuming only products gluten-free by nature. Furthermore, it must be noted that a large number of long-term treated celiac disease patients had high numbers of $\alpha \beta+$ IELs, irrespective of oats ingestion. Persistent small-bowel mucosal intraepithelial lymphocytosis proved here again to be a common finding in well-treated celiac disease patients [29,31]. Interestingly, our recent study suggested that oats might contribute to the duodenal lymphocytosis [31]. Nevertheless, when in that study, a lower cut of value 25 IELs per 100 enterocytes was used for defining intraepithelial lymphocytosis, no association between oat consumption and lymphocytosis was found [31]. Altogether, compared to the findings in the earlier studies the patients in the present study had shown an excellent response to a strict gluten-free diet, even though they had consumed commercial oats for years. As pure oat products are nowadays available, consumption of ordinary, non-dedicated oat products (having potential risk of 
being contaminated by gluten) is discouraged, especially if a celiac disease patient decides to consume high amounts of oats daily.

\section{Conclusions}

To conclude, long-term consumption of oats proved to be safe for celiac disease patients. Oats diversifies a gluten-free diet, and enhances its nutritional quality by increasing the intake of dietary fiber. When allowed, most celiac disease patients in this country prefer to consume some oats. Pure oat products with strictly controlled production systems are nowadays available endorsing their use more widely. Long-term regular follow-up of celiac disease patients is recommended; those using oats may safely be followed up similarly to non-users.

\section{Acknowledgments}

This work was supported by grants to the Celiac Disease Study Group from the Competitive State Research Financing of the Expert Responsibility Area of Tampere University Hospital, the Academy of Finland, the Sigrid Juselius Foundation, Elna Kaarina Savloainen's fund allocated for the development of cancer treatment.

\section{Conflicts of Interest}

The authors have none to declare. All authors have made substantial contributions to all of the following: (1) the conception and design of the study, or acquisition of data, or analysis and interpretation of data; (2) drafting the article or revising it critically for important intellectual content; (3) final approval of the version submitted.

\section{References}

1. Storsrud, S.; Hulthen, L.R.; Lenner, R.A. Beneficial effects of oats in the gluten-free diet in adults with special reference to nutrient status, symptoms and subjective experiences. $B r . J$. Nutr. 2003, 90, 101-107.

2. Kemppainen, T.A.; Heikkinen, M.T.; Ristikankare, M.K.; Kosma, V.M.; Julkunen, R.J. Nutrient intakes during diets including unkilned and large amounts of oats in celiac disease. Eur. J. Clin. Nutr. 2010, 64, 62-67.

3. Lovik, A.; Gjoen, A.U.; Morkrid, L.; Guttormsen, V.; Ueland, T.; Lundin, K.E.A. Oats in a strictly gluten-free diet is associated with decreased gluten intake and increased serum bilirubin. e-SPEN 2009, 4, e315-e320.

4. Holm, K.; Mäki, M.; Vuolteenaho, N.; Mustalahti, K.; Ashorn, M.; Ruuska, T.; Kaukinen, K. Oats in the treatment of chilhood coeliac disease: A two-year controlled and a long-term clinical follow-up study. Aliment. Pharmacol. Ther. 2006, 23, 1463-1472.

5. Janatuinen, E.K.; Kemppainen, T.A.; Julkunen, R.J.K.; Kosma, V.-M.; Mäki, M.; Heikkinen, M.; Uusitupa, M.I.J. No harm from five year ingestion of oats in coeliac disease. Gut 2002, 50, $332-335$. 
6. Pulido, O.M.; Gillespie, Z.; Zarkadas, M.; Dubois, S.; Vavasour, E.; Rashid, M.; Switzer, C.; Godefroy, S.B. Introduction of oats in the diet of individuals with celiac disease: A systematic review. Adv. Food. Nutr. Res. 2009, 57, 235-285.

7. Sey, M.S.; Parfitt, J.; Gregor, J. Prospective study of clinical and histological safety of pure and uncontaminated Canadian oats in the management of celiac disease. JPEN 2011, 35, 459-464.

8. Butzner, J.D. Pure oats and the gluten-free diet: Are they safe? JPEN 2011, 35, 447-448.

9. Fric, P.; Gabrovska, D.; Nevoral, J. Celiac disease, gluten-free diet, and oats. Nutr. Rev. 2011, 69, 107-115.

10. Parakkal, D.V.; Du, H.; Semer, R.; Ehrenpreis, E.D.; Guandalini, S. Do gastroenterologists adhere to diagnostic and treatment guidelines for celiac disease? J. Clin. Gastroenterol. 2012, 46, e12-e20.

11. Hernando, A.; Mujico, J.R.; Juanas, D.; Mendez, E. Confirmation of the cereal type in oat products highly contaminated with gluten. J. Am. Diet. Assoc. 2006, 106, 665-666.

12. Koerner, T.B.; Cléroux, C.; Poirier, C.; Cantin, I.; Alimkulov, A.; Elamparo, H. Gluten contamination in the Canadian commercial oat supply. Food Addit. Contam. 2011, 28, 705-710.

13. Arentz-Hansen, H.; Fleckenstein, B.; Molberg, O.; Scott, H.; Koning, F.; Jung, G.; Roepstorff, P.; Lundin, K.E.; Sollid, L.M. The molecular basis for oat intolerance in patients with celiac disease. PLoS Med. 2004, 1, e1.

14. Lundin, K.E.A.; Nilsen, E.M.; Scott, H.G.; Loberg, E.M.; Gjoen, A.; Bratlie, J.; Skar, V.; Mendez, E.; Lovik, A.; Kett, K. Oats induced villous atrophy in coeliac disease. Gut 2003, 52, $1649-1652$.

15. Vader, L.W.; Stepniak, D.T.; Bunnik, E.M.; Kooy, Y.M.C.; de Haan, W.; Drijfhout, J.W.; van Veelen, P.A.; Koning, F. Characterization of cereal toxicity for celiac disease patients based on protein homology in grains. Gastroenterology 2003, 125, 1105-1113.

16. Hogberg, L.; Laurin, P.; Falth-Magnusson, K.; Grant, C.; Grodzinsky, E.; Jansson, G.; Ascher, H.; Browaldh, L.; Hammersjo, J.A.; Lindberg, E.; et al. Oats to children with newly diagnosed coeliac disease: A randomised double blind study. Gut 2004, 53, 649-654.

17. Peräaho, M.; Kaukinen, K.; Mustalahti, K.; Vuolteenaho, N.; Mäki, M.; Laippala, P.; Collin, P. Effect of an oats-containing gluten-free diet on symptoms and quality of life in coeliac disease. A randomized study. Scand. J. Gastroenterol. 2004, 39, 27-31.

18. Peräaho, M.; Collin, P.; Kaukinen, K.; Kekkonen, L.; Miettinen, S.; Mäki, M. Oats can diversify a gluten-free diet in celiac disease and dermatitis herpetiformis. J. Am. Diet. Assoc. 2004, 104, $1148-1150$.

19. Järvinen, T.T.; Kaukinen, K.; Laurila, K.; Kyrönpalo, S.; Rasmussen, M.; Mäki, M.; Korhonen, H.; Reunala, T.; Collin, P. Intraepithelial lymphocytes in celiac disease. Am. J. Gastroenterol. 2003, 98, 1332-1337.

20. Dimenäs, E.; Carlsson, H.; Glise, H.; Israelsson, B.; Wiklund, I. Relevance of norm values as part of the documentation of quality of life instruments for use in upper gastrointestinal disease. Scand. J. Gastroenterol. 1996, 31, 8-13.

21. Midhagen, G.; Hallert, C. High rate of gastrointestinal symptoms in celiac patients living on a gluten-free diet: Controlled study. Am. J. Gastroenterol. 2003, 98, 2023-2026. 
22. Mustalahti, K.; Lohiniemi, S.; Collin, P.; Vuolteenaho, N.; Laippala, P.; Mäki, M. Gluten-free diet and quality of life in patients with screen-detected celiac disease. Eff. Clin. Pract. 2002, 5, 105-113.

23. Mälkki, Y. Trends in dietary fibre research and development. Acta Aliment. 2004, 33, 39-62.

24. Comino, I.; Real, A.; de Lorenzo, L.; Cornell, H.; López-Casado, M.Á.; Barro, F.; Lorite, P.; Torres, M.I.; Cebolla, A.; Sousa, C. Diversity in oat potential immunogenicity: Basis for the selection of oat varieties with no toxicity in coeliac disease. Gut 2011, 60, 915-922.

25. Catassi, C.; Fabiani, E.; Iacono, G.; D’Agate, C.; Francavilla, R.; Biagi, F.; Volta, U.; Accomando, S.; Picarelli, A.; de Vitis, I.; et al. A prospective, double-blind, placebo-controlled trial to establish a safe gluten threshold for patients with celiac disease. Am. J. Clin. Nutr. 2007, 85, 160-166.

26. Collin, P.; Thorell, L.; Kaukinen, K.; Mäki, M. The safe threshold for gluten contamination in gluten-free products. Can trace amounts be accepted in the treatment of coeliac disease? Aliment. Pharmacol. Ther. 2004, 19, 1277-1283.

27. Kanerva, P.M.; Sontag-Strohm, T.S.; Ryöppy, P.H.; Alho-Lehto, P.; Salovaara, H.O. Analysis of barley contamination in oats using R5 and omega-gliadin antibodies. J. Cereal Sci. 2006, 44, 347-352.

28. Storsrud, S.; Malmheden Yman, I.; Lenner, R.A. Gluten contamination in oat products and products naturally free from gluten. Eur. Food Res. Technol. 2003, 217, 481-485.

29. Lanzini, A.; Lanzarotto, F.; Villanacci, V.; Mora, A.; Bertolazzi, S.; Turini, D.; Carella, G.; Malagoli, A.; Ferrante, G.; Cesana, B.M.; et al. Complete recovery of intestinal mucosa occurs very rarely in adult coeliac patients despite adherence to gluten-free diet. Aliment. Pharmacol. Ther. 2009, 29, 1299-308.

30. Lee, S.K.; Lo, W.; Memeo, L.; Rotterdam, H.; Green, P.H.R. Duodenal histology in patients with celiac disease after treatment with a gluten-free diet. Gastrointest. Endosc. 2003, 57, 187-191.

31. Ilus, T.; Lähdeaho, M.-L.; Salmi, T.; Haimila, K.; Partanen, J.; Saavalainen, P.; Huhtala, H.; Mäki, M.; Collin, P.; Kaukinen, K. Persistent duodenal intraepithelial lymphocytosis despite a long-term strict gluten-free diet in celiac disease. Am. J. Gastroenterol. 2012, 107, 1563-1569. 
Reprinted from Nutrients. Cite as: Gatti, S.; Caporelli, N.; Galeazzi, T.; Francavilla, R.; Barbato, M.; Roggero, P.; Malamisura, B.; Iacono, G.; Budelli, A.; Gesuita, R. Oats in the Diet of Children with Celiac Disease: Preliminary Results of a Double-Blind, Randomized, Placebo-Controlled Multicenter Italian Study. Nutrients 2013, 5, 4653-4664.

Article

\section{Oats in the Diet of Children with Celiac Disease: Preliminary Results of a Double-Blind, Randomized, Placebo-Controlled Multicenter Italian Study}

Simona Gatti ${ }^{1{ }^{*} \text {, Nicole Caporelli }}{ }^{1}$, Tiziana Galeazzi ${ }^{1}$, Ruggiero Francavilla ${ }^{2}$, Maria Barbato ${ }^{3}$, Paola Roggero ${ }^{4}$, Basilio Malamisura ${ }^{5}$, Giuseppe Iacono ${ }^{6}$, Andrea Budelli ${ }^{7}$, Rosaria Gesuita $^{8}$, Carlo Catassi ${ }^{1}$, Elena Lionetti ${ }^{9}$

1 Department of Pediatrics, Università Politecnica delle Marche, 60121 Ancona, Italy;

E-Mails: nicole.capo@hotmail.it (N.C.); t.galeazzi@univpm.it (T.G.); catassi@tin.it (C.C.)

2 Interdisciplinary Department of Medicine, University of Bari, 70124 Bari, Italy;

E-Mail: rfrancavilla@gmail.com

3 Department of Pediatrics, "Sapienza" University of Rome, 00161 Roma, Italy;

E-Mail: Maria.Barbato@uniroma1.it

4 Neonatal Intensive Care Unit, Department of Clinical Sciences and Community Health,

Fondazione IRCCS Ca' Granda Ospedale Maggiore Policlinico, University of Milan, 20122

Milano, Italy; E-Mail: paola.roggero@unimi.it

5 Department of Pediatrics, S. Maria dell'Olmo Hospital Cava de' Tirreni, 84013 Salerno, Italy;

E-Mail: basiliomalamisura@libero.it

6 Pediatric Gastroenterology Unit, “G. Di Cristina” Children Hospital, 90134 Palermo, Italy;

E-Mail: stoai@inwind.it

7 R\&D Heinz Italia S.p.A, 04100 Latina, Italy; E-Mail: Andrea.Budelli@it.hjheinz.com

8 Department of Epidemiology, Biostatistics and Medical Information Technology, Università

Politecnica delle Marche, 60121 Ancona, Italy; E-Mail: r.gesuita@univpm.it

9 Department of Pediatrics, Università di Catania, 95123, Catania, Italy;

E-Mail: elenalionetti@inwind.it

* Author to whom correspondence should be addressed; E-Mail: simona.gatti@hotmail.it;

Tel.: +39-071-5962370; Fax: +39-071-36281.

Received: 30 September 2013; in revised form: 13 November 2013 / Accepted: 13 November 2013 / Published: 20 November 2013

Abstract: A gluten-free diet (GFD) is currently the only available treatment for patients with celiac disease $(C D)$. Several clinical trials have demonstrated that most celiac 
patients can tolerate a medium-high quantity of oats without any negative clinical effects; however, the inclusion of oats in GFD is still a matter of debate. In this study, Italian children with $\mathrm{CD}$ were enrolled in a 15-month, randomized, double-blind, placebo-controlled multicenter trial. Participants were randomized in two groups following either A-B treatment (6 months of diet "A", 3 months of standard GFD, 6 months of diet "B"), or B-A treatment (6 months of diet "B", 3 months of standard GFD, 6 months of diet "A"). A and B diets included gluten-free (GF) products (flour, pasta, biscuits, cakes and crisp toasts) with either purified oats or placebo. Clinical data (Gastrointestinal Symptoms Rate Scale [GSRS] score) and intestinal permeability tests (IPT), were measured through the study period. Although the study is still blinded, no significant differences were found in GSRS score or the urinary lactulose/mannitol $(\mathrm{L} / \mathrm{M})$ ratio between the two groups after 6 months of treatment. These preliminary results suggest that the addition of non-contaminated oats from selected varieties in the treatment of children with $\mathrm{CD}$ does not determine changes in intestinal permeability and gastrointestinal symptoms.

Keywords: oats; celiac disease; gluten-free diet; intestinal permeability; gastrointestinal symptoms

\section{Introduction}

Celiac disease (CD) is an immune-mediated disorder, triggered in genetically susceptible individuals by ingested gluten, the alcohol-soluble complex present in wheat, rye, and barley. The clinical spectrum of CD is extremely variable, including (a) typical CD, with the classical features of intestinal malabsorption; (b) atypical CD (characterized by extra-intestinal manifestations); (c) silent $\mathrm{CD}$, (occasionally found following serological screening in subjects who are asymptomatic); (d) potential CD, showing positivity of celiac serology associated with a normal (or nearly normal) intestinal mucosa at the small intestinal biopsy. The cornerstone of treatment is the lifelong exclusion of gluten-containing cereals from the diet, the gluten-free diet (GFD).

Oats were originally excluded from the diet of people with $\mathrm{CD}$. However, evidence supporting the toxicity of oats for CD individuals was poor. More recently, several studies have shown that medium-high amounts of gluten-uncontaminated oats can be safely ingested by patients with CD. In 1995, Finnish investigators compared the effect of 50-70 g/day of oats to placebo in 92 adults with CD on a GFD at diagnosis or in follow-up. They found no difference in clinical and laboratory outcomes and, more importantly, there was no sign of histological damage after 12 months of an oat-containing GFD [1]. These data have been replicated in other studies conducted in adults and children affected with CD or dermatitis herpetiformis [2-11], thereby confirming the safety of oat-based products for CD patients, provided that gluten contamination is avoided in the production chain. Recent studies suggest that some oats varieties may show a degree of residual toxicity in vitro, suggesting that there are differences between oat varieties in relation to their safety/toxicity for people with CD [12-16]. 
Official recommendations acknowledge the safety of products containing purified oats, and several national associations for CD allow inclusion of oats in the diet of people with CD [17,18]. The European Commission Regulation No. 41/2009 has included oats among allowed ingredients when the gluten content does not exceed 20 parts per million (ppm). During the last years, thousands of CD patients have been consuming large amounts of oat-based products in Northern European countries and Canada without any reported major side effects.

Oat is not a staple food in the diet of Mediterranean populations. This is probably the main reason why an oats "resurrection" in the GFD has not raised immediate interest in Southern European countries. In Italy, products containing gluten-uncontaminated oats are not currently available. This situation will hopefully change in the near future; rich in soluble dietary fiber, vitamins and minerals, the inclusion of oats unquestionably improves the nutritional value and increases the palatability of the GFD, while expanding food choices and ultimately improving the quality of life for people with CD [17].

For these reasons, we undertook a prospective, multicenter investigation on the safety and acceptance of gluten-free (GF) oat-based products from selected oat varieties in the diet of Italian CD children in treatment with the GFD. Clinical monitoring during the study was based on: (a) score of intestinal symptoms, (b) serological CD markers (IgA class anti-transglutaminase antibody), and (c) results of the double sugar intestinal permeability test (IPT), as a marker of mucosal integrity of the small intestine. In this work, we present the preliminary clinical and intestinal permeability results of this multicenter, placebo-controlled and double-blind study.

\section{Patients and Methods}

\subsection{Study Population}

Children (age range: 4-14 years) with biopsy-proven diagnosis of CD, on a GFD for at least 2 years, were recruited in 7 different Pediatric Gastroenterology Services in Italy (Ancona, Bari, Catania, Monza, Palermo, Roma, Cava de' Tirreni). Patients who (1) have other chronic conditions (including type 1 diabetes or inflammatory bowel disease), or (2) did not adhere to the GFD (as demonstrated by elevation of serological markers at enrollment) or (3) were on a GFD for less than 2 years were excluded.

\subsection{Study Design and Diets}

The protocol of the study is shown in Figure 1.

Clinical data (gastrointestinal [GI] symptoms, growth data) and IPT by measurement of urinary lactulose/mannitol (L/M) ratio were monitored at 0, 3, 6, 9, 12 and 15 months. Serological (IgA-antitransglutaminase-TTG, IgG-deamidated gliadin peptide-DGP, and anti-avenin antibodies) and biochemical data were measured at 0, 6, 9, and 15 months. An accurate food diary was completed in the 3 days preceding each visit at 3, 6, 12 and 15 months. Symptoms and/or side effects related to the ingestion of the products under investigation were promptly recorded and the decision to continue or withdraw from the study protocol was made after discussion with the children and their families. 
In this preliminary report, we describe the GI symptoms and IPT findings after 6 months of participation in this study.

Figure 1. Flow-chart of the multicenter trial ( $\mathrm{t} 0$ : baseline, $\mathrm{t} 3$ : 3 -month follow-up, t6: 6-month follow-up, t9: 9-month follow-up, t12: 12-month follow-up, t15: 15-month follow-up; GSRS: Gastrointestinal Symptoms Rate Scale, IgA tTG: IgA class anti-transglutaminase antibody, IgG DGP: IgG class deamidated gliadin peptides antibody).

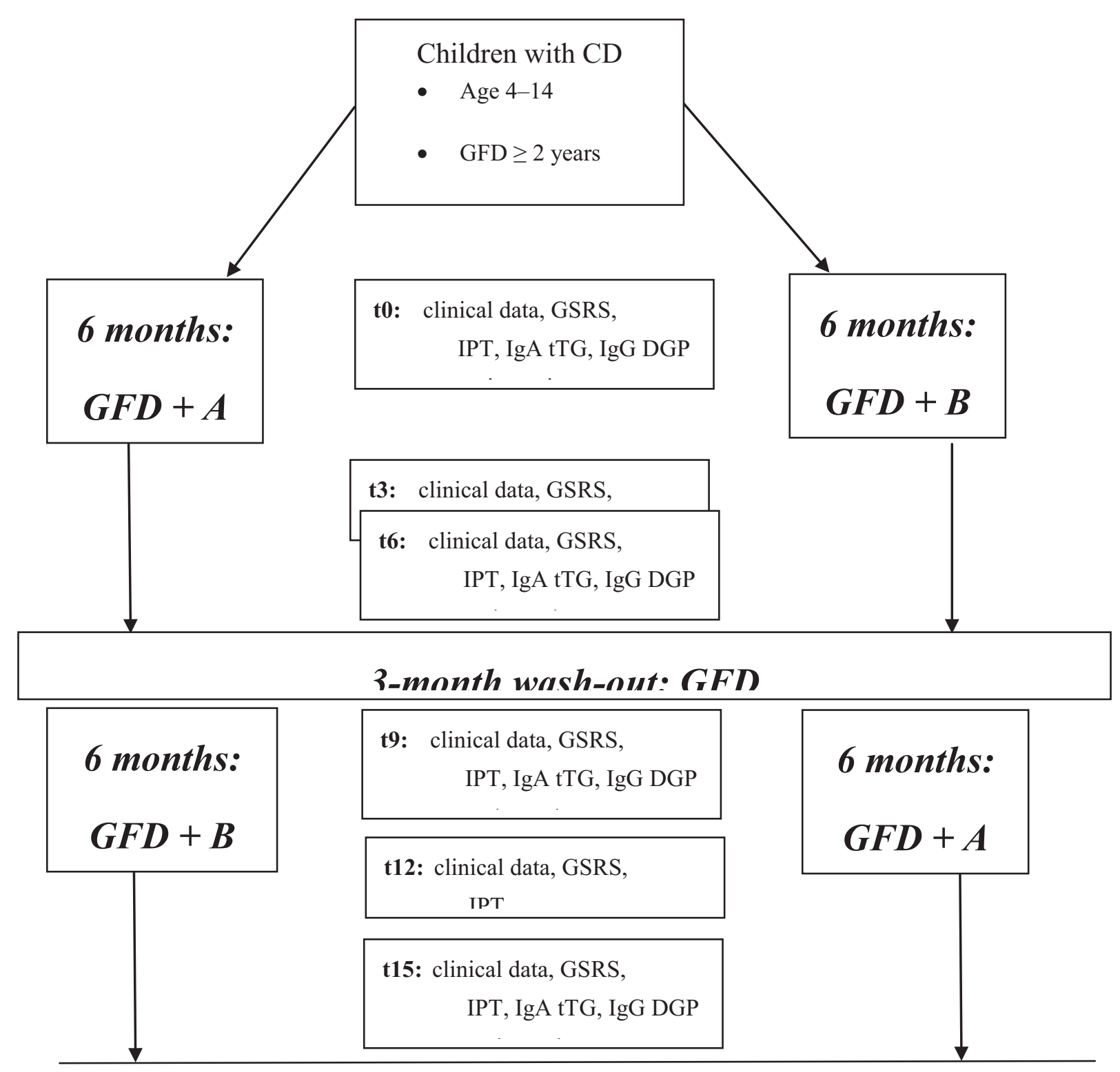

\subsection{Methods}

The occurrence of GI symptoms was monitored through the Gastrointestinal Symptoms Rate Scale (GSRS) at $0,3,6,9,12$ and 15 months.

The IPT was performed as previously described [19]. After an overnight fast and bladder emptying, an oral solution containing $5 \mathrm{~g}$ of lactulose and $2 \mathrm{~g}$ of mannitol was administered. Urine was collected during the following $5 \mathrm{~h}$. An aliquot was preserved at $-20^{\circ} \mathrm{C}$ with sodium azide. 
Urinary excretion of each sugar was assessed using a high performance anion-exchange Chromatography (Dionex DX-500). The ratio of recovered to ingested sugar was reported as ratio of lactulose $\%$ to mannitol\% (L/M). According to our own reference values, a urinary $\mathrm{L} / \mathrm{M}$ ratio $>$ 0.08 was considered abnormal (data not published). All IPTs were performed in the Laboratory of the Department of Pediatrics, Università Politecnica delle Marche, Ancona.

\subsection{Ethics}

Patients and families received appropriate information and informed consent was obtained. The protocol was approved by the Ethical Committee of Università Politecnica delle Marche, Ancona. The trial was registered on www.clinicaltrials.gov (identifier: NCT00808301).

\subsection{Statistical Analysis}

Statistical analyses were performed using GraphPad Prism version 5 (GraphPad Software, San Diego, CA, USA). Non-parametric tests were used because variables were not normally distributed and did not have equal variance. Results are presented as median (interquartile range [IQR]). Differences between groups were assessed by the Mann-Whitney U test. The Wilcoxon matched pairs test was used to calculate the differences for pair data. A $p$ value $<0.05$ was considered significant.

\section{Results}

Overall, 306 children (group A-B: 154, group B-A: 152) were enrolled in the study (median age = 9.62, IQR = 7.23-11.9 years). Patients' enrollment was concluded in March 2013. Fifty-five out of 154 patients enrolled in group A-B (35.7\%) and 42/152 patients in group B-A (27.6\%) dropped out from the study within the first 6 months of treatment $(p=0.14)$. One hundred-seventy one children received at least 6 months of treatment, 75 patients received A treatment (group A), and 96 received B treatment (group B). Clinical and biochemical data from these subgroups were considered for the purpose of this preliminary analysis.

Table 1 summarizes clinical and demographic characteristics of the study-group at enrollment.

Table 1. Clinical and demographic features of children in group A and B at enrollment.

$\mathrm{M}=$ males, $\mathrm{F}=$ females, $\mathrm{IQR}=$ interquartile range.

\begin{tabular}{cccc}
\hline & $\begin{array}{c}\text { Group A } \\
(\boldsymbol{N = 7 5 )}\end{array}$ & $\begin{array}{c}\text { Group B } \\
(\boldsymbol{N}=\mathbf{9 6})\end{array}$ & $\boldsymbol{p}$ \\
\hline Gender distribution (M:F) & $1: 2.5$ & $1: 1.9$ & 0.50 \\
Age at diagnosis Median (IQR) & $3.48(1.98-6.36)$ & $2.84(1.83-6.03)$ & 0.38 \\
Age at enrollment Median (IQR) & $8.76(7.07-11.38)$ & $9.35(7.24-12.01)$ & 0.54 \\
Duration of diet Median (IQR) & $4.25(2.11-6.04)$ & $4.49(1.25-6.55)$ & 0.06 \\
GSRS score at enrollment Median (IQR) & $3(0-5.25)$ & $2(0-4.5)$ & 0.36 \\
L/M at enrollment Median (IQR) & $0.055(0.030-0.083)$ & $0.052(0.026-0.088)$ & 0.62 \\
\hline
\end{tabular}


In both groups, a significant decrease in gastrointestinal symptoms was recorded through the 6-months period (Figure 2), with no differences detected comparing the delta-GSRS score $(\Delta$-GSRS score) between the 2 groups (Figure 3 ).

Figure 2. GSRS score in group A and B at enrollment and after 6 months (median and IQR): in both groups a significant reduction in GI symptoms was observed.

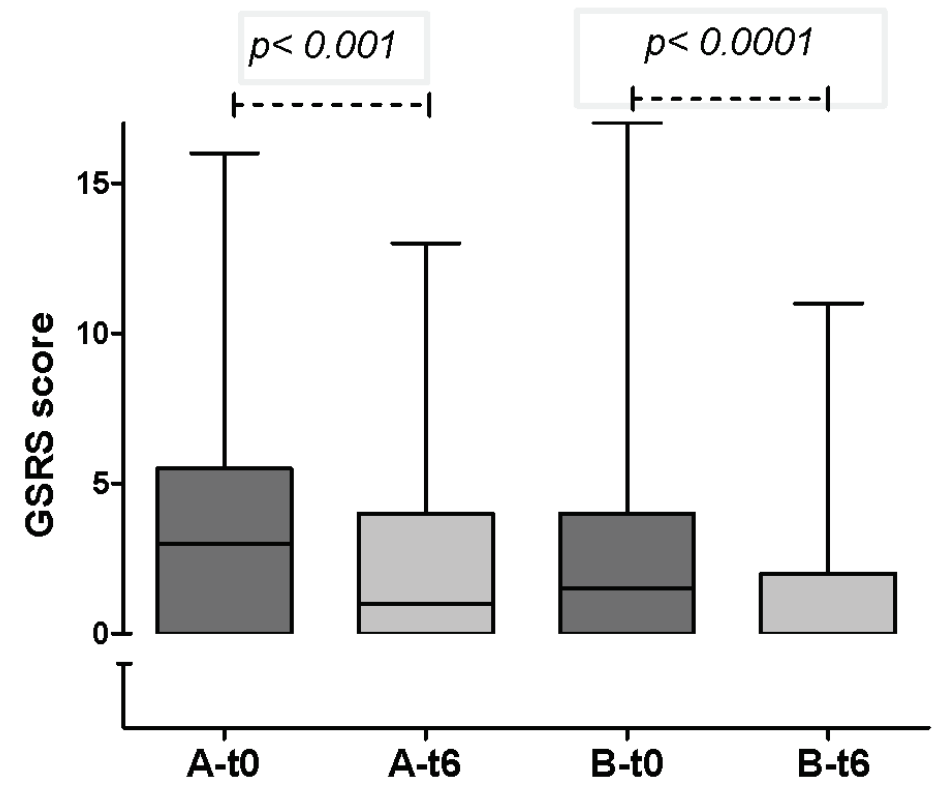

Figure 3. Change in GSRS score ( $\Delta$-GSRS score, median and IQR) between t6 and t0 in the 2 groups.

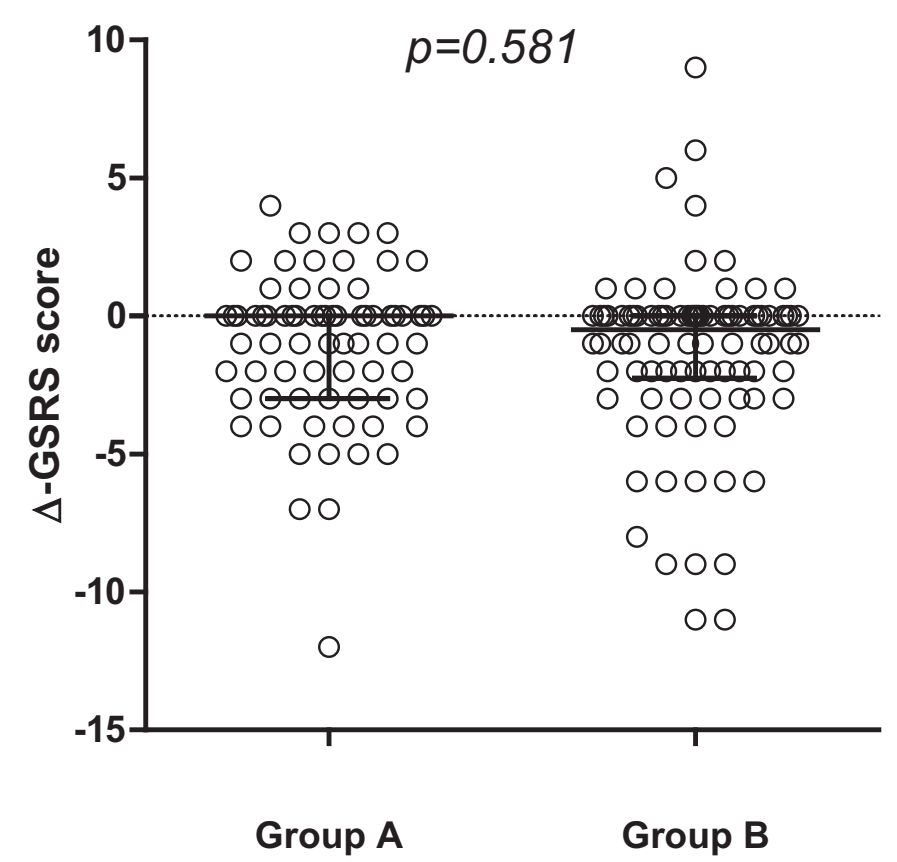

In both $\mathrm{A}$ and $\mathrm{B}$ groups, there was no significant change in urinary $\mathrm{L} / \mathrm{M}$ ratio after 3 (data not shown) and 6 months of treatment (Figure 4). 
Figure 4. Urinary L/M ratio in group $\mathrm{A}$ and $\mathrm{B}$ at $\mathrm{t} 0$ and t6 (median, IQR): in both groups no significant difference was observed after 6 months of treatment.

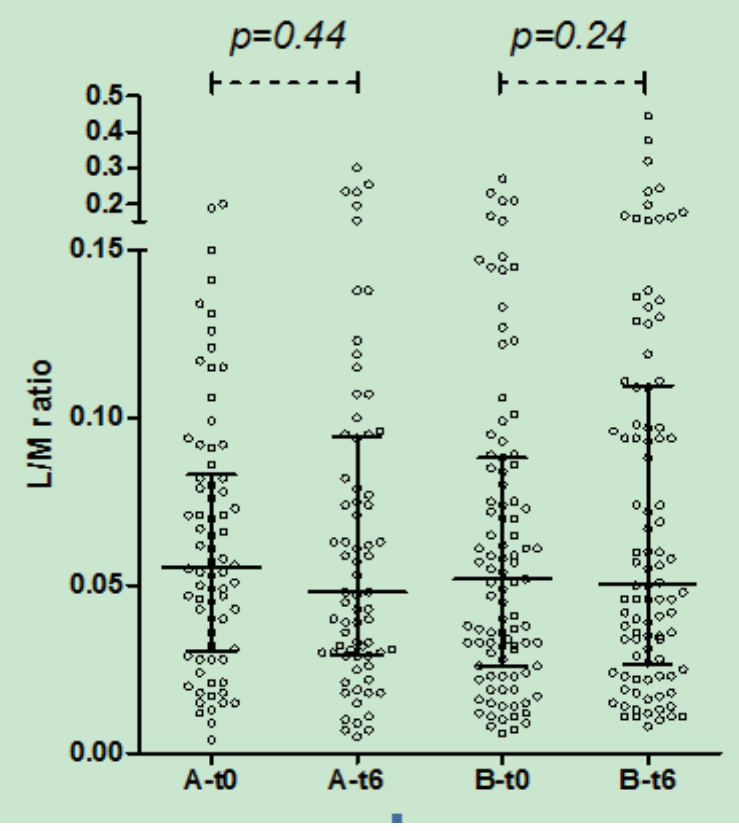

Furthermore, comparing the 6-month change in urinary $\mathrm{L} / \mathrm{M}$ ratio $(\Delta-\mathrm{L} / \mathrm{M})$, no difference was found between groups $\mathrm{A}$ and $\mathrm{B}$, suggesting no relevant impact of a 6-month period of either oats or placebo-added GFD on the intestinal permeability test (Figure 5).

Figure 5. Comparison between $\mathrm{t} 6$ and $\mathrm{t} 0$ urinary $\mathrm{L} / \mathrm{M}$ values in groups $\mathrm{A}$ and $\mathrm{B}(\Delta \mathrm{t} 6-\mathrm{t} 0$, median and IQR).

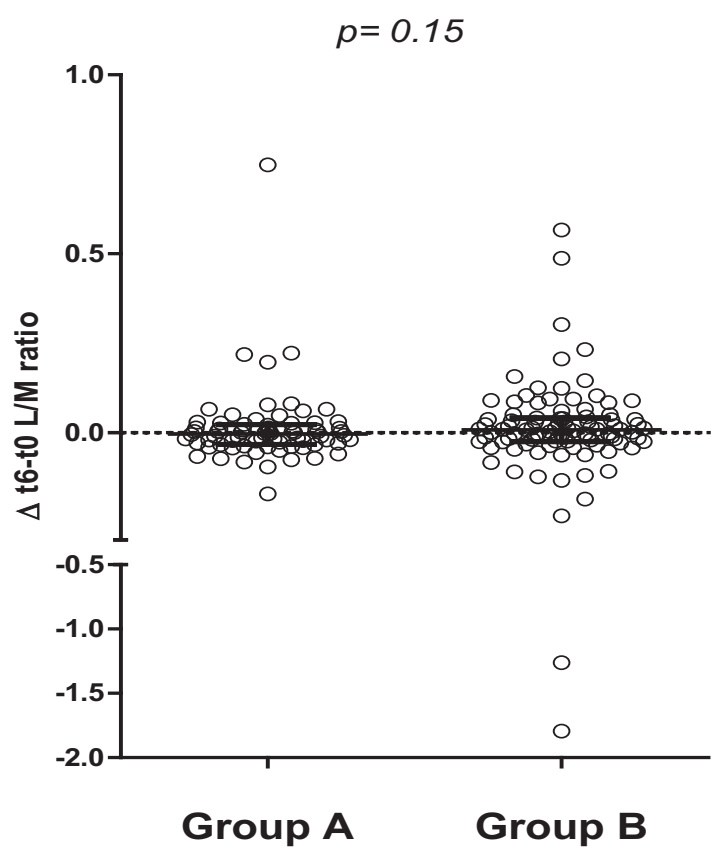

The 6-month $\Delta \mathrm{L} / \mathrm{M}$ value did not show a significant trend according to the different age groups (corresponding to different amount of oats or placebo) (Figure 6a,b). 
Figure 6. Changes in $\mathrm{L} / \mathrm{M}$ values (median, IQR) according to the 3 age classes, in groups A and B, respectively.
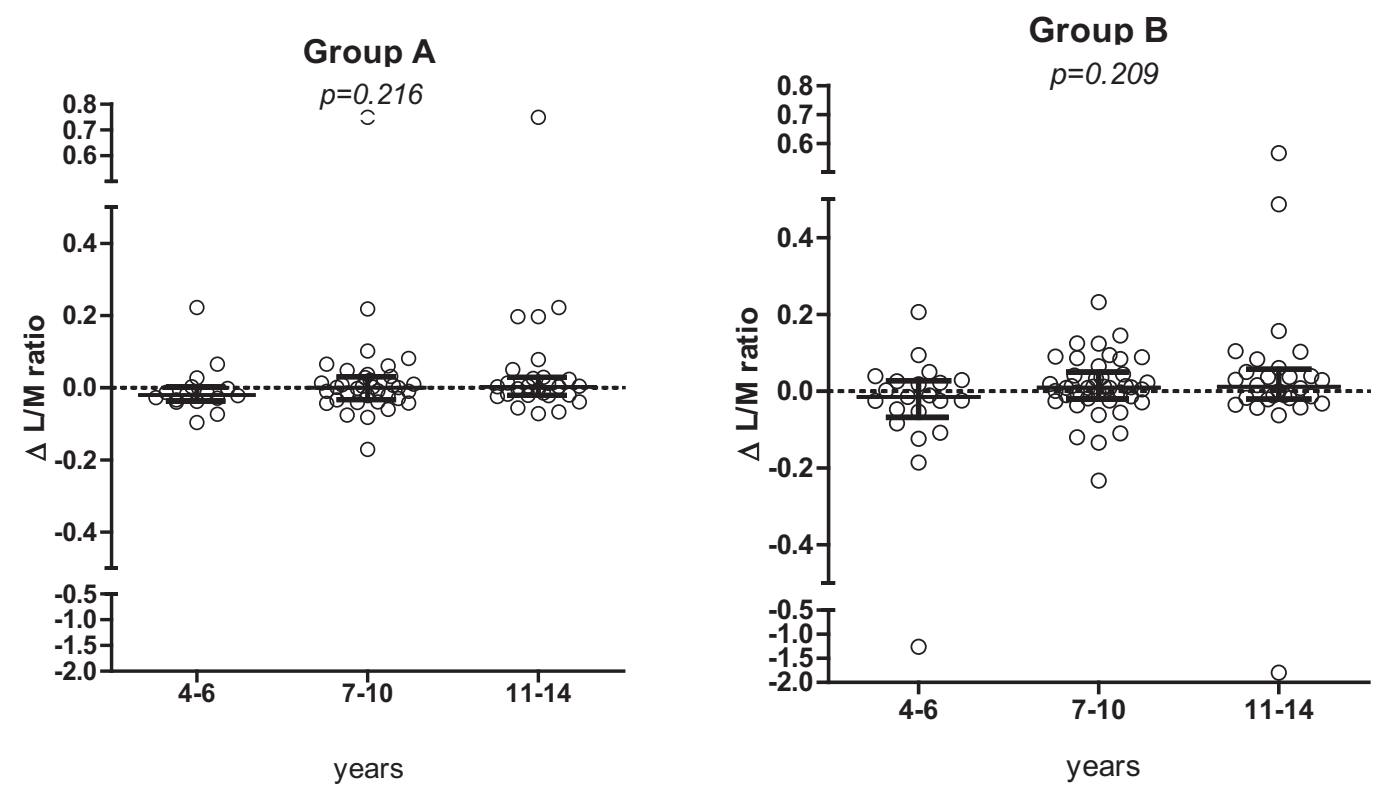

\section{Discussion}

In a large group of $\mathrm{CD}$ children that had not previously consumed oats, we found that the prolonged intake of a considerable amount of daily oats did not determine any relevant change in terms of clinical symptoms and intestinal permeability, even though the results were analyzed in a blind fashion.

Gastrointestinal symptoms were evaluated using the GSRS, that is an interview-based rating scale consisting of 15 items, validated and widely used for assessment of GI symptoms both in adults and children [20-24]. In both groups, there was a significant reduction in GI symptoms during the 6-month period of observation. Such a finding could simply reflect the effect of "being enrolled in a trial." The addition of non-contaminated oats from selected varieties in one of the two groups apparently had no impact on this clinical trend. Dyspeptic symptoms (described in other studies as related to the high amount of fiber in oats) were not recorded in our population. Although the number of dropouts was not significantly different in group A and B during the first 6 months of dietary intervention, the final analysis of this study, including reasons for withdrawal, will clarify the possible impact of oats ingestion on GI symptoms.

Besides a few exceptional cases reported in the literature [25], previous studies showed that the prolonged administration of oats does not induce any mucosal change at the intestinal level. Since performing repeated follow-up small intestinal biopsies was deemed unethical in our study, we evaluated the mucosal integrity by a non-invasive procedure, i.e., the IPT. The integrity of the intestinal barrier can be evaluated by the IPT, by quantifying the amount of orally administered substances recovered in urine. Mannitol (monosaccharide) and lactulose (disaccharide) are largely used as permeability probes due to their different intestinal permeability index. Mannitol is absorbed through enterocyte membrane's pores (transcellular pathway), while lactulose is absorbed through tight junctions (paracellular pathway). Ratio between the percentage of lactulose and 
mannitol excretion represents the permeability index that reflects the intestinal epithelium integrity. The non-invasive functional IPT is a sensitive tool for both triage of active celiac disease, as well as for monitoring celiac patients on a GFD [26]. In patients with active CD, a sugar "paradox" pathway is usually observed: the amount of urinary recovered mannitol is decreased due to the small intestine reduced absorptive surface (villous atrophy), while urinary recovered lactulose is increased because of damage of the TJs and consequent widening of paracellular spaces. For these reasons, an increased excretion disaccharide/monosaccharide ratio is the usual finding associated with CD damage of the small intestinal mucosa. In adults with CD, the IPT has been shown to be more sensitive than serological methods in monitoring patients during follow-up and detecting minimal changes related to gluten ingestion [27,28]. Conversely, the same test was not a reliable instrument to detect asymptomatic CD subjects in a mass screening project [29]. Furthermore, IPT did not perform optimally in two recent trials of larazotide acetate in measuring changes in intestinal permeability after a 14-day [30] or 6-week [31] gluten challenge.

In our study, IPT was chosen as a non-invasive surrogate measure to detect minimal damages of the small intestinal mucosa at different time points. Thus far, no study has been performed to investigate the effects of prolonged oats ingestion on the intestinal permeability test in patients with $\mathrm{CD}$. We did not find significant changes in urinary $\mathrm{L} / \mathrm{M}$ ratio either in group $\mathrm{A}$ or $\mathrm{B}$. This finding is further supported by the comparison of the $\Delta \mathrm{L} / \mathrm{M}$ in the two groups, again failing to show any difference between groups. However, the final results of our large study will give more information about the possible effect of oats ingestion on the results of the intestinal permeability. More data will also be available on the correlation between permeability indexes and serological CD markers.

The daily amount of suggested intake of oats products was calculated to guarantee medium-high amounts of oats (up to $40 \mathrm{~g}$ /day for older children). Although it was not possible to analyze the data according to the amount of ingested oats, the evaluation of the IPT results in the three age groups (corresponding to the different quantity of study products suggested) again showed no significant differences between groups. Overall, the lack of changes in intestinal permeability suggests that the varieties of gluten-uncontaminated oats chosen for production of these oat-based items are safe. The sample analyzed for the purpose of this analysis represents more than $50 \%$ of the enrolled study group. However, this analysis has not considered the original cross-over design of the protocol. Such a limitation implies caution in the interpretation of the results.

\section{Conclusions}

The preliminary results of this investigation suggest that the oats varieties used for producing the gluten-uncontaminated products used in this study are safe when administered for a 6-month period of time. These preliminary conclusions are based on both clinical data and the results of a non-invasive test for intestinal mucosa barrier function (IPT). The final analysis of the data collected in this study will provide conclusive data on the safety and the acceptance of these oat-based products in Italian children in treatment with the GFD. 


\section{Acknowledgments}

The intervention trial "Oats products in the treatment of Coeliac Disesase in children" is sponsored by Heinz Italia S.p.A. (Latina, Italy). The authors wish to express their gratitude to all the members of the Italian Working Group on "Oats for Children with Celiac Disease, including: Michela Grilli (Department of Pediatrics, Università Politecnica delle Marche, Ancona); Lisa Tonelli (Department of Pediatrics, Università Politecnica delle Marche, Ancona); Claudia Fontana (Interdisciplinary Department of Medicine, University of Bari, Bari); Chiara Di Camillo (Department of Pediatrics, "Sapienza" University of Rome, Roma); Stefania Leoni (Department of Pediatrics, "Sapienza" University of Rome, Roma); Roberto Panceri (Department of Pediatrics, Ospedale di Monza, Monza); Tiziana Passaro (Department of Pediatrics, S. Maria dell'Olmo Hospital Cava de' Tirreni, Salerno); Sara Manferdelli (R\&D Heinz Italia S.p.A, Latina); Alessia Grinzato (R\&D Heinz Italia S.p.A, Latina); Flavia Carle (Department of Epidemiology, Biostatistics and Medical Information Technology, Università Politecnica delle Marche, Ancona); Mario La Rosa (Department of Pediatrics, Università di Catania, Catania).

\section{Conflicts of Interest}

Carlo Catassi received consultAing fees from Heinz, Schär and Menarini Diagnostics. Elena Lionetti received consulting fees from Heinz. Andrea Budelli is an employee of Heinz.

\section{References}

1. Janatuinen, E.K.; Pikkarainen, P.H.; Kamppainen, T.A.; Kosma, V.M.; Jarvinen, R.M.; Uusitupa, M.I.; Julkunen, R.J. A comparison of diets with and without oats in adults with celiac disease. N. Engl. J. Med. 1995, 333, 1033-1037.

2. Srinivasan, U.; Leonard, N.; Jones, E.; Kasarda, D.D.; Weir, D.G.; O’Farrelly, C.; Feighery, C. Absence of oats toxicity in adult coeliac disease. BMJ 1996, 313, 1300-1301.

3. Hardman, C.M.; Gariogh, J.J.; Leonard, J.N.; Thomas, H.J.; Walker, M.M.; Lortan, J.E.; Lister, A.; Fry, L. Absence of toxicity of oats in patients with dermatitis herpetiformis. N. Engl. J. Med. 1997, 337, 1884-1887.

4. Reunala, T.; Collin, P.; Holm, K.; Pikkarainen, P.; Miettinen, A.; Vuolteenaho, N.; Mäki, M. Tolerance to oats in dermatitis herpetiformis. Gut 1998, 43, 490-493.

5. Hoffenberg, E.J.; Haas, J.; Drescher, A.; Bamhurst, R.; Osberg, I.; Bao, F. A trial of oats in children with newly diagnosed celiac disease. J. Pediatr. 2000, 137, 361-366.

6. Janatuinen, E.K.; Kemppainen, T.H.; Julkunen, R.J.; Kosma, V.M.; Maki, M.; Heikkinen, M.; Uusitupa, M.I. No harm from five year ingestion of oats in coeliac disease. Gut 2002, 50, 332-335.

7. Storsrud, S.; Olsson, M.; Arvidsson Lenner, M.; Nilsson, L.A.; Nilsson, O.; Kilander, A. Adult coeliac patients do tolerate large amounts of oats. Eur. J. Clin. Nutr. 2003, 57, 163-169.

8. Högberg, L.; Laurin, P.; Falth-Magnusson, K.; Grant, C.; Grodzinsky, E.; Jansson, G.; Ascher, H.; Browaldh, L.; Hammersjö, J.A.; Lindberg, E.; et al. Oats to children with newly diagnosed coeliac disease: A randomised double blind study. Gut 2004, 53, 649-654. 
9. Holm, K.; Maki, M.; Vuolteenaho, N.; Mustalahti, K.; Ashorn, M.; Ruuska, T.; Kaukinen, K. Oats in the treatment of childhood coeliac disease: A 2-year controlled trial and a long-term clinical follow-up study. Aliment. Pharmacol. Ther. 2006, 23, 1463-1472.

10. Sey, M.S.; Parfitt, J.; Gregor, J. Prospective study of clinical and histological safety of pure and uncontaminated Canadian oats in the management of celiac disease. J. Parenter. Enteral. Nutr. 2011, 35, 459-464.

11. Cooper, S.E.; Kennedy, N.P.; Mohamed, B.M.; Abuzakouk, M.; Dunne, J.; Byrne, G.; McDonald, G.; Davies, A.; Edwards, C.; Kelly, J.; et al. Immunological indicators of coeliac disease activity are not altered by long-term oats challenge. Clin. Exp. Immunol. 2013, 171, 313-318.

12. Silano, M.; di Benedetto, R.; Maialetti, F.; De Vincenzi, A.; Calcaterra, R.; Cornell, H.J.; de Vincenzi, M. Avenins from different cultivars of oats elicit response by coeliac peripheral lymphocytes. Scand. J. Gastroenterol. 2007, 42, 1302-1305.

13. Ballabio, C.; Uberti, F.; Manferdelli, S.; Vacca, E.; Boggini, G.; Redaelli, R.; Catassi, C.; Lionetti, E.; Penãs, E.; Restani, P. Molecular characterisation of 36 oat varieties and in vitro assessment of their suitability for coeliacs' diet. J. Cereal Sci. 2011, 54, 110-115.

14. Comino, I.; Real, A.; de Lorenzo, L.; Cornell, H.; López-Casado, M.Á.; Barro, F.; Lorite, P.; Torres, M.I.; Cebolla, A.; Sousa, C. Diversity in oat potential immunogenicity: Basis for the selection of oat varieties with no toxicity in coeliac disease. Gut 2011, 60, 915-922.

15. Real, A.; Comino, I.; de Lorenzo, L.; Merchán, F.; Gil-Humanes, J.; Giménez, M.J.; López-Casado, M.Á.; Torres, M.I.; Cebolla, Á.; Sousa, C.; et al. Molecular and immunological characterization of gluten proteins isolated from oat cultivars that differ in toxicity for celiac disease. PLoS One 2012, 7, e48365.

16. Mujico, J.R.; Mitea, C.; Gilissen, L.J.W.J.; de Ru, A.; van Veelen, P.; Smulders, M.J.M.; Koning, F. Natural variation in avenin epitopes among oat varieties: Implications for celiac disease. J. Cereal Sci. 2011, 54, 8-12.

17. Rashid, M.; Butzner, D.; Burrows, V.; Zarkadas, M.; Case, S.; Molloy, M.; Warren, R.; Pulido, O.; Switzer, C. Consumption of pure oats by individuals with celiac disease: A position statement by the Canadian Celiac Association. Can. J. Gastroenterol. 2007, 21, 649-651.

18. American Dietetic Association. Evidence-based nutrition practice guidelines on celiac disease. Available online: http://www.guideline.gov/content (accessed on 17 November 2013).

19. Generoso, M.; de Rosa, M.; de Rosa, R.; de Magistris, L.; Secondulfo, M.; Fiandra, R.; Carratù, R.; Cartenì, M.J. Cellobiose and lactulose coupled with mannitol and determined using ion-exchange chromatography with pulsed amperometric detection, are reliable probes for investigation of intestinal permeability. J. Chromatogr. B Analyt. Technol. Biomed. Life Sci. 2003, 783, 349-357.

20. Dimenas, E.; Glise, H.; Hallerback, B.; Hernqvist, H.; Svedlund, J.; Wiklund, I. Well-being and gastrointestinal symptoms among patients referred to endoscopy owing to suspected duodenal ulcer. Scand. J. Gastroenterol. 1995, 30, 1046-1052. 
21. Dimenas, E.; Carlsson, G.; Glise, H.; Israelsson, B.; Wiklund, I. Relevance of norm values as part of the documentation of quality of life instruments for use in upper gastrointestinal disease. Scand. J. Gastroenterol. Suppl. 1996, 221, 8-13.

22. Lohiniemi, S.; Mäki, M.; Kaukinen, K.; Laippala, P.; Collin, P. Gastrointestinal symptoms rating scale in coeliac disease patients on wheat starch-based gluten-free diets. Scand. J. Gastroenterol. 2000, 35, 947-949.

23. Hallert, C.; Grännö, C.; Grant, C.; Hultén, S.; Midhagen, G.; Ström, M.; Svensson, H.; Valdimarsson, T.; Wickström, T. Quality of life of adult coeliac patients treated for 10 years. Scand. J. Gastroenterol. 1998, 33, 933-938.

24. Thomson, M.; Fritscher-Ravens, A.; Hall, S.; Afzal, N.; Ashwood, P.; Swain, C.P. Endoluminal gastroplication in children with significant gastro-oesophageal reflux disease. Gut 2004, 53, 1745-1750.

25. Arentz-Hansen, H.; Fleckenstein, B.; Molberg, Ø.; Scott, H.; Koning, F.; Jung, G.; Roepstorff, P.; Lundin, K.E.; Sollid, L.M. The molecular basis for oat intolerance in patients with celiac disease. PLoS Med. 2004, 1, e1.

26. Vogelsang, H.; Schwarzenhofer, M.; Oberhuber, G. Changes in gastrointestinal permeability in celiac disease. Dig. Dis. 1998, 16, 333-336.

27. Duerksen, D.R.; Wilhelm-Boyles, C.; Parry, D.M. Intestinal permeability in long-term follow-up of patients with celiac disease on a gluten-free diet. Dig. Dis. Sci. 2005, 50, 85-90.

28. Vilela, E.G.; Ferrari, M.L.; de Gama Torres, H.O.; Martins, F.P.; Goulart, E.M.; Lima, A.S.; da Cunha, A.S. Intestinal permeability and antigliadin antibody test for monitoring adult patients with celiac disease. Dig. Dis. Sci. 2007, 52, 1304-1309.

29. Catassi, C.; Fabiani, E.; Rätsch, I.M.; Bonucci, A.; Dotti, M.; Coppa, G.V.; Giorgi, P.L. Is the sugar intestinal permeability test a reliable investigation for coeliac disease screening? Gut 1997, 40, 215-217.

30. Kelly, C.P.; Green, P.H.; Murray, J.A.; Dimarino, A.; Colatrella, A.; Leffler, D.A.; Alexander, T.; Arsenescu, R.; Leon, F.; Jiang, J.G.; et al. Larazotide Acetate in patients with celiac disease undergoing a gluten-challenge: A randomized placebo-controlled study. Aliment. Pharmacol. Ther. 2013, 37, 252-262.

31. Leffler, D.A.; Kelly, C.P.; Abdallah, H.Z.; Colatrella, A.M.; Harris, L.A.; Leon, F.; Arterburn, L.A.; Paterson, B.M.; Lan, Z.H.; Murray, J.A. A randomized, double-blind study of larazotide acetate to prevent the activation of celiac disease during gluten challenge. Am. J. Gastroenterol. 2012, 107, 1554-1562. 
MDPI AG

Klybeckstrasse 64

4057 Basel, Switzerland

Tel. +41616837734

Fax +41613028918

http://www.mdpi.com/

Nutrients Editorial Office

E-mail: nutrients@mdpi.com

http://www.mdpi.com/journal/nutrients 

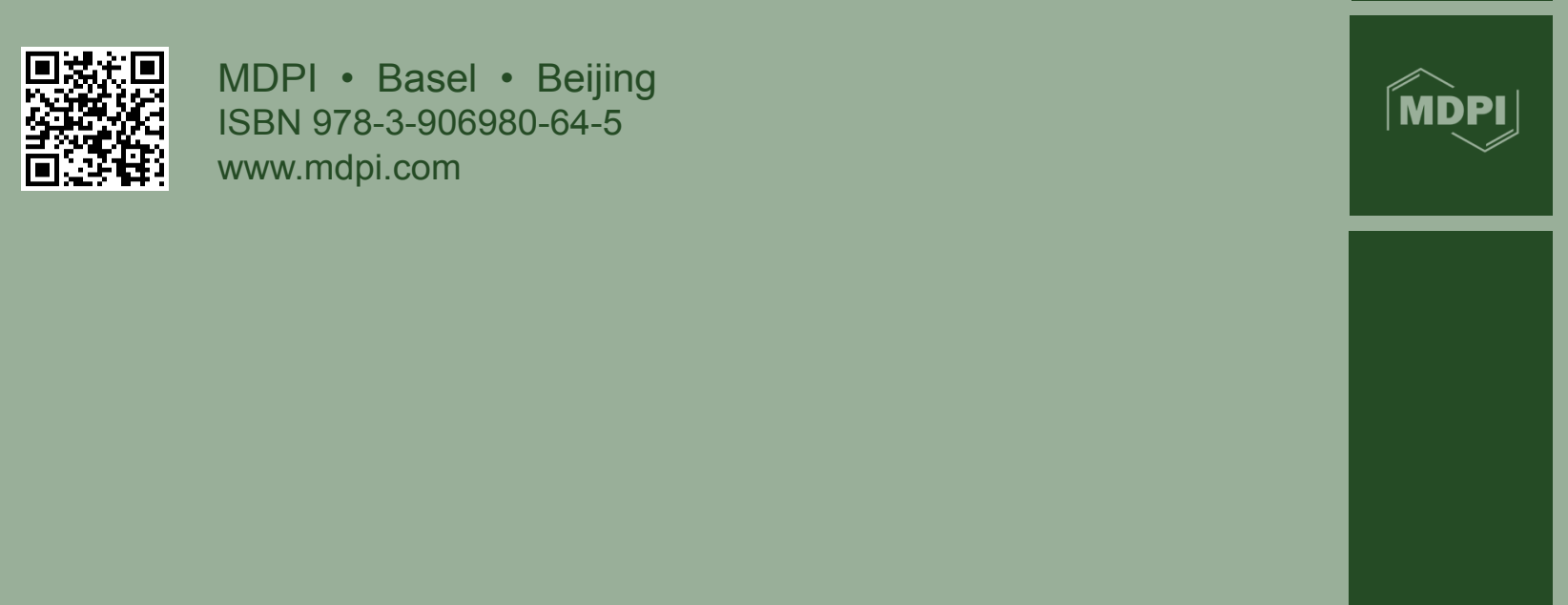